\title{
Beratung im Kontext des Lebenslangen Lernens
}

Konzepte, Organisation, Politik, Spannungsfelder

Olaf Dörner, Carola Iller, Ingeborg Schüßler, Cornelia Maier-Gutheil, Christiane Schiersmann (Hrsg.)

Schriftenreihe der Sektion Erwachsenenbildung der Deutschen Gesellschaft für Erziehungswissenschaft 
Schriftenreihe der Sektion Erwachsenenbildung in der Deutschen Gesellschaft für

Erziehungswissenschaft (DGfE) 
Olaf Dörner

Carola Iller

Ingeborg Schüßler

Cornelia Maier-Gutheil

Christiane Schiersmann (Hrsg.)

Beratung im Kontext

des Lebenslangen Lernens

Konzepte, Organisation,

Politik, Spannungsfelder

Verlag Barbara Budrich

Opladen • Berlin • Toronto 2019 
Bibliografische Information der Deutschen Nationalbibliothek

Die Deutsche Nationalbibliothek verzeichnet diese Publikation in der Deutschen Nationalbibliografie; detaillierte bibliografische Daten sind im Internet über http://dnb.d-nb.de abrufbar.

(C) 2019 Dieses Werk ist beim Verlag Budrich UniPress erschienen und steht unter der Creative Commons Lizenz Attribution-ShareAlike 4.0 International (CC BY-SA 4.0): https://creativecommons.org/licenses/by-sa/4.0/

Diese Lizenz erlaubt die Verbreitung, Speicherung, Vervielfältigung und Bearbeitung bei Verwendung der gleichen CC-BY-SA 4.0-Lizenz und unter Angabe der UrheberInnen, Rechte, Änderungen und verwendeten Lizenz.

\section{(c) (i) (2)}

Dieses Buch steht im Open-Access-Bereich der Verlagsseite zum kostenlosen Download bereit (https://doi.org/10.3224/84742204).

Eine kostenpflichtige Druckversion kann über den Verlag bezogen werden. Die Seitenzahlen in der Druck- und Onlineversion sind identisch.

$\begin{array}{ll}\text { ISBN } & 978-3-8474-2204-4 \text { (Paperback) } \\ \text { eISBN } & 978-3-8474-1230-4 \text { (PDF) } \\ \text { DOI } & 10.3224 / 84742204\end{array}$

Lektorat: Dr. Andrea Lassalle, Berlin - andrealassalle.de; Alison Romer, Lancaster, UK

Satz: Birgit Cirksena, Berlin - info@satzfein.de

Umschlaggestaltung: Bettina Lehfeldt, Kleinmachnow - www.lehfeldtgraphic.de Druck: Paper \& Tinta, Warschau 


\section{Inhalt}

Cornelia Maier-Gutheil/Christiane Schiersmann/Carola Iller/

Olaf Dörner/Inge Schüßler

Beratung im Kontext des Lebenslangen Lernens: Konzepte,

Organisation, Politik, Spannungsfelder - eine Einführung

Bernd Käpplinger

Beratung im Rampenlicht - Von Sisyphos zu Prometheus?

\section{Theoretische Zugänge zum Beratungshandeln: Verortung und Diskurse}

Jana Wienberg

Eine resonanztheoretische Betrachtung von Beratung 35

Julia Franz/Stephanie Welser/Annette Scheunpflug

Lernberatung im Diskurs der Erwachsenenbildung:

Eine systematische Reflexion

\section{Mediale Beratungsformate: Vergleiche - Spezifika - Intentionen}

Tim Stanik/Cornelia Maier-Gutheil

Computervermittelte, asynchrone Beratungen in der Weiterbildung im Spannungsfeld von Anliegen und Prozessierungstransfer

Sebastian Zick

Bildung, Beratung, Bewegung - Die Steuerung der Bildungsreise zwischen Apodemik und europäischer Mobilitätspolitik

\section{Beratung in der wissenschaftlichen Weiterbildung: Strukturen - Praxis - Herausforderungen}

Nico Sturm/Heike Rundnagel Optimierung von Beratungsstrukturen für nicht-traditionell Studieninteressierte in der wissenschaftlichen Weiterbildung am Beispiel von Studiengangkoordinierenden auf Programmebene 
Christoph Damm

Anrechnungsberatung als Möglichkeit der Öffnung von Hochschulen?

Struktur und Praxis der Anrechnungsberatung in der

wissenschaftlichen Weiterbildung

Katharina Koller

Beratung, Betreuung, wissenschaftliche Weiterbildung:

Herausforderung angenommen!?

\section{Beratungskompetenz und Professionalisierung}

Christiane Schiersmann/Peter Weber

Instrumente zur Erfassung der Kompetenzen von Beratenden im

Feld Bildung, Beruf und Beschäftigung - Konzept und Erprobung

Marc Weinhardt

Beratungskompetenzerwerb im Studium: Lern- und Bildungsprozesse

im Horizont subjektorientierter Professionalisierung

Johannes Wahl

Beratung zwischen biographischen Negativerfahrungen und offener biographischer Zukunft. Eine komparative Sicht auf pädagogische

Arbeitsfelder

Birgit Schmidtke/Roland Lehner

Das Pilotprojekt Bildungsberatungs-Radar - Bildungsbarrieren

sichtbar machen.

\section{Beratung in unterschiedlichen Alters- und Lebensphasen: Bedarfe - Anlässe - Übergänge}

Simone Hocke

Beratung in beruflichen Übergängen als Reflexionsraum für

und Initiierung von Lernprozessen

Caroline Kruse

„Bildung, das ist unser Auftrag!“ - Beratung im Freiwilligen Sozialen Jahr im Spannungsfeld zwischen außerschulischen Bildungszielen und psychosozialen Problemlagen 
Heike Chyle/Nina Wlassow/Christiane Dittrich

Ein ethnografischer Blick auf Materialität in der Beratung am Beispiel

von Coaching und Transfergesellschaft

Franziska Schulze-Stocker/Christian Schäfer-Hock/Robert Pelz

Intervention in den Studienverlauf und Beratung

221

\section{Gesprächsanalytische Zugänge zu Beratungsinteraktionen: Handlungstypik und Interaktionsmodi}

Farina Wagner

Subjektivierung in der Weiterbildungsberatung -

Beratungsgespräche in erzählanalytischer Perspektive

Christian Schröder/Claudia Muche

Professionalität im Coaching von Führungskräften:

Der organisationale Rahmen als konstituierende Prämisse

professionellen Handelns

\section{Beratung in organisationalen Kontexten: Lernen - Steuerung - Methoden}

Henning Pätzold

Beratung im Kontext organisationalen Lernens

Hildegard Macha

Das Konzept der transformativen Beratung in Unternehmen

und seine wissenschaftliche Evaluation

Kira Nierobisch

Beratung im Kontext Hochschule. Eigen- und Steuerungslogiken

zwischen Emanzipation und Entmündigung?

Shahinaz Khalil

Impact? Whose Impact? A Youth Career Guidance Program's effects on Parents, Teachers and Staff

Autorinnen und Autoren 

Cornelia Maier-Gutheil/Christiane Schiersmann/Carola Iller/ Olaf Dörner/Inge Schüßler

\section{Beratung im Kontext des Lebenslangen Lernens: Konzepte, Organisation, Politik, Spannungsfelder - eine Einführung}

Beratung ist ein Handlungsphänomen, das anthropologisch betrachtet recht alt ist, in transitiver (jemanden beraten) und reflexiver (sich beraten) Form wie auch gleichermaßen in alltagsweltlichen Zusammenhängen und professionellen Settings vorkommt und sich als Dienstleistung an einzelne Personen, Gruppen, und Organisationen (in Wirtschaft, Politik, Kultur u.Ä.) richtet (vgl. Gieseke/Nittel 2016).

Als eine der Grundformen pädagogischen Handelns (vgl. Giesecke 2000, 87 ff.) steht sie bereits seit den 1960er Jahren - korrespondierend mit einer ersten Welle des institutionellen Ausbaus - im Fokus erwachsenenbildnerischer Forschungsbemühungen und Erkenntnisinteressen. Ab Mitte der 1990er Jahre finden im Zuge des so genannten Beratungsbooms Diversifizierungen und Erosionsprozesse statt, die den Blick vor allem auf die Professionalisierung im Feld der Beratung lenken (vgl. Käpplinger/Maier-Gutheil 2015). Entsprechende Versuche, die Wissenschaft der Beratung weiterzuentwickeln, haben nicht nur die Einrichtung entsprechender Studiengänge zur Folge, sondern befördern insbesondere Fragen nach der Qualität von Beratung (vgl. Schiersmann/Weber 2013), den Möglichkeiten und Bedingungen, Beratungskompetenzen zu entwickeln (vgl. Weinhardt 2015; Weinhardt 2018) sowie den Wirkfaktoren im Kontext von Beratungsinteraktionen (vgl. Schiersmann/ Maier-Gutheil/Weber 2016).

Diese Entwicklungen stehen im paradigmatischen Kontext des Lebenslangen Lernens wodurch Beratung in modernen Gesellschaften in unterschiedlicher Weise bedeutsam wird. Seitens gesellschaftspolitischer Akteure wird Beratung einerseits als Steuerungsinstrument eingesetzt, etwa im Zusammenhang mit neoliberalen Entwicklungen (z.B. wenn Beratung ein verpflichtendes Element bei der Vergabe öffentlicher Fördergelder darstellt). Andererseits gibt es entsprechende (Beratungs-)Angebote, die diesen Tendenzen etwas entgegen zu setzen versuchen (z.B. Beratung im Kontext von Antifa-Arbeit bzw. Angebote für „Aussteiger"). Für Individuen ist mit Beratung prinzipiell die Möglichkeit verbunden, in einem von vielfältigen Anforderungen geprägten Leben, das sich im Spannungsfeld von Chancen und Zumutungen bewegt, Unterstützungsgelegenheiten für lebenslaufrelevante 
Entscheidungen zu finden. Auch hinsichtlich der Beratung in Bildung, Beruf, Beschäftigung hat sich das Feld weiter ausdifferenziert. So gibt es eigenständige professionelle Angebote z.B. bei der Karriereberatung, bei der Beratung bestimmter Zielgruppen (z. B. Frauen, Rehabilitand*innen, Migrant*innen), teilweise ist Beratung als Teilangebot von Weiterbildungseinrichtungen oder anderen öffentlich-rechtlichen Trägern verankert (z. B. Agenturen für Arbeit, Industrie- und Handelskammern). Schließlich weist auch die Lernberatung als zentraler Bestandteil pädagogischen Handelns eine lange Tradition auf. Darüber hinaus adressiert Beratung, neben Personen auch Organisationen (z. B. Qualifizierungs-, Politikberatung) und es zeigen sich interessante neue Entwicklungsperspektiven - auch über Grundbildungskontexte hinaus. Neben der klassischen persönlichen Beratung entwickeln sich neue Formate sowohl der aufsuchenden als auch der technologiegestützten Beratung (z. B. Telefon- bzw. Onlineberatung). Und nicht zuletzt fungieren Akteur*innen im unmittelbaren Lebensumfeld von Erwachsenen (z. B. Freund*innen, Kolleg*innen, Vorgesetzte, Familie, Vereine) als Berater*innen in Erwachsenenbildungs- und Weiterbildungsangelegenheiten, die nicht unwesentlich für die Inanspruchnahme und Nichtinanspruchnahme von institutionellen Beratungsangeboten relevant sind.

Die Jahrestagung der Sektion Erwachsenenbildung der Deutschen Gesellschaft für Erziehungswissenschaft (DGfE), die 2017 in Heidelberg stattfand, fokussierte die wechselseitigen Bezüge der Themen Beratung - Organisation - Politik im Kontext der Erwachsenenbildung, in denen sich die genannten Entwicklungen vollziehen. Das Feld Beratung in der Erwachsenenbildung wird dabei aus den unterschiedlichen und zum Teil widersprüchlichen disziplinären, institutionellen, (organisationalen), gesellschaftlichen und bildungspolitischen Perspektiven und damit verbundenen Spannungsfeldern beleuchtet.

- Im Anschluss an den Grundgedanken, Bildungsmöglichkeiten zu eröffnen, gehört Beraten als Bestandteil pädagogischen Handelns zu den zentralen Handlungsformen neben solchen wie Informieren, Unterrichten, Arrangieren. In den Blick kommen gleichermaßen Settings, in denen Beraten die Kernaktivität darstellt wie auch solche, bei denen Beraten als eine Teilaktivität (und vielfach parallel) neben anderen Aktivitäten zu beobachten ist. Gerade im Kontext der Unterstützung, Begleitung und Ermöglichung von Lern- und Bildungsprozessen zeigen sich Bedarfe für Beratung. Genannt seien hier unterschiedliche Ausprägungen/Varianten von Lernberatung, etwa im Rahmen selbstgesteuerter Lernprozesse, der didaktischen Gestaltung und Nutzung von Lernumgebungen (Stichwort: Selbstlernarchitekturen) oder auch der Dezentralisierung von Lernorten. Von Interesse sind auch Mikroanalysen, die der Prozesshaftigkeit von Beratung gerecht werden. Fragen richten sich darauf, wie die Relation von 
Beraten und anderen pädagogischen Handlungsformen in unterschiedlichen Settings ausgestaltet ist, welche Spannungsfelder sich möglicherweise ergeben (z. B. mit diagnostizierenden Aktivitäten) oder auch, welche Rolle Beraten in pädagogischen Handlungsfeldern vor dem Hintergrund der Verbreitung ökonomischer Orientierungen und Maßstäbe spielt.

- Beratung stellt eine anspruchsvolle soziale Dienstleistung dar. Wie bei vergleichbaren Berufen findet auch hier ein Professionalisierungsdiskurs statt. Dabei werden verschiedene Ebenen angesprochen: Kompetenzen als Kern der Professionalität müssen theoretisch begründet und empirisch validiert werden. Mit Blick auf (vorhandene oder zukünftige) Aus- und Weiterbildungsmöglichkeiten rückt auch die Frage nach spezifischen (Teil-) Curricula für das Beratungshandeln bzw. deren Integration in bestehende Curricula von Weiterbildungs- bzw. erziehungswissenschaftlichen Studiengängen in den Fokus. Darüber hinaus stellt sich die Frage nach weiteren Professionalisierungsprozessen, etwa durch Verbände, Publikationen oder Standards. Schließlich kommen Wechselwirkungen zwischen unterschiedlichen Systemebenen in den Blick (z. B. Weiterbildungsorganisationen als Adressat*innen von Organisationsberatung).

- Permanenter Wandel in der Folge von Technologisierung und damit verbundene Veränderungsprozesse/-notwendigkeiten betreffen auch die (Weiter-)Entwicklung von Beratungsformaten und -settings. Dies betrifft nicht nur medial vermittelte Formen (Telefon- bzw. Online-Beratung), sondern auch neuere Handlungsformate wie Gruppenberatung und Coaching. Zentral werden Fragen danach, ob und inwiefern sich die technologiebasierten Veränderungen auf Struktur- und Ablaufmuster, Anforderungen an Rolle und Funktion seitens der Beratenden wie auch der Ratsuchenden auswirken. Damit einher geht auch die Frage nach Möglichkeiten zum Abbau von Zugangshindernissen für pädagogische Beratung wie auch die Nutzung entsprechender Erkenntnisse für andere Akteursebenen.

- Der Blick richtet sich zudem auch auf die große und heterogene Gruppe der potenziellen Ratsuchenden. Unterschiede beziehen sich auf Beratungsanlässe, Teilnahmemotive wie auch etwaige Barrieren, beispielsweise mit Blick auf neue Zielgruppen im Kontext globaler Migrationsbewegungen oder Personen, die zur Gruppe der so genannten Bildungsfernen gezählt werden können. Hier werden wissenschaftlich tragfähige und nutzbare Modelle der Systematisierung von Zielgruppen der Beratung relevant. Ebenfalls mit Blick auf Adressat*innen ist danach zu fragen, inwieweit welche Erwachsenen überhaupt Beratungsangebote von Erwachsenen-/ Weiterbildungsanbietern oder anderen Einrichtungen (Organisationen) in Anspruch nehmen und welche Beratungsangebote für Weiterbildungsaktivitäten bedeutsam sind.

- Hinsichtlich von Beratung als eigenständigem, institutionalisiertem Angebot bzw. als Teilaufgabe von Weiterbildungsanbietern geht es einerseits 
um ein spezifisches trägerübergreifendes Verständnis von Beratung wie auch die Struktur von Beratungsanbietern im Feld Bildung, Beruf und Beschäftigung. Hervorzuheben sind in vielen Fällen die prekären finanziellen und institutionellen Rahmenbedingungen der Beratungsanbieter (z. B. Projektförmigkeit). Andererseits ergeben sich vielfältige Herausforderungen für die Ausgestaltung im Zusammenhang mit Beratung in betrieblichen Kontexten, etwa im Rahmen von Personalentwicklung/Human Ressource Management oder Organisationsentwicklung. Es differenzieren sich auch institutionalisierte Beratungsfelder aus, wie zum Beispiel in der Hochschulberatung, wo neben der klassischen Studienberatung durch den Bologna-Prozess und „heterogene(re)n“ Studierenden solche Felder wie Übergangs- und Laufbahnberatung, Lernberatung und Mentoring oder Organisationsberatung im Zuge von Akkreditierungs- und Qualitätsentwicklungsprozessen hinzutreten.

- Mit Blick auf die politischen Ebenen ist Beratung insbesondere im Kontext der Politik Lebenslangen Lernens bedeutsam. Aus europäischer wie auch länderspezifischer Perspektive sind Fragen der Wirkung von Beratung sowie diesbezüglich geeignete Forschungsdesigns (Input-, Prozess-, Output-, Outcome-Dimensionen) virulent. Im Zusammenhang mit (bildungs-) politischen Steuerungsmechanismen kommen die Folgen in den Blick, die sich etwa auf der Ebene der Organisation auswirken können (Stichworte: Orientierung an wirtschaftlichen Effizienzkriterien und unklare Finanzierungsmodelle). Das Spannungsfeld zwischen Individualisierung und Ökonomisierung kann aber auch auf der Interaktionsebene Einfluss nehmen, etwa hinsichtlich der (theoretischen) Fundierung von Beratung an Aufklärung, Mündigkeit und Autonomisierung. Hier stellen sich Fragen nach der gesellschaftlichen Verortung von Beratung, ihrer Rolle und Funktion(en), die im Kontext gesellschaftskritisch-reflexiver Zugänge diskutiert werden könnten.

Der vorliegende Tagungsband beleuchtet die genannten Perspektiven und Spannungsfelder in 22 Beiträgen. Den Anfang macht die Keynote von Bernd Käpplinger, in der anhand des systemischen Kontextmodells arbeitsweltbezogener Beratung nach Schiersmann das Feld der Bildungsberatungsforschung aufgespannt wird. Anhand von Bezügen zu hellenischen Mythengestalten werden dabei grundlegende Erkenntnisse wie auch Forschungsdesiderata benannt und hinsichtlich der einhergehenden Herausforderungen und offenen Fragen zugespitzt.

Kapitel eins widmet sich den theoretischen Zugängen und beinhaltet zwei Beiträge, die das Phänomen Beratung aus sehr unterschiedlichen Perspektiven analysieren. Jana Wienberg betrachtet Beratung vor dem Hintergrund resonanztheoretischer Bezüge und arbeitet heraus, inwiefern sich die zeitdiagnostisch beobachtbaren Beschleunigungen wie auch zunehmende Wett- 
bewerbsorientierung auf Beratung auswirken. Im Beitrag von Julia Franz, Stephanie Welser und Annette Scheunpflug wird der Diskurs um Lernberatung einer systematischen Analyse im Anschluss an Luhmann unterzogen, indem - exemplifiziert an drei Lernberatungskonzeptionen - rekonstruiert wird, welche Sinnkonstruktionen diesen in sachlicher, sozialer und zeitlicher Dimensionalität zugrunde liegen.

Den Besonderheiten medial vermittelter Beratungsformate wird sich im zweiten Kapitel ebenfalls in zwei Beiträgen genähert. Wie unterschiedlich Berater*innen die Anliegen von Ratsuchenden prozessieren, untersuchen Tim Stanik und Cornelia Maier-Gutheil am Beispiel von E-Mail-Weiterbildungsberatungen mit der Methode der qualitativen Korrespondenzanalyse. An zwei kontrastiv angelegten Fallbeispielen können sie entsprechende Varianzen aufzeigen und die Bedeutung etwa von Reflexionsfragen, Handlungsempfehlungen und Lösungsvorschlägen herausarbeiten. Sebastian Zick widmet sich in seinem Beitrag den subjektiven Aneignungsprozessen im Kontext des Reisens und wendet seine durch Foucault inspirierte Analyse der damit potenziell verbundenen Beratungsbedarfe und -angebote auf unterschiedliche zeitliche Kontexte an (Apodemiken und Reiseberichte im Internet). Auf diese Weise arbeitet er unter anderem die Folgen der europäischen Mobilitätspolitik und der durch sie (mit-)erzeugten Beratungsbedarfe heraus.

Im dritten Kapitel geht es in drei Beiträgen um Beratung im Kontext der wissenschaftlichen Weiterbildung. Nico Sturm und Heike Rundnagel fokussieren hier auf die spezifischen Bedarfe nicht-traditioneller Studierender und beantworten damit verbundene Fragen zur Konzeptionalisierung von Beratungsangeboten wie auch organisationaler Rahmenbedingungen auf der Ebene der Programmplanung. Erkenntnisse aus einem aktuellen BMBF-Verbundprojekt zur Anrechnungsberatung für beruflich Qualifizierte werden von Christoph Damm präsentiert, insbesondere zu bedeutsamen Handlungsmustern im Rahmen der Anrechnung und Anerkennung von außerhochschulisch erworbenen Leistungen. Im dritten Beitrag thematisiert Katharina Koller ebenfalls die Zielgruppe der nicht-traditionellen Studierenden und stellt Ergebnisse einer mixed-methods Bedarfserhebung sowie darauf basierende Angebotsentwicklungen aus einem zwei Hochschulen übergreifenden Verbundprojekt zum Thema Durchlässigkeit vor.

Der Themenkomplex Beratungskompetenz und Professionalisierung beinhaltet vier Beiträge (Kapitel 4). Zwei Aufsätze befassen sich mit Beratungskompetenzen. Mit welchen Instrumenten solche Kompetenzen angemessen erfasst und analysiert werden können, wurde in einem Projekt des Nationalen Forums Beratung und der Forschungsgruppe Beratungsqualität der Universität Heidelberg entwickelt. Christiane Schiersmann und Peter Weber stellen das Konzept für die Erfassung der Instrumente wie auch Ergebnisse zu deren Erprobung vor. Demgegenüber beschäftigt sich Marc Weinhardt in seinem Beitrag mit empirischen Ergebnissen zu den Lern- und Bildungsprozessen 
im Kontext von Beratungskompetenzerwerb und entwickelt das Modell einer subjektorientierten Professionalisierung und dafür notwendiger Strukturentwicklungen an der Hochschule. Der dritte Beitrag nimmt Bezug auf Ergebnisse aus einer komparativ angelegten Studie über pädagogische Arbeitsfelder, indem Johannes Wahl die vorliegenden Daten mit Blick auf die Kernaktivität des Beratens reanalysiert und etwa Beratungsanlässe aus den beruflichen Selbstbeschreibungen rekonstruiert. Schließlich eröffnen Birgit Schmidtke und Roland Lehner mit dem österreichischen Projekt Bildungsberatungs-Radar einen neuen Blick auf das in Beratungen generierte Wissen. Zentral geht es bei der Pilotstudie darum, wie die Beratungslandschaft als Radar genutzt werden kann, um etwa strukturelle Defizite oder Hindernisse über so genannte systemrelevante Fälle zu erfassen. Der damit generierbare Nutzen für die systematische Weiterentwicklung professioneller Beratung(slandschaften) verspricht auch für Deutschland Potenziale.

In Kapitel 5 sind ebenfalls vier Beiträge versammelt, in denen unterschiedliche Lebensphasen mit ihren je spezifischen Beratungsbedarfen und -anlässen behandelt werden. Simone Hocke lenkt mit der reflexiven Übergangsberatung den Blick auf sensible Phasen und Zeiten in beruflichen Kontexten am Beispiel betrieblicher Interessenvertreter*innen und arbeitet in ihrer Studie unter anderem drei methodenspezifische Wirkfaktoren heraus. Den Fragen nach Beratungsanlässen und -themen im Bereich der außerschulischen Jugendbildung wird bezogen auf den Kontext des Freiwilligen Sozialen Jahres von Caroline Kruse nachgegangen, die hierfür Bildungsreferent*innen interviewt hat. Übergänge in Arbeit und darauf bezogene Beratungsangebote haben Heike Chyle, Nina Wlassow und Christiane Dittrich in einem universitätsübergreifenden DFG-Projekt in Bezug auf darin sich zeigende pädagogische Rationalitäten erforscht. Im Beitrag werden am Beispiel von Coaching und Transfergesellschaft zwei spezifische Kontexte vor allem hinsichtlich der Materialität von Praktiken im Beratungssetting analysiert. Im letzten Beitrag von Franziska Schulze-Stocker, Robert Pelz und Christian Schäfer-Hock geht es um das Zusammenspiel von Beratung und Intervention im Hochschulkontext zum Aufbau eines so genannten Frühwarnsystems. Aus dem entsprechenden Projekt der TU Dresden werden Ergebnisse zu den Problemlagen der Studierenden, die zu einem Studienabbruch führen (können), wie auch deren Umgang damit, präsentiert.

Die Erforschung realer Beratungsinteraktionen anhand gesprächsanalytischer Zugänge verbindet die beiden Aufsätze im sechsten Kapitel. Aus einer an Foucault anschließenden Subjektivierungsperspektive werden von Farina Wagner authentische Weiterbildungsberatungsgespräche daraufhin untersucht, wie der Prozess der Entscheidungsgenerierung in den Interaktionen vor dem Hintergrund von Narrativen des Lebenslangen Lernens hergestellt wird. Auch Christian Schröder und Claudia Muche beschäftigen sich mit Gesprächsanalysen - jedoch bezogen auf Coaching von Führungskräften - mit 
dem Ziel, die Bedingungen professionellen Beratungshandelns zu rekonstruieren.

Im letzten Kapitel (7) richtet sich der Fokus der vier Beiträge auf Beratung in unterschiedlichen organisationalen Kontexten - auch aus spezifisch organisationspädagogischer Perspektive. Henning Pätzold lotet in seinem Aufsatz das Verhältnis von Organisationsberatung und organisationalem Lernen aus, indem er die These der Organisationsberatung als Lernberatung von Organisationen entwickelt. Auch Hildegard Macha setzt sich in ihrem Beitrag über transformative Beratung mit einem auf organisationale Lernprozesse zielenden Beratungskonzept auseinander. Am Beispiel zweier Interventionsprojekte mit dem Ziel, die Umsetzung von Diversity und Gleichstellung zu verbessern, werden Ergebnisse zum Beratungsverlauf wie auch der begleitenden Evaluation der Lerneffekte bei den beteiligten Gruppen vorgestellt. Kira Nierobisch widmet sich Prozessen der Fremd- und Selbststeuerung im Kontext Hochschule und analysiert aus einer gouvernementalitätssensiblen Perspektive ein universitätsspezifisches Projekt zur Institutionalisierung und Professionalisierung der Studien(fach)beratung vor dem Hintergrund des Konzepts einer rekonstruktiven pädagogischen Beratung. Der Beitrag von Shahinaz Khalil erforscht ebenfalls aus einer foucaultschen Perspektive die Einflussfaktoren eines konkreten ägyptischen Bildungs- bzw. Karriereberatungsprogramms für Jugendliche (career guidance) hinsichtlich von dessen Wirkungen auf die Jugendlichen selbst, deren Eltern, Lehrende und die Programmakteure. Im Fokus stehen die Transformationsprozesse, die sich im Verlauf des Programms ergeben, aus der Sicht der beteiligten erwachsenen Akteure.

Mit dem vorliegenden Tagungsband wollen die Herausgebenden nicht nur die Vielfalt der Beiträge der Jahrestagung 2017 dokumentieren, sondern zugleich die Bandbreite der Beratungsforschung in der Erwachsenenbildungswissenschaft aufzeigen. Dabei begegnen uns altbekannte Fragen nach der Professionalisierung, Institutionalisierung und Zielgruppenorientierung wieder, aber auch neue Zugänge und forschungsmethodische Ansätze geraten durch die Beratungsforschung in den Blick. Vor allem verdeutlicht uns der Tagungsband die reichhaltigen Bezüge und Schnittstellen zwischen verschiedenen Praxisfeldern der Begleitung und Beratung von Bildungsprozessen im Erwachsenenalter, die nun zunehmend in Forschungsprogrammen Eingang finden. Wir hoffen, mit dem vorliegenden Tagungsband zur Weiterentwicklung des wissenschaftlichen Diskurses wie auch des Praxisfeldes einen Beitrag leisten zu können. 


\section{Literatur}

Giesecke, Hermann (2000). Pädagogik als Beruf. Grundformen pädagogischen Handelns (7. Aufl.). Weinheim: Beltz, Juventa.

Gieseke, Wiltrud/Nittel, Dieter (Hg.) (2016). Handbuch - Pädagogische Beratung über die Lebensspanne. Weinheim: Beltz Juventa.

Käpplinger; Bernd/Maier-Gutheil, Cornelia (2015). Ansätze und Ergebnisse zur Beratung(sforschung) in der Erwachsenen- und Weiterbildung - Eine Systematisierung. In: Zeitschrift für Weiterbildungsforschung. Jg. 38, H. 2, S. 163-181. DOI 10.1007/s40955-015-0034-9

Schiersmann, Christiane, \& Weber, Peter (Hg.) (2013). Beratung in Bildung, Beruf und Beschäftigung. Eckpunkte und Erprobung eines Qualitätskonzepts. Bielefeld: W. Bertelsmann.

Schiersmann, Christiane/Maier-Gutheil, Cornelia/Weber, Peter (2016). Beratungsforschung im Kontext von Bildung, Beruf und Beschäftigung. In: Tippelt, Rudolf/ Schmidt-Hertha, Bernhard (Hg.), Handbuch Bildungsforschung, Wiesbaden, Springer (Reference Sozialwissenschaften, DOI 10.1007/978-3-531-20002-6_52-1)

Weinhardt, Marc (2018). Kompetenzorientiert systemisch Beraten lernen. Eine Gebrauchsanweisung für die eigene Professionalisierung. Heidelberg: Vandenhoeck \& Ruprecht.

Weinhardt, Marc (Hg.) (2015). Beratungskompetenzerwerb. Pilotstudien aus der Arbeitsstelle für Beratungsforschung. Weinheim: Beltz. 
Bernd Käpplinger

\section{Beratung im Rampenlicht - Von Sisyphos zu Prometheus?}

\section{Einleitung}

Dieser Text basiert auf einer Keynote bei der Jahrestagung 2017 der Sektion Erwachsenenbildung der Deutschen Gesellschaft für Erziehungswissenschaft. Der Bedeutungszuwachs von Bildungsberatung und damit verbundene Problematiken werden diskutiert sowie ältere Argumentationen neu erörtert. Zudem werden hellenische Mythengestalten herangezogen, um Fragen zur Rolle von Bildung und Wissenschaft zu sondieren, wie es bereits in der Vergangenheit geschah (vgl. Siebert 1992, Illich 2013).

\section{Prometheus - oder: über die Fortschritte der Beratungsforschung}

Der Name Prometheus steht für Fortschritt, Wissen und Zivilisation. Die antike mythische Gestalt wurde zum Symbol für den Menschen in seinem Aufbegehren und seinem Kampf gegen Widerstände. Fortschrittsoptimisten feierten den Triumph des ,prometheischen“ Menschen, der sich als autonomes Wesen konstituiert und sein Schicksal in die Hand nimmt.

Die Forschung zu Bildungsberatung in der Erwachsenenbildung kann man spätestens seit 2000 - dieses Bezugsjahr ist $u$. a. wegen eines facettenreichen REPORT-Themenhefts zu Beratung (Nuissl/Schiersmann/Siebert 2000) gewählt - als eine Geschichte des erheblichen wissenschaftlichen Fortschritts lesen. Das Handbuch von Gieseke/Nittel (2016) demonstriert dies in der Breite wie in der Tiefe. Es lassen sich in vielen Fachartikeln differenzierte Definitionen für die verschiedenen Formen und Ausprägungen von personen- oder organisationsbezogener Beratungen finden. Weitere Überblickstexte sind zu nennen (z. B. Käpplinger/Maier-Gutheil 2015, Maier-Gutheil 2012). Im Folgenden wird nun das Mehrebenenmodell von Schiersmann (2010, S. 30) als heuristische Systematisierungshilfe genutzt. Dies ist auch als Würdigung der Gastgeberin dieser Sektionstagung mit ihrer Heidelberger Forschungsgruppe intendiert, die zu den Fortschritten bei der Beratungsthematik in der Erwachsenenbildung wesentlich beigetragen haben. 


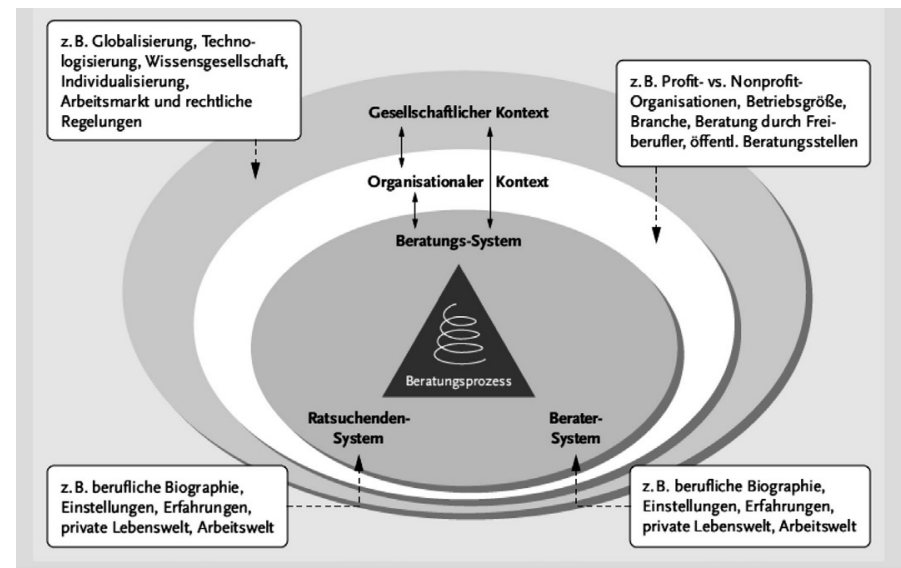

Abb. 1: Systemisches Modell arbeitsweltbezogener Beratung (Quelle: Schiersmann 2013, S. 30)

Dieses Modell ist von einer systemtheoretischen Perspektive auf Bildungsberatung informiert, wurde aber bislang nicht dafür genutzt, den Forschungsstand darzustellen. Mehrebenenmodelle haben in der Weiterbildungsforschung an Bedeutung gewonnen (Schrader 2011), wenngleich dies nicht gänzlich neu ist (vgl. Kowalski 1998, S. 9), was man so in der neueren Literatur ausweisen könnte, wenn man sich selbst in einer erwachsenenpädagogischen Traditionslinie begreifen würde.

Wichtig ist bezogen auf Mehrebenenmodelle u. a. die Frage nach dem $\mathrm{Zu}-$ sammenhang der Ebenen: Inwiefern kann Politik Praxis steuern oder werden Steuerungsversuche von einer autonomen Praxis abgewiesen? Dies als ein Beispiel, bei dem die Zusammenhänge der Ebenen interessieren.

Im „,Gesellschaftlichen Kontext“ des Modells könnten Arbeitstitel angesiedelt werden wie:

- „Zur Kontextualisierung des Beratungsdiskurses der Erwachsenenbildung"

- „Bildungsbarrieren sichtbar machen. Pilotprojekt Bildungsberatungs-Radar"

Diese zwei Titel aus diesem Sammelband und von der Sektionstagung können als exemplarisch angesehen werden. In Bezug auf den gesellschaftlichen Kontext werden politische, wirtschaftliche oder soziale Veränderungen thematisiert. Oft findet sich als Begründung von Bildungsberatung der zeitdiagnostische Verweis auf diskontinuierliche Erwerbsbiografien oder sogenannte Patchworkbiografien. Beratung über den Lebenslauf hinweg - und nicht nur an der ersten und zweiten Schwelle - sei notwendig. Kritische Denker 
bemängeln, dass der Beratungs- und Coachingboom Anzeichen einer neuen Form der Steuerung ist (Christof/Ribolits/Zuber 2007), beispielsweise verweisen die diskurskritischen Arbeiten von Kossack (2006) oder international Bengtson (2015) auf diese wichtige Lesart. Sie warnen davor, das gewachsene Interesse an Beratung nur positiv zu sehen und als emanzipatorische Subjektorientierung zu idealisieren. Im Kontext von Förderberatung wird der Begriff der regulativen Beratung (Käpplinger/Klein 2013) verwendet, da Bildungsberatung z. T. durch Zielvorgaben und Prüfroutinen samt Dokumentationspflichten durch Administrationen gekennzeichnet ist, die wenig mit dem Ideal einer klientenorientierten Beratung zu tun haben. Die externe Regulierung bzw. Regulierungsversuche von Beratungspraktiken sind in einer durchgesteuerten, risikoaversen Spätmoderne kritisch zu reflektierende Phänomene. Bildungsberatung bewegt sich in einem durch Zeitgeist, Politiken und gesellschaftliche, technische, wirtschaftliche oder soziodemographische Veränderungen gesteckten Rahmen. Die normative Forderung, sich dem Klienten verpflichtet zu fühlen und Hilfe zur Selbsthilfe zu leisten, sorgt für eine ethische Orientierung, da das Thema Beratungsethik wichtig ist und noch wichtiger wird.

Der „gesellschaftliche Kontext“ könnte mehr ausdifferenziert oder in Teilbereiche unterteilt werden, aber Modelle müssen sich, um als Heuristiken dienen zu können, der Anforderung von Sparsamkeit und Vereinfachung stellen (von Hippel/Käpplinger 2017). Megatrends wie Digitalisierung oder Flucht/Migration können als Schlagwörter Hinweise dafür bieten, dass sich Beratung immer intensiv mit gesellschaftlichen, technologischen und arbeitsmarktbezogenen Trends befassen muss (Käpplinger 2009). Es besteht die Herausforderung für Forschende, diesen Zeitgeist und Zeitdiagnosen (Wittpoth 2001) zu beachten und kritisch zu prüfen.

Im ,organisationalen Kontext “ bewegen sich Arbeiten mit Titeln wie:

- Optimierung von Beratungsstrukturen für nicht-traditionelle Studieninteressierte

- Anrechnungsberatung als Möglichkeit der Öffnung von Hochschulen?

- Beratung im Kontext organisationalen Lernens

Auch hier handelt es sich exemplarisch um Themen aus diesem Sammelband. Studien befassten sich mit Geschäftsmodellen der Bildungsberatung (Gieseke/Müller 2009); Trägerneutralität ist ein Dauer(streit)thema, wenngleich zumeist der nicht neutrale Einfluss der Förderer und ihrer normativen Programmatik ausgeblendet bleibt. Stanik (2016) hat die Bedeutung des institutionellen Kontexts anhand von Beispielen empirisch bearbeitet. Die Kommission Organisationspädagogik (Göhlich et al. 2010) hat des Öfteren das Thema Beratung aufgegriffen und aus ihrer organisationspädagogischen Perspektive beleuchtet. Der Fokus des Qualitätsdiskurses liegt eher auf Organisation, wobei der kritische Hinweis erlaubt sei, dass hier die Gefahr besteht, Organisa- 
tion wichtiger als Profession zu nehmen, zugespitzt Professionalisierung nur als Seitenstrang und Unterthema von Organisationsentwicklung zu begreifen (Egetenmeyer/Käpplinger 2011). Für den Weiterbildungsbereich und speziell LQW diskutieren die Studien von Hartz und die LQW-Implementationsstudie der damaligen Tübinger Forschungsgruppe um Schrader, dass die Qualitätsmanagements (QMs) den Kern, d. h. die Lehr-/Lernprozesse, kaum bis gar nicht erreichen (vgl. Hartz 2011). Warum werden jedoch immense Finanzsummen in QMs und darauf bezogene Praktiken (Zeit und Arbeit von PraktikerInnen) investiert, wenn sie kaum wirksam für die KlientInnen sind? Mittlerweile gibt es eigene QMs für Beratungsorganisationen (Schröder/Schlögl 2014, Schiersmann/Weber 2010). Auch hier sollte kritisch geprüft werden, was dadurch wirklich erreicht wird. Wurde eine Büchse der Pandora geöffnet? Wie stellt sich der Wettbewerb der QMs dar? Braucht es Zweit- und Drittzertifizierungen nebeneinander, weil öffentliche Auftraggeber verschiedene Standards verlangen? Was bringt die „Qualitätsberichtsprosa“ wirklich? Man legt als Beratungseinrichtung quasi eine Beichte über Qualitätsverfehlungen $a b$ und gelobt Besserung. Der Zertifizierer erteilt dann mit einem Zertifikat die Lossprechung - ggf. mit Bußauflagen - bis zur nächsten Beichte. Allerdings weiß „der Pfarrer“, dass das, was vor oder nach der Beichte passiert, oft nicht in seinem Einflussbereich liegt. Man kann sich an die „Pastoralmacht“ nach Forneck/Wrana (2006) in Qualitätsaudits erinnert fühlen. Gleichzeitig darf bei aller Kritik nicht vergessen werden, dass Institutionen und Organisationen professionelles Handeln entlasten und oft erst die Rahmenbedingungen sind und sächliche Ressourcen vorhalten, um Professionalität zu ermöglichen und zu erleichtern.

Mit dem „Beratungsprozess“ beschäftigen sich Studien mit Titeln wie in diesem Band:

- Computervermittelte, asynchrone Bildungsberatung im Spannungsfeld von Anliegen und Prozessierungstransfer

- Verhandlung von Differenz in der Weiterbildungsberatung

- Professionalität im Coaching von Führungskräften: Gemeinsame Herstellung eines Reflexionsraumes

Seit Gieseke et al. (2007) vor rund einer Dekade einen Aufsatz mit dem Titel „Prozessverläufe in der Beratung analysieren - Ein Desiderat“ überschrieben, hat sich viel getan. Maier-Gutheil (2009) hat Existenzgründerberatung interaktionsanalytisch untersucht und beschäftigt sich nun gemeinsam mit Stanik mit digitalen Kommunikationsformaten (s. Beitrag in diesem Band), einem wichtigen Thema in Zeiten der Digitalisierung, wo es mehr hybride Kommunikationsformen bzw. schon jetzt in vielen Beratungseinrichtungen ein $\mathrm{Ne}-$ beneinander von Telefon-, E-Mail-, Chat- oder Face-to-Face-Beratung gibt. Gieseke/Stimm (2016) haben eine Publikation mit Gesprächsanalysen vorgelegt. Schlüter (2014) legte einen Sammelband mit Fallanalysen der Bildungs- 
und Lernberatung vor. Es muss nicht auf Hidden-Client-Untersuchungen zurückgegriffen werden, da Praxis und Forschung kooperieren. Typischerweise werden mit expliziter Zustimmung aller Beteiligten Tonbandaufnahmen eingesetzt. Diese Forschung ist ein immenser Fortschritt, weil man Einblicke in die Beratungen mit ihrem Prozesscharakter erhält, statt über Interviews Konstruktionen und Rekonstruktionen $\mathrm{zu}$ analysieren. Methodenkritisch relativiert dies die eine oder andere Interviewaussage von Beratenden oder kollektiv getragene Mythen der Beratungsszene, die sich rhetorisch oft als grundsätzlich klientenorientiert einschätzt. Empirisch zeigen sich Beratungsverläufe als administrativ beeinflusst, aber nicht komplett durchgesteuert. Es existiert eine Eigenwilligkeit der Praxis und man sollte nicht unterschätzen, dass Klienten zumindest phasenweise maßgeblich mitsteuern können. Die rein governementalitätskritische Sicht oder eine nur soziologische Kritik können irreführend sein, weil sie zu sehr aus der Vogelperspektive auf die Beratung schauen und die Eigenwilligkeit der Subjekte und der Praxis unterschätzen. Gleichzeitig wäre es illusorisch anzunehmen, dass Beratende und Beratene ganz autonom sind und Kontext oder Vorgaben ignorieren können. Es braucht präzise, differenzierte empirische Forschung, bei der die Forschenden selbst bereit sein müssen, sich von schönen Hypothesen zu trennen und Neuem nachzuspüren. Frei nach den Frankfurter Poetik-Vorlesungen: „Das Material, das ich um mich anhäufe, ist mir aus der Kontrolle geraten" (Wolf 2008, S. 129). Inwiefern sind Forschende bereit, wirklich selbst lernend etwas Neues zu entdecken? Selbst manche induktive Arbeit - vermeintlich dem Grounded-Theory-Ansatz folgend - reproduziert eher vorgefasste Meinungen und Positionen der Forschenden statt Neues zu suchen.

Das „Beratersystem“ ist Thema von Beiträge mit Titeln wie:

- Instrumente zur Erfassung der Kompetenzen von Beratenden

- Beratungskompetenzerwerb im Studium

Es existieren einige Studiengänge und Qualifizierungsmöglichkeiten für Beratung (Schiersmann 2016). Die Universitäten Heidelberg, Münster, Duisburg-Essen, Mannheim/Schwerin oder die Aktivitäten der „Regionalen Qualifizierungszentren“ (RQZ) seien angeführt, wobei der Beratungsstudiengang in Heidelberg mittlerweile eingestellt wurde. Die Aktivitäten des Nationalen Forums tragen dazu bei, das Thema auf der politischen Agenda zu behalten und die Beratenden zu unterstützen. In Kooperation mit Schiersmann (2013) wurde das Kompetenzprofil für Beratende im Zuge einer sogenannten „Offenen Koordinierung“ formuliert. Die Frage, wie der Zugang zur Beratungstätigkeit (nicht) reguliert wird, ist ein Konfliktfeld. Momentan kann sich jeder „Bildungsberater“ nennen und ein Coachingbüro eröffnen, sodass ein Soziologe wie Kühl (2005) kritisch vom Scharlatanerieproblem für das Coaching spricht. Lediglich bei der öffentlichen Finanzierung wird der Coach auf Probleme stoßen, da oft eine QM-Zertifizierung verlangt wird, sodass man 
hier für diese Art der Regulierung des Zugangs zum Feld dankbar sein kann. Individuelle und kollektive Professionalisierung bleiben Daueraufgaben.

Die Beratenden selbst - Schiersmann nennt es ,Ratsuchendensystem “ sollten nicht vergessen werden:

- Beratung in beruflichen Übergängen als Reflexionsraum für Lernprozesse und Initiierung von Lernprozessen

- „Unzufrieden mit dem Studium, belastende Lebensumstände, falsche Studienwahl ..." - Was steckt hinter den Problemen im Studium?

Seit langen Jahren wird das Thema Lernberatung diskutiert (Klein/Kemper 1998, Harke/von Bialy 1991, Fuchs-Brüninghoff 2000 oder Ludwig 2012a, 2012b). Hier geht es um Beratung für Lernende direkt im Lernprozess und nicht um Beratung zu Bildungsentscheidungen im Vorfeld des Lernens. Es gibt Moden und Diskussionen, ob KlientIn, KundIn oder Ratsuchende die adäquaten Begriffe sind, um die Beratenen zu bezeichnen. Jeder Begriff hat seine Vor- und Nachteile und vielleicht müsste man je nach Kontext entscheiden. Jemand, der komplett freiwillig eine offene, unabhängige Beratungsstelle aufsucht, ist eher ein Ratsuchender als jemand, der in regulierten Kontexten zugewiesen wird und eigentlich selbst gar keinen Rat sucht. Der Kundenbegriff sollte kritisch geprüft werden, da häufig der/die KlientIn nicht der zahlende Kunde ist, sodass die beratende Person einen Auftraggeber und eineN MandantIn hat, die personell nicht identisch sind. Die Arbeiten von Schlutz (2006) zu Kundenbeziehungen in der Programmplanung sind übertragbar, da in der öffentlich finanzierten Beratung die/der (zahlende) KundIn und (beratene) KlientIn differieren. Es sollte nicht nur über trägerneutrale Beratung diskutiert werden, sondern auch über die Frage, inwiefern die Agenden und Programmatiken der politischen Administrationen eine unabhängige Beratung erschweren. Monitoring oder Zielvereinbarungen können sehr einflussreich sein. Statistiken werden administrativ gegen die Praxis und ohne Einfluss der Wissenschaft bemüht (Käpplinger/Bilger 2017), um selbst über verschiedene Förderwellen und Regierungen hinweg etablierte Beratungsstrukturen zusammenzustreichen. Dies haben die Weiterbildungsverbünde und ihre Beratungsstellen in Schleswig-Holstein gezeigt (Mikschl et al. 2018), die in ihrer Existenz maßgeblich auf ein Gutachten von Faulstich aus den 1990er Jahren zurückgingen.

Mit Blick auf die Sektionstagung ist es bemerkenswert, dass sich viele Beiträge mit den Beratenen befassen. Dies mag überraschen, weil mit Blick auf den Forschungsstand der Eindruck entstehen kann, dass die Beratenen manchmal etwas aus dem Blick geraten. Es gibt eine Konstruktion vermeintlich bildungsferner Menschen, die durch Bildungsberatung aus ihrer Orientierungslosigkeit befreit werden müssen und dann die Wichtigkeit des LLL erkennen sollen. Es wirkt paradox, dass bei der Aktivierungspolitik oft die Subjekte selbst aus dem Blick geraten und eher soziodemographische Merk- 
male interessieren. Eine primär an diesen orientierte Wirkungsforschung wäre wenig hilfreich, wenn sie als Nutzen nur das messen soll, was administrativ intendiert ist, wie die Beratungsforschung der letzten Jahre, bspw. der Sammelband von Schober/Langner (2017) oder international die ELGPN-Dokumentationen, zeigen. „Nutzen“ ist breiter und humanistisch zu konzipieren (Käpplinger 2010). Aufsuchende Beratung als Konzeption muss sich die kritische Frage gefallen lassen, was sie, wie man vielleicht überspitzt formulieren könnte, zu einer Kolonialisierung von Sozialwelten legitimiert? Studien von Bremer et al. (2015) sind anregend, weil sie die Eigenwilligkeit und Selbstbestimmung der Subjekte und ihrer Gemeinschaften respektieren. Es ist gut, dass der Begriff der Bildungsferne kritisch diskutiert wird. Dörner (2010) hat die Frage aufgeworfen, ob Annahmen des administrativen Diskurses zu lebenslanger Beratung mit der subjektiven Sicht der Bevölkerung übereinstimmen. Diese Frage wurde jedoch kaum rezipiert, vermutlich da es nicht konform mit administrativen Agenden ist. Wir haben partiell einen normativ überhöhten Beratungsdiskurs, in dem es kaum eine bildungspolitische Empfehlung gibt, die nicht zum Ausbau der Bildungsberatung rät. Empfehlungen durch mich selbst sehe ich hier mittlerweile selbstkritisch. AES-Daten liefern Irritationen, da der Wunsch nach Information und Beratung in der Bevölkerung abnimmt und bekundet wird, bereits einen guten Überblick zu haben:

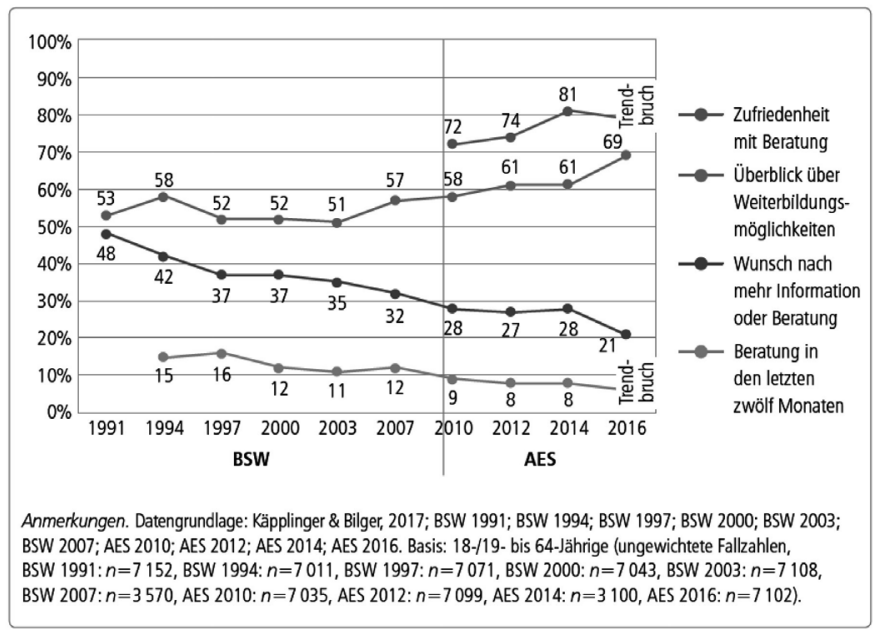

Abb. 2: Indikatoren zu Beratung im Kontext von Weiterbildung von 1991 bis 2016 (Quelle: Käpplinger/Bilger/Reuter 2018, S. 257)

Man sollte sich der tabufreien Diskussion stellen, was Bildungsberatung für das Leben vieler Menschen präzise bedeutet und für wen in welcher Lebens- 
situation sie eine Unterstützungsform sein kann. Beratung sollte nicht als Allheilmittel in Transformationsprozessen überschätzt werden oder als kurzfristiges Interventionsformat längere Lernprozesse ersetzen wollen. Ist Beratung so populär geworden, weil sie verspricht in wenigen Minuten und Stunden Wissen zu vermitteln und Lernerfolge zu zeigen, für die man früher Tage oder Wochen einplante? In der Beratung wird Wissen vermittelt, sie kann eine „Aufklärungsinstanz des Individuums“ sein (Enoch 2011), aber ist Wissensvermittlung zentral bei der Beratung? Es reicht nicht aus, pauschal zu sagen, Bildungsberatung sei positiv und wir müssten ein System der lebenslangen Beratung aufbauen. Vielleicht muss Beratung vor ihrem Erfolg geschützt und müssen ihre jeweiligen Funktionen und ethischen Grenzen präziser bestimmt werden?

Das Mehrebenenmodell von Schiersmann/Weber wurde in diesem Beitrag rekapituliert. Es ist als Heuristik und Systematisierungshilfe sinnvoll. Gleichzeitig kann man sich oft nicht allein auf einer Ebene bewegen und viele Studien bewegen sich eher „im Zwischen“ wie Beratung insgesamt oft ein Handeln im „Zwischen“ (Großmaß 2014) ist. Es wurde versucht aufzuzeigen, dass wir an beachtlichen Fortschritten in der Forschung erzielt haben. Oft ist es eher die mangelnde Kenntnis, Rezeption und Sichtbarkeit der Forschung, die dazu führt, dass vorschnell Forschungslücken proklamiert werden. Man will auf dem Markt der Aufmerksamkeit gehört werden und da ist die marktschreierische Klage oder sind die Untergangsszenarien populärer als das differenzierte, kleinteilige Schauen und Recherchieren, was es bereits gibt, und daran anzuschließen. Plecas/Sork (1986) haben dies als „Defnimania“ und „Lexicophilia“" bezeichnet, d. h. die Tendenz oder Willkür, das neu zu definieren, was schon definiert ist, und den Wunsch zu haben, ständig neue Begriffe in die Welt zu setzen. Man braucht neue Begriffe für neue Phänomene, aber sind es immer wirklich die Phänomene, die zu Neuschöpfungen führen, oder eher Disziplinlosigkeiten? Ungeachtet dessen hat es strukturelle Gründe, warum so manche Forschungsstudie sisyphosartig erscheint.

\section{Sisyphos - oder: das Fehlen von Kontinuität und wechselseitiger Bezugnahme}

Als Sisyphos(-arbeit) wird eine anstrengende, jedoch vergebliche Arbeit verstanden. Camus folgend wird Sisyphos verstärkt als Allegorie auf die Conditio Humana gelesen. Diese Deutung war bereits in der Antike vorhanden, durch Camus' Neubewertung erhielt sie einen positiven Gehalt. Sisyphos’ Tätigkeit wird bei ihm als sinnstiftend beschrieben, da die Gestalt des Sisyphos eine Wissenschaft symbolisiert, die sich selbst in Frage stellt. 
Auch Forschung in der Erwachsenenbildung sollte sich in Frage stellen. Mit historischem Bewusstsein zeigt sich, dass vieles Vorläufer hat, auf denen man aufbauen könnte. Wir müssen kritisch sein und Lücken aufzeigen, Diskurse, Debatten oder Streit in der Sache transparent ausfechten. Dies braucht eine solide - manchmal demütige - Analyse des Forschungsstands und nicht (über-)forsches Proklamieren von Lücken auf Basis einer nicht ausreichend intensiven Recherche. Ansonsten kommen wir als Sisyphos nicht weiter, weil wir nur den Stein erneut den Berg hochrollen. Eher die Sicht auf den Schultern von Riesen stehend zu suchen, sollte ein Ziel sein, um erneut eine andere alte Metapher zu bemühen (Käpplinger 2017).

Diese Diskontinuität hat strukturelle Gründe. Die Weiterbildungsforschung ist zumeist projektförmig angelegt, weshalb wir Drittmittelbewirtschafter versucht sind, „Projektprofessoren“ zu sein, die vor allem nach den neuesten bildungspolitischen Moden und dazu passenden Förderungen Ausschau halten. Das im Rahmen der Hamburger Tagung „Das Politische in der Erwachsenenbildung“ vom März 2017 erwähnte Zitat von Faulstich, „Die Leute denken zu wenig, die machen nur noch Projekte“, muss auch strukturell diskutiert werden und nicht allein als Schelte einzelner Individuen. Häufig werden Aufträge im Kontext von Evaluationsforschung erteilt und der „Projektprofessor“ ist ein Teilergebnis der Förderlandschaft. Die Zahl der Lehrstühle entspricht nicht der Bedeutung der EB/WB, wenngleich es Stolz machen kann, was in diesem großen Feld von uns doch relativ wenigen geleistet wird. Man sollte sich auf ein Forschungsfeld spezialisieren, aber es ist auf vielen Ebenen viel zu tun. Christine Zeuner hat in unserem Disput in Kassel bei der Mitgliederversammlung der Sektion einen wichtigen Punkt angesprochen. Die Beratungsforschung lebt stark von Qualifikationsarbeiten und es gibt nur sehr wenige große Forschungsprojekte mit mehreren Lehrstühlen wie das damalige Projekt „Bildungsberatung im Dialog“ von Arnold/Gieseke/Zeuner (2009). Aber gilt dies speziell für die Beratungsforschung? Oder ist es nicht ein generelles Problem der Weiterbildungsforschung? Man kann sich mehr Kooperationen von Lehrstühlen über „Schulen“ hinweg wünschen. Wir müssen intelligent Kontinuitäten und Kooperationen verfolgen. Das falsche Schisma zwischen qualitativer und quantitativer Forschung sollte vermieden werden. Wir müssen intelligent hinterfragen, ob und wo wir Allgemeinplätze pflegen: Trägerneutralität, Freiwilligkeit der Beratung, lebenslange Beratung als Wunsch aller, aufsuchende Beratung für Bildungsferne und Geringqualifizierte als primäre Zielgruppe. Dies alles sind durchaus gut gemeinte Prämissen, aber prüfen wir, ob sie in der Praxis noch zutreffen oder jemals zugetroffen haben? Pätzold hat in mehreren Publikationen aufgezeigt, dass das Thema Freiwilligkeit und Beratung komplex ist und man eher von einem Kontinuum zwischen Freiwilligkeit und Unfreiwilligkeit ausgehen sollte (Pätzold/Ulm 2015). Es sind solche differenzierenden Perspektiven, die wir brauchen, statt immer wieder mit ähnlichen Prämissen Variationen des Gleichen zu produzieren. 


\section{Von Epimetheus und Kassandra: Mitmenschlichkeit und dem Unbehagen nachspüren}

Epimetheus, der oft verspottete Bruder des Prometheus, öffnet schlecht bedacht die Büchse der Pandora, sodass angesichts vieler Übel nur die Hoffnung bleibt. Illich (2013, S. 144ff) hat ihn als ein Leitbild verwendet, um daran zu erinnern, was die negativen Wirkungen einer technisch überregulierten Welt sind. Einiges an den Entwicklungen um Dokumentationspflichten, Monitoring oder Wirkungsmessungen von Bildungsberatung ist problematisch und eine solche „nachbedenkende“, humane Haltung ist wichtig, damit man nicht Teil einer sich selbstständig machenden Maschinerie von Politikadministration, Unternehmensberatungen und Auftragsforschung wird, wo kaum noch Sinn- und Erkenntnisfragen ernsthaft gestellt, sondern primär Systemlogiken bedient werden. Wie viele Arbeitsressourcen werden mit Dokumentationsarbeit verschwendet? Wie viel Geld und Zeit fließen in beratende und zertifizierende Apparate statt in die Beratungsarbeit selbst? Der Epimetheus eines Illich kann daran erinnern, sich auf grundlegende Fragen des Menschseins zu besinnen und unsere Nächsten mehr als Regularien wahrzunehmen. Wahrnehmen zu können ist schließlich eine zentrale beraterische Kompetenz.

Zudem möchte ich auf eine andere Gestalt rekurrieren, die mich fasziniert in Bezug auf Bildungsberatung:

Kassandra, die von Apoll die Gabe der Weissagung bekam. Als sie seine Verführungsversuche zurückwies, verfluchte er sie, auf dass niemand mehr ihren Weissagungen Glauben schenken möge. Sie gilt in der Mythologie als tragische Heldin, die das Unheil voraussah, aber kein Gehör fand. Derart ungehörte Warnungen werden als Kassandrarufe eher abgewertet. Christa Wolf hat dagegen mit ihrem Roman „Kassandra“ eine feministische, positive Interpretation geliefert.

Bildungsberatung ist mehrheitlich weiblich - auf beiden Seiten des Beratungstisches; sie entstand maßgeblich im Kontext der Frauenemanzipation. Eine Gründerin der Berufsberatung, Josephine Levy-Rathenau, war nahezu vergessen und wurde erst vor kurzem durch eine Biografie gewürdigt (Nürnberger/Maier 2013). Strukturelle, genderbezogene Benachteiligungen existieren auch heute, wenngleich in anderer Form. Es mag unter jungen Frauen heute nicht en vogue sein, Genderperspektiven einzunehmen, aber sie bleiben gerade in der Beratung wichtig, wie u. a. Gieseke, Schiersmann und Schlüter gezeigt haben. Es muss neu gehandelt und es müssen Haltungen vertreten werden, wenn in interkulturellen Kontexten Beratungen für Frauen nur unter Anwesenheit von Männern durchgeführt werden sollen. Oder wenn Männer verlangen sich von einem Mann beraten zu lassen. Beratungsstellen kommen dem z. T. nach und begründen dies mit „Kundenorientierung“. Eine Ethik der Beratung sowie Haltung sind wichtig, jedoch ganz sicherlich nicht 
allein im Kontext von Migration. Ostendorf (2005) hat so Mängel in der Berufsberatung aus der Genderperspektive an Berufsinformationsmaterialien aufgezeigt. Wer sich z. B. die BIBB-Homepage ansieht und wie dort Berufe aus Gender- und Diversityperspektive textlich und ikonografisch präsentiert werden, wird feststellen, dass dort zumeist (,weiße“) Männer (an-)leitend im Mittelpunkt stehen:

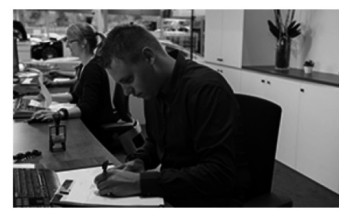

MITTWOCH, 12. APRIL 2017

Automobilkaufleute auf der Überholspur Ausbildungsordnung modernisiert

Digitalisierung, Vernetzung und Elektromobilität beschleunigen das ohnehin schon hohe Innovationstempo in der Automobilbranche. Dies wirkt sich auch auf den kaufmännischen Bereich aus. Die

Ausbildungsordnung für Automobilkaufleute wurde daher auf den neuesten Stand gebracht.

» weiterlesen

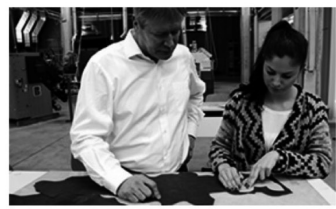

MONTAG, 27. MÄRZ 2017

Von High Heels bis zum Feuerwehrstiefel - Ausbildung für Schuhfertiger/-innen modernisiert

Für jeden Zweck die passenden Schuhe - von High Heels bis zum Feuerwehrstiefel. Schuhfertiger/-innen stellen sie in industrieller Produktion her. Diese Berufsausbildung wurde modernisiert und tritt zum 1. August 2017 in Kraft.

» weiterlesen

Abb. 3 und 4: Pressemitteilungen des Bundesinstituts für Berufsbildung (BIBB) (Quelle: https://www.bibb.de/de/pressemitteilung_60231.php vom 27. März 2017; https://www.bibb.de/de/pressemitteilung_60884.php vom 12. April 2017)

Es gibt weiterhin einiges zu tun, um Gleichberechtigung umzusetzen und nicht hinter Errungenschaften zurückzufallen. Aktuelle Migrationsbewegungen könnten eine Chance sein, Geschlechterverhältnisse zu thematisieren und den Status quo in der Mehrheitsgesellschaft, in migrantischen Kulturen sowie transkulturell kritisch zu bestimmen.

Bildungsberatung ist keine Prognose. Kassandra erahnte lediglich, dass etwas mit dem trojanischen Pferd nicht stimmt, und auch Beratende sollten der Versuchung wie Anmaßung widerstehen, detaillierte Prognosen zu treffen. Der selbstbewusste Beratende irrt oft genauso wie andere ExpertInnen, was Zukunftsprognosen betrifft. Berufe und Tätigkeiten, die heute nachgefragt sind, können morgen ihre Relevanz auf den Arbeitsmärkten verloren haben und vice versa. Man sollte Beratung als Moratorium nutzen, den Dingen nachzugehen, ohne zu wissen, was man genau sucht und wo man genau hingehen wird, wenngleich selbst manche Ratsuchende Orientierung einfordern. Wir Forschenden sollten der Versuchung widerstehen, für die Bildungspolitik und -administration nur das zu erforschen, was erwartet wird. Die 
Figur der Kassandra gemäß Christa Wolf kann anregen, das zu erforschen, was wir bei unserer Forschung zunächst nur vage vermuten. Dies mag nicht beliebt sein, aber geht es bei guter Forschung nicht darum, Neues zu entdecken, auch wenn es zunächst nicht opportun erscheint? Ich würde uns mehr Courage wünschen, das Unbequeme aufzuspüren, das öffentlich Relevante zu verfolgen, eigene wie fremde Tabus zu thematisieren und Studierende zum Non-Konformen anzuregen. Es bringt nicht weiter, wenn nur Erwartungen erfüllt werden. Die Forderungen, dass man den Bedeutungsaufschwung von Beratung „leidenschaftslos beschreiben“" (Gieseke/Nittel 2016, S. 12) solle, irritieren. Zumal wenn zuvor treffend kritisch erwähnt wird, dass die Beratung manchmal ein „manipulatives Interventionsformat“ (ebenda, S. 11) ist. Wo wir manipulative Interventionsformate nachweisen, ist da nicht durchaus leidenschaftlich und öffentlich zu widersprechen? Hier sei an eine Erkenntnis erinnert, die auch für Beratungsbemühungen gelten kann: „(Auch) die Nichtparteinahme ist eine Parteinahme, freilich eine verschleierte. Um die Parteinahme in jeder Bildungsbemühung, die überhaupt mit dem Problem unserer Gesellschaft zu tun hat, kommen wir also nicht herum.“ (von Oertzen 1977, S. 167) Es erscheint wichtig, dass die Sektion und ihre Mitglieder mit ihren Ergebnissen und Erkenntnissen auch die breitere Öffentlichkeit suchen. Welche Weiterbildungsstudie jenseits der Studien für den Alphabereich von Grotlüschen wurde in den letzten Dekaden von einer breiten Öffentlichkeit wahrgenommen? Wurde es versucht? Oder haben wir uns manchmal in Nischen jenseits der öffentlichen Wahrnehmung bequem eingerichtet?

\section{Vom Wert verschiedener Strategien}

Dieser Text ist inspiriert von dem Buch von Siebert (1992) „Bildung im Schatten der Postmoderne - Von Prometheus zu Sisyphos“. Er verabschiedet sich darin vom Leitbild des Prometheus aus einer Reihe ökologischer, politischer und auch teildisziplinärer Gründe. Die Ziele in der Weiterbildung sollten bescheidener und kleiner angelegt werden. Er regt zu einem „humorvollen Denken" eines lachenden, selbstironischen Sisyphos an. Dieses nahezu vergessene Buch ist ein zeithistorisches Dokument über die Popularisierung des Konstruktivismus. Nun wurde hier mit Prometheus, Sisyphos, Epimetheus und Kassandra argumentiert. Vielleicht hat das eine Resonanz in den Beiträgen zum Beratungsthema und seiner Aktualität. Anders als Siebert verschreibe ich uns keinen Paradigmenwechsel. Stattdessen wünsche ich uns

- den Mut und den Gestaltungswillen des Prometheus, um Strukturen zu schaffen und nicht nur Projekte zu machen; 
- die Ausdauer des Sisyphos, immer wieder Anlauf zu nehmen trotz Widerständen und geringer Nachhaltigkeit;

- die Mit-Menschlichkeit des Epimetheus, der nachbedenkend eigene Fehler erkennt, der sich um seine Mitmenschen sorgt und sich nicht technokratischen Organisationen und Prozessen unterordnet,

- und vor allem eine Kassandra, die ihrem Unbehagen nachspürt, das Unbequeme zu denken und auszusprechen wagt - gegen Widerstände und Schwarmdummheiten.

Es bedarf nicht allein einer Strategie, sondern sowohl in der Beratung als auch in der Weiterbildung, bzw. sowohl in der Forschung als auch in der Praxis sind viele Strategien situationsangemessen zu verfolgen. Darüber wäre dann offen zu diskutieren und manchmal konstruktiv zu streiten - wie wir es hoffentlich tun werden bei Sektionstagungen und Publikationen wie diesem Sammelband.

\section{Literatur}

Arnold, Rolf/Gieseke, Wiltrud/Zeuner, Christine (Hg.) (2009): Bildungsberatung im Dialog. 3 Bände, Baltmannsweiler: Schneider.

Bengtsson, Anki (2015): European career guidance policy: A focus on subtle regulatory mechanisms. In: Zeitschrift für Weiterbildungsforschung 38 (2), S. 241-250.

Bremer, Helmut/Kleemann-Göhring, Mark/Wagner, Farina (2015): Weiterbildung und Weiterbildungsberatung für „Bildungsferne“. Bielefeld: wbv.

Christof, Eveline/Ribolits, Erich/Zuber, Johannes (2007): Führe mich sanft. Beratung, Coaching \& Co. - Die postmodernen Instrumente der Gouvernementalität. Innsbruck: Studien-Verlag.

Dörner, Olaf (2010): Lifelong Guidance for all? In: DIE-Zeitschrift für Erwachsenenbildung 2, S. 28-31.

Egetenmeyer, Regina/Käpplinger, Bernd (2011): Professionalisation and Quality Management: Struggles, Boundaries and Bridges between two Approaches. In: RELA 2 (1), S. 21-35.

Enoch, Clinton (2011): Dimensionen der Wissensvermittlung in Beratungsprozessen. Wiesbaden: VS Verlag.

Forneck, Hermann/Wrana, Daniel (2005): Ein parzelliertes Feld. Bielefeld: wbv.

Fuchs-Brüninghoff, Elisabeth (2000): Lernberatung - die Geschichte eines Konzepts zwischen Stigma und Erfolg. In: Literatur- und Forschungsreport Weiterbildung 46, S. 81-92.

Gieseke, Wiltrud/Käpplinger, Bernd/Otto, Sylvana (2007): Prozessverläufe in der Beratung analysieren - Ein Desiderat. In: Report 30 (1), S. 33-42.

Gieseke, Wiltrud/Müller, Christina (2010): Die Realisierung und Konkretisierung von Dienstleistungsorganisationen für die Weiterbildungsberatung in Regionen. In: 
Dollhausen, Karin/Feld, Timm/Seitter, Wolfgang (Hg.): Erwachsenenpädagogische Organisationsforschung. Wiesbaden: VS Verlag, S. 143-161.

Gieseke, Wiltrud/Nittel, Dieter (Hg.) (2016): Pädagogische Beratung über die Lebensspanne - Handbuch. Weinheim: Beltz Juventa.

Gieseke, Wiltrud/Stimm, Maria (2016): Praktiken der professionellen Bildungsberatung - Innensichten auf die Entscheidungsfindung im Beratungsprozess. Wiesbaden: Springer VS.

Göhlich, Michael/Weber, S. M./Seitter, Wolfgang/Feld, Timm (Hg.) (2010): Organisation und Beratung. Wiesbaden: VS Verlag.

Großmaß, Ruth (2014): Arbeit im „Zwischen“- zur gesellschaftlichen Verortung von Beratung. Melter, Ingeborg et al. (Hg.): Zukunftsfeld Bildungs- und Berufsberatung III. Bielefeld: wbv, S. 15-30.

Harke, Dieter/Volk von Bialy, Helmut (Hg.) (1991): Erfahrungsberichte über Fortbildungen nach dem Konzept Lernberatung im Berufsförderungswerk. Hamburg: Beuth.

Hartz, Stefanie (2011): Qualität in Organisationen der Weiterbildung - Eine Studie zur Akzeptanz und Wirkung von LQW. Wiesbaden: VS Verlag.

von Hippel, Aiga/Käpplinger, Bernd (2017): Models of Program Planning in Germany and in North America - A Comparison. In: Käpplinger, Bernd et al. (Hg.): Cultures of Program Planning in Adult Education. Frankfurt/Main: Lang, S. 97-112.

Illich, Ivan (2013): Entschulung der Gesellschaft - Eine Streitschrift. München: Beck.

Käpplinger, Bernd (2009): Zukunft der personenbezogenen Bildungsberatung - Vier mögliche Szenarien. In: Arnold, Rolf et al. (Hg.): Bildungsberatung im Dialog Band I - Theorie - Empirie - Reflexion. Hohengehren: Schneider, S. 227-247.

Käpplinger, Bernd (2010): Nutzen von Bildungsberatung - Konzeptionelle Eckpunkte vor dem Hintergrund britischer Forschungsergebnisse. In: DIE-Zeitschrift für Erwachsenenbildung 17 (2), S. 32-35.

Käpplinger, Bernd/Maier-Gutheil, Cornelia (2015): Ansätze und Ergebnisse zur Beratung(sforschung) in der Erwachsenen- und Weiterbildung. In: Zeitschrift für Weiterbildungsforschung 38 (2), S. 163-181.

Käpplinger, Bernd (2017): Standing on the Shoulders of Giants - Building on Existing Knowledge. In: International Yearbook of Adult Education 40, S. 29-41.

Käpplinger, Bernd/Bilger, Frauke (2017): Erwartungswidriger Rückgang der Bildungsberatung? Der Adult Education Survey (AES) als Quelle für Analysen zur Bildungsberatung. In: Schober, Karin/Langner, Juia (Hg.): Wirksamkeit der Beratung in Bildung, Beruf und Beschäftigung. Bielefeld: wbv, S. 65-76.

Käpplinger, Bernd/Bilger, Frauke/Reuter, Martin (2018): Bildungsberatung und Transparenz des Bildungsangebots Erwachsener. In: Bilger, Frauke et al. (Hg.): Weiterbildungsverhalten in Deutschland 2016 - Ergebnisse des Adult Education Survey (AES). Bielefeld: wbv, S. 255-264.

Kemper, Marita/Klein, Rosemarie (1998): Lernberatung. Baltmannsweiler: Schneider. Kossack, Peter (2006): Lernen Beraten - Eine dekonstruktive Analyse des Diskurses zur Weiterbildung. Bielefeld: transcript-Verlag.

Kowalski, Theodore J. (1998): The Organization and Planning of Adult Education. Educational Leadership Faculty Publications. Paper 61. URL: http://ecommons. udayton.edu/eda_fac_pub/61 [Abruf: 21.04.2018] 
Kühl, Stefan (2005): Das Scharlatanerieproblem - Coaching zwischen Qualitätsproblemen und Professionalisierungsbemühung. Köln: Deutsche Gesellschaft für Supervision.

Ludwig, Joachim (Hg.) (2012a): Lernen und Lernberatung. Bielefeld: wbv.

Ludwig, Joachim (Hg.) (2012b): Lernberatung und Diagnostik. Bielefeld: wbv.

Maier-Gutheil, Cornelia (2009): Zwischen Beratung und Begutachtung. Wiesbaden: VS Verlag.

Maier-Gutheil, Cornelia (2012): Qualitativ-empirische Beratungsforschung in der Erwachsenen- und Weiterbildungsforschung. In: Schäffer, Burkhard/Dörner, Olaf (Hg.): Handbuch Qualitative Erwachsenen- und Weiterbildungsforschung. Opladen: Barbara Budrich, S. 489-501.

Mikschl, Josef/Neuner, Gerd/Viehöfer, Lothar (2018): Das Kieler Forum Weiterbildung, ein Ort der Kooperation und Beratung. In: Käpplinger, Bernd/Elfert, Maren (Hg.) Verlassene Orte der Erwachsenenbildung. Frankfurt/Main: Lang, S. 99-111.

Nuissl, Ekkehard/Schiersmann, Christiane/Siebert, Horst (Hg.) (2000): Beratung. REPORT - Zeitschrift für Weiterbildungsforschung. Band 46. URL: http://www. report-online.net/recherche/einzelhefte_inhalt.asp?id=430 [Abruf: 29.06.2018]

Nürnberger, Jürgen/Maier, Dieter G. (2013): Josephine Levy-Rathenau: Frauenemanzipation durch Berufsberatung. Berlin: Hentrich \& Hentrich.

von Oertzen, Peter (1977): Arbeiterbildung als kritisch-emanzipatorische Erwachsenenbildung. In: Siebert, Horst: Begründungen gegenwärtiger Erwachsenenbildung. Braunschweig: Westermann, S. 159-176.

Ostendorf, Helga (2005): Steuerung des Geschlechterverhältnisses durch eine politische Institution. Die Mädchenpolitik der Berufsberatung. Opladen: Barbara Budrich.

Pätzold, Henning/Ulm, Susanne (2015): Freiwilligkeit in der erwachsenenpädagogischen Beratung. In: Zeitschrift für Weiterbildungsforschung 2, S. 183-196.

Plecas, Darryl/Sork, Tom (1986): Adult Education: Curing the Ills of an Undisciplined Discipline. In: Adult Education Quarterly 37 (1), S. 48-62.

Sauer-Schiffer, Ursula (Hg.) (2004): Bildung und Beratung. Münster: Waxmann.

Schiersmann, Christiane (2013): Ein Kompetenzprofil für Beratende: Resultat eines offenen Koordinierungsprozesses. In: Käpplinger, Bernd et al. (Hg.): Engagement für die Erwachsenenbildung. Wiesbaden: VS Verlag, S. 157-168.

Schiersmann, Christiane/Remmele, Heide (2004): Beratungsfelder in der Weiterbildung: eine empirische Bestandsaufnahme. Baltmannsweiler: Schneider.

Schiersmann, Christiane (2010): Beratung im Feld Bildung, Beruf, Beschäftigung. In: Schiersmann, Christiane/Weber, Peter (Hg.): Beratung in Bildung, Beruf und Beschäftigung - Eckpunkte und Erprobung eines integrierten Qualitätskonzepts. Bielefeld: wbv, S. 25-32.

Schiersmann, Christiane (2016): Beratung als Gegenstand von Studiengängen. In: Gieseke, Wiltrud/Nittel, Dieter (Hg.): Handbuch Pädagogische Beratung über die Lebensspanne, Weinheim/Basel: Juventa, S. 818-827.

Schlüter, Anne (2014): Beratungsfälle - Fallanalysen für die Lern- und Bildungsberatung. Leverkusen: Barbara Budrich.

Schlutz, Erhard (2006): Bildungsdienstleistungen und Angebotsentwicklung. Münster: Waxmann. 
Schober, Karin/Langner, Judith (2017): Wirksamkeit der Beratung in Bildung, Beruf und Beschäftigung. Bielefeld: wbv.

Schrader, Josef (2011): Struktur und Wandel der Weiterbildung. Bielefeld: wbv.

Schröder, Frank/Schlögl, Peter (2014): Weiterbildungsberatung - Qualität definieren, gestalten, reflektieren. Bielefeld: wbv.

Siebert, Horst (1992): Bildung im Schatten der Postmoderne: von Prometheus zu Sisyphos. Frankfurt/Main: VAS.

Stanik, Tim (2016): Beratung in der Weiterbildung als institutionelle Interaktion. Frankfurt/Main: Peter Lang.

Wittpoth, Jürgen (Hg.) (2001): Erwachsenenbildung und Zeitdiagnose. Bielefeld: wbv.

Wolf, Christa (2008): Voraussetzungen einer Erzählung: Kassandra. Frankfurt/Main: Suhrkamp. 
I. Theoretische Zugänge zum Beratungshandeln: Verortung und Diskurse 



\section{Eine resonanztheoretische Betrachtung von Beratung}

\section{Abstract}

Der Beitrag befasst sich mit einer zeitdiagnostischen und resonanztheoretischen Betrachtung zunehmender Beschleunigungs- und Wettbewerbstendenzen sowie den damit einhergehenden Anforderungen und Auswirkungen auf Beratung. Beratung scheint sich nach Auffassung des österreichischen Bildungsforschers Erich Ribolits fest in den verschiedensten Lebensbereichen des postmodernen Menschen etabliert zu haben (Ribolits 2007). Angesichts dessen ließ sich bereits Anfang der 2000er Jahre eine räumliche Entgrenzung von Beratung erkennen (Sultana 2003: 31). Zudem zeichnen sich zeitgenössischen Diagnosen zufolge Entwicklungen einer zunehmenden Individualisierung (Beck 1986) und Modernisierung (Field, zit. n. Hösel u. a. 2017), Wettbewerbsund Optimierungslogik (Rosa 2016a, 2016b) und Beschleunigung (Rosa 2005) $\mathrm{ab}$, welche zu erhöhten Beratungs- und auch Coachingbedarfen führen. So lässt sich der Bedeutungszuwachs als Folge einer zunehmenden Komplexität des heutigen Lebens ${ }^{1}$ deuten (Ribolits 2007), gekennzeichnet durch eine $\mathrm{Zu}$ nahme von Informationen und ,informational overload“, verstärkte Unkalkulierbarkeit auf den Arbeitsmärkten sowie eine „steigende Unsicherheit in den (berufs-)biographischen Perspektiven“ (Arbeitsstab Forum Bildung 2001).

Im Rahmen des Beitrags wird unter resonanztheoretischer Perspektive zum einen die Frage aufgeworfen, inwiefern sich Auswirkungen und Folgen der Beschleunigung auf die Beratung identifizieren lassen. Zum anderen soll beleuchtet werden, ob Beratung eine instrumentalisierte Anpassung an den alternativlosen Mainstream erzeugt oder vielmehr eine Individualisierung verstärkt und die Optimierung, Beschleunigung und Flexibilität bis zur kritiklosen Selbstausbeutung steigert.

Vor dem Hintergrund zunehmender Ökonomisierungstendenzen wird kritisch hinterfragt, inwieweit Beratung als Katalysator des sozialen Wandels angesichts einer zunehmenden Beschleunigungs- und Wettbewerbslogik angesehen werden kann.

Der Beitrag schließt mit einer kritischen Schlussbetrachtung und einem Ausblick auf weiterführende Forschungsdesiderate.

1 Bereits in den 1990er Jahren kam im soziologischen Diskurs die Metapher einer „neuen Unübersichtlichkeit“ auf (Habermas 1985 in Ribolits 2007). 


\section{Einleitung}

Im internationalen Vergleich wird häufig mit Modernisierung und Individualisierung argumentiert, um den steigenden Beratungsbedarf bei beruflichen Übergängen zu erklären. So vergleichen beispielsweise Fanny Hösel und Carlo Terzaroli (2017) vor diesem Hintergrund Übergänge im Erwachsenenalter und entwickeln ein Analyseraster für eine komparative Erforschung der entsprechenden Beratung. In diesem Zusammenhang wird für Beratungsbedarfe konstatiert, dass zunehmende Unsicherheit $-u$. a. verursacht durch eine sich steigernde Schnelllebigkeit, Reichweitenvergrößerung und Optionenvielfalt den individuellen Bedarf an Beratung erhöht (insbesondere an biografischen Transitionen) (Hösel/Terzaroli 2017: 131-135).

Vor diesem Hintergrund finden sich auf Basis heutiger zeitdiagnostischer Betrachtung immer auch Bezüge auf Beratung, Coaching und Optimierung der Lebensführung. Beispielhaft lässt sich in diesem Zusammenhang Niklas Luhmanns Theorie der Sozialen Systeme (1987) nennen: Seine Grundannahme ist, dass nicht der Mensch kommuniziere, sondern die Kommunikation. Demzufolge reduziere das System Komplexität, tendiere zum Selbsterhalt und sei beendet, wenn es aufhöre zu kommunizieren. Im Zeitgeist des Kalten Kriegs bis zur Nachwendezeit, über das Ende der großen Erzählungen und der neoliberalen Alternativlosigkeitsthese werden diese konzeptionellen Überlegungen in die Erwachsenenbildung übertragen (vgl. u. a. Schiersmann 2007).

Beschleunigungstheoretisch wird kritisch auf den Versuch der individuellen Optimierung mittels Beratung rekurriert. Die Thesen des Soziologen Hartmut Rosa weisen darauf hin, dass ein Hamsterrad-Effekt entstehen könnte und dass trotz eifrigster Optimierung bei bestimmten Lebensbezügen - nämlich solchen mit hohem Entfremdungsanteil - ein Burnout eintreten könne. Rosa identifiziert hier fehlende „Resonanz“ als einen Auslöser. Neben dem Theorem der (reflexiven oder Spät-)Moderne provoziert Rosa (2009) mit der These, Beschleunigung erzeuge „,rutschende Abhänge“ (slippery slopes). Das Gefühl bzw. Phänomen der „rutschenden Abhänge“ kann aufgrund einer erhöhten Beschleunigung des Lebenstempos in der Wettbewerbsgesellschaft und dem damit einhergehenden Anpassungsdruck entstehen (Rosa 2016b: 43).

Fehlende Resonanz erzeugt demzufolge Burnouts (Rosa 2016b). Gerade hier sei ein möglichst rechtzeitiges Eingreifen nötig, oft kann eine Beratung vor einer ggf. dann doch notwendigen Therapie bewahren. Beschleunigung kann somit einen Grund für Beratung darstellen. 


\section{Beschleunigung und kein Ende in Sicht?}

Die Theorie der sozialen Beschleunigung Rosa (2005) geht davon aus, dass unsere heutige Gesellschaft einem allumfassenden Beschleunigungsphänomen unterliegt, das unweigerlich dazu führt, dass ,alles immer schneller und immer mehr wird", wodurch sich die Individuen zunehmend in Zeitnot fühlen. Es scheint, dass der „hochtourige Lerner zu Turbobedingungen Prototyp des effektiven Selbstlerners" geworden sei und im Zuge dessen Schnelligkeit zu einem unbefragten Konkurrenzprinzip wird (Meyer-Drawe 2008: 125).

Mit dieser lebensbereichsübergreifenden Beschleunigung und einer zunehmenden Technologisierung geht ein umfassender sozialer, kultureller und ökonomischer Wandel einher, der zu gesellschaftlichen Veränderungen führt (Blossfeld et al. 2017).

Jene Beschleunigungstendenzen vollziehen sich zum einen in Form einer Steigerung der Geschwindigkeit von Transport-, Kommunikations- und Produktionsprozessen durch technische Innovation. Die technische Beschleunigung, angetrieben vornehmlich durch ökonomische Motoren, führt jedoch zugleich zu einem Paradox: Es wird häufig eine Zeitknappheit trotz Zeitgewinn durch technische Beschleunigung wahrgenommen. Hieraus resultieren eine Reichweitenvergrößerung sowie die „moderne Grunderfahrung der Raumvernichtung" (Rosa 2016b: 21) im Sinne eines Schwindens der Bedeutung von Orten und damit einhergehend ein Verlernen von Raum und Entfernung. Zum anderen lassen sich (sich vergleichsweise langsamer vollziehende) Beschleunigungstendenzen des sozialen Wandels identifizieren, verbunden mit einer Steigerung des Tempos, mit dem sich soziale Strukturen, Praxisformen und Beziehungsmuster verändern. Dieser Wandel kann Selbstoptimierungsbestrebungen hervorbringen, die einen erhöhten Anpassungsdruck und das Gefühl, sich auf „rutschenden Abhängen“ (Rosa 2016b: 43) zu befinden, verstärken. Im Zusammenhang von neoliberalen, fremdbestimmten Wettbewerbs- und Optimierungszwängen besteht die Gefahr, dass Beratung „rein“ instrumentell, im Sinne einer Verwertungs- und Selbstoptimierungslogik, angesehen wird. In diesem Zusammenhang beschreibt der Beratungswissenschaftler Clinton Enoch eine zunehmende Marktkonkurrenz - im Sinne einer „verbetriebswirtschaftlichten“ Lebensführung - welche das Fürsorgeprinzip zu dominieren scheint (Enoch 2011).

Des Weiteren beschleunigt sich, neben der durch kulturelle Gegebenheiten bedingten technischen und sozialen Beschleunigung in einem selbstantreibenden Akzelerationszirkel (Rosa 2016a: 203), das Lebenstempo: Dieses zeigt sich u. a. in einer Steigerung der Anzahl an Handlungs- oder Erlebnisepisoden pro Zeiteinheit. Das impliziert, dass es zu einer objektiv wahrgenommenen Verkürzung oder Verdichtung von Handlungsepisoden (,the 
power of the deadline“") kommen und subjektiv eine enorme Zeitnot empfunden werden kann (,rasender Stillstand“) (Rosa 2016b: 27).

Diesen zunehmenden Zeitdruck und die steigende Aufgabenmenge beschreibt der Jenaer Soziologe Hartmut Rosa als scheinbare Hauptbelastungsquelle in der modernen Arbeitswelt.

Folgt man Rosas Argumentation, so könnte eine Resonanz als eine Antwort auf die Beschleunigung gesehen werden. Ihm zufolge stellt „Resonanz [...] eine durch Affizierung und Emotion, intrinsisches Interesse und Selbstwirksamkeitserwartung gebildete Form der Weltbeziehung [dar], in der sich Subjekt und Welt gegenseitig berühren und zugleich transformieren [...]. Resonanz ist [somit] keine Echo-, sondern eine Antwortbeziehung [...].

Resonanz ist kein emotionaler Zustand, sondern ein Beziehungsmodus. Dieser ist gegenüber dem emotionalen Inhalt neutral“" (Rosa 2016b: 298).

Das Pendant zur Resonanz wäre die Entfremdung als ,[...] eine spezifische Form der Weltbeziehung, in der Subjekt und Welt einander indifferent oder feindlich (repulsiv) und mithin innerlich unverbunden gegenüberstehen [...]. Entfremdung definiert damit einen Zustand, in dem die, Weltanverwandlung' misslingt, so dass die Welt stets kalt, starr, abweisend und nichtresponsiv erscheint [...]" (Rosa 2016b: 316).

Beschleunigungsprozesse sind (häufig) mit Eskalationstendenzen verbunden, so dass die Stellung des Subjekts stets essentiellen Veränderungen unterworfen ist - was wiederum eine fortwährende Störung des Resonanzverhältnisses darstellt. Dieses dialektische Verhältnis von resonanten und entfremdeten Momenten ist jedoch erforderlich. Denn als gelungene Lebensform kann nur ein „dialektisches Wechselverhältnis zwischen Entfremdungs- und Resonanzmomenten der Weltbeziehung" (Rosa 2016b: 298) angesehen werden.

Wie kann demzufolge auf die skizzierten Entwicklungen reagiert werden? Und wie können förderliche Bedingungen für ein „gelungenes Leben“ im Sinne einer Dialektik von resonanten und entfremdeten Momenten geschaffen werden? Anders formuliert:

„,...] wenn Beschleunigung [bzw. die (häufig) mit ihr verbundenen Eskalationstendenzen bzw. die fortwährende Störung des Resonanzverhältnisses] das Problem ist, dann ist Resonanz vielleicht die Lösung?" (Rosa 2016b: 13). Und welche Rolle könnte hierbei auf die unterschiedlichen (zeit- und ortsunabhängigen) Formate und Modi der Beratung zukommen?

Eine mögliche Reaktion auf die Beschleunigung der Umwelt und die daraus häufig resultierenden Entfremdungen wird oftmals in einer Verlangsamung des Subjekts gesehen. Eine Entschleunigung erscheint - den resonanztheoretischen Überlegungen von Hartmut Rosa folgend - jedoch keine adäquate Antwort darzustellen, wie beispielsweise im Leitgedanken des Achtsamkeitsdiskurses festhalten (Langemeyer 2015). In diesem Zusammenhang ist kritisch anzumerken, dass eine isolierte Betrachtung der Einheit 
,Zeit‘, ohne Berücksichtigung der politischen Dimension, von Aspekten der ökonomischen Verwertbarkeit und einer Betrachtung des Subjekts innerhalb seiner sozialen Weltverhältnisse und Rollenkonstellationen zu kurz greift.

Die Intention der Achtsamkeitsbewegung richtet sich auf den Aspekt der Entschleunigung. Dies steht in klarem Widerspruch zu Rosas Theorie, die als Antwort auf die Beschleunigung Resonanz ansieht. Denn Verlangsamung stellt seines Erachtens keine Gegenkraft zur Beschleunigung dar, sondern wird vielmehr als Folgewirkung der Beschleunigung angesehen (Rosa 2016b: 56). Nicht die Verlangsamung, sondern vielmehr der Beziehungsmodus steht demzufolge im Fokus.

In diesem Zusammenhang stellt sich die Frage, ob bzw. inwiefern die Beschleunigung einen Grund für die Beratung darstellt. Konkreter: Kann (und soll) Beratung jene Beschleunigungstendenzen tatsächlich aufhalten? Und inwiefern kann Beratung demzufolge (instrumentell) beschleunigungsfördernd wirken oder auch positiv eingesetzt vor einer ggf. notwendigen Therapie (beispielsweise bei Burnout) bewahren? Eine resonanztheoretische Ausdeutung von Beratung kann u. a. in der durch die Beschleunigung herbeigeführten Reichweitenvergrößerung gesehen werden. Ein Problem kann hierbei die Erzeugung eines Antwort-Verhältnisses in der Subjekt-(Um-)Welt-Beziehung darstellen. Gleichsam kann Beratung helfen, aus dem Beschleunigungszirkel ,auszusteigen“. So bilden Resonanz und Entfremdung in der Beratung notwendige Momente, die Irritationen ermöglichen und „unversöhnlich“ im Beratungsprozess sein können.

\section{Relevanz einer Resonanzachse ,Lebenslanges Lernen'}

Ausgehend von Hartmut Rosas Konzeption der Resonanzachsen soll im Folgenden die Bedeutung einer konzeptionellen Erweiterung des Modells durch eine Resonanzachse ,Lebenslanges Lernen', welche sowohl Bildungs- als auch Beratungsprozesse über die Lebensspannen impliziert, erläutert werden.

In diesem Zusammenhang erscheint der von Rosa verwendete Bildungsbegriff unzureichend, da dieser die Spezifika des Lernens im Erwachsenenalter nur ungenügend ausdeutet. Die (Weiter-)Bildung beziehungsweise das Lernen Erwachsener umfasst mehr als den schulischen oder institutionellen Bereich. Zudem sollten neben den „klassischen“ formellen zunehmend auch non-formelle und informelle Lernkontexte deutlicher Berücksichtigung finden. In diesem Zusammenhang lassen sich nur ungenügend Parallelen zu Rosas resonanztheoretischen Überlegungen herstellen und nur zu Teilen Übertragungen herleiten. Dementsprechend ist das Bildungsverständnis in Rosas 
Ausführungen meines Erachtens an einigen Stellen limitiert und reduziert Bildung auf die Institution Schule.

Ferner orientiert sich der normative Bildungsbegriff des Resonanztheoretikers Hartmut Rosa in erheblichem Maße an bildungsbürgerlichen Themen und Kategorien beziehungsweise am Habitus (Rosa 2016a: 420). Rosas Bildungsverständnis enthält, der subjektwissenschaftlichen Betrachtungsweise folgend und im Gegensatz zu identitätstheoretischen oder konstruktivistischen Ansätzen, eine inhaltliche Setzung und bringt Bildung und subjektive Entwicklung in einen engen Bezug (Initiative Kritische Erwachsenenbildung 2012: 19f.). Eine Kontextuierung in gesellschaftliche Zusammenhänge und Strukturen wird von Rosa zwar größtenteils vorgenommen, diese erscheinen meiner Auffassung nach jedoch in seinen Ausführungen zum Bildungsbegriff bisweilen von gesellschaftlichen Verhältnissen vom Bedingungshintergrund beziehungsweise von der Veränderung des Bildungsbegriffes im Verlauf der Modernen losgelöst. So werden in einer rückblickenden Betrachtung des Bildungsbegriffes weder seine Historizität noch seine Multiperspektivität (im Hinblick auf Interessenaushandlungen) und Heterogenität, im Sinne von Kontextuierung in kulturelle und gesellschaftliche Verhältnisse, ersichtlich (Faulstich 2016: 53) - angefangen vom Bildungsverständnis der Aufklärung, welches durch das Bestreben nach erweiterter Mündigkeit gegen feudale, absolutistische und totalitäre Herrschaftsformen gekennzeichnet ist (Faulstich 2016: 53), bis hin zum Neuhumanismus, in dem Bildungsbestrebungen den Wert der Individualität des Einzelnen und seine Entwicklung fokussierten (Faulstich 2016: 55). Zugleich wird „Bildung zum Privileg der Gebildeten und zum Legitimationsinstrument der groben ,feinen Unterschiede“" (Bourdieu 1982 in Faulstich 2016: 55) und losgelöst von der Herrschaftskritik beziehungsweise der Kritik bestehender Verhältnisse. Der Hamburger Erziehungswissenschaftler Peter Faulstich zeigt in diesem Zusammenhang einen aktuell wiederkehrenden radikalisierenden Individualismus auf, der im Kontrast zur Vorstellung eines ,vergesellschaftlichten“ Subjekts steht, welches durch seine milieu- beziehungsweise schichtspezifischen Erfahrungen geprägt und auch in diese Verhältnisse eingebunden ist (Faulstich 2016: 56).

\section{Wettbewerbs- und Selbstoptimierungslogik: höher, schneller, weiter}

Die Schaffung und Bereitstellung von verschiedenartigen (Weiter-)Bildungsund Beratungsstrukturen kann zweifelsohne grundlegend als (zunächst einmal) positiv bewertet werden, sofern diese als eine Chance zum Abbau von 
Bildungshemmnissen betrachtet wird und zu einer Aufweichung sozialer Selektivität beiträgt.

Die Ambivalenz des Lebenslangen Lernens birgt neben den Möglichkeiten, Lernen als positiv und als subjektiv nützlich zu empfinden, aber auch die Gefahr, Lebenslanges Lernen - beziehungsweise die Ausdehnung des Lernens auf die gesamte Lebensspanne und -welt - als eine Art Zwang zu empfinden, welcher gegebenenfalls einen Anpassungsdruck erzeugen kann, der durch Angst, Last, Überforderung und Fremdbestimmung gekennzeichnet ist (Faulstich 2006: 7; Erler 2012: 99). Ein solches Anpassungslernen lässt sich aufgrund der sogenannten „Wandelmetaphorik“ erklären: Das Subjekt fühlt sich in seiner Umwelt permanenten Veränderungen ausgesetzt, was wiederum einen Zwang zum „lebenslänglichen“ Lernen, um „,wettbewerbsfähig“ zu bleiben, erzeugen kann (Faulstich 2006: 11). Das heißt, wenn dem Lernen ausschließlich Individualisierungs- und Flexibilisierungsstrategien unterliegen, kann dieses zu einem Zwangsempfingen für den Lernenden führen (Faulstich 2006: 10).

Darüber hinaus kann eine geringe Bereitschaft, stetig weiter zu lernen, daraus resultieren, dass eine an den eigenen Lebensinteressen orientierte Zielrichtung fehlt (Faulstich 2006: 10). Die Auseinandersetzung mit dem jeweiligen Ausschnitt der Lebenswelt ist dabei immer an soziale Kontexte von Milieu und Gender gekoppelt (Faulstich 2006: 20).

Eine zunehmende Zahl von Rankings, Testverfahren, Evaluationen oder Qualitätsmessungen erzeugt zudem einen Druck, wie aktuell geführte Diskussionen zur Selbstoptimierung beziehungsweise zum Selbstoptimierungszwang verdeutlichen. Dieser basiert darauf, dass verstärkt das Individuum für die Gestaltung seines eigenen Berufs- und Lebensweges verantwortlich gemacht wird, sowie auf der Erkenntnis, dass die Anzahl an Normalbiografien abnimmt, Arbeitsverhältnisse ${ }^{2}$ destandardisiert werden und die Zahl der „Arbeitskraftunternehmer" zunimmt (Voß/Pongratz 2004). Diesen Wandel der Arbeitsstrukturen und den Anstieg sogenannter diskontinuierlicher Erwerbsbiografien - auch als Mosaik- oder Patchworkbiografien bezeichnet - bilden die von Hartmut Rosa angestellten resonanztheoretischen Überlegungen nur unzureichend $\mathrm{ab}$.

Als maßgeblich ursächlich die Veränderung von Arbeitsbiografien können die zunehmenden Beschleunigungstendenzen, die vergrößerten Wahlmöglichkeiten im räumlichen, zeitlichen und sozialen Handlungsspektrum angesehen werden.

2 Laut Statistischem Bundesamt hat der Anteil der atypischen Beschäftigten an allen Erwerbstätigen um mehr als die Hälfte zugenommen, von 13\% (1991) auf 21\% (2014): Mittlerweile arbeitet also jede/r fünfte nicht als „Normalarbeiter/in“(Wissenschaftlicher Beraterkreis der Gewerkschaften IG Metall und ver.di 2017: 10). 
Aufgrund einer deutlichen Zunahme von Lebensphasen, -ereignissen und Transitionen - wie z. B. Erwerblosigkeit, unfreiwillige Berufs-, Betriebsoder Arbeitsplatzwechsel - ist es erforderlich, diesen veränderten Lebensmustern, resultierend aus der Akkumulation, Verkürzung und Stückelung der individuellen und organisationalen Lebensphasen, zu begegnen und diese in theoretische Überlegungen und Ausdeutungen von Beratung zu berücksichtigen (Wissenschaftlicher Beraterkreis der Gewerkschaften IG Metall und ver. di 2017: 26).

\section{Die Resonanzachse, Lebenslanges Lernen'}

In Rosas Modell der Resonanzachsen werden diese Lern- und Bildungsprozesse zu unspezifisch abgebildet. Folglich bedarf es einer stärkeren Integration von Beratung und Bildung im Erwachsenenalter im Resonanzkonzept. Es scheint erforderlich, Rosas Konzeption der Resonanzachsen über die schulische Erstausbildung im Kindes- und Jugendalter mit einer Resonanzachse ,Lebenslanges Lernen“ (die u. a. Coaching und Beratung umfasst) zu ergänzen, da das Lernen und die Bildungsaktivitäten über die Phase des Erwachsenenalters in einer lebenslaufanalytischen Perspektive einen weitaus größeren Anteil haben. Das Lebenslange Lernen stellt eine übergeordnete Resonanzachse dar, die Rosas vorgesehene Resonanzachse ,Schule' als eine Unterkategorie beziehungsweise als einen Teil des Lernens im Laufe des Lebens begreift. Die Resonanzachse ,Lebenslanges Lernen“ umfasst demzufolge jegliche Bildungs- und Beratungsaktivitäten über die gesamte Lebensspanne und beinhaltet unterschiedliche Orte, Modi und Formate des Lernens (Trias des formalen, non-formalen, informellen Lernens) als auch ein mannigfaltiges Spektrum an Bereichen und Inhalten. Hierbei spannt sich die Resonanzachse ,Lebenslanges Lernen“ im Feld ,[... zwischen gesellschaftlichen, (bildungs-) politischen und ökonomischen Ansprüchen auf der einen, Zielsetzungen der jeweiligen Akteure - Lernende wie Lehrende, Repräsentanten von öffentlichen und privaten Bildungsinstitutionen, von Bildungspolitik oder Wissenschaft - auf der anderen Seite auf" (Zeuner 2008: 532).

Das Lebenslange Lernen und Beratung als Resonanzsphäre sind hierbei dem ständigen Prozess zwischen Widerstand und Anpassung im Sinne der Dialektik ausgesetzt. 


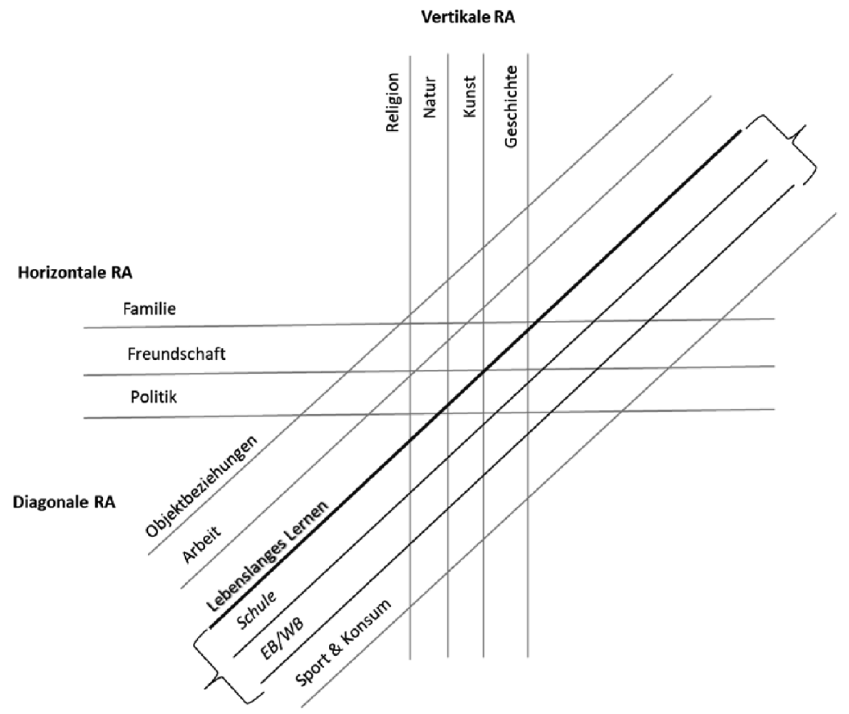

Abb. 1: Resonanzachse ,Lebenslanges Lernen“ (Quelle: eigene Darstellung in Anlehnung an Rosa)

In einer kritischen Schlussbetrachtung lässt sich die Resonanztheorie von Hartmut Rosa als ein multi- und interdisziplinärer Ansatz verstehen, der Leiblichkeit und Stofflichkeit einbindet und sich vom Kulturpessimismus der Kritischen Theorie und dem theorieimmanenten Anspruch, die Welt durch kritische Einsicht zu verändern, distanziert. So bezieht sich u. a. die Kritik des Erziehungswissenschaftlers Micha Brumlik an Rosas Resonanztheorie darauf, dass die Resonanztheorie nicht das (in der Kritischen Theorie verankerte) Interesse an der Aufhebung des gesellschaftlichen Unrechts in einer „unversöhnlichen“ Art impliziert. Brumlik merkt an, dass „,...] als Kennzeichen des Alters [...] gemeinhin Weisheit, Milde und Besonnenheit [gelten] - der mit Rosa altgewordenen Theorie fehlt daher das, was früher einmal die Schroffheit und Klarsicht der Kritischen Theorie, ihre unversöhnliche Haltung gegenüber den Verhältnissen, so jedenfalls noch Horkheimer und Adorno, ausmachte. In gewisser Weise hat diese letztmögliche Form einer Kritischen Theorie ihren Frieden mit den Verhältnissen gemacht", so Brumlik (Brumlik 2016: 123). Der Kritik, Rosas Theorie lasse sich nicht in Bezug zur Kritischen Theorie setzen, kann jedoch nicht uneingeschränkt zugestimmt werden: Zum einen gibt es innerhalb der Kritischen Theorie eine große Bandbreite der Verengung, Auslegung beziehungsweise der Ausübung „unversöhnlicher" gesellschaftlicher Kritik. Innerhalb des Diskurses lassen sich 
Differenzen im Hinblick auf die Theorieauslegung von Vertreterinnen und Vertretern der Kritischen Theorie erkennen (wie u. a. bei Christine Zeuner, Elke Gruber, Daniela Holzer, Ludwig A. Pongratz, bis hin zu Erich Ribolits sichtbar wird).

Bei der weiteren kritischen Übertragung des dem Konzept zugrundeliegenden Bildungsverständnisses werden Desiderate sichtbar: zum einen eine unzureichende kritische Perspektive auf Schicht- und Milieuunterschiede aufgrund eines „elitären“ Bildungsbegriffs, der sich stark auf das Bürgertum und nur unzureichend auf die Arbeiterbildung richtet. In diesem Zusammenhang ist es sinnvoll, Bezüge zu Bildungstheoretikern wie z. B. Heydorn herzustellen, nach deren Auffassung Bildung dazu beitragen soll, Bildungsunterschiede sowie Unterdrückung (gemäß dem Leitgedanken der Kritischen Theorie) entgegenzuwirken. Zum anderen bezieht sich Rosa auf (Teil-)Aspekte des humanistischen, humboldtschen Bildungsideals - dieses greift jedoch $\mathrm{zu}$ kurz. Eine Begriffserweiterung ist daher nötig - nicht eine Verengung des Bildungsverständnisses auf die Institution Schule - und zugleich eine stärkere Berücksichtigung von Weiterbildungsaktivitäten und mannigfaltigen Formen des informellen Lernens. Übertragen auf den arbeitsweltlichen Kontext zeigen sich weitere Forschungsdesiderate: Beispielweise bleibt zu diskutieren, inwiefern Resonanzverhältnisse kollektiver Gewerkschaftsbeziehungen im Aushandlungsprozess von Interessen resonanztheoretisch expliziter gedeutet werden können.

\section{Resümee und Ausblick}

Konstatierend lassen sich unterschiedliche bildungssoziologische Thesen im Zeitverlauf erkennen: von Diskursen zur Individualisierung und zum Arbeitskraftunternehmer (Käpplinger und Maier-Gutheil 2015) über die rutschenden Abhänge (Rosa 2016b) hin zu einer Gesellschaft der Angst (Bude 2014).

Zusammenfassend kann festgehalten werden, dass keine Beratung ohne gesellschaftstheoretische Kritik möglich ist. Gesellschaftsdiagnosen zeigen, dass das Individuum im 21. Jahrhundert nicht mehr uneingeschränkt positiv besetzt ist, wie es noch zu Rogers' Zeiten der Fall war.

Die Lösung der Probleme rutschender Abhänge (Rosa 2016b) kann nicht durch Beratung zu besserer Optimierung (Koppetsch 2013) gelingen. Folglich muss Beratung nicht als bloße Begleiterscheinung, sondern als ein wesentlicher Katalysator des gesellschaftlichen Wandels angesehen werden (Ribolits 2007). Es wird somit darauf ankommen, die Ratsuchenden auch mit Gesellschaftstheorie zu konfrontieren. Nur so können sie ihre Lage als struktu- 
relles Problem erkennen, welches mit individuellen Lösungen nur begrenzt beherrschbar wird. Dadurch kann Beratung reflexiv und erfüllend werden. Neben Beratung kann auch Bildung eine resonante Funktion einnehmen, die Frage einer „Resonanzachse Lebenslanges Lernen“ ließe sich in diesem $\mathrm{Zu}$ sammenhang weiterführend entfalten.

\section{Literatur}

Arbeitsstab Forum Bildung (Hg.) (2001): Lernen - ein Leben lang. Vorläufige Empfehlungen und Expertenbericht. Bonn.

Beck, Ulrich (1986): Risikogesellschaft - auf dem Weg in eine andere Moderne. Frankfurt a. M.: Suhrkamp.

Blossfeld, Hans-Peter et al. (2017): Bildung 2030 - veränderte Welt. Fragen an die Bildungspolitik. Gutachten. Vereinigung der Bayerischen Wirtschaft e. V. (Hg.). Münster: Waxmann.

Bude, Heinz (2014): Gesellschaft der Angst. Hamburg: Hamburger Edition, HIS.

Brumlik, Micha (2016): Resonanz oder: Das Ende der kritischen Theorie. Blätter für deutsche und internationale Politik, 5/2016, S. 120-123.

Enoch, Clinton (2011): Dimensionen der Wissensvermittlung in Beratungsprozessen. Gesprächsanalysen der beruflichen Beratung. Wiesbaden: VS Verlag.

Erler, Ingolf (2012): Das Lebenslange Lernen und die Wissensgesellschaft. In: Anzengruber, Grete et al. (Hg.): Kritisch denken: für eine andere Erwachsenenbildung. Schulheft 148. 37. Jg/ 2012. Innsbruck, Wien, Bozen: Studienverlag, S. 93-107.

Faulstich, Peter (2016): Das Politische in der Bildung. In: Hufer, Klaus-Peter/Lange, Dirk (Hg.): Handbuch politische Erwachsenenbildung. Reihe Politik und Bildung. Bd. 74. Schwalbach/Taunus: Wochenschau Verlag, S. 52-61.

Faulstich, Peter (2006): Lernen und Widerstände. In: Faulstich, Peter/Bayer, Mechthild (Hg.): Lernwiderstände. Anlässe für Vermittlung und Beratung. Eine Initiative von ver.di, IG Metall und GEW. Hamburg: VSA-Verlag, S. 7-25.

Graf, Anita (2011): Lebenszyklusorientierte Personalentwicklung als Ausgangspunkt für den Erhalt der Arbeitsmarktfähigkeit. In: Seyfried, Brigitte: Ältere Beschäftigte. Zu jung um alt zu sein. Konzepte - Forschungsergebnisse - Instrumente. Bielefeld: Bertelsmann Verlag, S. 93-106.

Hösel, Fanny/Terzaroli, Carlo (2017): Work Transitions in Adulthood: An Analytical Tool for Comparative Studies. In: Regina Egetenmeyer, Sabine Schmidt-Lauff und Vanna Boffo (Hg.): Adult learning and education in international contexts. Future challenges for its professionalization: comparative perspectives from the 2016 Wurzburg Winter School. Frankfurt am Main: Peter Lang (Studies in Pedagogy, Andragogy, and Gerontagogy, Vol. 69), S. 131-146.

Initiative Kritische Erwachsenenbildung (2012): Ansätze des Kritischen in der Erwachsenenbildung - eine Spurensuche. In: Anzengruber, Grete et al. (Hg.): Kritisch denken: für eine andere Erwachsenenbildung. Schulheft 148. 37. Jg/ 2012. Innsbruck, Wien, Bozen: Studienverlag, S. 9-23. 
Käpplinger, Bernd/Maier-Gutheil, Cornelia (2015): Ansätze und Ergebnisse zur Beratung(sforschung) in der Erwachsenen- und Weiterbildung - Eine Systematisierung. In: ZfW 38 (2), S. 163-181.

Koppetsch, Cornelia (2013): Die Wiederkehr der Konformität. Streifzüge durch die gefährdete Mitte. Frankfurt am Main: Campus Verlag.

Langemeyer, Ines (2015): Das Wissen der Achtsamkeit. Kooperative Kompetenz in komplexen Arbeitsprozessen. Münster, New York: Waxmann.

Luhmann, Niklas (1987): Soziale Systeme. Grundriss einer allgemeinen Theorie, Berlin: Suhrkamp.

Meyer-Drawe, Käte (2008): Diskurse des Lernens. München: Wilhelm Fink Verlag.

Ribolits, Erich (2007): Führe mich sanft. Beratung, Coaching \& Co. - die postmodernen Instrumente der Gouvernementalität. In: Schulheft (32) 126, 3/2007. Innsbruck, Wien, Bozen: Studienverlag.

Rosa, Hartmut (2016a): Weltbeziehungen im Zeitalter der Beschleunigung. Umrisse einer neuen kritischen Gesellschaftskritik. Berlin: Suhrkamp.

Rosa, Hartmut (2016b): Resonanz. Eine Soziologie der Weltbeziehung. Berlin: Suhrkamp.

Rosa, Hartmut (2009): Kritik der Zeitverhältnisse. Beschleunigung und Entfremdung als Schlüsselbegriffe der Sozialkritik.

Rosa, Hartmut (2005): Beschleunigung. Die Veränderung der Zeitstrukturen in der Moderne. Frankfurt a. M.: Suhrkamp.

Schiersmann, Christiane (2007): Systemische Beratung. Studienbrief: Universität Heidelberg.

Voß, Gerd-Günter/Pongratz, Hans J. (2004): Arbeitskraftunternehmer. 2. Auflage. Berlin: edition sigma.

Sultana, Ronald G. (2003): Strategien zur Bildungs- und Berufsberatung - Trends, Herausforderungen und Herangehensweise in Europa. Thessaloniki: CEDEFOP.

Wissenschaftlicher Beraterkreis der Gewerkschaften IG Metall und ver.di (2017): Berufsbildungsperspektiven 2017. Gute Arbeit braucht gute Weiterbildung. Berlin/ Frankfurt a. M.

Zeuner, Christine (2008): Erwachsenenbildung. In: Faulstich-Wieland, Hannelore/ Faulstich, Peter (Hg.): Erziehungswissenschaft. Ein Grundkurs. Hamburg: Rowohlt Verlag, S.532-555. 


\section{Lernberatung im Diskurs der Erwachsenenbildung: Eine systematische Reflexion}

\section{Einleitung}

Die pädagogische Handlungsform der Beratung hat eine lange Tradition (vgl. im Überblick Maier-Gutheil 2016) und gewinnt in der Erwachsenen- und Weiterbildung vor dem Hintergrund gesellschaftlicher Individualisierungsprozesse sowie durch die disziplinären Debatten um die Förderung selbstgesteuerten Lernens und einer „neuen Lernkultur“ zunehmend an Bedeutung. Gerade im Kontext der letztgenannten Diskurse spielen spezifische Formen von „Lernberatung“ eine zentrale Rolle.

Dabei zeigt sich zunächst, dass der Begriff Lernberatung äußerst unterschiedlich verwendet wird. So lassen sich zum einen enge Lernberatungsverständnisse, bei denen das Beratungsgespräch selbst im Mittelpunkt steht, von weiten Verständnissen, bei denen Lernberatung als ermöglichungsdidaktische pädagogische Haltung konzipiert wird, unterscheiden (vgl. Kollewe 2009, S. 127). Zum anderen wird zwischen expliziten (also intendierten) und impliziten (also mitgängigen und beiläufigen) Formen der Lernberatung differenziert (vgl. Maier-Gutheil 2016, S. 20). Diese Heterogenität möglicher Begriffsverständnisse setzt sich auch bei konzeptionellen Überlegungen zur Lernberatung fort. So liegen unterschiedliche Konzepte zur Lernberatung vor, die bislang kaum systematisch vergleichend betrachtet wurden (vgl. zu ersten Systematisierungen Ludwig 2016).

Vor diesem Hintergrund wird in diesem Beitrag mittels einer explorativen Analyse des Diskurses der Versuch unternommen, eine theoriegeleitete Systematisierung unterschiedlicher Konzeptionen von Lernberatung vorzunehmen. Dazu wird zunächst der aktuelle Forschungsstand zur Lernberatung skizziert. Anschließend wird eine systemtheoretisch orientierte Analyseperspektive entwickelt, mit Hilfe derer Sinnzuschreibungen von Lernberatungskonzeptionen untersucht werden können. Im Anschluss daran werden drei ausgewählte - enge und explizite - Lernberatungskonzeptionen analysiert und die Ergebnisse dieser Analyse im Lichte des Forschungsstands diskutiert, bevor abschließend Anschlussmöglichkeiten für weitere erwachsenenpädagogische Forschungen reflektiert werden. 


\section{Lernberatung: Ein Blick auf den Forschungsstand}

Der Diskurs zum Thema Lernberatung entwickelte sich explizit seit 1980er Jahren und kann insgesamt als überaus heterogen beschrieben werden (vgl. u. a. zur Entstehungsgeschichte Fuchs-Brüninghoff 2000). Matthias Rohs und Bernd Käpplinger haben den Begriff daher auch als einen „Omnibusbegriff auf Erfolgstour" (2004) bezeichnet. Im Vergleich zu der vielfältigen begrifflichen und konzeptionellen Auseinandersetzung erscheint die empirische Forschung jedoch eher unterrepräsentiert (vgl. Ludwig 2012; Käpplinger und Maier-Gutheil 2015). Insgesamt lassen sich hier überblicksartig zwei differente Forschungsstränge unterscheiden:

- Zum einen gibt es - wenige - Studien, die den Beratungsprozess oder das der Lernberatungspraxis zugrundeliegende Konzept empirisch untersuchen. Hier werden erstens mit Blick auf den Aspekt der Vermittlung Lehrende im Hinblick auf ihre Praxis der Lernberatung befragt bzw. es werden zweitens mit Blick auf Aneignungsprozesse Wahrnehmungen von Lernenden zum eigenen Lernen erhoben. Hinsichtlich der Lehrenden zeigt sich hier beispielsweise, dass Lernberatung vor allem als eine das Lehren ergänzende Praktik verstanden wird, die hohe Anforderungen an die Professionalität von Lehrenden stellt, welche insbesondere durch Berufserfahrung und eigenverantwortliche Professionalisierungsprozesse angeeignet wird (vgl. Pätzold 2004; Wrana und Maier Reinhard 2012; Springer und Forneck 2005). Hinsichtlich der Lernenden werden in einer Studie zu Alphabetisierungsprozessen und Lernberatung die Lernbegründungen von Teilnehmenden rekonstruiert, die dann als Anschlussmöglichkeiten zur Ausgestaltung von Lernberatung betrachtet werden (vgl. Ludwig 2012).

- Zum anderen gibt es Studien, die sich mit Diskursen und Programmen im Kontext von Lernberatung auseinandersetzen. So zeigt eine programmanalytische Studie, dass der durchaus komplexe Begriff der Lernberatung in Programmen der Erwachsenenbildung kaum Verwendung findet (vgl. Kollewe und Seitter 2009). Schließlich wird Lernberatung in diskursanalytischen Studien (vgl. z. B. Kossack 2006; Wrana 2012) als gouvernementale Subjektivierungspraktik dekonstruiert. Peter Kossack verweist in seiner dekonstruktiven Analyse auf spezifische Konstruktionsmuster von Lernberatungskonzeptionen. Deutlich wird dabei unter anderem, dass „das Steuerungsprinzip der konstruktivistischen Lernberatungskonzeptionen unterlaufen wird, indem ihm die Notwendigkeit der pädagogischen Außensteuerung entgegengesetzt wird“ (Kossack 2006, S. 184), dass die eingeforderte Symmetrisierung in der Beratungsinteraktion strukturell kaum eingelöst werden kann und der Übergang von einer defizitorientierten zu einer kompetenz- oder ressourcenorientierten Lernberatung als 
eine semantische Abfederung defizitärer Beratungsanlässe interpretiert werden muss (vgl. ebd.). Zudem liegen handlungstheoretische Systematisierungen von Lernberatungskonzepten vor (Ludwig 2016), die Lernberatungskonzepte nach defizitären oder subjektorientierten Ausgangspunkten als Grundlage des Beratungsgespräches unterscheiden.

Ergänzend zu diesen vorliegenden dekonstruktiven und handlungstheoretischen Systematisierungen wird im Folgenden eine weitere Möglichkeit der Systematisierung vorgeschlagen, mit der in den Blick genommen werden kann, welche (manifesten und latenten) Sinnstrukturen in Lernberatungskonzeptionen enthalten sind.

\section{Methodisches Vorgehen: Zur Operationalisierung theoretischer Sinndimensionen}

Erkenntnisleitend für die von uns vorgenommene systematisierende Analyse ist die Frage, welche Sinnkonstruktionen in unterschiedlichen Lernberatungskonzeptionen beobachtet werden können. Zur Bearbeitung dieser Fragestellung wurde eine deduktive Vorgehensweise gewählt, bei der in einem ersten Schritt theoriegeleitet eine Analyseperspektive entwickelt wird, die auf den Grundannahmen funktionaler, systemtheoretischer Theorieperspektiven zur Bedeutung von Sinn in sozialen Systemen aufbaut (vgl. Luhmann 1984). Mit diesem beobachtungstheoretischen Zugang können Lernberatungskonzeptionen als Beobachtungen des Interaktionssystems „Lernberatung“ verstanden werden, in denen - konzeptionelle - Sinnzuschreibungen sichtbar werden. Zur Analyse dieser Sinnzuschreibungen wird die theoretische Differenzierung einer sachlichen, sozialen und zeitlichen Sinndimension nach Niklas Luhmann im Hinblick auf Lernberatungskonzeptionen operationalisiert.

- Die sachliche Sinndimension verweist im Hinblick auf soziale Systeme auf Themen sinnhafter Kommunikation, die entweder als angemessen wahrgenommen werden und damit ,passen“ oder als unangemessen oder unpassend ausgeschlossen werden (vgl. Luhmann 1984, S. 114ff.). Im Hinblick auf Lernberatungskonzeptionen werden für die Analyse in dieser Sinndimension Themen und Gegenstände gefasst, die für das Beratungsgespräch konzeptionell inkludiert werden.

- Die soziale Sinndimension konstituiert sich im Möglichkeitshorizont der Kommunikationspartnerinnen und -partner. In der Sozialdimension wird das Zusammenspiel ihrer unterschiedlichen Perspektiven auf den und in dem Beratungsprozess relevant (vgl. Luhmann 1984, S. 119ff). Für die Analyse von Lernberatungskonzeptionen erscheint in dieser Dimension 
insbesondere die Frage relevant, welche Themen in der Kommunikation zwischen den Beteiligten auf der sachlichen Sinndimension in den Konzeptionen ein- oder ausgeschlossen werden und welche Rollenkonstrukte den Interaktionspartnerinnen und Interaktionspartnern konzeptionell zugeschrieben werden.

- Die zeitliche Sinndimension artikuliert sich im Horizont von Vergangenheit und Zukunft. Sie ermöglicht die differenzierte Beobachtung des Verstreichens von Zeit (vgl. Luhmann 1984, S. 115ff). Zur Analyse von Lernberatungskonzeptionen wird vorgeschlagen, hier die zeitliche Einund Zuordnung von Beratungsgesprächen innerhalb von strukturierten, formalen Lehr-Lernkontexten zu fokussieren.

Diese - für Lernberatungskonzeptionen operationalisierten - Sinndimensionierungen werden im zweiten Schritt als theoriegeleitete, deduktive Suchstrategien für die explorative Untersuchung dreier verschiedener Lernberatungskonzeptionen genutzt. Die untersuchten Konzepte wurden dabei nach zwei Kriterien ausgewählt. Zum einen sollte es sich um enge und explizite Lernberatungskonzeptionen handeln, die das Beratungsgespräch selbst fokussieren. Zum zweiten sollten die Konzeptionen eine explizite Bezugnahme zu formalen Lehr-Lernsettings (z. B. Kurse, Seminare, Trainings) aufweisen, da darüber die Anschlussmöglichkeiten zwischen didaktischem und beratendem Handeln sichtbar gemacht werden können. Diesen Kriterien entsprechend wurden folgende Lernberatungskonzeptionen für die Analyse ausgewählt: (a) das Konzept der „Lernerberatung“ (vgl. Kaiser u. a. 2007a, 2007b), (b) das Konzept von Lernberatung im Kontext von Selbstlernarchitekturen (vgl. Forneck 2005, 2006; Forneck und Springer 2005) und (c) das Konzept der rekonstruktiven Lernberatung (vgl. Ludwig 2012).

\section{Ergebnisse: Sinnkonstruktionen in Lernberatungskonzeptionen}

In der Darstellung der tentativen Analyseergebnisse wird zunächst das jeweilige Lernberatungskonzept kurz skizziert, bevor die Ergebnisse der Analyse anhand der operationalisierten Sinndimensionen dargestellt werden. 


\subsection{Lernerberatung}

Das Konzept der „Lernerberatung“ wurde im Rahmen des vom Bundesministerium für Bildung und Forschung (BMBF) geförderten Modellprojekts „Lernerfolg und Lernerfolgskontrolle“ (LeKo; 2003-2007) entwickelt. Lernerberatung zielt hier als Angebot in Seminaren auf die „Sicherung und Optimierung von Lernprozessen" ab (Hohenstein 2007, S. 147). Dabei stützt sich dieses Konzept auf die Analyse und Diagnose von „Lernereigenschaften" und damit verbundenen Lernschwierigkeiten, für die ein Fragebogen zur Erfassung von biografischen „Lernertypen und Lernereigenschaften“ als Diagnoseinstrument eingesetzt wird (FELTE, vgl. Hohenstein 2007). Die Auswertung dieses Instruments wird dann als Ausgangspunkt für das konkrete „Lernerberatungsgespräch“ genutzt. Die Lernberatung ist zwar in eine konkrete Kurs- oder Seminarveranstaltung eingebunden, wird aber als zusätzliches Element verstanden, das Teilnehmende bei Bedarf - außerhalb der Kurssituation - in Anspruch nehmen können. Die Beratung wird von fortgebildeten Kursleiterinnen und -leitern durchgeführt, die als „Experten für Lernen“ (Hohenstein 2007, S. 147) und weniger als Fachexperten und -expertinnen agieren. Gleichwohl sollen auch die Inhalte der jeweiligen Kurse in der Beratung berücksichtigt werden.

- Sachlich werden hier spezifische Aspekte einer Lernbiografie fokussiert und als Lerntypen, -eigenschaften und -schwierigkeiten konzeptualisiert. Diese werden über ein standardisiertes Instrument erfasst und als Kommunikationsgegenstand in das Interaktionssystem inkludiert. Es wird darauf verwiesen, dass eine Verknüpfung mit der inhaltlichen Lernsituation vorgesehen sei, allerdings wird konzeptionell nicht berücksichtigt, wie diese Verknüpfung erfolgt.

- In der Sozialdimension lässt sich festhalten, dass in dieser Konzeption Kursleitende als Beratende im Umgang mit dem Diagnoseinstrument geschult werden. Die Rolle kann daher - etwas überspitzt - als die von „lehrenden Diagnostikern“ beschrieben werden, die im Kontext der Beratungssituation als „Experten für Lernen“ fungieren. Lernende werden hier als „Experten der persönlichen Lernbiografie“ (ebd.) betrachtet, die dies in der Beratung klar explizieren, reflektieren und aktiv nach Optimierungen suchen.

- Zeitlich wird das Beratungsgespräch als ,aus dem Seminar ausgegliederte Intervention" (ebd. S. 148) verstanden und individualisiert nach den Bedarfen der Teilnehmenden realisiert. Wie die zeitliche Kopplung an die strukturierte Seminarsituation genau erfolgen soll, bleibt konzeptionell unklar. Es handelt sich damit um ein exkludierendes, unbestimmtes Zeitkonzept. 


\subsection{Lernberatung im Kontext von Selbstlernarchitekturen}

Ein zweites Konzept von Lernberatung wurde im Rahmen einer Theorie selbstsorgenden Lernens entwickelt (vgl. Forneck 2005, 2006). In dieser Konzeption wird von einer Selbstlernarchitektur ausgegangen, die auf vorstrukturierten und miteinander vernetzten Materialien basiert. In der Strukturierung der Selbstlernarchitektur werden inhaltliche Lernmaterialien als Lerneinheiten mit metakognitiven Lernpraktiken zur Bearbeitung des Lerngegenstandes verknüpft. Die Lerneinheiten werden durch Verweise miteinander sowie mit expliziten Lernberatungsgesprächen verbunden, bei denen der Gegenstand und die metakognitiven Bearbeitungsstrategien thematisiert werden. Entsprechend entstehen in dieser Struktur individuelle Lernwege, die Lernende selbstgesteuert durchlaufen. Lernberatung wird hier verstanden als ein fester Bestandteil einer didaktisch strukturierten Lernumgebung, für die methodische Hintergrundtexte als Hilfestellung für Beratende bereitgestellt werden. „Wir lassen nicht einen selbstgesteuerten Lernprozess ablaufen und bieten stattdessen Lernberatung an. Vielmehr beziehen wir Lernmaterial, Lernarchitekturen und die Beratungsangebote aufeinander" (Forneck 2005, S. 34). Lernberatung wird hier als ein Element innerhalb einer Selbstlernarchitektur konzeptualisiert, die auf die Reflexion des aktuell thematischen Lernprozesses abzielt.

- Als Themen der Lernberatung werden sachlich Inhalte und Lernstrategien konzeptionell in der Konstruktion einer thematischen Selbstlernumgebung verknüpft und als Gegenstand von Beratung inkludiert.

- Sozial werden Lehrende zunächst als didaktische Konstrukteure von Selbstlernumgebungen gefasst, die in der Begleitung der Selbstlernumgebung die Rolle der Lernberatung einnehmen. Die Interaktion erfolgt konzeptionell immer entlang eines thematischen Lernprozesses, für den Lernende unterschiedliche Strategien nutzen. Lernende werden hier als individualisierte Lernsubjekte konstruiert.

- Zeitlich werden durch die Einbindung in eine Selbstlernumgebung jeweils bestimmte Zeitpunkte für Beratungsgespräche konzeptionell integriert. In der zeitlichen Sinndimension handelt es sich damit um ein bestimmtes, integriertes Zeitkonzept von Lernberatung.

\subsection{Rekonstruktive Lernberatung}

Ein drittes Konzept - das der rekonstruktiven Lernberatung - wurde im Kontext empirischer Forschungen zu Alphabetisierungskursen entwickelt (Lud- 
wig 2012). Es zählt zu den fallorientierten Beratungsansätzen, bei denen - vor dem Hintergrund subjektwissenschaftlicher Ausdeutungen - Lernen als soziales und gesellschaftlich situiertes Handeln verstanden wird, dem spezifische Begründungen zu Grunde liegen. In der Lernberatung sollen nach diesem Konzept drei Aspekte des Lernens - Biografie, der fachliche Gegenstand und die Situation - bearbeitet werden. Die Lernberatung wird als „Grundform pädagogischen Handelns im Vermittlungsprozess“ (ebd. S. 193) verstanden und findet hier abwechselnd mit pädagogischen Handlungen des Lehrens statt. Anlass ist eine wahrgenommene Lernproblematik von Lernenden. Ziel der Beratung ist damit, ihre Lernproblematik umfassend zu verstehen und im Gespräch zu rekonstruieren, um im weiteren Verlauf Kernthemen sowie Gegenhorizonte herauszuarbeiten. Entsprechend wird hier Lernberatung als Prozess der Bedeutungsdifferenzierung gefasst, der in einem Beratungsschema mit dem Namen VIVA ${ }^{1}$ bearbeitet wird.

- Hinsichtlich der sachlichen Struktur werden in dieser Konzeption biografische, inhaltliche und situative Aspekte berücksichtigt. Konzeptuell wird davon ausgegangen, dass jeweils situativ bestimmt wird, welche Aspekte im Beratungsgespräch subjektiv bedeutsam und vor diesem Hintergrund konkret bearbeitet werden.

- Sozial wird eine Rollenkonstruktion sichtbar, bei der Lehrende als verstehende Rekonstruierende von Lernproblematiken auftreten, die Bildungsprozesse begleiten und unterstützen. Diese Rolle wird im Wechselspiel zu der Rolle als Lehrende situativ eingesetzt. Lernende werden hier also ebenfalls als individualisierte Lernsubjekte verstanden. Zusätzlich liegt aber auch ein Verständnis von Lernenden als Fallgebenden vor.

- Zeitlich erscheint die Lernberatung hier mit der Kurssituation verknüpft (vgl. ebd. S. 194). Dabei zeigen sich zwei unterschiedliche Möglichkeiten. Je nach Bedarf kann ein Reflexionsgespräch als Lernberatung in den Kurs integriert werden. In diesem Fall handelt es sich um ein unbestimmtes, integriertes Zeitkonzept. Gleichzeitig kann - wiederum nach dem individuellen Bedarf der Teilnehmenden - eine längere Beratung als Fallrekonstruktion aus dem Kurs exkludiert werden. Dabei handelt es sich um ein unbestimmtes, exkludierendes Zeitkonzept.

1 Unter VIVA sind folgende Prozesse subsumiert: Verstehen der Beratenden, Impulse und Gegenhorizonte setzen, Verstehen durch Ratsuchende, Alternative Handlungsmöglichkeiten aufzeigen. 


\section{Diskussion: Zur Relevanz beobachtungstheoretischer Analysen von Lernberatungskonzepten}

In der Zusammenschau der drei Konzepte zeigen sich unterschiedliche Ausdifferenzierungen der sachlichen, sozialen und zeitlichen Sinndimensionen von Lernberatung (siehe Abbildung 1).

Tab. 1: Ergebnisse der Analyse zu Lernberatungen im Überblick.

\begin{tabular}{|c|c|c|c|}
\hline $\begin{array}{l}\text { Sinn- } \\
\text { dimensionen }\end{array}$ & Lernerberatung & $\begin{array}{c}\text { Lernberatung in } \\
\text { Selbstlernarchitekturen }\end{array}$ & Rekonstruktive Lernberatung \\
\hline Sachlich & $\begin{array}{l}\text { - Fokussierung von Facetten } \\
\text { von Lernbiografie } \\
\text { (Lernertypen, } \\
\text { Lerneigenschaften und } \\
\text { Lernschwierigkeiten) }\end{array}$ & 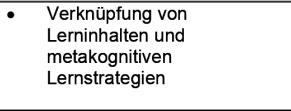 & $\begin{array}{l}\text { - } \quad \text { Berücksichtigung } \\
\text { biografischer, inhaltlicher und } \\
\text { situativer Aspekte im } \\
\text { Beratungsgespräch }\end{array}$ \\
\hline Sozial & $\begin{array}{l}\text { - Lehrende als Diagnostiker, } \\
\text { "Experten für Lernprozesse } \\
\text { (Hohenstein 2007, S. 147) } \\
\text { - Lernende als „Experten der } \\
\text { persönlichen Lernbiographie }{ }^{a} \\
\text { (ebd. S. 148) }\end{array}$ & $\begin{array}{ll}\text { - } & \text { Lehrende als didaktische } \\
\text { Konstrukteure in der Rolle } \\
\text { der Lernberatenden } \\
\text { - } \quad \text { Lernende als individualisierte } \\
\text { Lernsubjekte }\end{array}$ & $\begin{array}{ll}\text { - } & \text { Lehrende als } \\
& \text { Rekonstruierende von } \\
& \text { Lernproblematiken, in } \\
\text { situativem Rollenwechsel zum } \\
\text { Lehren } \\
\text { - } \quad \text { Lernende als individualisierte } \\
\text { Lernsubjekte und } \\
\text { „Fallgebende“ } \\
\end{array}$ \\
\hline Zeitlich & $\begin{array}{l}\text { - } \text { "aus dem Seminar } \\
\text { ausgegliederte Intervention }{ }^{a} \\
\text { (ebd. 148) } \\
\text { - Unbestimmtes, exkludiertes } \\
\text { Zeitkonzept }\end{array}$ & $\begin{array}{ll}\text { - } & \text { In einer didaktisch } \\
\text { strukturierten Selbstlern- } \\
\text { umgebung konzeptuell } \\
\text { inkludiert } \\
\text { - Bestimmtes, integriertes } \\
\text { Zeitkonzept }\end{array}$ & $\begin{array}{l}\text { - Unbestimmtes, integriertes } \\
\text { Zeitkonzept } \\
\text { ODER } \\
\text { - Unbestimmtes, exkludiertes } \\
\text { Zeitkonzept }\end{array}$ \\
\hline Modus & Diagnostik & Didaktik & Situative Fallarbeit \\
\hline
\end{tabular}

Quelle: eigene Darstellung

Betrachtet man die Sinndimensionen der Konzepte in Kontrast zueinander, lassen sich vor diesem Hintergrund drei verdichtete Modi der Sinngenerierung in Lernberatungskonzeptionen abstrahieren.

1. Lernberatung im Modus der Diagnose

2. Lernberatung im Modus der Didaktik

3. Lernberatung im Modus situativer Fallarbeit

Diese drei Modi setzen unterschiedliche Schwerpunkte; gleichzeitig werden bestimmte Aspekte jeweils ausgeblendet.

- Bei der Lernberatung im Modus der Diagnose wird der Schwerpunkt darauf gelegt, durch standardisierte Messinstrumente zur Erfassung von Lerntypen und Lernschwierigkeiten Lernproblematiken methodisch kontrolliert zu identifizieren. Nicht systematisch konzeptionell reflektiert wird hier, dass über die Schwerpunktsetzung auf diagnostisches Handeln die Verknüpfung zu den Inhalten in der jeweiligen Kurssituation verloren geht. Auf dieses Verschwinden des Inhalts auf der Sachebene der Lernbe- 
ratung wurde von verschiedenen Seiten schon hingewiesen (vgl. Ludwig 2012, Reutter 2011, Siebert 2001).

- Lernberatung im Modus von Didaktik fokussiert auf eine klare Strukturierung und konzeptionelle Einbindung von Lernmaterialien und Lernberatungsgesprächen. Diese Konzeption steht daher für eine sehr enge Verknüpfung von Inhalt und Prozessberatung. Im Hinblick auf die Frage nach der Verknüpfung von didaktischem und beratendem Handeln wird hier auf der Sozialebene die biografische Situiertheit des Subjekts nicht ausdifferenziert. Die Verknüpfung zwischen formalen und materialen Aspekten im Beratungsgespräch steht im Vordergrund, während biografische Thematisierungsperspektiven der zu Beratenden konzeptionell kaum in den Blick genommen werden.

- Lernberatung im Modus der situativen Fallarbeit legt den Schwerpunkt auf die situative Reflexion von Lernprozessen von Teilnehmenden und verknüpft didaktisches und beratendes Handeln über eine situative Perspektive. Konzeptionell weniger beleuchtet wird, wie hier auf der Zeitebene begründet werden kann, wann welche Form von Lernberatung notwendig erscheint.

Der Erkenntnisgewinn dieser Systematisierung und das Potenzial dieser Perspektive besteht in einer Erweiterung und Ergänzung handlungstheoretischer Ordnungen, mit denen beispielsweise kompetenz- von defizitorientierten Konzepten unterschieden werden können. Gerade für den Untersuchungsgegenstand „Lernberatung“, bei der didaktisches und beratendes Handeln in Verbindung gesetzt werden, bietet eine systemtheoretisch fundierte Analyse den Mehrwert, diese Verknüpfungen als Relationierung von Sinndimensionen differenziert erfassen zu können und damit Anschlussmöglichkeiten für weitere Formen der Lernberatung bereitzustellen.

Eine solche Systematisierung dient auch der konzeptionellen Weiterentwicklung von Konzepten der Lernberatung. Schließlich ermöglicht eine bewusste Entscheidung zwischen verschiedenen Konzepten eine präzisere Ausdifferenzierung und Weiterentwicklung von Lernberatungskonzepten als bisher möglich. Allerdings wäre es dazu hilfreich, Konzepte von Lernberatung stärker als bisher auf unterschiedliche Zielperspektiven zu beziehen, die durch Lernberatung direkt oder indirekt erreicht werden sollen. Schließlich kann Lernberatung sehr unterschiedlichen Zielperspektiven dienen: Sie kann die Anschlussmöglichkeit von Menschen an gesellschaftliche Bedarfe erhöhen, etwa wenn es darum geht, Migrantinnen und Migranten eine Lernberatung zukommen zu lassen oder zu gering Qualifizierte weiter zu schulen. Gleichzeitig kann es aber auch um eine unspezifische Wertschöpfung durch ein Mehr an Bildung gehen. Angesichts der immer geringeren Halbwertzeit von Wissensbeständen kann durch Lernberatung auf einen Qualifizierungsbedarf reagiert werden. Gerade in Kontexten allgemeiner Lernberatung mag 
es auch darum gehen, den Umgang mit divergierenden Wissensbeständen einzuüben, die durch gesellschaftliche Pluralisierungsformen bedingt sind. Diese jeweils unterschiedlichen Zielperspektiven von Lernberatung mögen - vermutlich auch noch je nach Inhaltsgebiet divergierend - unterschiedliche Formen von Lernberatung bedingen. Dieses konzeptionell deutlicher herauszuarbeiten und durch Forschung zu unterfüttern, ist jedoch nach wie vor ein Desiderat.

Eine konzeptionelle Weiterentwicklung der Lernberatung durch die Schärfung der jeweils verschiedenen Modi dürfte auch einen wichtigen Beitrag zur Professionalisierung des Feldes leisten. Bisher ist die Lernberatung ein Arbeitsfeld mit sehr unklarem Qualifikationsprofil und unbestimmtem Qualitätsmanagement. Erst eine konzeptionelle Präzisierung würde es ermöglichen, hier Qualitätsstandards zu entwickeln und bei den tätigen Professionellen als Qualifikation einzufordern.

Da es sich bei den vorgestellten Erkenntnissen um die Ergebnisse einer explorativen Analyse handelt, werden im Folgenden Anschlussmöglichkeiten für die weitere Forschung herausgearbeitet.

\section{Ausblick: Anschlussmöglichkeiten für beobachtungstheoretisch fundierte Analysen von Beratungskonzeptionen}

Die entwickelte Analyseperspektive ermöglicht eine beobachtungstheoretisch fundierte Betrachtung von Sinnzuschreibungen pädagogischer Beratungskonzepte. Um die Reichweite dieses angedachten Instrumentariums weiterzuentwickeln und zu prüfen, sollten im nächsten Schritt zunächst weitere Lernberatungskonzepte analysiert werden. Dazu würden sich „weite Konzeptionen“ (vgl. z. B. Klein/Reutter 1998) anbieten, in denen Beratung als pädagogische Haltung verstanden wird. So könnte gegebenenfalls der bislang nur wenig herausgearbeitete Modus der Didaktik weiter verfeinert werden. Bei solchen weiteren Analysen würde sich zudem anbieten, die in der explorativen Analyse nicht verwendete Sinndimension des „Raums“ zu integrieren.

Darüber hinaus könnte das Analyseinstrumentarium auch genutzt werden, um andere Beratungsfelder systematisch in den Blick zu nehmen. Hier bietet sich insbesondere das heterogene Feld der Forschungsberatung an, in dem vielfältige Praxisformen, aber kaum theoretische Reflexion und empirische Untersuchungen vorliegen. Für die Analyse von Beratungskonzepten könnten 
hier beispielsweise zunächst Konzepte strukturierter Promotionsprogramme untersucht werden, um zu analysieren, welcher Sinn dem Beratungskontext von Nachwuchsförderung hier zugeschrieben wird. ${ }^{2}$

Schließlich könnte die Analyseperspektive auch genutzt werden, um in einer größeren kontrastierenden Analyse differenzierte pädagogische Beratungsformen systematisch im Hinblick auf ihre jeweiligen impliziten und expliziten Sinnzuschreibungen zu untersuchen.

\section{Literatur}

Fuchs-Brüninghoff, Elisabeth (2000). Lernberatung - die Geschichte eines Konzepts zwischen Stigma und Erfolg. In E. Nuissl, C. Schiersmann \& H. Siebert (Hg.). In: Literatur- und Forschungsreport Weiterbildung, (46), S. 81-92.

Forneck, Hermann J. (2005). Gestaltung von Selbstlernarchitekturen. Eine integrative Konzeption für selbstgesteuertes Lernen. In S. Dietrich \& M. Herr (Hg.). Support für neue Lehr- und Lernkulturen. Bielefeld: W. Bertelsmann, S. 133 - 153.

Forneck, Hermann J. (2006). Selbstlernarchitekturen. Lernen und Selbstsorge. Baltmannsweiler: Schneider Verlag Hohengehren.

Forneck, Hermann J. \& Springer, Angela (2005). Gestaltet ist nicht geleitet - Lernentwicklungen in professionell strukturierten Lernarchitekturen. In P. Faulstich, H.J. Forneck, J. Hermann, P. Grell, K. Häßner, J. Knoll \& A. Springer (Hg.). Lernwiderstand - Lernumgebung - Lernberatung. Empirische Fundierungen zum selbstgesteuerten Lernen Bielefeld: W. Bertelsmann, S. 94-161.

Hohenstein, Kerstin (2007). Verständnis von Lernerberatung. In A. Kaiser, V. Buddenberg, K. Hohenstein, C. Holzapfel, M. Uemminghaus \& M. Wolter (Hg). Kursplanung, Lerndiagnose und Lernerberatung. Bielefeld. W. Bertelsmann, S. 147-157.

Kaiser, Armin; Kaiser, Ruth \& Hohmann, Reinhard (Hg.) (2007a). Lernertypen - Lernumgebung - Lernerfolg. Erwachsene im Lernfeld. Bielefeld: W. Bertelsmann.

Kaiser, Armin; Buddenberg, Verena; Hohenstein, Kerstin; Holzapfel, Cornelia; Uemminghaus, Monika \& Wolter, Maren (Hg.) (2007b). Kursplanung, Lerndiagnose und Lernerberatung. Handreichung für die Bildungspraxis. Bielefeld: W. Bertelsmann.

Käpplinger, Bernd \& Maier-Gutheil, Cornelia (2015). Ansätze und Ergebnisse zur Beratung(sforschung) in der Erwachsenen- und Weiterbildung - Eine Systematisierung. In: REPORT Zeitschrift für Weiterbildungsforschung 38(2), S. 163-181.

Klein, Rosemarie \& Reutter, Gerhard (Hg.) (1998). Lehren ohne Zukunft? Wandel der Anforderungen an das pädagogische Personal in der Erwachsenenbildung. Baltmannsweiler: Schneider Verlag Hohengehren.

2 Für die konstruktiven Rückmeldungen im Anschluss an den diesem Beitrag zugrundeliegenden Vortrag danken wir insbesondere Wolfgang Seitter. 
Kollewe, Lea (2009). Lernberatung: Begründungskontexte, Einflussgrößen und konstituierende Merkmale. In W. Seitter, Wolfgang (Hg.). Professionalitätsentwicklung in der Weiterbildung. Wiesbaden: VS Verlag, S. 117-134.

Kollewe, Lea \& Seitter, Wolfgang (2009). Lernberatung als empirische Leerstelle? Befunde aus Programmanalysen von Weiterbildungseinrichtungen. In: REPORT Zeitschrift für Weiterbildungsforschung 3/2009, S. 57-69.

Kossack, Peter (2006). Lernen Beraten. Eine dekonstruktive Analyse des Diskurses zur Weiterbildung. Bielefeld: transcript.

Ludwig, Joachim (2012). Rekonstruktive Lernberatung. In J. Ludwig (Hg.). Lernen und Lernberatung. Alphabetisierung als Herausforderung für die Erwachsenendidaktik. Bielefeld: W. Bertelsmann, S. 193-212.

Ludwig, Joachim (2016). Lernberatung. In W. Gieseke \& D. Nittel (Hg.). Handbuch Pädagogische Beratung über die Lebensspanne. Weinheim/Basel: Beltz Verlag, S. 304-312.

Luhmann, Niklas (1984). Soziale Systeme. Grundriß einer allgemeinen Theorie. Frankfurt a. M.: Suhrkamp.

Maier-Gutheil, Cornelia (2015). Beraten. Stuttgart: Kohlhammer.

Pätzold, Henning (2004). Lernberatung und Erwachsenenbildung. Baltmannsweiler: Schneider Verlag Hohengehren.

Rohs, Matthias \& Käpplinger, Bernd (2004). Lernberatung - ein Omnibusbegriff auf Erfolgstour. In M. Rohs \& B. Käpplinger (Hg.). Lernberatung in der beruflich-betrieblichen Bildung. Konzepte und Praxisbeispiele für die Umsetzung. Münster: Waxmann, S. 13-27.

Schiersmann, Christiane (2000). Beratung in der Weiterbildung - neue Herausforderungen und Aufgaben. In: REPORT Literatur- und Forschungsreport Weiterbildung (46), S. 18-32.

Wrana, Daniel (2012). Lernberatung als pädagogische Handlungsform und empirischer Gegenstand. In D. Wrana \& C. Maier Reinhard (Hg.). Professionalisierung in Lernberatungsgesprächen. Theoretische Grundlegungen und empirische Untersuchungen. Opladen u. a.: Budrich, S. 17-68.

Wrana, Daniel \& Maier Reinhard, Christiane (Hg.) (2012). Professionalisierung in Lernberatungsgesprächen. Theoretische Grundlegungen und empirische Untersuchungen. Opladen u. a.: Budrich. 
II. Mediale Beratungsformate:

Vergleiche - Spezifika - Intentionen 



\section{Computervermittelte, asynchrone Beratungen in der Weiterbildung im Spannungsfeld von Anliegen und Prozessierungstransfer}

\section{Einleitung}

Personenbezogene Beratungen zu Weiterbildungsfragen sind in den letzten Jahren vermehrt Gegenstand gesprächsanalytischer Studien und theoretischer Modellierungen geworden. Mit Hilfe dieser Studien konnten etwa die Funktionen der Wissensvermittlung (vgl. Enoch 2011), der Fragetechniken (vgl. Müller 2005) oder der institutionellen Einflüsse in bzw. auf Bildungsberatungen (vgl. Stanik 2015) rekonstruiert werden. Sowohl diese Studien als auch die theoretischen Modellierungen von Beratungen in der Weiterbildung (vgl. z. B. Meyer 1997; Kossack 2009) beziehen sich bislang ausschließlich auf Beratungsinteraktionen, bei denen die beteiligten Personen gleichzeitig körperlich anwesend sind.

Die vorliegende Studie greift dieses Desiderat insofern auf, als sie Prozesse von computervermittelten, asynchronen Weiterbildungsberatungen analysiert. Dies erscheint notwendig, da es sich um ein eigenständiges Format mit spezifischen Herausforderungen handelt (vgl. Brunner 2006) und hier noch eine „Forschungslücke für eine Professionalisierung der Beratung durch eine empirische Grundlagenarbeit zu schließen“" (Gieseke/Käpplinger/Otto 2007: 33 ) ist. Gegenstand der Studie sind E-Mail-Weiterbildungsberatungen, die sich mit individuellen Fallbezügen an Personen vor den Besuchen von Weiterbildungsmaßnahmen richten (vgl. Nittel 2009; Schiersmann/Remmele 2004).

Beratung definieren wir phänomenologisch als eine dyadische - in unserem Fall schriftliche - Kommunikation bzw. Korrespondenz zwischen einer ratsuchenden und einer beratenden Person, wobei erstere ein (Entscheidungs-) Problem im Hinblick auf eine Weiterbildungsfrage hat, für das sie zum Zeitpunkt der Beratung über keine Handlungsalternativen verfügt und sich daher professionelle Unterstützung sucht (vgl. Kraft 2011: 156f.).

Unter dem titelgebenden Begriff des „Prozessierungstransfers“" verstehen wir die Anforderung, die sich den Beratenden stellt, die mündlichen bzw. schriftlichen Anliegensdarstellungen, Problemschilderungen, Fallbeschreibungen der Ratsuchenden zunächst zu verstehen, um sie dann in professionell bearbeitbare Handlungsschritte zu überführen. Dieser Prozess umfasst im Anschluss an Kallmeyer (2000) die Entwicklung einer Problemsicht, die Fest- 
legung des Bearbeitungsgegenstandes und die Lösungsentwicklung durch die Beratenden.

Leitendes Erkenntnisinteressen der Studie ist es, mit diesem Fokus explorativ zu rekonstruieren, wie Beratende im Format von E-Mail-Weiterbildungsberatungen mit „Problempräsentationen“ von ratsuchenden Personen umgehen.

Im Folgenden werden zunächst E-Mails bzw. E-Mail-Beratungen als Korrespondenzen gegenstandstheoretisch verortet, um die Fragestellung der Studie zu präzisieren. Im Anschluss an die Darlegung des Untersuchungsdesigns werden die Analysen von zwei E-Mail-Weiterbildungsberatungen und deren Kontrastierung exemplarisch dargestellt, um abschließend einen Ausblick auf Forschungsdesiderate zu geben.

\section{E-Mail-Beratungen}

E-Mails sind asynchrone, computervermittelte Formen schriftlicher Kommunikation oder genauer gesagt Korrespondenzen, die sich aus mindestens zwei Korrespondenzexemplaren, in unserem Fall einer Anfrage durch eine ratsuchende Person und einer Antwort einer beratenden Person, zusammensetzen.

Im Unterschied zur klassischen Korrespondenzform des Briefes weisen computervermittelte Korrespondenzen ein höheres Maß an Anonymität auf und sind stärker dialogisch orientiert. In E-Mails wird dennoch in der Regel auf bestehende schriftsprachliche Konventionen zurückgegriffen, die vom formalen Duktus eines Geschäftsbriefes bis hin zu Umgangssprachlichkeiten im Telegrammstil unter Freunden reichen können (vgl. Wolf 2014: 53ff.).

Im Beratungsdiskurs werden E-Mail-Korrespondenzen sowohl Chancen eingeräumt als auch deren Herausforderungen problematisiert. Zunächst sind E-Mail-Beratungen anonymer als Face-to-Face Beratungsinteraktionen. Ratsuchende sind weder zeitlich noch örtlich an die Beratungseinrichtung gebunden. Diese Aspekte bezeichnet Knatz (2009: 61f.) als „äußere Niederschwelligkeit“, während mit der sogenannten ,inneren Niederschwelligkeit" gemeint ist, dass Ratsuchende weniger Hemmungen haben könnten, überhaupt eine Beratung in Anspruch zu nehmen. Eine Verschriftlichung von Beratungsanliegen kann außerdem einen Mehrwert bieten, da Ratsuchende zu Distanzierungen gegenüber ihren Problemen gezwungen werden und die notwendigen schriftlichen Strukturierungen Selbstreflexionsprozesse auslösen können (vgl. Knatz 2009: 64f.).

Diesen Chancen steht die Herausforderung gegenüber, dass E-Mail-Korrespondenzen im Vergleich zu Interaktionen prinzipiell kanalreduziert sind, da soziale Hinweisreize (z. B. Alter, Geschlecht), die Prosodie (z. B. Into- 
nation) oder die Kinesik (Gestik, Mimik) fehlen (vgl. Döring 2003: 149f.). Insbesondere Beratende benötigen daher eine professionelle Lesekompetenz (vgl. Brunner 2006), um hinreichende Klärungen der Beratungsanliegen vornehmen zu können. Während in Beratungskommunikationen/-interaktionen Beratende zum Beispiel ad hoc Nach- oder Verständnisfragen stellen können bzw. Anliegen im Gespräch ausgehandelt werden (vgl. Kallmeyer 2000), stehen bei E-Mail-Beratungen zunächst nur die schriftlichen Ausgangsschilderungen der Ratsuchenden zur Verfügung. Darüber hinaus benötigen Berater*innen für die Interventionen eine professionelle Schreibkompetenz, die an den Leser*innen bzw. Ratsuchenden orientiert ist, um an deren Vorwissen und Wortschatz anschlussfähig formulieren zu können (vgl. Becker-Mrotzek/ Schindler 2007: 13ff.).

An diesen skizzierten Herausforderungen setzt die Fragestellung des vorliegenden Beitrags insofern an, als dass analysiert wird, wie Berater*innen die schriftlichen Beratungsanliegen der Ratsuchenden in ihren Interventionen transformieren und prozessieren. Wichtig ist es in diesem Zusammenhang zu betonen, dass das Beratungshandeln nicht bewertet werden soll, sondern es soll auf Basis der empirischen Analysen ein erster explorativer Beitrag zur Formatlogik von E-Mail-Weiterbildungsberatungen geleistet werden.

\section{Untersuchungsdesign}

Zur Beantwortung der forschungsleitenden Fragestellung kann auf ein Korpus von 30 E-Mail-Weiterbildungsberatungen zurückgegriffen werden, die von vier Einrichtungen (zwei kommunale Weiterbildungsberatungsstellen, eine VHS, eine Online-Plattform mit Chatfunktion und E-Mail-Beratungen) angeboten worden sind. Alle Korrespondenzen des Korpus sind zweizügig und setzen sich aus einer E-Mail-Anfrage einer ratsuchenden Person und einer darauf bezogenen Antwort eines/einer Berater*in zusammen.

Für den vorliegenden Beitrag sind zwei Korrespondenzen als Eckfälle ausgewählt worden, die - was in der Folge zu zeigen sein wird - in den Anfragen der Ratsuchenden ähnliche Merkmale aufweisen, aber von den jeweils beratenden Personen in unterschiedlicher Weise prozessiert werden.

Für die Auswertung wurde das Verfahren der qualitativen Korrespondenzanalyse entwickelt, das methodologisch auf der linguistischen (Text-) Pragmatik basiert und sich in seinem methodischen Vorgehen an die Interpretationsprinzipien der strukturalen Hermeneutik anlehnt. Die linguistische Pragmatik betrachtet kommunikative Äußerungen als Sprech- bzw. als Schreibhandlungen und untersucht die zugrundeliegenden Prinzipien, an denen sich sprechende/schreibende und hörende/lesende Personen orientieren, 
damit ein gegenseitiges Verstehen gewährleistet wird (vgl. Ehrhardt/Heringer 2011: 14). Ziel von pragmatisch-linguistischen Analysen ist es, die Funktion von Gesprächen/Texten zu rekonstruieren, indem die Sprech-/Schreibhandlungen in ihren konkreten kommunikativen Verwendungszusammenhängen beschrieben werden. Beratungs-E-Mails lassen sich damit als Abfolge von Schreibhandlungen betrachten, die in spezifischen textuellen Relationen zueinander stehen, gemeinsame thematische Grundlagen haben und mit denen individuelle und gesellschaftliche Zwecke verfolgt werden (vgl. Langheine 1983: 197; Brünner/Graefen 1994: 14).

Für die Analyse sind zunächst die E-Mails der Ratsuchenden streng sequenziell und kontextfrei mit Hilfe einer extensiven Lesartendiskussionen durch die beiden Autor*innen rekonstruiert worden (vgl. Oevermann u.a. 1979), um daraufhin die Antwort-E-Mails der Beratenden mit dem gleichen Verfahren zu analysieren. Erst im Anschluss wurden die E-Mails der Ratsuchenden und die der Beratenden aufeinander bezogen, um dann Korrespondenzen des Korpus minimal miteinander zu kontrastieren (vgl. Strauss/ Corbin 1996).

Mit Hilfe dieses methodischen Zugriffs ist es weder möglich, die Intentionen der ratsuchenden noch die der beratenden Personen zu analysieren. Auch können keine Aussagen zum Beispiel über die Beratungsverständnisse der Beratenden oder über die Wirkungen und den Nutzen bei den Ratsuchenden getroffen werden. Eine weitere Einschränkung betrifft die Diskussion der Lesarten, da diese im Rahmen des Beitrags nicht vollständig dargestellt werden können. Außerdem wurden die Analysen durch die beiden Autor*innen angefertigt und nicht wie von Oevermann u. a. (1979) gefordert in einer Interpretationsgruppe, wenngleich Lesarten, die in der Diskussion des Vortrages auf der Sektionstagung formuliert worden sind, für den Beitrag berücksichtigt wurden.

\section{Ergebnisse einer qualitativen Korrespondenzanalyse - Ein Fallvergleich}

In der Folge werden ausgewählte Aspekte der Analysen am Beispiel von zwei Korrespondenzen dargestellt. Zunächst werden die Anfragen der Ratsuchenden analysiert, um anschließend den Prozessierungstransfer durch die jeweiligen Berater*innen miteinander zu vergleichen. Hierbei wird die Unterscheidung von informativen und situativen Beratungsbedarfen von Gieseke 
und Opelt (2004) als Heuristik aufgegriffen ${ }^{1}$, um insbesondere zu veranschaulichen, wie der/die Berater*in die Beratungsanliegen in ihren E-Mail-Antworten (nicht) prozessieren.

\subsection{Vergleich der E-Mail-Anfragen}

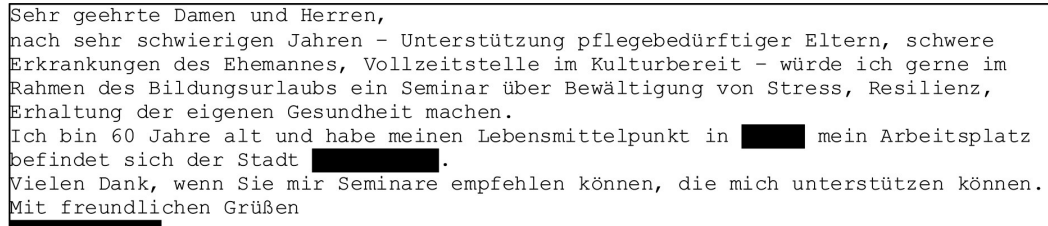

Abb. 1: Anfrage Korrespondenz 7

Die ratsuchende Person ${ }^{2}$ eröffnet die Beratungsanfrage, indem sie nach einer förmlichen Begrüßung direkt auf eine offensichtlich zurückliegende, belastende Lebenssituation stichwortartig Bezug nimmt, um daraufhin den Wunsch zu formulieren, eine Weiterbildung zu drei potentiellen Themenbereichen („Bewältigung von Stress, Resilienz, Erhaltung der eigenen Gesundheit“) im Rahmen eines „Bildungsurlaubs“ zu absolvieren. Sie ergänzt altersbezogene wie auch regionale Angaben, bevor sie sich abschließend prospektiv für eine mögliche Empfehlung bedankt. Es fällt auf, dass potenzielle Beratungsanliegen nicht explizit formuliert, sondern lediglich implizit angedeutet werden. So könnte die Ratsuchende entweder eine Unterstützung bei der Ermittlung einer zu den genannten Themen geeigneten Weiterbildung benötigen, und/oder sie möchte wissen, ob eine solche Weiterbildung über einen „Bildungsurlaub“ förderfähig ist bzw. welche Voraussetzungen hierfür zu erfüllen sind.

1 Während informative Beratungsanliegen dadurch gekennzeichnet sind, dass Ratsuchende bereits vor den Gesprächen klare Vorstellungen darüber haben, welche Weiterbildungsmaßnahmen sie aus welchen Motiven besuchen möchten, sprechen Ratsuchende in situativen Beratungen problematische Lebenssituationen an. Diese gehen zwar auch explizit mit Bedürfnissen nach Weiterbildungsmaßnahmen einher, wenngleich die Weiterbildungswünsche den Ratsuchenden noch unklar sind. Biografie orientierte Beratungen sind durch Lebenskrisen und Ängste der Ratsuchenden gekennzeichnet, wobei die widersprüchlichen Situationsbeschreibungen nicht ausreichen, um Weiterbildungsinteressen spezifizieren und Weiterbildungsmaßnahmen vorschlagen zu können (vgl. Gieseke und Opelt 2004: 34ff.).

2 Alle Angaben, die Rückschlüsse auf Personen, Orte, Einrichtungen usw. zulassen, wurden von uns oder den Berater*innen anonymisiert. 
Während diese Anliegen einen informativen Beratungsbedarf implizieren, kann jedoch auch ein situativer Beratungsbedarf nicht ausgeschlossen werden. Die in den Gedankenstrichen eingeschobenen Hintergrundinformationen (,Unterstützung pflegebedürftiger Eltern“, „,schwere Erkrankungen des Ehemannes“ bei Ausübung einer „Vollzeitstelle“) implizieren, dass die Ratsuchende Hilfe beim Umgang mit bzw. bei der Reflexion über diese Lebenssituation(en) wünscht. Dies könnte etwa die Frage betreffen, inwiefern eine der genannten Weiterbildungsmöglichkeiten dazu hilfreich wäre. Diese Lesart wird durch den mit dem Konditionalsatz erhofften Wunsch nach einer Unterstützung plausibel.

Dass es sich bei dieser prinzipiellen Uneindeutigkeit der Reichweite der Beratungsanliegen nicht um einen Einzelfall handelt, zeigt die zweite, im Folgenden analysierte Beratungsanfrage. Im Unterschied zum ersten Fall wird hier ein situativer Beratungsbedarf jedoch deutlicher markiert, da die ratsuchende Person sich offensichtlich in einer akut prekären Lebenssituation befindet, die sie mit Hilfe einer Weiterbildung zu lösen gedenkt.

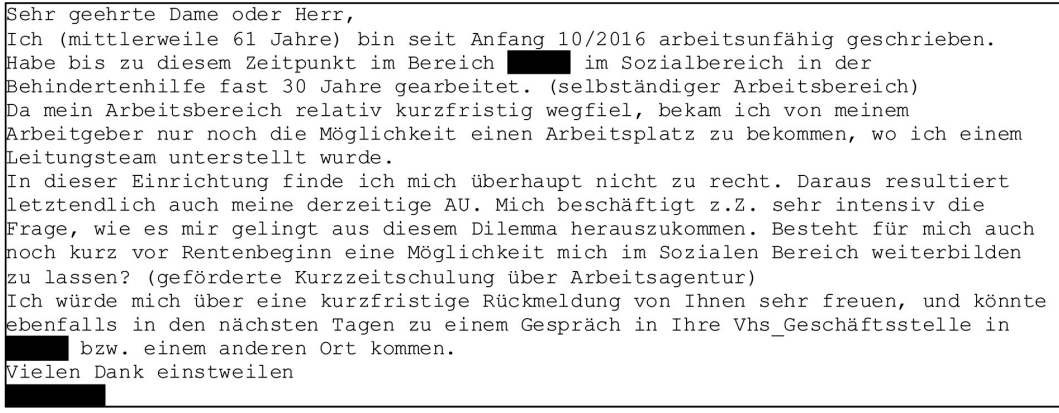

Abb. 2: Anfrage Korrespondenz 27

Zu Beginn fällt die ungewöhnliche Adressierung bei der Anrede (,Sehr geehrte Dame oder Herr") auf, die die Lesart eröffnet, dass der Ratsuchende nicht weiß, wer seine E-Mail-Anfrage bearbeiten wird. Durch die Anrede im Singular (üblich ist die Anrede im Plural) könnte auch markiert werden, dass die Korrespondenz vertraulich zu behandeln und nicht weiterzugeben oder im Team zu besprechen ist.

Im ersten Absatz stellt sich der Ratsuchende zunächst selbst als defizitäre Person vor, die - aus einer berufsbezogenen Perspektive - relativ alt und aktuell arbeitsunfähig ist. Daraufhin erläutert er seinen Arbeitskontext, wobei nicht die Art der Tätigkeit, sondern die Form ihrer Ausübung als selbstbestimmt hervorgehoben wird. Im zweiten Absatz geht er genauer auf seine 
Situation ein, indem angedeutet wird, warum er krankgeschrieben worden ist - sein langjähriger Arbeitsplatz ist weggefallen und er wurde einem Leitungsteam unterstellt. Die Passivkonstruktion (,unterstellt wurde") eröffnet die Lesart, dass er sich fremdbestimmt fühlt. Im Anschluss formuliert er die These, dass seine Arbeitsunfähigkeit ursächlich aus der neuen weisungsgebundenen Arbeitsplatzsituation resultiert ist, die er als "Dilemma“ charakterisiert. Hiermit könnte sich die Lesart der Markierung einer Vertraulichkeit bestätigen. Dadurch ist zudem ein offensichtlich situativer und/ oder latenter Biografie orientierter Beratungsbedarf offenkundig, wenngleich im Anschluss ein informatives Beratungsanliegen formuliert wird. So will der Ratsuchende wissen, inwiefern förderfähige Weiterbildungsangebote im „Sozialen Bereich“ existieren, die außerdem durch die Agentur für Arbeit finanziell gefördert werden. Die mit dem letzten Absatz eingeleitete Verabschiedung und das darin enthaltene Angebot, persönlich vorbeizukommen, wie auch die explizite Beendigung (,Vielen Dank einstweilen“) lassen die Lesart zu, dass die schriftliche Anfrage zur Kontaktanbahnung für einen persönlichen Beratungstermin dient - unter der Voraussetzung, dass förderfähige Weiterbildungen im gewünschten Bereich existieren.

Beide E-Mail-Anfragen weisen Anzeichen für ein situatives Beratungsanliegen auf, wobei mit den spezifischen Fragen der Ratsuchenden jeweils ein informatives Anliegen explizit thematisiert wird. Es stellt sich die Frage, wie die beiden Berater*innen diese Beratungen prozessieren. In beiden Fällen besteht die Möglichkeit, lediglich die informativen Fragen aufzugreifen und/ oder die prekären Lebenssituationen und deren Lösung durch den Besuch einer Weiterbildung zum Gegenstand der weiteren Korrespondenz werden zu lassen.

\subsection{Vergleich des Prozessierungstransfers}

Die beiden analysierten Korrespondenzen stehen für eine Korrespondenzsorte $^{3}$, die sich dadurch charakterisieren lässt, dass in den Anfragen bezüglich des Beratungsanliegens sowohl Hinweise auf informative als auch auf situative Beratungsanliegen vorhanden sind. Die Varianzen innerhalb der Korrespondenzsorte ergeben sich durch die je fallspezifische Prozessierung dieser Anliegen seitens der Beratenden. Ziel der vergleichenden Analyse ist es, die jeweiligen Prozessierungsaktivitäten der beiden Berater*innen in ihren Gemeinsamkeiten und Unterschieden zu rekonstruieren und mit Blick

3 Unter einer Korrespondenzsorte verstehen wir Beratungskorrespondenzen mit gleichen Merkmalen (vgl. Stanik/Maier-Gutheil 2018). 
auf die Potenziale und Spezifika computervermittelter asynchroner Bildungsberatung vor dem Hintergrund forschungsbezogener Konsequenzen einzuordnen.

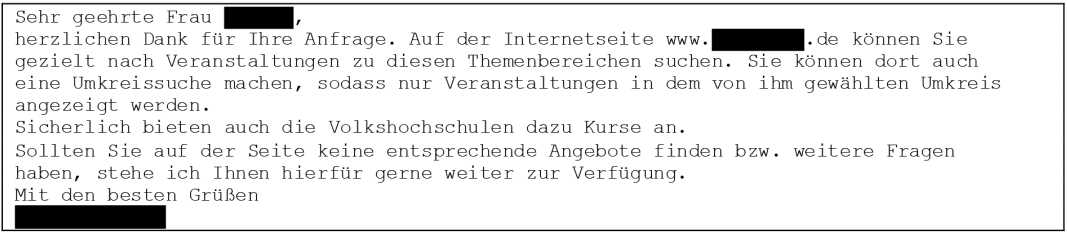

Abb. 3: Antwort Korrespondenz 7

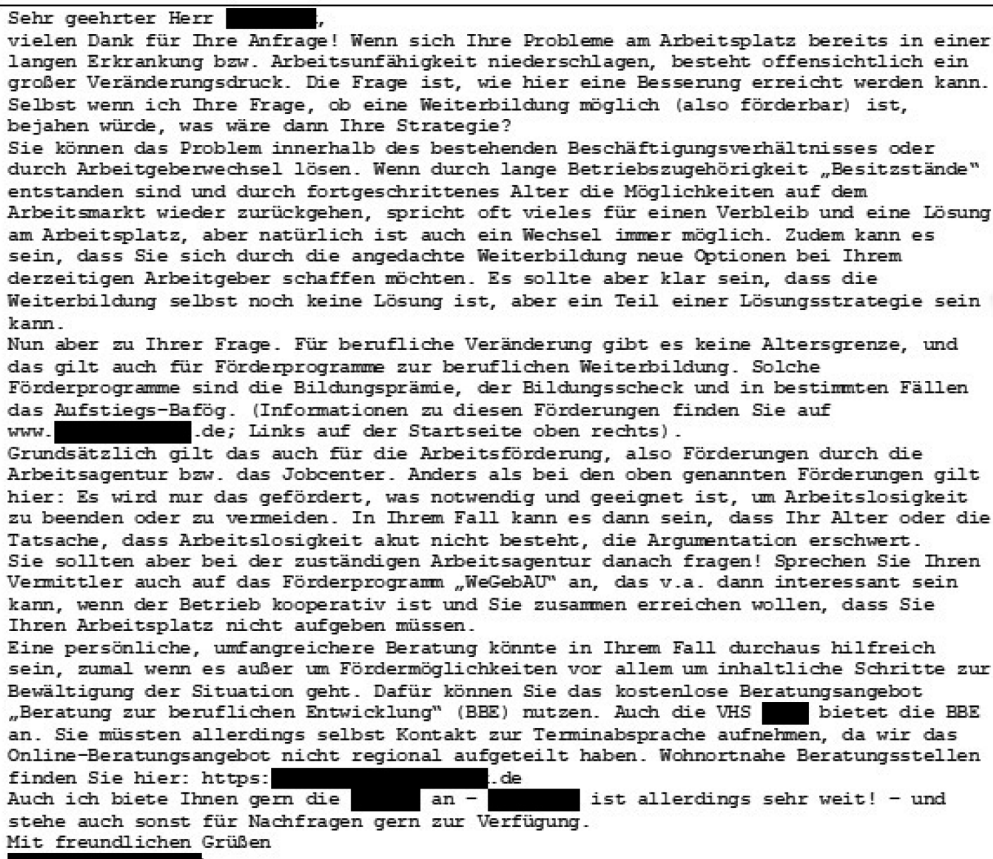

Abb. 4: Antwort Korrespondenz 27

In beiden Fällen bearbeiten die Berater*innen den informativen Bedarf, indem sie den Ratsuchenden Hinweise zu Recherchemöglichkeiten geben. Dabei werden jeweils Internetadressen genannt wie auch Hilfestellungen zur Suche angeboten: ,Sie können dort auch eine Umkreissuche machen, 
sodass nur Veranstaltungen in dem von ihm gewählten Umkreis angezeigt werden“ ( $\left.\mathrm{K}^{4} 7\right)$. Daneben werden auch allgemeine Informationen zum Beispiel über Finanzierungsmöglichkeiten unterbreitet: „Für berufliche Veränderung gibt es keine Altersgrenze, und das gilt auch für Förderprogramme zur beruflichen Weiterbildung. Solche Förderprogramme sind die Bildungsprämie, der Bildungsscheck und in bestimmten Fällen das Aufstiegs-Bafög“(K27). Die Hinweise auf Internetdatenbanken zeigen die Erwartungshaltung der Berater*innen, dass die ratsuchenden Personen in der Lage sind, selbständig nach Weiterbildungsangeboten für ihre individuellen Anliegen zu suchen wie auch eine diesbezügliche Entscheidung zu treffen. Damit dienen diese Prozessierungen offensichtlich dazu, die Handlungs- und Entscheidungsfähigkeit der Ratsuchenden herzustellen.

Hier enden die Gemeinsamkeiten auf der Ebene des Prozessierungstransfers der beiden Eckfälle.

Wenngleich auch in der Korrespondenz 7 ein situatives Anliegen erkennbar ist, wird es von der Beraterin nicht aufgegriffen. Stattdessen delegiert sie handlungskonstitutiv das Anliegen ,Empfehlung von Seminaren' an die Ratsuchende zurück und nennt dieser eine Möglichkeit, selbstgesteuert mithilfe einer regionalen internetbasierten Datenbank an die benötigten Informationen zu gelangen. Auffällig ist, dass die Beraterin weder Reflexionsfragen stellt, mit deren Hilfe die Ratsuchende ihre Weiterbildungsinteressen klären bzw. spezifizieren könnte noch den Aspekt einer potenziellen (Nicht-)Förderfähigkeit des von der Ratsuchenden erwähnten „Bildungsurlaubs“ aufgreift.

Demgegenüber prozessiert der Berater der Korrespondenz 27 einen situativ orientierten Beratungstyp und geht in den ersten beiden Absätzen deutend auf die aktuelle Situation des Ratsuchenden ein: ,besteht offensichtlich ein großer Veränderungsdruck“ (K27). Er nimmt damit eine Redefinition von dessen Anliegen ,zeitnahe Veränderung der aktuellen Beschäftigungssituation' vor, da sich dieser lediglich nach einer Weiterbildung und deren Förderung durch die Agentur für Arbeit erkundigt hatte. Mittels impliziter („Die Frage ist, wie hier eine Besserung erreicht werden kann“) und expliziter Reflexionsfragen (,was wäre dann Ihre Strategie?") geht er sodann auf die Frage des Ratsuchenden ein, wie dieser, aus diesem Dilemma herauskommen" könne. Insbesondere mit der zweiten Reflexionsfrage eröffnet der Berater einen Rahmen, der über das formulierte Anliegen hinausgeht. Er spitzt damit die Situation zu und markiert zugleich, dass eine Teilnahme per se aus seiner Experten-Sicht nicht ausreiche, sondern lediglich eine kurzfristige Lösung darstelle. Außerdem unterbreitet er dem Ratsuchenden auf der Basis seines Expertenwissens einen internen (,,innerhalb des 
bestehenden Beschäftigungsverhältnisses") und einen externen Lösungsweg (,Arbeitgeberwechsel“), wobei sich andeutet, dass er die interne Lösung präferiert.

Auch im Kontext der Prozessierung des informativen Anliegens (Absätze $3+4)$ erwähnt der Berater - neben den Informationen über Recherchemöglichkeiten und Förderprogrammen in Bezug auf die vom Ratsuchenden angesprochene „Arbeitsagentur“ - das Risiko, dass deren Förderungen an Bedingungen geknüpft sein könnten, die der Ratsuchende möglicherweise nicht erfüllt. Er formuliert eine explizite Handlungsempfehlung („Sie sollten aber bei der zuständigen Arbeitsagentur danach fragen!"), der durch das Ausrufezeichen Nachdruck verliehen wird. Indem er seine Empfehlung mit dem Verweis auf ein spezifisches Förderprogramm (,,WeGebAU“) ergänzt, verfolgt er handlungskonstitutiv seinen ohnehin präferierten internen Lösungsvorschlag mit dem aktuellen Arbeitgeber. Im letzten Absatz differenziert er abschließend noch einmal explizit zwischen Informationen über „Fördermöglichkeiten“ (informative Beratung) und „,inhaltliche Schritte zur Bewältigung der Situation“ (situative Beratung), wobei er für den letzteren Aspekt eine „persönliche, umfangreichere Beratung“ im Stil einer trägerunabhängigen Weiterbildungsberatung vorschlägt. Damit grenzt er das Format Face-to-Face-Beratung implizit und positiv(er) konnotiert vom Format E-Mail-Beratung ab.

Den Fallvergleich pointierend wird das Anliegen im ersten Eckfall primär informativ (kriteriengestützte Kurssuche) ohne die situativen Bezüge (familiäre Belastungssituation, Uneindeutigkeit des Weiterbildungswunsches) prozessiert. Im zweiten Eckfall wird das Anliegen sowohl situativ (berufliche Belastungssituation) als auch informativ (förderfähige Weiterbildungsmöglichkeiten) bearbeitet.

Gleichwohl gelingt es beiden Beratenden im Rahmen ihrer Prozessierungen der informativen Anliegen, durch Rückdelegationen an die Ratsuchenden (Verweis auf Internetdatenbanken und Recherchemöglichkeiten), diese zu selbständigen Aktivitäten aufzufordern, womit letztlich in beiden Fällen die Handlungsautonomie der Ratsuchenden unterstützt wird.

Während allerdings die Beraterin im ersten Eckfall die Komplexität des Beratungsanliegens eher ignoriert, greift der Berater im zweiten Eckfall die Komplexität auf und reflektiert diese darüber hinaus. Dies wird besonders deutlich, wenn man sieht, dass die Beraterin (K7) hier ohne Reflexionsfragen, Handlungsempfehlungen sowie Lösungsvorschläge auskommt, wohingegen der Berater (K27) mittels unterschiedlicher Fragen Reflexionsanlässe für den Ratsuchenden bietet und Handlungsempfehlungen sowie eigene Lösungsvorschläge entwickelt und dadurch explizit seine Expertensicht verdeutlicht. 


\section{Forschungsdesiderate}

Unsere Analysen zeigen, dass „,der Verstehensprozess immer als eine Wechselwirkung zwischen vorgegebenem Text und der Kognitionsstruktur des Lesers [bzw. der Leserin; TS/CMG] aufzufassen ist" (Christmann 2004: 33f.). Bislang liegen keine empirischen Erkenntnisse darüber vor, welche Prozesse sich seitens der Rezipient*innen, in unserem Fall der Beratenden, abspielen. Bedeutsam wäre es demnach, Verstehensprozesse beim Lesen genauer zu untersuchen, etwa mit Hilfe der Methode des lauten Denkens vor dem Hintergrund divergierender Ausgangssituationen mit Bezug auf das Vorwissen der Beratenden sowie ihrer Zielsetzungen, Erwartungen und Einstellungen (vgl. Christmann 2004: 34).

Weiter erscheint es notwendig, Beratungsverständnisse von Beratenden und Ratsuchenden in Bezug auf E-Mail-Weiterbildungsberatungen zu betrachten. So wird in der Korrespondenz 27 durch die Hinweise auf eine sich möglicherweise anschließende Face-to-Face-Beratung deutlich, dass für die Beteiligten E-Mail-Weiterbildungsberatungen bei komplexen Problemlagen ein Surrogat darstellen.

Auch wäre es notwendig, Interventionsstudien durchzuführen, um herauszufinden, inwiefern Qualifizierungen von Beratenden im Hinblick auf E-Mail-Beratungen dazu beitragen, dass textbasierte Anfragen in ihrer Komplexität angemessen erfasst und entsprechend bearbeitet werden. Hier könnten Studien, wie sie beispielsweise an der EH Darmstadt bezogen auf den Erwerb von Beratungskompetenzen (vgl. Weinhardt 2015) oder an der Universität Tübingen bezogen auf den Erwerb von Lehrkompetenzen durchgeführt werden (vgl. Hetfleisch/Goeze/Schrader 2017), praktische und methodologische Orientierungen bieten.

Schließlich müssten auch E-Mail-Beratungskorrespondenzen aus anderen Feldern (vgl. Überblick bei Pick 2017) systematisch untersucht und in maximalen Kontrastierungen miteinander verglichen werden, um auf einer solchen empirischen Basis ein Kernhandlungsschema für asynchrone digitale (Bildungs-)Beratungen zu entwickeln.

\section{Literatur}

Becker-Mrotzek, Michael/Schindler, Kirsten (2007): Schreibkompetenz modellieren. In: Becker-Mrotzek, M./Schindler, K. (Hg.): Texte Schreiben. Köln: Gilles \& Francke, S. 7-26. 
Brunner, Alexander (2006): Methoden des digitalen Lesens und Schreibens in der Online-Beratung. In: e-beratungsjournal.net, 4, 2. http://www.e-beratungsjournal.net/ausgabe_0206/brunner.pdf [Zugriff: 05.12.2017].

Brünner, Gisela/Graefen, Gabriele (1994): Einleitung: Zur Konzeption der Funktionalen Pragmatik. In: Brünner, G./Graefen, G. (Hg.): Texte und Diskurse. Methoden und Forschungsergebnisse der funktionalen Pragmatik. Opladen: Westdeutscher Verlag, S. 7-21.

Christmann, Ursula (2004): Verstehens- und Verständlichkeitsmessung: Methodische Ansätze in der Anwendungsforschung. In: Lerch, Kent D. (Hg.): Recht verstehen. Verständlichkeit, Missverständlichkeit und Unverständlichkeit von Recht. Berlin/New York: de Gruyter, S. 33-62.

Döring, Nicola (2003): Sozialpsychologie des Internets. Die Bedeutung des Internets für Kommunikationsprozesse, Identitäten, soziale Beziehungen und Gruppen. 2., vollständig überarbeitete und erweiterte. Göttingen: Hogrefe.

Ehrhardt, Claus/Heringer, Hans Jürgen (2011): Pragmatik. Paderborn: UTB.

Enoch, Clinton (2011): Dimensionen der Wissensvermittlung in Beratungsprozessen. Gesprächsanalysen der beruflichen Beratung. Wiesbaden: VS Springer.

Gieseke, Wiltrud/Käpplinger, Bernd/Otto, Sylvana (2007): Prozessverläufe in der Beratung analysieren - Ein Desiderat. Begründung und Entwicklung eines Forschungsdesigns. In: Report. Zeitschrift für Weiterbildungsforschung, 30, 1, S. 33-42.

Gieseke, Wiltrud/Opelt, Karin (2004): Weiterbildungsberatung. 2. Studienbrief. Zentrum für Wissenschaftliche Weiterbildung. Kaiserslautern.

Hetfleisch, Petra/Goeze, Annika/Schrader, Josef (2017): Wie PraktikerInnen wissenschaftliche Befunde verwenden. Selektions- und Rezeptionsprozesse bei der Implementation eines evidenzbasierten Trainingskonzepts. Zeitschrift für Pädagogik, 63, 2, S. 182-205.

Kallmeyer, Werner (2000): Beraten und Betreuen. Zur gesprächsanalytischen Untersuchung von helfenden Interaktionen. In: Zeitschrift für qualitative Bildungs-, Beratungs- und Sozialforschung (ZBBS) 1, 2, S. 227-252.

Knatz, Birgit (2009): Die webbasierte Mail-Beratung. In: Kühne, S./Hintenberger, G. (Hg.): Handbuch Online Beratung. Psychosoziale Beratung im Internet. 2. Auflage. Göttingen: Vandenhoeck und Ruprecht, S. 59-67.

Kraft, Volker (2011): Beraten. In: Kade, J./Helsper, W./Lüders, C./Egloff, B./Radtke, F.-O. /Thole, W. (Hg.): Pädagogisches Wissen: Erziehungswissenschaft in Grundbegriffen. Stuttgart: Kohlhammer S. 155-161.

Kossack, Peter (2009): Bildungsberatung revisited. Ein Strukturmodell zur Bildungsberatung. In: Rolf, A./Gieseke, W./Zeuner, C. (Hg.): Bildungsberatung im Dialog. Theorie - Empirie - Reflexion. Band 1. Baltmannsweiler: Schneider Verlag, S. 45-67.

Langheine, Volker (1983): Textpragmatische Analyse schriftlicher Kommunikation am Beispiel des Briefes. In: Grosse, S. (Hg.): Schriftsprachlichkeit. Düsseldorf, S. 190-211. https://ids-pub.bsz-bw.de/files/1977/Langeheine_Textpragmatische Analyse_1983.pdf [Zugriff: 05.12.2017].

Nittel, Dieter (2009): Beratung - eine (erwachsen-)pädagogische Handlungsform. Eine definitorische Verständigung und Abgrenzung. In: Hessische Blätter für Volksbildung 59, 1, S. 5-18. 
Meyer, Katharina (1997): Weiterbildungsberatung - Aktuelle Aufgaben und Probleme. In: Hessische Blätter für Volksbildung 47, 3, S. 224-233.

Müller, Andrea (2005): Weiterbildungsberatung. Qualitative Analyse von Interaktions- und Prozessverläufen situativer und biographieorientierter Weiterbildungsberatungsgespräche. Berlin: Rhombos-Verlag.

Oevermann, Ullrich/Allert, Tilman/Konau, Elisabeth/Krambeck, Jürgen (1979): Die Methodologie einer ,objektiven Hermeneutik“ und ihre allgemeine forschungslogische Bedeutung in den Sozialwissenschaften. In: Soeffner, H.-G. (Hg.): Interpretative Verfahren in den Sozial- und Textwissenschaften. Stuttgart: Metzler, S. $352-434$.

Pick, Ina (2017) (Hg.): Beraten in Interaktion. Eine gesprächslinguistische Typologie des Beratens. Frankfurt a. M.: Peter Lang.

Schiersmann, Christiane/Remmele, Heide (2004): Beratungsfelder in der Weiterbildung. Eine empirische Bestandsaufnahme. Baltmannsweiler: Schneider Verlag Hohengehren.

Stanik, Tim (2015): Beratung in der Weiterbildung als institutionelle Interaktion. Frankfurt a. M.: Peter Lang.

Stanik, Tim /Maier-Gutheil, Cornelia (2018): E-Mail-Weiterbildungsberatungen. Ergebnisse einer qualitativen Korrespondenzanalyse. Medienpädagogik 30, (2. März), 73-93. doi:10.21240/mpaed/30/2018.03.02.X.

Strauss, Anselm/Corbin, Juliet (1996): Grounded Theory: Grundlagen Qualitativer Sozialforschung. Weinheim: Beltz.

Weinhardt, Marc (2015) (Hg.): Psychosoziale Beratungskompetenz. Pilotstudien aus der Arbeitsstelle für Beratungsforschung. Weinheim: Beltz.

Wolf, Marc (2014): E-Mail in der Psychotherapie - Entwicklung und Evaluation eines E-Mail basierten Nachsorgeprogramms für die stationäre Psychotherapie. Heidelberg. http://archiv.ub.uni-heidelberg.de/volltextserver/16930/1/WOLF Dissertation_final.pdf [Zugriff: 05.12.2017]. 



\section{Bildung, Beratung, Bewegung - Die Steuerung der Bildungsreise zwischen Apodemik und europäischer Mobilitätspolitik}

\section{Einleitung}

Der Beitrag thematisiert die Frage, wie (Bildungs-)Reisende in informellen Kontexten Rat für ihr Reisevorhaben finden und wie durch unterschiedliche Modi von Beratung subjektive Reiseerfahrungen vorstrukturiert werden. Dafür wird ein weiter Begriff von Beratung zugrunde gelegt, der Beratung nicht an formale, professionelle (pädagogische) Settings bindet, in denen sich Beratende und Ratsuchende gegenüberstehen, sondern diese überall dort verortet, wo sich Subjekte externe Expertisen oder Dienstleistungen als Hilfe zur Bearbeitung individueller Sorgen aneignen.

Dieser Wandel von Beratung lässt sich auch in der sozialen Praxis des Reisens im Streben nach Bildung aufzeigen. Dabei stellt sich die Frage, wie und in welchen Formaten subjektive Bewegung, als Scharnier zwischen Bildung und Reisen, ,richtig“ eingesetzt wird, um dem Anspruch der Bildung auf Reisen zu entsprechen (vgl. Schroeder 2009). Beratung zielt in diesem Kontext demgemäß auf subjektive Mobilitätskompetenzen.

Reiseberichte und Reiseführer sind hierfür bereits seit Jahrhunderten ein Medium der Beratung. Entlang einer machttheoretischen Perspektive kann konstatiert werden, dass veränderte Subjektivierungsformen dabei stets neue Möglichkeiten der Beratung von Reisenden durch die Vermittlung von Wissen oder veränderte Formen der Adressierung von potentiell Reisenden evozieren.

Der Beitrag beschäftigt sich mit der Verbindung von Reisen, die in der Erwartung von Bildung angetreten werden, und Beratungsformen, in denen die Frage, wie sich Subjekte auf Reisen bewegen können oder sollen, bearbeitet werden. Ich werde darauf eingehen, wie und wodurch auf Subjekte von Beratung in ihren subjektiven Aneignungsprozessen von Welt durch Beratung eingewirkt wird, welche Verschiebungen sich im Rat zum richtigen Reisen zeigen und wie ein erwachsenenbildungsforschender Beratungsbegriff dies reflexiv aufnehmen kann.

Dazu werde ich zunächst eine bildungstheoretisch-ideengeschichtliche Perspektive auf den Nexus Bildung-Reisen entwickeln, welche die Kategorie der Bewegung als Verbindung setzt (2.). Darauf folgt eine Thematisierung von Beratung, bei der diese unter der doppelten Differenz von Rat und Tat 
sowie Selbst- und Fremdbestimmung betrachtet wird (3.). Diese Bindung von Bildungsreisegeschehen an Beratungsakte werde ich im Folgenden anhand der Apodemik, einer im 16. Jahrhundert entstehenden Kunstlehre des richtigen Reisens, darstellen (4.). Davon ausgehend endet der Beitrag mit einer Reflexion des (Bildungs-)Beratungsbegriffs im Kontext von Bildungsreisen vor dem Hintergrund einer Thematisierung der Mobilitätspolitik der Europäischen Union als aktueller Form der Adressierung potentieller Bildungsreisender (5.).

\section{Bildung, Reisen und Bewegung}

Die Verbindung von Bildung und Reisen zu untersuchen mag zunächst wenig innovativ wirken, da beide Konzepte fast schon „natürlich“ aneinander gekoppelt zu sein scheinen. Die romantisierende Grundannahme fußt dabei darauf, dass das Reisen die Wahrnehmung von ,irritierender Fremdheit“ (Schäfer 2011) ermögliche, Subjekte dadurch in Differenz zu ihren gewohnten Selbst- und Weltverhältnissen treten und im Zuge dessen ein reflexives Verhältnis zu sich selbst entwickeln können, welches transformative Bildungserfahrungen (u. a. Koller 2012) zu evozieren vermag.

Wirft man einen Blick auf die Geschichte des (Bildungs-)Reisens, zeigt sich, dass das Reisen historisch über einen langen Zeitraum primär funktional-materiell bestimmt wurde. Die Legitimierung der Bewegung konnte dabei nur durch das Erfüllen mit ihr verbundener politischer, ökonomischer oder militärischer Zwecke erfolgen (vgl. Hlavin-Schulze 1998). Die ideelle Zusammenführung von Bildung und Reisen als ,nicht-utilitäres“ Phänomen (Stagl 2002, 71) entpuppt sich so als moderne Erfindung, die erst auf der Grundlage eines Fortschritts an materiellen und technologischen Ressourcen sowie einer Erhöhung unterschiedlicher Qualifikationsbedarfe in bestimmten Bereichen erklärbar wird (ebd.). Die ideengeschichtliche Zusammenführung der Leitkategorien ergibt sich dabei aus der jeweiligen Referenz auf das Moment der Bewegung (vgl. Hlavin-Schulze 1998: 17ff), aus der Notwendigkeit des physischen Bewegens beim Reisen sowie der theoretisch-ideellen Ausprägung eines spezifischen Begriffs von Bildung.

Bildungstheoretisch zeigen sich Bildung und Bewegung so bei Wilhelm von Humboldt in der dritten Bestimmung der Wechselwirkung von Ich und Welt als rege verbunden. Während die beiden übrigen Bestimmungen vor allem die Vielfältigkeit der Beschäftigungen mit Welt (allgemein) sowie die äußeren Ermöglichungsbedingungen von Bildungsprozessen (frei) thematisieren, beschreibt Humboldt mit dem Attribut rege den Prozesscharakter von Bildung als bewegt (Humboldt 1793: 236). Ex negativo findet sich diese 
Denkfigur von Bildung als bewegter Tätigkeit auch in Theodor W. Adornos Theorie der Halbbildung, in welcher er den von ihm konstatierten Zustand von Halbbildung als degenerierter Bildung u. a. über die Zuschreibung einer defensiven Haltung des Stillstands, also über das Fehlen von Bewegung, charakterisiert (Adorno 1972: 108). In beiden Fällen bietet das verwendete bildungstheoretische Vokabular Anschlussmöglichkeiten für die Vorstellung, Bildung sei über das Mittel der Reise zu „erreichen“.

Aus einer sozialgeschichtlichen Perspektive zeigt sich zudem ein Wandel darin, wie Bildung und Reisen historisch zueinandergefunden haben. Bildung und Reisen wurden seit der Spätrenaissance für höchst differente soziale Gruppen in unterschiedlichen Reiseformen möglich (vgl. Hlavin-Schulze 1998). Die jeweiligen Ansprüche an Bildung unterschieden sich entsprechend. So ist es naheliegend, dass die Grand Tour der jungen Adligen Europas in der Renaissance sich so sehr von den heutigen Erasmus-Studierenden unterscheidet, wie dies bei der Walz im 18. oder den politischen Wanderbewegungen des 19. Jahrhunderts der Fall ist (ebd.).

Gleichzeitig wird daraus ersichtlich, dass das, was jeweils als Bildung galt, nicht für alle zu allen Zeiten in gleichem Maße einlösbar war und ist. Mit Bildungsreisen gehen stets auch Sorgen oder Unsicherheiten über den sinnvollen Gebrauch subjektiven Mobilitätspotentials einher. Bildungsreisen sind so in einem Doppelsinn auf die Kategorie der Sicherheit verwiesen: Reisen stellten über einen historisch langen Zeitraum zunächst ein Risiko für die leibliche Sicherheit dar. Reiseanleitungen dienten so dem Ziel des ungefährlichen Reisens. Sicherheit beschreibt jedoch auch die Gewissheit über die Richtigkeit des Reisens. Es geht im Kontext Bildungsreise nicht nur um bloße Bewegung, sondern eben um das richtige Bewegen der Richtigen zum richtigen Zweck. Akteur*innen der Vermittlung von Sicherheit richten sich hier auf Sorgen über die potentielle Uneinlösbarkeit des Erfahrenen. Daran schließen sich Fragen der Anerkennung des Reisens an, was sowohl die Resonanz im sozialen Umfeld als auch den Kontext formaler oder informeller Qualifizierungsbestrebungen tangiert. Bildungsreisen werden über diese Unsicherheiten somit auch Gegenstand des Suchens und Findens von Rat, was sie für Beratungskontexte zugänglich macht.

Die in Bildungsreisen zum Ausdruck kommenden Formen von Bewegung sind so stets im Spannungsfeld von Individualisierung und Zertifizierung zu betrachten. Keine Reise ohne mich selbst zu einem Anderen, einem Besseren zu entwickeln - keine gelungene Reise ohne die Anerkennung, mich zu einem Anderen, Besseren entwickelt zu haben. Reiseberatungen können dem sich in Sorge um die Richtigkeit seines Bildungsreisens befindlichen Subjekt helfen, indem sie eine Richtschnur anerkennungsfähigen Bildungsreisens anbieten. Folgt das Subjekt ihrem Rat, so steht diese Ausrichtung an Direktiven jedoch in Gegensatz zu einer Vorstellung des Bildungsreisens, welche vor allem die Spontaneität der Erfahrung hochschätzt und sich demgemäß nicht unter das 
Regiment einer Reiseleitung begeben kann. Innerhalb dieses Spannungsfelds lässt sich auch der Zusammenhang von Bildungsreise und Beratungsgeschehen im Folgenden verorten.

\section{3. (Reise-)Beratung und die doppelte Differenz}

An Beratung kommt man nur schwer vorbei. Die Zunahme und Ausweitung von Beratungsangeboten führt tendenziell dazu, dass sich Subjekte überall, jederzeit und lebenslänglich ${ }^{1}$ beraten lassen können. Beratung kann folglich betrachtet werden , als ein Schema der Kommunikation, das in allen gesellschaftlichen Kontexten anwählbar geworden ist" (Fuchs/Mahler 2000: 358).

Was mit Beratung jedoch angewählt wird, soll im Folgenden zunächst unter dem Postulat einer doppelten Differenz von Beratung betrachtet werden. Dabei werde ich bei der Thematisierung von Beratung zwischen Rat und Tat (vgl. ebd.) sowie zwischen Selbst- und Fremdbestimmung (Duttweiler 2004) unterscheiden.

Die Unterscheidung von Rat und Tat nähert sich dem Thema Beratung unter einer systemtheoretischen Perspektive über einen zeitlichen Abstand zwischen dem Akt der Beratung und einer nachgelagerten beratenen Tat (vgl. ebd.: 350f). Das Einholen von Rat als „Aufschub“ des Tuns geht dabei davon aus, dass in der Zukunft liegende Handlungen durch Rat potentiell optimierbar sind. Rat impliziert hier „das Motiv des Aufschubs, der Verzögerung [...] im Blick auf eine Zukunft, in der in bestimmten Hinsichten eine Palette von Handlungsmöglichkeiten enthalten ist" (ebd.: 354). Beratung geht so vom Vorhandensein mehrerer (richtiger oder falscher, sinnvoller oder sinnloser, rationaler oder irrationaler) Entscheidungsoptionen aus. „Beraten werden kann nur, wer abweichende Optionen wahrnehmen könnte und für sich selbst noch Freiheitsgrade sieht" (ebd.: 352).

Die Vielfalt von Optionen erfordert jedoch eine „(riskante) Auswahl, die eben durch das Begehren nach und Gewähren von Rat aufgeschoben wird“ (ebd.: 355). Gesellschaftliche Ordnungsverschiebungen, welche Leistungen zunehmend dem Individuellen statt dem Kollektiv zuschreiben, konfrontieren Subjekte dabei mit einer Permanenz eben dieses „Regime des Risikos“ (Fach 2004: 232). Beratung wird für das sich selbst verantwortende Subjekt zum individuellen Kompensationsinstrument der Risikogesellschaft (vgl. Ri-

1 Schon 2004 hat die EU zum Konzept des Lifelong Learning auch die Idee der Lifelong Guidance gesellt, welchen den enormen Stellenwert von Beratung auch auf politischer Ebene untermauert (vgl. Schiersmann 2010: 747; Ribolits 2007). 
bolits 2007: 21), in welchem die in die Autonomie entlassenen Subjekte (vgl. Dzierzbicka/Sattler 2004) verloren geglaubte Sicherheit wiederzugewinnen hoffen.

Beratung setzt entsprechend in ihrer Eigenlogik Selbstbestimmung und Freiwilligkeit (vgl. Pätzold/Ulm 2015) voraus, wobei sich machttheoretisch ein ständiges Oszillieren zwischen Selbst- und Fremdbestimmung innerhalb des Beratungsprozesses feststellen lässt (vgl. Duttweiler 2004): Subjekte entschließen sich selbstbestimmt dazu, Rat zu suchen, geben im konkreten Beratungsprozess einen Teil ihrer Autonomie ab, um als verbesserte eigenverantwortlich Handelnde gestärkt aus dem Beratungsgeschehen hervortreten zu können. Angelehnt an eine Perspektive auf die Subjektivierungsform des unternehmerischen Selbst (Bröckling 2002), gerät das Sich-beraten-lassen(-können) zu einer bedeutenden Selbstkompetenz für ein Subjekt, welches sich darum sorgt, dass seine Handlungen nicht deckungsgleich mit den marktgängigen Voraussetzungen sein könnten. „Wer sich beraten lässt, sucht (auch) Entlastung von der Selbstbestimmung" (Duttweiler 2004: 25), ohne dabei das Heft des Handelns völlig aus der Hand geben zu müssen. Der Möglichkeit individueller Fehlentscheidungen tritt Beratung in Form einer Absicherungsinstanz entgegen. Beratungen bieten so temporaltheoretisch betrachtet einen Vorschub an Anerkennung für zukünftig zu vollziehende Handlungen.

Im Kontext des Bildungsreisens lassen sich die hier stattfindenden Beratungsakte auf den Bereich der informellen Bildungsberatung eingrenzen (Ewers 2014). Reiseentscheidungen sind demgemäß Bildungsentscheidungen. Bezieht man die oben skizzierten Voraussetzungen einer doppelten Differenz des Beratungsbegriffs auf Bildungsreisen, so lassen sich die Formen der Adressierung von potentiellen (Bildungs-)Reisenden dahingehend befragen, wie potentielle Reisende in informellen Ratgebern adressiert und wie subjektive Aneignungsprozesse von Welt durch spezifische Beratungssettings präformiert werden.

\section{Apodemiken - Kunstlehren des richtigen Reisens}

Um einen Einblick in Modi der Adressierung und Beratung von Reisenden zu ermöglichen, wird im Folgenden das historische Phänomen der Apodemiken vorgestellt. Diese bezeichnen eine literarische Gattung, welche im Ende des 16. Jahrhunderts entstand. Apodemiken stellten reisespezifische Formen von Ratgeberliteratur dar, deren Lektüre Reisende im „richtigen“ Erfahren von Unbekanntem schulten (vgl. Stagl 2002).

Auch wenn die Apodemik mit der Zunahme touristischen Reisens und der Popularisierung von massenmedial produzierten Reiseführern im 19. 
Jahrhundert wieder verschwand, liefert sie dennoch einen Zugang zur Bearbeitung der Frage von Ratsuchen und Beratungen rund um das Thema Bildungsreise.

Apodemiken unterscheiden sich stark von dem, was heute unter Reiseführern verstanden wird. Sie widmen sich primär der Schilderung und Anweisung davon, wie Subjekte reisen und die Fremdheitserfahrungen einordnen sollen (vgl. Stagl 1983: 7). Das Verfassen von Apodemiken kann dabei als Manifestierung der Reflexion der Reise sowie als Beratungsakt für potentielle Bildungsreisende betrachtet werden. Sie wurden in aller Regel nicht als ortsbezogene Reiseführer verfasst. Beschreibungen einzelner Destinationen dienten eher als beispielhafte Schulungen des richtigen Beobachtens und Erfahrens. Ziel der Apodemiken war dementsprechend nicht die Kenntnis einzelner Orte, sondern eine bildungsreisespezifische Mobilitätskompetenz, die dann allerorts anwendbar sein sollte.

Dabei adressierten die Apodemiken die potentiell Mobilen direkt, wodurch sie dem Feld der ,personenbezogenen Beratung“ (Schiersmann 2010: 750) im Kontext informeller Bildungsberatung zugeordnet werden können. Sie zeugen hierin von einer Festsetzung des Ziels des Reisens als Bildungsreise: „Das Reisen zur Vervollkommnung der Persönlichkeit und zur Erlangung neuer Kenntnisse (peregrinari) wird dem zweck- und nutzlosen Umherschweifen (vagari, errare) stets scharf entgegengesetzt; die Reise, von der die Apodemiken handeln, wird als Bildungsreise bestimmt" (Hlavin-Schulze 2007: 84).

Drei wesentliche Merkmale der Veränderung des Anspruchs an Reisen sind für Apodemiken charakteristisch:

- Bildungsreisen erfahren durch Apodemiken eine zunehmende Methodisierung (vgl. Stangl 1983: 9; Brenner 1989: 31). Indem Apodemiken mit präzisen Schilderungen zur Durchführung des Reisens zum Zweck möglichst „wahrer“ Erfahrung aufwarten, entlasten sie die Reisenden von Sorgen des „falschen“ Reisens.

- Mit der Methodisierung geht zudem eine tendenzielle Entempirisierung von Bildungsreisen einher (Brenner 1989: 31f; Zick 2015: 82). Die Apodemik bricht mit der romantisierten Vorstellung von Bildungsreisen als möglichst spontanem Erfahren von Fremdheit und Differenz, indem sie eben diesem Erfahren eine methodische Richtschnur bestmöglicher Bildungsreisepraxis entgegenhält. Bildung wird so im Spannungsfeld von präformierter und wahrer Erfahrung konstruiert. Der Rat zum richtigen Reisen steht der romantisierten Vorstellung unvermittelter Differenzerfahrung entgegen.

- Daran anschließend lässt sich schließlich eine Pädagogisierung von Bildungsreisen konstatieren (vgl. Hlavin-Schulze 1998: 83; vgl. Zick 2015: 84). Reiseberatung operiert hier durch die Vermittlung sozialer Erwar- 
tungsvorstellungen an individuelle Bildungsreiseleistung. Apodemik ,war zunächst und vor allem Erziehungsform“ (Hlavin-Schulze 1998: 83), innerhalb derer eine Hierarchie von Beratenden und Beratenen hervortritt.

Den Lohn für das Befolgen des Rats stellt die Apodemik den potentiell Reisenden in Form einer erhöhten Sicherheit der Anerkennungsfähigkeit des subjektiven Bildungsreisens bereit. Apodemiken bildeten zu ihrer Zeit den sozialen Standard der von Bildungsreisenden zu erwartenden Leistung ab und konnten als Beratungsinstanz auf die sich um ihre Bildung sorgenden Reisesubjekte einwirken.

Die Mechanismen, mit welchen Apodemiken ihre Wirkung entfalteten, lassen sich machttheoretisch in Anlehnung an Foucault über den Begriff der Disziplin reflektieren (vgl. Foucault 1976). Diese richtet sich auf die Steigerung des produktiven Vermögens von Individuen. Die Machttechnik der Disziplin installiert dabei ,ein Regime minutiöser Reglementierungen und fortwährenden Übens“" (Bröckling 2017: 176). Wie die Apodemik überlässt die Disziplin nichts dem Zufall, indem sie „die Zeit, den Raum, die Bewegungen bis ins Kleinste codiert" (Foucault 1976: 175). Beratung wirkt dabei insofern individualisierend, als dass Subjekten im Rahmen des Disziplinierungsregimes zunächst eine, je individuelle, Abweichung von einer zu erreichenden Norm unterstellt wird - in diesem Fall zur Norm des bildenden Reisens. Der darin postulierten „Gefahr“ des falschen Gebrauchs individuellen Mobilitätspotentials wird in der Apodemik durch Schilderungen des einübbaren den Bildungsnormen entsprechenden Reisens beigekommen.

Anhand von Apodemiken lassen sich so Mechanismen der Unterweisung im richtigen Gebrauch des subjektiven Umgangs mit den je eigenen Mobilitätspotentialen herausstellen. Apodemiken können dementsprechend als beratende Hilfestellung für Reisende begriffen werden, mit welchen diese im sozial angemessenen Umgang mit den ihnen eigenen Kräften der Bildung qua Bewegung geschult werden (vgl. Zick 2015: 85).

\section{5. (Europäische) Apodemiken heute, oder: Wie weiter mit Beratung?}

Auch wenn von Apodemiken heutzutage keine Antwort mehr auf das Problem der sich um ihre Mobilität sorgenden Subjekte zu erwarten ist, kann nicht davon ausgegangen werden, dass diese Frage mit dem Ableben der Apodemik verschwunden ist. Im Gegenteil: Unter Vorzeichen eines sich vergrößernden Mobilitätsaufkommens, einer tendenziellen Omnipräsenz des Mobilitätsimperativs (vgl. Pongratz 2003: 149) sowie einer enorm gesteigerten Bedeutung 
von Mobilität im Rahmen formalisierter Bildungswege (vgl. Liesner 2008) stellt sich das Problem umso dringender.

Vor allem im Rahmen der Mobilitätspolitik der EU, innerhalb welcher Bildungsreisebewegungen als learning mobility bzw. Mobilität zu Lernzwecken auftreten, lässt sich eine postmoderne Nachfolgerin apodemischen Wirkens identifizieren. Auch hier handelt es sich um einen informellen Beratungskontext, innerhalb dessen die zu Beratenden jedoch nur nachgelagert adressiert werden. Die Wirkweise europäischer Mobilitätspolitik vollzieht sich zunächst nicht über die unmittelbare Interaktion von Beratenden und Beratenen.

Die Rede von „Mobilität zu Lernzwecken“ verdeutlicht zunächst, dass eben nicht alle Bewegungen den angestrebten Zwecken dienlich sind, was eine definitorische Rahmung der Mobilitäten notwendig macht, innerhalb derer sich anerkennungsfähige und förderfähige Bildungsbewegungen abspielen können:

\begin{abstract}
„Die Mobilität zu Lernzwecken - also ein Auslandsaufenthalt mit dem Ziel, neues Wissen sowie neue Fähigkeiten und Kompetenzen zu erwerben - ist eine der grundlegenden Möglichkeiten, mit denen junge Menschen ihre künftige Beschäftigungsfähigkeit erhöhen, ihr interkulturelles Bewusstsein und ihre persönliche Entwicklung voranbringen sowie ihre Kreativität und ihren Bürgersinn stärken können. Europäer, die als junge Lernende mobil sind, sind zumeist auch später im Arbeitsleben mobil.“ (Amtsblatt der Europäischen Union 2011/C 199/01: 1)
\end{abstract}

Im Gegensatz zur Apodemik finden sich hier keine präzisen Schilderungen und Anweisungen zum richtigen Reisen, sondern zunächst eine politische Setzung über den Gegenstand, worauf im Folgenden (vgl. ebd.: 2) organisationsbezogene Zielsetzungen zur Erleichterung von Mobilität folgen. Erreicht werden soll diese primär durch den Abbau von Mobilitätshindernissen, wobei die EU hier organisationsbezogenen Rat für die personenbezogenen Beratungen ihrer Mobilitätspartner liefert.

Die diversen strategischen Ziele zum Abbau von Mobilitätshindernissen (vgl. ebd.) eint das Bemühen, die Voraussetzungen zu schaffen, um dem Imperativ zur Bewegung möglichst umfassend Wirksamkeit zu verleihen. Zumindest rhetorisch inkludiert der Mobilitätsimperativ dabei alle sozialen Gruppen mit dem Ziel, „,dass Lernphasen im Ausland für alle jungen Menschen in Europa allmählich die Regel werden und nicht mehr die Ausnahme sind" (ebd.: 2). Die Unterstellung einer Förderfähigkeit scheinbar jeder gewünschten Mobilität verschiebt wiederum die Verantwortlichkeit für einen Mangel an Bewegung vom bloßen Nicht-Vorhandensein in ihre Verweigerung.

Aus einer Perspektive auf Mobilität als unternehmerische Selbstkompetenz ist die Botschaft von ERASMUS-Slogans wie „Wer sich bewegt, bewegt Europa" überdeutlich: Wer sich dem Sollwert der Mobilität verweigert, muss sich später nicht über etwaige Konsequenzen wundern. „Immobile Bürger 
gefährden nicht nur die Wettbewerbsfähigkeit Europas und seiner Nationalstaaten, sondern auch ihre eigene" (Liesner 2006, 175).

Vergleicht man europäische Mobilitätspolitik und Apodemik hinsichtlich der ihnen eingelagerten Beratungsmomente, so zeigen sich grundlegende Differenzen. Während in der Apodemik die richtige Auslegung von Bewegung disziplinierend in die sich bildenden Körper eingeschrieben wurde, agiert das gouvernementale Führen im Rahmen der EU-Mobilitätspolitik subtiler. Der Imperativ der Mobilität erfährt seine Wirksamkeit eher durch permanentes Hinweisen auf das Richtige als durch konkretes Unterweisen im Richtigen, was sich als anschlussfähig an Strategien des neoliberalen, sanften Führens zeigt (Bröckling 2017). Der Umgang mit subjektiven Mobilitätspotentialen in Beratungsformaten lässt sich so machttheoretisch als stark von differenten sozialen Konstruktionen von Selbst- und Fremdbestimmung abhängig beschreiben.

Es zeigt sich, abschließend, dass soziale Adressierungsformen von Beratung in unterschiedlichen historischen Kontexten beträchtlich differieren. Dies gilt sowohl für die Suche nach Rat, welche historisch möglich wird, als auch für die Angebotsstruktur von Beratung. Dabei offenbart sich ein Verhältnis von Ratsuche und Ratgabe, in dem nicht davon ausgegangen werden kann, dass Beratungsangebote den Artikulationen von Sorge oder den Bedürfnissen nach Rat nur reaktiv gegenüberstünden. Im Gegenteil: Die Unausweichlichkeit der Konfrontation mit Darstellungen gelungener Mobilität sowie die Omnipräsenz von Mobilitätsangeboten können auch als Anlässe betrachtet werden, durch welche das Bedürfnis nach Mobilität zu Lernzwecken sowie die Sorge um die Erreichung dieser Zwecke erst hervorgebracht werden. Subjektive Bildungsansprüche werden so durch Bildungsberatungsformate präformiert. Weiterbildungswiderstand (Holzer 2017) gegenüber diesen Praxen der Präformation von Erfahrung durch Beratung müsste sich, in Anlehnung an Foucaults Kritikbegriff (Foucault 1992: 8), auf das Bemühen oder die Kunst, nicht dermaßen beraten zu werden konzentrieren. Auch Bildungsberatung kommt demgemäß nicht umhin, ihre Involviertheit in das Zusammenspiel der Evokation von Sorge und ihrer Bearbeitung reflexiv aufzunehmen.

\section{Literatur}

Adorno, Theodor W. (1972): Theorie der Halbbildung, in: Ders.: Gesammelte Schriften Bd. 8, Frankfurt a. M.: Suhrkamp, S. 93-121.

Amtsblatt der Europäischen Union (2011): Empfehlung des Rates vom 28. Juni 2011 Jugend in Bewegung - die Mobilität junger Menschen zu Lernzwecken fördern (2011/C 199/01). 
Brenner, Peter J. (1989): Die Erfahrung der Fremde. Zur Entwicklung einer Wahrnehmungsform in der Geschichte des Reiseberichts, in: Ders. (Hg.): Der Reisebericht, Frankfurt a. M.: Suhrkamp, S. 14-49.

Bröckling, Ulrich (2007): Das unternehmerische Selbst - Soziologie einer Subjektivierungsform, Frankfurt a. M.: Suhrkamp.

Bröckling, Ulrich (2017): Nudging: Gesteigerte Tauglichkeit, vertiefte Unterwerfung, in: Ders.: Gute Hirten führen sanft. Frankfurt a. M.: Suhrkamp, S. 175-196.

Duttweiler, Stephanie (2004): Beratung, in: Bröckling, Ulrich et al. (Hg.): Glossar der Gegenwart. Frankfurt a. M.: edition suhrkamp, S. 23-30.

Dzierzbicka, Agnieszka / Sattler, Elisabeth (2004): Entlassung in die Autonomie Spielarten des Selbstmanagement, in: Pongratz, Ludwig A. et al. (Hg.): Nach Foucault - Diskurs- und machtanalytische Perspektiven der Pädagogik. Wiesbaden: VS Verlag für Sozialwissenschaften, S. 114-133.

Ewers, Nicole (2014): Bildungsberatung, in: Glossar Oekonomisierung von Bildung. Online: $\quad$ http://www.gloeb.de/index.php?title=Bildungsberatung\&oldid=918 [Stand: 25.04.2018].

Fach, Wolfgang (2004): Selbstverantwortung, in: Bröckling, Ulrich / Krasmann, Susanne / Lemke, Thomas (Hg.): Glossar der Gegenwart. Frankfurt a. M.: Suhrkamp, S. 228-235.

Foucault, Michel (1994): Überwachen und Strafen: Die Geburt des Gefängnisses. Frankfurt a. M.: Suhrkamp.

Foucault, Michel (1992): Was ist Kritik? Berlin: Merve Verlag.

Fuchs, Peter / Mahler, Enrico (2000): Form und Funktion von Beratung, in: Soziale Systeme 6, H.2, 2000, S. 349-368.

Hlavin-Schulze, Karin (1998): „Man reist ja nicht, um anzukommen“: Reisen als kulturelle Praxis. Frankfurt a. M./New York: Campus Verlag.

Holzer, Daniela (2017): Weiterbildungswiderstand. Eine kritische Theorie der Verweigerung. Bielefeld: transcript.

Humboldt, Wilhelm von (1793/1980): Theorie der Bildung des Menschen [Fragment], in: Flitner, Andreas et al. (Hg.): Wilhelm von Humboldt: Werke in fünf Bänden. Bd. IV. Darmstadt: WBG, S. 234-240.

Koller, Hans-Christoph (2012): Bildung anders denken - Einführung in die Theorie transformatorischer Bildungsprozesse. Stuttgart: Kohlhammer.

Liesner, Andrea (2008): Mobilität, in: Dzierzbicka, Agnieszka et al. (Hg.): Pädagogisches Glossar der Gegenwart. Von Autonomie bis Zertifizierung. Wien: Erhard Löcker.

Pätzold, Henning / Ulm, Susanne (2015): Freiwilligkeit in der erwachsenenpädagogischen Beratung, in: Zeitschrift für Weiterbildungsforschung - Report (38), 2/2015, S. 183-196.

Pongratz, Ludwig A. (2003): Zeitgeistsurfer. Beiträge zur Kritik der Erwachsenenbildung, Weinheim: Beltz 2003.

Ribolits, Erich (2007): Lifelong guidance. Die sanfte Art, Menschen zum Funktionieren zu bringen, in: schulheft 127, 3/2007, S. 18-29.

Schäfer, Alfred (2011): Irritierende Fremdheit: Bildungsforschung als Diskursanalyse. Paderborn: Ferdinand Schönigh.

Schiersmann, Christiane (2010): Beratung im Kontext lebenslangen Lernens, in: Tippelt, Rudolf et al. (Hg.): Handbuch Erwachsenenbildung/Weiterbildung, 4., 
durchgesehene Aufl., Wiesbaden: VS Verlag für Sozialwissenschaften, S. 747767.

Schroeder, Jörg (2009): Subjektivierung im Grenzland. Zur Gouvernementalität und Hegemonietheorie von Reisen und Bildung. Hamburg: Verlag Dr. Kovac.

Stagl, Justin (2002): Eine Geschichte der Neugier. Die Kunst des Reisens 1550-1800. Wien: Böhlau Verlag.

Stagl, Justin (1983): Apodemiken - Eine räsonnierte Bibliographie der reisetheoretischen Literatur des 16., 17. und 18. Jahrhunderts. Paderborn: Ferdinand Schöningh.

Zick, Sebastian (2015): Bildung, Reisen und Mobilität - ,,je beweglicher, desto edler"??, http://www.fabrico-verlag.de/online-texte/thesis-paedagogik/ [Stand: 25.04.2018]. 

III. Beratung in der wissenschaftlichen Weiterbildung: Strukturen - Praxis Herausforderungen 



\section{Optimierung von Beratungsstrukturen für nicht-traditionell Studieninteressierte in der wissenschaftlichen Weiterbildung - am Beispiel von Studiengangkoordinierenden auf Programmebene}

\section{Einleitung}

Die wissenschaftliche Weiterbildung hat innerhalb der deutschen Hochschullandschaft in den vergangenen Jahren einen rasanten Bedeutungszuwachs erfahren. ${ }^{1}$ Dazu haben die von Bund und Ländern aufgelegten Förderprogram$m^{2}$ einen wesentlichen Beitrag geleistet. Trotz dieser Entwicklungen kann Weiterbildung an deutschen Hochschulen aktuell als organisationskultureller Fremdkörper betrachtet werden (vgl. Seitter/Schemmann/Vossebein 2014: 3). Wesentliche Gründe hierfür sind in den für staatliche Hochschulen weitestgehend unbekannten Feldern der Nachfrageorientierung, der Erhebung vollkostendeckender Entgelte sowie der Entwicklung und Implementierung kundenbezogener Serviceleistungen zu sehen. Diese stehen im Gegensatz zur disziplinär abgeleiteten Angebotsorientierung staatlich finanzierter grundständiger und konsekutiver Studienprogramme (vgl. Seitter 2014: 143).

Die Zielgruppe der weiterbildendenden Studienprogramme sind in der Regel nicht-traditionell Studierende, die „,in verschiedenen Lebensphasen, mit individuellen beruflichen und familiären Verpflichtungen sowie sehr divergenten Leistungsvoraussetzungen an die Hochschule gelangen“" (Lewin/Vierthaler/Fraunhofer 2017: 160). Diese Zielgruppe weist spezielle Beratungs- und Unterstützungsbedarfe auf, wozu es jedoch noch an empirisch gesichertem Wissen fehlt (vgl. ebd.).

Der vorliegende Beitrag greift diese Erkenntnisse auf und geht am Beispiel der Serviceleistung der Beratung nicht-traditionell Studieninteressierter

1 Trotz der beschriebenen Entwicklungen stellt die wissenschaftliche Weiterbildung nach wie vor einen randständigen Bereich neben Forschung und Lehre dar und erhält in Praxis und Wissenschaft vergleichsweise wenig Aufmerksamkeit (vgl. Franz/Feld 2014: 30).

2 Exemplarisch sei hier auf den seit 2011 vom Bundesministerium für Bildung und Forschung (BMBF) geförderten Bund-Länder-Wettbewerb „Aufstieg durch Bildung: offene Hochschulen" hingewiesen

(vgl. hierzu: http://www.wettbewerb-offene-hochschulen-bmbf.de/ [Zugriff 25.01.2018]). 
in der wissenschaftlichen Weiterbildung der Frage nach, welche Beratungsbedarfe ${ }^{3}$ auf der Programmebene bearbeitet werden (sollten) und wie ein zielgruppenorientiertes Beratungssetting auf dieser Ebene organisiert werden kann. Daraus abgeleitet wird diskutiert, welche intraorganisationalen Rahmenbedingungen zu einer Optimierung der vorhandenen Beratungsstrukturen beitragen können.

Die Beantwortung dieser Fragen erfolgt auf der Grundlage einer kategoriengeleiteten inhaltsanalytischen Auswertung von Experteninterviews mit Studiengangkoordinierenden, die in zwei Teilprojekten des Verbundprojekts „WM ${ }^{3}$ Weiterbildung Mittelhessen ${ }^{4}$ durchgeführt wurden. Abbildung 1 visualisiert, wie aus der kombinatorischen Gesamtschau dieser zwei Projekte Erkenntnisse für die Optimierung von Beratungsstrukturen abgeleitet werden.

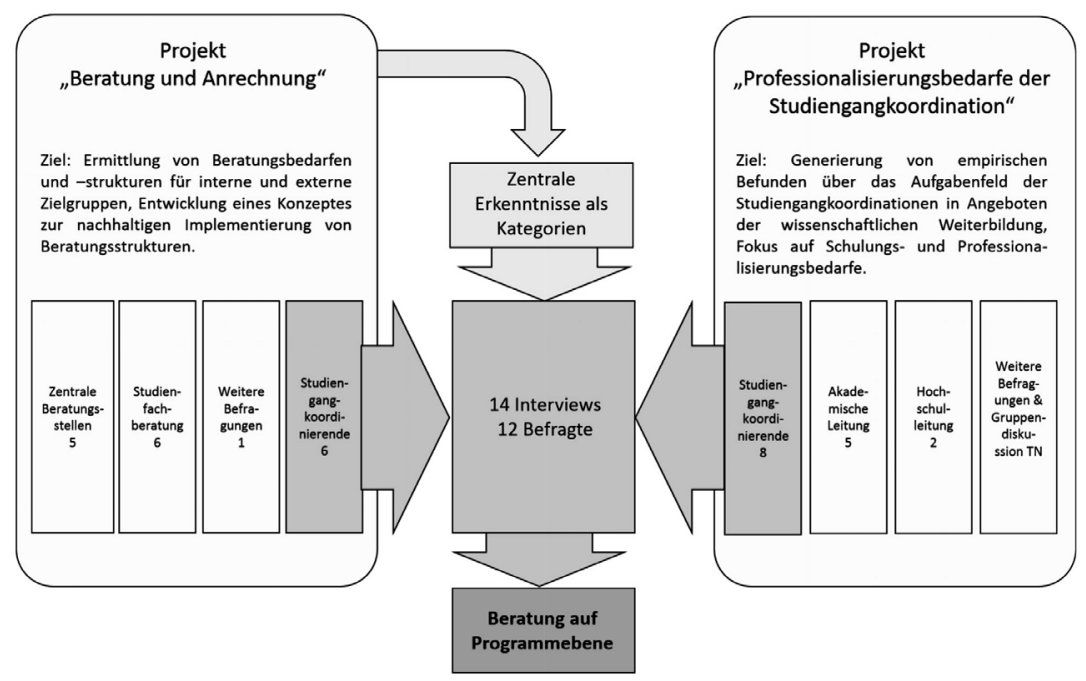

Abb. 1: Methodisches Vorgehen (Quelle: eigene Darstellung)

Dazu untergliedert sich der Beitrag in drei Teile: In einem ersten Teil werden sowohl die besonderen Beratungsbedarfe nicht-traditionell Studieninter-

3 Beratungsbedarfe können informativ, situativ oder biographie-orientiert ausgeprägt sein. Für ausführlichere Darstellungen siehe Gieseke 2000.

4 Hierbei handelt es sich um das Entwicklungsprojekt „Beratung und Anrechnung“ sowie das Forschungsprojekt „Professionalisierungsbedarfe der Studiengangkoordination“ des durch den Bund-Länder-Wettbewerb „Aufstieg durch Bildung: offene Hochschulen!“ geförderten Verbundprojektes „WM3 Weiterbildung Mittelhessen“. Für weitere Informationen siehe: www.wmhoch3.de [Zugriff 02.07.2018]. 
essierter (2.1) als auch generalisierbare hochschulische Beratungsstrukturen herausgearbeitet (2.2). Im zweiten Teil erfolgt eine Fokussierung auf die Beratungsleistungen, die auf der Ebene der weiterbildenden Studienprogramme durch die Studiengangkoordinierenden erbracht werden (3). Dabei finden sowohl die Beratungsanliegen Berücksichtigung, die auf dieser Ebene an die Hochschulprofessionellen adressiert werden (3.1), als auch die strukturellen Besonderheiten, die bei der Beratung nicht-traditionell Studieninteressierter zu berücksichtigen sind (3.2). Im dritten Teil des Beitrages erfolgt eine Rückbindung der dargestellten Ergebnisse auf die Ausgangsfragen, indem Potentiale für eine Optimierung der Beratungsstrukturen formuliert werden (4).

\section{Beratungsbedarfe und Beratungsstrukturen}

Da Programme der wissenschaftlichen Weiterbildung für öffentliche Hochschulen eine weitestgehend neue Angebotssparte darstellen, geraten nicht-traditionell Studieninteressierte als neue Zielgruppe in den Fokus. Insbesondere die mit den Beratungsanliegen konfrontierten Stellen innerhalb der Hochschulen sollten daher in den Blick genommen werden. Um eine effiziente Beratung ${ }^{5}$ realisieren zu können, spielen sowohl die Frage nach den gruppenspezifischen Beratungsbedarfen (2.1) als auch nach den intraorganisationalen Strukturen (2.2) eine große Rolle. Im Folgenden wird daher eine Darstellung der zentralen Aspekte zu diesen beiden Fragen vorgenommen, um daran anschließend vertiefende Betrachtungen vorzunehmen.

\subsection{Beratungsbedarfe}

Eine Befragung von Studienberatenden mit unterschiedlichen Funktionen (zentrale Studienberatung, Studienfachberatung, Studiengangkoordinierende) und unterschiedlicher organisationaler Verankerung (Zentrale Allgemeine Studienberatung, Studienberatung auf Fachbereichsebene, Studienberatung auf Ebene der Studienprogramme) zeigt, dass bei der Artikulation der Beratungsbedarfe Besonderheiten in der Gruppe der nicht-traditionell Studieninteressierten vorliegen. Diese Besonderheiten lassen sich in zwei Kategorien

5 Effiziente Beratung bezieht sich darauf, mit einem möglichst geringen Ressourcenaufwand und mit möglichst geringer Zeitverausgabung, sowohl für Beratende als auch auf Ratsuchende, ein optimales Beratungsergebnis zu erzielen. 
unterteilen. In der Kategorie der zielgruppenübergreifenden Beratungsfelder konnten Themenfelder identifiziert werden, in denen sowohl von traditionell als auch von nicht-traditionell Studieninteressierten Beratungsbedarfe artikuliert wurden: Dies betrifft die Felder Studienwahl, Passung, Bewerbung, Zulassung, Anrechnung und Finanzierung. Bei genauerer Betrachtung dieser Themenfelder werden jedoch zielgruppenspezifische Ausprägungen deutlich. Abbildung 2 zeigt die zielgruppenübergreifenden Beratungsfelder mit ihren jeweiligen zielgruppenspezifischen Ausprägungen.

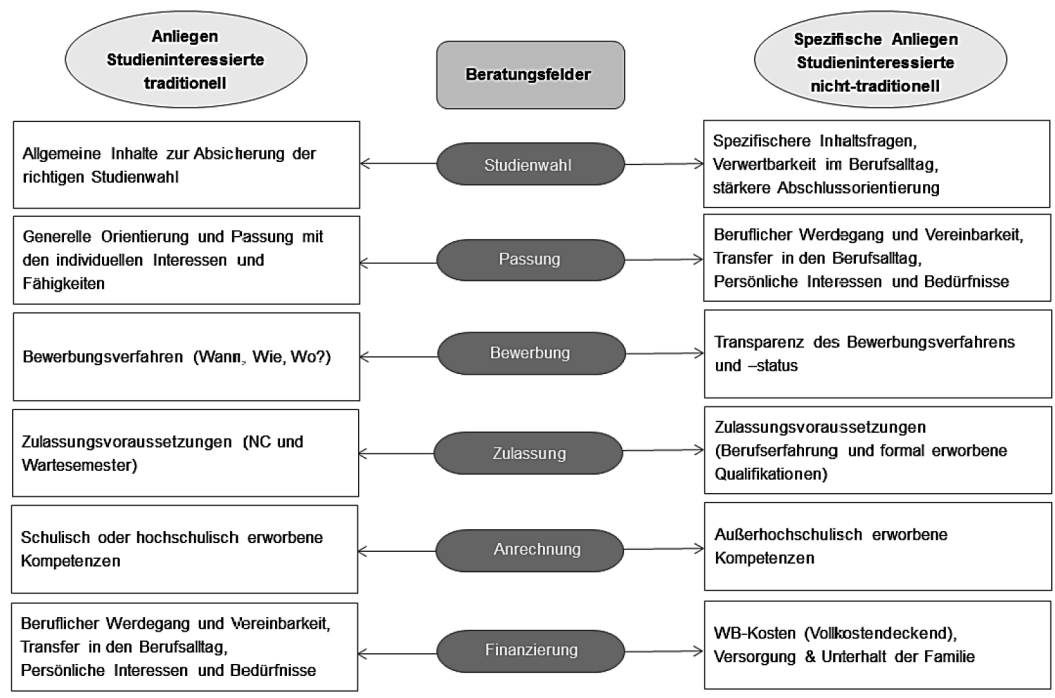

Abb. 2: Zielgruppenübergreifende Anliegen mit den jeweiligen Ausprägungen (Quelle: Sturm/Bopf 2017)

In der Kategorie der zielgruppenspezifischen Beratungsfelder der nicht-traditionell Studieninteressierten konnten neben den dargestellten spezifischen Bedarfen zusätzliche Beratungsbedarfe identifiziert werden, welche innerhalb der Gruppe der traditionell Studieninteressierten nicht artikuliert wurden. Dies ist die Frage nach der notwendigen Zeitverausgabung für ein weiterbildendes Studienprogramm sowie die Frage danach, ob die Studieninteressierten die individuellen Voraussetzungen zum Absolvieren des Studienprogramms erfüllen. Ein weiteres beratungsfeldübergreifendes Querschnittsthema bei der Beratung nicht-traditionell Studieninteressierter ist in der Frage nach der Vereinbarkeit des weiterbildenden Studienprogramms mit Familienpflichten und Erwerbsarbeit zu sehen. 
Zusammenfassend kann festgestellt werden, dass nicht-traditionell Studieninteressierte auf den gleichen Feldern Beratungsbedarfe artikulieren wie traditionell Studieninteressierte. Allerdings zeigen sich gruppenspezifische Ausprägungen bei den artikulierten Beratungsanliegen. Darüber hinaus konnten gruppenspezifische Beratungsfelder nicht-traditionell Studieninteressierter identifiziert werden. Themenunspezifisch gerahmt werden die Beratungsanliegen beider Zielgruppen oft durch die Frage nach der Vereinbarkeit.

\subsection{Beratungsstrukturen}

Am Beispiel einer dezentral organisierten Verankerung weiterbildender Studienangebote innerhalb einzelner Fachbereiche lassen sich hochschulweit drei Organisationseinheiten herausstellen, die wesentlich mit Beratungsanliegen nicht-traditionell Studieninteressierter im Feld der wissenschaftlichen Weiterbildung konfrontiert sind. ${ }^{6}$ Auf Hochschulebene werden entsprechende Beratungsanliegen an die zentralen Studienberatungen adressiert. An Fachbereichen, an denen weiterbildende Studienprogramme organisational verankert sind, werden die Fachstudienberatungen mit den Beratungsbedarfen konfrontiert. Und auf der Ebene der weiterbildenden Studienprogramme sind die Studiengangkoordinierenden die zentralen Ansprechpersonen.

Bei einer gleichzeitigen Betrachtung der Beratenden auf den drei Organisationsebenen können vier wesentliche ebenenspezifische Merkmale (Beratungsstruktur, Beratungstätigkeit, Beratungsinhalt und Implementierungsgrad) differenziert werden. Tabelle 1 zeigt die Charakteristika und unterschiedlichen Rahmenbedingungen der Beratenden auf diesen drei Ebenen.

6 Die drei identifizierten Organisationseinheiten werden auch im Falle einer zentralen organisationalen Verankerung von wissenschaftlicher Weiterbildung mit Beratungsanliegen nicht-traditionell Studieninteressierter konfrontiert. Hier entspricht die Fachbereichsebene der jeweils für wissenschaftliche Weiterbildung verantwortlichen Organisationseinheit (Zentrum für Weiterbildung). Für weitere Informationen siehe Sturm/Bopf 2017. 
Tab. 1: Charakteristika der auf verschiedenen Ebenen angesiedelten Organisationseinheiten (Quelle: Sturm/Bopf 2017)

\begin{tabular}{|l|l|l|l|}
\hline & $\begin{array}{l}\text { Hochschulebene } \\
\text { (Zentrale } \\
\text { Studienberatung) }\end{array}$ & $\begin{array}{l}\text { Fachbereichsebene } \\
\text { (Fachstudienberatung) }\end{array}$ & $\begin{array}{l}\text { Programmebene } \\
\text { (Studiengangkoordina- } \\
\text { tion) }\end{array}$ \\
\hline $\begin{array}{l}\text { Beratungs- } \\
\text { struktur }\end{array}$ & $\begin{array}{l}\text { zentral organisierte } \\
\text { Beratung }\end{array}$ & $\begin{array}{l}\text { Hybridform zentral / } \\
\text { dezentral }\end{array}$ & $\begin{array}{l}\text { dezentral organisierte } \\
\text { Beratung }\end{array}$ \\
\hline Beratungs- & $\begin{array}{l}\text { Beratung als wesentliche } \\
\text { tätigkeit }\end{array}$ & $\begin{array}{l}\text { Beratung als wesentliche } \\
\text { Tätigkeit }\end{array}$ & $\begin{array}{l}\text { Beratung als } \\
\text { Aufgabenfeld } \\
\text { vielen }\end{array}$ \\
\hline Beratungs- \\
inhalt
\end{tabular}

Im Folgenden wird die Beratung auf Programmebene durch die auf dieser Ebene tätigen Studiengangkoordinierenden vertieft betrachtet, da diesen als zentrale Ansprechpersonen sowohl für Weiterbildungsstudierende als auch für Studieninteressierte eine besondere Bedeutung zukommt.

\section{Programmebene: Beratung durch Studiengangkoordinierende}

Studiengangkoordinierende ${ }^{7}$ übernehmen die dezentral organisierte Beratung quasi direkt vor Ort und sind als „One-Stop-Office“ (Hanft 2014: 115) Haupt-Ansprechpersonen über den gesamten Student-Life-Cycle hinweg. Sie übernehmen eine Vielzahl von Aufgaben und sind die zentrale Schnittstelle in dem jeweiligen weiterbildenden Studienprogramm (vgl. Gronert/Rundnagel 2018: 201). Eine Aufgabe innerhalb dieses umfangreichen Tätigkeitsfel-

7 Studiengangkoordinierende, wie sie im Projekt „WM3 Weiterbildung Mittelhessen“ etabliert wurden, sind auf Programmebene die Personen, die das Angebot koordinieren und weitestgehend alle Aufgaben in der operativen Abwicklung, Finanzadministration, Marketing, Qualitätsmanagement, Prüfungsadministration, Studierendensupport, Dozierendenmanagement und Kooperationsmanagement übernehmen (weiterführend siehe Gronert/ Rundnagel 2017). 
des besteht in der Beratung von nicht-traditionell Studieninteressierten. Da Studiengangkoordinierende aufgrund ihrer dezentralen Verortung direkt im weiterbildenden Studienprogramm angesiedelt sind, ist anzunehmen, dass sie als Expertinnen und Experten sowohl mit organisationalem als auch mit programm- und fachspezifischem Wissen besonders bedeutsame Beratende für die Anliegen von Studieninteressierten sind. Um Potentiale zur Optimierung hochschulischer Beratungsstrukturen ableiten zu können, werden im Folgenden zunächst Beratungsbedarfe identifiziert, die an die Studiengangkoordinierenden adressiert werden (3.1). Daran anschließend werden die Anforderungen an Rahmenbedingungen der Beratung auf Programmebene herausgearbeitet (3.2).

\subsection{Beratungsbedarfe auf Programmebene}

Aus der Analyse der Befragungsergebnisse des in der Einleitung dargestellten Forschungsprojekts wird ersichtlich, dass Beratung von Studieninteressierten nur ein Aufgabenfeld von vielen darstellt. Die Studiengangkoordinierenden benennen jedoch die Zeit vor bzw. während der Bewerbung als ,sehr intensive telefonische Beratungszeit" (Studiengangkoordinierende 1, Abs. 7). Eine weitere Studiengangkoordinierende führt aus, dass zusätzlich zu individuellen Beratungsanliegen eine für die Studieninteressierten verpflichtende „Fachstudienberatung" durchgeführt wird, die sie verantwortet (Studiengangkoordinierende 6, Abs. 31).

Die Studiengangkoordinierenden benennen die in Kapitel 2.1 identifizierten Beratungsbedarfe nicht-traditionell Studieninteressierter ausgenommen den der Anrechnung ${ }^{8}$. Diese Bedarfe lassen sich entsprechend der Häufigkeit ihrer Nennung gewichten. Insbesondere Beratungsanliegen bezüglich der $\mathrm{Zu}-$ lassung wurden in den Interviews mehrfach thematisiert. Für die Studieninteressierten ist es von besonderer Bedeutung, ob ihre bereits erworbenen Qualifikationen für die Zulassung zum entsprechenden weiterbildenden Studienprogramm ausreichen, als eine zentrale Frage von Studieninteressierten formuliert eine Studiengangkoordinierende: „Also bringe ich die notwendigen Voraussetzungen mit, um da teilnehmen zu können?" (Studiengangkoordinierende 1, Abs. 13). Als ebenso wichtig werden studienorganisatorische Aspekte wie „Zeitpläne und Fehlzeiten“ (Studiengangkoordinierende 7, Abs. 4), aber

8 Fragen der Anrechnung außerhochschulisch erworbener Kompetenzen wurden von den Befragten so eng an die Frage der Zulassung gekoppelt, dass sie nicht eindeutig dem Beratungsfeld der Anrechnung zugeordnet werden konnten. Für die hier verwendete Definition von Anrechnung siehe Hanak/Sturm 2015: 19. 
auch die zeitliche Vereinbarkeit des Studiums mit bestehenden Verpflichtungen sowie spezifische Inhaltsfragen benannt. Die kommunikative Bearbeitung der Studienwahl spielt daher in der Beratung ebenfalls eine große Rolle.

Einen weiteren hohen Beratungsbedarf stellt die Finanzierung des weiterbildenden Studienprogramms dar. Hier spielen sowohl die hohen Kosten als auch generelle Fragen zur Finanzierung, Kostenübernahme, Stipendien und steuerlicher Absetzbarkeit eine besondere Rolle; grundsätzlich also „Fragen rund um Weiterbildungsfinanzierung“" (Studiengangkoordinierende 1, Abs. 13). Weniger häufig wurde von den Studiengangkoordinierenden benannt, dass diese die Studieninteressierten auf deren Passung hin beraten. Lediglich zwei Koordinierende gaben an, dass sie daraufhin beraten, „ob der Kurs für sie [die Studieninteressierten] in Frage kommen würde" (Studiengangkoordinierende 9, Abs. 33). Auf Programmebene wird ebenfalls seltener zur Bewerbung allgemein beraten, wobei es sich bei den von den Studiengangkoordinierenden dargestellten Anliegen eher um Informationsweitergabe als um Beratung handelt.

Abbildung 3 zeigt die von nicht-traditionell Studieninteressierten an Studiengangkoordinierende adressierten Beratungsbedarfe auf Programmebene absteigend gewichtet nach ihrer Häufigkeit und ergänzt durch exemplarische Beratungsanliegen.

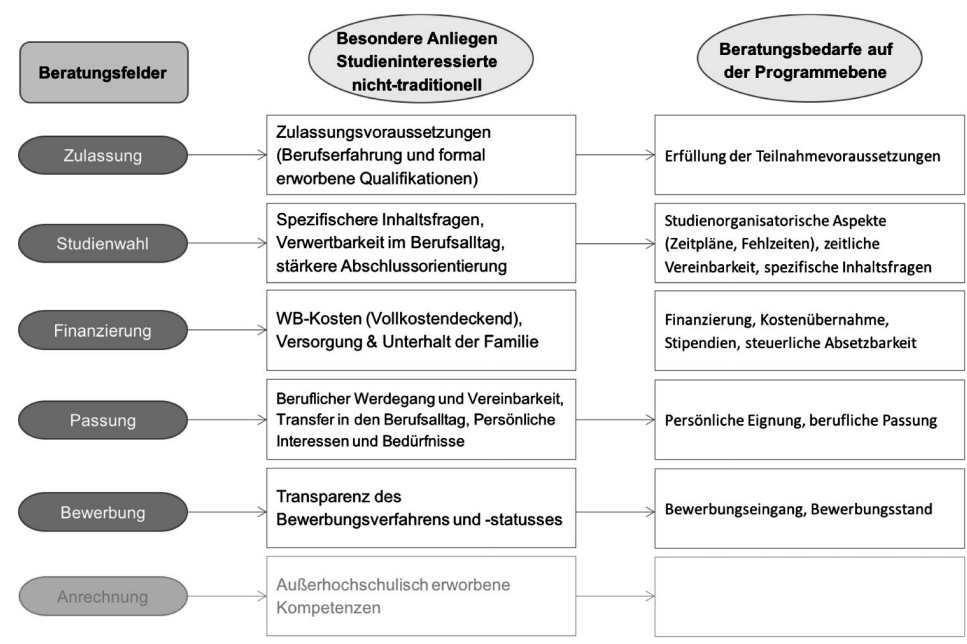

Abb. 3: Beratungsbedarfe auf Programmebene (Quelle: eigene Darstellung)

In einem nächsten Schritt erfolgt nun die Fokussierung der identifizierten hochschulischen Beratungsstrukturen auf die besonderen Spezifika innerhalb der Programmebene. 


\subsection{Beratungsstrukturen auf Programmebene}

Neben den identifizierten Beratungsbedarfen nicht-traditionell Studieninteressierter auf Programmebene und den sich daraus ergebenden Anforderungen an das Beratungswissen der Studiengangkoordinierenden, kommt der Schaffung entsprechender Rahmenbedingungen für die Beratung eine besondere Rolle zu. So formulieren nahezu sämtliche der befragten Studiengangkoordinierenden, dass die Beratung der nicht-traditionell Studieninteressierten unter besonderen Bedingungen abläuft. Während bei regulären Studierenden in der grundständigen Lehre die Beratung primär ,Face-to-Face' oder ggf. im Rahmen einer telefonischen Beratung durchgeführt wird, findet die Beratung nicht-traditionell Studieninteressierter in der wissenschaftlichen Weiterbildung häufig telefonisch, per E-Mail oder auch via Videochat statt. Dies ist zum einen auf die geografische Entkopplung zwischen dem Wohn- und dem Studienort der Ratsuchenden zurückzuführen, da diese aus allen Teilen Deutschlands oder anderen Ländern stammen. Zum anderen liegt es auch an Anforderungen der Zeitverausgabung, da nicht-traditionell Studieninteressierte aufgrund von Berufstätigkeit und Familienpflichten eher abends oder am Wochenende Zeit finden ihre Beratungsanliegen zu kommunizieren. So führt eine Studiengangkoordinierende aus: „Also sie schreiben halt vielleicht spät am Abend, ich antworte auch spät am Abend“" (Studiengangkoordinierende 6, Abs. 17). Aus dem gleichen Grund bietet eine andere Studiengangkoordinierende eine Telefonsprechstunde ,,von vier bis sechs [an], damit auch die Berufstätigen da eine Chance haben sich zu melden" (Studiengangkoordinierende 1, Abs. 7). Insgesamt kann festgestellt werden, dass die Arbeitszeiten der Studiengangkoordinierenden stark an den Zeitbudgets orientiert sind, die den Studieninteressierten zur Verfügung stehen. Dies erfordert oftmals Beratungsleistungen außerhalb der Kernarbeitszeiten in den Abendstunden und an den Wochenenden.

Als weiteren Aspekt betonen die befragten Studiengangkoordinierenden insbesondere die Bedeutung der Rücksprache mit der jeweiligen akademischen Leitung. ${ }^{9}$ Ein Austausch oder eine Zusammenarbeit mit der Zentralen Allgemeinen Studienberatung auf Hochschul- oder auch der Fachstudienberatung auf Fachbereichsebene wird durch die Studiengangkoordinierenden sehr selten benannt. In dieser Hinsicht wird lediglich der Wunsch nach einem

9 Die akademische Leitung des weiterbildenden Studienprogramms ist in der Regel ein Professor oder eine Professorin, der oder die einschlägig im Bereich des Angebots ist. Die Leitung übernimmt auf übergeordneter Ebene vor allem die Verantwortung für finanzielle Ressourcen, die Kontaktpflege und Auswertung des Angebots. Meist initiiert sie die Implementierung des Angebots und verantwortet dessen Durchführung (weiterführend siehe Gronert/Rundnagel 2017). 
stärkeren Austausch mit den anderen Beratungsebenen (2.2) formuliert, um Redundanzen zu minimieren und Fehlinformationen zu vermeiden (Studiengangkoordinierende 8, Abs. 77).

\section{Optimierung von Beratung}

Abschließend wird durch die gleichzeitige Betrachtung der bisherigen Ergebnisse eine Rückbindung auf die Ausgangsfrage vorgenommen, wie ein zielgruppenorientiertes Beratungssetting auf Programmebene organisiert werden sollte und welche intraorganisationalen Rahmenbedingungen zu einer Optimierung der vorhandenen Beratungsstrukturen auf dieser Ebene beitragen können. Dabei wurden in der Analyse der Ergebnisse vier zentrale Aspekte herausgearbeitet, denen eine besondere Relevanz zukommt: Die Beratungsstrukturen, das Beratungswissen, die Beratungskompetenz und die Beratungsinstrumente auf Programmebene.

Beratungsstrukturen

Charakteristisch für die Rolle von Studiengangkoordinierenden als Akteurinnen und Akteure mit zentraler Schnittstellenfunktion innerhalb eines weiterbildenden Studienprogramms ist, dass sie neben der Beratung von nicht-traditionell Studieninteressierten eine Vielzahl weiterer Aufgaben wahrnehmen. Darüber hinaus ist zu berücksichtigen, dass die Beratung von Studieninteressierten zeitlich verdichtet vor Beginn des weiterbildenden Studienprogramms ansteht.

Um diese besonders beratungsintensiven Phasen optimal zu gestalten, bedarf es zum einen entsprechender Zeitbudgets für die Beratung der nicht-traditionell Studieninteressierten und zum anderen der Schaffung möglichst effizienter Beratungsstrukturen, um der bedeutsamen Aufgabe der Beratung auf Programmebene hinreichend gerecht werden zu können. Unter Rückbindung auf die hochschulischen Beratungsstrukturen (2.2) ist für die Beratung auf Programmebene bedeutsam, welche Beratungsbedarfe bis zu welcher Beratungstiefe ${ }^{10}$ auf welcher Beratungsebene bearbeitet werden und ab wann an welche Organisationseinheit zur abschließenden Beratung weiterverwiesen wird. ${ }^{11}$ Eine eindeutige Klärung der Verantwortlichkeiten minimiert Redundanzen, reduziert das Risiko von fehlerhaften Beratungsinhalten und macht

10 Mit „Beratungstiefe“ ist der inhaltliche Detailgrad des jeweiligen Beratungsgegenstandes gemeint.

11 Für weiterführende Informationen zur Frage der hochschulischen Organisation von Beratungsleistungen für nicht-traditionell Studierende siehe Sturm/Bopf 2018. 
die Beratung sowohl für die Studiengangkoordinierenden als auch für die Ratsuchenden effizienter.

Beratungswissen

Auf der Grundlage zuvor geklärter Beratungsstrukturen ist sicherzustellen, wie die Studiengangkoordinierenden auf das Beratungswissen zugreifen können. Unter Berücksichtigung dessen, dass Beratung nur eine Aufgabe des vielfältigen Aufgabenspektrums von Studiengangkoordinierenden darstellt, erscheint es bedeutsam, sowohl das Wissen abrufbar zu machen, als auch Mechanismen zur fortlaufenden Aktualisierung des Beratungswissens zu implementieren. Wie dargestellt, liegen umfassende Erkenntnisse zu den artikulierten Beratungsbedarfen nicht-traditionell Studieninteressierter im Allgemeinen (2.1) und für die Beratung auf Programmebene auch im Speziellen (3.1) vor. Ausgehend von diesen Bedarfen ist das nötige Beratungswissen zu rekonstruieren, organisational zu festigen und ein Modus zu implementieren, wie bei Veränderungen eine Aktualisierung des Wissenspools erfolgt. Im Idealfall können sowohl die Studiengangkoordinierenden als auch andere organisationsinterne Akteurinnen und Akteure, die für die Beratung auf Programmebene relevantes Wissen beisteuern, den konservierten Wissensbestand aktualisieren und bei entsprechendem Bedarf abrufen. Neben der Aktualität des Beratungswissens kann somit auch die dauerhafte Verfügbarkeit sichergestellt werden. Dies hat für die Beratung durch Studiengangkoordinierende auf dem Feld der wissenschaftlichen Weiterbildung besondere Relevanz, da die Beschäftigungsverhältnisse oft in Gestalt befristeter Projektstellen organisiert sind, die i. d. R. mit einer wissenschaftlichen Qualifizierung verknüpft werden. Eine solche Befristung hat eine höhere personelle Fluktuation zur Folge als in den Organisationseinheiten, in denen auf Fachbereichs- oder Hochschulebene beraten wird, und macht ein integriertes Wissensmanagement umso notwendiger. Ein entsprechendes Wissensmanagement stellt sicher, dass das Beratungswissen nicht mit Befristungsende oder Abschluss der Qualifizierungsphase der Organisation unwiderruflich verloren geht.

Beratungskompetenz

Zur Sicherung eines qualitativ hochwertigen Beratungsniveaus bedarf es methodischen Beratungswissens sowie einer entsprechenden Professionalisierung. Während Mitarbeitende in zentral für Beratung zuständigen Organisationseinheiten (Zentrale Studienberatung) oder Funktionsstellen (Studienfachberatung) in der Regel über umfangreiche methodische Kompetenzen auf dem Feld der Beratung verfügen, sind diese methodischen Kompetenzen bei den Studiengangkoordinierenden auf Programmebene deutlich schwächer ausgeprägt. Dies begründet sich sowohl aus der stärker disziplinär abgeleiteten Kopplung an das jeweilige Studienprogramm als auch daraus, dass Beratung nur einen Baustein ihres umfangreichen Aufgabenportfolios darstellt. Die Notwendigkeit einer stärkeren Professionalisierung wird auch darin deutlich, dass Studiengangkoordinierende auf dem Feld der Beratung Fort- und 
Weiterbildungsbedarfe artikulieren (vgl. Gronert/Rundnagel 2017: 63). Die Berücksichtigung der nicht-traditionell Studieninteressierten als Zielgruppe sowie die Entwicklung der wissenschaftlichen Weiterbildung als Geschäftsfeld von Hochschulen machen es notwendig, entsprechende zielgruppen- und geschäftsfeldspezifische Schulungsangebote zur Professionalisierung von Beratung innerhalb der Organisation zu entwickeln und anzubieten. ${ }^{12}$

Beratungsinstrumente

Sowohl die geografische Entkopplung der an Angeboten der wissenschaftlichen Weiterbildung Interessierten als auch die Anforderung, das weiterbildende Studienprogramm mit Familienpflichten und Erwerbsarbeit zu harmonisieren, stellt eine besondere Herausforderung dar. Diese resultiert in Synchronisationsanforderungen unterschiedlicher Temporalordnungen von studieninteressierten Ratsuchenden und hochschulangehörigen Beratenden. ${ }^{13}$ Demnach ist auf Beratungsinstrumente zurückzugreifen, die räumliche Distanzen überbrücken (Telefonberatung, Videochat etc.) oder ungleiche Zeitbudgets oder Zeitfenster innerhalb des Tagesablaufs tolerieren (E-Mail, Chat, Forum etc.). Neben der technischen Implementierung bedarf es entsprechender Schulungen für die Beratenden, um die Beratungsinstrumente sowohl bedarfsgerecht als auch zielgruppenorientiert einzusetzen.

Zusammenfassend kann festgestellt werden, dass vielfältige Aspekte berücksichtigt, intraorganisationale Strukturen und Zuständigkeiten geklärt sowie entsprechende Rahmenbedingungen geschaffen werden müssen, um durch die Optimierung vorhandener Strukturen ein zielgruppenorientiertes Beratungssetting für die wissenschaftliche Weiterbildung auf Programmebene zu organisieren. Wesentliche Ansätze liegen hierbei in der Klärung von Verantwortlichkeiten und Verweisstrukturen, im Wissensmanagement, in der Umsetzung von Professionalisierungsangeboten sowie in der Auswahl zielgruppenspezifischer und bedarfsgerechter Beratungstools.

Eine Berücksichtigung dieser Aspekte könnte wesentlich zur Sicherung des zielgruppenspezifischen Beratungswissens auf der einen Seite und zur Optimierung der Beratungsstrukturen für nicht-traditionell Studieninteressierte auf der anderen Seite und somit zu einer Professionalisierung von öffentlichen Hochschulen auf dem Feld der wissenschaftlichen Weiterbildung insgesamt beitragen.

12 Exemplarisch für ein Qualifizierungsprogramm im Feld der wissenschaftlichen Weiterbildung siehe Braun/Rumpf 2018.

13 Für weiterführende Informationen zur Zeitverausgabung nicht-traditioneller Zielgruppen in der wissenschaftlichen Weiterbildung siehe Schirmer 2017 und Denninger/Kahl/Präßler 2017. Für umfassende Betrachtungen zur Bedeutung von Zeit für das Feld der wissenschaftlichen Weiterbildung siehe Seitter 2017. 


\section{Literatur}

Braun, Monika/Rumpf, Marguerite (2018): Qualifizierung des Personals in der wissenschaftlichen Weiterbildung: Das Zertifikatsprogramm und die Modulwerkstatt von WM3 Weiterbildung Mittelhessen. In: Seitter, Wolfgang/Friese, Marianne/Robinson, Pia (Hg.): Wissenschaftliche Weiterbildung zwischen Entwicklung und Implementierung. Wiesbaden: Springer VS, S. 95-128.

Denninger, Anika/Kahl, Ramona/Präßler, Sarah (2017): Individuumsbezogene Zeitbudgetstudie - Konzeptionen zur Erhebung der Zeitverausgabung von Teilnehmenden wissenschaftlicher Weiterbildung. In: Seitter, Wolfgang (Hg.): Zeit in der wissenschaftlichen Weiterbildung. Wiesbaden: Springer VS, S. 59-96.

Gieseke, Wiltrud (2000): Beratung in der Weiterbildung - Ausdifferenzierung der Beratungsbedarfe. In: Nuissl, Ekkehard/Schiersmann, Christiane/Siebert, Horst (Hg.): Literatur- und Forschungsreport Weiterbildung. Bielefeld: Bertelsmann Verlag, S. 10-18.

Gronert, Laura/Rundnagel, Heike (2017): Studiengangkoordination in der wissenschaftlichen Weiterbildung. Forschungsbericht. http://www.wmhoch3.de/ images/Studiengangkoordination_in_der_wissenschaftlichen_Weiterbildung. pdf [Zugriff: 10.01.2018].

Gronert, Laura/Rundnagel, Heike (2018): Studiengangkoordination in dezentral organisierten Weiterbildungsprogrammen. Eine zentrale Schnittstelle mit vielfältigem Aufgabenspektrum. In: Seitter, Wolfgang/Friese, Marianne/Robinson, Pia (Hg.): Wissenschaftliche Weiterbildung zwischen Implementierung und Optimierung. Wiesbaden: Springer VS, S. 179-203.

Franz, Melanie/Feld, Timm C. (2014): Steuerungsproblematiken im Prozess der Implementierung wissenschaftlicher Weiterbildung an Universitäten. In: Report Zeitschrift für Weiterbildungsforschung, Heft 4, S. 28-40.

Hanak, Helmar/Sturm, Nico (2015): Anerkennung und Anrechnung außerhochschulisch erworbener Kompetenzen. Eine Handreichung für die wissenschaftliche Weiterbildung. Wiesbaden: Springer VS.

Hanft, Anke (2014): Management von Studium, Lehre und Weiterbildung an Hochschulen. Münster: Waxmann.

Lewin, Dirk/Vierthaler, Barbara/Fraunhofer, Andreas (2017): Zielgruppen erreichen. In: Pohlmann, Stefan/Vierzigmann, Gabriele/Doyé, Thomas (Hg.): Weiter denken durch wissenschaftliche Weiterbildung. Wiesbaden: Springer VS, S.159-202.

Schirmer, Kerstin (2017): Work-Learn-Life-Balance. Temporale Vereinbarkeitsstrategien von berufsbegleitenden Studierenden in der wissenschaftlichen Weiterbildung. In: Seitter, Wolfgang (Hg.): Zeit in der wissenschaftlichen Weiterbildung. Wiesbaden: Springer VS, S. 21-46.

Seitter, Wolfgang (2014): Nachfrageorientierung als neuer Steuerungsmodus. Wissenschaftliche Weiterbildung als organisationale Herausforderung universitärer Studienangebotsentwicklung. In: Weber, Susanne Maria/Göhlich, Michael/Schröer, Andreas/Schwarz, Jörg (Hg.): Organisation und das Neue. Beiträge der Kommission Organisationspädagogik. Wiesbaden: Springer VS, S. 141-150.

Seitter, Wolfgang (Hg.) (2017): Zeit in der wissenschaftlichen Weiterbildung. Wiesbaden: Springer VS. 
Seitter, Wolfgang/Schemmann, Michael/Vossebein, Ulrich (2014): Bedarf - Potential - Akzeptanz. Integrierende Zusammenschau. In: Dies. (Hg.): Zielgruppen in der wissenschaftlichen Weiterbildung. Wiesbaden: Springer VS, S. 23-59.

Sturm, Nico / Bopf, Noell (2017): Beratung und Anrechnung. Entwicklung von Beratungstools für nicht-traditionelle Studieninteressierte in der wissenschaftlichen Weiterbildung am Beispiel der Anerkennung und Anrechnung außerhochschulisch erworbener Kompetenzen. http://www.wmhoch3.de/images/Beratung und_Anrechnung.pdf [Zugriff: 10.01.2018].

Sturm, Nico/Bopf, Noell (2018): Optimierung von Beratungsstrukturen für Studieninteressierte in der wissenschaftlichen Weiterbildung. In: Seitter, Wolfgang/Friese, Marianne/Robinson, Pia (Hg.): Wissenschaftliche Weiterbildung zwischen Implementierung und Optimierung. Wiesbaden: Springer VS, S. 255-282. 


\section{Anrechnungsberatung als Möglichkeit der Öffnung von Hochschulen? Struktur und Praxis der Anrechnungsberatung in der wissenschaftlichen Weiterbildung}

Im Beitrag wird der Frage nachgegangen, wie sich Anrechnungsberatung in Bezug auf die Öffnung von Hochschulen darstellt. Hergeleitet wird zunächst (1) Öffnung der Hochschulen für beruflich Qualifizierte sowie die Relevanz von Anrechnungsberatung. Anschließend wird (2) Anrechnungsberatung als Interaktionszusammenhang an den Grenzen der sozialen Welt wissenschaftliche Weiterbildung konstruiert. Dem folgen (3) Ausführungen zu einer bundesweiten Studie, die im Hinblick auf anrechnungsbezogene Interaktionszusammenhänge zwischen Ratsuchenden und -gebenden vorgestellt wird. Der Beitrag schließt (4) mit dem Fazit, dass Anrechnungsberatung dann zur Öffnung der Hochschulen beiträgt, wenn dies im Interesse des Studienangebots bzw. der Hochschule ist.

\section{Öffnung der Hochschulen für beruflich Qualifizierte}

Für die Teilnahme an wissenschaftlicher Weiterbildung mehr als bisher beruflich Qualifizierte mit geringer akademischer Vorerfahrung zu gewinnen, ist erklärtes Ziel bildungs- und wirtschaftspolitischer Akteure in Deutschland. Dem geht die Diagnose geringer Quoten an Akademiker_innen in OECD $^{1}$-Vergleichen voraus (vgl. Freitag 2011, S. 35). Verorten lässt sich die Debatte im Kontext lebenslangen Lernens im Sinne der Europäischen Kommission (2000, S. 3): Allen Europäer_innen soll ermöglicht werden, sich sozialen und wirtschaftlichen Veränderungen anzupassen. Ausdruck finden entsprechende Bemühungen in Programmen wie „Aufstieg durch Bildung: offene Hochschulen“ (vgl. BMBF² 2016), deren Ziel die Verbesserung der Durchlässigkeit von der beruflichen Praxis in die Hochschule ist, etwa indem Zugangsmöglichkeiten durch Anrechnung beruflicher Erfahrungen po-

1 Organisation for Economic Co-operation and Development.

2 Bundesministerium für Bildung und Forschung. 
tenziellen Teilnehmer_innen durch Beratungsangebote transparent gemacht werden (vgl. Lewin/Vierthaler/Fraunhofer 2017, S. 193 f.). Ulf Banscherus, Caroline Kamm und Alexander Otto (2016) zeigen anhand von NEPS ${ }^{3}$-Daten, dass sogenannte nicht-traditionell Studierende Informations- und Beratungsangebote häufiger als andere Studierende bei der Zulassung zum Studium nutzen (vgl. ebd., S. 301, 304).

Anzunehmen sind Herausforderungen sowohl bei der Aufnahme als auch bei der Bewältigung eines weiterbildenden Studiums, also neben Beruf und familiären Verpflichtungen. Anrechnung von außerhochschulischen, etwa beruflichen Vorleistungen (z. B. Kenntnisse, Fähigkeiten, Qualifikationen), soll diese Herausforderungen abmildern und damit mehr beruflich Qualifizierten den Zugang zum weiterbildenden Studium eröffnen. In allen Studiengängen, die seit 2015 akkreditiert oder reakkreditiert wurden, solle geprüft werden, inwiefern Möglichkeiten der Anrechnung von Vorleistungen vorgehalten werden (vgl. Akkreditierungsrat 2014). Vor dem Studium erworbene Fähigkeiten und Fertigkeiten sollen bei Gleichwertigkeit verwendet werden können, um ein wiederholtes Studieren von bereits Bekanntem zu vermeiden sowie eine Reduzierung von Studieninhalten und/oder -kosten zu ermöglichen (Milderung zeitlicher Herausforderungen und finanzielle Anreize). Neben dieser Anrechnung von Vorleistungen auf Inhalte geht es auch um die Anrechnung von beruflichen Vorleistungen, um einen Zugang zum Studium zu ermöglichen. Das zentrale Thema bei der Zulassungsfrage ist die Schließung der sogenannten „Bachelor-Master-Lücke“. Üblicherweise werden für einen weiterbildenden Master $60 \mathrm{CP}^{4}$ bzw. $90 \mathrm{CP}$ vergeben, um reduzierte Studienzeiten $\mathrm{zu}$ erreichen, die insbesondere bei berufsbegleitenden Formaten erforderlich sind. Der Master-Abschluss setzt allerdings einen Gesamterwerb von $300 \mathrm{CP}$ voraus. Durch ein Bachelor-Studium werden mindestens $180 \mathrm{CP}$, teilweise $210 \mathrm{CP}$ und selten $240 \mathrm{CP}$ erreicht. Dies hat je nach Kombination von Bachelor- und Master-Studium zur Folge, dass eine Lücke von $30 \mathrm{CP}$ bis $60 \mathrm{CP}$ geschlossen werden muss (vgl. Hanak/Sturm 2013, S. 14; KMK ${ }^{5}$ 2002, S. 2). Allerdings gäbe es für berufliche Qualifizierte die Möglichkeit, bis zu $30 \mathrm{CP}$ für jedes anerkannte Jahr einschlägiger beruflicher Tätigkeit angerechnet zu bekommen (vgl. HRK 2008, S. 71).

Es würden jedoch kaum spezifische Beratungsangebote für diese Zielgruppe vorgehalten (vgl. Banscherus/Pickert 2013, S. 13). Barbara Vierthaler und Gabriele Vierzigmann (2017, S. 461) weisen darauf hin, dass an Hochschulen traditionell erst beraten werde, wenn Studierende immatrikuliert seien. Der Zugang zum weiterbildenden Studium (vgl. Faulstich et al. 2007,

3 Nationale Bildungspanel (National Educational Panel Study).

4 Credit Points im European Credit Transfer System (ECTS).

5 Kultusminister_innenkonferenz. 
S. 140) sowie die Anerkennung von außerhochschulischen Vorleistungen werden als Nadelöhr identifiziert (vgl. Dörner 2016, S. 15). Ziel von Beratung müsse es in diesem Zusammenhang sein, so Nico Sturm und Noell Marie Bopf (2017), Fragen und Unklarheiten bei der Anrechnung zu bearbeiten, indem nicht-traditionell Studierenden die Studien- sowie Auswahlentscheidung durch das Aufzeigen von Anrechnungsmöglichkeiten erleichtert werde (vgl. ebd., S. 26).

\section{Anrechnungsberatung an den Grenzen wissenschaftlicher Weiterbildung}

Entgegen Entwicklungen in übergangsbezogener Weiterbildungsberatung, durch kommunale Vernetzung angebots- und trägerübergreifend zu beraten (vgl. Schlüter 2014, S. 264), ist die Beratung in Anrechnungsfragen häufig ein Aufgabenbereich von Mitarbeiter_innen der wissenschaftlichen Weiterbildung. Ein Problem können dabei eigene Absichten der Ratgebenden darstellen, aufgrund derer sie etwa Lösungen anbieten, die im Sinne der Angebote und nicht der Ratsuchenden sind (vgl. Maier-Gutheil 2016, S. 18). Grund für solche Absichten können bspw. Legitimationsanforderungen sein, denen sich eine marginalisierte wissenschaftliche Weiterbildung in Hochschulen ausgesetzt sieht (vgl. Kondratjuk 2017, S. 154).

Wolfgang Jütte und Karl Weber (2006, S. 11) fassen unter wissenschaftlicher Weiterbildung Hochschulstudiengänge, die sich an Personen mit tertiären Bildungsabschlüssen nach einer ersten Phase der Erwerbstätigkeit richten. Inhalte werden auf wissenschaftlichem Niveau angeboten. Grundlagentheoretisch kann wissenschaftliche Weiterbildung mit Maria Kondratjuk (2017) konzeptualisiert werden als soziale Welt, die in ihrem Kern auf die Bereitstellung von Weiterbildungsangeboten für Berufstätige an Hochschulen ausgerichtet ist. Sowohl die Positionierung der wissenschaftlichen Weiterbildung in der Hochschule als auch das Verhältnis zwischen Hochschule und außerhochschulischen Akteur_innen werde in Arenen ausgehandelt (vgl. ebd., S. 97).

Anrechnungsberatung, die sich an eine erst noch zu erreichende Zielgruppe richtet, steht in diesem Kontext. Ratgebende sind Akteur_innen an der Grenze der sozialen Welt, die sich transitiv an ratsuchende beruflich Qualifizierte richten (vgl. Maier-Gutheil 2016, S. 18 f.). Letztere befinden sich als Studieninteressierte (noch) außerhalb der sozialen Welt oder als Studienanfänger_innen im Übergang in diese. Innerhalb der Hochschule ist wissenschaftliche Weiterbildung durch ihre besondere Nähe zum Beruflichen (vgl. Jütte 2016) grenznah positioniert. Ausdruck findet dies etwa in der Vereinbarung der KMK, an beruflichen Erfahrungen der Teilnehmer_innen anzuknüpfen 
(vgl. KMK 2001, S. 3), beispielsweise durch verbesserte Möglichkeiten der Anerkennung und Anrechnung von außerhochschulischen Vorleistungen.

So zeigt sich auf der einen Seite die Herausforderung, einer nicht-traditionellen Zielgruppe den Zugang zum weiterbildenden Studium zu ermöglichen und durch Anrechnungsberatung Zugangsmöglichkeiten aufzuzeigen. Auf der anderen Seite ist wissenschaftliche Weiterbildung gefordert, sich innerhalb von Hochschule als legitimes Studienangebot darzustellen. Dieses Spannungsfeld wird im Folgenden empirisch beleuchtet und dabei der Frage nachgegangen, wie sich Anrechnungsberatung als grenzbezogener Interaktionszusammenhang zwischen Ratgebenden und -suchenden darstellt.

\section{Beratung und der Umgang mit Anerkennung und Anrechnung}

In einem BMBF-geförderten Verbundprojekt (vgl. Bechmann et al. 2016) wurde $u$. a. eine bundesweite Studie zur Anerkennung und Anrechnung von außerhochschulischen Vorleistungen in der wissenschaftlichen Weiterbildung durchgeführt. ${ }^{6}$ In einer quantitativen Teilstudie wurde der Frage nachgegangen, welche Relevanz dem Thema Anrechnung von Akteur_innen in der wissenschaftlichen Weiterbildung beigemessen wird. Vorgestellt werden von dieser Teilstudie im Folgenden nur die beratungsbezogenen Aspekte. ${ }^{7}$ Umfangreicher wird auf eine qualitative Teilstudie eingegangen, in der gefragt wurde, wie Akteur_innen der wissenschaftlichen Weiterbildung mit der Anerkennung und Anrechnung außerhochschulischer Vorleistungen umgehen. Fokussiert werden ebenfalls nur jene Aspekte, die sich auf Anrechnungsberatung beziehen.

\section{Beratungsangebote: Verbreitung und Umfang}

In der quantitativen Teilstudie nahmen 276 Personen, die nach eigenen Angaben in der wissenschaftlichen Weiterbildung an einer deutschen Hochschule für Anrechnungsfragen zuständig sind, an einer onlinebasierten Fragebogen-

6 Geleitet wurde das Teilforschungsprojekt von Prof. Olaf Dörner und Prof. Johannes Fromme.

7 Weitere Ergebnisse sind in einem Forschungsbericht veröffentlicht (Damm 2018, S. 3753). 
untersuchung teil. Erhoben wurden Daten der Befragten, der durch sie repräsentierten Studiengänge sowie zur Anrechnung von außerhochschulischen Vorleistungen. 200 Befragte (72,5\%) gaben an, im weiterbildenden Studiengang Möglichkeiten der Anrechnung zu bieten. In Bezug auf Anrechnungsberatung wurden zwei Aspekte deutlich: Der überwiegende Teil der Befragten bietet nach eigenen Angaben Möglichkeiten der Beratung bei Anrechnungsfragen (95\%). Wenige geben an, die Beratung zu planen (2\%). Ebenso wenige der Befragten mit Anrechnungsmöglichkeiten bieten keine Anrechnungsberatung $(2 \%)$ und ein kleiner Rest gibt keine Antwort (1\%). Der von den Befragten geschätzte Umfang der Beratung stellt sich vielfältiger dar. Es gibt einen hohen statistischen Zusammenhang zwischen der Matrikelgröße und der Einschätzung des Beratungsumfangs: Je kleiner die Matrikel, desto eher wird von einem hohen Beratungsumfang ausgegangen. Es ist also anhand der quantitativen Beschreibungen zunächst festzuhalten, dass eine große Zahl der Befragten zustimmt, Anrechnungsberatung zu bieten, es werden aber Unterschiede im geschätzten Umfang der Beratung deutlich.

\section{Beratung bei der Anrechnung}

In der qualitativen Teilstudie wurden bundesweit 21 offene leitfadengestützte Expert_innen-Interviews (vgl. Dörner 2012) mit Akteur_innen, die in Angeboten der wissenschaftlichen Weiterbildung für Anrechnung zuständig sind, geführt. Ausgewertet wurden die Daten mit einer handlungstheoretischen Perspektive mittels Kodierung entlang der methodologischen Überlegungen der Grounded Theory (vgl. Strauss/Corbin 1996). Als Ergebnis stellten sich vier Handlungsmuster, wie mit Anrechnung umgegangen wird, heraus. Deutlich wird in diesen Handlungsmustern u. a., inwieweit bei Anrechnungsfragen Anfragen von Studieninteressierten bzw. Studienanfänger_innen relevant sind und wie damit um- bzw. darauf eingegangen wird.

In zwei Handlungsmustern, die sich durch ein Geschlossenhalten der Zugangsmöglichkeiten auszeichnen, wird Beratung vor allem im Zusammenhang mit bereits eingeschriebenen Student_innen geschildert: Einmal werden dabei konkrete Anrechnungsmöglichkeiten aufgezeigt, ein anderes Mal wird es vermieden, Informationen zur Anrechnung transparent zu machen. In den anderen beiden Handlungsmustern, die eine Tendenz zur Öffnung für neue Zielgruppen haben, wird Beratung beim Übergang ins Studium thematisiert: Hier wird einmal die angebotsbezogene Passung von Bewerber_innen in intensiven persönlichen Gesprächen überprüft und einmal umfangreich über Anrechnungsmöglichkeiten in gruppenbezogenen Veranstaltungen informiert. Die vier Handlungsmuster werden im Folgenden kurz vorgestellt. 


\section{Handlungsmuster „Erforderliche Begrenzung"}

Das erste Handlungsmuster, „Erforderliche Begrenzung“, findet sich vor allem in Angeboten, die durch einen geringen Bedarf nach Veränderung charakterisiert sind. Die Öffnung für neue Zielgruppen ist kaum möglich und die personellen und zeitlichen Ressourcen sind stark darauf ausgerichtet, Student_innen von der Einschreibung bis zum Abschluss zu begleiten. Es besteht eine starke Bindung an den hochschulischen Rahmen und es werden viele Bezüge zu Unternehmen in einer bestimmten Region oder Branche hergestellt. Beratungszusammenhänge werden nach der Immatrikulation bis zum Übergang in eine Anschlussbeschäftigung nach dem Studium geschildert. Die Anrechnung von Vorleistungen ist aber vor allem beim Zugang zum Studium ein Thema: Es muss aus einer großen Zahl von Bewerber_innen eine begrenzte Zahl von Student_innen ausgewählt werden, wobei Beratung keine Rolle spielt. Die Verpflichtung, Anrechnungsmöglichkeiten zu schaffen, wird aufgrund begrenzter Kapazitäten unterbetont und eher als Pflicht bzw. zusätzlicher Aufwand verstanden. Da die Folgen von Anrechnung klein gehalten werden sollen, wird möglichst vermieden, Informationen zur Anrechnung zu geben und dazu zu beraten. Exemplarisch steht dafür die Aussage einer Studiengangskoordinatorin:

„die Unwissenheit [der Studierenden ist, C. D.] das, was uns natürlich auch davor schützt, dass wir uns damit beschäftigen müssen". ${ }^{8}$

Möglich wird dies durch die klare Trennung von Anrechnung beim Zugang und Beratung nach Immatrikulation. Eine Anrechnung auf Inhalte ist nicht möglich, letztlich um zu vermeiden, dass offizielle Anträge gestellt werden:

„meist läuft das auf=ner informellen Ebene ab [...] wir müssen das dann im Rahmen der Beratung schon vereinen und sagen: ,nein, das geht nich-““.

Beratungszusammenhänge werden in diesem Handlungsmuster deutlich, beziehen sich allerdings nicht auf Anrechnung.

8 In den Transkript-Auszügen werden Abbrüche von Wörtern durch einen Bindestrich (-), Zusammenziehungen mehrerer Wörter durch ein Gleichheitszeichen $(=)$ und Dehnungen in Wörtern je nach Länge durch mehrere Doppelpunkte (::) dargestellt. Sinkt die Intonation, wird ein Punkt (.) gesetzt, bei leicht steigender Intonation ein Komma (,), bei stark steigender ein Fragezeichen (?). Auslassungen sind durch drei Punkte in eckigen Klammern $[\ldots]$ angezeigt und Einfügungen durch den Autor sind ebenfalls durch eckige Klammern und die Initialen des Autors (C. D.) gekennzeichnet. Die Wiedergabe direkter Rede wird durch einfache Anführungszeichen markiert $\left(, x^{6}\right)$. 


\section{Handlungsmuster „Selbstverständliche Exklusivität”}

Das zweite Handlungsmuster, „Selbstverständliche Exklusivität“, findet sich ebenfalls in Angeboten, die durch einen geringen Bedarf an Veränderung charakterisiert sind. Allerdings sind diese Angebote in einem professionellen Kontext verortet. Es handelt sich um statusträchtige professionsbezogene Weiterbildungen auf Masterniveau in der Medizin, im Ingenieurbereich oder im Rechtswesen, die auf den Erwerb zusätzlicher Titel abzielen. Trotz institutioneller Zugehörigkeit zur Hochschule werden die Vorteile der relativen Autonomie von Hochschulstrukturen betont. Die Anrechnung von Vorleistungen ist eine Möglichkeit, um beruflich versierten Professionsvertreter_innen Anreize zur Aufnahme des Studiums zu geben. Es geht im Hinblick auf Anrechnungsberatung vor allem darum, den bereits immatrikulierten Student_innen bei konkreten Fragen und Anliegen Wege und Möglichkeiten der Umsetzung aufzuzeigen. Ein Studiengangskoordinator sagt:

„unsere Studenten sind ja älter und es sind auch zum Teil Prominente [die, C. D.] genau wissen in der Regel was sie möchten und dann auch ihre Fragen haben“.

Anrechnungsmöglichkeiten werden als Anreize für Student_innen thematisiert. Immer wieder geht es um die hohen Kosten der Weiterbildungsangebote, die durch Anrechnung etwas reduziert und die intensiven beruflichen Anforderungen, die durch Anrechnung mit dem Ziel der Studienzeitreduzierung kompensiert werden können. Problematisiert werden rechtliche Zugangsmöglichkeiten von Bachelor-Absolvent_innen mit wenigen Jahren Berufserfahrung. Vordergründig wird die Exklusivität des Angebots als Selbstverständlichkeit präsentiert und im Hintergrund alles so organisiert, dass die Grenzen trotz neuer Anforderungen geschärft bleiben. Anrechnungsberatung eröffnet hier also nicht Zugänge, sondern bietet einer exklusiven Zielgruppe Anrechnungsmöglichkeiten als Anreize an.

\section{Handlungsmuster „Gezielt genutzte Öffnung"}

Im dritten Handlungsmuster, „Gezielt genutzte Öffnung“, wird die Debatte um die Öffnung der Hochschulen als Chance gesehen, ein innovatives Studienangebot zu etablieren oder zur Professionalisierung eines Berufsfeldes beizutragen. Angebote der wissenschaftlichen Weiterbildung stehen hier vor der Aufgabe, sich innerhalb der Hochschule zu etablieren. Gleichzeitig werden klassische Zugangsmöglichkeiten hinterfragt: Berufspraktiker_innen ohne ersten Hochschulabschluss oder ohne Hochschulzugangsberechtigung soll aufgrund einer besonderen berufspraktischen Eignung der Zugang zum Studium ermöglicht werden. Anrechnung wird hier als Instrument genutzt, um Zulassungsvoraussetzungen zu erfüllen. Anrechnung auf die Studieninhalte wird hingegen abgelehnt, da die beruflichen Erfahrungen didaktisch in 
das Studienprogramm eingebettet werden sollen. Anrechnung und Anrechnungsberatung sind auf den Zugang zum Studienangebot und insbesondere die Ermöglichung des nicht-traditionellen Zugangs fokussiert. Es geht vor allem darum, die inhaltliche Passung der Bewerber_innen durch persönliche Beratungsgespräche vor der Immatrikulation zu überprüfen. So heißt es exemplarisch bei einer Studiengangskoordinatorin:

„dann findet eine Prüfung statt, ich führe übrigens auch sehr intensive Gespräche mit allen Kandidaten“.

Bevor es zu einer Immatrikulation kommt, wird versucht herauszufinden, wer in der Lage ist, das Studium erfolgreich zu absolvieren. Dies ist wesentlich, um nur Personen in das Studienprogramm aufzunehmen, die in der Lage sind, dieses bis zum Ende erfolgreich zu durchlaufen. Davon verspricht sich die Hochschule Kostendeckung und vorzeigbare Ergebnisse, es ist jedoch verbunden mit der die Schwierigkeit, zum einen Bewerber_innen zu gewinnen und zum anderen die Passung zu wahren:

„dann gibt es natürlich persönliche Beratungsgespräche [...] Das kann dann auch so passieren dass sie dann außerhalb [des Studienfachs, C. D.] dann landen, die Studenten“.

Neben dem Übergang vom Beruf ins Studium ist auch der zweite Übergang aus dem Studium in den Beruf relevant und wird nur in diesem Handlungsmuster so deutlich angesprochen. Erfolgreiche Professionalisierung wird als etwas behandelt, was beratungswürdig ist. Die Beratung selbst wird nicht thematisiert, wie bei einer Studiengangskoordinatorin exemplarisch deutlich wird:

„Ich berate auch zu dem Einstieg ins Berufsleben. Also dann nach dem Studium also [...] nach der Akademisierung Einstieg ins Berufsleben aber auch [möglicher anschließender, C. D.] Masterstudiengänge.“

Anrechnungsberatung bezieht sich in diesem Handlungsmuster also sowohl auf gezielte und geprüfte Übergänge in das Studium als auch aus dem Studium heraus.

\section{Zentral gewollte Öffnung}

Im vierten Handlungsmuster, „Zentral gewollte Öffnung“, geht es, anders als bei den bisherigen Handlungsmustern, nicht um die Planung und Durchführung von einzelnen Studienangeboten, sondern primär um die Etablierung von wissenschaftlicher Weiterbildung als dritter Säule innerhalb der Hochschule durch sogenannte Zentren für wissenschaftliche Weiterbildung. Charakteristisch ist die strategische Investition von Fördermitteln zur Entwicklung vollkostenfinanzierter Angebote der wissenschaftlichen Weiterbildung. Um dies zu erreichen, wird Anrechnung als eine Möglichkeit verstanden, den Zugang zum Studium zu erleichtern und somit neue Zielgruppen zu adressie- 
ren. Außerdem sollen durch Anrechnungsmöglichkeiten im Studium zusätzliche Anreize geschaffen werden. Durch Anrechnung wird also für die Aufnahme eines weiterbildenden Studiums geworben. Umfangreiche Informationen zur Anrechnung werden für die spezifische Zielgruppe im Internet und auf gruppenbezogenen Veranstaltungen gegeben. Eine Beratung von Einzelpersonen wird hingegen nicht geschildert. Durch allpräsente und umfassende Informationen sollen möglichst viele Personen gleichzeitig erreicht werden. Hierzu ein Zitat eines Studiendekans:

„hatten vor zwei Wochen eine Infoveranstaltung, [...] im Juli eine Infoveranstaltung, [...] im Au::gust noch eine Infoveranstaltung [...] und da weisen wir explizit auf Anrechnungsmöglichkeiten hin.“

Auch wenn sich die Medien der Information und Beratung in den einzelnen Interviews unterscheiden, so wird im Kern immer wieder deutlich, dass Anrechnungsinformationen umfassend publik gemacht werden.

\section{Schluss}

Beratung zu Anrechnungsfragen, die als übergangsrelevant in der wissenschaftlichen Weiterbildung anzunehmen ist, wird nach eigenen Angaben von einem Großteil der Befragten angeboten. Analysen von Handlungspraktiken zeigen allerdings im Hinblick auf die Öffnung von Hochschulen für beruflich Qualifizierte deutliche qualitative Unterschiede. Zunächst ist insgesamt festzuhalten, dass eine Kongruenz zwischen den Öffnungs- und Schließungstendenzen und der Beratung zu Anrechnungsfragen empirisch beobachtet werden kann. Dort, wo bestehende Grenzen erhalten bleiben sollen, wird Beratung in Bezug auf Student_innen geschildert, die bereits immatrikuliert sind. Sollen Zulassungsmöglichkeiten erweitert werden, wird zudem grenznah über Anrechnungsfragen informiert oder beraten. Die eingangs formulierte Problematik der negativen Beeinflussung von Weiterbildungsberatung durch angebots- bzw. trägereigene Interessen ist angesichts der empirischen Ergebnisse anzunehmen. Als Antwort auf die Frage des Beitrages ist festzuhalten, dass anrechnungsbezogene Interaktionszusammenhänge zwischen Ratsuchenden und Ratgebenden in der wissenschaftlichen Weiterbildung von der Durchsetzung angebotsbezogener Interessen bestimmt werden. Anrechnungsberatung trägt also dann zur Öffnung der Hochschulen bei, wenn dies im Interesse des Studienangebots bzw. der Hochschule ist. Vor diesem Hintergrund sollte überlegt werden, wie eine Beratung zu Anrechnungsfragen in der wissenschaftlichen Weiterbildung unabhängig von Interessen einzelner Hochschulen und Angebote organisiert werden kann. 


\section{Literatur}

Akkreditierungsrat (2014): Anrechnung außerhochschulischer Kenntnisse und Fähigkeiten. Online unter: http:/www.akkreditierungsrat.de/fileadmin/Seiteninhalte/ KMK/Vorgaben/KMK_Anrechnung_ausserhochschulisch_II.pdf [31.01.2018].

Banscherus, Ulf/Pickert, Anne (2013): Unterstützungsangebote für nicht-traditionelle Studierende. Stand und Perspektiven. (Thematischer Bericht der wissenschaftlichen Begleitung des Bund-Länder-Wettbewerbs „Aufstieg durch Bildung: offene Hochschulen").

Banscherus, Ulf/Kamm, Caroline/Otto, Alexander (2016): Gestaltung von Zu- und Übergängen zum Hochschulstudium für nicht-traditionelle Studierende. Empirische Befunde und Praxisbeispiele. In: Wolter, Andrä/Banscherus, Ulf/Kamm, Caroline (Hg.): Zielgruppen Lebenslangen Lernens an Hochschulen. Ergebnisse der wissenschaftlichen Begleitung des Bund-Länder-Wettbewerbs „Aufstieg durch Bildung: offene Hochschulen“ (Band 1). Münster: Waxmann. S. 295-320.

Bechmann, Martin/Damm, Christoph/Fredrich, Helge/Frosch, Ulrike/Krüger, Stina/ Vieback, Linda (2016): Ein Blick auf und in die Magdeburger Weiterbildungslandschaft. Forschungsbasierte Projektarchitekturen. In: Hochschule und Weiterbildung (Ausgabe 2), S. 32-40.

BMBF (Bundesministerium für Bildung und Forschung) (2016): Aufstieg durch Bildung. Online unter: https://www.bmbf.de/de/aufstieg-durch-bildung-1240.html [26.09.2016].

Damm, Christoph (2018): Anrechnung von außerhochschulischen Vorleistungen in der wissenschaftlichen Weiterbildung. Ergebnisse einer zweiteiligen empirischen Studie. Forschungsbericht. Unter Mitarb. v. Olaf Dörner u. Johannes Fromme. Magdeburg: Otto-von-Guericke-Universität Magdeburg. Online unter: http://nbn-resolving.de/urn:nbn:de:gbv:ma9:1-11408. DOI: 10.24352/UB.OVGU-2018-093.

Dörner, Olaf (2012): Experteninterviews. In: Dörner, Olaf/Schäffer, Burkhard (Hg.): Handbuch Qualitative Erwachsenen- und Weiterbildungsforschung. Opladen et al.: Budrich. S. 321-333.

Dörner, Olaf (2016): Nadelöhr Anerkennung: Wissenschaftliche Weiterbildung als bedingte Möglichkeit für Berufstätige. Studie zu Praxen der Anerkennung und Anrechnung außerhochschulisch erbrachter Leistungen. In: berufsbildung 161, S. $14-16$.

Europäische Kommission (2000): Memorandum über Lebenslanges Lernen. Brüssel.

Faulstich, Peter/Graeßner, Gernot/Bade-Becker, Ursula/Gorys, Biance (2007): Länderstudie Deutschland. In: Hanft, Anke/Knust, Michaela (Hg.): Weiterbildung und lebenslanges Lernen in Hochschulen. Eine internationale Vergleichsstudie zu Strukturen, Organisation und Angebotsformen. Münster: Waxmann. S. 88-157.

Freitag, Walburga K. (2011): Hochschulen als Orte lebenslangen Lernens? Analysen hochschulstatistischer Daten zum Hochschulstudium von Studierenden mit beruflicher Qualifikation. In: Freitag, Walburga K./Hartmann, Ernst A./Loroff, Claudia/Stamm-Riemer, Ida/Völk, Daniel/Buhr, Regina (Hg.): Gestaltungsfeld Anrechnung: Hochschulische und berufliche Bildung im Wandel. Münster et al.: Waxmann. S. 35-56. 
Hanak, Helmar/Sturm, Nico (2013): Anrechnung und Anerkennung außerhochschulisch erworbener Kompetenzen: Eine Handreichung für Studiengangsentwickler/innen im Rahmen des Projekts „WM ${ }^{3}$ Weiterbildung Mittelhessen“. Online unter: http://www.wmhoch3.de/images/Anrechnung_und_Anerkennung.pdf [31.01.2018].

HRK (Hochschulrektorenkonferenz) (2008): HRK-Positionspapier zur wissenschaftlichen Weiterbildung. Bonn.

Jütte, Wolfgang (2016): Beruf als zentraler Referenzrahmen für die wissenschaftliche Weiterbildung. In: Hochschule und Weiterbildung (Ausgabe 1), S. 7-8.

Jütte, Wolfgang/Weber, Karl (2006): Universitäre Weiterbildung international - ein Spiel ohne Grenzen. In: Jütte, Wolfgang/Weber, Karl (Hg.): Kontexte wissenschaftlicher Weiterbildung: Entstehung und Dynamik von Weiterbildung im universitären Raum. Münster: Waxmann. S. 9-13.

KMK (Kultusministerkonferenz) (2001): Sachstands- und Problembericht zur ,Wahrnehmung wissenschaftlicher Weiterbildung an den Hochschulen“. Beschluss der Kultusministerkonferenz vom 21.09.2001. Online unter: http://www.kmk.org/ fileadmin/Dateien/veroeffentlichungen_beschluesse/2001/2001_09_21-Problembericht-wiss-Weiterbildung-HS.pdf [31.01.2018].

KMK (Kultusministerkonferenz) (2002): Anrechnung von außerhalb des Hochschulwesens erworbenen Kenntnissen und Fähigkeiten auf ein Hochschulstudium (I): (Beschluss der Kultusministerkonferenz vom 28.06.2002). Online unter: http://www.kmk.org/fileadmin/Dateien/veroeffentlichungen_beschluesse/2002/2002_06_28-Anrechnung-Faehigkeiten-Studium-1.pdf [31.01.2018].

Kondratjuk, Maria (2017): Soziale Welt Hochschulweiterbildung. Figurationsmerkmale, Arenastruktur, Handlungsmodell. Bielefeld: W. Bertelsmann Verlag.

Lewin, Dirk/Vierthaler, Barbara/Fraunhofer, Andreas (2017): Zielgruppen erreichen. In: Pohlmann, Stefan/Vierzigmann, Gabriele/Doyé, Thomas (Hg.): Weiter denken durch wissenschaftliche Weiterbildung. Wiesbaden: Springer Fachmedien Wiesbaden. S. 159-202.

Maier-Gutheil, Cornelia (2016): Beraten. Stuttgart: Verlag W. Kohlhammer.

Schlüter, Anne (2014): Von der Transparenz der Weiterbildung zur lebensbegleitenden Übergangsberatung - zum Funktionswandel von Weiterbildungsberatung. In: Felden, Heide von/Schäffter, Ortfried/Schicke, Hildegard (Hg.): Denken in Übergängen: Weiterbildung in transitorischen Lebenslagen. Wiesbaden: Springer VS. S. 253-266.

Strauss, Anselm L./Corbin, Juliet M. (1996): Grounded Theory: Grundlagen qualitativer Sozialforschung. Unveränderter Nachdruck der ersten Auflage. Weinheim: Beltz.

Sturm, Nico/Bopf, Noell Marie (2017): Beratung und Anrechnung. Entwicklung von Beratungstools für nicht-traditionelle Studieninteressierte in der wissenschaftlichen Weiterbildung am Beispiel der Anerkennung und Anrechnung außerhochschulisch erworbener Kompetenzen. Marburg: $\mathrm{WM}^{3}$ Weiterbildung Mittelhessen.

Vierthaler, Barbara/Vierzigmann, Gabriele (2017): Bildungswege begleiten. In: Pohlmann, Stefan/Vierzigmann, Gabriele/Doyé, Thomas (Hg.): Weiter denken durch wissenschaftliche Weiterbildung. Wiesbaden: Springer Fachmedien. S. 461-502. 



\section{Beratung, Betreuung, wissenschaftliche Weiterbildung: Herausforderung angenommen!?}

\section{Einleitung}

Im Zuge der Öffnung der Hochschulen (KMK 2009) nimmt das Hochschulsystem neue Zielgruppen ins Blickfeld. Diese neuen Zielgruppen bringen aufgrund ihrer Bildungsbiografie heterogene Bedarfe mit. Bestehende Strukturen, v. a. hinsichtlich der Beratung und Betreuung von heterogenen Studierendengruppen müssen somit weiterentwickelt werden. Offen bleibt, wie sich bestehende Angebote und Strukturen ändern können.

Im Rahmen des Verbundprojekts OTH mind - modulares innovatives Netzwerk für Durchlässigkeit der OTH Amberg-Weiden und Regensburg wird ein über die Region angelegtes Maßnahmenkonzept für lebenslanges Lernen entwickelt. Dabei steht die Bedarfserhebung der Zielgruppen an erster Stelle, wobei spezifische Beratungs- und Betreuungsstrukturen, Gender Mainstreaming und Diversity Management zentrale Themen sind. Die Bildungsangebote adressieren vor allem beruflich Qualifizierte, Studienabbrecher/innen, Ingenieur/innen sowie technische Fachkräfte. Es gilt für die Gruppe der nicht traditionell Studierenden - auf Basis der Analyse und der vorhandenen Angebote - bedarfsgerechte professionelle Beratungs- und Betreuungsstrukturen $\mathrm{zu}$ installieren und weiterzuentwickeln sowie eine Infrastruktur zu schaffen, die zu einem erfolgreichen Studienzugang, Studienverlauf und Studienabschluss beiträgt.

\section{Forschungsstand}

Im Jahr 2015 studierten rund 51.000 beruflich Qualifizierte an deutschen Hochschulen. Der Anteil der neuen Zielgruppen unter allen Studierenden beträgt etwa $1,9 \%$. Es handelt sich somit im Vergleich zu traditionell Studierende um eine kleine Zielgruppe, welche in den vergangenen Jahren aber stark gewachsen ist (Nickel/Schulz 2017). Für die wissenschaftliche Weiterbildung liegen bisher wenige Daten vor, jedoch ist von einer ähnlichen Situation auszugehen. Dies kann laut Hörr und Jütter (2017) daran liegen, dass es schwierig ist, „wissenschaftliche Weiterbildung klar abzugrenzen bzw. die Ziel- 
gruppen wissenschaftlicher Weiterbildung eindeutig zu bestimmen" (ebd.: 30). Lebenslanges Lernen gewährleistet eine fortlaufende Aktualisierung des Bildungsniveaus und die kontinuierliche Anpassung an neue Aufgaben. Wissenschaftliche Weiterbildung ermöglicht dies auf Hochschulniveau und öffnet somit die Hochschullandschaft für neue Zielgruppen, die spezifische Beratungs- und Betreuungsstrukturen erfordern.

Die Work-Life-Education-Balance stellt eine große Herausforderung für alle Weiterbildungsbeteiligten, wie Teilnehmende, Dozierende und Organisatoren, dar. Informations-, Beratungs- und Betreuungsangebote beeinflussen den Weiterbildungszugang, -verlauf und -abschluss und tragen zu einer erfolgreichen Teilnahme bei. Die Heterogenität der Gruppe der Weiterbildungsteilnehmenden erfordert zudem eine zeitliche und inhaltliche Anpassung der (bestehenden) Serviceleistungen. Bisher sind diese überwiegend an traditionell Studierenden ausgerichtet und werden meist von hochschulinternen Einrichtungen, wie bspw. Studienservices, oder von externen Partnern, wie Studentenwerken, angeboten. Beruflich Qualifizierte unterscheiden sich jedoch von traditionell Studierenden in folgenden Merkmalen: Alter, Bildungsbiographie, berufliche Vorerfahrung, Bekanntheitsgrad an Hochschulen, Informationsbereitstellung, Bedarf an überfachlichen Kursen (z. B. Lernstrategien), finanzielle und zeitliche Ressourcen, sozialer Background (Brunner et al. 2015; Gierke 2013; Kamm et al. 2016; Otto/Kamm 2016; Hanft et al. 2013; Jürgens/Zinn 2015). Diese Gegebenheiten können im Kontext der bestehenden Hochschulstruktur u. a. Gründe für die oben beschriebenen niedrigen Beteiligungszahlen beruflich Qualifizierter sein. Eine rasche Reaktion auf die Heterogenität fällt den Hochschulen oftmals schwer (Wolter 2011). Um den neuen Bedarfen gerecht zu werden, müssen Angebote (weiter-)entwickelt werden, da, wie Jürgens und Zinn (2012) angeben, beruflich Qualifizierte ein besonders hohes Interesse an der entsprechenden Fachthematik aufweisen und somit auch eine große Bereitschaft zur Anstrengung besteht. Nach Otto und Schwaniger (2013) sind die Beweggründe berufliche Qualifizierter für ein Studium vor allem die persönliche Weiterentwicklung, die Erweiterung von Fachwissen und die berufliche Weiterentwicklung. Banscherus und Pickert (2013) behandelten das Thema Unterstützungsangebote für neue Zielgruppen der Hochschulen und zeigen unter anderem einige Hürden auf. Die Zielgruppe der Studierenden ohne schulische Studienberechtigung weist einen hohen Bedarf an Informationen und Beratung hinsichtlich Studienanforderungen, Studienfinanzierung, Vereinbarkeit von Studium mit beruflichen und/oder familiären Anforderungen, Zulassungs- und Zugangsbedingungen und Anrechnung von beruflichen Kompetenzen auf. Bei Fernweiterbildungen zeigte sich, dass v. a. telefonische oder E-Mail-Beratungen genutzt wurden (Fogolin 2010). „Fast zwei Drittel der Befragten recherchierten die von ihnen genutzten Informationsmöglichkeiten selbst; vielfach auf Basis von Werbung bzw. [...] auf Basis von Hinweisen aus dem persönlichen Umfeld. Nur wenig 
Hinweise auf Informationsquellen wurden von professionellen Beraterinnen und Beratern gegeben“ (ebd.: 29). Ergebnisse zu expliziten Herausforderungen hinsichtlich der Bedarfe beruflich Qualifizierter im Prozess der Eingliederung in das Hochschulsystem liegen bislang nicht vor.

\section{Beratung und Betreuung im Projekt OTH mind}

Die beiden Bereiche Beratung und Betreuung weisen eine große Schnittmenge auf, so dass sich eine Differenzierung, insbesondere in Bezug auf Ziele und Maßnahmengestaltung, als schwierig erweist. Beratung bezieht sich auf „konkrete Orientierungsangebote und Hilfestellungen [...] bei Entscheidungs- und Gestaltungsfragen“, unter Betreuung hingegen wird ein längerfristig angelegtes „Begleit- und Unterstützungsangebot“ verstanden (Jorzik 2013: 1). Im Rahmen des Projektes OTH mind wurden diesbezüglich zwei Definitionen festgelegt. Beratung umfasst eine Interaktion zwischen einem bzw. einer Ratsuchenden und einem Berater bzw. einer Beraterin, die zeitlich begrenzt ist und auf Freiwilligkeit basiert, sie kann auch in Form von Informationsbereitstellung erfolgen. Dieser Selbstinformationsprozess versteht sich als Hilfe zur Selbsthilfe (nach Pätzold 2004, zitiert in: Fischer 2008). Unter Betreuung wird hingegen eine Support-Struktur verstanden, die sowohl Angebote umfasst, die zeitlich länger laufen und sich direkt auf die Weiterbildung beziehen (z. B. Lernunterstützung), wie auch Angebote, die eher Serviceangebote darstellen, bspw. Bibliotheksführungen.

\section{Forschungsaktivitäten und -methoden}

Um die heterogenen Bedarfe der Projektzielgruppen bzgl. der Thematik Beratung und Betreuung zu erheben, wurden im Kontext von OTH mind unterschiedliche Erhebungsmethoden im Sinne eines Mixed-Methods-Ansatzes gewählt (Gläser-Zikuda et al. 2012). Ziel der empirischen Analysen war es, auf regionaler Ebene die vielfältigen und heterogenen Weiterbildungsbedarfe sowie das Bildungsinteresse zu ermitteln, um anschließend zielgruppenadäquate Qualifizierungsangebote zu entwickeln. Neben Dokumentenanalysen und Expert/inneninterviews wurden vor allem zielgruppenspezifische Umfragen, wie Unternehmens-, Führungskräfte-, Fachkräfte- und Ingenieur/ 
innenbefragungen durchgeführt. ${ }^{1}$ Aufgrund der Heterogenität der Zielgruppen erfolgte in einem Analysemodell (siehe Abb. 1) eine Aufteilung in zwei übergeordnete Gruppen. Zum einen ,,potentielle Teilnehmer/innen von Weiterbildungsangeboten“ und zum anderen „Teilnehmer/innen von Weiterbildungsangeboten“. Unter der Bezeichnung ,potentielle Teilnehmer/innen von Weiterbildungsangeboten" ${ }^{\text {" }}$ werden alle Personen gefasst, die ein generelles Interesse an Weiterbildung an Hochschulen haben, an einer solchen aber noch nicht teilgenommen haben. Dabei wurden sowohl die Fachkräfte- als auch die Ingenieur/innenbefragung herangezogen ${ }^{3}$. Ziel der Befragung aus Sicht von Beratung und Betreuung war, das Interesse, den Informationsstand, den Informationsweg, Barrieren und Bedarfe für die Weiterbildungsteilnahme zu identifzieren. Die Gruppe der „Teilnehmer/innen von Weiterbildungsangeboten ${ }^{\prime 4}$ umfasst Personen, die bereits an einer Weiterbildung an einer Hochschule teilgenommen haben oder zum Zeitpunkt der Befragung an einer solchen teilnehmen. Ihre Daten wurden in Form einer quantitativen Befragung im Rahmen des Propädeutikums ${ }^{5}$ an der OTH Amberg-Weiden sowie in Form einer Befragung mit Weiterbildungsteilnehmenden am Zentrum für Weiterbildung und Wissensmanagement in Regensburg (ZWW) erhoben. Ziel der Befragungen war aus Sicht von Beratung und Betreuung, die Informationswege der Teilnehmenden, ihre Kenntnisse über bestehende Angebote der Beratung und Unterstützung und deren Bewertung, sowie weitere Bedarfe für eine erfolgreiche Teilnahme an einer Weiterbildung an einer Hochschule zu ermitteln. Des Weiteren wurden Expert/innen herangezogen, die Weiterbildungsinteressierte und -teilnehmende an Hochschulen beraten und unterstützen. Ziel dieser Befragung war, ihre Erfahrungen zu ermitteln, die Inanspruchnahme der Beratungs- und Unterstützungsangebote einzuschätzen sowie weitere Beratungsfelder herauszufinden.

\footnotetext{
Vgl. Schnurer 2016.

2 Im Folgenden auch als ,potentielle Teilnehmende“ bezeichnet.

3 Zudem wurden qualitative Interviews mit erwerbslosen Ingenieur/innen geführt.

4 Im Folgenden auch als ,Teilnehmende“ bezeichnet.

5 Bei der Propädeutikumsbefragung handelte es sich um Teilnehmende des Studienvorbereitungskurses „Propädeutikum“ des OTH Professional-Bereiches der OTH Amberg-Weiden, bei der ZWW-Befragung um Teilnehmende eines Weiterbildungsangebotes des Zentrums für Weiterbildung und Wissensmanagement der OTH Regensburg.
} 


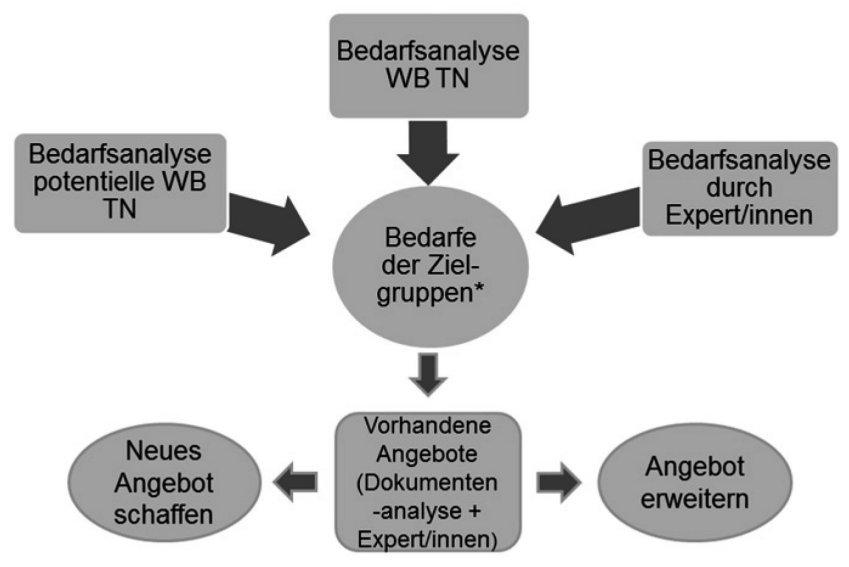

Abb. 1: Analysemodell (Quelle: eigene Darstellung 20176)

(* die Zielgruppen werden durch die Erhebungen genauer beschrieben)

\section{Ergebnisse des Beratungs- und Betreuungsbedarfs}

Stellenvertretend für die Gruppe der potentiellen Teilnehmenden wird im Folgenden die Bedarfserhebung der Fachkräfte herangezogen. Diese wurde gezielt für die Region Oberpfalz ausgewertet $(\mathrm{N}=224)$. Dabei waren 86,0\% männliche $(\mathrm{m})$ und $14,0 \%$ weibliche $(\mathrm{w})$ Personen vertreten. $81,1 \%$ davon waren zum Zeitpunkt der Erhebung zwischen 20 und 29 Jahre und 9,6\% hatten Familienpflichten. In einem sozialversicherungspflichtigen Beschäftigungsverhältnis (VZ und unbefristet) befanden sich $87,9 \%$ und $83,5 \%$ befanden sich zum Zeitpunkt der Erhebung in einer Aufstiegsfortbildung ${ }^{7}$ (beispielsweise Meister/in oder Techniker/in) bzw. hatten diese bereits absolviert. Interesse an Weiterbildung hatten 80,6\% aller befragten Frauen sowie 74,9\% der Männer. Fast $60 \%$ der Befragten war bekannt, dass sie Weiterbildungsangebote an Hochschulen besuchen können. Die drei meistgenannten Gründe gegen eine Weiterbildung bei Frauen waren zeitliche Gründe (Arbeit), finanzielle Gründe und keine passenden Angebote. Bei Männern waren es ebenfalls zeitliche

6 Bedeutung der Abkürzungen: WB - Weiterbildung, TN - Teilnehmende.

7 Die Teilnahme an Aufstiegsfortbildungen dient hierbei nicht als Indikator für Weiterbildungsinteresse sondern ergibt sich aus dem Kontext der Zielgruppenbefragung. 
und finanzielle Gründe sowie kein eigenes Zutrauen bzgl. der Anforderungen. 80,6\% der Frauen sowie 74,9\% der Männer äußersten Interesse an Weiterbildung an Hochschulen.

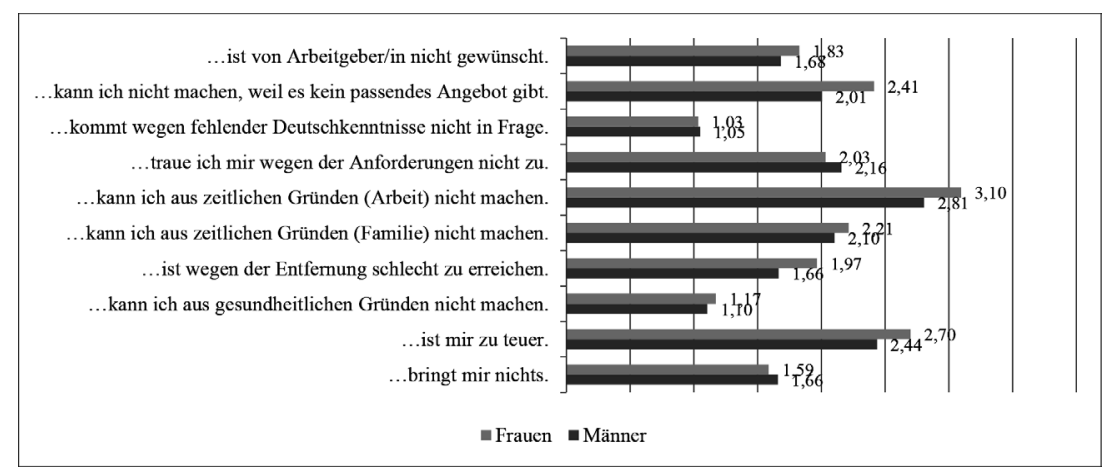

Abb. 2: Fachkräfteerhebung „Gründe gegen Weiterbildung an Hochschulen“ (Quelle: Fachkräfteerhebung, eigene Darstellung 2017. Skala: $1=$ trifft nicht zu, $4=$ trifft zu)

In einem zweiten Schritt erfolgte die Analyse von Personen, die ein Weiterbildungsinteresse geäußert haben $(\mathrm{N}=168)$. Insbesondere für Frauen sind folgende Aspekte bei einer Weiterbildung wichtig: ,sich persönlich weiterentwickeln', ,berufliche/fachliche Kenntnisse erwerben' und ,neue innerbetriebliche Tätigkeitfelder übernehmen'. Bei Männern verhält es sich ähnlich, auch sie wollen sich ,persönlich weiterentwickeln' und ,berufliche/fachliche Kenntnisse erwerben' und mehr Geld verdienen. 86,7\% der befragten Männer und $92,0 \%$ der befragten Frauen würden unterschiedliche Beratungs- und Unterstützungsangebote an Hochschulen in Anspruch nehmen. Von diesen $(\mathrm{N}=148)$ finden $88,5 \%$ die persönliche Beratung am hilfreichsten, danach folgen Beratungen per E-Mail $(53,4 \%)$ und Online-Informationsmaterialien $(51,4 \%)$. 


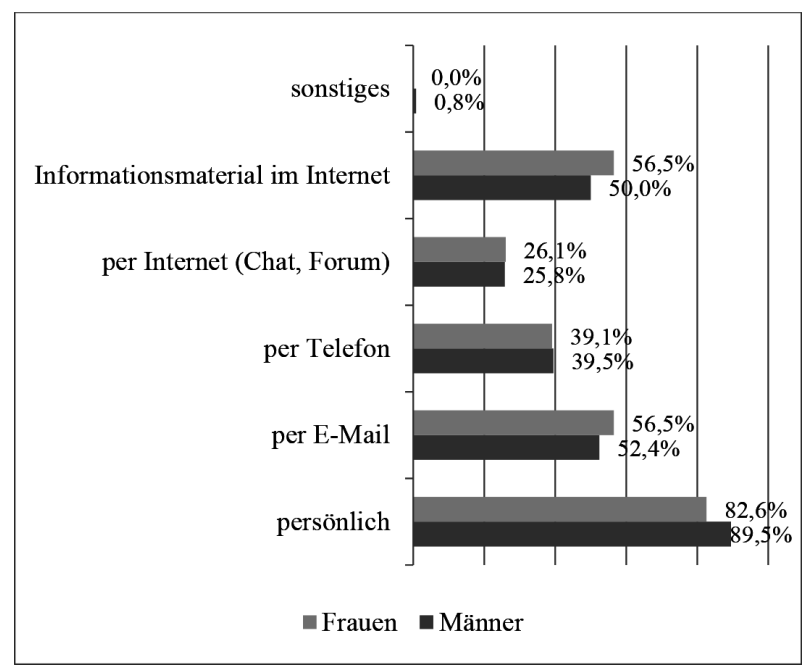

Abb. 3: Fachkräfteerhebung „Beratungsformen“(Quelle: Fachkräfteerhebung, eigene Darstellung 2017)

Keine geschlechterspezifischen Unterschiede zeigen sich bei Beratungsthemen wie finanzielle Förderung, berufliche Perspektiven und Karriereplanung sowie Zeitmanagement. Dies gilt auch für die Frage nach möglichen Unterstützungsangeboten: Männer und Frauen sehen vorrangig eine feste Ansprechperson für Fragen, Lernberatung und Verpflegung als hilfreich an. Bei den weiblichen Fachkräften waren die meistgenannten Hürden für die Aufnahme einer Weiterbildung zeitliche Gründe (Arbeit), finanzielle Gründe und keine passenden Angebote. Bei Männern waren es ebenfalls zeitliche und finanzielle Gründe sowie fehlendes Zutrauen bzgl. der Anforderungen.

Für die Gruppe der Teilnehmenden folgen die Ergebnisse der Propädeutikumsbefragung. An dieser Erhebung nahmen zehn Personen teil $(\mathrm{N}=10$; $10 \mathrm{~m}$ ), die im Schnitt zwischen 25 und 29 Jahre (60\%) alt waren und als höchsten Schulabschluss einen Realschulabschluss (50\%) angaben. Die Befragten sind über die Webseite der OTH Amberg-Weiden, Berufsberatung, Webseiten oder Studienberatungen anderer Hochschulen oder durch Sonstiges (wie bspw. Bundeswehr) auf das Angebot aufmerksam geworden. Ein weiterer Informationsgewinn erfolgte über persönliche Beratungsgespräche, Telefonberatung sowie die Teilnahme an Informationsveranstaltungen. Als Unterstützungsangebote wurden genannt: Lernunterstützung, Verpflegung am Hochschulstandort, Online-Selbsttest, Kinderbetreuung während der Weiterbildung, Bibliotheksführung sowie Lernräume vor Ort. 
Die Experten/inneninterviews wurden mit drei Personen (w) geführt, die u. a. Weiterbildungsberaterinnen an den Hochschulen bei OTH Professional und im ZWW sind. Die erste Kontaktaufnahme erfolgt überwiegend über Messen. Themen der Beratung sind Kosten, Organisatorisches (wie z. B. Termine, Stundenplan usw.) sowie oftmals konkrete Fragen zu einem Kurs. Die Befragten berichten, dass die eigentliche Beratung erst in der Entscheidungsphase einsetzt, d. h., wenn die Interessierten sich bereits für ein Weiterbildungsangebot entschieden haben.

Zusammenfassend kann zielgruppenübergreifend festgehalten werden, dass beruflich Qualifizierte v. a. folgende Unterstützungsangebote als hilfreich empfinden: Feste Ansprechperson, Lernberatung, Verpflegung und Betreuung von Kindern sowie Mentoring. Als Beratungsthemen wurden vorwiegend finanzielle Fördermöglichkeiten, Zeitmanagement sowie berufliche Perspektiven und Karriereplanung genannt, was sich wiederum mit den Erkenntnissen von Banscherus und Pickert (2013) deckt. Beruflich Qualifizierte sehen v. a. folgende Beratungsformen als hilfreich an (AES-Trendbericht): Informationen online, E-Mail, persönlich, telefonisch und Informationsveranstaltungen. Zudem wird deutlich, dass Hochschulen als Beratungsstellen im Mittelfeld angesiedelt sind, was weitere Schritte notwendig macht (siehe Tabelle 1).

Tab. 1: Deskriptive Ergebnisse: zusammenfassende Erkenntnisse aus den Erhebungen. Quelle: eigene Darstellung 2017

\begin{tabular}{|l|l|l|l|}
\hline $\begin{array}{l}\text { Gründe } \\
\text { Weiterbildung }\end{array}$ & $\begin{array}{l}\text { Unterstützungs- } \\
\text { angebote }\end{array}$ & Beratungsformen & Beratungsthemen \\
\hline $\begin{array}{l}\text { Zeit-Faktor (Arbeit und } \\
\text { Familie) }\end{array}$ & Feste Ansprechperson & Informationen online & $\begin{array}{l}\text { Finanzielle } \\
\text { Fördermöglichkeiten }\end{array}$ \\
\hline Finanzielle Gründe & Lernberatung & Per E-Mail & Zeitmanagement \\
\hline Selbstorganisation & $\begin{array}{l}\text { Verpflegung und } \\
\text { Betreuung von Kindern }\end{array}$ & Persönlich & $\begin{array}{l}\text { Berufliche Perspektiven } \\
\text { und Karriereplanung }\end{array}$ \\
\hline Passende Angebote & Mentoring & Telefonisch & \\
\hline
\end{tabular}




\section{Umsetzung}

Auf Grundlage der gewonnenen Erkenntnisse aus Literatur und eigenen Erhebungen wurden zielgruppenspezifische und bedarfsgerechte Beratungs- und Betreuungsangebote (weiter-)entwickelt. Die Angebotsentwicklung orientiert sich dabei an dem Konzept des Student-Life-Circle (siehe Abbildung 2).

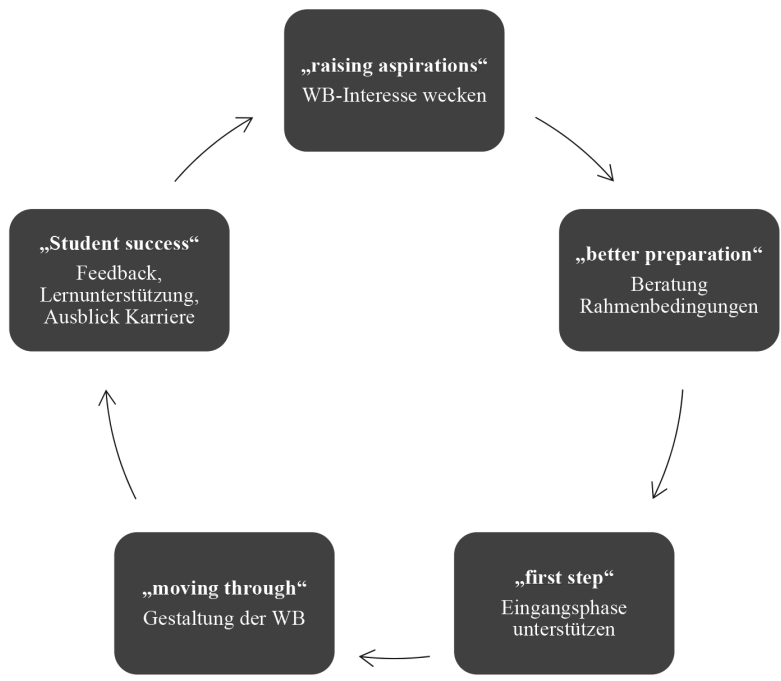

Abb. 4: Student-Life-Circle für Weiterbildungsteilnehmer/innen (Quelle: eigene Darstellung 2017 in Anlehnung an Schulmeister 2007, S. 49)

",raising aspiration“"

Das Weiterbildungsinteresse muss geweckt und die Öffnung der Hochschulen bekannter gemacht werden. Wie die Ergebnisse der Bedarfserhebung zeigen, weiß ein Großteil der Befragten nicht, dass Hochschulen Weiterbildungen anbieten. Potentielle Teilnehmenden gaben an, v. a. das Internet zur Informationsgewinnung zu nutzen, daher wurden insbesondere webbasierte Beratungsangebote entwickelt.

",better preparation“"

In dieser Phase steht vor allem die Beratungs- und Informationsbereitstellung im Fokus. Hierbei erfolgt meist die erste persönliche Kontaktaufnahme seitens der (potentiellen) Teilnehmenden. Beratungsformen wie E-Mail, Informationsveranstaltungen sowie persönliche und telefonische Beratung gelten als hilfreich, daher wurden auf den Projektwebseiten die Ansprechpersonen 
klar dargestellt und Flyer mit Kontaktmöglichkeiten an verschiedene Stellen verschickt und ausgelegt.

"first step"

$\mathrm{Zu}$ Beginn der Weiterbildung spielt die Bereitstellung von detaillierten Informationen die wichtigste Rolle. Deshalb wurde eine Informationsbroschüre mit Informationen zur Hochschule, hilfreichen Anlaufstellen und Angeboten wie Weiterbildung mit Kind sowie allgemeinen Campusinformationen erstellt. Diese Broschüre wurde den Kursteilnehmenden am ersten Weiterbildungstag gegeben.

"moving through"

Beratungsthemen wie finanzielle Fördermöglichkeiten, Zeitmanagement sowie berufliche Perspektiven und Karriereplanung waren stark nachgefragt. Daher wurde v. a. für die Teilnehmenden des „BeVorStudiums“ ein Vortrag zu Finanzierungsthemen in der Weiterbildung sowie im zukünftigen Studium zusammen mit dem Studien- und Career Service der OTH Amberg-Weiden organisiert.

"student success"

Um einen erfolgreichen Kursverlauf sowie einen erfolgreichen Übergang ins Studium zu gewährleisten, wurde das Modul „Studienkompetenz“ entwickelt, welches bereits vor Studienbeginn studienrelevante Inhalte wie Lernstrategien usw. vermittelt.

\section{Herausforderungen}

Im Rahmen der Bedarfserhebungen und der ersten Testphase der Angebote zeichneten sich verschieden Herausforderungen und (Weiter-)Entwicklungspotentiale ab. Im Folgenden werden sowohl zielgruppen- als auch hochschulspezifische Herausforderungen näher beleuchtet:

Akzeptanz im Hochschulsystem

Einem Großteil der Befragten war noch nicht bewusst, dass beruflich Qualifizierte Weiterbildungen an Hochschulen besuchen dürfen. Des Weiteren kann es unter Umständen vorkommen, dass gewisse Vorbehalte gegenüber beruflich Qualifizierten bestehen. Dies kann sich z. B. bezüglich eines möglichen schulischen Nachholbedarfs (z. B. Mathematikkenntnisse) zeigen. In der Entwicklungs- und Erprobungsphase können somit förderliche und hemmende Faktoren seitens der hochschulinternen Beteiligten bzgl. der Bereitschaft zur Beteiligung und Annahme der neuen Zielgruppe auftreten.

Beratungs- und Betreuungsangebote

Durch die gegebenen Hochschulstrukturen sind eine Vielzahl an Beratungsund Betreuungsangeboten auf traditionell Studierende ausgerichtet. Essenzi- 
elle Angebote bzgl. Informationen und Bedarfen von beruflich Qualifizierten können somit vom bestehenden System nicht abgedeckt werden - wie bspw. besondere Lernberatung und -betreuung, flexible Beratungszeiten, erweiterte Kinderbetreuung usw. Daher muss eine zeitliche und inhaltliche Anpassung der bestehenden Servicestrukturen vorgenommen werden, um die bestehenden Angebote in Bezug auf die neue Zielgruppe (weiter) zu entwickeln.

Digitalisierung

Digitalisierung wird im Bildungsbereich stark diskutiert, daher setzen Hochschulen auf E-Learning- und Blended-Learning-Konzepte. Weiterbildungsinteressierte bevorzugen jedoch oftmals klassische Lernszenarien wie Präsenzveranstaltungen. Daraus ergibt sich ein Ungleichgewicht, welches individuell je nach Hochschule und Region zu lösen ist.

Neue Strukturen

Das Hochschulsystem ist für einen Großteil der beruflich Qualifizierten im Gegensatz zum alltäglichen Arbeitsumfeld ein neues Umfeld mit neuen Strukturen. Faktoren wie Selbstorganisation und selbstverantwortliches Lernen sind darin unabdingbar. Diese Umstrukturierung und Selbstverantwortung birgt eine große Herausforderung. Aus diesem Grund ist es wichtig, Studienkompetenzen von Beginn an zu vermitteln.

All diese Aspekte lassen viele Fragen zur Umsetzung und Schaffung neuer Strukturen, zur Nachhaltigkeit der geschaffenen Strukturen sowie zur Implementierung und Verankerung in Regelstrukturen des Hochschulsystems offen.

\section{Fazit}

Anhand der Ergebnisse der Bedarfserhebungen und der Erkenntnisse aus der ersten Testphase im Projekt OTH mind kann resümiert werden, dass Herausforderungen im Bereich der Beratung und Betreuung in der wissenschaftlichen Weiterbildung von der zielgruppenspezifischen Heterogenität bis hin zur hochschulinternen Umstrukturierung reichen. Um die zuvor aufgeführten Faktoren bewältigen zu können und um schließlich die Beratungs- und Betreuungsangebote in der wissenschaftlichen Weiterbildung zielgruppengerecht zu etablieren, können folgende Lösungsansätze vorgeschlagen werden:

- Workshops und Schulungen für hochschulinterne Beteiligte (z. B. Beratungspersonal) zur Sensibilisierung für die neue Zielgruppe: Hierbei sollten vor allem die unterschiedlichen Bedarfe gegenüber traditionell Studierenden im Fokus stehen. Es können bspw. zeitliche und inhaltliche Anpassungen der (bestehenden) Serviceleistungen erfolgen. 
- Im Zuge dessen sollte auch eine hochschulinterne Zusammenarbeit stattfinden, um die Vielzahl der Angebote zu managen. Verschiedene Anlaufstellen und Einrichtungen sowie entsprechende Projekte können im regelmäßigen Austausch stehen und gemeinsam eine Strategie verfolgen.

- Brückenkurse konzipieren und langfristig etablieren: Diese Kurse können sowohl fachliche Kompetenzen (z. B. Mathematikkenntnisse) als auch studienrelevante Kompetenzen vermitteln.

- Als ein Bestandteil von Beratung sollten verschiedene Informationsmedien und -wege miteinbezogen und ausgebaut werden, v. a. die Onlinekanäle sind in der Einstiegsphase sehr hilfreich. Zugleich kann auf diesem Weg der Bekanntheitsgrad der „Öffnung der Hochschulen“ erhöht werden.

Offen bleibt folgende Frage:

Wie kann sichergestellt werden, dass nicht-traditionell Studierende in die bestehenden Strukturen integriert werden?

\section{Literatur}

Banscherus, Ulf/Kamm, Caroline/Otto, Alexander (2016): Gestaltung von $\mathrm{Zu}-$ und Übergängen zum Hochschulstudium für nicht-traditionelle Studierende. Empirische Befunde und Praxisbeispiele. In: Wolter, Andrä/Banscherus, Ulf/Kamm, Caroline (Hg.): Zielgruppen Lebenslangen Lernens an Hochschulen. Ergebnisse der wissenschaftlichen Begleitung des Bund-Länder-Wettbewerbs „Aufstieg durch Bildung: offene Hochschulen“. Münster: Waxmann, S. 295-320.

Banscherus, Ulf/Pickert, Anne (2013): Unterstützungsangebote für nicht-traditionelle Studierende - Stand und Perspektiven. Thematischer Bericht der wissenschaftlichen Begleitung des Bund-Länder-Wettbewerbs „Aufstieg durch Bildung: offene Hochschule“.

Brunner, Stefanie/Kretschmer, Stefanie/Hoffmann, John/Zawacki-Richter, Olaf (2015): Blended Counselling: Konzeption eines Online-Beratungsportals für beruflich qualifizierte Studieninteressierte. In Freitag, Walburga K./Buhr, Regina/ Danzeglocke, Eva-Maria/Schröder, Stefanie/Völk, Daniel (Hg.): Übergänge gestalten. Durchlässigkeit zwischen beruflicher und hochschulischer Bildung erhöhen. Münster: Waxmann, S. 31-48.

Fischer, Nina (2008): Weiterbildungsberatung in beruflich-betrieblichen Kontexten. MA Berlin: TU Berlin/Institut für Berufliche Bildung und Arbeitslehre. https:// depositonce.tu-berlin.de/bitstream/11303/2605/1/Dokument_18.pdf [letzter Zugriff: 25.01.2018].

Fogolin, Angela (2010): (Weiter-)Bildungsberatung im Fernlernen aus Sicht der Nachfragenden. Ergebnisse einer Onlinebefragung. Wissenschaftliche Diskussionspapiere, Schriftenreihe des Bundesinstituts für Berufsbildung (Heft 116, 71). Bonn: BiBB. 
Gierke, Willi B. (2013): Wege an die Hochschule für beruflich Qualifizierte - geschichtliche Aspekte der Entwicklung unter besonderer Berücksichtigung Niedersachsens und der Carl von Ossietzky Universität Oldenburg. In: Hanft, Anke/ Brinkmann, Katrin (Hg.): Offene Hochschulen. Die Neuausrichtung der Hochschulen auf Lebenslanges Lernen. Münster: Waxmann, S. 80-96.

Gläser-Zikuda, Michaela/Seidel, Tina/Rohlfs, Carsten/Gröschner, Alexander/Ziegelbauer, Sascha (2012): Mixed Methods in der empirischen Bildungsforschung. Münster: Waxmann.

Hanft, Anke/Maschwitz, Annika/Hartmann-Bischoff, Monika (2013): Beratung und Betreuung von berufstätigen Studieninteressierten und Studierenden zur Verbesserung des Studienerfolgs. In: Hanft, Anke/Brinkmann, Katrin (Hg.): Offene Hochschulen. Die Neuausrichtung der Hochschulen auf Lebenslanges Lernen. Münster: Waxmann, S. 110-119.

Hörr, Beate/Jütte, Wolfgang (Hg.) (2017): Weiterbildung an Hochschulen. Der Beitrag der DGWF zur Förderung wissenschaftlicher Weiterbildung. Bielefeld: W. Bertelsmann Verlag.

Jorzik, Bettina (2013): Charta guter Lehre. Grundsätze und Leitlinien für eine bessere Lehrkultur. Stifterverband für die Deutsche Wissenschaft. http://www.stifterverband.info/wissen-schaft_und_hochschule/lehre/charta_guter_lehre/charta_guter_lehre.pdf. [letzter Zugriff: 25.01.2018].

Jürgens, Alexandra/Zinn, Bernd (2015): Nicht-traditionell Studierende in Deutschland - Stand der empirischen Forschung und Desiderate. In: Elsholz, Uwe (Hg.): Beruflich Qualifizierte im Studium. Analysen und Konzepte zum Dritten Bildungsweg. Bielefeld: W. Bertelsmann Verlag, S. 35-56.

Jürgens, Alexandra/Bernd Zinn (2012): Nichttraditionell Studierende in ingenieurswissenschaftlichen Studiengängen - Zugangswege, Motive, kognitive Voraussetzungen. In: IHF (Hg.): Beiträge zur Hochschulforschung, 34 (4/2012). München, S. 34-53.

Kamm, Caroline/Spexard, Anna/Wolter, Andrä (2016): Beruflich Qualifizierte als spezifische Zielgruppe an Hochschulen. Ergebnisse einer HISBUS-Befragung. In: Wolter, Aandrä/Banscherus, Ulf/Kamm, Caroline (Hg.): Zielgruppen Lebenslangen Lernens an Hochschulen. Ergebnisse der wissenschaftlichen Begleitung des Bund-Länder-Wettbewerbs „Aufstieg durch Bildung: offene Hochschulen“. Münster: Waxmann, S. 165-196.

KMK (2009): Hochschulzugang für beruflich qualifizierte Bewerber ohne schulische Hochschulzugangsberechtigung. http://www.kmk.org/fileadmin/Dateien/veroeffentlichungen beschluesse/2009/2009 03 06-Hochschulzugang-erful-qualifizierte-Bewerber.pdf [letzter Zugriff: 25.01.2018].

Nickel, Sigrun/Schulz, Nicole (2017): Update 2017: Studieren ohne Abitur in Deutschland. Überblick über aktuelle Entwicklungen. http://www.che.de/ downloads/CHE_AP_195_Studieren_ohne_Abitur_2017.pdf. [letzter Zugriff: 25.01.2018].

Otto, Alexander/Kamm, Caroline (2016): „Ich wollte einfach noch eine Stufe mehr.“ Vorakademische Werdegänge und Studienentscheidungen von nicht-traditionellen Studierenden und ihr Übergang in die Hochschule. In: Wolter, Andrä/ Banscherus, Ulf/Kamm, Caroline (Hg.): Zielgruppen Lebenslangen Lernens an Hochschulen. Ergebnisse der wissenschaftlichen Begleitung des Bund-Länder- 
Wettbewerbs „Aufstieg durch Bildung: offene Hochschulen“. Münster: Waxmann, S. 197-224.

Otto, Christian/Schwaniger, Katja (2013): Motivlagen und berufliche Zielsetzungen von beruflich qualifizierten Studierenden. In: Agentur für Erwachsenen- und Weiterbildung (Hg.): Beruflich qualifiziert studieren - Herausforderungen für Hochschulen. Ergebnisse des Modellprojekts Offene Hochschule Niedersachsen. Bielefeld: Bertelsmann, S. 37-48.

Schulmeister, Rolf (2007): Der „Student Lifecycle“ als Organisationsprinzip für E-Learning. In: Keil, Reinhard/Kerres, Michael/Schulmeister, Rolf.: eUniversity - Update Bologna. Münster: Waxmann, S. 45-77.

Schnurer, Katharina (2016): Gesamtüberblick über das Vorgehen „Quantitative Befragung zur Erfassung des regionalen Weiterbildungsbedarfs“". OTH mind Methodenbericht. https://www.oth-aw.de/files/oth-aw/Forschung/Forschungsprojekte/ OTH_mind/Publikation_Methodenbericht_mit_Fragebogen.pdf [letzter Zugriff: 20.04.2018].

Wolter, Andrä (2011): Die Öffnung der Hochschulen in Deutschland. Vortrag vom 08.06.2011 auf der Tagung „Hochschulen öffnen ...“ - HRK Projekt nexus. Berlin. http://www.hrk-nexus.de/uploads/media/Tagung-nexus-Hochschulenoeffnen-Wolter_03.pdf [letzter Zugriff: 25.01.2018]. 
IV. Beratungskompetenz und Professionalisierung 



\section{Instrumente zur Erfassung der Kompetenzen von Beratenden im Feld Bildung, Beruf und Beschäftigung - Konzept und Erprobung}

\section{Einleitung}

Aus den aktuellen gesellschaftlichen Rahmenbedingungen für die Gestaltung der Bildungs- und Beschäftigungsbiografien, die eine hohe Individualisierung und Selbstverantwortung implizieren, resultiert ein wachsender Bedarf an professioneller Beratung. Dabei beschränkt sich Beratung nicht auf Übergänge, Sondersituationen und Krisen im Lebenslauf, sondern ist ausgerichtet auf Potenziale und Möglichkeiten, kann präventiv angelegt sein und ist dementsprechend als kontinuierliches, begleitendes Angebot zu verstehen (Schiersmann 2013, S. 25f.). Professionelle Beratung in Bildung, Beruf und Beschäftigung erfordert seitens der Beratenden spezifische und umfassende Kompetenzen, um eine qualitativ hochwertige soziale Dienstleistung zu erbringen. Die Aus- und Fortbildungswege für Beratende in diesem Feld sind vielfältig und nicht standardisiert. Häufig werden Beratungskompetenzen im Zuge der Beratungspraxis informell erworben und weiterentwickelt. U. a. in diesem Zusammenhang stellt sich die Frage nach geeigneten Instrumenten, mit deren Hilfe die Kompetenzen der Beratenden zuverlässig und nahe am situativen Kontext des Beratungsfeldes erfasst und bewertet werden können, um daraus Perspektiven für die Weiterentwicklung des persönlichen Kompetenzprofils erarbeiten zu können.

Gerade bei einer so anspruchsvollen Dienstleistung wie der Beratung besteht eine große Herausforderung (für die Forschung, aber z. B. auch in der Ausbildung von Beratenden) darin, die erforderlichen Kompetenzen übergreifend (d. h. auf verschiedene Settings, Zielgruppen oder Beratungsansätze ausgerichtet) und zugleich detailliert zu beschreiben, und darüber hinaus vor allem darin, diese angemessen zu dokumentieren und zu bewerten. Zurückblickend auf die letzten Jahre lassen sich in unterschiedlichen Fachdisziplinen eine Vielzahl von Forschungsvorhaben konstatieren, die sich intensiv mit dem Thema Kompetenzerfassung auseinandersetzen und das Kompetenzkonstrukt in seiner Komplexität anhand verschiedener methodischer Ansätze zu erfassen versuchen. Für das Beratungsfeld „Bildung Beruf und Beschäftigung" liegen allerdings bislang kaum Forschungserkenntnisse zur Erfassung der performanznahen Beratungskompetenz in der Praxis vor. In diesem Beitrag gehen wir der Frage nach, wie diese Lücke geschlossen werden kann. 
Grundlage für die Darstellung bildet ein Teilvorhaben im Rahmen des vom Institut für Bildungswissenschaft der Universität Heidelberg und dem Nationalen Forum Beratung $(n f b)$ durchgeführten und vom Bundesministerium für Bildung und Forschung geförderten Projektes zur Beratungsqualität (im Folgenden: $\mathrm{BeQu}$ ). Es wurden verschiedene Instrumente zur Erfassung und Bewertung der Kompetenzen von Beratenden entwickelt und erprobt. Dieser Prozess und seine Ergebnisse stehen im Mittelpunkt dieses Beitrags.

\section{Kompetenzverständnis, Kompetenzmodell und Kompetenzprofil für Beratende}

Kompetenzen können als Kern der Professionalität (von Beratenden) beschrieben werden. Das hier vertretene Kompetenzverständnis orientiert sich dabei an einem performanzorientierten Kompetenzbegriff, der den Zusammenhang von Dispositionen und Handeln fokussiert. Dies bedeutet, dass insbesondere die Performanz betrachtet werden soll, die allerdings auf vielfältigen Ressourcen bzw. Dispositionen (u. a. Wissen, Fertigkeiten, Emotionen und Motivation) basiert, die für eine kompetente Handlung notwendig sind. Dabei ist $\mathrm{zu}$ beachten, dass der Zusammenhang zwischen den erforderlichen Ressourcen und der Performanz in der Handlungssituation konzeptionell hergestellt bzw. theoretisch begründet werden muss. Weinert (2001, S. 54) schlägt vor, in diesem Zusammenhang bestimmte Metakompetenzen zu berücksichtigen, die es Individuen ermöglichen, in einer konkreten Situation adäquat auf Fähigkeiten und Wissen etc. zurückzugreifen, um die Situation erfolgreich zu bewältigen (Schiersmann/Weber/Petersen 2013, S. 196f.).

Kompetenz und spezifischer Beratungskompetenz wird als erfolgreiche, selbstorganisierte Bewältigung einer (und potentiell vieler) konkreten Problemstellung(en) (z. B. der Gestaltung eines Beratungsprozesses) unter Rückgriff auf verschiedene aktivierbare Ressourcen verstanden (s. Abbildung 1). Was dabei als erfolgreich zu bewerten ist, muss in dem Kompetenzkonzept definiert werden. 


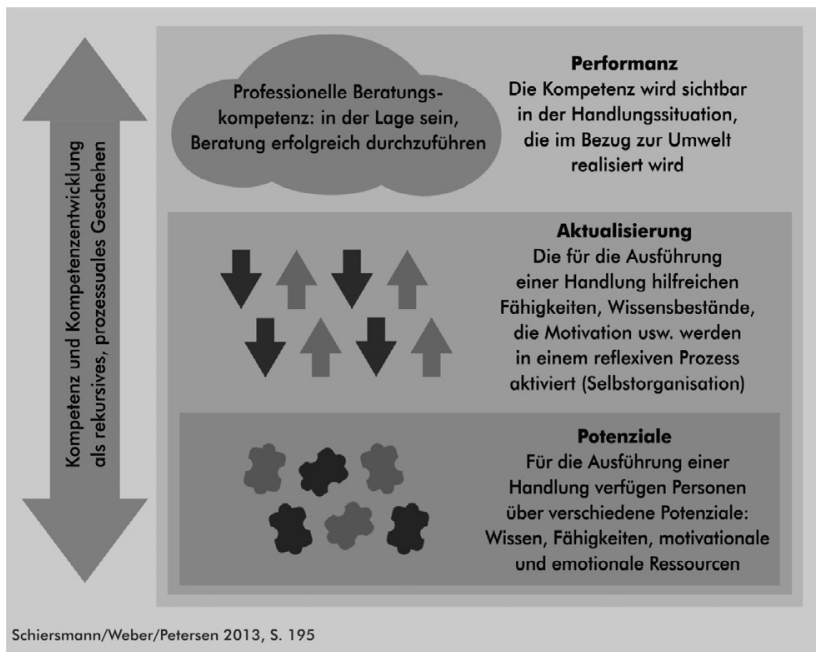

Abb. 1: Kompetenz und Kompetenzentwicklung als rekursives, prozessuales Geschehen

Weitere Voraussetzungen für die Erfassung von Kompetenzen stellen ein Kompetenzmodell im Sinne einer theoretisch begründeten Systematisierung der Handlungsbereiche sowie ein daraus abgeleitetes Kompetenzprofil dar. Hierfür wurde ein systemisches Modell arbeitsweltbezogener Beratung erstellt (s. Abbildung 2).

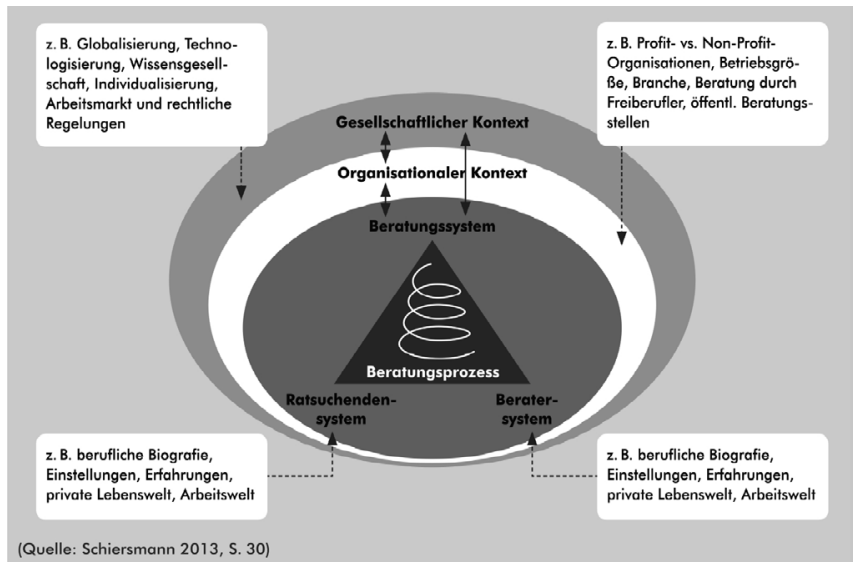

Abb. 2: Systemisches Modell arbeitsweltbezogener Beratung 


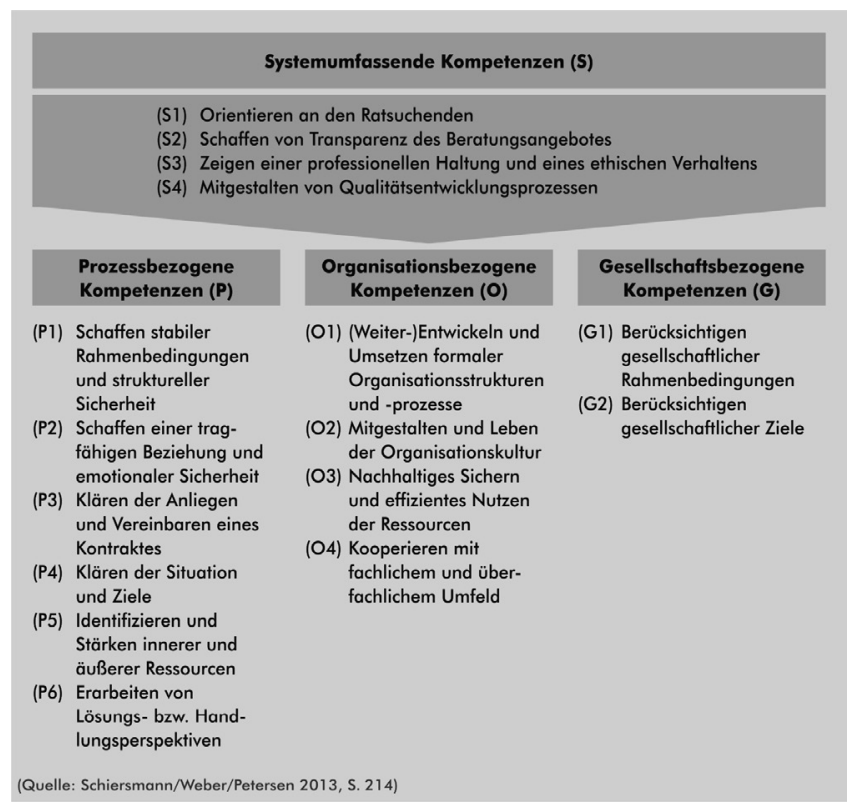

Abb. 3: Kompetenzprofil für Beratende in Bildung, Beruf und Beschäftigung

Basierend auf diesem Kompetenzmodell von Beratung (s. Abbildung 2) wurde ein Kompetenzprofil für Beratende - unter intensivem Einbezug von Fachleuten aus unterschiedlichen Beratungsfeldern - entwickelt (s. Abbildung 3). Die in der Abbildung 3 dargestellten Kompetenzgruppen wurden auf einzelne Kompetenzen heruntergebrochen und diese wiederum mit Indikatoren versehen sowie um Verhaltensmerkmale ergänzt, um die Kompetenzen messbar zu machen (Schiersmann/Petersen/Weber 2017, S. 22ff. inklusive der Online-Materialien zu dieser Publikation).

\section{Konstruktionsprinzipien der entwickelten Instrumente zur Kompetenzerfassung}

Aufbauend auf dem skizzierten Kompetenzmodell und dem daraus abgeleiteten Kompetenzprofil wurde im Rahmen des Projektes BeQu eine multimethodale Vorgehensweise zur empirischen Erfassung der Kompetenzen entwickelt, 
die mehrere Instrumente zur Kompetenzerfassung beinhaltet. Im Folgenden werden zunächst die theoretischen Annahmen beschrieben, die hinter dem Modell stehen. Anschließend werden diese Instrumente vorgestellt.

Ausgehend von den Überlegungen zum Kompetenzbegriff besteht die Anforderung an Instrumente zur Erfassung von Kompetenz, sowohl die Ressourcen, insbesondere Wissen, als auch die Performanz, also das kompetente beraterische Handeln zu erfassen. Die Verbindung von Wissen und Handeln bleibt dabei in der Regel implizit und muss für die Erfassung durch Reflexion expliziert werden. Daraus ergibt sich für die Erarbeitung von Instrumenten die Anforderung, die drei Aspekte Ressourcen, Handeln und die Reflexion über die situative Begründung des Handelns zu erfassen. Da die einzelnen Instrumente je spezifische Aspekte fokussieren, soll durch deren Kombination eine möglichst umfassende Abdeckung von Beratungskompetenz erreicht werden. Reflexion meint dabei das Überdenken von Erfahrungen, Ereignissen und Handlungen im Nachhinein (retrospektive Reflexion) sowie das Bedenken von zukünftigen Handlungen und ihren Folgen (prospektive Reflexion) (s. Abbildung 4) auf der Grundlage von bestehenden Normen, Werten, Wissens- und Erfahrungsbeständen (vgl. Gillen 2009, S. 108f.). Reflexion als Bewusstheit über die eigenen Kompetenzen und das eigene Tun wird als Kernelement von Professionalität aufgefasst (vgl. Combe/Kolbe 2008, S. 859).

Der herausgehobene Stellenwert von Reflexion begründet sich wie folgt: Wir gehen davon aus, dass professionell Beratende nicht nur kompetent handeln sollten, sondern auch ein professionelles Gespür sowie eine erhöhte kognitive Wahrnehmungsschärfe für die Qualität des eigenen Handelns benötigen (Dörr 2014, S. 18). Die Beratenden sollten in der Lage sein professionelles Handeln auf Basis von (Selbst-)Beobachtungen und dem eigenen theoretischen Wissen differenziert zu beschreiben. Hinzu kommt die erwartete Fähigkeit, den Handlungsprozess auf Basis wissenschaftlicher Theorien und Befunde zu erklären und zu begründen. Nicht zuletzt wird von professionell Handelnden erwartet, die potentiellen Wirkungen des situativen Handelns auf weitere Interaktionen vorherzusagen und auf dieser Annahme Handlungsalternativen und Handlungsvervollständigungen zu entwickeln. Aus diesem Dreiklang begründet sich die Strukturierung der Instrumente, die (in verschiedenen Varianten) sowohl Handlungsbeobachtung als auch Handlungsreflexion und die Vervollständigung von Handeln bzw. die Frage nach Handlungsalternativen beinhalten.

Die einzelnen Instrumente wurden zunächst auf der Basis vorhandener Beispiele aus verschiedenen professionellen Handlungsfeldern konzipiert (Schiersmann/Petersen/Weber 2017, S. 30-42). Berücksichtigt wurden dabei insbesondere die Bildung der Lehrpersonen, die Berufspädagogik, die betriebliche (Weiter-)Bildung sowie die Beratung. Neben der Berücksichtigung der Funktionalität der Instrumente wurde bei der Entwicklung auch auf die weitere Operationalisierung des Kompetenzprofils auf Basis von videogra- 
phierter Beratung und durch die Einbindung von fachkundigen Beratenden geachtet (Schiersmann/Petersen/Weber 2017 S. 47f.). Durch die letztgenannten Aktivitäten entstand in einer Reihe von Expertenworkshops und durch die inhaltsanalytische Auswertung von schriftlichem Beobachtungsmaterial eine strukturierte und validierte Sammlung von Verhaltensmerkmalen, welche die einzelnen Indikatoren der prozessbezogenen Beratungskompetenz (s. Abbildung 3) weiter konkretisiert. Darauf aufbauend wurden die Instrumente in einer Reihe von Anwenderworkshops erprobt und mit den beteiligten Beratenden diskutiert und weiterentwickelt.

\begin{tabular}{|c|c|}
\hline Instrument & Erfassungsgegenstand \\
\hline Selbsteinschätzungsbogen & Selbstwahrnehmung \\
\hline Textvignetten & Handlungsplanung \\
\hline Videovignetten & Handlungswahrnehmung \\
\hline Sitzungsbogen & Handlungsüberprüfung \\
\hline Simulation/Reale Beratungssituation & Handeln (Performanz) \\
\hline & \\
\hline & \\
\hline & \\
\hline & \\
\hline &
\end{tabular}

Abb. 4: Instrumente zur Erfassung von Handlungsaspekten der Beratungskompetenz

Ein Selbsteinschätzungsbogen als standardisierter Fragebogen mit vorgegebenen Kompetenz- und Ressourcenindikatoren dient dazu, dass sich die Beratenden auf der Ebene der Selbstwahrnehmung einstufen und sich so individuell mit den eigenen Kompetenzen und kognitiven Ressourcen auseinandersetzen (Schiersmann/Petersen/Weber 2017, S. 49). Den Bezugspunkt dieses Selbsteinschätzungsbogens stellt die bisherige Beratungspraxis dar. Ebenso kann der Bogen auch zur Fremdeinschätzung (z. B. durch andere Beratende oder Vorgesetzte) genutzt werden. Auch können die Selbsteinschätzungen verschiedener Berater (in einer Organisation) miteinander verglichen werden. Die Ausprägung der Kompetenz wird auf einer Skala von 1 bis 4 dokumentiert. Eine Auswertung mittels Excel erlaubt eine grafische Darstellung (Spinnennetz) der Ausprägung der Kompetenzen.

Textvignetten, die einen Beratungsfall enthalten, zielen auf die Betrachtung der Handlungsplanung (Schiersmann/Petersen/Weber 2017, S. 53). Sie werden dazu genutzt, die Beratenden zur Beschreibung situationsspezifischer Analyse- und Handlungsweisen anzuregen. Des Weiteren werden die Be- 
ratenden dazu aufgefordert, auf Grundlage der Fallbeispiele über mögliche Schritte bzw. Handlungsstrategien zur Gestaltung des fiktiven Beratungsprozesses nachzudenken. Durch das offene Bearbeitungsformat der Textvignette ohne vorgegebene Kategorisierungen oder Antwortformate wird eine Handlungsfreiheit während des Bearbeitungsprozesses gewährleistet. So werden Rückschlüsse bezüglich der hier relevanten Aspekte der Beratungskompetenzen und Wissensstruktur der jeweiligen Person möglich.

Bei der Auswertung wird in einem ersten Schritt geprüft, inwieweit die Beschreibungen der Beratenden, die sie in einem Erfassungsbogen vorgenommen haben, mit den in einem gesonderten Auswertungsbogen dargestellten Kompetenzindikatoren bzw. Verhaltensmerkmalen übereinstimmen. Dazu werden Punkte vergeben. In einem zweiten Schritt wird eine Gesamteinschätzung der beschriebenen Vorgehensweisen in Bezug auf die jeweiligen Kompetenzen vorgenommen. Die Ergebnisse können im Rahmen von kollegialer oder professioneller Supervision reflektiert werden (Schiersmann/ Petersen/Weber 2017, S. 55f.).

Videovignetten ermöglichen eine Beobachtung fremden Beratungshandelns. Auf der Basis der Handlungswahrnehmung bewerten und reflektieren die Beratenden das wahrgenommene Beratungshandeln einer anderen Person und formulieren ggf. Verbesserungsvorschläge bzw. weitere Handlungsstrategien in Bezug auf die Gestaltung der jeweiligen Beratungssituation (Schiersmann/Petersen/Weber 2017, S. 58). Das Instrument zielt darauf, durch die Bewertung der Einschätzung fremden beraterischen Handelns etwas über die Beratungskompetenzen der Einschätzenden aussagen zu können. Insbesondere wird die Wahrnehmungsfähigkeit der Person bzgl. des beraterischen Handelns erfasst und im Abgleich mit Verhaltensmerkmalen, die empirisch gewonnen wurden, bewertet (Schiersmann/Petersen/Weber 2017, S. 58f.).

Mit Hilfe eines leitfadengestützten Sitzungsbogens werden Beratende angeregt, gezielt über ihr kompetentes Handeln zu reflektieren. Basis ist ein selbst durchgeführtes Beratungsgespräch. Die Auswertung und Überprüfung des beraterischen Handelns kann im Abgleich mit möglichen Verhaltensweisen und Begründungen für das Handeln gewonnen werden (Schiersmann/ Petersen/Weber 2017, S. 64f.). Auf der Ebene der Handlungsüberprüfung/-begründung denken die Beratenden über ihre Kompetenzen in Bezug auf das selbst durchgeführte Beratungsgespräch sowie über das angewandte Fachwissen, die eingesetzten Methoden und Strategien im jeweiligen Beratungsprozess nach und dokumentieren diese Überlegungen. Die Reflexion auf der Ebene der Handlungsüberprüfung, insbesondere durch Transparentmachung von Begründungen für das eigene Handeln, soll Aussagen über die Beratungskompetenzen erlauben. Die „nachträgliche Begründungsverpflichtung“ (Oevermann 1996, S. 77f.) der professionellen Praxis nach dem Handlungsvollzug wird hier als wesentliches Merkmal des professionellen Handelns verstanden und im Instrument genutzt. 
Ein weiteres Instrument, das insbesondere die Erfassung der Performanz in den Mittelpunkt rückt, stellt die Beobachtung von vollständigen simulierten oder echten Beratungssituationen dar, die aber nicht zwingend selbst durchgeführt wurden. Hier können Beratungsgespräche durch den Einsatz von Simulationsklientel (z. B. in einem Rollenspiel) oder videographierten realen Beratungssituationen anhand eines Beobachtungsbogens dokumentiert und beurteilt werden. Bei dem Beobachtungsbogen handelt es sich, wie bei den vorigen Instrumenten, um eine Zusammenstellung von Kompetenzindikatoren, die Bewertungen des direkt beobachtbaren Handelns eines Beratenden (Performanz) erfordern. Der jeweilige Handlungsvollzug entwickelt sich - so wird angenommen - aus der Reflexion in der Situation in wechselseitiger Beziehung mit den eigenen Deutungen, Handlungsgewohnheiten, Erfahrungen usw. (Schön 1983, S. 67). Welche Handlung in der Situation realisiert wird, entscheidet sich durch ,eine reflexive Beziehung auf die in der Situation erlebte Herausforderung" (Joas 1996, S. 236).

Die Auswertung der drei letztgenannten Instrumente folgt ähnlichen Prinzipen und vergleichbaren Instrumenten. Grundlage ist jeweils das Kompetenzprofil für Beratende, das in einem Auswertungsbogen abgebildet ist. Dieser umfasst sieben Teilkompetenzen zur Gestaltung von Beratungsprozessen, die jeweils durch Kompetenzindikatoren und Verhaltensmerkmale operationalisiert sind. Diese Auswertungshilfe kann dazu beitragen, die Beurteilungsbreite sowie den Spielraum für subjektive Beurteilung zu reduzieren. Die Bewertung sollte dennoch von erfahrenen und geschulten Personen vorgenommen werden. Die Herausforderung besteht darin, die dargestellten Beobachtungen, Bewertungen und Reflexionen im Kompetenzmodell einzuordnen und dabei auch offen zu bleiben für sinnvolle Alternativen, die ggf. in den angebotenen Verhaltensmerkmalen nicht enthalten sind. Das Verfahren besteht aus zwei Schritten. Im ersten Schritt werden die von den Beratenden im jeweiligen Instrument dargestellten Verhaltensweisen (z. B. Handlungsstrategien) sowie die Begründung der Vorgehensweise im Beratungsprozess und die Handlungsoptionen dokumentiert, d. h. dieser Schritt basiert auf dem Abgleich mit den Kompetenzen und Verhaltensmerkmalen des zugrunde gelegten Modells. Im zweiten Schritt wird auf einer Skala eine Gesamteinschätzung vorgenommen, inwiefern der jeweilige Beratende die relevanten Kompetenzen bzw. deren Operationalisierungen erkannt bzw. dargestellt hat. Die Gesamteinschätzung erfolgt auf Basis der vom Beratenden zur Beschreibung genutzten Verhaltensmerkmale und der Begründung der Vorgehensweise bzgl. des jeweiligen Kompetenzaspektes. Hieraus ergibt sich ein differenziertes Gesamtergebnis, das z. B. im Gespräch zwischen auswertender Person und den Beratenden besprochen werden kann. 


\section{Erfahrungen aus der Erprobung}

Die Instrumente wurden mit Beratenden (Bildung, Beruf, Beschäftigung) sowie mit Studierenden in verschiedenen Projektphasen erprobt. Dabei wurde darauf geachtet, dass Beratende aus unterschiedlichen Feldern mit unterschiedlich umfassender Beratungserfahrung einbezogen wurden. Nach Abschluss der Entwicklungsarbeiten wurde das komplette Instrumentarium mit einer Gruppe von ca. 20 Beratenden angewandt und die Ergebnisse wurden ausgewertet (Schiersmann/Petersen/Weber 2017, S. 72). In dieser Erprobungsphase ging es darum, etwaige Zusammenhänge zwischen den durch die hier vorgestellten und erfassten Kompetenzdimensionen (Wissen, Performanz, Reflektion) und anderen Variablen (u. a. Aus- und Weiterbildung, Berufserfahrung) festzustellen. Um außerdem die Zusammenhänge zwischen Selbst- und Fremdeinschätzung zu untersuchen, wurden die quantifizierten Werte aus den Erhebungsinstrumenten mit Variablen wie der Dauer der professionellen Tätigkeit, Ausbildungsform- und -niveau oder der Erfahrung in (unterschiedlichen) Beratungsfeldern korreliert. Die erzielten Ergebnisse zeigen, dass die Instrumente einen fundierten Vergleich von Selbst- und Fremdeinschätzung ermöglichen, auch wenn aufgrund der Stichprobengröße keine Signifikanztests möglich waren. Auch weisen die ersten Befunde auf stichhaltige Zusammenhänge der gezeigten Kompetenz mit Vorerfahrungen und Ausbildung der Beratenden hin. Dabei ist nicht nur die lineare Zunahme der Kompetenzen zu beobachten, sondern es können auch Besonderheiten identifiziert werden. So scheint z. B. weniger die Dauer der Berufserfahrung ausschlaggebend zu sein, vielmehr hat die Varianz der Handlungsfelder, in denen jemand tätig war, einen Einfluss auf die Kompetenz.

In einer zweiten Stichprobe wurden 35 Beratende aus unterschiedlichen Handlungsfeldern und mit unterschiedlichen Vorkenntnissen und Erfahrungen berücksichtigt. Die Instrumente erlauben sowohl die Erstellung von individuellen Kompetenzprofilen als auch die Untersuchung der Zusammenhänge der genannten Variablen und die Signifikanz dieser Zusammenhänge. Neben den hier beschriebenen Instrumenten wurde ein Fragebogen zur Erfassung professioneller Selbstwirksamkeitserwartung sowie zur Erfassung der soziodemographischen Daten und der Beratungserfahrung und -Ausbildung eingesetzt (Dörr 2014, S. 47). Die Ergebnisse zeigen u. a. Zusammenhänge zwischen Ausbildungsniveau, Dauer von Berufserfahrung und Kompetenzergebnissen sowie eine relativ starke Korrelation von professioneller Selbstwirksamkeitserwartung und den Kompetenzergebnissen (Dörr 2014, S. 87). Es zeigen sich jedoch auch Ergebnisse, die einen solchen Zusammenhang nicht stützen. So scheint der Aspekt des Beziehungsaufbaus schwächer mit Faktoren wie Ausbildung oder Selbstwirksamkeitserwartung zusammenzuhängen als andere Kompetenzaspekte. Aufgrund der bisher geringen Stichprobe müssen die Er- 
gebnisse aus der Pilotierung jedoch noch mit Vorsicht interpretiert werden. Es zeichnet sich jedoch ab, dass sich in Bezug auf vertiefte statistische Untersuchungen interessante Fragestellungen ergeben können.

Gleichwohl gehört es auch zu den Erfahrungen aus der ersten Erprobung der Instrumente, dass Beratung ein wenig standardisierbares Handlungsfeld ist, was auch die Erfassung und Bewertung der Kompetenzen aufwändig und schwierig macht. Das Projekt hat sich mit dem breiten Kompetenzprofil und den anspruchsvollen Erhebungsinstrumenten bewusst für eine möglichst realitätsnahe und wenig reduktionistische Vorgehensweise entschieden. Trotzdem bleibt die Balance zwischen der Standardisierung durch die Instrumente versus die Aufrechterhaltung der notwendigen Offenheit (z. B. gegenüber unterschiedlichen Vorgehensweisen und Ansätzen der Beratung) eine Herausforderung. Auch das Ziehen von Rückschlüssen aus der Beobachtung von Performanz auf die Kompetenz von Beratenden bleibt eine methodische Herausforderung. Die Einbeziehung von (handhabbaren und validen) introspektiven Verfahren der Reflexion erscheint ein wichtiger und vielversprechender Weg zu sein. Die Entwicklung und Validierung von einerseits differenzierten und andererseits praxisangemessenen Verfahren bleibt für das Ziel einer umfassenden und professionsangemessenen Kompetenzerfassung eine anspruchsvolle Aufgabe.

\section{Fazit und Desiderate}

Mit dem Vorliegen des Kompetenzprofils für Beratende sowie des Verfahrens zur Kompetenzerfassung und -beurteilung ist ein wichtiger Schritt in Richtung Stärkung der Professionalität von Beratenden im Feld Bildung, Beruf und Beschäftigung getan. Gleichwohl bedarf es weiterer Schritte, um das Verfahren zur Kompetenzerfassung und -beurteilung inhaltlich weiterzuentwickeln und wissenschaftlich sowie für den Praxiseinsatz im Beratungsfeld Bildung, Beruf und Beschäftigung abzusichern. Anknüpfend an theoretische Überlegungen und praktische Erfahrungen aus den bisherigen Forschungen sollte aus unserer Sicht der Schwerpunkt der Weiterentwicklung des Verfahrens zur Kompetenzerfassung und -beurteilung im Rahmen weiterer Forschungen vorwiegend auf den folgenden Aspekten liegen:

- Entwicklung von zusätzlichen videographierten und schriftlichen Vignetten zur Kompetenzerfassung, die beratungsfeldspezifisch sind (z. B. für Berufs-, Weiterbildungs-, Laufbahnberatung), so dass das Feld der Beratung in Bildung, Beruf und Beschäftigung in seiner Breite bei der Kompetenzerfassung berücksichtigt bzw. einbezogen wird; 
- erweiterte Erprobung des Verfahrens zur Kompetenzerfassung hinsichtlich der Validität, Reliabilität und Praktikabilität mit einer größeren Stichprobe von Beratenden aus unterschiedlichen Beratungsfeldern in Bildung, Beruf und Beschäftigung;

- inhaltliche Weiterentwicklung und Erprobung des Konzeptes zur Beurteilung der Kompetenzen unter Einbindung von Beratungsfachleuten aus unterschiedlichen Beratungsfeldern in Bildung, Beruf und Beschäftigung;

- Optimierung der inhaltlichen und technischen Qualität der Instrumente zur Kompetenzerfassung und -beurteilung auf Basis der gewonnenen Erkenntnisse aus der Praxiserprobung;

- Entwicklung und Validierung von Niveaudifferenzierungen der Beratungskompetenz für Beratende in Bildung, Beruf und Beschäftigung;

- Klärung der Frage, welche Verfahren und Zuständigkeiten für die Anerkennung von Kompetenzen sinnvoll und umsetzbar sein könnten (z. B. Zertifizierung, Selbstbewertung).

Die Nutzung der Instrumente außerhalb der Forschung ist durch die Publikation der Materialien in verschiedenen Anwendungskontexten möglich. Die im Rahmen des $\mathrm{BeQu}$ Projektes entwickelten und hier vorgestellten Instrumente zur Kompetenzerfassung und -auswertung werden interessierten Beratenden, Beratungsanbietern, Anbietern von Aus- und Weiterbildung und weiteren Akteursgruppen im Beratungsfeld als Materialien zur Selbstreflexion und Selbstauswertung der Beratungskompetenz zur Verfügung gestellt (Schiersmann/Petersen/Weber 2017, S. 48). Das Download-Material zum Buch ist auf der Website des Verlages abrufbar. So können die Instrumente im Rahmen von Personalauswahl und Personalentwicklung eingesetzt werden. Nicht zuletzt eröffnen die Instrumente Wege zu einer fundierten und professionsangemessenen Zertifizierung von formell und informell erworbenen Kompetenzen.

Über den Nutzen der Erkenntnisse für das Beratungsfeld hinaus ergeben sich aus dem Projekt auch Anregungen für die allgemeinere Diskussion um die Erfassung und Anerkennung (beruflicher) Kompetenzen. Insbesondere in Berufsfeldern, in denen sozialkommunikative Kompetenzen im Mittelpunkt stehen, erweist sich die transparente und valide Erfassung als äußerst anspruchsvoll. Dies betrifft die Simulation von handlungs- bzw. praxisnahen Aspekten von Kompetenz, die auch im Projekt als besondere Herausforderung erfahren wurde. Durch die Erprobung verschiedener Instrumente konnten diese Probleme fokussiert und eingegrenzt werden. Dies betrifft z. B. die theoretisch zu erwartende Differenz von Wissen als Ressource und tatsächlicher Performanz, aber auch die Differenz zwischen Performanz (wie sie in Simulationen sichtbar wurde) und der Reflexion bzw. Reflexionstiefe, die Beratende aufweisen. 


\section{Literatur}

Combe, A./Kolbe, F.-U. (2008): Lehrerprofessionalität: Wissen, Können, Handeln. In: Helsper, W./Böhme, J. (Hg.): Handbuch der Schulforschung. Wiesbaden: VS Verlag, S. 857-875.

Dörr, E. (2014). Beratungsprozessbezogene Selbstwirksamkeitserwartung und professionelle Sensibilität von Beratenden - Erprobung eines Forschungsdesigns zur Erfassung von Kompetenzentwicklungspotentialen Professionalisierter und Auszubildender des Feldes Beratung in Bildung, Beruf und Beschäftigung. Heidelberg: Masterarbeit

Erpenbeck, J./Sauter, W. (2016): Stoppt die Kompetenzkatastrophe! Wege in eine neue Bildungswelt. Berlin, Heidelberg: Springer Verlag.

Gillen, J. (2009): Kompetenzfeststellung als Chance zur Selbstreflexion - was können und sollen Kompetenzfeststellungen zur Förderung von Reflexivität leisten? In: Münk, D./Severing, E. (Hg.): Theorie und Praxis der Kompetenzfeststellung im Betrieb - Status quo und Entwicklungsbedarf. Bonn: BIBB Verlag, S.107-120.

Joas, H. (1996): Die Kreativität des Handelns. Frankfurt a. M.: Suhrkamp.

Oevermann, U. (1996): Theoretische Skizze einer revidierten Theorie professionalisierten Handelns. In: Combe, Arno/Helsper, Werner (Hg.): Pädagogische Professionalität. Untersuchungen zum Typus pädagogischen Handelns. Frankfurt a. M.: Suhrkamp, S. 70-182.

Schiersmann, C. (2013): Beratung im Feld Bildung, Beruf, Beschäftigung. In: Schiersmann, C./Weber, P. (Hg.): Beratung in Bildung, Beruf und Beschäftigung. Eckpunkte und Erprobung eines integrierten Qualitätskonzeptes. Bielefeld: W. Bertelsmann Verlag, S. 25-32.

Schiersmann, C./Weber, P./Petersen, C.-M. (2013): Kompetenz als Kern von Professionalität. In: Schiersmann, C./Weber, P. (Hg.): Beratung in Bildung, Beruf und Beschäftigung. Eckpunkte und Erprobung eines integrierten Qualitätskonzeptes. Bielefeld: Bertelsmann Verlag, S. 195-222.

Schiersmann C., Petersen, C.-M., \& Weber, P. (2017). Kompetenzerfassung im Beratungsfeld Bildung, Beruf und Beschäftigung. Instrumente zur Dokumentation, Bewertung und Reflexion der Beratenden. Bielefeld: W. Bertelsmann Verlag.

Schön, D. A. (1983). The reflective practitioner: how professionals think in action. New York: Basic book.

Weinert, F. E. (2001): Concept of competence: A conceptual Clarification. In: Rychen, D. S. (Hg.): Defining and selecting Key Competencies, Seattle [u. a.]: Hogrefe \& Huber Verlag, S. 45-65. 


\section{Beratungskompetenzerwerb im Studium: Lern- und Bildungsprozesse im Horizont subjektorientierter Professionalisierung}

\section{Einleitung}

Beratung ist eine spezifische Handlungsform, die in nahezu allen pädagogischen Tätigkeitsfeldern verlangt wird. Sie kommt dort entweder als alleinige professionelle Tätigkeit oder verwoben mit anderen pädagogischen Prozessen wie Unterrichten, Informieren oder Arrangieren vor (Giesecke 2015; Maier-Gutheil 2016). Häufig unterbricht sie dort stetige Vorgänge der Erziehung, des Lernens und Lehrens (Mollenhauer 1973) durch den sie kennzeichnenden Modus des Fragens und der Eröffnung eines Reflexionsraumes (Weinhardt 2018a), ohne Antworten vorwegnehmen zu wollen. Sie wird erziehungswissenschaftlich systematisch und entlang unterschiedlicher Logiken behandelt, beispielsweise der Lebensspanne (Gieseke und Nittel 2016) oder methodischer und arbeitsfeldspezifischer Differenzierungen (Nestmann et al. 2014b, 2014a). In der Sozialpädagogik spielt sie in ihrer sehr prozessbezogenen Form als psychosoziale Beratung eine prominente Rolle, auch und gerade unter gesellschaftlichen Bedingungen, in denen die Gestaltung komplexer Übergänge und die Auswahl und Bewältigung unterschiedlicher Lebensoptionen und Existenzweisen (Walther und Weinhardt 2013) ausgeprägte Reflexions-, Orientierungs- und Entscheidungsfähigkeiten verlangt. Beispiele einer so verstandenen psychosozialen Beratung im Rahmen wohlfahrtsstaatlicher Daseinsvorsorge sind die allgemeine Jugend-, Familien- und Lebensberatung, aber auch die Sucht- oder Schwangerschaftskonfliktberatung oder Beratung im Kontext von Inklusion. Wesentliche Kernelemente von Beratung sind hier das reflexive Bilanzieren, das antizipierte oder realisierte Probehandeln sowie die Erlangung kognitiver und emotionaler Stabilität (Nestmann und Sickendiek 2011).

Eine solche Form psychosozialer Beratung erfordert auf Seiten der sozialpädagogischen Fachkräfte eine spezifische Beratungskompetenz (Bauer und Weinhardt; Weinhardt 2015), deren Erwerb im Zuge der Einführung der gestuften und auf unmittelbare Berufsbefähigung hin formierten Bologna-Studiengänge neu hinterfragt werden kann. Dem vorliegenden Beitrag liegt hier die These zugrunde, dass ein gelingender Kompetenzerwerb bereits im Studium, das im Falle der Sozialpädagogik auch genuin mit Praxisphasen ausgestattet ist, durchaus gelingen kann. Nimmt man die Komplexität von 
Professionalisierung als spezifischen Lern- und Bildungsprozess ernst, so ist $\mathrm{zu}$ fragen, wie die frühen Stadien von Professionalisierung im Rahmen des Studiums gestaltet und theoretisch und empirisch befriedigend modelliert werden können. Eine solche Modellierung muss dabei auch berücksichtigen, dass die aus der allgemeinen Expertise- und Kompetenzforschung bekannten Befunde zum zeitlichen Umfang des Entstehens von Expertise es erfordern, ein auf Handlungsbefähigung zielendes Studium konsequent im Rahmen Lebenslangen Lernens zu verorten.

Der Beitrag geht dabei in drei Schritten vor. Zunächst wird eine subjektorientierte Perspektive auf die Professionalisierungsprozesse (angehender) Beratungsfachkräfte entfaltet. Sie macht sichtbar, dass herkömmliche Professionalisierungsmuster im Zuge der nun vollständig auf Bologna-Studiengänge umgestellten Hochschullandschaft bezogen auf die Handlungsform Beratung besonders herausgefordert werden. Diese grundsätzlichen Überlegungen werden in einem zweiten Teil entlang von empirischen Daten aus einer hoch inferenten Videostudie zum Beratungslernen an der Hochschule konkretisiert und anschließend zu Überlegungen über eine subjektorientierte Professionalisierung verdichtet.

\section{Eine subjektorientierte Perspektive auf Kompetenzerwerb als zwangsläufige Veränderung von Professionalisierungsstrukturen in der Beratung}

Die im vorliegenden Beitrag untersuchte Handlungsform Beratung hat sowohl eine allgemeine als auch eine spezifische Bedeutung. Allgemein kann sie durchaus exemplarisch für andere pädagogische Handlungsformen wie das Erziehen und Bilden stehen, die als professionalisierungsbedürftig gelten, weil sie im Kontext akademischer Studienberufe auf Wissen und Können verweisen, das sich gerade nicht mit der Reproduktion alltäglicher Routinen im beruflichen Kontext erschöpft. Beratung in der hier gemeinten reflexiven Ausprägung (Nestmann et al. 2014b, 2014a) ist mehr als eine hypertrophierte Form sozialer Alltagskompetenzen und auch kein bloßes Rat geben im Sinne einer Informationsweitergabe. Obwohl ihre höchste Expertiseform phänomenologisch oft als spielerisch-leichte Interaktion erscheint, ist diese Mühelosigkeit gemäß der Expertiseforschung einer hochkomplexen, stark verkörperlichten und implizit gewordenen Wissensbasis geschuldet (Strasser 2006; Strasser und Gruber 2015).

Neben diesen allgemeinen Merkmalen professionalisierungsbedürftiger Tätigkeiten (Schwarz et al. 2014) bildet Beratung aber in der hier behandel- 
ten Form psychosozialer Beratung auch einen Sonderfall unter den pädagogischen Handlungsformen. Ihre Professionalisierungsgeschichte basiert in wesentlichen Teilen auf aufwendigen, aus der Psychotherapie entliehenen methodischen Konzepten, die für die Verwendung in wohlfahrtsstaatlichen Angeboten kompexitätsanreichernd weiterentwickelt und pädagogisiert wurden (Weinhardt 2016b). Dies war für die Form von Professionalisierungsprozessen psychosozialer Beratungsfachkräfte nicht folgenlos, denn das Resultat ist eine spezifische Form zweiphasiger Professionalisierung als Notwendigkeit, solche komplexen Handlungskompetenzen zu erwerben, wobei sich in der Regel das von der Expertiseforschung bekannte 10-Jahres-Fenster auch strukturell abgebildet hat: Nach einem in der Regel umfangreichen Diplomstudium schloss sich eine erste Phase der Berufstätigkeit an, nach der die eigentliche Spezialisierung und Professionalisierung hinsichtlich Beratung erst beginnt, nämlich eine zeitaufwendige mehrjährige Weiterbildung, die häufig um eine zentrale Beratungstheorie, beispielsweise die klientenzentrierte oder systemische Beratung, herum organisiert ist. Zwingend vorgesehen sind in den zugehörigen Curricula immer eine bereits kumulierte Berufspraxis hinsichtlich Beratung sowie das Tätigsein in der Beratung während der Weiterbildung. Solche Curricula weisen folgerichtig neben expliziten Wissensvermittlungsformen vor allem auch implizite Formen des Lehrens und Lernens auf, die sich mit dem gemeinsamen Tätigsein in einer Community of Practice (Lave und Wenger 1991; Wenger 1998), beispielsweise in Peergruppen- oder Supervisionsgruppen, sowie dem Lernen im Modus der Cognitive Apprenticeship (Collins et al. 1987) beschreiben lassen. Ein solches Gesamtarrangement von Professionalsierungsteilprozessen ist aber hoch voraussetzungsvoll: Gefordert wird die Einbettung der Weiterbildung in eine Arbeitsstelle mit Beratungselementen, der Zugriff auf Fälle, die Herstellung der unterschiedlichen Sozialformen mit zugehörigen lehrenden BeraterInnen etc. Eine solche Form der Beratungsprofessionalisierung vollzog sich vor Bologna ganz überwiegend berufsbiographisch spät und hatte sehr implizite Formen, die sich entlang der Strukturen der Beratungslandschaft ausrichteten. So erscheint Professionalisierung vorwiegend als weitgehend unbewusste Einsozialisation in diejenigen bald habituell übernommenen Deutungs- und Handlungskonstrukte, die das Arbeitsfeld Beratung eben ausmachen. Mit dieser Form der Zweiphasigkeit geht auch einher, dass Professionalisierungsprozesse in Deutschland sowohl in formalen (Hochschule) als auch in non-formalen (Weiterbildungs-)Settings sequenziell wechselnd stattfinden - im Gegensatz zum internationalen Diskurs, in dem der Beratungskompetenzerwerb schon immer und über seine gesamte Spanne des Lern- und Bildungsprozesses hinweg an akademische, also hochschulische Wissensvermittlung gebunden ist (Hohenshil et al. 2015), z. B. in den Vereinigten Staaten im Kontext des Boulder-Modells. Zusammenfassend lässt sich also sagen: Die Professionalisierung in der psychosozialen Beratung war lange Zeit sehr eng an bestimmte, nicht weiter explizie- 
rungsbedürftige Strukturen gebunden - generalistische Studiengänge, erste Berufserfahrungen, die Aufnahme einer Weiterbildung und dort der Erwerb spezifisch beraterischer Handlungskompetenz unter dauernder Anwendungsmöglichkeit und Reflexionsherausforderung im Zusammenspiel von beruflicher Arbeit und Weiterbildungscurriculum.

Dieser Primat der Struktur in der Beratungsprofessionalisierung wird aktuell durch drei Entwicklungen aufgebrochen, die hier nur kursorisch erwähnt werden können und jeweils einen eigenen Fachbeitrag wert wären: Erstens befindet sich derzeit die Hilfelandschaft in der Sozialpädagogik in einem gewaltigen Heterogenisierungsprozess (Nestmann et al. 2014c, Bauer und Weinhardt 2014). Organisationale und institutionelle Strukturen von Beratung ändern sich sehr rasch. Beispiele sind neue Aufgaben im Kinderschutz, das Hinzukommen medial vermittelter Beratung im Sinne von Blended Counseling und so weiter. Diese Heterogenisierung der Beratungslandschaft geht zusätzlich mit einer Zunahme von Beratungsangeboten einher. In der Folge können aktuelle Professionalisierungswünsche hinsichtlich Beratung nicht mehr auf den überlieferten Strukturkern einer im wesentlichen gesetzten Beratungsinfrastruktur aufsetzen. Beratung als Handlungsform ist angesichts dieser Heterogenisierung aus ihren Strukturen freigesetzt, ähnlich wie das Unterrichten, das sich angesichts rasch ausdifferenzierender Schulformen- und konzepte zunehmend auch wieder aus seiner eigentlichen pädagogischen Praxis heraus und nicht (nur) entlang der Institution Schule legitimieren muss. Zweitens bedeutet Bologna, dass Professionalisierungsprozesse schon früher zielgerichtet beginnen als im bisherigen berufsbiographischen Zweiphasenmodell. Nimmt man an dieser Stelle die Handlungsorientierung- und Befähigung ernst, so ist im Kontext von Hochschullehre zu fragen, wie Professionalisierungsprozesse in berufsbiographisch frühen Phasen und am Lernort Hochschule gestaltet werden können. Und schließlich gibt es drittens einige Befunde aus der Beratungsprofessionalisierungsforschung, die darauf hinweisen, dass aus einer empirischen Perspektive das bisherige Modell gar nicht immer das überlegene war. Beispielsweise ist umstritten, ob das berufsbiographisch späte Aufsetzen der Beratungsweiterbildung mit ihren spezifischen Lern- und Bildungsimpulsen immer zu synergetischer Wissensproduktion zwischen vorhandener und nicht immer domänengebundener Berufserfahrung und neuem Wissen führt oder nicht vielmehr Vorwissen einem weiteren Lernen auch im Wege stehen kann. Bruder, Hertel, Klug und Schmitz (2010) haben beispielsweise in ihrer Studie zum Beratungskompetenzerwerb von Lehrerinnen und Lehrern festgestellt, dass der Weiterbildungserfolg für das Beraten lernen mit steigender Berufserfahrung als LehrerIn sinkt. Ähnliche Befunde für das Beratungslernen, die in Richtung Immunisierung des Lernens aufgrund vorheriger Berufserfahrung gehen, haben auch de Shazer und Kollegen in ihrer Studie festgestellt (Shazer et al. 2001) - sie folgen sinngemäß den Befunden aus der allgemeinen Professionalisierungsforschung, die darauf hinweisen, dass es 
bestimmte Erlebensformen von Praxis gibt, die Lernende davon abhalten, sich weiteres Wissen anzueignen bzw. Relationierung und Transformation bestehenden Wissens zuzulassen. Nochmals sei an dieser Stelle betont, dass im internationalen Diskurs frühe Professionalisierungsprozesse in Studienprogrammen und durchgehend im Kontext wissenschaftlicher (Weiter-)Bildung die Regel sind (Hohenshil et al. 2015).

Änderungen in den Erwartungen an Hochschulbildung und Veränderungen auf Seiten der institutionalisierten und organisierten Beratungspraxis lassen zusammen mit den hier auszugsweise referierten Forschungsbefunden die Frage entstehen, ob ein überwiegend strukturbezogener Blick auf Professionalisierung nicht einer Sichtweise weichen müsste, in der die je individuellen und berufsbiographisch früh entfalteten Perspektiven von sich hinsichtlich Beratung bildenden Subjekten im Vordergrund stehen sollten. Damit würde einhergehen, sich weitaus umfassender als bisher diesen individuellen Lernund Bildungsprozessen zuzuwenden. Hierauf basierend stellt sich dann die empirische Frage, ob eine frühe Professionalisierung an der Hochschule überhaupt möglich ist und wie sie sinnvoll auszugestalten und zu theoretisieren sei.

\section{Empirische Daten zum Beratungskompetenzerwerb an der Hochschule}

Im Folgenden werden Ergebnisse eigener Studien referiert (Weinhardt 2013, 2014a, 2014b), in denen ein spezifisches Verfahren zur Erfassung von Beratungskompetenz im Rahmen von Videostudien unter einer auf Performanz orientierten Perspektive vorgeschlagen wird. Dieses Verfahren erscheint sowohl für die Grundlagenforschung als auch für die unmittelbare Einbindung in innovative Lehrformate reizvoll und gab den Ausschlag, vermehrt über die Idee subjektorientierter Professionalisierung nachzudenken. Aus den Ergebnissen dieser Studien lassen sich durch statistische Aggregation allgemeine Entwicklungsgesetze hinsichtlich des zeitlichen Verlaufs des Kompetenzerwerbs an der Hochschule abbilden (Weinhardt und Bauer 2016), Effekte von Lernangeboten feststellen (Weinhardt 2016a) sowie die Struktur von Beratungskompetenz in frühen Expertisestadien aufklären (Weinhardt und Kelava 2016). Aufgrund der Anlage als Videostudien besteht in einer zweiten Verwendungslinie des empirischen Materials die Möglichkeit, individuelle Lern- und Bildungsprozesse im Rahmen eines entwicklungsaufgabenorientierten Modells (Weinhardt 2017, 2018b) entlang der videographierten Gespräche zu strukturieren und mit teilnehmerInnenspezifischem Material anzureichern. Zentral für die hier thematischen Untersuchungen ist das BeraLab 
als Mittelpunkt von Forschung und Lehre. Im BeraLab können Studierende während des gesamten Studiums psychosoziale Beratungserfahrungen unter stark realitätsangenäherten Bedingungen machen. Hierzu stehen neben der Nachbildung einer klassischen Beratungsstelle mit schriftlicher Voranmeldung, Wartebereich und Beratungszimmer ausgebildete SimulationsklientInnen bereit, die prototypische Beratungsanliegen aus dem Kontext der Sozialen Arbeit darstellen. Derzeit sind diese Fallszenarien auf den Bereich der Jugendberatung beschränkt und umfassen beispielsweise Ärger mit den Eltern oder Schwierigkeiten in der Ausbildung. Die Ausbildung der SimulationsklientInnen erfolgt entlang stark verdichteter Fallstrukturvorgaben, die aus echten Fällen gewonnen sind und im Rahmen einer theaterpädagogischen Ausbildung von den einzelnen SimulationsklientInnen zu spezifischen Rollenbiographien verarbeitet werden, die nach erfolgtem Schauspieltraining standardisiert präsentiert werden. Durch den vielfachen Einsatz der SimulationsklientInnen lassen sich nach einigen Durchgängen die einzelnen Fälle als spezifische Aufgaben auffassen, deren Schwierigkeitsgrad und Konstanz der Darstellung näherungsweise sehr gut bestimmt werden kann. Dies stellt für manche Auswertungsverfahren einen großen Vorteil dar und eröffnet in der Lehrkomponente die Möglichkeit, bestimmte Beratungsaufgaben gezielt zum Weiterlernen anbieten zu können. Zudem können durch die im BeraLab mögliche Simulation auch Kompetenzwerte von beratungsunerfahrenen Studierenden gewonnen werden, die aus ethischen Gründen noch nicht mit echten Beratungsfällen arbeiten sollten.

Neben den videographierten Gesprächen erfasst ein Basisfragebogen soziodemographische Hintergrundvariablen, Vorerfahrung, Selbstwirksamkeit sowie zentrale Persönlichkeitsmerkmale im Rahmen eines Big-Five-Kurzinventars. In unterschiedlichen Vertiefungsstudien kommen weitere quantitative und qualitative Instrumente zum Einsatz, beispielsweise zur Erfassung kristalliner Intelligenz oder ein Think-Aloud-Interview nach der BeraLab-Teilnahme entlang der eigenen Videographie.

Die videographierten Beratungsgespräche werden von einem Team geschulter RaterInnen auf einem hoch inferenten Instrument, der TBKS (Tübinger Beratungskompetenz-Skala, Weinhardt 2014), erfasst. Die TBKS ist eine übersetzte und adaptierte Version der US-amerikanischen Counseling Skills Scale (Eriksen und McAuliffe 2006, 2003). Sie operationalisiert Beratungskompetenz in sechs Facetten: Interesse und Anerkennung zeigen (IAZ), Problem explorieren (EXP), Sitzung vertiefen (VER), Veränderungen initiieren (INT), beraterisch-therapeutische Beziehung entwickeln (BEZ) sowie Sitzung strukturieren (STR). Das Skalenformat ist dabei anhand konkret operationalisierter Stufen kompetenzorientiert formuliert (von -2, keine Kompetenzen vorhanden bzw. schädliches Beratungsverhalten, bis zu +2 , vollständig ausgeprägte Kompetenz), so dass mit diesem Instrument eine empirisch tragfähige und psychometrisch belastbare (Weinhardt und Kelava 2016) Lösung 
für die Erfassung von Beratungskompetenz in Forschung, Lehre und Praxis realisiert werden kann.

Mit den so gewonnenen Daten sollen hier ausschnittsweise zwei zentrale Punkte beleuchtet werden: Zum einen die Entwicklungsperspektive über das Studium hinweg, zum anderen ein teilnehmerInnenspezifischer Blick auf die Heterogenität von Professionalisierungsprozessen entlang der TBKS-modellierten Eingangsbedingungen für ein Beratungsseminar. Auf diese Weise wird auch deutlich, dass das BeraLab als Simulationsumgebung eine produktive Verbindung zwischen Lernen, Lehren und Forschen herzustellen vermag und sich aus dieser Verbindung naturwüchsig ein stark subjektorientiertes Lern- und Bildungsverständnis ergibt.

Bezüglich der Frage nach der Entwicklungsperspektive zeigt Abbildung 1 eine Trendstudie über die Semester 1 bis 12 von angehenden SozialpädagogInnen (BA, MA, Dipl.), die am BeraLab teilgenommen haben. Zur besseren Übersichtlichkeit wurden hier die TBKS-Daten faktorenanalytisch noch weiter zu einer Zwei-Faktor-Lösung (ausführlich Weinhardt 2016a) verdichtet, in der der Faktor TM (Techniken und Methoden) die technisch-methodischen Aspekte psychosozialer Beratung sowie der Faktor IAB (Interesse, Anerkennung und Beziehung) Beziehungsgestaltungaspekte abbildet.

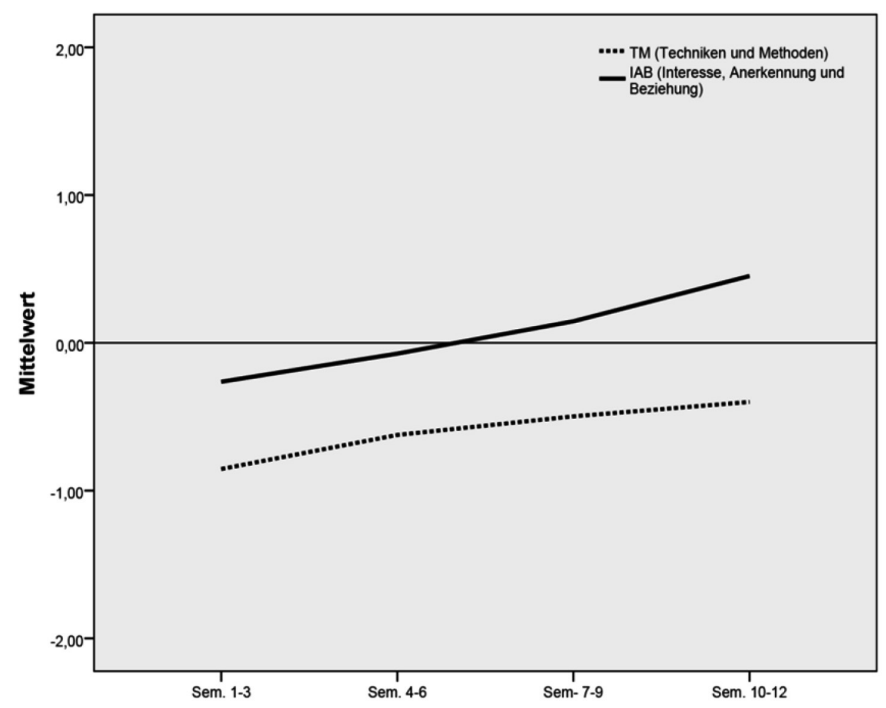

Abb. 1: Beratungskompetenzentwicklung über den Studienverlauf, $n=206$ Erstgespräche aus dem BeraLab (Quelle: eigene Darstellung) 
In beiden Bereichen - sowohl der Beziehungsgestaltungsfähigkeit als auch dem technisch-methodischen Handeln - zeigen sich Lerneffekte. Beratungslernen findet also nachweislich bereits im Studium und in den hier untersuchten generalistischen Studiengängen der Sozialpädagogik statt. Auffällig am Zuwachs bezüglich der Beziehungsgestaltungskompetenzen ist die Tatsache, dass es sich um eine fast perfekt lineare Funktion der Zeit handelt mit einer durchschnittlichen Effektstärke $\mathrm{d}_{\text {cohen }}$ von .21 pro Hochschuljahr, was entlang gängiger Einteilungen einem kleinen Effekt entspricht (ausführliche interferenzstatistische Auswertungen finden sich in Weinhardt 2016a). Ein anderes Bild zeigt sich im zeitlichen Verlauf des technisch-methodischen Handelns. Auch hier lassen sich Lerneffekte über die Zeit nachweisen, allerdings flachen sich die Zuwächse mit zunehmender Studiendauer ab. Diese empirischen Daten lassen sich nun unterschiedlich interpretieren. Aus Sicht der allgemeinen Professionalisierungsforschung verwundert beispielsweise nicht, dass die Beziehungsgestaltungskompetenz mit einem eher kleinen Effekt, aber linear zunimmt. Unterstellt wird hier oft, dass sich gerade in dieser Kompetenzdimension das Zusammenspiel unterschiedlicher Lern- und Entwicklungseffekte zeigt, beispielsweise Einsozialisation in zunehmend komplexere Situationen, aber auch persönliche biographische Entwicklung, insbesondere wenn man sich vergegenwärtigt, dass hier ein Lebensalter von ungefähr 20 bis etwa 27 Jahren abgebildet ist. Für den nichtlinearen Verlauf im Kompetenzzuwachs des technisch-methodischen Handelns lautet eine gängige These, dass für komplexe Interventionstechniken, wie sie sich in dieser Teilkompetenz abbilden, die Curricula generalistisch angelegter Studiengänge nicht mehr genügend Lernmöglichkeiten bieten. Hierfür spricht, dass in einer Interventionsstudie, in der zwischen zwei BeraLab-Gesprächen ein 24 UE umfassendes entwicklungsaufgabenorientiertes Blockseminar zur Vermittlung von Beratungstechniken unter Nutzung der Videographien absolviert wurde, ein Lernzuwachs messbar war, der etwa drei Semestern herkömmlichem Hochschulbesuchs entspricht (Weinhardt 2016a). Dies könnte bedeuten, dass durchaus Spielraum in den theoretisch denkbaren Ressourcen von Hochschulbildung vorhanden ist, diese aber mehr in Passung auf die unterschiedlichen Bedürfnisse der LernerInnen gebracht werden müssen, was vor allem in der Beratung, in der die Person der Fachkräfte selbst zentrales Mittel der Veränderungsarbeit ist, besonders produktiv sein könnte.

An dieser Stelle leitet die Betrachtung statistisch aggregierter Werte auf eine Betrachtung einzelner Kompetenzprofile von Beraterungslernenden über, die in Abbildung 2 dargestellt sind. Es handelt sich dabei um die TBKS-Werte zu Beginn eines regulären Beratungsseminars an der Hochschule. Zwar sind die im BeraLab erfassten Kompetenzen immer Momentaufnahmen, in die situative und fallbezogene Varianzen mit eingehen (die Nutzung des BeraLab als Assessment würde z. B. systematische Mehrfachmessungen erfordern). In der naturalistischen Studienumgebung von Hochschulstudiengängen sind 
diese Daten aber trotz oder gerade wegen ihrer situativen Natur die naturwüchsige Ausgangsbasis, von der aus sich auch im Falle echter Beratungen das Lernen und die Bildung hin orientieren würden. Bereits auf den ersten Blick sticht die Vielfalt der individuellen Kompetenzprofile hervor. Neben der unterschiedlichen quantitativen Ausprägung - es gibt hier StudentInnen, die in der BeraLab-Situation schon insgesamt gut ausgeprägte Kompetenzen zeigen und solche, die wenig Kompetenzen zeigen - ist auch die Heterogenität der Profilzusammensetzungen beeindruckend. So gibt es StudentInnen, denen die Problemexploration gut gelingt, die aber beispielsweis mit der Strukturierung Mühe haben, andere scheinen von Natur aus BeziehungskünstlerInnen zu sein, es fehlt aber an Teilkompetenzen, um Veränderungen $\mathrm{zu}$ initiieren etc. In einer der bereits erwähnten Vertiefungsstudien sind wir solchen Unterschieden im Handeln unter der Perspektive der Selbstreflexion der LernerInnen nachgegangen.
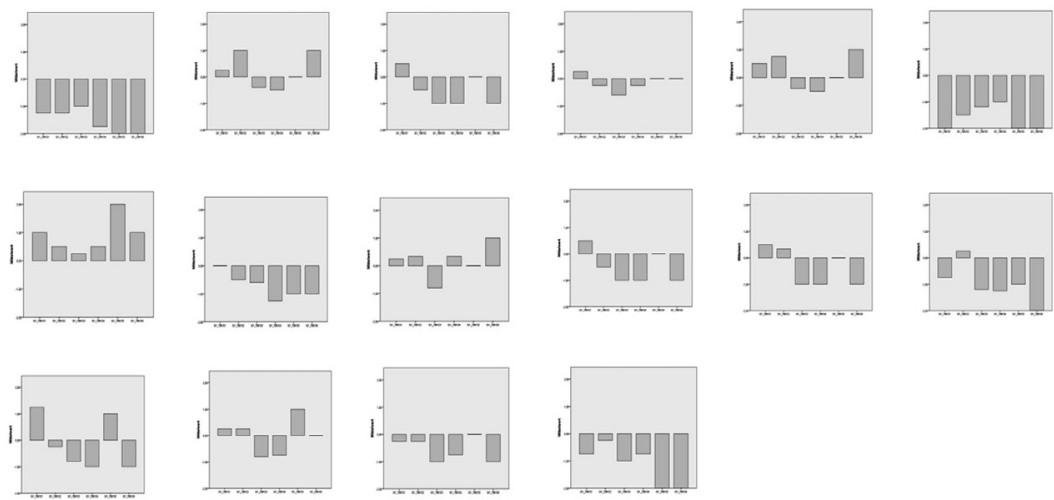

Abb. 2: TBKS-Kompetenzprofile von 16 StudentInnen zu Beginn eines Beratungsseminars (Quelle: Eigenen Darstellung)

Die an dieser Untersuchung teilnehmenden StudentInnen konnten nach einer definierten Zeitspanne von einigen Stunden ihr BeraLab-Video zusammen mit ForscherInnen nochmals anschauen und wurden in diesen Think-Aloud-Interviews zu zwei Dingen aufgefordert: Nämlich die Aufnahme anzuhalten, wenn aus ihrer Sicht etwas Relevantes, das mit Beratungslernen zu tun hat, passiert, und sodann das Geschehene laut denkend zu kommentieren. Die detaillierten Ergebnisse dieser Studie werden derzeit aufbereitet. Als zentrales Ergebnis lässt sich postulieren, dass Beratungslernende schon in frühen Kompetenzerwerbsstadien potentielle Lernmomente und Schlüsselsituationen in den Aufzeichnungen erkennen können. An nicht wenigen Stellen (hier 
müssen zukünftig noch gezielte Mixed-Method-Studien abgesicherte Verbindungen zwischen den quantitativen und qualitativen Daten herstellen) zeigt sich, dass relevante Momente aus Sicht der Studierenden auch diejenigen waren, die die externen CodiererInnen dazu bewogen haben, einen bestimmten Kompetenzwert zuzuweisen. Weiterhin zeigt sich in der detaillierten Auswertung der Think-Aloud-Transkripte, dass Studierende im Anschluss an das Identifizieren relevanter Stellen sehr differenzierte Reflexionen über ihr Handeln abgeben können. Empirisch erfahrbar wird dann in einer faszinierend tiefenscharfen Sicht, dass nicht nur sehr individuelle Kompetenzprofile in LernerInnengruppen existieren, sondern diese Differenzen von den LernerInnen ganz unterschiedlich erklärt werden. So können Momente, an denen z. B. der Redefluss oder das Schweigen aus Sicht der StudentInnen besonders kompetent oder insuffizient im BeraLab-Gespräch gelöst wurde und an denen sie aus ihrer Sicht folgerichtig gestoppt und erzählt haben, ganz unterschiedliche Narrationen erzeugen: beispielsweise die eines fehlenden Selbstwirksamkeitserlebens, einer fehlenden Technik, um das Problem zu vermeiden, oder die Schilderung der versuchten Anwendung von bereits gehörtem Wissen, was aber situativ aufgrund fehlender Routine scheitert - und vieles mehr. Hier entsteht dann sowohl im Forschungsprozess auf der Ebene der von uns untersuchten Beratungslernenden als auch in der modellhaften, theoretischen Verdichtung insgesamt ein Umschlagpunkt, an dem das Erfassen und Bilanzieren individueller Unterschiede in der Beratungskompetenz die Chance birgt, tatsächlich in subjektorientierte Professionalisierungsprozesse einzutreten: StudentInnen erschließen sich in diesen Prozessen das Thema Beratung intensiv und direkt auf ihre Person bezogen und werden gleichzeitig selbst für Beratung erschlossen, wenn solche stark sinngenerierenden Subjektivierungen des eigenen Lernprozesses in Bildung umschlagen.

\section{Subjektorientierte Professionalisierung als Programm für handlungswissenschaftliche Studiengänge}

In der weiteren Forschungs- und Theoriearbeit wird zu klären sein, ob eine berufsbiographisch früh einsetzende, bewusst gestaltete und konsequent subjektorientiert ausgerichtete Professionalisierung kompetente, akademisierte Beratungsfachkräfte hervorzubringen vermag. In den Handlungswissenschaften könnte das Ausloten einer solchen Perspektive den Brückenschlag zwischen bereits existierenden und breit etablierten Professionalisierungsmodellen wie beispielsweise der reflexiven Professionalisierung nach Dewe (Schwarz et al. 2014) einerseits und der bisher nicht vollständig eingelösten Kompetenzorientierung auf der Handlungsebene andererseits leisten (Otto 
2014). Der im vorliegenden Artikel nachgezeichnete Transformationsprozess einer Abkehr von der bisher als gesetzt geltenden Zweiphasenstruktur von Bildungsprozessen von Beratungsfachkräften verweist jedenfalls darauf, dass Hochschulen und die von ihnen angebotenen Studiengänge Chancen bieten, solche neuen und subjektiven Professionalisierungspraktiken für das Thema Beratung zu ermöglichen. Auf der Ebene der Didaktik wäre ein solches Programm gar nicht neu, sondern lediglich eine Systematisierungsoption für viele bereits vorhandene Instrumente der Erwachsenenbildung, z. B. der Portfolioarbeit oder Lernarrangements, die konsequent auf Nutzung von Digitalisierung setzen, um möglichst individuelle Lern- und Bildungsprozesse zu initiieren. In verschiedenen Projektzusammenhängen hat sich für die didaktische Konkretisierung subjektorientierter Professionalisierung ein Modell bewährt, in dessen Zentrum individuelle fachliche Entwicklungsaufgaben stehen, die im Spannungsfeld zwischen personengebundenen Eingangsvoraussetzungen und strukturellen Setzungen eines Bildungsgangs auf der Basis eines konkreten Anforderungsmodells gelingenden Handelns von den LernerInnen selbst (re-)konstruiert und gelöst werden (Bauer et al. 2017; Weinhardt 2017, 2018b). Mit einer so verstandenen Subjektorientierung wäre auch verbunden, dass Praxisformationen, in denen bisher Beratung gelernt wurde und die bisweilen auch ungewollte Normierungseffekte gezeigt haben, ersetzt werden durch stark reflexive und bewusst gestaltete individuelle Kombinationen von Zugangswegen zum Wissenserwerb - ein Prozess, in dem die Essenz von Beratung dann auch in die Bildungsprozesse der Fachkräfte selbst Eingang finden würde. Dieses Thema wird bisher im Professionalisierungsdiskurs nur wenig bedacht.

\section{Literatur}

Bauer, Petra; Weinhardt, Marc (2016): Professionalisierungs- und Kompetenzentwicklungsprozesse in der sozialpädagogischen Beratung. Baltmannsweiler: Schneider Hohengehren.

Bauer, Petra; Weinhardt, Marc (2014). Perspektiven sozialpädagogischer Beratung. Empirische Befunde und aktuelle Entwicklungen. Weinheim: Beltz.

Bauer, Petra; Weinhardt, Marc; Carfagno, Kristin; Christ, Aline; Kniep, Kathrin; Thomas, Marie; Urban, Markus (2017): Posterpräsentation ProfiL - Professionalisierung durch Beratung im Lehramtsstudium. Abschlusstagung der DFG-Forschergruppe „Analyse und Förderung effektiver Lehr- Lernprozesse“. Tübingen. Online verfügbar unter https://www.researchgate.net/publication/316687708 ProfiL___Professionalisierung_durch_Beratung_im_Lehramtsstudium. (Datum des letzten Abrufs: 30.07.2018) 
Bruder, Simone; Klug, Julia; Hertel, Silke; Schmitz, Bernhard (2010): Modellierung der Beratungskompetenz von Lehrkräften. In: Zeitschrift für Pädagogik. Beiheft 56, S. 274-284.

Collins, Allan; Brown, John Seely; Newman, Susan E. (1987): Cognitive apprenticeship. Teaching the craft of reading, writing and mathematics: Technical Report 403. Cambridge: BBN Laboratories.

Eriksen, Karen; McAuliffe, Garrett (2003): A measure of counselor competency. In: Counselor Education and Supervision 43 (2), S. 120-133.

Eriksen, Karen; McAuliffe, Garrett (2006): Constructive development and counselor competence. In: Counselor Education and Supervision 45 (3), S. 180-192.

Giesecke, Hermann (2015): Pädagogik als Beruf. Grundformen pädagogischen Handelns. Weinheim: Beltz.

Gieseke, Wiltrud; Nittel, Dieter (Hg.) (2016): Handbuch Pädagogische Beratung über die Lebensspanne. Weinheim: Beltz.

Hohenshil, Thomas H.; Amundson, Norman E.; Niles, Spencer G. (2015): Counseling Around the World. An International Handbook. Hoboken: Wiley.

Lave, Jean; Wenger, Etienne (1991): Situated learning. Legitimate peripheral participation. Cambridge: Cambridge University Press.

Maier-Gutheil, Cornelia (2016): Beraten. Stuttgart: Kohlhammer.

Mollenhauer, Klaus (1973): Einführung in die Sozialpädagogik. Weinheim: Beltz.

Nestmann, Frank; Engel, Frank; Sickendiek, Ursel (2014a): Das Handbuch der Beratung. Ansätze, Methoden und Felder. Tübingen: DGVT.

Nestmann, Frank; Engel, Frank; Sickendiek, Ursel (2014b): Das Handbuch der Beratung. Disziplinen und Zugänge. Tübingen: DGVT.

Nestmann, Frank; Sickendiek, Ursel (2011): Beratung. In: Hans Thiersch und HansUwe Otto (Hg.): Handbuch Soziale Arbeit. München: Reinhardt, S. 109-119.

Otto, Hans-Uwe (2014): Akademisiert - aber nicht professionalisiert. Essay zur Situation der Sozialen Arbeit als personenbezogene Dienstleistung. In: Martin P. Schwarz, Wilfried Ferchhoff, Ralf Vollbrecht (Hg.): Professionalität: Wissen - Kontext. Sozialwissenschaftliche Analysen und pädagogische Reflexionen zur Struktur bildenden und beratenden Handelns. Bad Heilbrunn: Klinkhardt, S. 750-755.

Schwarz, Martin P.; Ferchhoff, Wilfried; Vollbrecht, Ralf (Hg.) (2014): Professionalität: Wissen - Kontext. Sozialwissenschaftliche Analysen und pädagogische Reflexionen zur Struktur bildenden und beratenden Handelns. Bad Heilbrunn: Klinkhardt.

Shazer, Steve de; Weakland, John H.; Hoyt, Michael F. (2001): On the importance of keeping it simple and taking the patient seriously: A Conversation with Steve de Shazer and John Weakland. In: Michael F. Hoyt (Hg.): Interviews with brief therapy experts. Philadelphia: Routledge, S. 1-33.

Strasser, Josef (2006): Erfahrung und Wissen in der Beratung. Theoretische und empirische Analysen zum Entstehen professionellen Wissens in der Erziehungsberatung. Göttingen: Cullivier.

Strasser, Josef; Gruber, Hans (2015): Learning processes in the professional development of mental health counselors: knowledge restructuring and illness script formation. In: Advances in Health Sciences Education 20 (2), S. 515-530. 
Walther, Andreas; Weinhardt, Marc (Hg.) (2013): Beratung im Übergang. Weinheim: Beltz.

Weinhardt, Marc (2014): Kompetenzentwicklung in der psychosozialen Beratung am Beispiel von Studierenden der Erziehungswissenschaft. In: Petra Bauer, Marc Weinhardt (Hg.): Perspektiven sozialpädagogischer Beratung. Empirische Befunde und aktuelle Entwicklungen. Weinheim: Beltz, S. 214-231.

Weinhardt, Marc (Hg.) (2015): Psychosoziale Beratungskompetenz. Pilotstudien aus der Arbeitsstelle für Beratungsforschung. Weinheim: Beltz.

Weinhardt, Marc (2016a): Haltung und Methode, Person und Technik: Empirische Perspektiven auf Struktur und Vermittlung von Beratungskompetenz. In: Petra Bauer, Marc Weinhardt (Hg.): Professionalisierungs- und Kompetenzentwicklungsprozesse in der sozialpädagogischen Beratung, S. 75-91.

Weinhardt, Marc (2016b): Jugendhilfe und Systemische Beratung. In: Dieter Nittel, Wiltrud Gieseke (Hg.): Pädagogische Beratung über die Lebensspanne. Weinheim: Beltz, S. 599-610.

Weinhardt, Marc (2017): Subjektorientierte Professionalisierung, Lebenslanges Lernen und der EQR/DQR in der Systemischen Fort- und Weiterbildung. In: Kontext 47 (3), 262-277.

Weinhardt, Marc (2018a): Beraten. In: Gunther Graßhoff, Anna Renker, Wolfgang Schröer (Hg.): Soziale Arbeit. Eine elementare Einführung. Wiesbaden: VS.

Weinhardt, Marc (2018b): Kompetenzorientiert systemisch beraten lernen. Eine Gebrauchsanweisung für die eigene Professionalisierung. Heidelberg: Vandenhoeck \& Ruprecht.

Weinhardt, Marc; Bauer, Petra (2016): Die Professionalisierung von Personal als Leitungsaufgabe. In: Michael Vilain, Sebastian Wegener (Hg.): Social Talk 2015. Am Wendepunkt? Innenperspektiven der Sozialwirtschaft. Tagungsband des Instituts für Zukunftsfragen der Gesundheits- und Sozialwirtschaft (IZGS) der Evangelischen Hochschule Darmstadt. Frankfurt a. M., S. 44-51.

Weinhardt, Marc; Kelava, Augustin (2016): Die performanzorientierte Erfassung psychosozialer Beratungskompetenz. In: neue praxis 2016 (4), S. 363-377.

Wenger, Etienne (1998): Communities of practice. Learning, meaning, and identity. Cambridge: University Press. 



\section{Beratung zwischen biographischen Negativerfahrungen und offener biographischer Zukunft. Eine komparative Sicht auf pädagogische Arbeitsfelder}

\section{Beratung im Horizont des Lebensverlaufs}

Aus erziehungswissenschaftlicher Perspektive lässt sich das Thema Beratung unter vielfältigen Gesichtspunkten erforschen. So können bspw. die gesellschaftlichen und organisationalen Kontexte von Beratung (Schiersmann 2013: 31-32) oder diverse Handlungsfelder, wie sie exemplarisch von Gieseke und Nittel (2016) gesammelt wurden, untersucht werden. Darüber hinaus besteht die Möglichkeit, die spezifischen Interaktionsmodalitäten in Beratungssituationen (Maier-Gutheil 2009: 125-170), die dabei eingesetzten Praktiken und Medien (Seel 2014: 57-130) oder die Anforderungen für Berater*innen im Kontext ihres professionellen Handelns (Gröning 2011: 107-126) näher zu betrachten. Zudem lassen sich auch die Beratenen selbst in den Fokus des erziehungswissenschaftlichen Interesses rücken und dabei bspw. erforschen, inwiefern ihre Wunsch- und Zielvorstellungen im Beratungsprozess berücksichtigt werden (Gieseke \& Stimm 2016: 183-232).

Diese Sicht auf die Relevanzsetzungen von Beratenen im Rahmen dieser pädagogischen Kernaktivität (Nittel et al. 2014: 86) wird auch im vorliegenden Beitrag eingenommen. Dabei erfolgt keine unmittelbare Analyse von Aussagen der Beratenen selbst. Stattdessen werden die beruflichen Selbstbeschreibungen von Beratenden aus der PAELL-Studie herangezogen, in denen auch Beratungsanlässe der Ratsuchenden thematisiert werden. Bei den untersuchten Personen handelt es sich nicht um pädagogisch Tätige (Nittel 2017: 136-138), die ausschließlich beraten, sodass sich durch diesen empirischen Zugang die Chance ergibt, die Verwobenheit der Beratung mit anderen pädagogischen Kernaktivitäten zu erforschen. In diesem Zusammenhang lassen sich die Beratungsanliegen im Hinblick auf die Bezüge zur biographischen Situation der Ratsuchenden untersuchen, wodurch sich ein Analysefokus auf die Zeitdimension von Beratung ergibt.

Gemäß dieser Konstellation werden im vorliegenden Beitrag zwei Fragestellungen diskutiert: 
1. Wie wird die Kernaktivität des Beratens in den beruflichen Selbstbeschreibungen von pädagogisch Tätigen mit anderen pädagogischen Kernaktivitäten relationiert?

2. Welche Beratungsanlässe und Zeitbezüge lassen sich in den beruflichen Selbstbeschreibungen von pädagogisch Tätigen erfassen?

Zur Bearbeitung dieser Fragestellungen wird Zeit zunächst als erziehungswissenschaftlicher Forschungsgegenstand umrissen, wobei ein Schwerpunkt auf Untersuchungen zum Thema Beratung liegt. Daran anschließend wird die empirische Basis der Befunde dargelegt und ein wissenssoziologisch orientiertes Differenzierungsschema für Zeitbezüge vorgestellt. Danach werden die Ergebnisse präsentiert, anhand derer die zwei aufgeworfenen Fragestellungen diskutiert werden. Den Abschluss des Beitrags bildet eine Reflexion der zentralen Erkenntnisse.

\section{Zeit als erziehungswissenschaftlicher Forschungsgegenstand}

Zeitphänomene werden im Rahmen erziehungswissenschaftlicher Studien in vielfältiger Weise erforscht. Die entsprechenden Kontexte sind so unterschiedlich, dass an dieser Stelle aus Platzgründen keine systematische Aufarbeitung aller erziehungswissenschaftlich relevanten Ansätze erfolgen kann. Stattdessen sollen die nachfolgend thematisierten Bezugspunkte zum Thema Zeit auf die Vielfalt des Diskurses verweisen. Zeit stellt einen zentralen Faktor für transformatorische Bildungsprozesse dar, wobei mit dem Verstreichen von Zeit und der Auseinandersetzung mit Bildungsstoffen Veränderungen des Selbst einhergehen (Zirfas 2011: 14). Verschiebt sich der Fokus von Bildung auf Lernen, so wird temporalen Bezügen ebenfalls eine wichtige Bedeutung beigemessen. Dies betrifft bspw. die Einsicht, dass die Bereitstellung bzw. Verfügbarkeit von Zeit ein wesentliches Merkmal lernförderlicher Umgebungen darstellt (Göhlich \& Zirfas 2007: 193). Die elementare Bedeutung von Zeit wird zudem deutlich, wenn die Lebens- als Lernzeit konzipiert wird. Im Kontext lebenslangen Lernens wird demensprechend eine zeitliche Entgrenzung des Lernens diagnostiziert, wobei das Zeitfenster für Lernen auf die gesamte Lebenszeit ausgedehnt wird (Hof 2009: 58).

Betrachtet man Studien mit Bezug zum Erwachsenenalter, so wird die Bedeutung zeitlicher Ressourcen ebenfalls bestätigt. Dies zeigt sich exemplarisch an der essentiellen Bedeutung von Zeit als Gelingensbedingung für informelles Lernen. In diesem Kontext wird erkennbar, dass die Investition eigener zeitlicher Ressourcen für Tätigkeiten wie Zeitungslektüre oder Inter- 
netnutzung zu deutlichen Lernerträgen führen kann (Brödel \& Yendell 2008: 110). Einen weiteren Bezugspunkt bilden die Eigenzeiten von Lernimpulsen im Erwachsenenalter. Sie verweisen auf die subjektiven Interessen und Relevanzsetzungen bei der Deklaration von Zeiträumen als Lernzeiten (SchmidtLauff 2008: 121).

Auch in Bezug auf Beratung werden Zeitphänomene relevant, so etwa in der Mikroanalyse von Beratungsgesprächen. Dabei lässt sich bspw. eine zeitliche Situationskonstitution rekonstruieren, wodurch die temporale Befristung und Rahmung von Gesprächsterminen in den Fokus rückt (Maier-Gutheil 2009: 96). Ebenso wird das Thema Zeit herangezogen, um die Konfiguration von Beratung im Sinn einer Prozesschronologie zu charakterisieren, wodurch die (Un-)Gleichzeitigkeit verschiedener Dimensionen von Beratung erkennbar wird (Kossack 2016: 127-128). Zusätzlich zu dieser konstitutiven Funktion von Zeit für die Existenz von Beratungsinteraktionen kann ihre Verfügbarkeit als Qualitätskriterium von Beratung konzipiert werden. Dabei zeigt sich, dass eine gelingende Beratung mit der Passung von Zeit für die Beratenen (Seel 2014: 169-170) einhergeht. Zudem kann die Konstellation eintreten, dass sich das erwünschte Ergebnis des Beratungsprozesses erst nach längerer Zeit einstellt, wodurch das Phänomen der Ungewissheit zu einem immanenten Bestandteil dieser sozialen Dienstleistung wird (Schiersmann \& Weber 2013: 42). Schließlich können Temporalbezüge in Beratungsinteraktionen auch über die Rekonstruktion von biographischen Mustern sozialer Zeit durch die Methode der biographischen Exploration (Bauer et al. 2012: 25-26) rekonstruiert werden.

Wie diese exemplarischen Bezüge zu Zeitphänomenen als Gegenstand erziehungs- und beratungswissenschaftlicher Forschung zeigen, wird dem Faktor Zeit in Beratungskontexten sowie den damit verbundenen Lernprozessen eine wichtige Rolle zugeschrieben. Mit Bezug auf die Ausgangsfragestellungen dieses Beitrags soll nun geklärt werden, welche chronologischen Kontextualisierungen pädagogisch Tätige nutzen, die die Kernaktivität des Beratens ausführen.

\section{Vergleich pädagogischer Arbeitsfelder}

Eine Möglichkeit, sich dem faktischen Beratungshandeln von pädagogisch Tätigen zu nähern und gleichzeitig die damit verbundenen Wissensbestände der Berufsrollenträger zu erforschen, eröffnet sich durch die Analyse von beruflichen Selbstbeschreibungen. Diese Textgattung bietet sich vor allem deswegen zur Analyse von Berufskulturen aus unterschiedlichen pädagogischen Arbeitsfeldern an, weil sich in ihr zentrale Elemente der jeweili- 
gen sozialen Einheit wie bspw. Lizenz, Mandat sowie das damit verbundene Selbstverständnis als Kollektiv dokumentieren (Hartig 2008: 87). Der in diesem Beitrag vorgenommene Vergleich von pädagogisch Tätigen aus verschiedenen Arbeitsfeldern kann dem Ansatz der komparativen pädagogischen Berufsgruppenforschung (Nittel, Schütz \& Tippelt 2014: 15) zugeordnet werden, womit auch hier der Fokus auf der Identifizierung von arbeitsfeldübergreifenden Schnittmengen pädagogischen Handelns liegt. Im vorliegenden Beispiel dient die Orientierung an diesem Ansatz dem Zweck, arbeitsfeldspezifische und -übergreifende Kontextualisierungen von Beratung zu rekonstruieren.

Als Datengrundlage wird eine Sekundäranalyse der Studie „Pädagogische Erwerbsarbeit im System des lebenslangen Lernens" (PAELL) herangezogen. In der Primärstudie, die zwischen 2009 und 2011 stattfand, wurden die beruflichen Selbstbeschreibungen von pädagogisch Tätigen aus acht verschiedenen pädagogischen Arbeitsfeldern in Bayern und Hessen miteinander verglichen. Die Studienteilnehmenden aus dem Elementar- und Primarbereich, den Sekundarbereichen I und II, der beruflichen Bildung, dem tertiären Bereich sowie die Beschäftigten der Erwachsenenbildung und der außerschulischen Jugendbildung wurden zu unterschiedlichen Facetten ihres beruflichen Handelns befragt (Nittel \& Schütz 2010: 50-51). Den analytischen Fokus bildeten dabei die individuellen Einstellungen und kollektiven Orientierungen der Studienteilnehmenden hinsichtlich Themen wie den Kooperationsaktivitäten und der arbeitsfeldspezifischen Verankerung des lebenslangen Lernens. $\mathrm{Zu}$ diesem Zweck wurden sowohl schriftliche Befragungen als auch Gruppendiskussionen durchgeführt. Im Rahmen dieser Studie wurde deutlich, dass die Kernaktivität des Beratens für die Verwirklichung des jeweiligen Mandats bspw. in biographisch wichtigen Entscheidungssituationen hinsichtlich der Auswahl weiterführender Lernkontexte von zentraler Bedeutung ist (Nittel et al. 2014: 88). Damit zeigt sich, dass dem Beratungshandeln eine entscheidende Bedeutung bei der Prozessierung des lebenslangen Lernens zukommt. Dieser Zusammenhang zeigt sich auch in anderen Kontexten, bspw. im Fall der Lernberatung im Erwachsenenalter (Ludwig 2016: 308-309) oder im Zuge der Übergangsberatung zur Förderung von biographischer Reflexivität (Walther 2016: 651).

An einem der zentralen Ergebnisse der Primärstudie, nämlich der je nach pädagogischem Arbeitsfeld unterschiedlich stark ausgeprägten Orientierungskraft des lebenslangen Lernens (Wahl, Nittel \& Tippelt 2014: 134-135), schloss die Sekundäranalyse an und differenzierte die zuvor entwickelten Ausprägungen der Orientierungskraft (hoch, moderat, niedrig) weiter aus. Dabei lag der Fokus der Untersuchung auf denjenigen Arbeitsfeldern, in denen eine hohe Orientierungskraft des lebenslangen Lernens diagnostiziert wurde. Dementsprechend wurden in der Sekundäranalyse die Wissensbestände und die damit verbundenen geschlossenen Sinnbereiche (Schütz 1971: 263) 
von pädagogisch Tätigen aus dem Elementarbereich, der beruflichen Bildung und der Erwachsenenbildung untersucht.

Zur Analyse der Zeitbezüge von Beratungsanlässen wird ein wissenssoziologisch orientiertes Differenzierungsschema herangezogen. Es basiert auf einem zentralen Ergebnis der Sekundäranalyse, nämlich der Diagnose, dass lebenslanges Lernen arbeitsfeldspezifisch kontextualisiert ist. Dabei lassen sich die Bezüge der drei sozialen Einheiten hinsichtlich zweier Dimensionen untergliedern.

Das erste Unterscheidungsmerkmal bildet die formale Referenzebene des Lernens. Damit werden die Kontextualisierungen dahingehend unterscheidbar, ob das lebenslange Lernen eher mit den Lernaktivitäten der pädagogisch Tätigen oder der pädagogisch Anderen, also der Adressat*innen des pädagogischen Handelns, verbunden wird. Diesbezüglich zeigt sich in den Daten, dass das lebenslange Lernen arbeitsfeldübergreifend überwiegend mit der Prozessierung von Lernprozessen der pädagogisch Anderen verbunden und die Ermöglichung des lebenslangen Lernens von den Befragten als wichtiger Teil des beruflichen Mandats dargestellt wird (Wahl 2017: 182). Im Detail wird das lebenslange Lernen im Elementarbereich primär mit der Aufgabe verbunden, die eigenen pädagogisch Anderen auf den anstehenden Übergang in den Primarbereich vorzubereiten und die dazu nötigen Kompetenzen sowie eine basale Lernmotivation zu fördern. Anhand der Angaben von Lehrkräften aus der beruflichen Bildung zum lebenslangen Lernen wird ersichtlich, dass sie ihre pädagogisch Anderen auf die Übernahme einer Berufsrolle vorbereiten und einen Beitrag zu deren Persönlichkeitsentwicklung leisten möchten. Schließlich wird das lebenslange Lernen im pädagogischen Arbeitsfeld der Erwachsenenbildung mit der Förderung von Lernmotivation bei den pädagogisch Anderen angesichts heteronomer Anforderungen sowie milieu- und altersspezifischer Problemlagen kontextualisiert. In allen drei pädagogischen Arbeitsfeldern werden zur Prozessierung des Lernens verschiedene pädagogische Kernaktivitäten genutzt, zu denen auch das Beraten zählt.

Das zweite Unterscheidungsmerkmal bildet die Zeitebene des Lernens (Schmidt-Lauff 2008: 121). Die beruflichen Selbstbeschreibungen aus den drei untersuchten pädagogischen Arbeitsfeldern unterscheiden sich dahingehend, inwiefern das lebenslange Lernen mit Lernanlässen und ihrem chronologischen Kontext im Horizont der Lebensspanne verbunden werden. In Anlehnung an die wissenssoziologische Differenzierung zwischen Um-zu-Motiven und Weil-Motiven (Schütz \& Luckmann 2017: 286-304) konnten in diesem Kontext sowohl prospektiv-teleologisch ausgerichtete als auch retrospektiv-kompensatorisch ausgerichtete Lernanlässe rekonstruiert werden. Die beruflichen Selbstbeschreibungen der Studienteilnehmenden, in denen das lebenslange Lernen angesprochen wird, sind diesbezüglich primär prospektiv-teleologisch ausgerichtet, es werden also überwiegend Lernanlässe thematisiert, die in der biographischen Zukunft der pädagogisch Anderen 
liegen (Wahl 2017: 183). So bildet der Übergang in den lebensgeschichtlich nachgeschalteten Primarbereich den zentralen Kontextualisierungshorizont im Elementarbereich. Eine vergleichbare temporale Ausrichtung zeigt sich in der beruflichen Bildung, in der die Vorbereitung der pädagogisch Anderen auf die zukünftig anstehende Übernahme einer Berufsrolle im Mittelpunkt der Aussagen zum lebenslangen Lernen steht. Diese Fokussierung auf die Zukunft der pädagogisch Anderen zeigt sich auch in der Erwachsenenbildung. Hier verweisen die Studienteilnehmenden überwiegend auf den Umstand, dass sich die Bedeutung des lebenslangen Lernens aufgrund zukünftig auftretender berufsbezogener Lernanforderungen ergibt. Deren Form lässt sich aus Sicht der Befragten zwar noch nicht näher konturieren, doch wird das Eintreten derartiger Konstellationen ebenso prognostiziert wie die Ungewissheit von zukünftigen Herausforderungen einer individualisierten Lebensführung. Im letztgenannten pädagogischen Arbeitsfeld zeigen sich zudem verstärkt retrospektiv-kompensatorische Bezüge, wobei das lebenslange Lernen als wichtig für die Kompensation negativer schulischer Lernerfahrungen erachtet wird. Auch bei der Analyse dieses Unterscheidungsmerkmals zeigt sich, dass dem Beraten bspw. hinsichtlich zukünftiger Gestaltungsoptionen ein wichtiger Stellenwert zukommt.

\section{Relationierung pädagogischer Kernaktivitäten im Kontext von individueller und kollektiver Fallarbeit}

Durch die Sekundäranalyse, in der auch Ergebnisse zum Thema Beratung erzielt wurden, lassen sich die oben genannten Fragestellungen bearbeiten. In Bezug zur ersten Frage nach der Relationierung der verschiedenen pädagogischen Kernaktivitäten zeigt sich arbeitsfeldübergreifend die Tendenz, dass Beratung primär flankierend zur Erfüllung eines Mandats (bspw. im Sinne des lebenslangen Lernens) genutzt wird, das erst durch die Kombination mehrerer Kernaktivitäten vollständig umgesetzt werden kann. Dies dokumentiert sich in den drei pädagogischen Arbeitsfeldern in unterschiedlicher Weise. So ist die Beratung im Elementarbereich nicht auf die pädagogisch Anderen, sondern auf deren Erziehungsberechtigte ausgerichtet. Durch diese spezifische Intervention wird die Entwicklung der Kinder zwar nicht direkt durch die pädagogisch Tätigen beeinflusst, doch lassen sich die familialen Erziehungsaktivitäten aus Sicht der Studienteilnehmenden durch diese Beratungsaktivitäten unterstützen. Dabei erbringen die elementarpädagogischen Fachkräfte Beratungsleistungen, die im Kontext von Erziehungsberatung (Lux \& Walper 2016: 170) und Schuleingangsberatung (Kucharz 2106: 178) angesiedelt sind. Die eigentliche Interaktion mit den pädagogisch Anderen 
und die damit verbundenen pädagogischen Kernaktivitäten der kollektiven Fallarbeit sind von diesem Beraten nicht unmittelbar betroffen und können parallel durchgeführt werden. In der beruflichen Bildung werden beratende Aktivitäten verstärkt mit der Kernaktivität des Unterrichtens in Verbindung gebracht. Dabei berichten die Lehrkräfte bspw. von Anfragen der pädagogisch Anderen, die jenseits des Unterrichts von den pädagogisch Tätigen zu den Gestaltungsoptionen ihrer Bildungs- bzw. Berufskarrieren beraten werden möchten. Hier zeigt sich erneut die Verwobenheit von individueller und kollektiver Fallarbeit zugunsten der Mandatserfüllung. Die pädagogisch Tätigen beraten in diesem Kontext im Bereich der Berufsorientierung an den Übergängen zwischen Schule und Beruf bzw. zwischen Schule und Hochschule (Brüggemann \& Sauer-Schiffer 2016: 203-204). Während die Kombination der unterschiedlichen Kernaktivitäten in den beiden bereits thematisierten pädagogischen Arbeitsfeldern im Mittelpunkt der beruflichen Selbstbeschreibungen steht, zeigt sich in der Erwachsenenbildung ein differenzierteres Bild. Auch hier verweisen die Studienteilnehmenden auf Konstellationen, in denen sie zusätzlich zu anderen Kernaktivitäten wie bspw. dem Unterrichten beratend tätig werden. Darüber hinaus verweisen sie auf die Existenz institutionalisierter Beratungssettings im Bereich der Schuldnerberatung (Ebli/Herzog 2016: 724) oder Suchtberatung (Morgenstern/Tögel-Lins/Werse 2016), sodass hier eine klassische individuelle Fallarbeit vorliegt. Vor dem Hintergrund dieser arbeitsfeldspezifischen Ergebnisse und ihres Vergleichs zeigt sich, dass die Kernaktivität des Beratens in pädagogischen Handlungsfeldern in unterschiedlichsten Konstellationen eingesetzt werden kann. So können damit wie in der beruflichen Bildung und der Erwachsenenbildung die pädagogisch Anderen direkt adressiert oder wie im Elementarbereich deren Angehörige beraten werden. Zudem ergibt sich aus der Kontrastierung der drei pädagogischen Arbeitsfelder die Differenz zwischen dem Beraten als eigenständiges Setting der individuellen Fallarbeit auf der einen und dem Beraten im Verbund mit weiteren Kernaktivitäten, die eher auf kollektive Fallarbeit ausgerichtet sind, auf der anderen Seite.

\section{Zeitbezüge von Beratungsanlässen}

Der in der Studie verwendete Zugang zum Thema Zeit eröffnet zudem die Möglichkeit, Beratungsanlässe unter dem Aspekt der subjektiven Handlungsmotivation und der damit verbundenen zeitlichen Verortung im Horizont des Lebenslaufs zu analysieren. Dementsprechend lassen sich sowohl Temporalbezüge von Beratungsanliegen identifizieren, die sich auf die biographische Vergangenheit beziehen, als auch Zeitkontexte rekonstruieren, die auf die bio- 
graphische Zukunft verweisen. Anhand des Datenmaterials der Primärstudie lassen sich diese beiden Optionen weiter präzisieren und damit die zweite Fragestellung nach den konkreten Beratungsanlässen und den damit verbundenen Zeitbezügen bearbeiten. Im Elementarbereich werden ausschließlich Beratungsanliegen der Erziehungsberechtigten thematisiert, die jedoch direkt an die biographische Entwicklung der pädagogisch Anderen gekoppelt sind. Die dabei identifizierten Anliegen umfassen einerseits den korrekten Umgang mit erwartungswidrigem Verhalten der Kinder in der Vergangenheit sowie andererseits den Wunsch nach Empfehlungen für die angemessene Gestaltung des perspektivisch anstehenden Übergangs der pädagogisch Anderen in den Primarbereich. Somit zeigen sich in diesem Arbeitsfeld sowohl retrospektiv-kompensatorische als auch prospektiv-teleologische Beratungsanlässe. Der Blick auf die Daten aus der beruflichen Bildung zeigt eine vergleichbare Konstellation. Wie bereits bei der ersten Fragestellung herausgearbeitet wurde, werden die pädagogisch Anderen in diesem Arbeitsfeld durch die Kernaktivität des Beratens direkt adressiert. Als Beratungsanlässe fungieren hier bspw. negative Erfahrungen am Ausbildungsplatz. Diese können im Rahmen der dualen Ausbildung in solchen Fällen auftreten, in denen die Interaktion zwischen den pädagogisch Anderen und den Ausbilder*innen im Betrieb konflikthaft verläuft. Darüber hinaus vollziehen sich beratende Aktivitäten auch am Lernort Berufsschule. In diesem Kontext werden die pädagogisch Tätigen aufgesucht und um ihren Rat zur Gestaltung des weiteren Lebenswegs gebeten. Im Material tauchen diesbezüglich Konstellationen auf, in denen einerseits die Gestaltung der biographischen Zukunft allgemein oder andererseits konkrete Gestaltungsoptionen für die jeweilige Berufs- und/oder Bildungsbiographie erörtert werden. In der Erwachsenenbildung werden die retrospektiv-kompensatorischen Beratungsanlässe exklusiv den institutionalisierten Beratungssettings zugeordnet. Demgegenüber werden in den Konstellationen, in denen die pädagogisch Anderen nicht ausschließlich beraten, sondern bspw. im Rahmen von Kursen vorwiegend unterrichtet werden, eher Gestaltungsoptionen für den weiteren Verlauf der Berufs- und/oder Bildungsbiographie diskutiert. Der Vergleich der pädagogischen Arbeitsfelder zeigt, dass die pädagogisch Tätigen zwei grundlegende Beratungsanlässe beschreiben, die sich je einem Zeitbezug zuordnen lassen. So werden Anliegen, in denen der Umgang mit negativen (Lern-)Erfahrungen im Mittelpunkt steht, als retrospektiv-kompensatorische Anliegen konzipiert. Während sich diese auf die biographische Vergangenheit beziehen, werden in den beruflichen Selbstbeschreibungen der Studienteilnehmenden auch Temporalbezüge zur biographischen Zukunft deutlich. Diese zeigen sich in Konstellationen, in denen die Gestaltungsoptionen für die weitere Berufs- und/oder Bildungsbiographie als Anliegen dargestellt werden, sodass sie als prospektiv-teleologische Beratungsanlässe bezeichnet werden können. 


\section{Biographische Vergangenheit und Zukunft als Ausgangspunkt von Beratung}

Der Vergleich der beruflichen Selbstbeschreibungen von pädagogisch Tätigen aus drei pädagogischen Arbeitsfeldern ermöglicht einen Einblick in die Vielfalt von Beratungsanlässen, mit denen die pädagogisch Anderen bzw. deren Angehörige sie aufsuchen. Durch diese komparative Sicht wird deutlich, dass Beraten zwar in speziell für diesen Zweck institutionalisierten Settings allein zur Erfüllung des Mandats eingesetzt wird. Jedoch zeigt sich ebenfalls, dass Beraten in anderen pädagogischen Kontexten erst in Kombination mit anderen pädagogischen Kernaktivitäten wie bspw. dem Unterrichten dazu führt, dass der pädagogische Auftrag erreicht wird. Somit wird der unmittelbare Zusammenhang zwischen dem Beraten als „Inkarnation der individuellen Fallarbeit" (Nittel et al. 2014: 86) und anderen Formen der kollektiven Fallarbeit erkennbar. Zudem ergibt die Sekundäranalyse der PAELL-Studie, dass die pädagogisch Tätigen aus allen drei pädagogischen Arbeitsfeldern sowohl prospektiv-teleologische als auch retrospektiv-kompensatorische Beratungsanlässe wahrnehmen. So weisen Anliegen, die den Umgang mit negativen (Lern-)Erfahrungen umfassen, einen Zeitbezug zur biographischen Vergangenheit auf. Demgegenüber wird die biographische Zukunft in solchen beruflichen Selbstbeschreibungen chronologisch kontextualisiert, in denen mögliche Gestaltungsoptionen für die weitere Berufs- und/oder Bildungsbiographie den Beratungsanlass darstellen.

Darüber hinaus lässt sich aus den Ergebnissen eine professionstheoretisch anschlussfähige Folge ableiten. Die Pluralität der möglichen Negativerfahrungen und die potentiell zunehmende Vielfalt der berufs- und bildungsbiographischen Gestaltungsoptionen führen zu steigenden Anforderungen an die pädagogisch Tätigen, die ihren Wissensstand entsprechend anpassen müssen; eine Konstellation, die sich für diejenigen verschärft, die im Rahmen ihres beruflichen Handelns nicht ausschließlich beratend tätig sind.

\section{Literatur}

Bauer, Annemarie/Gröning, Katharina/Hoffmann, Cornelia/Kunstmann, AnneChristin (Hg.) (2012): Grundwissen Pädagogische Beratung. Göttingen: Vandenhoeck \& Ruprecht.

Brödel, Rainer/Yendell, Alexander (2008): Weiterbildungsverhalten und Eigenressourcen. NRW-Studie über Geld, Zeit und Erträge beim lebenslangen Lernen. Bielefeld: Bertelsmann. 
Brüggemann, Tim/Sauer-Schiffer, Ursula (2016): Beratung am Übergang Schule-Beruf. In: Gieseke, W./Nittel, D. (Hg.): Handbuch Pädagogische Beratung über die Lebensspanne. Weinheim: Beltz Juventa, S. 203-211.

Ebli, Hans/Herzog, Kerstin (2016): Schuldnerberatung. In: Gieseke, W./Nittel, D. (Hg.): Handbuch Pädagogische Beratung über die Lebensspanne. Weinheim: Beltz Juventa, S. 724-732.

Gieseke, Wiltrud/Nittel, Dieter (Hg.) (2016): Handbuch Pädagogische Beratung über die Lebensspanne. Weinheim: Beltz Juventa.

Gieseke, Wiltrud/Stimm, Maria (2016): Praktiken der professionellen Bildungsberatung. Innensichten auf die Entscheidungsfindung im Beratungsprozess. Wiesbaden: Springer VS.

Göhlich, Michael/Zirfas, Jörg (2007): Lernen. Ein pädagogischer Grundbegriff. Stuttgart: Kohlhammer.

Gröning, Katharina (2011): Pädagogische Beratung. Konzepte und Positionen. 2. Auflage. Wiesbaden: VS.

Hartig, Christine (2008): Berufskulturelle Selbstreflexion. Selbstbeschreibungslogiken von ErwachsenenbildnerInnen. Wiesbaden: VS.

Hof, Christiane (2009): Lebenslanges Lernen. Eine Einführung. Stuttgart: Kohlhammer.

Kossack, Peter (2016): Die pädagogische Binnenstruktur der Beratung. In: Gieseke W./Nittel, D. (Hg.): Handbuch Pädagogische Beratung über die Lebensspanne. Weinheim: Beltz Juventa, S. 122-130.

Kucharz, Diemut (2016): Schuleingangsberatung. In: Gieseke, W./Nittel, D. (Hg.): Handbuch Pädagogische Beratung über die Lebensspanne. Weinheim: Beltz Juventa, S. 177-185.

Ludwig, Joachim (2016): Lernberatung. In: Gieseke, W./Nittel, D. (Hg.): Handbuch Pädagogische Beratung über die Lebensspanne. Weinheim: Beltz Juventa, S. 304-312.

Lux, Ulrike/Walper, Sabine (2016): Erziehungsberatung. In: Gieseke, W./Nittel, D. (Hg.): Handbuch Pädagogische Beratung über die Lebensspanne. Weinheim: Beltz Juventa, S. 168-176.

Maier-Gutheil, Cornelia (2009): Zwischen Beratung und Begutachtung. Pädagogische Professionalität in der Existenzgründungsberatung. Wiesbaden: Springer VS.

Morgenstern, Cornelia/Tögel-Lins, Karsten/Werse, Bernd (2016): Online-Drogenberatung. In: Gieseke, W./Nittel, D. (Hg.): Handbuch Pädagogische Beratung über die Lebensspanne. Weinheim: Beltz Juventa, S. 715-723.

Nittel, Dieter (2017): Ein System im statu nascendi. Auf dem Weg zum pädagogisch organisierten System des lebenslangen Lernens? In: Arnold, R./Neuser, W. (Hg.): Beobachtung des Wissens - Das Wissen des Beobachters: Annäherung an eine systemische Hermeneutik. Baltmannsweiler: Hohengehren, S. 119-165.

Nittel, Dieter/Schütz, Julia (2010): Komparative Berufsgruppenforschung: Pädagogische Erwerbsarbeit im System des Lebenslangen Lernens. In: Zeitschrift für Bildungsverwaltung 26, 1, S. 49-56.

Nittel, Dieter/Schütz, Julia/Tippelt, Rudolf (2014): Einleitung: Impuls für eine komparative Berufsgruppenforschung in den Erziehungswissenschaften - Die PAELL-Studie. In: Nittel, D./ Schütz, J./Tippelt, R. (Hg.): Pädagogische Arbeit im 
System des lebenslangen Lernens. Ergebnisse komparativer Berufsgruppenforschung. Weinheim: Beltz Juventa, S. 13-19.

Nittel, Dieter/Tippelt, Rudolf/Dellori, Claudia/Siewert-Kölle, Andrea (2014): Gemeinsamkeiten und Unterschiede der pädagogischen Berufsgruppen. In: Nittel, D./ Schütz, J./Tippelt, R. (Hg.): Pädagogische Arbeit im System des lebenslangen Lernens. Ergebnisse komparativer Berufsgruppenforschung. Weinheim: Beltz Juventa, S. 60-101.

Schiersmann, Christiane (2013): Beratung im Feld Bildung, Beruf, Beschäftigung. In: Schiersmann, C./Weber, P. (Hg.): Beratung in Bildung, Beruf und Beschäftigung. Eckpunkte und Erprobung eines integrierten Qualitätskonzepts. Bielefeld: Bertelsmann, S. 25-32.

Schiersmann, Christiane \& Weber, Peter (2013): Gesamtkonzept für Qualität von Beratung. In: Schiersmann, C./Weber, P. (Hg.): Beratung in Bildung, Beruf und Beschäftigung. Eckpunkte und Erprobung eines integrierten Qualitätskonzepts. Bielefeld: Bertelsmann, S. 41-54.

Schmidt-Lauff, Sabine (2008): Zeit für Bildung im Erwachsenenalter. Interdisziplinäre und empirische Zugänge. Münster: Waxmann.

Schütz, Alfred (1971): Gesammelte Aufsätze I. Das Problem der sozialen Wirklichkeit. Den Haag: Nijhoff.

Schütz, Alfred/Luckmann, Thomas (2017): Strukturen der Lebenswelt. 2. Auflage. Konstanz: UVK.

Seel, Hans-Jürgen (2014): Beratung. Reflexivität als Profession. Göttingen: Vandenhoeck \& Ruprecht.

Wahl, Johannes (2017): Lebenslanges Lernen zwischen Bildungspolitik und pädagogischer Praxis. Die Verankerung in pädagogischen Arbeitsfeldern. Bielefeld: Bertelsmann.

Wahl, Johannes/Nittel, Dieter/Tippelt, Rudolf (2014): Lebenslanges Lernen: Die Verankerung einer Wissens- und Institutionalisierungsform in den sozialen Welten pädagogisch Tätiger. In: Nittel, D./ Schütz, J./Tippelt, R. (Hg.): Pädagogische Arbeit im System des lebenslangen Lernens. Ergebnisse komparativer Berufsgruppenforschung. Weinheim: Beltz Juventa, S. 120-164.

Walther, Andreas (2016): Übergangsberatung. In: Gieseke, W./Nittel, D. (Hg.): Handbuch Pädagogische Beratung über die Lebensspanne. Weinheim: Beltz Juventa, S. 644-655.

Zirfas, Jörg (2011): Bildung. In: Kade, J./Helsper, W./Lüders, C./Egloff, B./Radtke, F.O./ Thole, W. (Hg.): Pädagogisches Wissen. Erziehungswissenschaft in Grundbegriffen. Stuttgart: Kohlhammer, S. 13-19. 



\section{Das Pilotprojekt Bildungsberatungs-Radar - Bildungsbarrieren sichtbar machen.}

\section{Einleitung}

Beratung eröffnet individuelle Perspektiven und gibt damit zugleich Aufschluss über gesellschaftliche Verhältnisse. Durch die Unterstützung der selbständigen Entscheidungsfähigkeit zielt Beratung auf neue Optionen und die Erweiterung von Autonomiespielräumen ab (vgl. Gieseke 2016: 31ff.). Den AkteurInnen in der Beratungspraxis sind jedoch vielfach Fälle bekannt, in denen trotz Eigeninitiative und Selbstbestimmung die getroffenen Entscheidungen aufgrund struktureller Hindernisse wie zum Beispiel fehlender Weiterbildungsangebote oder rechtlicher Einschränkungen nicht umgesetzt werden können. Die längerfristigen Wirkungen individueller Beratung werden durch den fehlenden Handlungsspielraum bei der Umsetzung der geplanten Handlungsschritte eingeschränkt oder verhindert. Besonders relevant sind diese Einschränkungen beispielsweise für geflüchtete Menschen im Asylverfahren, deren Alltag und Zugang zum Bildungssystem und Arbeitsmarkt rechtlich stark reglementiert ist. Auch Menschen, die sich weiterbilden oder beruflich verändern möchten, sind immer wieder mit einschränkenden Regelungen und Hindernissen im Bildungs- und Fördersystem konfrontiert. Individuelle Entscheidungen können nicht umgesetzt werden, weil beispielsweise bestimmte Förderbedingungen nicht zutreffen oder in Abhängigkeit vom Wohnort unterschiedliche rechtliche Voraussetzungen für eine Weiterbildung gelten. Für die BeraterInnen im Feld der Bildungs- und Berufsberatung ist diese Situation vielfach mit einem Gefühl der „Ohnmacht“ verbunden, da die sozialstrukturellen Rahmenbedingungen zunächst als unveränderlich erlebt werden. Die eigene Rolle wird dadurch auf das „Überbringen schlechter Nachrichten“ reduziert.

Dem stehen Beratungsansätze gegenüber, zu deren Selbstverständnis auch die Auseinandersetzung mit gesellschaftlichen Rahmenbedingungen und deren Veränderung gehört. Dies zeigte sich bereits in den Anfängen der Bildungs- und Berufsberatung in der Entwicklung feministischer Beratung. Auf institutioneller Ebene stand die Gründung der ersten Berufsberatungsstellen in Deutschland und Österreich in Verbindung zur Frauenbewegung, die sich gesellschaftlich und politisch für Bildungs- und Berufsrechte für Frauen einsetzte (Gröning 2010: 21f.; Gugitscher 2016: 3). Gröning (2010) rekonstruiert, dass die Frauenbewegung die Praxis und Theoriebildung pädagogischer Beratung durch ihren aufklärenden und emanzipativen Anspruch beeinflusst 
hat. In ihrer Analyse betont sie bei den ersten Beratungsstellen für Frauen die „ethischen Grundlagen von Parteilichkeit und Solidarität jenseits des Therapiemodells" (ebd.: 140) und sieht in diesen die gemeinsame gesellschaftskritische Fundierung pädagogischer und feministischer Beratung. Damit findet ein Perspektivenwechsel von einem defizitorientierten Beratungszugang zu einer kritischen Auseinandersetzung mit den gesellschaftlichen Verhältnissen und individuellen Lebensbedingungen statt. In ihrer Rekonstruktion der Entwicklungslinien feministischer Beratung wird besonders deutlich, wie aus der Beratungspraxis gewonnenes Wissen als Grundlage für die Formulierung von gesellschaftlichen Reformbedarfen genutzt wird.

In der Nachbardisziplin, der Sozialen Arbeit, wird im Ansatz der lebensweltorientierten sozialen Arbeit ebenfalls die Aufgabe, sich in die soziale und politische Diskussion zur Veränderung der gesellschaftlichen Bedingungen einzumischen, explizit formuliert. Das Prinzip der Einmischung wird verstanden ,als parteiliche Vertretung lebensweltlicher Erfahrungen und Probleme in z. B. Arbeitsmarkt-, Familien-, Sozial- und Wohnungsbaupolitik auf den unterschiedlichen politischen Ebenen von Bund, Ländern, Kommunen und Stadtteilen" (Grunwald/Thiersch 2008: 23). Auch in Beratungsfeldern wie der Anerkennungsberatung für Personen mit im Ausland erworbenen Qualifikationen, die mit restriktiven und oftmals einschränkenden rechtlichen Rahmenbedingungen konfrontiert sind, erfolgt vielfach eine aktive Auseinandersetzung mit den rechtlichen Rahmenbedingungen und deren Weiterentwicklung (vgl. Schmidtke 2017).

Während in diesen Ansätzen ein gesellschaftlicher Auftrag zum professionellen Selbstverständnis gehört, liegt der Schwerpunkt in der Bildungsund Berufsberatung auf der individuellen Wirkungsebene von Beratung. Aus erwachsenenpädagogischer Perspektive stehen die Prinzipien der „Hilfe zur Selbsthilfe" und der Respekt vor der individuellen Entscheidungsfähigkeit der KlientInnen im Sinne eines nicht-direktiven Vorgehens (vgl. Rogers 2007) im Mittelpunkt der Beratung. Entsprechend beschäftigt sich auch die Beratungsforschung primär mit dem Beratungskontext auf individueller Ebene, während die gesellschaftlichen Strukturen weiterhin ,ein randständiges Forschungsfeld" (Enoch 2011: 82) bleiben. Aus professionstheoretischer Perspektive gehören dagegen ein gesellschaftlich anerkanntes Selbstverständnis und die Übernahme gesellschaftlicher Aufgaben zu den Merkmalen einer professionellen Beratung (vgl. Seel 2013: 1646ff.). Es gibt in der Bildungs- und Berufsberatung bisher jedoch kaum systematische Möglichkeiten, um auf überindividueller Ebene aus dem Verständnis einer gesellschaftlich anerkannten Position zu intervenieren. Direkte Einwirkungen auf die sozialstrukturellen Rahmenbedingungen beschränken sich auf individuelles Handeln von BeraterInnen, wenn beispielsweise über einen Anruf direkt Kontakt zur Behörde aufgenommen wird. Es sind gerade diese Interventionen, welche aus erwachsenenpädagogischer Perspektive zugleich in einem Spannungsverhältnis zur 
emanzipativen Zielsetzung einer Handlungsermächtigung stehen. Vor diesem Hintergrund ist die Idee für das Pilotprojekt Bildungsberatungs-Radar entstanden. Die Anforderung an professionelle Beratung, sich gesellschaftlich zu positionieren, wird umgesetzt, indem das Beratungssystem als Radar verstanden wird, welches aktuelle Entwicklungen, Bedarfe und strukturelle (Weiter-)bildungshindernisse „ortet“ und an das System der Erwachsenenbildung zurückmeldet. In diesem Beitrag wird zunächst ein Einblick in die Projektstruktur, die Modellbildung und die praktische Umsetzung des Bildungsberatungs-Radars gegeben. Darauf aufbauend werden die ersten Ergebnisse und Erfahrungen aus der Pilotphase hinsichtlich der Definition von gesellschaftlichen Aufgaben professioneller Bildungs- und Berufsberatung und wissenschaftlicher (Begleit-)Forschung dargestellt.

\section{Projektstruktur}

Die Idee für den Bildungsberatungs-Radar wurde 2014 im Netzwerk Bildungsberatung Oberösterreich ${ }^{1}$ entwickelt. Seit 2015 erfolgt die Umsetzung als ein Pilotprojekt mit wissenschaftlicher Begleitung. Methodisch wurde der Schwerpunkt auf ein partizipatives Forschungsdesign gelegt. Unter der Projektleitung der Arbeiterkammer Oberösterreich (gesetzliche Interessenvertretung der ArbeitnehmerInnen) wird das Projekt gemeinsam von den Beratungseinrichtungen des Netzwerks ${ }^{2}$ und der wissenschaftlichen Begleitung ${ }^{3}$ durchgeführt. Mit der zyklischen Abfolge von Aktion und Reflexion orientiert sich die Projektstruktur an der Aktionsforschung (vgl. Lewin 1946; Unger 2014: 51ff.). Die erhobenen Daten werden in einer kollektiven Wissensbasis gesammelt und anschließend gemeinsam im Team aus BeraterInnen, ProjektleiterInnen und wissenschaftlicher Begleitung ausgewertet und analysiert. Die Dokumentation der Ergebnisse erfolgt über schriftliche Fallanalysen, welche als Intervention in Form von Handlungsempfehlungen an relevante AdressatInnen übermittelt werden (vgl. Dworschak et al. 2016: 3).

1 Das Netzwerk Bildungsberatung Oberösterreich gehört zu der vom Bundesministerium für Bildung, Wissenschaft und Forschung, dem Europäischen Sozialfonds und aus Ländermitteln geförderten Initiative Bildungsberatung Österreich.

2 Die beteiligten Beratungseinrichtungen sind: ALOM - Verein für Arbeit und Lernen Oberes Mühlviertel, Berufsförderungsinstitut Oberösterreich, Frauenstiftung Steyr/Verein Frauenarbeit Steyr, Migrare - Zentrum für MigrantInnen Oberösterreich, Volkshochschule Oberösterreich und Wirtschaftskammer Oberösterreich.

3 Die wissenschaftliche Begleitung besteht aus LISA - Linzer Institut für soziale Analysen und dem Österreichischen Institut für Berufsbildungsforschung (öibf) in Kooperation mit Prof. Carola Iller, Stiftung Universität Hildesheim. 


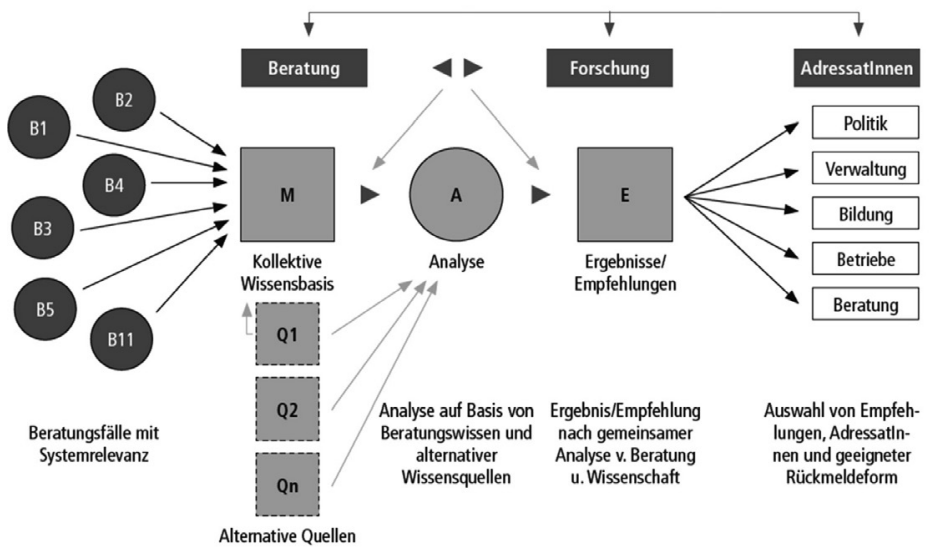

Abb. 1: Das Ablaufmodell des Bildungsberatungs-Radars (Quelle: Dworschak et al. 2016: 4)

\subsection{Aufbau einer kollektiven Wissensbasis}

In einem ersten Schritt werden in Anlehnung an die Datensammlung in der Aktionsforschung durch die auf dem Bildungsberatungs-Radar geschulten BeraterInnen Beratungsfälle in einem vorstrukturierten webbasierten Tool dokumentiert. Der Fokus liegt dabei auf Fällen mit möglicher Systemrelevanz. Als systemrelevante Fälle werden Beratungsfälle bezeichnet, die auf strukturelle Defizite oder Hindernisse hinweisen und die auf individueller Ebene bzw. durch ein Beratungsgespräch nicht gelöst oder beeinflusst werden können. Neben der Selbsteingabe der systemrelevanten Beratungsfälle durch die BeraterInnen wurde während der Pilotphase seitens der wissenschaftlichen Begleitung auch das Angebot gemacht, mögliche Fälle mittels leitfadengestütztem qualitativem Interview zu erheben. Der dabei verwendete Leitfaden orientierte sich am Eingaberaster des webbasierten Erfassungstools im Bib-Radar. Im Anschluss an die Interviews wurden diese transkribiert und die mit den BeraterInnen gemeinsam definierten systemrelevanten Fälle von den ForscherInnen in das Erfassungstool eingepflegt. Die eingegebenen anonymisierten Fälle können über eine Kommentarfunktion um bereits recherchierte oder mögliche Quellen und Hinweise ergänzt werden. Voraussetzung für den Aufbau einer kollektiven Wissensbasis sind neben den strukturellen Voraussetzungen entsprechende zeitliche Ressourcen und Kompetenzen der BildungsberaterInnen sowie die individuelle Bereitschaft zu einer kooperativen Zusammenarbeit auf überinstitutioneller Ebene und im Projektkontext. 


\subsection{Analyse ausgewählter Fälle}

Die eigentliche Identifikation und Analyse der systemrelevanten Fälle erfolgt gemeinsam im Kontext einer Team- oder Fallbesprechung. Die Auswahl der eingegebenen Fälle orientiert sich an einem gemeinsam vereinbarten Kriterienraster, das die Kriterien quantitative Einschätzung betroffener Personen, räumliche Reichweite (Landes- oder Bundesebene), mit dem Fall verbundene Motivation bzw. Herausforderung und Ideen für konkrete Handlungsempfehlungen beinhaltet. In der Pilotphase wurden die Fälle zudem in Bezug auf die thematisierten Rahmenbedingungen den Ebenen „institutionelle Praxis“, „Angebotsstruktur" und „gesetzlicher Rahmen“" zugeordnet. Damit ist die Annahme verbunden, dass mit den unterschiedlichen Strukturebenen auch die Entwicklung spezifischer Formen von Rückmeldungen notwendig ist. In der Pilotphase erfolgte die gemeinsame Analyse exemplarisch für einen Fall auf jeder Strukturebene. Zu den ausgewählten Fällen gehörten die fehlende Transparenz bei spezifischen Prüfungsanforderungen im zweiten Bildungsweg, bundeslandspezifische Unterschiede in den Voraussetzungen für die außerordentliche Lehrabschlussprüfung und der fehlende Zugang bestimmter Berufsgruppen zur Berufsreifeprüfung und damit zu einer akademischen Qualifikation.

\subsection{Entwicklung von Rückmeldeverfahren als Interventionen}

Eine besondere Herausforderung stellt im Projekt die Identifizierung von AdressatInnen der Handlungsempfehlungen und von Kommunikationswegen dar. Im Pilotprojekt wurden unterschiedliche Rückmeldeverfahren von schriftlicher bis persönlicher Kontaktaufnahme in Abhängigkeit von den AdressatInnen und vom eigenen institutionellen Hintergrund diskutiert und entwickelt. Eine wichtige Bedeutung kommt dabei immer der Darstellung über einzelfallorientierte „Stories“ zu, die anschließend hinsichtlich der strukturellen Faktoren analysiert werden. Dabei ist zu berücksichtigen, dass es sich bei den eingebrachten Fällen oftmals um sehr aktuelle oder emotional belastende Themen handelt. Der Prozess von der gemeinsamen Identifikation und Analyse des Falls bis hin zur Entwicklung von Handlungsempfehlungen und Rückmeldeformen ist dagegen ein zeitlich längerfristiger. Auch die Übermittlung und Einbindung von AdressatInnen kann längere Zeit in Anspruch nehmen. Nachdem drei Fälle ausgewählt und bearbeitet wurden, ist das Projekt jetzt auf die Rückmeldungen der AdressatInnen angewiesen. Während einige AdressatInnen großes Interesse und Offenheit für die Erfahrungen und Ergebnisse aus dem Bildungsberatungs-Radar zeigen und vor allem die spezi- 
fische Expertise der BeraterInnen wertschätzen, wird in anderen Fällen auch eine entmutigende Rückmeldung und Abhängigkeit von der Bereitschaft der AdressatInnen deutlich. Für die künftige Weiterentwicklung des Projekts ist daher eine frühzeitige Einbindung relevanter AkteurInnen aus dem System des Lebenslangen Lernens anzudenken. An den unterschiedlichen Erfahrungen des Pilotprojekts zeigt sich, dass neben der Übermittlung an AdressatInnen zusätzliche Öffentlichkeitsarbeit wie beispielsweise ein Jahresbericht oder eine quartalsweise Darstellung ausgewählter Fälle in Fachzeitschriften sinnvoll wäre. Hinsichtlich der Entwicklung eines gemeinsamen Beratungsverständnisses und der Sensibilisierung für die gesellschaftliche Rolle von Beratung lassen sich aus der Pilotphase jedoch bereits erste Ergebnisse formulieren.

\section{Aufgaben- und Rollenklärung professioneller Beratung}

Während der Umsetzung des Pilotprojektes hat sich auch außerhalb des Landesnetzwerkes im Feld der Bildungs- und Berufsberatung ein großes Interesse an dem Projekt gezeigt. Die Regelungen und Grenzen im Bildungs- und Fördersystem und die einrichtungsübergreifende Reflexion der gesellschaftlichen Rolle und Position von Bildungsberatung sind demnach Themen, welche die BeraterInnen in ihrer alltäglichen Beratungsarbeit beschäftigen. Im Mittelpunkt steht dabei das Bedürfnis, der „Ohnmacht“ individueller Beratung entgegenzuwirken, indem das in der Beratung generierte Wissen wirksam auf struktureller Ebene weitergegeben und damit die eigene Handlungsfähigkeit und die der KlientInnen erweitert wird. Aus beratungstheoretischer Perspektive greift das Pilotprojekt durch die Verständigung über einen gemeinsamen Beratungsbegriff und die Formulierung eines klaren Selbstverständnisses bzw. einer transparenten Rolle und Aufgabe in der Gesellschaft die wissenschaftliche Debatte zur Professionalisierung auf. Nachfolgend werden die Erfahrungen aus dem Pilotprojekt hinsichtlich dieser Entwicklungen reflektiert und die Ergebnisse in Bezug auf die Professionalisierung von Beratung dargestellt.

Der Bildungsberatungs-Radar unterstützt zunächst als gemeinschaftlich entwickeltes und durchgeführtes Projekt die überinstitutionelle Zusammenarbeit zwischen den Einrichtungen, welche in Österreich in Netzwerken strukturiert wird. Die Identifikation von Fällen mit Systemrelevanz, die Analyse und die Entwicklung von Handlungsempfehlungen setzt ein gemeinsames Beratungsverständnis und übereinstimmende Zielsetzungen voraus, welche für jeden Fall neu verhandelt und konkretisiert werden müssen. Sultana (2017) beschreibt für einen emanzipatorischen Ansatz in der Karriereberatung die 
Verpflichtung für Mitglieder einer Profession „einen Standpunkt zu beziehen, der sich grundlegend der sozialen Gerechtigkeit verpflichtet sieht" (ebd.: 27). Das Pilotprojekt macht deutlich, dass dieser gemeinsame Standpunkt im Feld der Bildungs- und Berufsberatung nicht als gegeben vorausgesetzt werden kann. Stattdessen sind systematische Kommunikationsstrukturen erforderlich, um eine kritische Auseinandersetzung mit den „Paradoxien des Feldes“ (Enoch 2013: 191) zu ermöglichen. In Abhängigkeit von den institutionellen Anforderungen, den Erwartungen der KlientInnen und den Ansprüchen der BeraterInnen können unterschiedliche Zielsetzungen auf struktureller Ebene verfolgt werden. Es kann sich dabei um eine anwaltschaftliche Intervention im Interesse der KlientInnen handeln, um die (Wieder-)Herstellung sozialer Gerechtigkeit für eine größere Gruppe von Betroffenen oder um die Verbesserung zukünftiger Beratungssituationen durch die Schaffung systematischer Wissensbestände für die BeraterInnen. Obwohl die Zielsetzungen größtenteils übereinstimmen oder sich gegenseitig beeinflussen, zeigt sich aus der Erfahrung der Pilotphase auch ein möglicher Widerspruch zwischen diesen Zielen. Die Komplexität der Aushandlung von Zielsetzungen wird beispielsweise deutlich, wenn eine Angleichung von gesetzlichen Regelungen in verschiedenen Bundesländern in Einzelfällen auch zum Nachteil von KlientInnen führen kann. Voraussetzung für die Formulierung von gemeinsamen Zielsetzungen ist eine systematische Einbindung von Reflexion als ein mehrstufiges Verfahren von der Fallbesprechung über die Supervision bis zur fachöffentlichen Diskussion. Die wissenschaftliche Begleitung bildet hier eine zusätzliche Reflexionsebene, die unabhängig vom Handlungsdruck der konkreten Beratungssituation neue Perspektiven eröffnen kann (vgl. Schrödter 2014: 811f.). Der Bildungsberatungs-Radar bietet durch den zyklischen Forschungsprozess von Reflexion und Aktion eine Struktur für die Entwicklung eines überinstitutionellen Selbstverständnisses und von Zielsetzungen im Feld der Bildungs- und Berufsberatung, welche aufgrund der heterogenen Angebotsstruktur nicht selbstverständlich sind.

Seel (2013) beschreibt die Entwicklung einer anerkannten gesellschaftlichen Rolle und Position von Beratung als eines der größten Problemfelder in der Professionalisierung von Beratung. Eine Profession setzt demnach auch die Übernahme von gesellschaftlichen Aufgaben voraus (vgl. ebd.: 1653). Der Bildungsberatungs-Radar ermöglicht eine gesellschaftliche Positionierung, indem er über die individuelle Ebene zur Unterstützung der Handlungs- und Entscheidungsfähigkeit hinausgeht und stattdessen die gesellschaftlichen und strukturellen Bedingungen von Beratung fokussiert. Dieser kritische Ansatz entspricht der Zielsetzung der Aktionsforschung, konkrete Veränderungen in der Praxis zu bewirken (vgl. Bortz/Döring 2003: 345). Die Erfahrungen aus dem Pilotprojekt zeigen, dass damit jedoch auch neue Aufgaben und Herausforderungen der Abgrenzung für die BeraterInnen verbunden sind. Besonders deutlich wird die Notwendigkeit von klaren Grenzziehungen bei den Rück- 
meldungen an relevante AkteurInnen als Intervention. Zur „Entlastung“ der BeraterInnen übernimmt hier die wissenschaftliche Begleitung eine wichtige Funktion durch die Aufbereitung und Kommunikation der Ergebnisse. Entsprechend dem methodischen Zugang der Aktionsforschung ist damit auch ein verändertes, emanzipatorisches Verständnis der wissenschaftlichen Forschung verbunden (vgl. ebd.: 344). Während in Österreich die Beratungsforschung bisher vor allem Aufgaben in der Begleitung der Beratungspraxis mit Schwerpunkt auf der Wissensaufbereitung und Ausarbeitung begrifflicher Differenzierungen übernommen hat (vgl. Gugitscher et al. 2018: 144f.), erhält sie dadurch eine explizit gesellschaftskritische Rolle:

„Notwendig ist dafür eine Beratungswissenschaft, die sich nicht darauf beschränkt, Beratung zu optimieren, sondern darüber hinaus aus Beratungserfahrungen gesellschaftliche Problemlagen systematisch herausarbeitet, d. h. Erfahrungen aus den einzelnen, individuellen Beratungskommunikationen im Sinne einer gesellschaftskritischen Analyse auf der Makroebene verwertet und in politische Diskurse über die Möglichkeiten gelingender Lebensgestaltung einfüttert“" (Seel 2013: 1658).

Wissenschaftliche Forschung setzt in dem Pilotprojekt an der Schnittstelle $\mathrm{zu}$ den gesellschaftlichen Bedingungen an und stellt eine Verbindung zu den AdressatInnen und zur (Fach-)Öffentlichkeit her. Die Begleitforschung wird hier zu einer kritischen Beratungsforschung, indem Beratungsrealität durch die gemeinsame Analyse in strukturelle Zusammenhänge eingeordnet und Bildungs- und Berufsberatung in ihrer gesellschaftlichen Funktion als „Aufklärungsinstanz" (Enoch 2013: 194) nicht nur auf individueller, sondern auch auf struktureller Ebene unterstützt wird (Hofer/Wimplinger 2017: 23f.).

\section{Ausblick}

Der Bildungsberatungs-Radar zeigt auf, wie durch die Übernahme gesellschaftlicher Aufgaben im Sinne eines „Ortungs- und Erkennungsverfahrens“ das durch die Beratung generierte Wissen und die Expertise der BeraterInnen für das System der Erwachsenenbildung nutzbar gemacht werden können. Die Erfahrungen aus dem Pilotprojekt machen jedoch zugleich deutlich, wie wichtig eine transparente Rollen- und Aufgabenklärung für die Umsetzung einer professionellen Beratung ist. Die Beratungsforschung erhält hier einen explizit gesellschaftskritischen Auftrag. Das Pilotprojekt zielt demnach nicht auf ein zusätzliches Aufgabengebiet von BeraterInnen im Feld der Bildungsund Berufsberatung ab, sondern auf eine Auseinandersetzung mit der eigenen professionellen Rolle innerhalb der Rahmenbedingungen und der bewussten Abgrenzung einer gesellschaftskritischen Bildungs- und Berufsberatung gegenüber Formen der Organisations- oder Politikberatung. 
Aus erwachsenenpädagogischer Perspektive bietet das Projekt Bildungsberatungs-Radar mehrere Anknüpfungspunkte für weitere Forschungen. Der Bildungsberatungs-Radar richtet den Blick auf strukturelle Bildungsbarrieren und gesellschaftliche Ausgrenzungsmechanismen und stellt damit ein zusätzliches Evaluierungs- und Qualitätsförderungsinstrument für ein lernendes System der Erwachsenenbildung dar. Insbesondere für Weiterbildungseinrichtungen kann der Bildungsberatungs-Radar nützliche Informationen über die Nachfrage von Weiterbildungen oder Hindernisse für die Inanspruchnahme von Angeboten zur Verfügung stellen. AdressatInnen des Bildungsberatungs-Radars stellen jedoch nicht nur Politik, Verwaltung, Bildung und Betriebe dar, sondern auch das Beratungssystem selbst, welches kritisch hinterfragt und weiterentwickelt wird.

Der Bildungsberatungs-Radar steht nicht zuletzt für einen Perspektivenwechsel, indem Beratung als Generierung von zielgruppenspezifischem Wissen verstanden wird. Der strukturierte Prozess des Bildungsberatungsradars ermöglicht damit auch einen Rahmen für die überinstitutionelle Systematisierung von Wissensbeständen. Durch die Dokumentation und Aufbereitung bestehender und neuer Wissensbestände erfolgt eine Anerkennung der spezifischen Expertise der BeraterInnen. Der Bildungsberatungs-Radar stellt damit eine mögliche Lernquelle für BildungsberaterInnen dar. Mit Bezug auf die von Käpplinger und Maier-Gutheil (2015) formulierten Herausforderungen für die Beratungsforschung (vgl. ebd.: 178) könnte der Bildungsberatungs-Radar damit eine systematische und kontinuierliche Verbindung von Forschungsergebnissen und der Aus- und Fortbildung von BeraterInnen ermöglichen. Voraussetzung hierfür bleiben jedoch Förderkontexte, die den Freiraum für innovative Entwicklungen aus der Beratungspraxis heraus ermöglichen.

\section{Literatur}

Bortz, Jürgen /Döring, Nicola (2003): Forschungsmethoden und Evaluation für Human- und Sozialwissenschaftler. 3. Auflage. Heidelberg: Springer Verlag.

Dworschak, Helmut/Hofer, Gerhard/Iller, Carola/Lehner, Roland/Schmidtke, Birgit/ Wimplinger, Johanna (2016): Das Pilotprojekt „Bildungsberatungs-Radar“. In der Beratung generiertes Wissen nützen und weitergeben. In: Magazin erwachsenenbildung.at. Das Fachmedium für Forschung, Praxis und Diskurs, Ausgabe 29, Thema 13, S. 1-6.

Enoch, Clinton (2011): Dimensionen der Wissensvermittlung in Beratungsprozessen. Gesprächsanalysen der beruflichen Beratung. Wiesbaden: VS Verlag für Sozialwissenschaften.

Enoch, Clinton (2013): Beratung als Aufklärungsinstanz des Individuums - demokratietheoretische Aspekte einer kritischen Beratungstheorie. In: Käpplinger, 
Bernd/Robak, Steffi/Schmidt-Lauff, Sabine (Hg.): Engagement für die Erwachsenenbildung. Ethische Bezugnahmen und demokratische Verantwortung. Wiesbaden: Springer Fachmedien, S. 187-195.

Gieseke, Wiltrud (2016): Beratung über die Lebensspanne: Zwischen Steuerung, neuen Optionen und Erweiterung von Autonomiespielräumen - Wechselwirkungen. Gieseke, Wiltrud/Nittel, Dieter (Hg.): Handbuch Pädagogische Beratung über die Lebensspanne. Weinheim, Basel: Beltz Juventa, S. 31-41.

Gröning, Katherina (2010): Entwicklungslinien pädagogischer Beratungsarbeit: Anfänge - Konflikte - Diskurse. Wiesbaden: VS Verlag für Sozialwissenschaften.

Grunwald, Klaus/Thiersch, Hans (2008): Praxis lebensweltorientierter sozialer Arbeit. Handlungszugänge und Methoden in unterschiedlichen Arbeitsfeldern. 2. Auflage. Weinheim: Juventa-Verlag.

Gugitscher, Karin (2016): Bildungs- und Berufsberatung in Österreich historisch betrachtet. Vom Fürsorgewesen über individualisierte Bildungsberatung zur lebensbegleitenden Beratung und Kompetenzvermittlung. In: Magazin erwachsenenbildung.at. Das Fachmedium für Forschung, Praxis und Diskurs. Ausgabe 29, 2016. Wien. Thema 03, S. 1-13.

Gugitscher, Karin/Schmidtke, Birgit/Schlögl, Peter/Kellner, Wolfgang (2018): Von frühen Formen der Beratung über deren Professionalisierung hin zu aktuellen Forschungs- und Entwicklungsfeldern. Ein Überblick zu Beratung, Beratungsangeboten und Beratungsforschung in der österreichischen Erwachsenen- und Weiterbildung. In: Holzer Daniela, Dausien Bettina, Schlögl Peter, Schmid Kurt (Hg.): Forschungsinseln. Beobachtungen aus der österreichischen Erwachsenenbildungsforschung. Münster u. a.: Waxmann (in Veröffentlichung), S. 133-149.

Hofer, Gerhard/Wimplinger Johanna (2017): „Wenn ihr wüsstet, was ihr wisst!“ Aus der Praxis des Bildungsberatungsradars. In: Bildungsberatung im Fokus. Bildungsberatung wirkt. 2/2017, S. 23-25.

Käpplinger, Bernd/Maier-Gutheil, Cornelia (2015): Ansätze und Ergebnisse zur Beratung(sforschung) in der Erwachsenen- und Weiterbildung - Eine Systematisierung. In: Zeitschrift für Weiterbildungsforschung - Report (38), S. 163-181.

Lewin, Kurt (1946): Action research and minority problems. In Kurt Lewin/Gertrud Weiß Lewin (Hg.): Resolving social conflicts. New York: Harper \& Brothers, S. 201-216.

Rogers, Carl R. (2007): Die nicht-direktive Beratung. Counseling and Psychotherapy. 12. Aufl. Frankfurt: Fischer

Schmidtke, Birgit (2017): Anerkennungsberatung. Anerkennungstheoretische Begründung pädagogischer Praxis. Diss. Linz: Johannes Kepler Universität Linz / Institut für Pädagogik und Psychologie.

Schrödter, Wolfgang (2014): Beratungsforschung. In: Frank Nestmann, Frank Engel und Ursel Sickendiek (Hg.): Das Handbuch der Beratung. Band 2. Ansätze, Methoden und Felder. 3. Auflage. 3 Bände. Tübingen: dgvt-Verlag, S. 809-824.

Seel, Hans-Jürgen (2013): Aufgaben und Probleme der Professionalisierung von Beratung. In: Frank Nestmann, Frank Engel und Ursel Sickendiek (Hg.): Neue Beratungswelten. Fortschritte und Kontroversen. Tübingen: dgvt-Verlag, S. 16451661.

Sultana, Ronald G. (2017): Laufbahnberatung und der Gesellschaftsvertrag in einer flüchtigen Welt. Übersetzung: Frank Nestmann/Bearbeitung: Ursel Sickendiek. 
In: Marika Hammerer, Erika Kanelutti-Chilas, Gerhard Krötzl, Ingeborg Melter (Hg.): Zukunftsfeld Bildungs- und Berufsberatung IV. Schwierige Zeiten - Positionierungen und Perspektiven. Bielefeld: W. Bertelsmann-Verlag, S. 17-30.

von Unger, Hella (2014): Partizipative Forschung. Einführung in die Forschungspraxis. Wiesbaden: Springer VS. 

V. Beratung in unterschiedlichen Altersund Lebensphasen:

Bedarfe - Anlässe - Übergänge 



\section{Beratung in beruflichen Übergängen als Reflexionsraum für und Initiierung von Lernprozessen}

Berufliche Übergänge im Sinne von Berufs-, Betriebs- und Funktionswechseln innerhalb des Erwerbsverlaufs werden bisher in der Übergangsforschung nur wenig betrachtet. ${ }^{1}$ Dabei hat die Zahl der Wechsel innerhalb der Erwerbsphasen merklich zugenommen und Flexibilisierungstendenzen führen auch innerhalb der Stammbelegschaften zu Diskontinuitätserfahrungen. Zudem steigt der Anteil an Beschäftigungswechseln, die mit Verschlechterungen in der Art der Tätigkeit einhergehen (vgl. Trischler/Kistler 2010: 36). Die erwachsenenpädagogische Übergangsforschung fordert ein „Denken in Übergängen“ für eine professionelle Entwicklungsbegleitung im Kontext lebenslangen Lernens (vgl. Felden/Schäffter/Schicke 2014: 15). Sie zielt daher ,,auf Übergangszeit als einem [sic!] persönlich bedeutsamen Entwicklungs- und Bildungsprozess ab, der nach einer lernförderlichen Supportstruktur verlangt" (ebd.: 13). Reflexive Übergangsberatung stellt ein Format einer lernförderlichen Supportstruktur dar, was im Folgenden am Beispiel des Praxisforschungsprojekts „,Spurwechsel - Gestaltung von Übergängen in der Bildungs- und Erwerbsbiografie betrieblicher Interessenvertreter/innen“ ${ }^{\text {“2 }}$ dargestellt wird.

Die Bildungs- und Berufsbiografien von betrieblichen Interessenvertreter*innen (Mitgliedern von Betriebs- und Personalräten und Mitarbeitendenvertretungen) ist zunehmend von Brüchen, Ausstiegen, Umstiegen und Wiedereinstiegen und damit von Übergängen geprägt (vgl. Tietel/Hocke 2015). Betriebsrats- und Personalratsarbeit wurde bisher in erster Linie als politisches Ehrenamt betrachtet. Für freigestellte Betriebsratsmitglieder wird sie zugleich zur Hauptbeschäftigung und damit eine eigene Phase in der Erwerbsbiografie. Aufgrund wirtschaftlicher und betrieblicher Veränderungen finden Übergänge wider Willen statt bzw. wird Betriebsratsarbeit von vornherein als eine zeitlich begrenzte Phase verstanden und nach einer gewissen Zeit ein beruflicher Wechsel angestrebt. Betriebsratsarbeit stellt aufgrund

1 Als exemplarisch für die Unterbelichtung dieses Themas kann angesehen werden, dass in dem umfassenden „Handbuch Übergänge“ (Schröer u. a. 2013) mit seinen 54 Artikeln lediglich ein Artikel zu finden ist, der berufliche Übergänge thematisiert: „Innerbetriebliche Übergänge“" (Ebbers/Langanka 2013).

2 Durchgeführt am Zentrum für Arbeit und Politik Universität Bremen, Laufzeit Januar 2015 bis August 2017, gefördert von der Hans-Böckler-Stiftung. 
komplexer werdender Problemstellungen, damit wachsenden Anforderungen und gesetzlich geregelter Lernzeitansprüche auch eine intensive Lern- und Weiterbildungsphase dar. Von diesen Entwicklungen ausgehend stellt sich die Frage, wie die entstehenden Übergänge gelingend gestaltet werden können.

Nach der Vorstellung des Projekts Spurwechsel und des Forschungsdesigns thematisiert der Beitrag auf der Grundlage methodenspezifischer Wirkfaktoren und eines subjektwissenschaftlichen Lernverständnisses empirische Fallbeispiele mit Beratungsanlass, Methoden und Ergebnissen zur Reflexion und Initiierung von Lernprozessen.

\section{Projekt Spurwechsel und Forschungsdesign}

Ausgangspunkt des Projekts ist die Stärkung der Perspektive, dass betriebliche Interessenvertretung eine berufs- und bildungsbiografische Phase ist, deren Übergangskonstellationen aktiv zu gestalten sind. Es wurden unter anderem Bildungs- und Beratungsprozesse mit betrieblichen Interessenvertreter*innen, die sich in beruflichen Übergängen befinden, durchgeführt und evaluiert (vgl. Hocke 2018). Hierbei wurde ein reflexives Beratungsverständnis zugrunde gelegt. Reflexive Beratung definiert Pongratz als „Entwicklung von Problemlösungen (Beratung) oder von Problemlösefähigkeit (Bildung) durch dialogische Aufklärung der eigenen Verwicklungen und Verstrickungen in aktuelle und frühere Handlungskonstellationen und Beziehungsmuster" (2011: 32). Danach kann reflexive Beratung unterschieden werden von Wissensvermittlung und Expertenberatung, aber auch von Selbsthilfe und informellem Lernen (vgl. ebd.).

Im Fokus des vorliegenden Beitrags steht die Frage: Welche Wirkfaktoren werden in den Beratungen mit welchen Methoden aktiviert und inwiefern werden damit Lernprozesse für eine gelingende Übergangsgestaltung gefördert? Rekurriert wird auf die empirischen Ergebnisse der Pilotierung und Evaluation der Beratungsprozesse. Insgesamt wurden mit 10 Interessenvertreter*innen Übergangsberatungen im Umfang von 67 Beratungsgesprächen konzipiert, durchgeführt und evaluiert. Das Forschungsdesign umfasste mehrere Schritte:

1. Sondierungsgespräch/Interview: Die Erhebung der Beratungsanliegen, der speziellen Herausforderungen und der Kontextbedingungen der Beratungsteilnehmenden erfolgte mittels leitfadengestützter fokussierter Interviews (vgl. Hopf 2004). Die Interviews waren biografisch angelegt und stellten damit zugleich eine Methode in der Berufswegberatung dar (vgl. Mörth/Söller 2011: 169ff.). Die Sondierungsgespräche dienten der 
Datensammlung und bildeten die Grundlage für die Beratungsvereinbarung (vgl. Rappe-Giesecke 2009). Die Interviews wurden transkribiert, verdichtet und inhaltsanalytisch ausgewertet.

2. Beratungsvereinbarung mit Beratungszielen und -anliegen: Auf Grundlage der herausgearbeiteten Anliegen und Themen erfolgte eine personenspezifische Konzeption des Beratungsverlaufs. Die Beratungsanliegen und der geplante Beratungsverlauf wurden auf Moderationskarten visualisiert und im ersten Beratungsgespräch mit den Teilnehmenden besprochen sowie gegebenenfalls angepasst. Diese sind Bestandteil der Beratungsvereinbarung, die Beratende und Teilnehmende abschließen.

3. Begleitende Dokumentation und Evaluation: Jedes durchgeführte Beratungsgespräch wurde von der Beraterin und von der bzw. dem Teilnehmenden mittels eines Auswertungsbogens reflektiert, dokumentiert und bewertet. Diese fortlaufende Prozessevaluation floss direkt in die weitere Planung des Beratungsverlaufs ein.

4. Evaluierendes Interview mittels Strukturlegetechnik (in Anlehnung an Scheele/Groeben 1988; Greif/Runde/Seeberg 2004): Vier bis sechs Wochen nach dem letzten Beratungsgespräch wurde ein leitfadengestütztes evaluierendes Interview durchgeführt, um subjektive Bewertungsmerkmale und Erfolgsfaktoren zu erheben. Im Fokus standen der subjektiv hergestellte Zusammenhang zwischen Beratungsprozess und Übergangsgestaltung sowie der Zusammenhang zwischen Beratungsanliegen und angewendeten Methoden. Hierzu legten die Teilnehmenden ihr subjektives Strukturbild aus den Anliegen-Karten des Sondierungsgesprächs und den Methodenkarten des Beratungsprozesses. Zudem wurden die evaluierenden Interviews nach methodenspezifischen Wirkfaktoren ausgewertet, um Zusammenhänge zwischen den verwendeten Methoden und der subjektiven Anliegenbearbeitung beschreiben zu können.

Die Sondierungsgespräche und die Beratungsvereinbarung wurden verwendet, um eine teilnehmenden- und anliegenorientierte Beratung sicherzustellen und die abschließende Evaluation auf Grundlage vorher vereinbarter Ziele durchführen zu können. Die Wahl der Beratungsmethoden erfolgte auf der Basis einer vorangehenden Literaturrecherche zu vorhandenen Methoden der Berufswegberatung, der Karriereberatung und der Kompetenzbilanzierung sowie konkret anhand der Beratungsanliegen. Hierbei fand eine zielgruppenspezifische Anpassung statt.

Durch die Projektkonzeption entstand eine Doppelrolle von Beraterin und Forscherin. Unterschiedliche Erwartungen an die jeweilige Rolle führten zu Spannungen und Herausforderungen, z. B. als Forscherin bestimmte Methoden auszuprobieren und als Beraterin an aktuellen Anliegen der Beratenen zu arbeiten. Die zeitnahe Reflexion und Bearbeitung der entstandenen Spannungsfelder fand in einer projektbegleitenden externen Forschungssuper- 
vision statt. Dadurch konnten die herausgearbeiteten Aspekte für den Beratungs- und Forschungsprozess fruchtbar gemacht werden.

Sinnvoll wäre darüber hinaus, eine wiederholte Evaluation nach erfolgtem Übergang durchzuführen, um längerfristige Wirkungen der Beratungsprozesse auf die Übergangsgestaltung erheben zu können. Dies war wegen des zeitlich begrenzten Projektrahmens nicht möglich.

\section{Das Konzept der Wirkfaktoren}

Wirkfaktoren bezeichnen „Merkmale, die im allgemeinen [sic!] in der Interaktion zwischen Klient, Coach und ihrer Umgebung realisiert und aufrechterhalten werden müssen, damit das Coaching zum Erfolg führt" (Greif 2008: 278). Die Herleitung der Wirkfaktoren erfolgt aus psychotherapeutischen Wirksamkeitsuntersuchungen, in denen allgemeine und schulenübergreifende Wirkfaktoren untersucht werden, von denen der Erfolg einer Therapie abhängt (vgl. Greif/Schmidt/Thamm 2012). Diese Erkenntnisse werden auf das Coaching übertragen und angepasst. Coaching nach Greif ist „,eine intensive und systematische Förderung der Reflexionen und Selbstreflexionen sowie Beratung von Personen oder Gruppen zur Verbesserung der Erreichung selbstkongruenter Ziele oder zur bewussten Selbstveränderung und Selbstentwicklung" (Greif 2008: 69). Coaching in diesem Sinne stellt ein reflexives Beratungsformat dar. ${ }^{3}$

Greif/Schmidt/Thamm (2012) benennen sieben Coaching-Wirkfaktoren. Diese Wirkfaktoren arbeitet Wechsler (2012) methodenspezifisch aus. Im Projekt Spurwechsel dienten die methodenspezifischen Wirkfaktoren als Auswertungskategorien für den Teilbereich der Methodenevaluation. ${ }^{4}$ Daher werden nachfolgend die beim Einsatz von Beratungsmethoden relevanten Wirkfaktoren kurz erläutert (vgl. Wechsler 2012: 14ff.):

- Ergebnisorientierte Selbstreflexion: Reflexionen zu Vorstellungen und Handlungen mit Bezügen zum realen und idealen Selbstkonzept sowie Ableitung von Folgerungen

3 Im Projekt Spurwechsel wurden die Begriffe reflexive Übergangsberatung und Übergangscoaching synonym verwendet.

4 Die Methodenevaluation stellt nur einen Teilbereich der empirischen Ergebnisse dar. Weitere Ergebnisse bestehen zu Beratungshaltung und -kompetenz sowie zu Beratung in unterschiedlichen Übergangskonstellationen. Zu Letzterem wurden Falldarstellungen erstellt (vgl. Hocke 2018). 
- Ergebnisorientierte Problemreflexion: Analysen und Reflexionen zu nicht selbstkonzeptbezogenen Problemen oder Situationen sowie Ableitung von Folgerungen

- Zielklärung: Konkretisierung und genaue Beschreibung von Zielen oder Problemlösungen

- Umsetzungsunterstützung: Unterstützung der Umsetzung von Zielen durch übende Maßnahmen oder das Aufstellen von Plänen

- Ressourcenaktivierung: Identifizierung und genaue Beschreibung von Ressourcen sowie Überlegungen zu deren Nutzung zur Zielerreichung

- Erlebnisaktivierung (nur als begleitender Wirkfaktor neben anderen): erlebnismäßige Aktualisierung der im Coaching behandelten Themen/Probleme

Ausgewählte Wirkfaktoren werden im Folgenden anhand von Fallbeispielen und empirischen Ergebnisse aus der Übergangsberatung konkreter vorgestellt.

\section{3. Übergangsberatung und Lernprozesse}

Das hier vorliegende Lernverständnis bezieht sich auf die subjektwissenschaftliche Lerntheorie (Holzkamp 1995). Ausgangspunkt der Lernkonzeption sind die Problemstellungen der Subjekte. Wenn alltägliche Handlungsroutinen scheitern, kann dies subjektiv als Diskrepanz empfunden werden zwischen dem, was man kann, und dem, was man will. Wird antizipiert, dass diese erlebte Diskrepanz durch Lernen überwunden werden kann, wird aus der Handlungsproblematik eine Lernproblematik. Lernen stellt dann den dominanten Aspekt einer Handlung dar und ist damit eine spezifische Form von Handeln. Holzkamp bezeichnet dies als intentionales Lernen (vgl. ebd., S. 183).

Beratung als „Lern(unterstützungs)praxis“ (Göhlich/König/Schwarzer 2007: 7) bedeutet unter subjektwissenschaftlicher Perspektive, dass die Beratungsteilnehmenden sich bewusst für eine Beratung entscheiden, weil sie in Handlungsproblematiken Diskrepanz erfahren haben. Mit der Teilnahme an dem Beratungsangebot verlassen die Teilnehmenden ihren unmittelbaren Handlungszusammenhang, um die eigenen Anliegen und Fragen (Handlungsproblematiken) mit pädagogischer Unterstützung zu reflektieren, Erkenntnisse zu generieren und ihre Kompetenzen zu erweitern. Es wird eine „Lernschleife“ (Faulstich/Ludwig 2004: 20) eingelegt, um die eigenen Handlungsmöglichkeiten zu erweitern: 
„Die zu erwartenden Anstrengungen und Risiken des Lernens werden hier also unter der Prämisse von mir motiviert übernommen, dass ich im Fortgang des Lernprozesses in einer Weise Aufschluss über reale Bedeutungszusammenhänge gewinnen und damit Handlungsmöglichkeiten erreichen kann, durch welche gleichzeitig eine Entfaltung meiner subjektiven Lebensqualität zu erwarten ist“" (Holzkamp 1995: 190).

Lerngegenstände in der Übergangsberatung sind u. a. die eigene Biografie, Entscheidungskonstellationen, soziale Strukturen und die konkrete Übergangsgestaltung. Es gilt, die „kurzschlüssige Zielgerichtetheit“ des bisherigen Handelns durch „Dezentrierung, Standpunktwechsel, gedankliche Variation“ reflektierbar zu machen (Holzkamp 1995: 184). Wie in den empirischen Fallbeispielen gezeigt wird, bietet sich reflexive Beratung hierfür als eine angemessene Lernform an.

Die folgenden Fallbeispiele sind ausgehend von jeweils einem methodenspezifischen Wirkfaktor dargestellt. Es erfolgt eine kurze Darstellung des Falls, der verwendeten Methode, eines exemplarischen Zitats aus dem evaluierenden Interview und eine Interpretation in Bezug auf reflektierte bzw. initiierte Lernprozesse.

\subsection{Wirkfaktor: ergebnisorientierte Selbstreflexion}

Der erste Fall betrifft ein freigestelltes Betriebsratsmitglied (49 Jahre, weiblich). Sie ist seit 11 Jahren im Betriebsrat eines Großbetriebs und seit 3 Jahren freigestellt. Nebenberuflich ist sie bei der Gewerkschaft als Teamerin tätig. Mit der Arbeitsweise im Betriebsrat ist sie seit einiger Zeit unzufrieden, die Zusammenarbeit belastet sie und sie stellt rückwirkend in Frage, ob dies überhaupt der richtige berufliche Weg für sie war. Nach längeren Überlegungen hat sie sich entschieden aus dem Betriebsrat auszusteigen. Dieser Entschluss stand zu Beginn der Beratung fest, sie wusste aber noch nicht, wie sie den Übergang gestalten und wohin sie beruflich gehen will. Es gab bereits einige mögliche Vorstellungen, allerdings war sie dabei sehr unsicher, vor allem in Bezug auf ihre vorhandenen Kompetenzen.

Als Auftakt der Beratung wurde daher mit der Methode „Lebensprofil“ (Zukunftszentrum Tirol 2009: 35ff.) gearbeitet. Hierbei erfolgt eine bildhafte Darstellung der eigenen Biografie, der unterschiedlichen Lebensbereiche und Etappen. Anschließend wird reflektiert, was in den einzelnen Bereichen jeweils gelernt wurde. Persönlichen Ressourcen und biografische Kontinuitäten sollen herausgearbeitet und sichtbar gemacht werden, damit sie für die Gestaltung der eigenen Biografie genutzt werden können. Im evaluierenden Interview sagt die Teilnehmerin dazu: 
„Wir haben tatsächlich Kompetenzen nochmal von Kindheit an aufgeführt, die für mich bei der Suche nach einem Job völlig in den Hintergrund getreten sind. Also ich mache Bildungsarbeit. Ich kann vor Menschen sprechen. Die verstehen mich. Die lernen von mir. Das hatte ich nicht präsent. Also nicht vordergründig präsent. Natürlich weiß ich, dass ich das gut kann, aber es war nicht in Verbindung mit der Jobsuche. Ich hatte völlig verdrängt, welche Kompetenzen ich besitze. Und dass es vielleicht auch jetzt nochmal gehen könnte etwas Neues zu beginnen. Da ist Verschüttetes nach oben geholt worden und die ganzen Verwirrungen in meinem Kopf haben sich sortiert.“

Die Beratungsteilnehmerin lernte in dieser Beratung ihre vorhandenen Kompetenzen wieder wahrzunehmen und $\mathrm{zu}$ benennen. Ihre anfängliche Leidensgeschichte durch die aktuelle Belastungssituation konnte sie anschließend als Lerngeschichte erzählen. Sie bettete die nebenberufliche Bildungstätigkeit in ihre Biografie ein und stellte einen Zusammenhang zu Gegenwart und Zukunft her. In der rückblickenden Reflexion von Biografie und Lernergebnissen wurden ihr Lernprozesse bewusst und dadurch neue Optionen eröffnet.

Mittels der Methode Lebensprofil können die Selbstreflexion angeregt und biografische Lernprozesse unterstützt werden. Das Selbstkonzept kann dadurch neu interpretiert und es können Schlussfolgerungen für die Zukunft gezogen werden. Insofern befördert das Lebensprofil den Wirkfaktor „ergebnisorientierte Selbstreflexion“.

\subsection{Wirkfaktor: Ressourcenaktivierung}

Den zweiten Fall stellt ein seit fünf Jahren freigestellter Betriebsratsvorsitzender (39 Jahre, männlich). Trotz des Stresses, den die Funktion des Vorsitzes mit sich bringt, hat er viel Freude an der Arbeit und möchte diese weiterhin ausüben. Er hat jedoch Zweifel, ob er bis zum Renteneintritt wiedergewählt wird (alle vier Jahre erfolgt die Betriebsratswahl). Eine unvorbereitete Abwahl macht ihm Sorge, aber er sieht momentan keine machbaren beruflichen Alternativen. Vor der Freistellung hatte er nach einer Berufsausbildung in der Produktion gearbeitet. Eine Rückkehr an seinen früheren Arbeitsplatz kann er sich nicht vorstellen. In der Beratung möchte er berufliche Alternativen zur Betriebsratsfunktion entwickeln und vorbereiten sowie Weiterbildungsmöglichkeiten klären. Mögliche zukünftige Übergänge sollen antizipiert und bearbeitet werden.

Um das Spannungsfeld von Vergangenheit und Zukunft gegenwärtig reflektierbar zu machen, wurde im Beratungsprozess der Blick zunächst mit dem Lebensprofil in die Vergangenheit gelenkt, um danach mit einer verkürzten Kompetenzbilanzierung (in Anlehnung an Lang-von Wins/Triebel (2012) und den ProfilPass des DIE) vorhandene Fertigkeiten zu analysieren und zu 
bewerten sowie letztlich mögliche Zukünfte zu entwickeln. Die Fertigkeitenanalyse und -bewertung stellte einen sehr intensiven Arbeitsschritt dar. Der Teilnehmer sagt im evaluierenden Interview hierzu:

„Für mich war sehr interessant, die Fähigkeiten oder auch das Wissen aufzuschreiben. Es war dann immer die Frage: Kann ich es umsetzen? Kann ich es weitervermitteln? Und ich habe festgestellt, dass ich doch viele Sachen auch jemand anderem beibringen kann. Das war so ein Aha-Erlebnis, wo man sagt: ja das hast du auch irgendwo unterschwellig gemacht, aber nie bewusst. Da ist mir klar geworden, dass ich mich in den letzten sechs Jahren, seit es den Betriebsrat gibt, massiv weiterentwickelt habe. Und das hat diese Beratung konkret ans Licht gebracht. Man macht sich kleiner, als man ist. Die Beratung hat gezeigt, da ist wesentlich mehr, als nur das, was man persönlich einschätzt. Und wenn man das erstmal auf Papier sieht, was man alles schon gemacht hat und wie weit man sich entwickelt hat, dann nimmt man auch gern einen Schwung mit und sagt: ja, ich traue mir noch ein Studium zu."

Der Beratungsteilnehmer reflektierte in dieser Beratung die bereits stattgefundenen zum Teil inzidentellen Lernprozesse und lernte die eigenen Fähigkeiten mittels des Bewertungsrasters des Profilpasses (vgl. Neß/Bretschneider/ Seidel 2007: 394) einzuordnen. Dadurch gewann er Sicherheit in den eigenen Kompetenzen und seine Selbstwirksamkeit konnte gestärkt werden, sodass letztlich neben anderen beruflichen Optionen selbst ein Studienabschluss in einen erreichbaren Horizont rückte. Der Teilnehmer konnte in der Beratung seine Unsicherheit zu beruflichen Perspektiven positiv wenden und eine bewusste „Multioptionalität“ entwickeln, welche eine relevante Ressource in biografischen Übergängen darstellt (vgl. Schicke/Gorecki/Schäffter 2014).

Die Kompetenzbilanzierung ermöglicht die Reflexion, Bewusstwerdung und Beschreibung eigener Kompetenzen, wodurch Unsicherheit zu Multioptionalität gewendet werden kann. Kompetenzen und Multioptionalität können als Ressourcen für die Übergangsgestaltung genutzt werden. Daran wird deutlich, dass Kompetenzbilanzierungen den Wirkfaktor „Ressourcenaktivierung" fördern.

\subsection{Wirkfaktor: ergebnisorientierte Problemreflexion}

Der dritte Fall bezieht sich auf ein seit zwei Jahren freigestelltes Personalratsmitglied (40 Jahre, männlich). Neben seiner Personalratstätigkeit ist er parteipolitisch engagiert und mit drei Kindern stark familiär eingebunden. Im Moment pausiert sein Personalratsmandat, weil er für eine Führungslaufbahn im öffentlichen Dienst eine Bewährungszeit als Führungskraft nachweisen muss. Diese absolviert er aktuell. Für ihn stellt sich die Frage, ob er in den Personalrat zurückkehren, also einen Übergang wieder hinein oder ganz heraus aus dem Personalrat gestalten will. Speziell diese Entscheidungskonstellation, 
aber auch generell seine beruflichen Entwicklungsmöglichkeiten möchte er in der Beratung reflektieren.

Gegen Ende des Beratungsprozesses nach u. a. einer verkürzten Kompetenzbilanzierung wird seine aktuelle Lebenssituation mit der Methode „Lebensrad“" (Gasteiner 2014: 84f.) reflektiert, um daraus Schlussfolgerungen für die zukünftige Gestaltung ableiten zu können. Bei der Methode Lebensrad stellt der Teilnehmende seine unterschiedlichen Lebensbereiche (z. B. Personalratstätigkeit, berufliche Tätigkeit, politisches Engagement, Familie) mit aktuellen Zeitanteilen in Form eines Kuchens visuell dar und erläutert, wie zufrieden er mit dieser Verteilung ist bzw. welche Problemsicht vorliegt. Im evaluierenden Interview sagt er dazu:

„Das hat bei mir eine Erleuchtung gebracht, was ich für Themen gerade bewege und auf welchen Feldern ich mich bewege. Ich habe für mich festgestellt: es gibt eigentlich zu viel. Als ich das so visualisiert habe, konnte ich sehen, wenn ich zurückgehe in den freigestellten Personalratsbereich, dann würde ich wieder Zeit gewinnen, wenn nicht, müsste ich mich von meinen Ehrenämtern und Nebenämtern trennen. Das hat mir eine Hilfe gegeben, weil ich momentan den Eindruck habe, das geht zu Lasten meiner Familie und auch meiner Gesundheit. Ich fühle mich schon ein bisschen schlapp und ausgelaugt. Von dem her hat mir dieses Lebensrad eine gute Erkenntnis gebracht: darzustellen, was gerade so ist, und auch darüber zu sprechen. Auch um jetzt sagen zu können: Was könnte man denn verändern? Wo sind da überhaupt Stellschrauben vorhanden?“

Der Beratungsteilnehmer kam in dieser Beratung zu der Erkenntnis, dass er aktuell in seinem Leben zu viele Themen und Felder für die zeitlich begrenzten Ressourcen bearbeitet. Dies unterstützte ihn dabei, seine Belastungen zu thematisieren und zu überlegen, wo Veränderungsmöglichkeiten liegen.

Mittels der Methode Lebensrad können Reflexionen über die aktuelle Handlungsproblematik und Lernprozesse bezüglich eigener Lebensschwerpunkte unterstützt werden. Dadurch können Veränderungsbedarfe deutlich und Lösungsperspektiven entwickelt werden. Insofern besitzt das Lebensrad den Wirkfaktor „ergebnisorientierte Problemreflexion“. Die durch die Reflexion gewonnenen Erkenntnisse können für Entscheidungskonstellationen in Übergängen genutzt werden, um mehr subjektive Lebensqualität entfalten zu können.

\section{Fazit}

Die empirischen Ergebnisse im Projekt Spurwechsel haben gezeigt, dass wechselseitige Zusammenhänge zwischen Beratungsmethoden, Wirkfaktoren, Lernprozessen und Übergangskompetenz bestehen. Beratungsmethoden legen jeweils einen Schwerpunkt auf unterschiedliche Wirkfaktoren, z. B. 
sind die methodenspezifischen Wirkfaktoren des „Lebensprofils“ vor allem Selbstreflexion und Ressourcenaktivierung. Zugleich werden damit spezifische Lernprozesse gefördert, weil bestimmte Lerngegenstände im Fokus stehen und Reflexionsprozesse dazu angeregt werden. Speziell fördert das Lebensprofil vor allem biografisches Lernen und das Bewusstwerden von Kompetenzen, welche für die Übergangsgestaltung genutzt werden können. In diesem Sinne bietet Übergangsberatung Reflexionsraum und Zeit für Bilanzierung für in der Vergangenheit stattgefundene Lernprozesse.

Die verwendeten Methoden mit ihren spezifischen Wirkfaktoren können durch die eröffneten Reflexionsmöglichkeiten und angeregten Perspektivwechsel aber auch Lernprozesse initiieren, in denen beispielsweise Erkenntnisse über Handlungsproblematiken und Lösungsperspektiven gewonnen werden oder diffuse Unbestimmtheit zu „beruflicher Multioptionalität“ gewendet wird.

Je nach Beratungsanliegen der Teilnehmenden und der Phase im Übergangsprozess (u. a. Abwägung, Entscheidung, Handlungsplanung oder Umsetzung) sollte daher die Auswahl der Methoden auf Grundlage der Wirkfaktoren und damit der zu reflektierenden bzw. initiierenden Lernprozesse erfolgen. Übergeordnetes Ziel der Beratung ist die gelingende Gestaltung von Übergängen und die Förderung von Übergangskompetenz, d. h. im Verständnis von Schicke/Gorecki/Schäffter von einem ,situationsadäquaten Umgang mit biografischen Transitionen in einem gering strukturierten und somit offen angelegten zielgenerierenden Prozessverlauf" (Schicke/Gorecki/Schäffter 2014: 191).

Die Ergebnisse zeigen, dass die Entwicklung einer Übergangskompetenz mit reflexiver Beratung gefördert werden kann. Offen bleibt, wie langfristig die gewonnene Ressource zur Verfügung steht. Übergangsberatung ist eine lernförderliche Supportstruktur, weil Lernprozesse reflektiert und initiiert und dadurch Ressourcen für eine gelingende Übergangsgestaltung aktiviert werden. In Bezug zu Vergangenheit und gegenwärtigen Umständen können zukünftige Möglichkeiten entwickelt und ausgelotet werden, um Übergänge nicht unbegriffen zu erleiden, sondern in Richtung gelingendes Leben gestalten zu können.

\section{Literatur}

Ebbers, Ilona; Langanka, Alexander (2013): Innerbetriebliche Übergänge. In: Schröer, Wolfgang; Stauber, Barbara; Walther, Andreas; Böhnisch, Lothar; Lenz, Karl (Hg.): Handbuch Übergänge. Weinheim: Beltz Juventa, S. 705-720.

Faulstich, Peter; Ludwig, Joachim (2004): Lernen und Lehren - aus „subjektwissenschaftlicher Perspektive“. In: Faulstich, Peter; Ludwig, Joachim (Hg.): Expansi- 
ves Lernen. Grundlagen der Berufs- und Erwachsenenbildung; 39. Baltmannsweiler: Schneider-Verl. Hohengehren, S. 10-28.

Felden, Heide von; Schäffter, Ortfried; Schicke, Hildegard (Hg.) (2014): Denken in Übergängen. Weiterbildung in transitorischen Lebenslagen. Wiesbaden: Springer VS.

Gasteiger, Rosina M. (2014): Laufbahnentwicklung und -beratung - Berufliche Entwicklung begleiten und fördern. Göttingen: Hogrefe.

Greif, Siegfried (2008): Coaching und ergebnisorientierte Selbstreflexion. Theorie, Forschung und Praxis des Einzel- und Gruppencoachings. Göttingen: Hogrefe.

Greif, Siegfried; Runde, Bernd; Seeberg, Ilka (2004): Change Explorer. In: Rauen, Christopher (Hg.): Coaching-Tools. Erfolgreiche Coaches präsentieren 60 Interventionstechniken aus ihrer Coaching-Praxis. 2. Aufl. Bonn: ManagerSeminare-Verl., S. 317-325.

Greif, Siegfried; Schmidt, Frank; Thamm, André (2012): Warum und wodurch Coaching wirkt. In: Organisationsberatung, Supervision, Coaching, Jg. 19, H. 4, S. $375-390$.

Göhlich, Michael; König, Eckard; Schwarzer, Christine (2007): Beratung, Macht und organsiationales Lernen. Eine Einführung. In: Michael. Göhlich, Eckard. König und Christine. Schwarzer (Hg.): Beratung, Macht und organisationales Lernen. Wiesbaden: VS Verlag für Sozialwissenschaften, S. 7-19.

Hocke, Simone (2018): Spurwechsel - Übergänge in der Bildungs- und Erwerbsbiografie von Betriebs- und Personalräten aktiv gestalten: ein Manual zur Berufsweg- und Übergangsberatung. Düsseldorf: Hans-Böckler-Stiftung (Hg.). Online verfügbar unter https://www.boeckler.de/pdf/p_study_hbs_388.pdf (zuletzt geprüft am 27.08.2018).

Holzkamp, Klaus (1995): Lernen. Subjektwissenschaftliche Grundlegung. Studienausgabe. Frankfurt a. M. [u. a.]: Campus-Verlag.

Hopf, Christel (2004): Qualitative Interviews - ein Überblick. In: Flick, Uwe; Kardorff, Ernst v.; Steinke, Ines (Hg.): Qualitative Forschung. Ein Handbuch. Reinbek bei Hamburg: Rowohlt, S. 349-360.

Lang-von Wins, Thomas; Triebel, Claas (2012): Karriereberatung. Coachingmethoden für eine kompetenzorientierte Laufbahnberatung. 2. Aufl. Berlin: Springer.

Mörth, Martina; Söller, Imke (2011): Handbuch für die Berufs- und Laufbahnberatung. Göttingen: Vandenhoeck \& Ruprecht.

Neß, Harry; Bretschneider, Markus; Seidel, Sabine (2007): ProfilPass - Weiterbildungspass mit Zertifizierung informellen Lernens. In: Erpenbeck, John; Rosenstiel, Lutz von (Hg.): Handbuch Kompetenzmessung. Erkennen, Verstehen und Bewerten von Kompetenzen in der betrieblichen, pädagogischen und psychologischen Praxis. 2., überarb. und erw. Aufl. Stuttgart: Schäffer-Poeschel, S. 388-411.

Pongratz, Hans (2011): Reflexive Beratung und gewerkschaftliche Interessenvertretung - Hindernisse und Chancen. In: Tietel, Erhard; Kunkel, Roland (Hg.): Reflexiv-strategische Beratung. Gewerkschaften und betriebliche Interessenvertretungen professionell begleiten. Wiesbaden: VS Verlag für Sozialwissenschaften, S. 29-46.

Rappe-Giesecke, Kornelia (2009): Sondierung - von der Beratungsfrage zum Kontrakt. In: Pühl, Harald (Hg.): Handbuch der Supervision 3. Grundlagen, Praxis, Perspektiven. Berlin: Leutner, S. 64-80. 
Scheele, Brigitte; Groeben, Norbert (1988): Die Heidelberger Struktur-Lege-Technik (SLT). Eine Dialog-Konsens-Methode zur Erhebung subjektiver Theorien mittlerer Reichweite. Weinheim: Beltz.

Schicke, Hildegard; Gorecki, Claudia; Schäffter, Ortfried (2014): Berufliche Multioptionalität als Ressource in biographischen Übergängen. Ergebnisse einer projektevaluierenden Begleitforschung. In: Felden, Heide von u. a. (Hg.): Denken in Übergängen. Weiterbildung in transitorischen Lebenslagen. Wiesbaden: Springer VS, S. 173-207.

Schröer, Wolfgang; Stauber, Barbara; Walther, Andreas et al. (Hg.) (2013): Handbuch Übergänge. Weinheim: Beltz Juventa.

Tietel, Erhard; Hocke, Simone (2015): Nach der Freistellung. Beruflich-biografische Perspektiven von Betriebsratsmitgliedern. Baden-Baden: Nomos Verlagsgesellschaft.

Trischler, Falko; Kistler, Ernst (2010): Gute Erwerbsbiographien. Arbeitspapier 1: Erwerbsverläufe im Wandel. Gefördert von der Hans-Böckler-Stiftung. Online verfügbar unter https://www.boeckler.de/pdf_fof/91966.pdf (zuletzt geprüft am 23.01.2018).

Wechsler, Theresa (2012): Coaching-Tools in der Wirkfaktorenanalyse. In: Rauen, Christopher (Hg.): Coaching-Tools III. Erfolgreiche Coaches präsentieren 55 Interventionstechniken aus ihrer Coaching-Praxis. Bonn: managerSeminare-Verlag, S. 11-21.

Zukunftszentrum Tirol (2009): Kombi Handbuch - Kompetenzorientierte Beratung für MigrantInnen und Personen mit Basisbildung. Online verfügbar unter http:// www.forschungsnetzwerk.at/downloadpub/KOMBI_Handbuch2009_innsbruck. pdf (zuletzt geprüft am 23.01.2018). 


\section{"Bildung, das ist unser Auftrag!" - Beratung im Freiwilligen Sozialen Jahr im Spannungsfeld zwischen außerschulischen Bildungszielen und psychosozialen Problemlagen}

\section{Überblick}

Das Freiwillige Soziale Jahr (FSJ) wird inzwischen jährlich von fast 60.000 Heranwachsenden ${ }^{1}$ als außerschulisches Bildungs- und Orientierungsangebot genutzt (vgl. Autorengruppe Bildungsberichterstattung 2016: 90). Das Interesse junger Menschen an diesem gesetzlich geregelten Jugendfreiwilligendienst wächst stetig (vgl. Rauschenbach 2015: 210). Erklärt wird dies mitunter durch einen gestiegenen Orientierungsbedarf Heranwachsender aufgrund veränderter Dynamiken in Gesellschaft und Arbeitswelt (vgl. ebd.: 211). Das FSJ bietet jungen Menschen einen ,weichen“ Übergang zwischen Schule, Ausbildung und Beruf (vgl. BMFSFJ 2015: 24), der sowohl der persönlichen und beruflichen Orientierung als auch der Übernahme gesellschaftlicher Verantwortung dienen soll (vgl. ebd.: 25). Zur Unterstützung dieser Bildungsziele ist die pädagogische Begleitung der Freiwilligen gesetzlich vorgeschrieben, die zuvorderst von den Bildungsreferent*innen der FSJ-Träger verantwortet wird (vgl. ebd.: 283).

Inwieweit Beratung für die Bildungsreferent*innen in der pädagogischen Begleitung der Freiwilligen eine Rolle spielt, ist die übergeordnete Fragestellung eines Dissertationsprojektes an der WWU Münster. Es existiert allerdings ein großes Forschungsdesiderat zum Thema Beratung in der Außerschulischen Jugendbildung (AJB) allgemein und speziell in ihrem Handlungsfeld des FSJ. Der bald 15 Jahre alte von Ziemons (2004) konstatierte (Miss-)Stand, dass es grundlegend an einem Verständnis von Anlässen, Funktion und Wirkung von Beratung in der AJB fehle, hat bis heute nicht an Aktualität eingebüßt.

Im Rahmen des vorliegenden Beitrags wird die Frage nach Beratungsanlässen bzw. -themen fokussiert, die die Bildungsreferent*innen bei den Teilnehmenden im FSJ wahrnehmen. Dafür wird im Folgenden ein kurzer

1 In Anlehnung an die Altersbegrenzung in Jugendfreiwilligendiensten (vgl. §2 Abs. 4 JFDG) werden hier mit den Begriffen Heranwachsende und Jugendliche Personen im Alter zwischen 16 und 27 Jahren angesprochen. 
Überblick sowohl über das FSJ als Handlungsfeld der AJB (1) als auch über Beratung als pädagogische Handlungsform (2) gegeben und das methodische Vorgehen der Studie skizziert (3). Im Mittelpunkt des Beitrags steht die Darstellung ausgewählter Forschungsbefunde am Beispiel von drei Schlüsselfällen aus der qualitativen Interviewstudie (4) des Dissertationsprojektes.

\section{Das Freiwillige Soziale Jahr als Handlungsfeld der Außerschulischen Jugendbildung - Bildungsverständnis und (Bildungs-)Ziele}

Da das noch zu explizierende Verständnis von Beratung als pädagogische Handlungsform (vgl. Kap. 2) nach einer Klärung des Bildungsverständnisses als grundlegender Orientierung für eine mögliche Beratungsarbeit verlangt, werden hier neben grundlegenden Rahmenbedingungen insbesondere die (Bildungs-)Ziele und das (mögliche) Bildungsverständnis des FSJ vorgestellt.

Im FSJ engagieren sich junge Menschen zwischen 16 und 27 Jahren freiwillig, in der Regel für 12 Monate, in gemeinwohlorientierten Einrichtungen. Die pädagogische Begleitung wird hierbei von den FSJ-Trägern verantwortet. Von zentraler Bedeutung für diesen Bildungs- und Orientierungsdienst sind 25 gesetzlich vorgeschriebene Seminartage, die insbesondere der Reflexion des Praxiseinsatzes und der Persönlichkeitsbildung dienen sollen (vgl. BMFSFJ 2015: 20ff.).

Mit dem FSJ werden verschiedene Ziele und Hoffnungen verknüpft. Beispielsweise wird im Jugendfreiwilligendienstegesetz (JFDG) von 2008 die Förderung von „Bildungsfähigkeit“ ( $\$ 1$, Abs.1) und von sozialen, kulturellen und interkulturellen Kompetenzen sowie des Verantwortungsbewusstseins für das Gemeinwohl ( $\$ 3$, Abs.2) benannt. Angesprochen werden hier Bildungsziele, die schematisch voneinander getrennt als subjektbezogene „Persönlichkeitsbildung“ und als auf die Gesellschaft bezogene „soziale Bildung“ charakterisiert werden können (vgl. Münchmeier 2010: 58). Über dieses Bildungsverständnis humanistischer Prägung hinaus wird im JFDG auch der inzwischen gewollte berufsqualifizierende Charakter von Jugendfreiwilligendiensten offenbar: „[I]n das Zeugnis [sind] berufsqualifizierende Merkmale des Jugendfreiwilligendienstes aufzunehmen“ (§11, Abs.4). Kritische Stimmen warnen hier vor der Gefahr einer Indienstnahme der Bildungspotentiale des FSJ für ökonomische Zwecke (vgl. Hübner, Maedler 2015: 242).

Dieser Kritik folgend, wird ein subjektorientierter Bildungsbegriff für das FSJ favorisiert (vgl. ebd.; Münchmeier 2010). Bildung darf demnach nicht mit beruflicher Qualifizierung gleichgesetzt werden, sondern bedeutet „Selbst- 
bildung als Aneignung des eigenen Selbst in der Welt und Reflexion dieser Aneignungsprozesse" (Macha, Wischmeier 2012: 75). Eine so verstandene Bildung weist der AJB die Aufgabe zu, junge Menschen zu unterstützen, selbstbestimmte und gemeinschaftsfähige Mitglieder der Gesellschaft zu werden (vgl. ebd.: 9). Der Reflexion von Aneignungsprozessen wird hierbei eine zentrale Rolle zugesprochen. Subjektorientierte Bildungsarbeit heißt also vorrangig Räume für Diskurse, Austausch und für Selbstreflexion und -vergewisserung zu schaffen (vgl. Hübner, Maedler 2015: 240).

Das FSJ bietet ideale Potentiale für eine so verstandene Jugendbildung. Die besonderen Bildungschancen liegen vor allem in der Kombination des Praxiseinsatzes, durch den in Ernstsituationen informelle Bildungsprozesse ermöglicht werden und der nicht formellen Seminararbeit, die Raum für die Reflexion der in der Praxis gemachten Aneignungsprozesse bietet.

Inwieweit diese Bildungspotentiale ausgeschöpft werden, hängt maßgeblich von der pädagogischen Begleitung ab (vgl. Eberhard 2002: 13), d. h. zuvorderst von den Bildungsreferent*innen der FSJ-Träger. Die zurzeit aktuellste Evaluationsstudie zum FSJ (vgl. BMFSFJ 2015) betont mit Hinblick auf die pädagogischen Aufgaben der Bildungsreferent*innen, dass Konfliktmanagement und (kollegiale) Beratung typische Angebote dieser pädagogisch Tätigen darstellen (vgl. ebd.: 283ff.). Über diesen ersten Hinweis hinaus existieren jedoch keine weiteren Untersuchungsergebnisse zum Thema Beratung im FSJ. Deshalb wird der Blick auf ihre wissenschaftliche Bezugsdisziplin, die AJB, gerichtet.

\section{Beratung in der Außerschulischen Jugendbildung}

Es finden sich nur wenige wissenschaftliche Abhandlungen, die sich explizit mit Beratung in der AJB beschäftigen. ${ }^{2}$ Über Jugend(en) und ihre spezifischen Herausforderungen, insbesondere vor dem Hintergrund aktueller gesellschaftlicher Dynamiken, wurde hingegen viel diskutiert. ${ }^{3}$ Weitestgehende Einigkeit herrscht darüber vor, dass das Jugend- und junge Erwachsenenalter mit einer besonderen Dynamik der Identitätssuche und mit spezifischen Entwicklungsaufgaben verbunden ist (vgl. Hurrelmann, Quenzel 2016). Vor diesem Hintergrund werden Pädagog*innen in der AJB, die einen direkten

2 Ausnahmen sind bspw. die Beiträge von Weide (2004), Ziemons (2004) und Sauer-Schiffer (2004; 2010).

3 Siehe z. B. die Projekte und Publikationen des Deutschen Jugendinstituts (vgl. URL: https://www.dji.de, Stand: 26.04.2018). 
Zugang zu den jugendlichen Lebenswelten haben (können), häufig von den Teilnehmenden als Ratgebende aufgesucht (vgl. Sauer-Schiffer 2010: 34).

Bei der Suche nach Beratungskonzepten für Jugendliche werden vor allem institutionalisierte und problemspezifische Beratungsansätze mit überwiegend psychologisch-psychotherapeutischen Definitionen von Beratung offenbar (vgl. Reutlinger 2007). Diese scheinen aber für den Bildungskontext der AJB wenig hilfreich und eine diesbezügliche Forderung lautet, für die Felder der AJB die Bildungsaspekte von Beratung stärker in den Blick zu nehmen (vgl. Ziemons 2004: 256). Hierfür kann auf erziehungswissenschaftliche Ansätze von Beratung zurückgegriffen werden (z. B. Mollenhauer 1965; Aurin 1984; Kraft 1993; Sauer-Schiffer 2004, 2006; Hechler 2010).

Den genannten Abhandlungen ist gemeinsam, dass sie Beratung als pädagogische Handlungsform mit dem Ziel der Initiierung von Selbstbildungsprozessen verstehen. In Anlehnung an Sauer-Schiffer (2006), die auf Aurin (1984) rekurriert, wird hier unter Beratung in der AJB folgendes gefasst: Sie

- ist ein Kommunikations- und Interaktionsprozess zwischen Beratenden (Bildungsreferent*innen u. ä.) und Ratsuchenden (Teilnehmende, Dozierende u. ä.)

- bei dem es um Hilfe zur Selbsthilfe und um

- einen gemeinsamen Austausch- und Reflexionsprozess geht, der

- Menschen beim Prozess der Urteilsbildung im Rahmen eines Anliegens unterstützt und der auf die Aktivierung der Fähigkeiten zur

- Selbstbestimmung,

- Neuorientierung von Einstellungen und Haltungen und

- Erkundung möglichst eigenständiger Lösungsmöglichkeiten zielt (vgl. Sauer-Schiffer 2006: 16f.).

Beratung wird also zusammengefasst als eine pädagogische Interventionsform zur Unterstützung der subjektorientierten Bildungsziele der AJB verstanden.

\section{Beratung im Freiwilligen Sozialen Jahr? Vorannahmen und methodisches Vorgehen}

Wie bereits erläutert, existieren bisher keine grundlegenden Untersuchungen zum Thema Beratung im FSJ. Folgende Annahmen über dieses Forschungsfeld können jedoch formuliert werden:

1. Es wird der Hypothese gefolgt, dass (halbformaler) Beratung wie in nahezu jedem pädagogischen Handlungsfeld (vgl. Sauer-Schiffer 2010: 33) 
auch in der Bildungs- und Begleitungsarbeit im FSJ eine gewisse Relevanz zukommt.

2. Darüber hinaus verweist die Konzeption des FSJ als Bildungs- und Orientierungsangebot auf Bedürfnisse, bei denen Beratung als pädagogische Unterstützungsleistung grundsätzlich greifen kann. So hält z. B. Weide (2004) für ihre Tätigkeit als Bildungsreferentin in der Deutschen Pfadfinderschaft fest, dass diese zu ,neunzig Prozent eine beratende“ (ebd.: 261) ist, da die jungen Menschen hier „Fragen und Antworten zu sich selbst, zu ihrer Lebensplanung, zu den von ihnen favorisierten Lebensaufgaben und -zielen" (ebd.) suchen und finden können. In diesem Sinne wird das FSJ als mögliches Vehikel für eben solche Fragen betrachtet.

Aufbauend hierauf musste sich dem Thema Beratung im FSJ explorativ genähert werden. In der Hauptstudie des Dissertationsprojektes wurden dazu u. a. elf qualitative, leitfadengestützte Interviews mit Bildungsreferent*innen in Leitungspositionen geführt. Die Leitfragen für den Themenkomplex Beratung lauteten hierbei: „Wenn Sie spontan einen Fortbildungskurs für ihre pädagogischen Mitarbeitenden anbieten würden, wie würde der Titel lauten?“ und „Welche Rolle spielt Beratung innerhalb des Aufgabenspektrums ihrer pädagogischen Mitarbeitenden?““. Die Antworten wurden vollständig transkribiert und in einem ersten Schritt in Anlehnung an die inhaltlich strukturierende qualitative Inhaltsanalyse nach Kuckartz (2016) ausgewertet. Diese Methode erlaubte eine systematische, kategoreinbasierte Rekonstruktion der subjektiven Wahrnehmungen der Leitungspersonen. Induktiv konnten sieben Unterkategorien zum Thema Beratung im FSJ gebildet werden: „Beratungsanlässe/-themen“, „Stellenwert von Beratung“, „Beratende und Zu-Beratende“, „Umgang \& Reaktion mit Beratungsanlässen/-themen“, „Beratungsorte/-zeiten“, „Beratungsgrenzen“" und „Professionalisierung von Beratung“.

Im Rahmen des vorliegenden Beitrags wird die Kategorie „Beratungsanlässe/-themen" anhand von drei exemplarischen Interviews ${ }^{4}$ fokussiert und mit Hilfe falltypischer Interviewzitate näher vorgestellt. Zum Zeitpunkt des Interviews ist Frau A, studierte Sozialpädagogin, 45 Jahre alt und übt ihre Funktion als Teamleitung von 14 Bildungsreferent*innen seit fünf Jahren bei einem öffentlichen Träger aus. Frau R, ebenfalls Sozialpädagogin, ist zum Zeitpunkt des Interviews 53 Jahre alt und übt seit neun Jahren ihre Funktion als Teamleitung von 17 Bildungsreferent*innen bei einem öffentlichen Träger aus. Herr D, studierter Soziologe, ist zum Zeitpunkt des Interviews 36 Jahre alt und hat die Leitung des 14 Personen starken pädagogischen Teams bei einem kirchlichen Träger ein halbes Jahr inne.

4 Kriterien zur Auswahl der drei von insgesamt elf Interviews waren, dass sowohl männliche als auch weibliche Interviewte sowohl eines öffentlichen wie auch eines kirchlichen FSJ-Trägers vertreten sein sollten. 


\section{Beratungsthemen und Beratungsanlässe im Freiwilligen Sozialen Jahr aus Sicht der Bildungsreferent*innen}

Durch die Analyse der drei Interviews konnten zwei Subkategorien der Kategorie „Beratungsanlässen/-themen im FSJ“ ermittelt werden: (a) „Psychische und psychosoziale Problemlagen“ und (b) „Auseinandersetzung mit der eigenen Identität" (vgl. Abbildung 1).

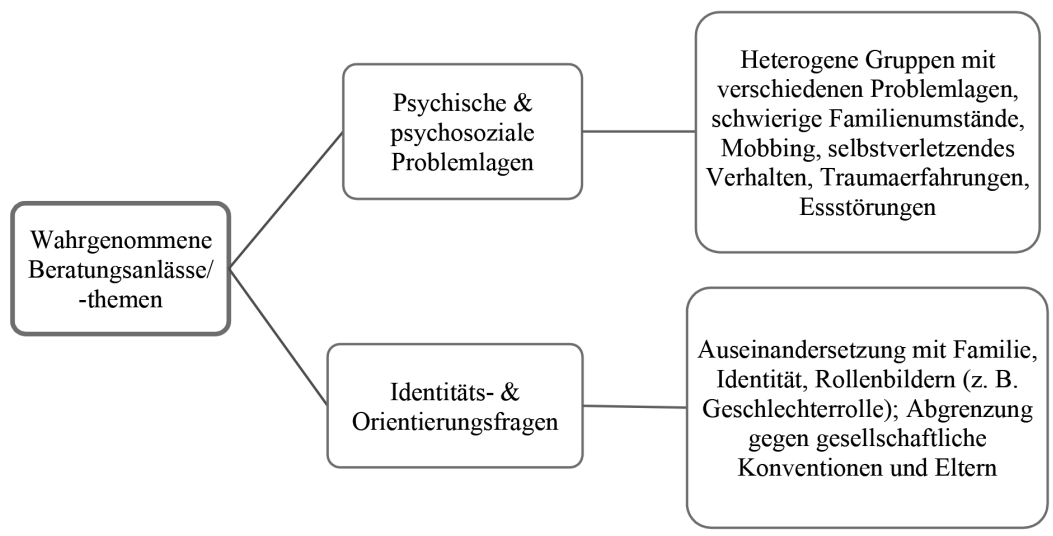

Abb. 1: Auszug Kategoriensystem: Wahrgenommene Beratungsanlässe und -themen von Frau A, Frau R, Herrn D (Quelle: eigene Darstellung)

(a) Mit Blick auf die Seminargruppen im FSJ beschreibt Frau A den „Umgang mit sehr heterogenen Gruppen mit immer mehr verschiedenen Problemlagen der Jugendlichen" als eine aktuelle Herausforderung für ihre pädagogischen Mitarbeitenden. Diesen Punkt führt sie wie folgt aus:

„Also wir machen ja keine klassische Jugendsozialarbeit, sondern Jugendbildung [...]. Nichtsdestotrotz kommen wir immer mehr in den Bereich, dass ganz viele Jugendliche auch psychische Auffälligkeiten mitbringen, auch keine einfache Situation zuhause haben. Und da geht es nicht darum, dass wir praktisch die Profis werden, aber dass wir Beratung noch qualifizierter und professioneller anbieten können."

An späterer Stelle des Interviews führt sie ihr Verständnis von „psychischen Auffälligkeiten“ mit der Nennung von „Mobbing“ und „selbstverletzendes Verhalten" beispielhaft aus. Die benannten Problemlagen sollen laut Frau A im Rahmen des FSJ möglichst qualifiziert und professionell beraten werden, obwohl das FSJ in seiner Funktion als Jugendbildung betont und ausdrücklich darauf verwiesen wird, dass das FSJ kein Feld der Jugendsozialarbeit sei. 
Auch Frau R betont den Bildungsauftrag des FSJ, sie zieht hieraus allerdings eine gänzlich andere Konsequenz für Beratung im FSJ:

„Also Bildung, das ist unser Auftrag, die Persönlichkeitsbildung. [...] Und dass wir nicht abschweifen in die Beratungstätigkeit, da gibt es andere, die haben den Auftrag und machen das gut. [...] Weil, die haben ja dann auch Probleme meistens, wenn das so ist, dass die Pädagogen sagen, da brauchen wir Beratung, das heißt, die sind dann auffällig und ich glaube, dass das denen auch nichts nutzt im FSJ weiter zu bleiben, weil es da auch nicht die Möglichkeit gibt, denen zu helfen.“

An späterer Stelle des Interviews führt sie bezüglich der angesprochenen „auffälligen“ Probleme der Freiwilligen genauer aus: „Teilnehmer, die noch nicht in Behandlung sind, Essstörungen haben, sich ritzen, also das ist total vielfältig“. Frau R fallen beim Thema Beratung also ähnliche Problemlagen der Freiwilligen ein wie Frau A. Allerdings schließt sie im Gegensatz zu Frau A im FSJ jede Möglichkeit aus, Jugendlichen mit den beschriebenen Problemlagen zu „,helfen“. Das begründet sie damit, dass der Auftrag des FSJ „Persönlichkeitsbildung“" sei. Die Beratung von Jugendlichen mit psychosozialen bzw. psychischen Problemlagen stellt für Frau R also keine zum Bildungsauftrag des FSJ gehörende Aufgabe dar und sollte deshalb von ,anderen“ übernommen werden, die das „gut machen“. Zusammenfassend betont sie an anderer Stelle des Interviews: „Wir sind hier keine Beratungsstelle“.

Herr D nimmt ebenfalls Themen der Freiwilligen wahr, die er dem Bereich der ,psychologischen Dispositionen“ zuordnet:

„Traumaerfahrungen und so weiter. Das kann auch ein Schwerpunkt sein. Das wäre dann allerdings unsere Aufgabe, die Leute dann weiterzuverweisen und nicht diesen Dienst zu haben oder festzuhalten."

Wie Frau R, möchte Herr D Jugendliche mit gewissen psychischen Belastungen nicht im FSJ „,festhalten“ und betont als Aufgabe der Bildungsreferent*innen die Weitervereisung solcher Freiwilligen.

Alle drei Interviewten nehmen also psychische und/oder psychosoziale Problemlagen als Beratungsthema im FSJ wahr, allerdings werden konträre Einschätzungen bezüglich des Umgangs mit dieser Thematik offensichtlich. Während in einem Fall die Professionalisierung von Beratung von Freiwilligen mit den benannten Problemlagen befürwortet wird, sprechen sich zwei Interviewte dafür aus, dass solche Freiwilligen weiterverwiesen werden sollten.

(b) Die Auseinandersetzung mit Identitäts- und Orientierungsfragen wird von einem der drei Interviewten im Zusammenhang mit dem Thema Beratung im FSJ genannt. Als „Beratungsfelder“ im FSJ nennt Herr D zuvorderst: „Auseinandersetzung mit der eigenen Familie, mit der eigenen Identität". An anderer Stelle beschreibt er beispielhaft das mögliche Hinterfragen der eigenen Geschlechterrolle: 
„Also es ist ja ein Orientierungsjahr für junge Leute [...] und bei dieser Orientierung hängt auch ganz stark eine persönliche Orientierung mit drin. Viele Leute hinterfragen sich nochmal in ihrer Rolle, in ihrer Frauen- oder Männerrolle, vielleicht sogar in ihrem Geschlecht und müssen sich da teilweise auch abgrenzen, gegen ihre Eltern, gegen die Konventionen. [...] In so einer [...] Orientierungsphase wird so etwas manchmal nochmal ganz neu und ganz individuell verhandelt. Da kommt das meistens dann in Einzelgesprächen dann vor. Manchmal auch in Gruppengesprächen.“

Hier betont Herr D die Funktion des FSJ als persönliche Orientierungsmöglichkeit für junge Menschen. Und in einer solchen „Orientierungsphase“ können dem Interviewten zufolge persönliche Themen wie z. B. die eigene (Geschlechter-)Rolle neu hinterfragt und Abgrenzungsnotwendigkeiten zu Eltern oder zu gesellschaftlichen Konventionen offenbar werden. Diese Themen werden laut Herrn D in Einzel- oder manchmal auch in Gruppengesprächen thematisiert.

\section{Diskussion und Ausblick}

Auf Grundlage der drei exemplarischen Interviews konnten zwei Themenbereiche identifiziert werden, die mit dem Thema Beratung im FSJ in Zusammenhang stehen.

(a) Alle drei Interviewten treffen Aussagen zu ,psychischen und psychosozialen Problemlagen“, wobei der beschriebene Umgang mit diesen variiert. Inwieweit Freiwillige mit psychischen und/oder psychosozialen Problemlagen von den pädagogisch Tätigen selbst beraten werden sollten oder nicht wird unterschiedlich eingeschätzt.

Über diese differierende Beurteilung hinaus kann an dieser Stelle festhalten werden, dass die drei Interviewten grundlegend ein sehr ähnliches Phänomen im FSJ wahrnehmen. Bildungsreferent*innen werden nach den hier analysierten Aussagen vor die beraterische Herausforderung gestellt, mit Teilnehmenden umgehen zu müssen, die in der einen oder anderen Form psychosoziale und/oder psychische Belastungen mitbringen. Auch die Entscheidung für einen Ausschluss bzw. für eine Weiterverweisung dieser Jugendlichen setzt voraus, dass im Vorfeld (Beratungs-)Gespräche geführt werden, um ,zuerst einmal heraus[zu]kriegen, was ist überhaupt?“, wie Frau A betont. Auch Frau R verweist auf die von ihr wahrgenommene Notwendigkeit, auf die Jugendlichen mit den beschriebenen Problemlagen zu reagieren: „Viele Pädagogen, die auch sagen, ich muss ja mit denen was machen, ich muss die ja beraten“".

Es kann hier also konstatiert werden, dass psychische und/oder psychosoziale Problemlagen für die drei Leitungspersonen einen Beratungsanlass im 
FSJ darstellt. Bereits die grundlegende Klärung der individuellen Problemlage - „was ist überhaupt“ - sowie das Erfordernis, zumindest zunächst einmal reagieren zu müssen - ,,ich muss die ja beraten“ -, verweist auf das Vorkommen und die persönlich gefühlte Notwendigkeit beraterischer Tätigkeiten der Pädagog*innen.

Weiterführend wäre zu diskutieren, inwiefern das oben aufgeworfene Beratungsverständnis, das Beratung als Bildungsgeschehen ausweist, pädagogisch begründbare Beratungsgrenzen im FSJ aufzeigen könnte, die für oder gegen eine Weiterverweisung von Teilnehmenden mit psychischen und/oder psychosozialen Beratungsanliegen sprechen würden. Demnach wäre es eine erste Aufgabe der Bildungsreferent*innen im (Beratungs-)Anliegen des*der Freiwilligen einen Bildungsbedarf im Rahmen seiner*ihrer individuellen Haltungen und Einstellungen zu lokalisieren (vgl. hierzu Hechler 2010). Würde ein solcher Bildungsbedarf nicht aufzuspüren sein, könnte dies für andere, eben nicht pädagogische, Beratungsverfahren sprechen.

(b) Der zweite Bereich „Identitäts- und Orientierungsfragen“ wird von einem Interviewten in Zusammenhang mit Beratung im FSJ ausgeführt. Das FSJ wird von Herrn D als Gelegenheitsraum für Fragen zur eigenen Identität und zur persönlichen Orientierung beschrieben, die in Einzel- oder Gruppensettings hinterfragt und neu verhandelt werden (können).

Die oben formulierte Annahme, dass das FSJ als mögliches Vehikel für jugendspezifische (Identitäts-)Fragen genutzt wird, findet in diesem Fall Bestätigung. Die Hinterfragung und Neuverhandlung solcher Themen wird von Herrn D als Beratungsthematik ausgewiesen.

Obwohl alle drei Interviewten die Rolle des FSJ als außerschulisches Bildungsjahr und die Unterstützung der Persönlichkeitsbildung als Aufgabe im FSJ betonen, werden Orientierungs- und Identitätsfragen der jungen Menschen von Frau A und Frau R nicht als (potentielle) Beratungsthematik genannt.

Methodenkritisch zu diskutieren ist in diesem Zusammenhang die in den Interviews zu Beginn gestellte Frage nach möglichen Fortbildungsbedarfen der pädagogischen Mitarbeitenden. Diese lenkt die Aufmerksamkeit der befragten Leitungspersonen auf Themen, die als Herausforderungen für die Pädagog*innen erlebt werden, und schränkt damit wahrscheinlich eine weitestgehend ungefilterte Aufzählung von wahrgenommenen Beratungsthemen der Freiwilligen ein. Vor diesem Hintergrund erscheint es nachvollziehbar, dass ,psychische und psychosoziale Auffälligkeiten“ der Teilnehmenden einen recht großen Raum bei den Interviewantworten einnehmen und weniger Orientierungs- und Identitätsfragen genannt werden, die möglicherweise als selbstverständlicher(er) und weniger herausfordernder Teil der pädagogischen (Beratungs-)Arbeit erlebt werden. Inwieweit die hauptsächliche Benennung von psychischen und psychosozialen Problemlagen als Beratungsthema/-anlass die Hypothese erlaubt, dies als einen Hinweis auf ein eher problem- 
orientiertes Beratungsverständnis von Frau A und Frau R zu deuten, bleibt in weiteren Analyseschritten zu prüfen.

Insgesamt kann die bisherige Analyse der drei Interviews erste Hinweise darauf liefern, dass Beratung eine gewisse Relevanz in der pädagogischen Arbeit der FSJ-Bildungsreferent*innen zukommt. Als beraterische Herausforderung im FSJ konnten bisher insbesondere psychische und psychosoziale Problemlagen der Teilnehmenden lokalisiert werden. Unter bildungstheoretischen Gesichtspunkten und im Hinblick auf das Selbstverständnis des FSJ als außerschulisches Bildungsjahr wird es lohnenswert sein, pädagogisch begründbare Grenzen der Beratungsarbeit im FSJ vertiefend zu diskutieren.

\section{Literatur}

Aurin, Kurt/Furch-Krafft, Elisabeth (Hg.) (1984): Beratung als pädagogische Aufgabe. Bad Heilbrunn: Klinkhardt.

Autorengruppe Bildungsberichterstattung (2016): Allgemeinbildende Schule und non-formale Lernwelten im Schulalter. Nationaler Bildungsbericht 2016. Bielefeld: Bertelsmann.

Bundesministerium für Familie, Senioren, Frauen und Jugend (BMFSFJ) (Hg.) (2015): Abschlussbericht der gemeinsamen Evaluation des Gesetzes über den Bundesfreiwilligendienst (BFDG) und des Gesetzes zur Förderung von Jugendfreiwilligendiensten (JFDG). Frankfurt a. M.

Eberhard, Angela (2002): Engagement für andere und Orientierung für sich selbst. Gestalt, Geschichte, Wirkungen des freiwilligen sozialen Jahres. München: Landesstelle für Katholische Jugendarbeit in Bayern.

Hechler, Oliver (2010): Pädagogische Beratung. Theorie und Praxis eines Erziehungsmittels. Stuttgart: Kohlhammer.

Hübner, Kerstin/Maedler, Jens (2015): Prozesse informeller und non-formaler Bildung in Jugendfreiwilligendiensten. In: Bibisidis, T./Bibisidis, J./Klein, A./Perabo, C./Rindt, S. (Hg.): Zivil - Gesellschaft - Staat. Freiwilligendienste zwischen staatlicher Steuerung und zivilgesellschaftlicher Gestaltung. Wiesbaden: Springer VS, S. 237-247.

Hurrelmann, Klaus/Quenzel, Gudrun (2016): Lebensphase Jugend. Eine Einführung in die sozialwissenschaftliche Jugendforschung. Weinheim: Beltz Juventa.

Kraft, Volker (1993): Probleme einer pädagogischen Theorie der Beratung. Bildung und Erziehung 46, 3, S. 345-359.

Kuckartz, Uwe (2016): Qualitative Inhaltsanalyse. Methoden, Praxis, Computerunterstützung. Weinheim: Beltz Juventa.

Mollenhauer, Klaus (1965): Das pädagogische Phänomen „Beratung“. In: Mollenhauer, K./Müller, C. W. (Hg.): Führung und Beratung in pädagogischer Sicht. Heidelberg: Quelle \& Meyer, S. 25-50.

Münchmeier, Richard (2010): Bildungschancen in freiwilligen Engagements junger Menschen. In: Schmidle, M./Slüter, U. (Hg.): Das Freiwillige Soziale Jahr zeigt 
Wirkung! Freiwilligenbefragungen im Kontext der Qualitätsentwicklung im FSJ. Freiburg im Breisgau: Lambertus Verlag, S. 50-62.

Rauschenbach, Thomas (2015): Bildung in Jugendfreiwilligendiensten. In: Bibisidis,T./Bibisidis, J./Klein, A./ Perabo, C./Rindt, S. (Hg.): Zivil - Gesellschaft Staat. Freiwilligendienste zwischen staatlicher Steuerung und zivilgesellschaftlicher Gestaltung. Wiesbaden: Springer VS, S. 209-223.

Reutlinger, Christian (2007): Beratung für Jugendliche. In: Nestmann, F./Engel, F./ Sickendiek, U. (Hg.): Das Handbuch der Beratung. Band 1. Disziplinen und Zugänge. Tübingen: dgvt-Verlag, S. 269-279.

Sauer-Schiffer, Ursula (2010): Beratungskompetenz für die pädagogische Intervention am Übergang. In: Sauer-Schiffer. U./Brüggemann, T. (Hg.): Der Übergang Schule-Beruf. Münster: Waxmann, S. 21-55.

Sauer-Schiffer, Ursula (Hg.) (2006): In der Balance liegt die Chance. Themenzentrierte Interaktion in Bildung und Beratung. Münster: Waxmann.

Sauer-Schiffer, Ursula (2004): Beratung in der Erwachsenenbildung und außerschulische Jugendbildung: Eine Einführung in Theorie und Praxis. In: Sauer-Schiffer, U. (Hg.): Bildung und Beratung. Beratungskompetenz als neue Herausforderung für Weiterbildung und außerschulische Jugendbildung? Münster: Waxmann, S. 9-65.

Weide, Angelika (2004): Beratung in der außerschulischen Jugendbildung am Beispiel der DPSG im Diözesenverband Münster. In: Sauer-Schiffer, U. (Hg.): Bildung und Beratung. Beratungskompetenz als neue Herausforderung für Weiterbildung und außerschulische Jugendbildung? Münster: Waxmann, S. 261-272.

Wischmeier, Inka/Macha, Hildegard (2012): Außerschulische Jugendbildung. Eine Einführung. München: Oldenbourg.

Ziemons, Michael (2004): Überblick über das Praxisfeld: Wandel und Aufbruch der außerschulischen Jugendbildung. In: Sauer-Schiffer, U. (Hg.): Bildung und Beratung. Beratungskompetenz als neue Herausforderung für Weiterbildung und außerschulische Jugendbildung? Münster: Waxmann, S. 236-259. 



\section{Ein ethnografischer Blick auf Materialität in der Beratung am Beispiel von Coaching und Transfergesellschaft}

\section{Einleitung}

In der erziehungswissenschaftlichen Übergangsforschung wird Angeboten zur Begleitung von und Beratung zu Übergängen in Erwerbsarbeit aus einer ethnografischen Perspektive kaum Aufmerksamkeit gewidmet. Im Kontext beruflicher Veränderungsprozesse von Erwachsenen finden sich ethnografische Betrachtungen bislang lediglich in ethnografisch orientierter Organisationsforschung mit Fokus auf Organisationsberatung im Weiterbildungsbereich und im Rahmen aktivierender arbeitsmarktpolitischer Maßnahmen aus machtanalytischer Perspektive (vgl. beispielsweise Ott 2011; Dollhausen 2010). Mit Blick auf die personenbezogenen Dienstleistungen der Begleitung und Beratung liegt jedoch ein analytischer Zugang nahe, der eine prozessuale Perspektive auf die organisationale Rahmung von Übergängen in Erwerbsarbeit einnimmt und dabei die Innenperspektive von Beratungsprozessen und deren Praktiken in den Fokus rückt.

Vor diesem Hintergrund beschäftigt sich der Beitrag aus ethnografischer Perspektive mit der Herstellung und Gestaltung von Beratungssettings im Kontext der Beratungsangebote Coaching und Transfergesellschaft. Im Rahmen des DFG-Forschungsprojekts „Transition Processing“ werden hierfür mittels teilnehmender Beobachtung angebotstypische Situationen und reale Handlungsvollzüge der Beteiligten empirisch erfasst. In diesem Zusammenhang liegt der Fokus der Analyse auf dem Umgang mit Artefakten, die - wie sich im empirischen Datenmaterial zeigt - sowohl im Coaching als auch in der Transfergesellschaft für die Inszenierung einer Beratung eine zentrale gestalterische Rolle spielen.

Im Folgenden wird zunächst das Projekt „Transition Processing“, dem der thematische Zuschnitt dieses Beitrags entlehnt ist, vorgestellt (2.). Daran anschließend werden die theoretischen und methodischen Grundlegungen dieses Beitrags eingegrenzt (3.). Im 4. Abschnitt wird beispielhaft skizziert, wie sich Materialität als Steuerungselement in der Beratung des Coachings und der Transfergesellschaft zeigt. Abschließend werden die beiden Handlungsfelder Coaching und Transfergesellschaft im Fazit vergleichend betrachtet (5.). 


\section{Forschungskontext}

Übergänge in Arbeit sind in ihrer Zunahme, Ausdehnung und Verdichtung zwischen Lebensphasen bzw. Berufspositionen zunehmend durch pädagogische Zugänge charakterisiert, wodurch sich Beratung am Übergang in Arbeit als zentraler Handlungsmodus pädagogischer Praxis erweist (vgl. z. B. Helsper 2013; Stauber et al. 2007; Walther 2008). Ziel des DFG-Forschungsprojektes „Transition Processing - Pädagogische Rationalitäten in personenbezogenen Dienstleistungen zur Begleitung und Beratung von Übergängen in Arbeit" ${ }^{\text {"1 }}$ ist es daher, eine grundlagentheoretische vergleichende Analyse fünf verschiedener Angebote zur Beratung und Begleitung am Übergang in Arbeit ${ }^{2}$ vorzunehmen. Analyseleitend sind dabei die Fragen, wie sich pädagogische Rationalitäten in den Wissensbeständen der Akteur*innen zeigen, wie pädagogische Rationalitäten in den unterschiedlichen Organisationsformen dieser Dienstleistungen prozessiert werden und ob sich gemeinsame und/oder feldspezifische Deutungsmuster zeigen. Diesen Fragen liegt die Annahme zugrunde, dass diese Dienstleistungen sozial geprägt sind, d. h. Gestaltung, Nutzung und Rahmung der Dienstleistungen auf sozial geteiltem Wissen beruhen, auf welches die Akteur*innen mit ihren Handlungen zurückgreifen. Sie sind soziale Vollzugswirklichkeit, also Ergebnis und Prozess einer sozialen Herstellungsleistung (vgl. Karl 2014).

\section{Theoretische und methodologische Grundlegung}

In „Transition Processing“ wird Beratung als gesprächsförmig gestaltete soziale Interaktion verstanden, die auf Augenhöhe und mit gegenseitiger Anerkennung der Beteiligten stattfindet. Diese Interaktion ist zeitlich umrissen, prozesshaft, interessensensibel und ergebnisoffen (vgl. Gieseke 2016: 102; Schiersmann 2011: 429). Ratsuchende werden unterstützt, ihre Interessen, Ressourcen und Kompetenzen zu erkennen und zur eigenverantwortlichen Gestaltung ihrer Lebensentwürfe oder im Fall von „Transition Processing“

1 Transition Processing wurde in folgender Kooperation durchgeführt: Prof. Dr. Barbara Stauber, Nina Wlassow (Uni Tübingen), Prof. Dr. Andreas Walther, Christiane Dittrich (Uni Frankfurt a. M.), Prof. Dr. Ute Karl, Dr. Christian Schröder (Uni Luxemburg), Prof. Dr. Inga Truschkat, Prof. Dr. Wolfgang Schröer, Dr. Claudia Muche (Uni Hildesheim), Prof. Dr. Ilona Ebbers, Heike Chyle (Uni Flensburg); Laufzeit: 01.01.2015-31.05.2018.

2 Berufsberatung der Agentur für Arbeit, Berufsorientierungsmaßnahme an einer Schule, Maßnahme für Early School Leaver, Coaching und Transfergesellschaft. 
und damit im Kontext von Erwachsenenbildung zur Gestaltung spezifischer Bildungs- und Berufsbiografien weiterzuentwickeln (vgl. Tippelt 2013: 71; vgl. Schiersmann 2011: 429). Es geht um Entscheidungsprozesse und die Bewältigung von Problemen, wofür die Bedingungen im Beratungsprozess zu gestalten sind (vgl. Gieseke 2016: 102; Schiersmann 2011: 429). Beratung beinhaltet zum einen eine strukturierte Form der Informations- und Wissensvermittlung, geht zum anderen jedoch über diese hinaus. Sie schließt eine subjektiv relevante Reflexion der Sachverhalte ein und ist durch das Wechselspiel von Reflexion und Informationsvermittlung charakterisiert (vgl. Tippelt 2013: 71; Schiersmann 2011: 429).

Der Beitrag betrachtet Beratung als soziale Situation mit dem Fokus auf ihre Herstellung und Gestaltung. Ziel ist es aufzuzeigen, wie die Situation über Materialität, hier insbesondere über den Einsatz von Artefakten, hergestellt wird. Als theoretischer Analyserahmen erscheinen in diesem Zusammenhang Goffmans Situationsanalyse (1959) und Reckwitz' Praxistheorie (2003) als gegenstandsangemessen, deren für den Beitrag wesentliche Punkte im Folgenden knapp umrissen werden.

Für die Situationsanalyse bei Goffman kommt seine Theatermetapher zum Tragen, mit der er in der Analyse das Geschehen zwischen Alltagswelt und Theaterbühne unterscheidet. Auf der Theaterbühne agieren Schauspieler*innen in künstlichen Rollen, die ein Stück vorschreibt. In der Alltagswelt hingegen beschreibt der Begriff Rolle soziale Rollen, die in bestimmten Kontexten Handlungsweisen vorgeben, die mit dem Ziel der (Selbst-)Darstellung genutzt werden können und müssen. Gleichzeitig finden in sozialen Situationen Adressierungen von Akteur*innen in Hinblick auf bestimmte Rollen statt (vgl. Goffman 1959): In Beratungssituationen im Coaching und in der Transfergesellschaft sind die Rollen des*der Beraters*in (Coach bzw. Transfer-Mitarbeiter*in) und des*der Ratsuchenden (Coachee bzw. Teilnehmer*in) konstitutiv, die aus ihren Rollenzuweisungen heraus für den jeweils anderen ihre Performance in dieser Situation, spielen'. Durch Regeln, denen soziale Rollen unterliegen, wird eine Interaktionsordnung hergestellt (vgl. Bausch 2001: 204). Die Inszenierung der einzelnen Rollen kann durch intentionales Handeln und unbewusste Praktiken die Situation gestalten. Dabei ist ausschlaggebend, inwiefern Rollenerwartungen, Verhaltensregeln und Rollenverpflichtungen aufgeführt bzw. dargestellt werden (vgl. Reckwitz 2003; Bausch 2001: 219). Mit Rückbezug auf die Theorie sozialer Praktiken (vgl. Reckwitz 2003) erfolgt eine Abgrenzung von der Reduktion des Handelns auf Intentionalität. Soziale Ordnungen gelten hier als Konstrukt, das durch sozial geteilte Wissensordnungen hergestellt wird. Soziale Praktiken werden gefasst als Verhaltensroutinen, deren Wissen einerseits in den Körpern der Subjekte inkorporiert und andererseits im Umgang mit Artefakten routinisiert ist. Damit ist aus praxeologischer Perspektive auf die zentrale Bedeutung der so gefassten Materialität von Praktiken verwiesen (vgl. ebd.: 289 ff.). 
Zur Analyse der hier dargestellten theoretischen Referenzpunkte der Inszenierung von Rollen und der sozialen Praktiken orientiert sich die Auswertung des ethnografischen Studienteils von „Transition Processing“ methodologisch an der Grounded Theory (vgl. Glaser/Strauss 2010). Für die Auswertung bilden Protokolle aus teilnehmenden Beobachtungen von feldtypischen Situationen den Analysegegenstand. Entsprechend des Erkenntnisinteresses und der Datensorte wurden in Anlehnung an Charmaz' Vorschlag zum gegenstandsangemessenen Umgang mit dem Kodierparadigma (2006) praxistheoretische Leitfragen entwickelt und zugrunde gelegt, welche die Analyse von Praktiken der Beratung ermöglichen. Die Besonderheit der Ethnografie, Nicht-Versprachlichtes zu versprachlichen, ermöglicht einen Einblick in unausgesprochene und unhinterfragte Routinen in der Beratungssituation (vgl. Breidenstein et al. 2013: 95) sowie die Analyse der interaktiven Herstellung organisationalen Wissens. Somit bereichert diese Perspektive andere Analysezugänge wie beispielsweise die Analyse von Diskursen, gesprächsinteraktiven und biographischen Wissens.

Für die praxistheoretischen Leitfragen der ethnografischen Auswertung sind die Aspekte Materialität - Raum, Körper, Artefakte -, Interaktion - Adressierung, (Selbst-)Positionierung - und Wissen - implizites und explizites Wissen - zentral. Zusätzlich zur codierenden Gesamtauswertung wurde der Blick auf spezifische Feldausschnitte gelenkt und eine Detailanalyse durchgeführt, die sich methodisch auf die Fallanalyse nach Breidenstein et al. (2013) stützt. Zur Operationalisierung der zugrunde liegenden Fragestellung, wie Beratung hergestellt und gestaltet wird, dienen folgende Fragen: Welche Rollen werden von wem wie ,gespielt"? Worin zeigen sich Rollenerwartungen, Verhaltensregeln und Rollenverpflichtungen? Wie wird Beratung durch Körper, Raum und Artefakte gestaltet?

\section{Materialität von Praktiken im Coaching und in der Transfergesellschaft als Steuerungselement}

Im Hinblick auf die oben genannte Fragestellung wird im Folgenden anhand von empirischen Auszügen die Bedeutung von Raum und Artefakten zur Herstellung sozialer Rollen und zur Initiierung, Strukturierung und Steuerung von Beratung aufgezeigt. 


\subsection{Coaching}

Strukturelle Veränderungen in der Arbeitswelt, nämlich die Zurückdrängung und Aufweichung der starr segmentierten und bürokratisch reglementierten Top-down-Steuerung zugunsten der Selbststeuerung sowohl auf der Ebene des*der Einzelnen als auch auf der Ebene der Organisationseinheiten, führen zu veränderten Anforderungen u. a. an Führungspersonen (vgl. Geißler 2010: 181). Vor diesem Hintergrund hat sich Coaching als individuelle Maßnahme der Personalentwicklung seit einigen Jahrzehnten in der Arbeitswelt mit Erfolg etabliert und wird beispielsweise von neu ernannten Führungspersonen für deren relevante Fragestellungen genutzt (vgl. Schreyögg 2010: 19 ff; Böning 2005: 21). Im Rahmen des Projektes „Transition Processing“ wurde für den Fall Coaching mit einem privatwirtschaftlichen Personal- und Organisationsdienstleister, der ,berufliches Coaching für Führungspersonen ${ }^{6}$ anbietet, kooperiert. Bereits während des Feldaufenthaltes stellte sich heraus, dass Coaching weniger zur Vorbereitung und Umsetzung von beruflichen Veränderungsprozessen nachgefragt wurde als vielmehr nachdem bereits eine neue berufliche Position eingenommen wurde. Somit handelt es sich um eine Art nachhaltiger Absicherung dieser Position und wird insbesondere im Folgenden als Unterstützung im Umgang mit den neuen Anforderungen (vgl. Schreyögg 2010: 9) gesehen. Übergänge von Arbeit in Arbeit sind also nicht zwingend mit der Übernahme der neuen Position abgeschlossen, sondern es zeigen sich weitergehende Übergangsprozesse, welche individuell bewältigt werden müssen.

\subsubsection{Raum generiert Atmosphäre und soziale Rollen}

Im Zuge der Analyse der ethnografischen Protokolle wurde deutlich, dass der (Beratungs-)Raum und dessen Gestaltung konstitutiv für das Setting des Coachings sind:

„Im Raum steht ein großer ovaler Tisch mit sechs Stühlen. Der Raum wirkt sachlich und nüchtern, er strahlt eine Arbeitsatmosphäre aus. An den Wänden hängen Plakate. Es scheinen Arbeitsplakate von anderen oder einer anderen Sitzung zu sein. Auf dem Tisch stehen Getränke. [...] Herr Cremers [Coach] sitzt am Kopf des Tisches, Herr Günther [Coachee] rechts von ihm.“(BP 10)

Der Auszug verdeutlicht, dass durch die räumliche Gestaltung sowohl die Kompetenz des Coachinganbieters als auch dessen Kundenorientierung zum Ausdruck gebracht wird. Zum erfolgreichen Verkauf von Coaching scheint neben der Fachlichkeit ein bestimmter, allgemein üblicher Service unerlässlich.

Mit Hilfe der Sitzposition des Coachs am Kopfende des Tisches präsentiert dieser sich als kompetente, strukturierende und leitende Instanz, welche gleichzeitig den Kunden im Blick hat. Die Sitzposition des Coachees deu- 
tet darauf hin, dass dieser Besucher ist, der die unausgesprochenen Regeln dieses Settings kennt und akzeptiert. Die ovale Tischform lockert jedoch die Sitzpositionen auf, da die ,harten' Ecken fehlen und dadurch eine informelle Atmosphäre hergestellt wird. Die Positionierungen im Raum und die damit verbundenen sozialen Rollen werden von beiden Interaktionspartnern nicht hinterfragt und offenbar akzeptiert.

\subsubsection{Artefakte generieren Struktur}

Die folgende Sequenz zeigt den weiteren Verlauf der Coachingsitzung mit Herrn Günther, der seinen formal vollzogenen Übergang in eine neue berufliche Position mit Hilfe von Coaching absichern möchte. Die Analyse zeigt, dass verschiedene Artefakte zur Steuerung und Strukturierung der Sitzung genutzt werden. Der Coach schickte im Vorfeld der Sitzung einen $\mathrm{KODE}^{\mathbb{R}}$-Fragebogen ${ }^{3}$ an den Coachee, überlässt diesem jedoch die Entscheidung, ob der Bogen in der Sitzung bearbeitet werden soll. Dadurch wird suggeriert, dass der Coachee die methodische und inhaltliche Gestaltung der Sitzung bestimmt, jedoch wird die oben beschriebene Rollenverteilung mit dem Coach aufrechterhalten: Zwar entscheidet sich der Coachee für die Bearbeitung des Bogens in der Sitzung, dies geschieht aber unter der Voraussetzung der Steuerung durch den Coach, da diese Entscheidung für den Coachee nur möglich ist, indem ihm der Coach durch das Zuschicken des Bogens überhaupt erst die Möglichkeit gibt, diese Variante zu wählen. Gleichzeitig wird durch die Eröffnung von Wahlmöglichkeiten nochmals deutlich, dass der Coach sich weiterhin als kompetenten, flexiblen und kundenorientierten Akteur darstellt. Das asymmetrische Verhältnis zwischen den Akteuren wird durch die Entscheidungsfreiheit des Coachees zwar punktuell aufgebrochen, jedoch strukturiert der Coach weiterhin die Sitzung und hält so seine Position bzw. Rolle aufrecht:

„Herr Günther bekommt nun einen dritten Bogen mit einzelnen Aussagen, die anzukreuzen sind, er soll die Aussagen, die für ihn zutreffen, ankreuzen. Dafür bekommt er etwas Zeit. Es ist still im Raum. Herr Günther liest und kreuzt an, Herr Cremers schaut auf seinen Block und auf den 2. Bogen. Herr Günther hat zu einem Begriff [...] eine Frage. Diese stellt Herr Cremers hinten an, er soll zunächst den Bogen machen, das würden sie später klären. Er soll sich Zeit lassen und den Bogen auf sich wirken lassen. [...] Herr Cremers verlässt den Raum und kommt mit Papieren zurück. Er hat ein Trainingsprogramm zum Begriff „Delegieren“ für Herrn Günther ausgedruckt. Dies könnte er lesen, wenn's ihm nicht gefällt, soll er es weglegen." (BP 10)

3 KODE® steht für KOmpetenzDiagnostik und -Entwicklung. Es handelt sich um ein Analyseverfahren, bestehend aus mehreren Bögen, zur Messung von individuellen Handlungsfähigkeiten und arbeitet ausschließlich mit ausbaufähigen Stärken und Potenzialen (vgl. https://www.kodekonzept.com/de/kode/ [Zugriff: 13.01.2018]). 
Durch die vorgegebene Art und Weise der Bearbeitung des Bogens ähnelt die Sitzung einer Schulstunde, wodurch das eingangs beschriebene asymmetrische Arbeitsverhältnis reproduziert wird. Im Coaching zeigt sich der Einsatz von Artefakten als strukturierendes Element, mit dem gleichzeitig die Expertise des Coachs hervorgehoben werden kann. Das Coaching wird durch die Artefaktnutzung zu einem fremdgesteuerten Setting. Es sind also nicht allein das Verhalten und die Aussagen des Coachees, welche den Verlauf der Sitzung bestimmen, sondern der Coach leitet mit Hilfe der Artefakte aktiv den Coachingprozess. Standardisierte und damit in der Nutzung routinisierte Artefakte zeigen sich dafür als ein akzeptiertes und geeignetes Instrument. Sie scheinen kontrollierbar, nachvollziehbar, wiederholbar und erleichtern das Sichtbarmachen des Coachingverlaufs und der Inhalte. Das Angebot des Trainingsprogramms impliziert erneut Symmetrie in der Sitzung, womit der freiwillige Charakter und der Dienstleistungsaspekt bedient werden. Außerdem zeigt sich darin ein weiterer Aspekt: Das Coachingunternehmen versucht mithilfe bedarfsgerechter Angebote, den Kunden für weitere Sitzungen zu gewinnen. Dadurch wird zum einen der Bedarf des Kunden gedeckt, zum anderen wird die Finanzierung des Unternehmens sichergestellt.

\subsubsection{Artefakte stellen Sichtbarkeit von Erfolg/Misserfolg her}

Die Nutzung von Artefakten bietet nicht nur Gesprächsanlass in der Coachingsitzung, sondern auch Diskussionsanlass in den regelmäßig stattfindenden Teamsitzungen des Coachingunternehmens:

„Frau Becker [Coach] sagt, es sei wichtig zu präsentieren, was alles passiert ist ,über die Beratung'. Herr Cremers [Coach] ergänzt: ,Nutzen erfahrbar machen‘. Manchmal war der Nutzen so groß, dass ein neues Leitbild für den Kunden entstanden ist. Herr Cremers erzählt weiter, dass er mit einer Skalierung von 1-10 arbeitet. Dafür hat er einen Klebestreifen auf den Fußboden im Flur geklebt und die Coachees sollen sich an der jeweiligen Stelle von 1-10 positionieren. Er macht damit ein ,Vorher-Nachher-Bild‘. Damit arbeitet er einerseits während des Prozesses, also wo wollen die Coachees hin, z. B. von drei auf acht, manchmal macht er dieses Bild zum Abschluss des Prozesses.“ (BP 09)

Diese Sequenz zeigt eine Thematisierung des Einsatzes von Artefakten auf der Metaebene. Hier dienen Artefakte in Form von Methoden als Instrumente, um Erfolge des Coachings sichtbar und „erfahrbar“ zu machen. Damit hat das Artefakt einerseits Einfluss auf die Coachingsitzung, indem es beispielsweise den Schlusspunkt einer Sitzung oder eines Prozesses markiert, und gleichzeitig ist es ein Instrument, um die Wirksamkeit von Coaching zu visualisieren. Damit tangiert der Einsatz der Artefakte nicht nur die direkte Ebene des Coachings, sondern auch die organisationale Ebene, hier die betriebswirtschaftliche Seite des Unternehmens. 


\subsection{Transfergesellschaft}

Im Rahmen einer aktivierenden Arbeitsmarktpolitik gewinnen Transfergesellschaften zunehmend an Bedeutung im Übergang von drohender Arbeitslosigkeit in neue Arbeit. Als Auftakt einer solchen Transfergesellschaft muss vom privaten Träger der Transfergesellschaft als einem Contracting-Out-Partner des öffentlichen Trägers ein sogenanntes Profiling durchgeführt werden (vgl. Peters et al. 2014: 231). Wie aus dem Feldaufenthalt bekannt ist, unterziehen sich alle gekündigten Arbeitnehmer*innen eines Kündigungsbetriebs diesem Profiling-Prozess und entscheiden sich danach für oder gegen die Teilnahme an einer Transfergesellschaft. Das Profiling dient dabei der Erfassung der für die Vermittlung in neue Arbeit relevanten beruflichen Fähigkeiten der Teilnehmenden.

\subsubsection{Transfergesellschaft als institutioneller Lernort}

Für die Herstellung des Settings für den Profiling-Prozess spielt die räumliche Gestaltung eine spezifische Rolle:

„Wir gehen dann alle gemeinsam in den größeren Raum, in dem die Teilnehmenden bereits größtenteils sitzen. Der Aufbau ähnelt einem Klassenzimmer. Tische und Stühle sind frontal zu einem einzelnen Tisch mit Tafel sowie Flipchart aufgebaut. Außen stehen die Tische dann in einem großen U zusammen“ (BP 01).

Die räumliche Gestaltung scheint im Fall dieses Gruppentermins für alle Teilnehmer*innen den Rahmen der Veranstaltung zu setzen, wobei das Arrangement der Tische und Stühle eine Klassenzimmeratmosphäre suggeriert, die klare Rollenverteilungen vorgibt: Die Transferberater*innen stehen als Prozesssteuernde und Inhaltsvermittelnde frontal zu den Teilnehmer*innen als Lernende im Raum. Diese wiederum entsprechen den Rollenerwartungen, die durch die räumliche Gestaltung transportiert werden, und nehmen routinisiert die (Sitz-)Plätze der Lernenden ein. Durch die frontale Sitzordnung im Raum und die daraus resultierende Position der Körper wird der Eindruck eines Lehrer*innen-Schüler*innen-Verhältnisses vermittelt. Durch die spezifische räumliche Anordnung vermittelt die Situation des Profilings den Eindruck eines schulischen Lernorts für Erwachsene, in welchem ein asymmetrisches Lehr-Lernverhältnis inszeniert wird.

\subsubsection{Artefakte als Beratungsanlass}

Die Profiling-Bögen der Agentur für Arbeit zeigen sich als das zentrale Artefakt, welches über beide Tage des Profiling-Prozesses hinweg bearbeitet wird: 
„Dann werden die ,Profiling-Bögen“ der Arbeitsagentur ausgeteilt. Herr Teschner [Transferberater] erklärt, hierbei gehe es um die Vorbereitung auf den Termin bei der Arbeitsagentur. Bei dem Bogen geht es auch darum zu sehen, ,wo stehe ich'. Letztlich müsse man auf dieser Grundlage dann jeweils einen Weg mit den jeweiligen Sachbearbeitern dort finden.“(BP 01)

Am Einsatz der Profiling-Bögen zeigt sich die Kooperation der Transfergesellschaft mit der Agentur für Arbeit, für die sie im Rahmen formalisierter Abläufe nach einer Kündigung eine spezifische Aufgabe übernimmt. Die oben beschriebene, spezifische Einführung der Bögen lässt die Lesart zu, dass eine Verharmlosung (zugespitzt: eine Verschleierung) des eigentlichen Ziels der Bögen (Selektion auf dem Arbeitsmarkt) erfolgt. Die Bögen werden so gerahmt, als ob es lediglich um die Erfassung des aktuellen beruflichen Stands der Teilnehmenden und die Vorbereitung des Termins bei der Agentur für Arbeit ginge. Diese Kontextualisierung lässt den Profiling-Bogen als ein wenig machtvolles Instrument erscheinen, wohingegen der Zweck des Bogens in der arbeitsmarktpolitischen Verwertungslogik darin liegt, die Teilnehmenden hinsichtlich ihrer Fähigkeiten und Qualifikationen in Bezug auf deren Employability zu kategorisieren, d. h. sie damit für den Arbeitsmarkt zu selektieren bzw. ein entsprechendes Matching zu ermöglichen (vgl. Balzereit/ Kolbe 2014). Der Transferberater scheint sich in obenstehender Sequenz vorsichtig von der Agentur für Arbeit zu distanzieren, indem er sich durch den Hinweis auf den weiteren Prozess mit dem jeweiligen Sachbearbeiter lediglich als Experte für den Verfahrensablauf darstellt, sich jedoch nicht zur inhaltlichen Weiterarbeit mit der zuständigen Person bei der Agentur für Arbeit äußert. Er scheint sich also von der Organisation, die Sanktionsmacht besitzt, $\mathrm{zu}$ distanzieren, indem er lediglich auf eine bestehende Verbindung zwischen der Transfergesellschaft und der Agentur für Arbeit hinweist, die parallele Betreuung in unterschiedlicher Weise jedoch möglichst intransparent hält. Zwar ist der Einsatz der Profiling-Bögen ein strukturell verbindliches Element einer Transfergesellschaft, wodurch eine Thematisierung von Übergängen in der Logik der öffentlichen Arbeitsverwaltung erfolgt. Die Transfergesellschaft scheint jedoch auf Abgrenzung bedacht und versucht dadurch möglicherweise, eine Übertragung negativer Einstellungen der gekündigten Arbeitnehmer*innen gegenüber der Agentur für Arbeit auf das Beratungsangebot der Transfergesellschaft zu verhindern, um sich selbst als hilfreiches Beratungsangebot zu positionieren.

\subsubsection{Artefakte zur systematischen Gestaltung der Beratungssituation}

Das Artefakt Profiling-Bogen wird als Einstieg in die Beratungstätigkeit und zur systematischen Gestaltung der Beratungssituation verwendet: 
„Es wird von Frau Garbes erläutert, dass man nun die Einzelgespräche mit den Teilnehmern führen werde. Dabei geht es um Fragen zum Profiling-Bogen und was sonst noch offen sei. Die BeraterInnen werden sich gleich an drei Plätzen im Gebäude verteilen und dann könne immer nacheinander jemand zu ihnen kommen.“ (BP 01)

Die Bögen erfüllen neben der verpflichtenden Weitergabe an die Agentur für Arbeit auch den Zweck, die Beratungssituation einer Transfergesellschaft zu strukturieren, indem zunächst in der Großgruppe damit gearbeitet und dann eine individuelle Beratung angeschlossen wird. Die Profiling-Bögen bieten also einen Anlass, mit den Teilnehmenden den Übergang zu thematisieren und werden dazu methodisch vielfältig eingesetzt. Deutlich werden dadurch die Bemühungen der Transfergesellschaft, die Profiling-Bögen zum persönlichen Vorteil der Teilnehmenden zu gestalten und der verbindlichen Durchführung des Profilings einen individuellen Nutzen der Transfergesellschaft in Form von Kurzprofilen abzugewinnen.

\section{Vergleichendes Fazit}

Werden die beiden Fälle Coaching und Transfergesellschaft mit dem Fokus auf Materialität von Praktiken vergleichend in den Blick genommen, ergeben sich ähnliche Einsichten: Die Materialität in Form des Raumes zeigt sich sowohl im Coaching als auch in der Transfergesellschaft als konstitutives Element für Atmosphäre, Positionierung der Körper und die Etablierung der damit verbundenen sozialen Rollen. Dabei dominiert in der Transfergesellschaft der schulähnliche Charakter, im Coaching wird gleichzeitig der Dienstleistungscharakter hervorgehoben.

Die Beratungssituation wird zudem durch den Einsatz von Artefakten systematisch gestaltet und gesteuert. Artefakte dienen in beiden Fällen als Medium der Gestaltung, als Redeanlass und somit zur methodischen Umsetzung von Beratungsgesprächen. Insbesondere im Coaching generiert das Artefakt eine Ordnung des Sprechens und bietet objektiven, durch den Arbeitsbogen generierten Gesprächsanlass und -inhalt. Die Artefakte stellen einerseits das Beratungssetting her und bieten andererseits über den Beratungsanlass hinaus weitere Verwertbarkeit. In der Transfergesellschaft werden beispielsweise zum einen den Teilnehmer*innen, zum anderen aber auch der Agentur für Arbeit und potentiellen neuen Arbeitgebern in Form von Kurzprofilen vorhandene berufliche Fähigkeiten der Arbeitnehmer*innen sichtbar gemacht. Im Coaching wird die Artefaktnutzung dem Coachee angeboten, er hat die Wahl, diese im Beratungssetting und darüber hinaus für eine Reflexion der eigenen Fähigkeiten zu nutzen. In beiden Fällen geht es dabei um eine Kompetenzbilanzierung der zu Beratenden, die allerdings in divergenter Weise 
verwertet wird: In der Transfergesellschaft werden die ermittelten Kompetenzen an übergeordnete und sonstige relevante Institutionen weitergeleitet. Im Coaching dagegen liegt die Verantwortung für die weitere Verwendung allein beim Coachee. Es zeigt sich hier ein relevanter Unterschied: innerhalb der Transfergesellschaft erzielte Ergebnisse müssen für Dritte verwertbar sein, dagegen sind Coaching-Inhalte ausschließlich für die eigenverantwortliche Nutzung durch den Coachee vorgesehen, es gibt keine strukturellen Vorgaben. Im Coaching zeigt sich gleichfalls die Wahlmöglichkeit des Coachs, ihm passend erscheinende Artefakte in einem Beratungsprozess einzusetzen, in der Transfergesellschaft ist zumindest die Nutzung des Artefakts Profiling-Bogen strukturell vorgegeben. In beiden Fällen ermöglicht die Verwendung von Artefakten den Berater*innen jedoch gleichermaßen, sich in ihrer Rolle als steuernde Expert*innen für die Übergangsbearbeitung zu positionieren.

Der in Kapitel 2 angedeutete Problemaufriss, dass die Bewältigung von Übergängen in Arbeit im Verlauf einer Biografie zunehmend schwieriger und unsicherer wird und Beratung politisch als Lösungsansatz angesehen wird, verdeutlicht die Relevanz der Forschung im Handlungsfeld Beratung. Wie die vorgestellte empirische Analyse zeigt, lässt sich Beratung am Übergang in Arbeit sowohl im Fall Transfergesellschaft als auch im Fall Coaching in den Kontext von Lern- und Bildungsprozessen stellen (vgl. Maier-Gutheil 2016: 103; 2012: 489), d. h. Übergänge in Arbeit und deren Begleitung können eng mit der Frage des Lernens bzw. des Lernens Erwachsener verknüpft werden (vgl. Hof/Maier-Gutheil 2014: 150). Damit wird Beratung am Übergang in Arbeit zum Thema der Erwachsenenbildung. Im Hinblick auf die explizierten Analyseergebnisse erscheint es ergiebig, beispielsweise die Rolle und die Relevanz der professionellen Berater*innen im Beratungsprozess weitergehend zu beleuchten, da diesen, wie bereits angedeutet, eine erhebliche Steuerungsfunktion im Beratungsprozess zukommt.

\section{Literatur}

Balzereit, Marcus/Kolbe Christian (2014): Mechanismen der Zurichtung und Ausschließung an Übergängen in Schule und Arbeitsmarkt. In: Hof, Christiane/ Meuth, Miriam/Walther, Andreas (Hg.): Pädagogik der Übergänge. Weinheim Basel: Beltz Verlag, S. 49-77.

Bausch, Constanze (2001): Die Inszenierung des Sozialen. Erving Goffman und das Performative. In: Wulf, Christian/Göhlich, Michael/Zirfas, Jörg: Grundlagen des Performativen. Eine Einführung in die Zusammenhänge von Sprache, Macht und Handeln. Weinheim und München: Juventa Verlag, S. 203-225.

Böning, Uwe (2005): Coaching: Der Siegeszug eines Personalentwicklungs-Instruments - Eine 15-Jahres-Bilanz. In: Rauen, Christopher (Hg.): Handbuch Coaching. 3. Auflage. Göttingen: Hogrefe Verlag, S. 21-54. 
Breidenstein, Georg/Hirschauer, Stefan/Kalthoff, Herbert/Nieswand, Boris (2013): Ethnografie. Die Praxis der Feldforschung. Konstanz mit UVK/Lucius \& München, UVK Verlagsgesellschaft.

Charmaz, Kathy (2006): Constructing Grounded Theory: A Practical Guide Through Qualitative Analysis. Los Angeles, Calif. [u. a.]: Sage.

Dollhausen, Karin (2010): Ethnografisch orientierte Organisationsforschung als Ressource der Organisationsberatung in Weiterbildungseinrichtungen. In: Göhlich, Michael/Weber, Susanne M./Seitter, Wolfgang/Feld, Timm C. (Hg): Organisation und Beratung. Wiesbaden: VS Verlag für Sozialwissenschaften, S. 69-77.

Geissler, Harald (2010): Business Coaching als Kommunikationsgattung pädagogischer Beratung - eine empirische Rekonstruktion. In: Göhlich, Michael/Weber, Susanne M./Seitter, Wolfgang/Feld, Timm C. (Hg): Organisation und Beratung. Wiesbaden: VS Verlag für Sozialwissenschaften, S. 179-190.

Gieseke, Wiltrud (2016): Entscheidungstheoretische Grundlagen der Beratung. In: Gieseke, Wiltrud/Nittel, Dieter (Hg.): Handbuch Pädagogische Beratung über die Lebensspanne. Weinheim: Beltz Juventa, S. 102-111.

Glaser, Barney G./Strauss, Anselm L. (2010): Grounded theory: Strategien qualitativer Forschung. 3. unveränd. Auflage. Bern: Huber.

Goffman, Erving (1959): The Presentation of Self in Everyday Life. New York: Doubleday.

Helsper, Werner (2013): Die Bedeutung von Übergängen im Bildungsverlauf. In: Siebholz, Susanne/Schneider, Edina/Schippling, Anne/Busse, Susann/Sandring, Sabine (Hg.): Prozesse sozialer Ungleichheit. Wiesbaden: VS Verlag für Sozialwissenschaften, S. 21-28.

Hof, Christiane/Maier-Gutheil, Cornelia (2014): Übergänge im Erwachsenenalter. Befunde und offene Fragen für die Erwachsenenbildung. In: Hof, Christiane/ Meuth, Miriam/Walther, Andreas (Hg.): Pädagogik der Übergänge. Übergänge in Lebenslauf und Biografie als Anlässe und Bezugspunkte von Erziehung, Bildung und Hilfe. Weinheim: Beltz Juventa, S. 146-167.

Karl, Ute (2014): Rationalitäten des Übergangs in Erwerbsarbeit. Weinheim und Basel: Beltz Juventa Verlag.

Maier-Gutheil, Cornelia (2016): Beraten. Stuttgart: Kohlhammer.

Maier-Gutheil, Cornelia (2012): Qualitativ-empirische Beratungsforschung in der Erwachsenen- und Weiterbildung. In: Schäffer, Burkhard/Dörner, Olaf (Hg.): Handbuch Qualitative Erwachsenen- und Weiterbildungsforschung. Opladen: Budrich, S. 489-501.

Ott, Marion (2011): Aktivierung von (In-)Kompetenz. Praktiken im Profiling - eine machtanalytische Ethnografie. Konstanz : UVK Verlagsgesellschaft mbH.

Peters, Luisa/Truschkat, Inga/Herz, Andrea (2014): Die Entwicklung arbeitsmarktpolitscher Übergangsgestaltung am Beispiel von Transfergesellschaften. In: Karl, Ute (Hg.): Rationalitäten des Übergangs in Erwerbsarbeit. Weinheim: Beltz Juventa, S. 222-237.

Reckwitz, Andreas (2003): Grundelemente einer Theorie sozialer Praktiken. Eine sozialtheoretische Perspektive. In: Zeitschrift für Soziologie, Jg. 32, Heft 4, S.282301. Online verfügbar unter: http://www.zfs-online.org/index.php/zfs/article/ view/1137/674 [Zugriff: 13.12.2017] 
Schiersmann, Christiane (2011): Beratung im Kontext von Lebenslangem Lernen und Life Design - ein Generationen übergreifendes Konzept. In: Eckert, Thomas/ von Hippel, Aiga/Pietraß, Manuela/Schmidt-Hertha, Bernhard (Hg.): Bildung der Generationen. Wiesbaden: VS Verlag für Sozialwissenschaften, S. 427-440.

Schreyögg, Astrid (2010): Coaching für die neu ernannte Führungskraft. Wiesbaden: VS Verlag für Sozialwissenschaften.

Stauber, Barbara/Walther, Andreas/Pohl, Axel (Hg.) (2007): Subjektorientierte Übergangsforschung. Weinheim: Juventa.

Tippelt, Rudolf (2013): Lebenswelten und Lebenslagen - Der Nutzen empirischer Milieuforschung für die Bildungsberatung. In: Hammerer, Marika/Kanelutti-Chilas, Erika/Melter, Ingeborg (Hg.): Zukunftsfeld Bildungs- und Berufsberatung II. Das Gemeinsame in der Differenz finden. Bielefeld: W. Bertelsmann Verlag, S. 71-82.

Walther, Andreas (2016): Übergangsberatung. In: Gieseke, Wiltrud/Nittel, Dieter (Hg.): Handbuch pädagogische Beratung über die Lebensspanne. Weinheim: Beltz Juventa, S. 644-655.

Walther, Andreas (2008): Die Entdeckung der jungen Erwachsenen: eine neue Lebensphase oder die Entstandardisierung des Lebenslaufs? In: Rietzke, Tim/ Galuska, Michael (Hg.): Lebensalter und Soziale Arbeit, Band 4: Junges Erwachsenenalter. Baltmannsweiler: Schneider Verlag Hohengehren, S. 10-36. 



\section{Intervention in den Studienverlauf und Beratung}

\section{Einleitung}

Vor dem Hintergrund des Lebenslangen Lernens und des Fachkräftemangels stellt die akademische Bildung (junger) Erwachsener eine zentrale Quelle für Produktivität und Wachstum dar. Lehr- und Lernkulturen und ihre Ausprägungsformen an Hochschulen in Deutschland sind daher von besonderem Interesse für die Forschung im Bereich der Erwachsenenbildung (Rhein 2016). Insbesondere problematische Studienverläufe und Studienabbrüche sind derzeit ein wichtiges Thema in bildungspolitischen Debatten (Heublein et al. 2017). Probleme im Studium können eine Studienabbruchneigung bedingen (für den Zusammenhang mit Studienabbruch: Bean 1982; Blüthmann 2012; Gold 1988), die als ,die Häufigkeit bzw. die Ernsthaftigkeit von Gedanken an das Verlassen der Universität“ (Blüthmann 2012: 89) operationalisiert wird.

Stimmt man mit Schüßler (2016) überein, dass Erwachsenenbildung die Lernkulturen der Gesellschaft aktiv mitgestalten soll, muss sie gerade solche Projekte und Vorhaben kritisch-konstruktiv begleiten, in denen präventiv an der Vermeidung von Studienabbrüchen gearbeitet wird. Dazu gehören vor allem sog. Frühwarnsysteme, die an vielen deutschen Hochschulen gegenwärtig im Rahmen von Projekten aufgebaut und getestet werden. Mit ihnen kann der Anspruch verwirklicht werden, ,in und mit der Praxis zu experimentieren“ (Schüßler 2016: 24).

Für den vorliegenden Beitrag soll das Zusammenspiel von Beratung und Intervention in Frühwarnsystemen am Beispiel der Technischen Universität Dresden (TU Dresden) behandelt werden, wobei die Problemlagen der Studierenden und ihr Umgang mit diesen im Mittelpunkt stehen. Diese sind die Basis für den Aufbau von wirksamen Frühwarnsystemen an Hochschulen. Im Folgenden werden sowohl Rahmenbedingungen von Frühwarnsystemen und ihre theoretischen Grundlagen als auch die Spezifika der methodischen Herangehensweise und die Ergebnisse des Frühwarnsystems der TU Dresden vorgestellt. 


\section{Beratung und Intervention im Hochschulkontext}

Laut Schubert (2015) bemüht sich die Beratung derzeit um Professionalisierung hinsichtlich eines gemeinsamen Ausbildungs- und Beratungsverständnisses sowie der Qualitätssicherung unter Einbindung von Wissenschaft. „Beratung ist [...] als professionelles Angebot zu ko-konstruktiven, reflexiven Hilfestellungen in der Bewältigung krisenhafter moderner Lebens- und Arbeitsbedingungen“ (ebd.: 41) zu verstehen. Der Beratung kommt daher ein besonderer Stellenwert im Rahmen der Erwachsenenbildung an Hochschulen $\mathrm{zu}$.

Verschiedene Präventions- und Interventionsmaßnahmen werden derzeit mit dem Ziel, Studierende über die Beratung hinaus bei ihrem Studium zu unterstützen und Abbrüche zu vermeiden, an deutschen Universitäten erprobt, eingerichtet oder evaluiert (Ahles et al. 2016; Blüthmann et al. 2008; Kolb et al. 2006; Pelz/Schulze-Stocker 2018).

$\mathrm{Zu}$ diesen Maßnahmen, die zum Teil auch als zeitlich begrenzte Projekte angeboten werden, zählen auch Frühwarnsysteme. Sie entstammen dem Risikomanagement (Hahn/Krystek 1984) und dienen dort als Informationssysteme für mögliche Gefahren, die mit zeitlichem Vorlauf angezeigt werden. Das soll die Möglichkeit schaffen, rechtzeitig geeignete Maßnahmen zur „Umgehung, Abwehr oder Minderung der signalisierten Gefährdung ergreifen zu können" (ebd.: 4). In Deutschland werden derzeit an einigen Hochschulen Frühwarnsysteme erprobt (TU Dresden 2017; BMBF 2016). Für die Etablierung eines Frühwarnsystems ist es zentral zu wissen, welche Probleme Studierende an Hochschulen haben und wie sie mit diesen Schwierigkeiten umgehen.

\section{Theoretische Grundlagen}

\subsection{Ursachen für Studienabbruch}

Studienabbruch ist nicht nur von dem oder der Studierenden allein abhängig (bspw. Vorgeschichte, Lebensumstände, kognitive Fähigkeiten etc.), sondern auch von Merkmalen der Hochschulen (z. B. Lehrkapazitäten und -qualitäten, Ausstattung, formalrechtliche Studienstrukturen, Lehr- und Studienkultur) und gesellschaftlichen Einflüssen. Die Gewichtung der einzelnen Einflussfaktoren variiert dabei je nach Forschungsfokus und zugrundeliegender theoretischer Perspektive, also bspw. interaktionistischer, psychologischer, sozio- 
kultureller Perspektive oder rationaler Wahl (weiterführend: Sarcletti/Müller 2011; andere Differenzierungen: Schuemer/Ströhlein 1991).

Es gibt theoretische Modelle, die Studienabbruch als multifaktoriellen Prozess begreifen. Beispielsweise werden individuelle, institutionelle und gesellschaftliche Faktoren (Heublein/Wolter 2011) oder psychologische, soziodemografische und institutionelle Einflussfaktoren (Schiefele et al. 2007), aber auch Studienvoraussetzungen, Studien- und Kontextbedingungen, Studier- und Lernverhalten und Studienerfolg (Blüthmann et al. 2008) unterschieden.

\subsection{Handlungsmöglichkeiten der Studierenden bei Schwierigkeiten}

Nicht minder wichtig als die Frage nach den Ursachen für Schwierigkeiten im Studium ist die Frage nach ihren Folgen: Welche Möglichkeiten haben Studierende, auf Schwierigkeiten zu reagieren? Zur Erklärung und Vorhersage von Handlungen lassen sich verschiedene klassische Theorien heranziehen, die auf dem Rational-Choice-Ansatz aufbauen. Dazu gehören bspw. die Spieltheorie (Hargreaves Heap/Varoufakis 2004), die Theory of reasoned action/of planned behavior (Ajzen 1991) oder die Exit-Voice-Theorie (Hirschman 1974). Während die ersten beiden eine große Zahl an Annahmen für die Analyse von Entscheidungssituationen voraussetzen, ist die dritte prädestiniert für Zwecke eines Frühwarnsystems an Hochschulen. ${ }^{1}$

Hirschmans Theorie ist ein Schlüsselwerk der Wirtschaftssoziologie (Knoll 2017) und beruht auf der Annahme, dass eine Person, die unzufrieden mit einer Institution bzw. einer bestimmten Situation ist, zwei Möglichkeiten hat zu reagieren: Entweder sie wählt Exit, verlässt also diese Situation auf der Suche nach einer anderen, oder sie wählt Voice, erhebt - bildlich gesprochen die Stimme und versucht, die Situation aktiv zu verändern (Hirschman 1974; Barry 1974). Auf Studierende angewendet, könnte das bedeuten, dass sie etwa mit den Leistungsanforderungen im Studium unzufrieden sind und daher überlegen, das Studienfach zu wechseln, das Studium abzubrechen, eine längere Pause bspw. mit einem Praktikum einzulegen oder sich zurückzuziehen und das Studium z. B. mit Hilfe von Ablenkung zu verdrängen (Exit). Es könnte aber auch bedeuten, sich hochschulpolitisch für bessere Studienbedingungen einzusetzen, einen Workshop zum Selbstmanagement zu besuchen oder eben auch an einem Frühwarnsystem teilzunehmen (Voice). Welche Re-

1 Zumal Hirschman seine Theorie in einer Makroperspektive auf Bildungsthemen übertragen hat (Hirschman 1984). 
aktion gezeigt wird bzw. in welcher Reihenfolge welche Reaktionen auftreten (auch Gleichzeitigkeit ist möglich), hängt neben Persönlichkeitsmerkmalen wie Selbstständigkeit, Zielorientierung oder Motivation (in-/extrinsisch) auch von der sogenannten Loyalität ab, die laut Hirschman (1974) einer Institution entgegengebracht wird (z. B. Fachbindung).

\section{Fragestellung}

Auf Grundlage dieser theoretischen Zugänge zu Ursachen von Schwierigkeiten und Handlungsmöglichkeiten der Studierenden sind zwei Fragen zentral für die Etablierung eines Frühwarnsystems:

1. Welche Probleme haben Studierende während des Studiums?

2. Wie gehen Studierende mit Schwierigkeiten im Studium um?

Im Folgenden geht es um die an der TU Dresden beschrittenen methodischen Wege, diese Fragen zu beantworten.

\section{Methode}

\subsection{Kontext: Das Frühwarnsystem an der TU Dresden}

Das Frühwarnsystem „Partnerschaft · Studienerfolg · TU Dresden“ (kurz: PASST?!)2 ist Teil des Studienerfolgskonzepts der TU Dresden und wird kooperativ von der Zentralen Studienberatung und dem Zentrum für Qualitätsanalyse koordiniert. Aus seinen drei zentralen Aufgabenbereichen (Abbildung 1) resultieren die unterschiedlichen Zugänge zu den für das Frühwarnsystem grundlegenden Informationen.

2 Mit einer Laufzeit von 03/2016 bis 12/2020 wird PASST?! vom Freistaat Sachsen aus dem Hochschulpakt 2020 finanziert. Weitere Informationen: http://tu-dresden.de/passt (17.07.2018). 


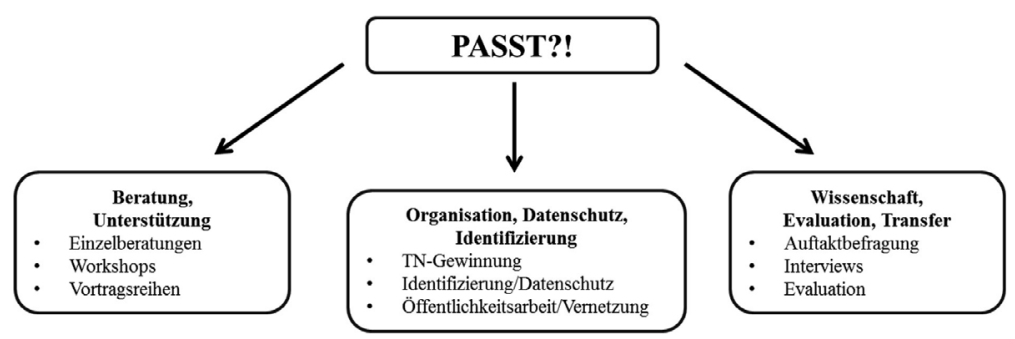

Abb. 1: Arbeitsbereiche des PASST?!-Programms (Quelle: Schulze-Stocker et al. 2017)

Für das Frühwarnsystem wurden aufbauend auf den Erfahrungen der Zentralen Studienberatung der TU Dresden und der Forschungslage (u. a. Kolb et al. 2006; Hörnstein et al. 2016) 5 Identifizierungsmerkmale festgelegt, die den Studienverlauf betreffen und Hinweise auf Probleme sein können (Details: Schulze-Stocker et al. 2017). Freiwillig Teilnehmende (rund 5.800 $=17 \%$ der TU-Studierenden; Stand: 01.07.2018) bekommen immer dann eine E-Mail, wenn eines oder mehrere Merkmale auf sie zutreffen. Diese enthält Empfehlungen zu individuell zugeschnittenen (PASST?!-)Beratungs- und Unterstützungsangeboten. Die Teilnahme an diesen ist ebenfalls freiwillig.

Um die Brauchbarkeit der Identifizierungsmerkmale zu prüfen und Informationen zu den TU-spezifischen Problemlagen (Schwerpunkt: studienstrukturelle Aspekte und Studienverlauf) zu erhalten, wurde eine quantitative Befragung TU-Studierender durchgeführt. Anschließend fokussierten qualitative Interviews (persönliche) Hintergründe für Schwierigkeiten der Studierenden und ihre Bewältigungsstrategien. Aktuell wird das Frühwarnsystem evaluiert. Zudem sollen zukünftig die gewonnenen Daten in das Qualitätsmanagementsystem der TU Dresden einfließen.

\subsection{Anlage \& Stichprobe}

\subsubsection{Auftaktbefragung}

Die Auftakterhebung wurde als quantitative, onlinebasierte Studierendenbefragung im Sommersemester 2016 durchgeführt. Die gestellten Fragen waren an das Modell von Blüthmann et al. (2008) angelehnt und beinhalteten Aspekte zu Wechsel-, Unterbrechungs- oder Abbruchgedanken (mit Fokus auf den Studiengang), Eingangsvoraussetzungen, Studier- und Lernverhalten sowie Kontextbedingungen. 
Studierende von 11 Studiengängen ${ }^{3}$ (rund 10\% der TU-Studierenden) wurden angeschrieben, von denen rund $13 \%$ an der Befragung teilnahmen $(\mathrm{n}=463)^{4}$. Zum annähernd gleichen Anteil haben Frauen wie Männer die gestellten Fragen beantwortet. Im Mittel waren die Befragten 22 Jahre alt und studierten im 4. (Bachelor) bzw. 6. (Diplom) Fachsemester (Details: Schulze-Stocker et al. 2017; Pelz/Schulze-Stocker 2018).

\subsubsection{Interviewstudie}

Als Folgebefragung wurden qualitative Interviews im Sommersemester 2017 mit 17 (von 72 angeschriebenen) Personen durchgeführt, die in der Auftaktbefragung Abbruchgedanken formulierten. Das Ziel der Interviews lag in der Erforschung der Gründe für Probleme im Studium sowie der Strategien des Umgangs mit diesen Problemen und Schwierigkeiten bzw. unsicheren Entscheidungssituationen (auch: Meinefeld 2007; zur Methode: Nawratil 2008). Zentrale Aspekte des zugrunde liegenden teilstandardisierten Leitfadens waren die Studienmotivation, Schwierigkeiten und Herausforderungen im Studium sowie Bedarfe, Ressourcen und wahrgenommene/genutzte Hilfen im Umgang mit den Schwierigkeiten.

Die Befragten stammten aus 8 der 11 Studiengänge, die in der Auftaktbefragung vertreten waren. Der Frauenanteil lag bei 52,9\%.

3 Die Auswahl erfolgte im Rahmen der Pilotphase des Projektes problem- und vielfaltsbezogen. Es wurden folglich jene Studiengänge ausgewählt, die hohe Abbruchquoten oder andere spezifische Problemlagen aufwiesen: Bauingenieurwesen (Diplom), Elektrotechnik (Diplom), Mechatronik (Diplom), Regenerative Energiesysteme (Diplom), Soziologie (Diplom und Bachelor), Biologie (Bachelor), Informatik (Bachelor), Medieninformatik (Bachelor), Philosophie (Bachelor) und Sprach-, Literatur- und Kulturwissenschaften (Bachelor).

4 Diese recht geringe Ausschöpfungsquote ist bei der vielbefragten Gruppe der Studierenden nicht überraschend (Engel/Schmidt 2014). Die Stichprobe ist repräsentativ für die Pilotstudiengänge: Verzerrungen der Stichprobe bzgl. Geschlecht und Studiengang treten nur minimal durch eine leichte Überrepräsentation der Soziologie auf. In den Ergebnissen zeigt sich, dass der Umfang der Problemlagen der Studierenden an der TU Dresden nicht von den Ergebnissen anderer Forschungsgruppen (z. B. Heublein et al. 2017; Römer et al. 2013) abweicht. Obwohl die Befragten also aus rein methodischen Überlegungen nicht repräsentativ für alle TU-Studierenden stehen, zeigt sich eine hohe externe Validität. 


\section{Ergebnisse}

\subsection{Auftaktbefragung}

Rund 30\% der Befragten gaben Studienabbruchgedanken an. Diese beziehen sich sowohl auf den Wechsel des Studiengangs oder der Universität als auch auf eine Unterbrechung des Studiengangs oder einen Abbruch des Studiengangs ohne Wiedereinstieg. Für die Analyse der Daten der Auftaktbefragung wurden zuerst bivariate statistische Verfahren mit dem Ziel eingesetzt, erste Anhaltspunkte für mögliche Einflussvariablen auf Studienabbruchgedanken an der TU Dresden zu erlangen (Schulze-Stocker et al. 2017). Als Aspekte des individuellen Studier- und Lernverhaltens stehen bisher erworbene Leistungspunkte, die nicht den Studienempfehlungen entsprechen $($ Cramer-V $=.15, \mathrm{p}<.05)$, und nichtbestandene Module (Cramer-V=.19, $\mathrm{p}<.05)$ in einem vergleichsweise schwachen, aber statistisch signifikanten Zusammenhang mit diesen Gedanken. Etwas stärkere Effekte lassen sich für die Einschätzung des eigenen Leistungsstands im Vergleich zu anderen Studierenden aufzeigen (Cramer-V=.24, $\mathrm{p}=.00$ ). Gleiches lässt sich für die $\mathrm{Zu}$ friedenheit mit den eigenen Leistungen im Studium (Cramer-V=.23, $\mathrm{p}=.00$ ) nachweisen. Zudem wurden die Bedarfe hinsichtlich möglicher Unterstützung erfragt. Rund $2 / 3$ der Studierenden wünschen sich mehr Unterstützung bei der Studien- und Prüfungsorganisation; 63\% formulierten einen Bedarf an besserer Betreuung.

Anschließend wurde als multivariates Verfahren eine binär-logistische Regression mit einem hierarchischen Einschlussverfahren von drei Variablenblöcken (Eingangsvoraussetzungen zum Studium [z. B. Bildungsherkunft, Informationsstand]; Studienbedingungen und Studien- und Lernverhalten; Kontextbedingungen [bspw. Kinderbetreuung, Pflegeverantwortung]) gerechnet (Pelz/Schulze-Stocker 2018). Dabei zeigte sich, dass ungünstige Eingangsvoraussetzungen für das Studium in Form eines schlechten Informationsstandes über das gewählte Studienfach sowie die Studienwahlmotivation die Studienabbruchneigung beeinflussen. Einen nicht zu unterschätzenden Effekt haben zudem die Studienbedingungen - im Besonderen die Transparenz der Studienanforderungen - und die Zufriedenheit mit diesen Faktoren sowie Leistungsschwierigkeiten. Hinsichtlich der belastenden Lebensumstände, die mögliche Einflussfaktoren sein können, zeigten sich an der TU Dresden nur für psychische Belastungen signifikante Effekte. 


\subsection{Interviewstudie}

Das vorliegende Interviewmaterial wurde inhaltsanalytisch auf der Basis eines induktiv-deduktiv erstellten Kategoriensystems ausgewertet (Nawratil/ Schönhagen 2008). Die in den Interviews genannten Schwierigkeiten der Studierenden umfassen u. a. Leistungsprobleme (26 Nennungen), Probleme bei der Studienorganisation (21 Nennungen), Unvereinbarkeit von Studium und Familie, Beruf oder Freundschaften (11 Nennungen), aber auch Probleme mit den Studieninhalten (11 Nennungen), wobei häufig Mehrfachnennungen vorkommen.

Bei den Reaktionen auf Probleme und Schwierigkeiten - im Sinne von Voice - werden genannt (Mehrfachnennungen):

- Konsultation von und Austausch mit Kommilitoninnen und Kommilitonen (9 Nennungen),

- Nutzung von Beratung (15 Nennungen),

- Strategische Studienorganisation (12 Nennungen, wie z. B. Teilnahme an Lerngruppen - 4 Nennungen).

Daneben stellt das aktive Einfordern von Unterstützung eine weitere Voice-Strategie dar, indem Unterstützungsbedarfe klar benannt werden (13 Nennungen).

Der Studienabbruchgedanke (eine Nennung) oder die Recherche nach Alternativen zur jetzigen Studiensituation (8 Nennungen) können als Exit verstanden werden.

\section{Zusammenführung und Diskussion der Ergebnisse}

Forschungsfrage 1: Welche Probleme haben Studierende während des Studiums?

Die dargestellten Ergebnisse zeigen, dass die Gründe für Schwierigkeiten im Studium an der TU Dresden sowohl auf der studienstrukturellen als auch auf der persönlichen Seite gelagert sind. Insofern unterscheiden sich die hier gewonnenen Erkenntnisse nicht merklich von bundesweiten Studien (etwa Heublein et al. 2017), was im Vorfeld zum Aufbau eines Frühwarnsystems aber nicht einfach angenommen werden konnte (Stichwort: ökologischer Fehlschluss). Insgesamt lässt sich beobachten, dass Gedanken an einen Studienabbruch im hier definierten Sinne von einem Faktorenbündel beeinflusst werden. Dies stellt Institutionen, die intervenierend tätig sein wollen, vor komplexe Herausforderungen. 
Forschungsfrage 2: Wie gehen Studierende mit Schwierigkeiten im Studium um?

Die quantitative Befragung und auch die qualitativen Interviews zeigen, dass sich die Studierenden der TU Dresden sowohl für Voice- als auch für Exit-Optionen entscheiden, wenn Schwierigkeiten im Studium auftreten. In welchem Verhältnis und in welcher Reihenfolge dies geschieht, kann mit den bisher erhobenen Daten noch nicht geklärt werden. Das betrifft auch die Bedeutung der Loyalität, also der Bindung an die TU Dresden als Institution und den gewählten Studiengang. Im Detail zeigt sich die Unzufriedenheit bezüglich der Studieninhalte, -bedingungen, -leistungen und -anforderungen und des Informationsstands zu Beginn des Studiums sowie bei psychischen Belastungen und der Vereinbarkeit von Studium, Beruf, Familie und sozialem Umfeld. Die Studienabbruchgedanken oder auch das Recherchieren nach Alternativen zeugen von einer Vorstufe der Exit-Strategie, wohingegen die Inanspruchnahme von Beratung, das Beschweren, die Teilnahme am Frühwarnsystem, die Gründung von Lerngruppen und das Anzeigen von Unterstützungsbedarfen für Voice stehen (Abbildung 2).

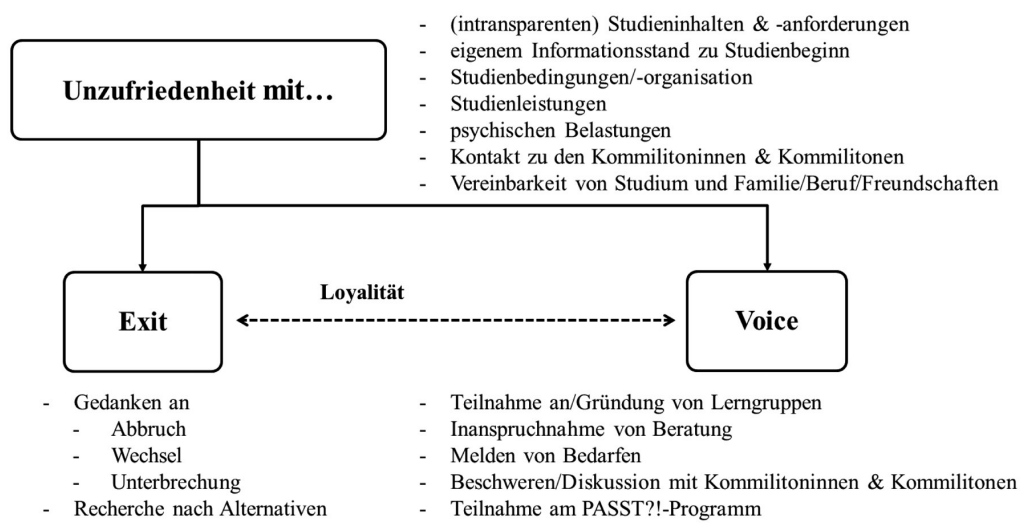

Abb. 2: Zusammenführung der PASST?!-Ergebnisse mit dem Exit-Voice-Modell (Quelle: eigene Darstellung)

Frühwarnsysteme als verwaltungstechnische Eingriffe in das Studium führen nicht nur zu intendierten Reaktionen, die den Studienabbruch verhindern sollen, sondern - wie fast immer bei rationalen Eingriffen in bestehende Institutionen - unter gewissen Umständen auch zum Gegenteil (Seibel 2016). Je nach Kontext, Persönlichkeitstyp und individueller Motivationslage kann 
eine frühzeitige Intervention den Abbruchwunsch sogar erst hervorrufen oder einen vorhandenen Wunsch in diese Richtung verstärken. Für die persönliche Entwicklung und für den weiteren Bildungsweg kann das aber auch eine Chance zum Neubeginn sein. Daraus ergeben sich hohe Ansprüche an die professionellen Kompetenzen von Studienberaterinnen und Studienberatern und deren Qualifizierung (z. B. Hoff/Zwicker-Pelzer 2015; Stiehler 2007).

\section{Fazit \& Ausblick}

Im Namen des Programms „Partnerschaft - Studienerfolg - TU Dresden“ spiegelt sich Beratung als „fachkundige Partnerschaft auf Zeit“ (Hoff/Zwicker-Pelzer 2015: 16) wider, die durch Identifizierung und wissenschaftliche Begleitung ergänzt wird. Im Zuge der Professionalisierung von Beratung bieten Frühwarnsysteme wichtige Unterstützungsleistungen für die Beratung an, wenn es darum geht, Studierende mit Problemlagen zu erreichen. Dies geschieht jedoch nicht im Sinne einer ,Klientifizierung“ oder als ,Erfüllungsgehilfen' im Rahmen von Ökonomisierungstendenzen, wie etwa von Wilhelm (2017) befürchtet, sondern auf freiwilliger Basis und mit der Akzeptanz, dass individuelle Bildungswege auch abseits des ,objektiven` Studienerfolgs möglich und nötig sind.

Da die Erprobung des Programms erst 2020 endet, sind die Antworten auf die gestellten Fragen nach Problemen der Studierenden und deren Umgang vorläufig und weiter offen für Experimente mit der Praxis (Schüßler 2016). Gewissermaßen experimentell ist auch die Verwendung der im Rahmen der Forschung zur Erwachsenenbildung bisher kaum genutzten Exit-Voice-Theorie (Hirschman 1974). Ihre Übertragung kann auch auf anderen Feldern der Erziehungswissenschaft fruchtbar sein - auf jeden Fall immer dann, wenn der konkrete Umgang mit Schwierigkeiten handlungstheoretisch erfasst werden soll.

Um das Frühwarnsystem im dargelegten Sinne weiter zu verbessern und den Bedürfnissen der TU Dresden und ihrer Studierenden anzupassen, werden im Rahmen regelmäßiger Erhebungen die Wünsche der PASST?!-Teilnehmerinnen und -Teilnehmer berücksichtigt und zugleich überprüft, wie sie mit der Intervention und den Angeboten umgehen: Welche weiteren Identifizierungsmerkmale wünschen sie sich? Welche Beratungs- und Unterstützungsangebote vermissen sie? In welchen Situationen zeigen sie Exit- oder Voice-Reaktionen? In welchen Situationen bewirkt PASST?! vielleicht nicht-intendierte Effekte? Tritt durch die verstärkte und hochschulweite, offene Auseinandersetzung mit dem Thema Studienprobleme vielleicht eine Enttabuisierung 
beim Umgang damit ein? Letzteres wäre dann keine negative, sondern - gerade für junge Menschen am Anfang ihres Bildungsweges - eine positive, nicht-intendierte Folge der Einführung eines Frühwarnsystems an einer deutschen Hochschule.

\section{Literatur}

Ahles, Lisa/Köstler, Ursula/Vetter, Nicole/Wulff, Anne (2016): Studienabbrüche an deutschen Hochschulen. Stand der Thematisierung und strategische Ansatzpunkte. Baden-Baden: Nomos.

Ajzen, Icek (1991): The theory of planned behavior. In: Organizational Behavior and Human Decision Processes 50, 2, S. 179-211.

Barry, Brian (1974): Review Article: ,Exit, Voice, and Loyalty‘. In: British Journal of Political Science 4, 1, S. 79-107.

Bean, John P. (1982): Student Attrition, Intentions, and Confidence: Interaction Effects in a Path Model. In: Research in Higher Education, 17, 4, S. 291-320.

Blüthmann, Irmela (2012): Studierbarkeit, Studienzufriedenheit und Studienabbruch: Analysen von Bedingungsfaktoren in den Bachelorstudiengängen. Dissertation. FU Berlin. https://d-nb.info/1051812437/34 [Zugriff: 29.01.2018].

Blüthmann, Irmela/Lepa, Steffen/Thiel, Felicitas (2008): Studienabbruch und -wechsel in den neuen Bachelorstudiengängen. Untersuchung und Analyse von Abbruchgründen. In: Zeitschrift für Erziehungswissenschaft 11, 3, S. 406-429.

BMBF (2016): Richtlinie zur Förderung von Forschung über „Studienerfolg und Studienabbruch“. Bundesanzeiger vom 24.02.2016. https://www.bmbf.de/foerderungen/bekanntmachung-1151.html [Zugriff: 29.03.2018].

Engel, Uwe/Schmidt, Björn O. (2014): Unit- und Item-Nonresponse. In: Baur, Nina/ Blasius, Jörg (Hg.): Handbuch Methoden der empirischen Sozialforschung, Wiesbaden: Springer VS, S. 331-346.

Gold, Andreas (1988): Studienabbruch, Abbruchneigung und Studienerfolg: Vergleichende Bedingungsanalysen des Studienverlaufs. Frankfurt a. M. u. a.: Peter Lang.

Hahn, Dietger/Krystek, Ulrich (1984): Frühwarnsysteme als Instrument der Krisenerkennung. In: Staehle, Wolfgang H./Albach, Horst (Hg.): Betriebswirtschaftslehre und ökonomische Krise: Kontroverse Beiträge zur betriebswirtschaftlichen Krisenbewältigung. Wiesbaden: Gabler, S. 3-24.

Hargreaves Heap, Shaun P./Varoufakis, Yanis (2004): Game Theory. A critical text. London: Routledge.

Heublein, Ulrich/Ebert, Julia/Hutzsch, Christopher/Isleib, Sören/König, Richard/ Richter, Johanna/Woisch, Andreas (2017): Zwischen Studienerwartungen und Studienwirklichkeit. Ursachen des Studienabbruchs, beruflicher Verbleib der Studienabbrecherinnen und Studienabbrecher und Entwicklung der Studienabbruchquote an deutschen Hochschulen. In: Forum Hochschule 1. http://www. dzhw.eu/pdf/pub_fh/fh-201701.pdf [Zugriff: 01.06.2017]. 
Heublein, Ulrich/Wolter, Andrä (2011): Studienabbruch in Deutschland. Definition, Häufigkeit, Ursachen, Maßnahmen. In: Zeitschrift für Pädagogik 57, 2, S. 214 236.

Hirschman, Albert O. (1974): Abwanderung und Widerspruch: Reaktionen auf Leistungsabfall bei Unternehmungen, Organisationen und Staaten. Tübingen: Mohr.

Hirschman, Albert O. (1984): University Activities Abroad and Human Rights Violations: Exit, Voice, or Business as Usual. In: Human Rights Quarterly 6, 1, S. 21-26.

Hörnstein, Elke/Kreth, Horst/Blank, Christian/Stellmacher, Carolin (2016): Studiengang-Monitoring. Studienverlaufsanalysen auf Basis von ECTS-Punkten. Aachen: Shaker Verlag.

Hoff, Tanja/Zwicker-Pelzer, Renate (2015): Gegenwärtige Entwicklungen in der Profession und Wissenschaft von Beratung. In: Hoff, Tanja/Zwicker-Pelzer, Renate (Hg.): Beratung und Beratungswissenschaft. Baden-Baden: Nomos, S. 13-28.

Knoll, Lisa (2017): Albert O. Hirschman: Abwanderung und Widerspruch. In: Kraemer, Klaus/Brugger, Florian (Hg.): Schlüsselwerke der Wirtschaftssoziologie. Wiesbaden: Springer VS, S. 185-193.

Kolb, Monika/Kraus, Michael/Pixner, Johann/Schüpbach, Heinz (2006): Analyse von Studienverlaufsdaten zur Identifikation von studienabbruchgefährdeten Studierenden. In: Das Hochschulwesen 54, 6, S. 196-201.

Meinefeld, Werner (2007): Studienabbruch und Studienfachwechsel in der Soziologie: Ein Blick hinter die Zahlen. In: Soziologie 36, 1, S. 45-62.

Nawratil, Ute (2008): Das qualitative Interview: Die Darstellung von Erfahrungen. In: Wagner, Hans (Hg.): Qualitative Methode in der Kommunikationswissenschaft. München: Reinhard Fischer, S. 319-332.

Nawratil, Ute/Schönhagen, Philomen (2008): Die qualitative Inhaltsanalyse: Rekonstruktion der Kommunikationswirklichkeit. In: Wagner, Hans (Hg.): Qualitative Methoden in der Kommunikationswissenschaft. München: Reinhard Fischer, S. 333-346.

Pelz, Robert/Schulze-Stocker, Franziska (2018): Determinanten der Studienabbruchneigung von Studierenden. Ergebnisse einer Befragung im Rahmen des PASST?!-Programms der TU Dresden. Arbeitspapier Nr. 2018-01. http://tlp.de/p19w [Zugriff: 16.04.2018].

Rhein, Rüdiger (2016): Die Universität als Lernort. In: Dörner, Olaf/Iller, Carola/Pätzold, Henning/Robak, Steffi (Hg.): Differente Lernkulturen - regional, national, transnational, Opladen: Budrich, S. 205-216.

Römer, Jasmin/Drews, Frauke/Rauin, Udo/Fabricius, Dirk (2013): Riskante Studienund berufsrelevante Merkmale von Studierenden: Ein Vergleich von Lehramtsund Jurastudierenden. In: Zeitschrift für Bildungsforschung 3, 2, S. 153-173.

Sarcletti, Andreas/Müller, Sophie (2011): Zum Stand der Studienabbruchforschung. Theoretische Perspektiven, zentrale Ergebnisse und methodische Anforderungen an künftige Studien. In: Zeitschrift für Bildungsforschung 1, 3, S. 235-348.

Schiefele, Ulrich/Streblow, Lilian/Brinkmann, Julia (2007): Aussteigen oder Durchhalten. Was unterscheidet Studienabbrecher von anderen Studierenden? In: Zeitschrift für Entwicklungspsychologie und Pädagogische Psychologie 39, 3, S. $127-140$. 
Schubert, Franz-C. (2015): Die historische Dimension von Beratung. In: Hoff, Tanja/ Zwicker-Pelzer, Renate (Hg.): Beratung und Beratungswisenschaft. Baden-Baden: Nomos, S. 28-44.

Schuemer, Rudolf/Ströhlein, Gerhard (1991): Dropout-Forschung und Dropout-Prophylaxe: Zur Theorie und Methodologie. In: Holmberg, Börje/Orthner, Gerhard E. (Hg.): Research into Distance Education/Fernlehre und Fernlehrforschung. Essen: Peter Lang Verlag, S. 196-222.

Schulze-Stocker, Franziska/Schäfer-Hock, Christian/Pelz, Robert (2017): Weniger Studienabbruch durch Frühwarnsysteme - Das Beispiel des PASST?!-Programms an der TU Dresden. In: Zeitschrift für Beratung und Studium 12, 1, S. 26-32.

Schüßler, Ingeborg (2016): Lernkulturen in Transformationsgesellschaften. Paradoxien, Herausforderungen und Gestaltungsoptionen. In: Dörner, Olaf/Iller, Carola/ Pätzold, Henning/Robak, Steffi (Hg.): Differente Lernkulturen - regional, national, transnational, Opladen: Budrich, S. 15-26.

Seibel, Wolfgang (2016): Verwaltung verstehen. Eine theoriegeschichtliche Einführung. Berlin: Suhrkamp.

Stiehler, Sabine (2007): Studien- und Studentenberatung. In: Nestmann, Frank/Engel, Frank/Sickendiek, Ursel (Hg.): Das Handbuch der Beratung. Tübingen: dgvt-Verlag (2. Auflage), S. 877-889.

TU Dresden (2017): Mit Frühwarnsystemen Studienabbrüchen an Sachsens Hochschulen vorbeugen. http://t1p.de/xa3o [Zugriff: 28.01.2018].

Wilhelm, Daniel (2017): Kritische Reflektion einiger Ökonomisierungstendenzen in der Studienberatung. In: Zeitschrift für Beratung und Studium 12, 4, S. 106-109. 

VI. Gesprächsanalytische Zugänge zu Beratungsinteraktionen: Handlungstypik und Interaktionsmodi 

Farina Wagner

\section{Subjektivierung in der Weiterbildungsberatung - Beratungsgespräche in erzählanalytischer Perspektive}

\section{Entscheidungen Lebenslangen Lernens im Feld der Weiterbildungsberatung}

Weiterbildungsberatung kommt im Kontext von Beruflichkeit und Lebenslangem Lernen die Funktion zu, Veränderungen in den Verhältnissen der Ratsuchenden zu ihrem Beruf herbeizuführen und dabei „Entscheidungen für weitergehende Bildungswege vorzubereiten“ (Schlüter 2017: 216). Das Moment der Entscheidung bzw. der Entscheidungsvorbereitung konturiert das Feld der Weiterbildungsberatung (vgl. Gieseke/Stimm 2016), welche darin unterstützen soll, ,Bildungs- und Berufsbiographien eigenverantwortlich zu gestalten“ (Schiersmann 2011: 429) und zur „Regulierung von Lebensläufen“ (Käpplinger/Maier-Gutheil 2015) beiträgt. Auf der einen Seite geht es also um eigenverantwortliches Entscheiden, was den Ratsuchenden in der Figur Lebenslangen Lernens Selbstbestimmung und Selbststeuerung beimisst. Auf der anderen Seite wird durch das Beratungsangebot ,eine spezifische Nicht-Autonomie unterstellt" (Forneck/Wrana 2005: 159), da es bei der Entscheidung einer Unterstützung oder Begleitung bedarf. Damit setzt Weiterbildung in dem Verhältnis von Selbststeuerung und gleichzeitiger Nicht-Autonomie eine Freiheit ein, ,die unablässig bedroht ist und damit zur Grundlage immer neuer, somit nie endender, also lebenslanger Interventionen wird“ (ebd.: 154). Freie Entscheidungen, die den Ratsuchenden in der Beratung ermöglicht und ihnen nicht von den Berater_innen abgenommen werden sollen, sind in ein produktives Machtverhältnis eingebunden, denn „Entscheidungen sind nur innerhalb eines entschiedenen Feldes möglich, das nicht ein für allemal entschieden ist" (Butler 2006, S. 221). Es herrschen diskursive Ordnungen, die das Feld und seine Entscheidungsmöglichkeiten begrenzen, jedoch nur dadurch, dass diese durch ein immer wiederkehrendes Treffen von Entscheidungen aufrechterhalten werden. Weiterbildungsberatung als zentrales Instrument zur Umsetzung des Konzepts Lebenslangen Lernens (vgl. Schiersmann 2009: 747) wird damit gleichzeitig zu einer An- und Aufforderung gouvernementaler Selbstführung (Foucault 2000), d. h. der Auswahl und Abwägung von richtigen, also im Sinne der Ordnung legitimen Entscheidungen. Mit dem Begriff der Gouvernementalität bezeichnet Foucault die Verbindung zwischen Regierung (gouvernement) und Denkweise (mentalité) und ,analysiert die gegenwärtige 
Regierungskunst als Form der Machtausübung, die - scheinbar paradox - auf der freien Entscheidung der Individuen beruht" (Klingovsky 2017: 34). Diskursanalytisch gerahmte Betrachtungen markieren den Entscheidungsraum Lebenslangen Lernens im Sinne einer „Regierung durch Individualisierung“ und der damit einhergehenden „Responsibilisierung der Individuen für ihre eigenen Lebenschancen" (Klingovsky 2017: 40). Beschäftigtsein und Arbeitslossein wird zu einer Frage des (selbstgesteuerten) Lernens (vgl. Rothe 2011: 262), weshalb nahezu alle Programme und Strategien auf die Drohung mit Exklusion setzen (vgl. ebd.: 291), wenn im Lebensverlauf die falschen Entscheidungen getroffen werden. Damit bildet Weiterbildungsberatung als Hervorbringungspraxis von Differenz- und Normalisierungsordnungen Lebenslangen Lernens (vgl. Klingovsky/Pawlewicz 2014: 95) und als Ort (der Anbahnung) von Entscheidungsprozessen eine zentrale Scharnierstelle, die einen Austragungs- und Verhandlungsort spezifischer Adressierungs- und Anerkennungsprozesse formiert, in dem die Person zu Weiterbildung und Beruf ins Verhältnis gesetzt wird, um einen spezifischen Subjektstatus zu konstituieren. Nittel verweist darauf, dass „die soziale Identität einer Person, die mit einem partikularen Problem in die Beratung hineingeht und später mit Hilfe des Ratschlags die anstehende Handlungskomplikation bzw. Krise aufzulösen versucht, [...] eine andere [ist] als die, welche akut mit dem Problem konfrontiert war" (Nittel 2016: 23). Veränderungsprozesse werden in der Weiterbildungsberatung vollzogen, wenn unterschiedliche Positionierungen des Selbst durch Ratsuchende und Beratende verhandelt werden, wenn unterschiedliche situative Identitätskonstruktionen zur Disposition stehen. Es stellt sich die Frage, wie diese Relationen in Weiterbildungsberatungsgesprächen lokal erzeugt und ausgestaltet werden, wie das Selbst, die eigene Biographie im Modus von Weiterbildung und Weiterbildungsberatung erzählt wird, wie die Thematisierung von Selbstverhältnissen der_des Ratsuchenden durch Erzählungen von ratsuchender und beratender Person verhandelt, also im Gespräch gemeinsam hervorgebracht werden. Biographische Elemente werden vor diskursiven Hintergründen bzw. einnehmbaren Subjektpositionen thematisiert, geordnet oder verworfen - in Erzählungen als bzw. über situative Identitätskonstruktionen, im Positionieren in und zu diesen.

Diese diskursiv gerahmte Ereignishaftigkeit wird im vorliegenden Beitrag in Anlehnung an mein Dissertationsprojekt als eine Empirie der Subjektivierung untersucht, welche Selbstverhältnisse bzw. das „Umgehen mit Selbstverhältnishaftigkeit“ (Ricken et al. 2017: 216) auf eine bestimmte Weise in den Blick nimmt (Kapitel 2). Die Fragestellung kann wie folgt zugeschnitten werden: Wie wird Entscheidbarkeit in der Weiterbildungsberatung durch Differenz- und Normalisierungsordnungen und ent- oder widersprechende Subjektivierungsvollzüge hergestellt? Eine Antwort verweist auf die Bedeutung von Erzählungen in den Beratungsgesprächen, welcher hier nachgegangen werden soll. Methodisch wird an ein erzählanalytisches Verfahren 
angeschlossen, welches die Re-Konstruktion ${ }^{1}$ von situativen Identitätskonstruktionen in subjektivierungstheoretischer Perspektive ermöglicht (Kapitel 3). In der exemplarischen (Sequenz-)Analyse (Kapitel 4) geht es um die Konstruktion von Entscheidbarkeit durch die Verschränkung von Erzählungen vor dem Hintergrund von Narrativen Lebenslangen Lernens und Beruflichkeit, welche abschließend in ihrer Bedeutung für die Weiterbildungsberatung noch einmal betont wird (Kapitel 5).

\section{Eine Empirie der Subjektivierung}

Im Anschluss an Foucaults Begriff des ,assujettissements“ (1976) ist der Konstitutionsprozess des Subjekts als ein Vorgang zu verstehen, der in der gleichzeitigen Entstehung und Unterwerfung „aus Individuen Subjekte macht“ (Foucault 1994: 246). Butler übersetzt dies in "subjectivation“" (Butler 2001: 187) und beschreibt einen Prozess gleichzeitiger Unterwerfung unter sozial etablierte intelligible Normen und deren Überschreitung (vgl. Ricken et al. 2017: 200) als Möglichkeit von Handlungsfähigkeit im Vollzug bis hin zu Subversion. In regulierten Wiederholungsprozessen nimmt das Subjekt in der Unterwerfung die Macht an (vgl. Butler 2001: 16), empfängt oder erleidet sie jedoch nicht nur, sondern eignet sie sich durch das Aufführen performativ an. Es deutet sich die Frage an, warum ein Subjekt sich wie subjektivieren lässt und verweist auf Aneignungs-, Handlungs- oder Verhandlungsspielräume, die hier innerhalb von Weiterbildungsberatungsgesprächen betrachtet werden sollen.

Im Folgenden wird das Vorhaben vor dem Hintergrund unterschiedlicher Suchbewegungen im Feld empirischer Subjektivierungsforschung kurz skizziert. In Foucaultscher Tradition zeigen die Analysen häufig die Tendenz, eher ein subjektiviert-werden auszumachen und große, oftmals dichotome Modell-Subjekte, z. B. „Leistungsträger vs. Arbeitslose“ (Bosančić 2014: 172) zu benennen. Diskursanalytisch geprägt wird dazu angehalten, zunächst mögliche Subjektpositionen, die also potenziell einnehmbar sein könnten, zu analysieren um darauf individuelle Aneignungsweisen betrachten zu können. In der Analyse von Gesprächen der Weiterbildungsberatung erscheint es jedoch zentral, Subjektivierungsprozesse nicht über bestimmte Setzungen von erwartbaren Positionen zu großen Modellsubjekten vorwegzunehmen. Diese

1 Mit dieser Schreibweise möchte ich darauf hinweisen, dass in der interpretativen Rekonstruktion gleichzeitig Konstruktionen im Sinne performativer Hervorbringungen und Aktualisierungen durch die Forschenden stattfinden. 
können den Blick auf kleinere, vielleicht noch nicht beschriebene Subjektivierungsprozesse verstellen und entbehren einer Offenheit, gerade auch Störungen, Abweichungen, Subversionen analytisch zuzulassen, die sich nicht an den großen Figuren, sondern an anderen Positionen abarbeiten oder diese erst entwerfen. Damit wird vor allem der Vollzugs- und Hervorbringungscharakter des Subjektivierungskonzepts betont (vgl. auch Ricken et al. 2017, Wrana 2015), welchem außerdem dadurch Rechnung getragen wird, dass keine zu Forschungszwecken initiierten, sondern natürliche Situationen als Material herangezogen werden. Damit kann die konkrete Prozessierung von Subjektivierungen in der Weiterbildungsberatung betrachtet werden, was bspw. in Interviews mit gewissen Problemlagen behaftet und durch die Positionierung gegenüber den von den Forschenden eingebrachten Adressierungen anders gelagert ist.

Gespräche in der Weiterbildungsberatung lassen die Betrachtung von Verhandlungen im Kontext von Subjektivierung im Rahmen kleiner, großer, zunächst unbestimmter Subjektpositionen wie auch deren Erzählungen innerhalb der Interaktion gleichermaßen zu. Wie sich diese Verhältnisse von Verhandlungen ausgestalten, soll im Rahmen des Dissertationsprojekts betrachtet und hier durch eine ausschnitthafte Analyse skizziert werden.

Vielen der methodologischen und methodischen Überlegungen ist gemeinsam, dass sie eine analytische Trennung von zwei Seiten des Subjektivierungsprozesses vornehmen. Dabei wird in unterschiedlichen Ausgestaltungen und Differenzierungen mit den Begriffen der Position und Positionierung gearbeitet (so u. a. Ricken et al. 2017, Wrana 2015). Im Rahmen der Analyse wird die diskursiv gerahmte Ereignishaftigkeit und Verhandlung von Selbstverhältnissen in Subjektposition und Positionierung aufgespalten. Der Fokus auf die Selbstverhältnisse ist hier entscheidend, da im Unterschied dazu auch eine bestimmte Gegenstandsbezogenheit zum Einsatz kommen kann. Wrana geht es bspw. um „Positionierungen, die im Diskurs in Bezug auf eine Thematik oder eine Sache vorgenommen werden" (Wrana 2015: 128). Es werden „gleichzeitig die Gegenstände, von denen die Rede ist und die Subjektpositionen, in denen gesprochen wird, produziert" (ebd.). In der vorliegenden Analyseperspektive ist der Gegenstand gewissermaßen die Subjektposition als Norm, als intelligible Anrufung von Identität, als anerkennbarer Subjektstatus. Diese Position wird nun in den Positionierungen als situativer Vollzug, Identifikation oder Verwerfung, verhandelt: als Verhandlung von Selbstverhältnissen. 


\section{Subjektivierung und Erzählung in der Weiterbildungsberatung: Der Zugang der Positionierungsanalyse}

Die Thematisierung und Verhandlung von Selbstverhältnissen findet in Gesprächen der Weiterbildungsberatung besonderen Eingang, da die Ratsuchenden sich in Bezug zu Beruflichkeit auf eine bestimmte Weise erzählen bzw. durch die Beratenden erzählt werden. Biographische Elemente werden vor diskursiven Hintergründen bzw. einnehmbaren Subjektpositionen thematisiert, geordnet oder verworfen - in Erzählungen als bzw. über situative Identitätskonstruktionen, im Positionieren in und zu diesen. Dabei geht es um eine gemeinsame, interaktionistisch gedachte Hervorbringung von Erzählungen, die eine Vielfalt von Deutungen verhandeln (vgl. Dinkelaker/Wyßuwa i. E.). Während das biographisch-narrative Interview durch das Interesse an der Lebensgeschichte vor allem formal abgeschlossene Erzähltexte der interviewten Person in den Blick nimmt (vgl. Schütze 1983), wird der Fokus in der hier eingenommen erzählanalytischen Perspektive verschoben. Alltäglich situierte Erzählungen, die dadurch häufig unvollständig oder brüchig sein können, werden als „,small stories“ in Abgrenzung zu „,big stories“ der Biographien als Ganzes aufgegriffen und als Identitäten ,in-the-making“ (Bamberg/Georgakopoulou 2008: 379) verstanden. Es wird somit an einen weiten Erzählbegriff angeschlossen: „At any point of time, our sense of entities, including ourselves, is an outcome of our subjective involvement in the world. Narrative mediates this involvement." (Ochs/Capps 1996: 21). Beratung in der Weiterbildung kann also nicht nur als institutionelle Interaktionsform (vgl. Stanik 2016) betrachtet werden, sondern sie formiert weiterhin Erzählfiguren, in denen das Leben in seiner Eingebundenheit in bestimmter Weise erzählt werden kann, in denen die Bedeutung von Beruf und Weiterbildung in der eigenen Biographie aufgerufen und gemeinsam bearbeitet wird. Diese Bearbeitung, in der die Selbstverhältnisse der Ratsuchenden durch diese selbst wie auch durch die Beratenden thematisch werden, wird als Prozess der Subjektivierung verstanden.

Für eine empirische Re-Konstruktion in dem dargelegten Verständnis wird die erzählanalytische Positionierungsanalyse von Bamberg (u. a. Bamberg/Georgakopoulou 2008) aufgegriffen. Sie stellt eine Verbindung zwischen poststrukturalistisch informierten diskurs- wie auch konversations- bzw. narrationsanalytischen Elementen her und fasst situative Identitätskonstruktionen über „small stories“ als „narratives-in-interaction“ (Bamberg/Georgakopoulou 2008: 379). Diese schließen an eine subjektivierungstheoretische Forschungsperspektive an, die zunächst die kleineren Ausgestaltungsprozesse gegenüber den großen Modellsubjekten betont. Es lassen sich im Anschluss an Spies (2011) Verknüpfungen mit Stuart Halls Identitätskonzept herstellen, welches eine Verbindungslinie zwischen Identi- 
täts- und Subjektkonzepten zieht und Positionen und Positionierungen noch einmal näher bestimmt: „Identität ist eine Erzählung (narrative) vom Selbst; sie ist die Geschichte (story), die wir uns vom Selbst erzählen, um zu erfahren, wer wir sind. Wir zwingen ihr eine Struktur auf.“ (Hall 1999: 94) Identität lässt sich als situationsbezogener, temporärer "Punkt des Vernähens" (Hall 2004: 173, Hervorh. i.O.) zwischen Subjektposition und Positionierung verstehen. Spies hält im Anschluss an Hall fest: ,,der/die Einzelne [geht] nicht in den Subjektpositionen auf, sondern konstruiert sich - aus einer Vielzahl verschiedener, intersektionell verschränkter Diskurse und abhängig von der individuellen Erfahrungsgeschichte sowie den jeweiligen situativen und kommunikativen Bedingungen - eine Identität" (Spies 2011: 67). In dieser Konstruktion, die nie eine vollständige Einheit von Subjektposition und Positionierung hervorbringen kann, lässt sich die Iterabilität (vgl. Derrida 1988, im Anschluss Butler 2006) als wiederholende Bezeichnungspraktik verorten, deren Zukunft und Verlauf z. T. noch offen ist. Erzählungen vollziehen sich vor einem diskursiven Hintergrund, zitieren in der Iterabilität große wie klei$n e$ Erzählungen dadurch, dass im Vollzug des Verweisens auf große Narrative kleine Erzählungen erst konstituiert werden können, durch den Verweis auf Subjektpositionen Positionierungen eingenommen werden können.

\section{Exemplarische Analyse: Dynamiken von Entscheidbarkeit und Verletzbarkeit}

Für die Positionierungsanalyse trennt Bamberg analytisch Erzähltes, also die erzählte Person, und Erzählen, also die erzählende Person. Wenn auch auf einer anderen Ebene, lassen sich Parallelen zu Un-Einheit von Subjektposition und Positionierung ziehen, da hier die Einheit der Person analytisch aufgebrochen wird. Die aufgerufene Subjektposition als implizite oder explizite Norm kann sich formal in Erzähltem wie im Erzählen ansiedeln lassen, wird aber in der Art und Weise der Hervorbringung von Positionierungen erst aufgerufen. Bezogen auf das Feld der Weiterbildungsberatung wird re-/ konstruiert, welche Selbstverhältnisse wie artikuliert, wie diese im Verlauf des Beratungsgesprächs über Positionierungen verhandelt werden und was dies zu einem Verständnis von Entscheidbarkeit im Kontext Lebenslangen Lernens beiträgt. Bamberg/Georgakopoulou (2008) halten nun für die Positionierungsanalyse drei unterschiedliche Ebenen fest, die hier knapp skizziert werden:

Ebene 1 beschreibt, wie innerhalb der Erzählung die Figuren (characters) räumlich und zeitlich positioniert sind, markiert also das Erzählte.

Ebene 2 betrachtet, wie die Erzählung interaktiv durch Positionierungen 
der Gesprächsbeteiligten ermöglicht wurde, markiert also das Geschehen des Erzählens.

In Ebene 3 wird aus 1 und 2 interpretativ erschlossen, wie welche Aspekte von Selbstverhältnissen her- und dargestellt werden, als Identitätskonstruktion über Positionierungen, welche hier subjektivierungstheoretisch interpretiert werden.

Zur Illustration des Vorgehens wird eine Anfangssequenz eines auf Tonband aufgezeichneten Weiterbildungsberatungsgesprächs vorgestellt, in welcher biographisches Entscheiden bzw. Entscheidbarkeit von dem Hintergrund von Beruf und Lebenslangem Lernen durch den Ratsuchenden (R) und die Beraterin (B) bearbeitet werden.

Ebene 1

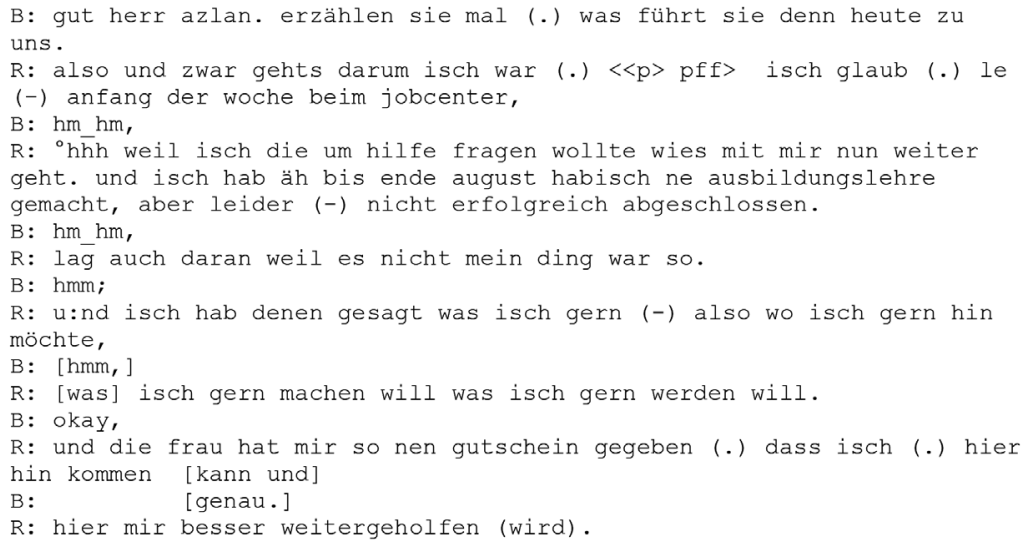

In der Erzählaufforderung von B (Z. 1-2) geht es nach Ebene 1 um Figuren eines Einzelnen (Sie) und einer Gruppe (uns). Aus der aufgeforderten Erzählung soll hervorgehen, warum die einzelne Person „heute“, also zeitlich, bei „uns“, also örtlich zumindest angedeutet, ist. „Uns“ kann auf die Institution verweisen, welche aber nicht örtlich gebunden sein muss. Das Verhältnis von „Sie“, „uns“ und „heute“ wird klärungsbedürftig und bildet die Rahmung der Positionierung der eingeforderten Erzählung. Das Thema bleibt offen und muss nun von R inhaltlich gefüllt werden. Jedoch zieht das „,heute“ eine zeitliche Begrenzung ein, welche eine Relation zwischen dem „heute“ der Institution und dem „heute“ von Rs Biographie herstellt, in der sich R erzählen soll. Diese Verschränkung bietet eine Rahmung für bestimmte Anrufungen von Subjektpositionen, welche sich daraufhin entfalten können. R schließt mit einer Erzählung an, die das ,ich“ zeitlich und räumlich über zwei Kontexte, also durch zwei stories, entfaltet:

In der ersten Geschichte geht der Protagonist als aktiv Hilfesuchender, der 
weiß, was er will, zum Jobcenter, weil er wiederum nicht weiß, wie es mit ihm, mit seiner Zukunft weitergeht (Z. 3-7 und Z. 12-20). Er geht davon aus, dass letztlich die Entscheidungen nicht von ihm, sondern von den Institutionen (Jobcenter und Beratungseinrichtung) getroffen werden. Eigeninitiative und Ohnmacht, Klarheit und Unbestimmtheit werden erzählt und die Trennungslinie verläuft entlang der Institutionen als Orten, an denen der weitere Lebensverlauf entschieden wird. Es muss gefragt werden, wie es weitergeht und es kann nicht einfach frei entschieden werden, wodurch ein bestimmtes Machtverhältnis anklingt.

In der zweiten Geschichte geht es um denselben Protagonisten, der vorher eine Ausbildung gemacht, aber keinen Abschluss bekommen hat (Z. 7-10). Auffällig ist, dass hier die Institution nicht auftaucht, sondern nur der Protagonist als Akteur. Seine Aktivität als Scheitern tritt hervor, wobei diese der Nicht-Passung mit der Ausbildung zugerechnet wird, das Problem also nicht bei allen Ausbildungen bestehen muss.

Die zweite Erzählung bildet zudem den Hintergrund der Elaboration zu der Frage, wie es mit ihm nun weitergeht, was auf Ebene 2 verweist.

\section{Ebene 2}

In Ebene 2 geht es um die Positionierungen der erzählenden Personen, was vor allem auf die Interaktionsebene abhebt. Das ,also“ (Z. 3) gibt das Signal für die Erzählung, wodurch der Anfrage, dem Impuls (,erzählen Sie mal“, Z. 1) entsprochen wird. Mit ,gehts darum“ (Z. 3) wird nun das Thema eingeführt, als eine Problempräsentation im Sinne der Elaboration eines Hilfeanliegens im Beratungssetting. Durch Zuhörsignale (Z. 5, 9, 11, 14) und das ,genau“ ( $Z$. 19 ) erfolgt eine Bestätigung des Vorgangs durch B. Die Erzählung hat somit den richtigen Informationsgehalt im Sinne einer Bewertung nach einem institutionellen Ablaufschema. Die Positionierung von R als nicht wissendem Erfüller von Erwartungen wird bestärkt. Bs Positionierung verfügt durch die Rahmung und die Bewertung über das Legitimieren der Erwartungen. Im Hinblick auf den Gutschein (Z. 17) wird das Differenzverhältnis noch einmal deutlich: B kennt die institutionellen Abläufe, während $\mathrm{R}$ diesen eher offen bis naiv begegnet, da sie für ihn etwas Neues zu sein scheinen.

Ebene 3

Die Problempräsentation etabliert sich über eine spezifische Ordnung der Anerkennbarkeit in der Weiterbildungsberatung: über eine Trennung in Problemträger_in und Lösungsfinder_in als große Subjektfiguren.

Diese Position der Aktivität erscheint als Identitätskonstruktion bzw. anerkennbarer Subjektstatus, da sie handlungsmächtig erzählbar ist: eine Person, die etwas leisten will, die einen Beruf will und sich daher einen Platz in der Gesellschaft erwarten kann im Sinne eines meritokratischen Gesellschaftsbilds, welches die Responsibilisierung der Individuen im Lebenslan- 
gen Lernen stützt; als Lösungsfinder_in. Ein Bruch mit der Einnehmbarkeit dieser Subjektposition findet sich in dem Einschub der Zwischenerzählung, in welcher das Prinzip nicht mehr funktioniert: Der Protagonist hat überspitzt formuliert alles gemacht um der Exklusion aus der Beruflichkeit zu entgehen, aber das meritokratische Versprechen geht nicht auf, die Anerkennung wird trotz der Anstrengung verweigert. Als Problemträger_in wird eine eher prekär eingesetzte Grenzposition eingenommen. Die Erzählung wird an dieser Stelle brüchig, aber wieder aufgenommen durch eine erneute Anstrengung, als jemand, der Hilfe sucht in einem anerkannten Rahmen, innerhalb der Anrufung: Gestalte dein Leben durch Aus- und Weiterbildung. Die Erzählung gewinnt nahezu lebensentscheidende Bedeutung, weil sie in diesem Moment dort einsetzt, wo der Fortgang des Lebens thematisch wird. Es wird dabei nicht über irgendjemanden gesprochen, sondern ich spreche von meinem Scheitern und meinen Aktivitäten. Der Ratsuchende legt seine Geschichte offen, die B nun weitererzählen soll im Sinne einer gemeinsamen Hervorbringung von Selbstverhältnissen. Durch das Weitererzählen als Versuch eines Zukunftsentwurfs wird das Selbstverhältnis im Als-ob-Modus vollziehbar. Damit ist es gleichzeitig offen für verschiedene Zukünfte und durch die zu erzählende Geschichte begrenzt.

Möglicherweise zeigt sich in diesem Positionierungsakt ein Konstitutionsmoment von Weiterbildungsberatung, wenn Ebene 1 und 2 sich verschränken: Das Erzählte wird auf einmal zum Moment des Erzählens, es entsteht eine Gleichzeitigkeit: die Vergangenheit wird in die Gegenwart eingeführt, wird zum Moment der Gegenwart. Das ,heute“ markiert die in der Interaktion anwesende Person R (Ebene 2), die eine Geschichte von sich im gestern (Ebene 1) erzählt, um eine Problemelaboration (Ebene 2) als Selbstelaboration (Ebene 3) aufzuführen.

$\mathrm{R}$ vollzieht bestimmte Bewegungen zwischen aktiver Zielgerichtetheit als handlungsermächtigende Erzählung und dem Hilfegesuch als Erzählung und Aufführung von Verletzbarkeit und Bedrohung, als „discursive maneuvers between these two positions“ (Bamberg/Georgakopoulou 2008: 392). Handlungsfähigkeit ist damit prekär gelagert, ein anerkennbarer Subjektstatus steht bei einer Wiederholung des Scheiterns auf dem Spiel. Um aber im Spiel zu bleiben, muss sich $\mathrm{R}$ in dieser risikobehafteten Positionierungsbewegung anbieten, verbunden mit der Hoffnung, damit eine anerkennbare Subjektposition gemeinsam mit B entwerfen und verhandeln zu können. Denn die Position des beruflich Handelnden steht ihm nicht zur Verfügung. Das zugrundeliegende Anerkennungsproblem wurde durch das Aus- und Weiterbildungssystem erzeugt und soll nun durch dieses in der Beratung gelöst werden, als gemeinsames Weitererzählen von Selbstverhältnissen. 


\section{Weiterbildungsberatung: Erzählen um zu entscheiden}

Die Erzählanalyse bearbeitet eine Schnittstelle von Biographie und Diskurs, in welcher durch die Verhandlung von diskursiven Subjektpositionen über Positionierungen, durch die Verschränkung der Erzählebenen, ein fluides Ineinandergreifen beschreibbar wird. Die Bewegung zwischen Entscheidbarkeit durch Handlungsmacht und drohender Ohnmacht durch prekäre Verletzbarkeit lässt sich nachzeichnen und weiterverfolgen. In der Weiterbildungsberatung wird damit die Entscheidbarkeit über die Verschränkung von Erzählungen vor dem Hintergrund von Narrativen Lebenslangen Lernens verhandelt.

Erzählungen bilden in der Weiterbildungsberatung eine konstitutive Bedingung der Herstellung von Entscheidbarkeit im Kontext Lebenslangen Lernens. Dies geschieht in den Gesprächen eingangs häufig durch ein Erzählenlassen und im Verlauf bzw. im Vergleich lassen sich weitere Varianten wie das Weitererzählen, Wiedererzählen oder Umerzählen ausmachen. Die Herstellung anerkennbarer Erzählbarkeiten im Sinne von Subjektpositionen bildet die Entscheidungsgrundlage. Durch häufig breit, aber bruchstückhaft angelegte biographische Erzählungen wird das Feld der Entscheidungen immer enger und geschlossener.

Durch die von Ratsuchenden und Beratenden gemeinsam geleistete Arbeit am Selbst der Ratsuchenden wird deutlich, dass Entscheidungen, ob im Sinne einer Responsibilisierung oder einer unterstellten freien Entscheidungsmöglichkeit, nicht allein von den Ratsuchenden getroffen werden (können), nicht von einer „subjektiven Entscheidungsfähigkeit“ (Gieseke/Stimm 2016: 167) ausgegangen werden kann. Die eingangs beschriebene eigentümliche Freiheit zwischen Bedrohung, lebenslanger Intervention und Selbststeuerung findet in den dargestellten (Erzähl-)Bewegungen eine Ausgestaltung. Koproduktive Erzählungen aktualisieren oder transformieren ein „entschiedenes Feld“ (Butler 2006, S. 221), welches die Grenzen der Entscheidbarkeit im Hinblick auf Berufs- und Bildungsentscheidungen markiert und damit auch das Verständnis von Weiterbildungsberatung verschiebt. Ermöglicht wird durch die subjektivierungstheoretisch orientierte Erzählanalyse eine re-/konstruktive Betrachtung der in Beratungspraktiken ,notwendige[n] (notwendig falsche[n]!) Ontologie, die die in der Beratungskommunikation selbst konstruierte soziale Adresse (das Selbst qua Therapie oder Beratung) mit ,Bewusstsein' und ,kausaler Wirkung von (Entscheidungs-)Handeln“ verwechselt" (Maasen, 2011: 22). Die individuelle Entscheidung wird gemeinsam als eine Entscheidung Lebenslangen Lernens im Prozess der Subjektivierung hergestellt - durch Erzählungen des Selbst. 


\section{Literatur}

Bamberg, Michael/Georgakopoulou, Alexandra (2008): Small stories as a new perspective in narrative and identity analysis. In: Text \& Talk 28 (3), S. 377-396.

Bosančić, Saša (2014): Arbeiter ohne Eigenschaften. Über die Subjektivierungsweisen angelernter Arbeiter. Wiesbaden: Springer VS.

Butler, Judith (2006): Haß spricht. Zur Politik des Performativen. Frankfurt a. M.: Suhrkamp.

Butler, Judith (2001): Psyche der Macht. Das Subjekt der Unterwerfung. Frankfurt a. M.: Suhrkamp.

Derrida, Jacques (1988): Signatur Ereignis Kontext. In: Derrida, J. (Hg.): Randgänge der Philosophie. 1. Auflage. Wien: Passagen-Verlag, S. 291-314.

Dinkelaker, Jörg/Wyßuwa, Franziska (i.E.): Lebenslauf und Bildungspraxis. Verschränkte Fluchtpunkte erziehungswissenschaftlicher Interaktions- und Biographieanalysen. In: Wiezorek, C./Kreitz, R./Demmer, C./Fuchs, T. (Hg.): Das Erziehungswissenschaftliche qualitativer Forschung. Opladen: Budrich.

Forneck, Hermann/Wrana, Daniel (2005): Ein parzelliertes Feld. Eine Einführung in die Erwachsenenbildung. Bielefeld: wbv.

Foucault, Michel (1994): Das Subjekt und die Macht. In: Dreyfus, H./Rabinow, P. (Hg.): Michel Foucault. Jenseits von Strukturalismus und Hermeneutik, Weinheim: Beltz, S. 243-261.

Foucault, Michel (2000): „Die Gouvernementalität“. In: Bröckling, U./Krasmann, S./ Lemke, T. (Hg.): Gouvernementalität in der Gegenwart. Studien zur Ökonomisierung des Sozialen. Frankfurt a. M.: Suhrkamp, S. 41-67.

Gieseke, Wiltrud/Stimm, Maria (2016): Praktiken der professionellen Bildungsberatung. Innensichten auf die Entscheidungsfindung im Beratungsprozess. Wiesbaden: Springer VS.

Hall, Stuart (1999): Ethnizität: Identität und Differenz. In: Engelmann, J. (Hg.): Die kleinen Unterschiede. Der Cultural Studies Reader. Frankfurt a. M.: Campus, S. 83-98.

Hall, Stuart (2004): Wer braucht Identität? In: Hall, S.: Ideologie, Identität, Repräsentation. Ausgewählte Schriften 4, hg. J. Koivisto und A. Merkens. Hamburg: Argument Verlag, S. 167-187.

Käpplinger, Bernd/Maier-Gutheil, Cornelia (2015): Ansätze und Ergebnisse zur Beratung(sforschung) in der Erwachsenen- und Weiterbildung. Eine Systematisierung. In: Zeitschrift für Weiterbildungsforschung - Report, (2015) 2, S. 163 181.

Klingovsky, Ulla (2017): Lebenslanges Lernen revisited - Zur Strukturlogik der New Educational Governance in der Wissensgesellschaft. In: Bauer, U./Bolder, A./ Bremer, H./Epping, R. (Hg.): Bildung für Arbeit unter neuer Steuerung. Wiesbaden: Springer VS, S. 2744.

Klingovsky, Ulla/Pawlewicz, Susanne (2014): Untiefen im Diskurs um das Lebenslange Lernen. Zur Transformation erwachsenenpädagogischer Handlungsformen. In: Holzer, D./Kloyber, C./Schuster, W./Vater, S. (Hg.): Wenn Weiterbildung die Antwort ist, was war die Frage? Innsbruck, Wien, Bozen: Studienverlag, S. 8597.

Maasen, Sabine (2011): Das beratene Selbst. Zur Genealogie der Therapeutisierung 
in den >langen< Siebzigern: Eine Perspektivierung. In Maasen, S./Elberfeld, J./ Eitler, P./Tändler, M. (Hg.): Das beratene Selbst. Zur Genealogie der Therapeutisierung in den >langen< Siebzigern. Bielefeld: Transcript, S. 7-34.

Nittel, Dieter (2016): Der erziehungs- und bildungswissenschaftliche Zugang zur Handlungsform „Beratung“. In: Gieseke, W./Nittel, D. (Hg.): Handbuch Pädagogische Beratung über die Lebensspanne. Weinheim/Basel: Beltz, S. 20-30.

Ochs, Elinor/Capps, Lisa (1996): Narrating the Self. In: Annual Review of Anthropology, Vol. 25 (1996), S. 19-43.

Ricken, Norbert/Rose, Nadine/Kuhlmann, Nele/Otzen, Anne (2017): Die Sprachlichkeit der Anerkennung. $\mathrm{Zu}$ den theoretischen und methodologischen Perspektiven auf ,Anerkennung in der Erforschung von Unterrichtspraktiken. In: Vierteljahrsschrift für wissenschaftliche Pädagogik 93 (2017), Heft 2, S. 193-233.

Rothe, Daniela (2011): Lebenslanges Lernen als Programm. Eine diskursive Formation in der Erwachsenenbildung. Frankfurt a. M.: Campus.

Schiersmann, Christiane (2009): Beratung im Kontext lebenslangen Lernens. In: Tippelt R./von Hippel A. (Hg.): Handbuch Erwachsenenbildung/Weiterbildung. Wiesbaden: Springer VS, S. 747-767.

Schiersmann, Christiane (2011): Beratung im Kontext von Lebenslangem Lernen und Life Design. Ein Generationen übergreifendes Konzept. In: In: Eckert, T./von Hippel, A. /Pietraß, M./Schmidt-Hertha, B. (Hg.): Bildung der Generationen, S. 427-439. Wiesbaden: Springer VS.

Schlüter, Anne (2017): Bildungsberatung als elementarer Baustein für erwachsenenbildnerische Professionalität. In: Münk, D./ Walter, M. (Hg.): Lebenslanges Lernen im sozialstrukturellen Wandel - Ambivalenzen der Gestaltung von Berufsbiografien in der Moderne. Wiesbaden: Springer VS, S. 215-230.

Schütze, Fritz (1983): Biographieforschung und narratives Interview. In: Neue Praxis, H.3, S. $283 \mathrm{ff}$.

Spies, Tina (2011): ,Alte‘ Männlichkeiten und ,neue‘ Ethnizitäten - Positionierungen junger Migranten in transnationalen Räumen. In: Gender. Zeitschrift für Geschlecht, Kultur und Gesellschaft, Heft 1/2011, S. 65-80.

Stanik, Tim (2016): Beratung in der Weiterbildung als institutionelle Interaktion. Frankfurt a. M.: Peter Lang Verlag.

Wrana, Daniel (2015): Zur Analyse von Positionierungen in diskursiven Praktiken. Methodologische Reflexionen anhand von zwei Studien. In: Fegter, S./Kessl, F./ Langer, A./Ott, M./Rothe, D./Wrana, D. (Hg.): Erziehungswissenschaftliche Diskursforschung. Empirische Analysen zu Bildungs- und Erziehungsverhältnissen. Wiesbaden: Springer VS, S. 123-142. 


\section{Professionalität im Coaching von Führungskräften: Der organisationale Rahmen als konstituierende Prämisse professionellen Handelns}

\section{Einleitung}

Seit den 1990er Jahren wird Coaching als personenbezogene Beratungsform von verschiedenen privaten Unternehmen angeboten. In den letzten 30 Jahren hat Coaching einen enormen Bedeutungszuwachs erfahren, die Entwicklung verlief rasant und die Angebotsvielfalt ist heute kaum zu überblicken: ,[...] das Format Coaching hat einen Blitzstart hingelegt und sich, sozusagen aus dem Nichts kommend, innerhalb einer überraschend kurzen Zeit als Beratungsformat etabliert"، (Liska 2010: 27). Auch die Professionsbildung im Feld des Coachings schreitet voran. Berufsverbände haben sich etabliert. Auch wenn in Fach- und Wissenschaftsliteratur versucht wird, Kompetenzanforderungen für Coaches zu definieren (Fritsch 2010; Steinke 2015; Birgmeier 2011; Loebbert 2015), ist „Coach“ grundsätzlich keine geschützte Berufsbezeichnung; ,Jeder kann sich Coach nennen oder das, was er tut, als Coaching bezeichnen" (Greif 2008: 53). Dadurch ist die Angebotsstruktur heterogen und es werden Wissensbestände aus ganz unterschiedlichen Disziplinen im Coaching angewandt. Dies wirft Fragen nach der Professionalität auf, die aufgrund fehlender allgemein verbindlicher Standards und fehlender homogener Ausbildungsstruktur - auf freiwilliger Selbstverpflichtung basiert.

In diesem Beitrag wird Professionalität in einer differenztheoretischen Perspektive als die Bearbeitung von in sich widersprüchlichen Strukturproblemen im interaktiven Vollzug der Coaching-Sitzungen betrachtet. Ziel des Beitrags ist es, professionelles Beratungshandeln in situ zu erforschen, um „Spannungsfelder und Kernprobleme des (situativen) professionellen Beratungshandeln[s]" zu ermitteln und daran die performativen Gestaltungsanforderungen aufzuzeigen sowie Berater*innen zu Selbstbeobachtungen und -reflexion durch einen fremden Blick auf ihre Praxis anzuregen (Maier-Gutheil 2013: 182). 


\section{Datenerhebung und -analyse}

Empirisch analysiert wird die Coaching-Praxis mit Führungskräften, bei der es um die Bearbeitung persönlicher Handlungsprobleme bezüglich beruflicher bzw. arbeitsfeldbezogener Themen geht (Fietze 2015). Coaching mit Führungskräften zielt darauf ab, in personenbezogenen Beratungsgesprächen Handlungsfähigkeit im beruflichen Kontext zu verbessern (Riedel 2003). Die Beratung findet in Form einer dyadischen Gesprächssituation statt, an der unternehmensexterne Coachs und Führungskraft teilnehmen. In Abgrenzung $\mathrm{zu}$ anderen Beratungsformaten vollzieht sich Coaching mit einer „ausgeprägten Prozess- und Personen-Orientierung" schleifenartig entlang folgender Basisaktivitäten: „Situation definieren, Beziehung gestalten, Veränderung konstruieren und Coaching evaluieren" (Graf 2017: 81).

Unsere Analysen basieren auf einer Datenerhebung in einem kleineren privaten Coaching-Unternehmen in Westdeutschland, das insbesondere soziale Einrichtungen berät und dabei u. a. Coaching für Führungskräfte anbietet. Der Bildungshintergrund und Erfahrungshorizont der im Unternehmen arbeitenden Coaches variiert stark. Entweder wird auf der Homepage auf eine über Jahrzehnte hinweg ausgeübte praktische Tätigkeit als Coach und Organisationsberater*in verwiesen oder es werden einschlägige absolvierte Studiengänge aufgelistet, die die fachliche Kompetenz der Coachs ausweisen sollen.

Die Daten stammen aus dem kooperativen, grenzüberschreitenden DFG/ FNR-Projekt Transition Processing, in dem pädagogische Rationalitäten im Kontext personenbezogener Beratungsdienste vergleichend untersucht wurden. ${ }^{1}$

Mit der Gesprächsanalyse rekonstruieren wir Rationalitäten auf der Ebene der Einzelberatungsgespräche und verfolgen die Frage, wie in konkreten Situationen soziale Wirklichkeit hergestellt wird (Garfinkel 1967). Wir gehen also davon aus, dass vermeintlich objektive Tatsachen nicht per se existieren, sondern erst durch unser Handeln erzeugt werden. Neben der Frage, wie etwas gesagt wird, interessiert uns vor allem auch wozu, d. h. in welcher Logik der Interaktion, etwas gesagt wird. Wir fragen also auch nach dem Zweck bestimmter Aussagen in der jeweiligen Handlungsabfolge und rekonstruieren so, wie institutionelle Strukturprobleme bearbeitet werden (Deppermann 2008: 79ff.).

Als Datenbasis dienen fünf Aufzeichnungen von Einzelcoachings mit einer Dauer von je 90 bis 120 Minuten. Die Gespräche wurden nach dem Transkriptionssystem „GAT 2“ vollständig transkribiert (Selting et al. 2009).

1 Am Projekt beteiligte Universitäten sind die Universitäten Flensburg, Hildesheim, Frankfurt, Tübingen sowie Luxemburg. Der luxemburgische Anteil wurde vom luxemburgischen Fonds National de la Recherche unter der Fördernummer INTER/DFG/14/8888406/ TransPro/Schroeder und die deutschen Partner wurden von der Deutschen Forschungsgemeinschaft unterstützt. 
Zunächst wurden die Gesprächstranskripte makroskopisch bearbeitet, d. h., für jedes Gespräch wurde ein thematischer Verlauf erstellt, nach welchem das Gespräch sequenzartig unterteilt und die jeweiligen Textpassagen paraphrasiert wurden. Diese makroskopische Arbeit an den Transkripten diente als Überblick über die gesamte Struktur der Gespräche sowie als Hilfestellung bei der Auswahl von Sequenzen für die mikroskopische Feinanalyse (Deppermann 2008: 51f.). Die mikroskopische Analyse erfolgte in einem festen Team und in Kooperation der Universitäten Luxemburg und Hildesheim. ${ }^{2}$ Die Ergebnisse wurden in konzeptionellen Memos festgehalten, die die Grundlage für die weitere Auswahl von Textsequenzen bildeten.

\section{Ergebnisse}

Die fünf aufgezeichneten Beratungsgespräche wurden von zwei unterschiedlichen männlichen Coachs ${ }^{3}$ geführt. Ausgewählt für die Ergebnisdarstellung wurden drei Gespräche, die sich durch den Zeitpunkt des Beratungsprozesses unterscheiden. Ein Erst- und ein Abschlussgespräch sowie ein Gespräch mitten im Beratungsprozess bilden den Verlauf des Coachings über einen Zeitraum von 5 bis 10 Gesprächen ab. Alle Coachees sind in sozialen Dienstleistungseinrichtungen oder Bildungseinrichtungen beschäftigt und haben dort jeweils eine Leitungsfunktion inne.

Mindestens in zwei Fällen gibt es Hinweise darauf, dass dabei die Impulse zum Coaching von dritter Seite bzw. aus der jeweiligen Organisation heraus entstanden sind. Dies wird teilweise auch an den Äußerungen der Coachees deutlich, die vermitteln, dass kein direkter Anlass in ihrem beruflichen Alltag sie dazu bewegt, ein Coaching in Anspruch zu nehmen.

\subsection{Makroskopische Analyse}

Mit Blick auf die Gesamtstruktur der Coaching-Gespräche zeigt sich die Rollenverteilung, dass sich der*die Coach für die methodische Ausgestaltung der

2 An den Analysesitzungen waren neben den Autor*innen des Beitrags Ute Karl und Inga Truschkat die studentischen Mitarbeiterinnen Claudine Bastian und Mieke Mierzowsky beteiligt.

3 Im Text werden primär die Funktionsbezeichnungen „Coach“ für die beratende Person und „Coachee“ für die zu beratende Person verwendet. 
Gespräche verantwortlich zeigt, während der*die Coachee die inhaltlichen Themen setzt (siehe Tabelle 1). Deutlich wird jedoch auch, dass die methodische Rahmung durch die Coachs insgesamt offen gestaltet ist und auch die thematischen Vorstellungen der Coachees stark variieren. So möchte in Gespräch 1 Herr G ein berufliches Coaching in Anspruch nehmen, um Reflexion und Unterstützung für seine neue berufliche Funktion zu bekommen. Er ist vor etwa einem halben Jahr zu Träger X gewechselt und hat damit einen Karriereaufstieg vollzogen. In seiner neuen Position ist er Geschäftsführer einer größeren sozialen Einrichtung. Das vorliegende Gespräch fand in der ersten Sitzung des Coachings statt, in der u. a. die künftige Zusammenarbeit mit dem*der Berater*in und die Bezahlung des Coachings verhandelt werden. Herr D (Gespräch 2) wurde erst vor kurzen zum Teamleiter in einer sozialen Einrichtung befördert, woraufhin ihm sein Chef die Teilnahme am Coaching vorschlug. In einer vorangegangenen Coaching-Sitzung wurde das Thema Mitarbeitergespräche besprochen und in der aufgezeichneten Sitzung soll ein solches Gespräch ,durchgespielt" werden. Frau N ist Schulleiterin und nimmt bereits seit einiger Zeit am Coaching teil. Im Gespräch geht es um den aktuellen Stand im beruflichen Kontext. Dazu wird auf eine Frau N bereits bekannte Methode, das „Brett“, zurückgegriffen. Frau N beginnt zunächst ihre Rolle im Zuge der Aufstellung auf dem Brett sowie aktuelle bestehende Probleme zu reflektieren. Der Coach macht verschiedene Angebote, wie Frau N damit umgehen kann.

Tab. 1: Auswahl der Gespräche (eigene Darstellung)

\begin{tabular}{|l|l|l|l|}
\hline $\begin{array}{l}\text { Rollen- und } \\
\text { Erwartungsklärung }\end{array}$ & $\begin{array}{l}\text { Gespräch 1 } \\
\text { Erstgespräch/ } \\
\text { Coach 1 und Herr G }\end{array}$ & $\begin{array}{l}\text { Gespräch 2 } \\
\text { Im Beratungsprozess } \\
\text { Coach 2 und Herr D }\end{array}$ & $\begin{array}{l}\text { Gespräch 3 } \\
\text { Abschlussgespräch } \\
\text { Coach 2 und Frau N }\end{array}$ \\
\hline $\begin{array}{l}\text { Coach: } \\
\text { methodische } \\
\text { Gestaltung }\end{array}$ & $\begin{array}{l}\text { klarer in der metho- } \\
\text { dischen Rahmung }\end{array}$ & $\begin{array}{l}\text { Offen und wenig } \\
\text { eindeutig }\end{array}$ & $\begin{array}{l}\text { Offen und wenig } \\
\text { eindeutig }\end{array}$ \\
\hline $\begin{array}{l}\text { Coachee: } \\
\text { inhaltliche } \\
\text { Gestaltung }\end{array}$ & $\begin{array}{l}\text { Reflexion und Begleitung } \\
\text { in eine neue Position } \\
\text { (Supervision) }\end{array}$ & $\begin{array}{l}\text { Mitarbeitergespräche } \\
\text { (Schulung) }\end{array}$ & $\begin{array}{l}\text { Rückblick auf den } \\
\text { Beratungsprozess }\end{array}$ \\
\hline
\end{tabular}

Im Gespräch 1 und Gespräch 2 weicht der Gesprächsverlauf von der (offensichtlichen) Vorstellung des Coachs von einem Coaching - die in einer Reflexion der eigenen Führungsposition in der Organisation besteht - ab. Die Coachees verlangen eher nach einer Supervision oder einer Schulung in der Führung von Mitarbeitergesprächen. Wenngleich die Coachs in diesen Gesprächen versuchen, ein offenes Gespräch über die Position der Coachees in ihrer Organisation zu initiieren und thematisch auszuhandeln, finden keine 
eindeutigen Prozesse der Grenzziehung seitens der Coaches statt, die das Gespräch inhaltlich begrenzen würden. Ferner ist festzustellen, dass zu Beginn des Coachings die Gesprächsthemen bzw. Coaching-Ziele, wenn überhaupt, dann nur sehr vage festgelegt werden.

\subsection{Mikroskopische Analyse}

In den mikroskopischen Analysen wurde neben der Frage nach dem „Wie etwas gesagt wurde" auch untersucht, welche Funktion das Gesagte im Gesprächsverlauf erfüllt. So konnten insgesamt drei Strukturprobleme des Coachings herausgearbeitet werden. Wie anhand von Gesprächsauszügen zu zeigen sein wird, besteht ein Strukturproblem darin, die fachliche Expertise als Coach ausweisen zu müssen, aber dabei zugleich einen möglichst offenen Raum für inhaltliche Themensetzung und Selbstreflexion zu lassen. Ein weiteres Strukturproblem zeigt sich in einer Beratungsbedürftigkeit, die hergestellt werden muss, um die Sinnhaftigkeit von Beratung zu rechtfertigen, die jedoch nicht die schon vorhandene Kompetenz des Coachees in Frage stellen darf. Zuletzt besteht das Strukturproblem in einer unbedingten Orientierung an den Bedürfnissen und Bedarfen des/der Klient*in und gleichzeitig in der Notwendigkeit, auch einen Folgeauftrag für die Beratungsorganisation zu sichern.

\section{Empirisches Beispiel I: Fachexpertise vs. Raum für Selbstreflexion}

Der folgende Sequenzauszug stammt aus dem Beratungsgespräch 1. Dieses Gespräch ist ein Erstgespräch, was erklärt, dass es zunächst zu einer etwas allgemeineren Auftragsklärung zwischen Coach 1 und Herrn G kommt.

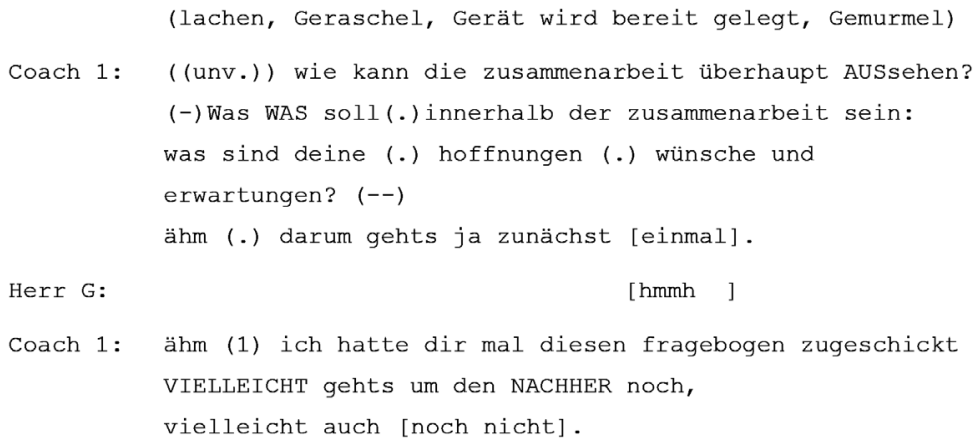




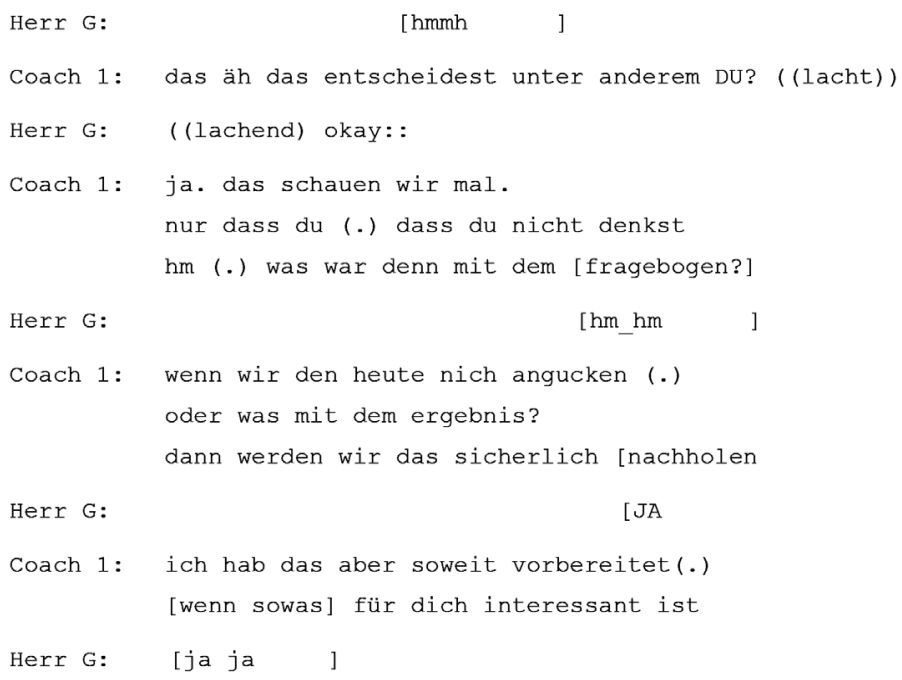

(Eingangssequenz, Gespräch 1, Erstgespräch)

Der Gesprächseinstieg erfolgt durch eine indirekte Erzählaufforderung des Beraters, die nach der Art und Weise der Zusammenarbeit fragt. Der vom Coach gewählte Begriff der „Zusammenarbeit“ verweist zunächst auf den augenscheinlichen Aspekt der Freiwilligkeit (es gibt keinen Zwangskontext in der Beratung), die jeweiligen Rollen können dennoch in einem hierarchischen Verhältnis stehen (Experte-Laie). Mit der Formulierung des Beraters „WAS soll(.) innerhalb der zusammenarbeit sein:" wird ein Raum eröffnet, in dem die Beratung stattfinden soll. Gefragt wird nach den Hoffnungen, Wünschen und Erwartungen des Coachees. Diese erscheinen als eine noch nicht feststehende Figur, die im Entstehungsprozess ist. Der Berater eröffnet so einen positiv besetzten Raum, den der Kunde auch gestalten und mitbestimmen kann (z. B. „das entscheidest unter anderem DU“), zugleich werden dabei die Grenzen des Raums gezogen, u. a., wenn der Berater sein Rollenverständnis darlegt (z. B. „ich hab das aber soweit vorbereitet"). Der Coach konstituiert sich hier als kompetenter und vorbereiteter Berater, der zugleich flexibel ist. Die Eröffnung des Coachings erinnert insgesamt an das Heraufbeschwören eines gemeinsamen Weges, an dem beide Akteure mitentscheiden können, wobei die Gestaltungsmacht im Sinne der Rollen- und Auftragsklärung aber letztlich beim Berater liegt.

Die nachfolgende Textsequenz stammt aus einem Gespräch am Ende des Beratungsprozesses: 


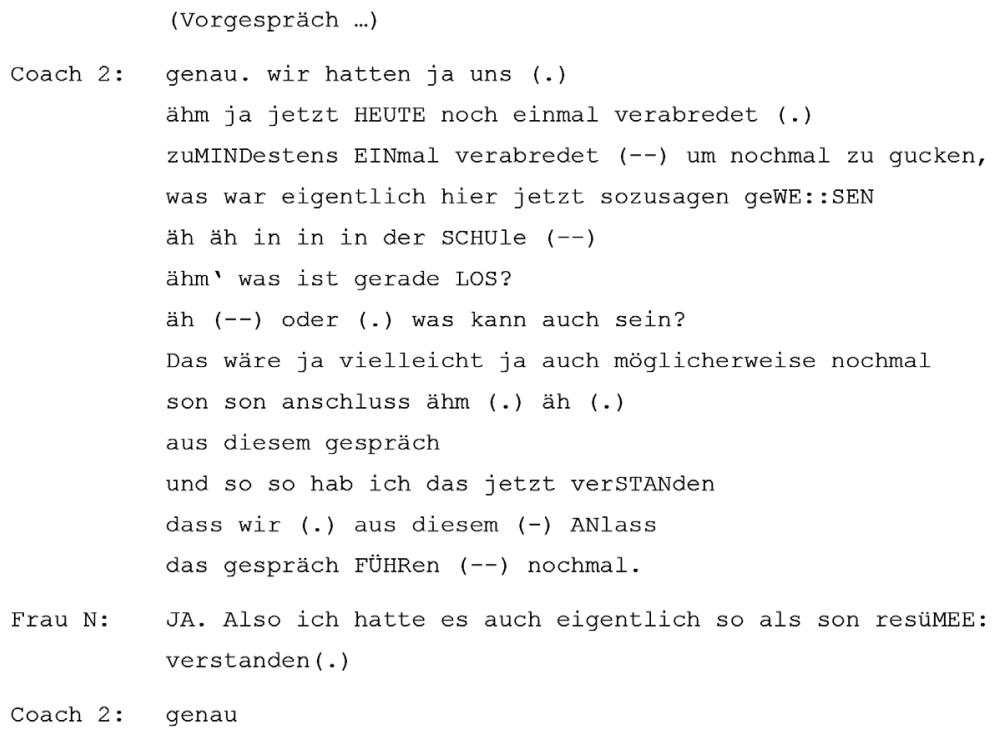

(Eingangssequenz, Gespräch 3, Ende des Beratungsprozesses)

Der Berater spricht von der Schule ohne ein bestimmtes Thema daran zu knüpfen. Der Rahmen bleibt offen, die Coachee kann frei entscheiden, ob sie nun ein fallbezogenes Thema aufgreift oder ein neues Thema besprechen will. Nachdem der Anlass geklärt scheint, macht der Berater eine weitere Option auf, indem er anfügt: „möglicherweise nochmal son son anschluss“. Er schlägt damit eine Brücke zwischen zwei Alternativen, der des Beratungsabschlusses und eines Folgetermins, der jedoch nicht vorgesehen ist. Er überprüft, ob der uns unbekannte Ausgangsauftrag abgeschlossen werden kann oder ob es zu einem Folgeauftrag kommt.

Der Berater schließt seinen Part mit der gleichen Unsicherheit, mit der er ihn begonnen hat. Er gibt den aktiven Gesprächspart recht spät an die Coachee $a b$, was darauf hindeutet, dass er sich in der Verantwortung sieht, das Gespräch zu führen. Für die Coachee scheinen der Bezugsrahmen und die Verständigung mit dem Berater hingegen klar zu sein. Sie gibt an, dass sie die derzeitige Sitzung ,auch eigentlich so als son resümEE verstanden“ hatte, als Rückblick und nicht als Klärung zukünftiger Optionen. Im weiteren Verlauf wird die Rolle des Beraters als Strukturgeber immer konfuser, während die Coachee als Inhaltsgeberin immer stärker auftritt.

Es zeigt sich ein Spannungsfeld darin, dass der Coach seine Expertise in der fachlichen Gesprächsführung einerseits gegenüber der Coachee beweisen möchte, andererseits aber möglichst Themen anregen möchte, die sich aus 
einem reflexiven Selbstbezug der Coachee gleichsam organisch entwickeln sollen. In einem Erstgespräch - genauso wie in einem Gespräch am Ende des Beratungsprozesses - eröffnet der Berater einen tentativen Rahmen, der für eine große Bandbreite an möglichen Themen seitens der Coachee offenbleibt.

\section{Empirisches Beispiel II: Bedürftigkeit vs. Kompetenz des Coachees}

In der folgenden Schlusssequenz einer Beratungssitzung im Beratungsprozess gibt es (noch) keine organisationale Vereinbarung, die einen weiteren Termin selbstverständlich vorgibt (wie in Gespräch 1).

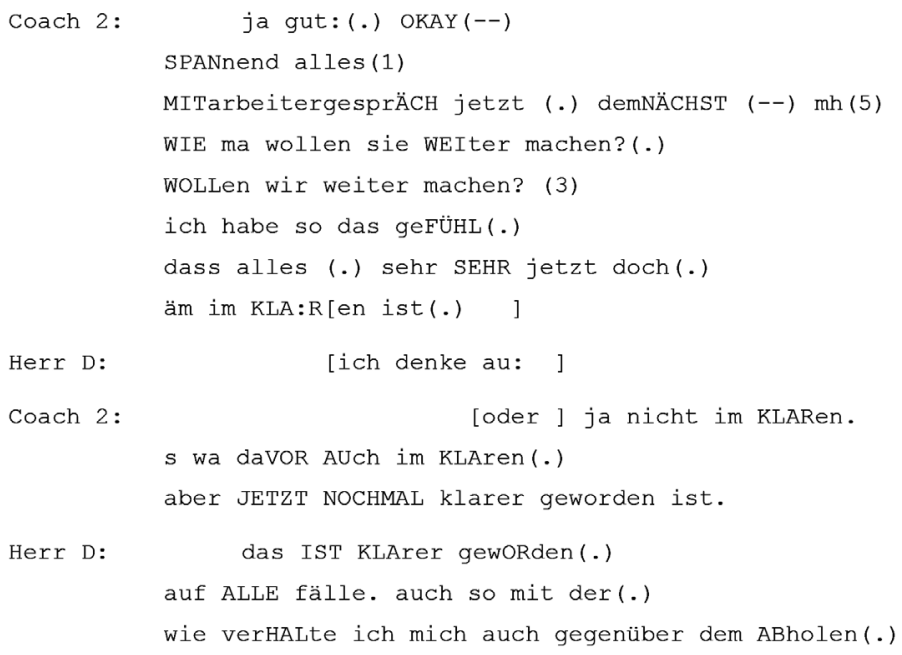

(Gespräch 2, Beratungsprozess)

Der Berater leitet mit seinen Fragen eine abschließende Sequenz ein: „WIE ma wollen sie WEIter machen? (.) WOLLen wir weiter machen? (3)“. Anschließend kommt es zunächst zu einer gegenseitigen Bestätigung davon, dass die Beratung sinnvoll war. In der Formulierung des Coaches wird deutlich, dass er ein mögliches Defizit beim Coachee verdecken will. Seine Selbstreparatur (Egbert 2009) zielt nicht nur auf eine Verbesserung des Verständnisses, sondern auch auf das Vermeiden einer möglichweise schon abschätzig wirkenden Formulierung. Der Coach korrigiert sich von klar auf klarer und fügt hinzu, dass es auch davor schon klar war. An dieser Selbstreparatur wird deutlich, wie wichtig es für ihn ist, am Ende die Kompetenz des Coachees herauszustellen, aber zugleich auch die Sinnhaftigkeit des Beratungsgesprächs hervorzuheben, um schließlich weiteren Bedarf für kommende Gespräche zu 
generieren. Auch der Coachee sieht sich in der Bewertung des Gesprächs nun in der Situation, etwas zu finden, dass er aus dem Coaching mitnimmt. Diese abschließende Bewertung scheint eine günstige Vorbereitung dafür zu sein, eine Anschlussmöglichkeit für ein weiteres Gespräch bzw. einen Folgeauftrag zu schaffen. Obwohl alles noch klarer geworden ist, wird wieder ein neuer Bedarf bzw. Termin seitens des Beraters generiert.

Es verdeutlicht sich insgesamt das Dilemma, dass Beratung - und folglich auch Coaching - sich eigentlich nur über etwas Defizitäres auf Seiten des Ratsuchenden legitimiert. Im Fall des Coachings muss die Beratung jedoch zugleich als überflüssig darstellt werden, um nicht am Selbstbild einer (immer schon) kompetenten Führungskraft zu nagen. Coaching muss also einen Bedarf erzeugen, indem potentielle Defizite aufgezeigt und problematisiert werden, möglichst ohne dass dieser Bedarf vom Coachee als defizitär empfunden wird.

Auch die gegenseitige Bewertung von Coach und Coachee erfolgt in einem Balanceakt. Es muss gelingen, einen Bedarf zu erzeugen, ohne dass dieser Bedarf als defizitär empfunden wird. Der Coach muss daher die Kompetenz des Coachees hervorheben und zugleich von einer weiteren Verbesserung auf einem hohen Niveau ausgehen.

\section{Empirisches Beispiel III: Klientenorientierung vs. Folgeaufträge auf der organisationalen Ebene absichern}

Im folgenden Gesprächsauszug handelt es sich um die Schlusssequenz eines abschließenden Gesprächs im Beratungsprozess. Zunächst wird ganz unverbindlich ein Termin vorgeschlagen:

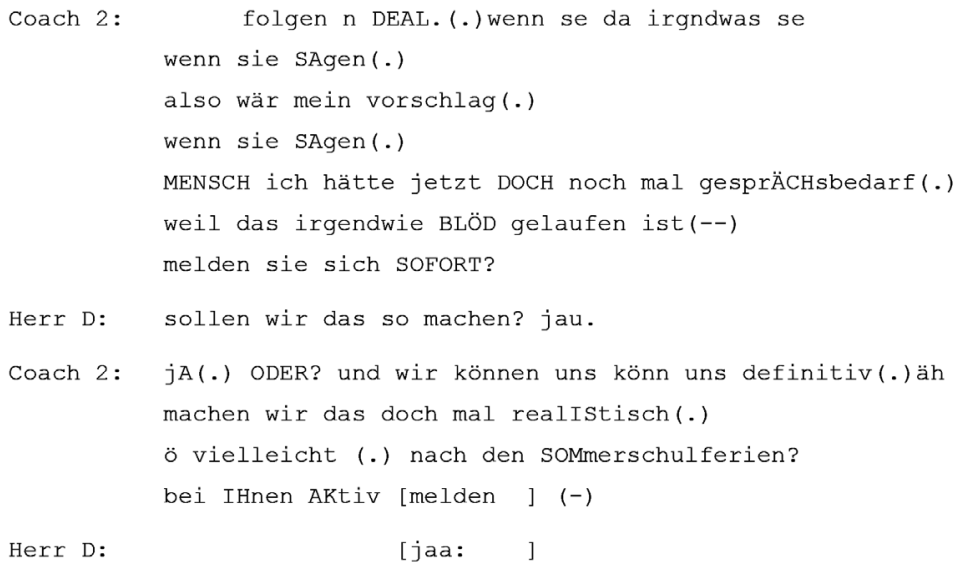




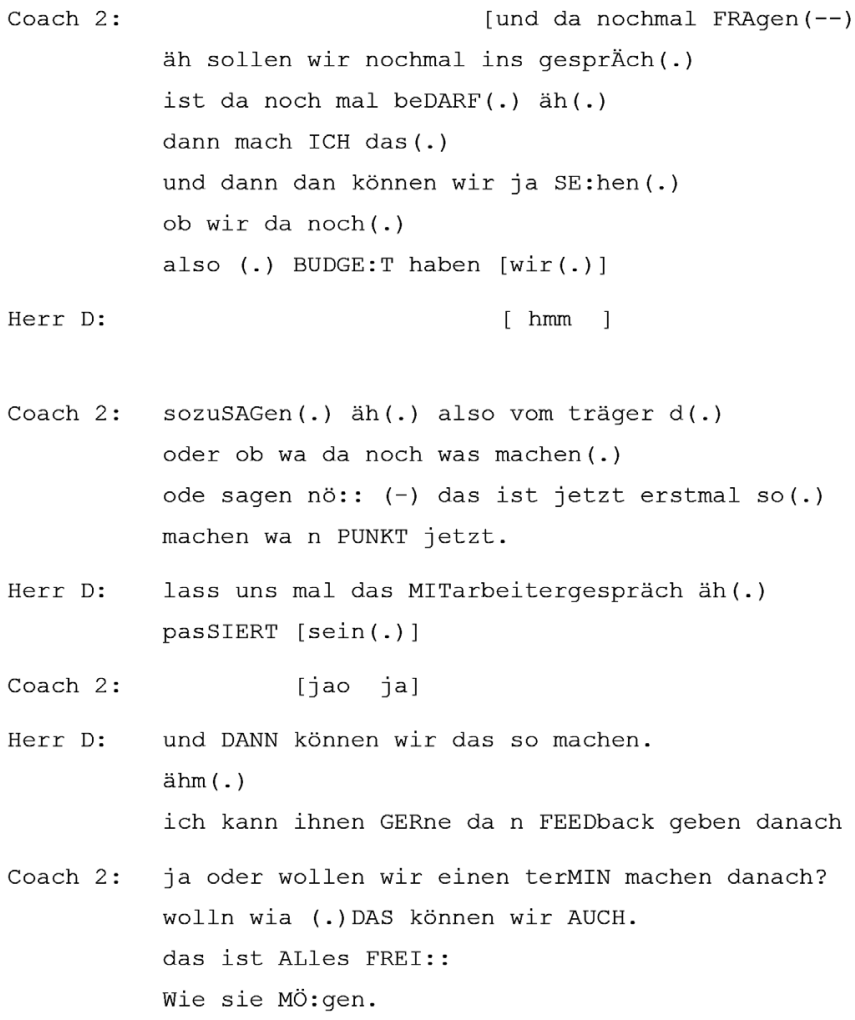

(Gespräch 3, abschließendes Gespräch)

Der Berater belässt es nicht beim unverbindlichen Terminangebot, sondern schlägt vor, sich selbst beim Coachee zu melden. Schließlich bietet er an, einen Termin verbindlich zu vereinbaren. Nachdem die Vorbereitung der Mitarbeitergespräche abgeschlossen ist, die vom Coachee in der Eingangssequenz eingefordert wurde, muss nun daran gearbeitet werden, wie man wieder daran anschließt und das Coaching am Laufen hält. Hier deutet sich das dritte Strukturproblem an, einerseits klientenorientiert zu arbeiten, andererseits aber auch auf der organisationalen Ebene Folgeaufträge sichern zu müssen.

Im Vorfeld der folgenden Schlusssequenz eines Gesprächs am Ende des Beratungsprozesses wurde bereits vorab mehrfach von der Coachee das Signal gesendet, dass es keinen besonderen Redebedarf mehr gibt. U. a. kommt es gegen Ende des Gesprächs zu einer 13-Sekündigen Pause. Dennoch stellt der Coach eine offene Frage, auf die man nicht mit ja oder nein antworten kann, sondern eher zu einer weiteren Erzählung aufgefordert wird. 


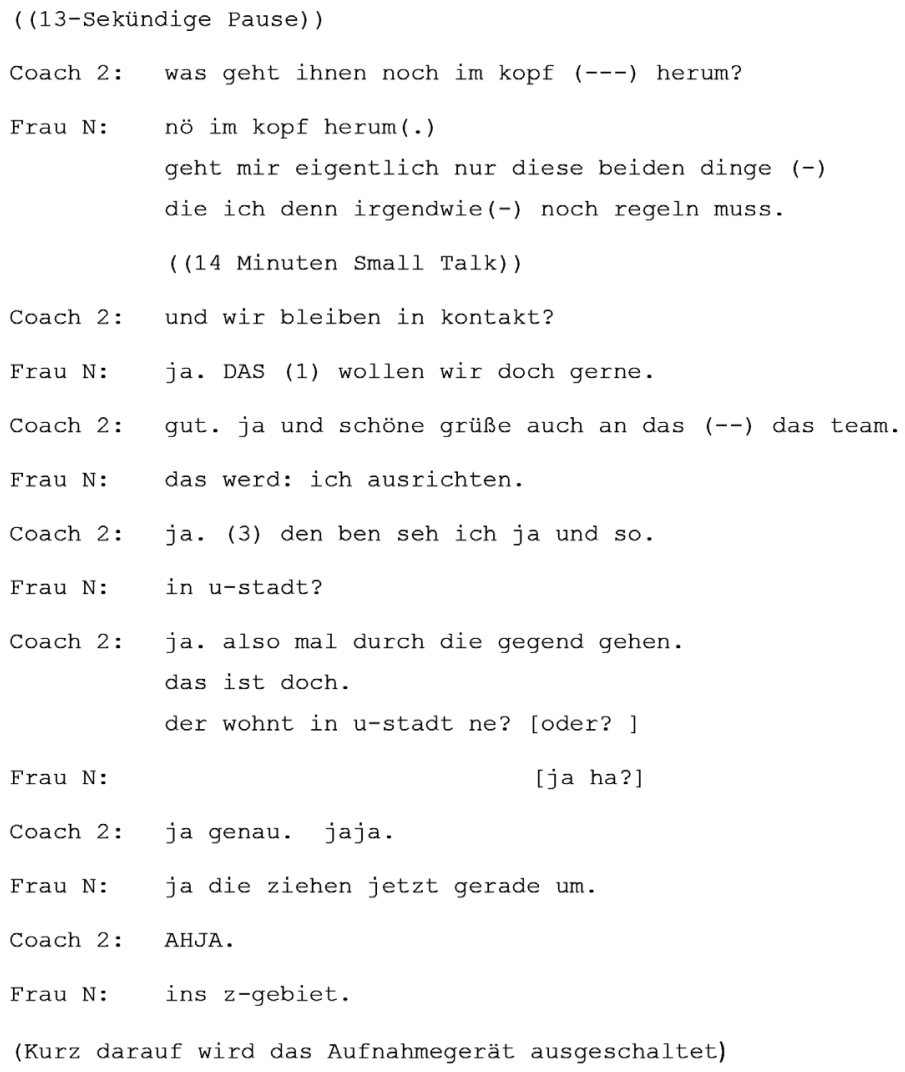

(Schlusssequenz, Gespräch 3)

Mit der Eingangsfrage wird vom Coach suggeriert, es müsse doch noch etwas Berichtenswertes geben. Auch der weitere Verlauf weist auf eine bestimmte Fortsetzungsidee des Coachings hin, die der Coach zu verfolgen scheint. Nachdem die Coachee geäußert hat, dass es eigentlich keinen Gesprächsbedarf mehr gebe, versucht der Coach einen weiteren Termin für ein Gespräch $\mathrm{zu}$ vereinbaren. In der 14-minütigen Auslassung im Transkript folgen verschiedene Terminangebote, die auch mit ,Small Talk' verbunden werden und weder inhaltliche noch methodische Elemente enthalten.

Nach dieser langen Phase des Small Talks fragt der Berater erneut: „Und wir bleiben in Kontakt"“. Es verdeutlicht sich eine informelle Ebene. Die Bezugnahme auf ein weiteres Treffen ist nicht zuvorderst ziel- und bedarfsorientiert für die Coachee. Vielmehr erscheint es so, als ob der Berater Beziehungsarbeit leisten muss, um den Kontakt zu halten. Auffällig ist, dass das 
Gespräch über Persönliches seitens des Beraters am Laufen gehalten wird. Dies zeigt sich u. a. daran, dass der Berater der Coachee nicht die Möglichkeit bietet, letztlich ein Ende zu setzen. Die Beziehungsarbeit kann auch strategisch betrachtet werden, da über Beziehungen auch Folgeaufträge generiert und sich Bekanntheit des Beratungsdienstleisters auch über Mundpropaganda erreichen lässt. In diesem Sinn kann das private Gespräch als Teil des Coachings betrachtet werden, in dem es nicht mehr um eine Klientenorientierung, sondern um den organisationalen Rahmen dreht.

Ein*e Coach ist auf organisationaler Ebene darauf angewiesen, Aufträge und Kunden zu akquirieren. In den Schlusssequenzen zeigt sich, dass - sofern die organisationale Rahmung ohnehin einen weiteren Termin vorsieht, z. B. nach dem ersten Gespräch oder inmitten eines über mehrere Sitzungen ablaufenden Beratungsprozesses - das Gespräch relativ schnell beendet werden kann. Anders verhält es sich im abschließenden Gespräch eines Beratungsprozesses. Hier steht die Beziehungsarbeit im Vordergrund, um den Kontakt zum Kunden möglichst verbindlich zu halten. Es zeigt sich ein Balanceakt zwischen einerseits einem erfolgreichen Prozessabschluss und andererseits einem Offen- und Anschlussfähighalten für weitere Aufträge.

\section{Resümee}

Die herausgearbeiteten Strukturprobleme basieren auf der fallanalytischen Untersuchung eines Coaching-Anbieters. Die Fallstudie ist daher ein erstes exploratives Aufspüren von Strukturproblemen und ihrer professionellen Bearbeitung. Die herausgearbeiteten handlungsübergreifenden typischen Kernprobleme und Spannungsfelder können in weiteren Studien überprüft und erweitert werden. Dabei wäre es auch interessant herauszuarbeiten, wie unterschiedliche organisationale Rahmungen, z. B. Beratung in Sanktionskontexten (Böhringer u. a. 2012; Wolff 2014) oder in privat-öffentlich finanzierten Kontexten in Transfergesellschaften (Muche u. a. i. E.) Spannungsfelder für einen professionellen Umgang in der Beratung entstehen lassen.

In Bezug auf Professionalisierungstendenzen wird in sozialwissenschaftlichen Zusammenhängen die Debatte um „Organisation und Profession“ seit langem geführt (Klatetzki/Tacke 2012; Klatetzki/Nokielski 2010). Argumentiert wird, dass professionelles Handeln durch organisationale Rahmungen und Strukturierungen eingeschränkt oder gar verunmöglicht werde. Auch Beratung weist stets sowohl professionsförmige als auch organisationsförmige Elemente auf (Brüsemeister 2004). In den Beratungsgesprächen des untersuchten Coaching-Unternehmens zeigte sich eine professionsförmige Ausrichtung darin, eine Art Moratorium für Selbstreflexionsprozesse zu schaffen. 
Wie mit der Beschreibung des ersten Strukturproblems deutlich wurde, ist der Coach bemüht, den alltäglichen Entscheidungsdruck außen vor zu lassen und Gelegenheit für die organische Entwicklung von Themen zur Selbstreflexion der eigenen (Führungs-)Position des Coachees zu geben. Dabei scheint es jedoch gleichermaßen zentral, durch das Einbringen von Beraterwissen die eigene Fachexpertise zu unterstreichen. Ein freier Markt, auf dem sich ein Coaching-Unternehmen behaupten muss, führt im untersuchten Fall dazu, eine thematische Offenheit bis hin zur Beliebigkeit scheinbar immer wieder durch den Verweis auf methodische Elemente zu rahmen und Coaching dadurch auch als solches (etwa im Unterschied zu Alltagsgesprächen) zu kennzeichnen. Als „ergebnisorientierte Selbstreflexion“ (Graf 2017: 78) geht es auch darum, ein positives Ergebnis interaktiv zu konstruieren, das Kostenund Zeitaufwand rechtfertigt.

Gleichwohl wird dadurch ein Expert*innen-Lai*innen-Verhältnis hergestellt. Ein solch asymmetrisches Verhältnis ist in den untersuchten Beratungssitzungen für sich gesehen schon problematisch. Denn tunlichst soll vermieden werden, dem*der Coachee den Expert*innenstatus abzusprechen. Dies ist jedoch nur durch einen Balanceakt zu bewerkstelligen. Nicht nur muss sich der Coach durch das Ausweisen von Fachexpertise ausreichend Legitimation verschaffen, auch die Beratungssituation rechtfertigt sich selbst nur dadurch, dass überhaupt eine Beratungsbedürftigkeit beim Coachee besteht.

Die Strukturproblematik, Coaching als Coaching-Gespräch durch Fachexpertise ausweisbar zu machen und trotz implizit unterstellter Beratungsbedürftigkeit den Coachee nicht als bedürftig erscheinen lassen zu dürfen, verweist auf die organisationsförmigen Aspekte des Coachings. Denn neben einer Kundenorientierung geht es für ein auf einem freien Anbietermarkt operierenden Unternehmen auch um die Möglichkeit, Folgeaufträge zu generieren. So dienen Beziehungsarbeit und die gemeinsame Bewertung des Coachings durch Coachee und Coach auch dazu, eine Arbeitsbeziehung aufrechtzuerhalten oder bestenfalls auszubauen. Zugespitzt deuten unsere Erkenntnisse darauf hin, dass „Profession“ im Kontext von Coaching nicht durch „Organisation“" eingeschränkt wird, sondern vielmehr der organisationale Rahmen die konstituierende Prämisse professionellen Handelns bildet.

\section{Literatur}

Birgmeier, Bernd (2011): Coachingwissen. Wiesbaden: VS Verlag für Sozialwissenschaften.

Böhringer, Daniela/Karl, Ute/Müller, Hermann/Schröer, Wolfgang/Wolff, Stephan (2012): Den Fall bearbeitbar halten. Gespräche in Jobcentern mit jungen Menschen. Opladen: B. Budrich. 
Böning, Uwe/Fritschle, Brigitte (2008): Coaching fürs Business. Was Coaches, Personaler und Manager über Coaching wissen müssen. 2. Aufl. Praxishandbuch Coaching. Bonn: ManagerSeminare-Verl.-GmbH.

Böning, Uwe/Kegel, Claudia (2015): Ergebnisse der Coaching-Forschung. Aktuelle Studien - ausgewertet für die Coaching-Praxis. [ohne Ort]: Springer Science and Business Media.

Brüsemeister, Thomas (2004): Beratung zwischen Profession und Organisation. In: Schützeichel, R./Brüsemeister, T. (Hg.): Die beratene Gesellschaft. Zur gesellschaftlichen Bedeutung von Beratung. 1. Aufl. Wiesbaden: VS Verlag für Sozialwissenschaften.

Deppermann, Arnulf (2008): Gespräche analysieren. Eine Einführung. 4. Aufl. Qualitative Sozialforschung, Bd. 3. Wiesbaden: VS Verlag für Sozialwissenschaften.

Egbert, Maria (2009): Der Reparatur-Mechanismus in deutschen und interkulturellen Gesprächen. Mannheim: Verlag für Gesprächsforschung.

Fietze, Beate (2015): Coaching auf dem Weg zur Profession? Eine professionssoziologische Einordnung. In: Schreyögg, A./Schmidt-Lellek, C. (Hg.): Die Professionalisierung von Coaching. Ein Lesebuch für den Coach. Wiesbaden: Springer, S. 3-22.

Fritsch, Michael (2010): Professionalität im Coaching. Beiträge der Coachingverbände zur Professionsbildung und Professionalisierung in Deutschland. Münster: MV.

Galdynski, Karolina/Kühl, Stefan (2009): Black-Box Beratung? Empirische Studien zu Coaching und Supervision. Wiesbaden: VS Verlag für Sozialwissenschaften.

Garfinkel, Harold (1967): Studies in ethnomethodology. Reprinted. Cambridge: Polity Press.

Geißler, Harald (2017): Die Grammatik des Coachens. Eine empirische Rekonstruktion. Wiesbaden: Springer.

Graf, Eva (2017): Management-Coaching: Ein arbeitsweltliches Beratungsformat zwischen Selbst-Reflexion und Output-Orientierung. In: Pick, I. (Hg.): Beraten in Interaktionen. Eine gesprächslinguistische Typologie des Beratens. Frankfurt a. M. u. a.: Peter Lang, S. 77-96.

Greif, Siegfried (2008): Coaching und ergebnisorientierte Selbstreflexion. Theorie, Forschung und Praxis des Einzel- und Gruppencoachings. Innovatives Management. Göttingen u. a.: Hogrefe.

Klatetzki, Thomas/Nokielski, Hans (2010): Soziale personenbezogene Dienstleistungsorganisationen als bürokratisch-professionelle Handlungszusammenhänge: Weber und die Folgen. In: Klatetzki, T. (Hg.): Soziale personenbezogene Dienstleistungsorganisationen. Soziologische Perspektiven. 1. Aufl. Organisation und Gesellschaft. Wiesbaden: VS Verlag für Sozialwissenschaften, S. 25-60.

Klatetzki, Thomas/Tacke, Veronika (Hg.) (2012): Organisation und Profession. 1st ed. Organisation und Gesellschaft. Wiesbaden: VS Verlag für Sozialwissenschaften.

Liska, Gerhard (2010): Zur Bedeutungszunahme der Beratungsformate Supervision und Coaching aus organisationstheoretischer Sicht. In: Schreyögg, A./ Schmidt-Lellek, C. (Hg.): Die Organisation in Supervision und Coaching. Wiesbaden: VS Verlag für Sozialwissenschaften, S. 27-40.

Loebbert, Michael (2015): Coaching Theorie. Eine Einführung. Aufl. 2015. Wiesbaden: Springer Fachmedien Wiesbaden GmbH. 
Maier-Gutheil, Cornelia (2013): Professionalität in der Beratung - erwachsenenbildnerische Analysen und Reflexionen. In: Käpplinger, B./Robak, S./Schmidt-Lauff, S. (Hg.): Engagement für die Erwachsenenbildung. Ethische Bezugnahmen und demokratische Verantwortung; Festschrift für Wiltrud Gieseke. Wiesbaden: Springer, S. 179-186.

Muche, Claudia/Truschkat, Inga/Schröder, Christian/ Karl, Ute (i. E.): Beratung in der Transfergesellschaft als gemeinsame Herstellung eines Schonraums. In: Peters, L./Muche, C./Truschkat, I. (Hg.): Personen(un)bezogene Dienstleistungen am Arbeitsmarkt. Der Beschäftigungstransfer im Spannungsfeld von Staat, Markt und Gemeinschaft. Weinheim: Juventa.

Rauen, Christopher (2003): Coaching. Innovative Konzepte im Vergleich. 3., unv. Aufl. Schriftenreihe Psychologie und innovatives Management. Göttingen: Verlag für Angewandte Psychologie.

Riedel, Jens (2003): Coaching für Führungskräfte. Erklärungsmodell und Fallstudien. Wiesbaden: Deutscher Universitäts-Verlag.

Schreyögg, Astrid (2016): Individuelle Personalentwicklung von Fach- und Führungskräften: Coaching. In: Gieseke, W./Nittel, D. (Hg.): Handbuch Pädagogische Beratung über die Lebensspanne. 1. Auflage. Weinheim: Beltz Juventa, S. 784-794.

Schröder, Christian/Karl, Ute (2017): On being guided - a typology of career guidance in EU discourses. In: British Journal of Guidance \& Counselling, S. 1-10.

Selting, Margret et al. (2009): Gesprächsanalytisches Transkriptionssystem 2 (GAT 2). In: Gesprächsforschung - Online-Zeitschrift zur verbalen Interaktion, 10, S. $1617-1837$.

Steinke, Ingo (2015): Kompetenzanforderungen an Coaches. In: Schreyögg, A./ Schmidt-Lellek, C. (Hg.): Die Professionalisierung von Coaching. Ein Lesebuch für den Coach. Wiesbaden: Springer, S. 257-282.

Wolff, Joachim (2014): Sanktionen im SGB II und ihre Wirkungen. http://doku.iab.de/ stellungnahme/2014/SN0214.pdf [Zugriff: 29.03.2017]. 

VII. Beratung in organisationalen Kontexten: Lernen - Steuerung - Methoden 

Henning Pätzold

\section{Beratung im Kontext organisationalen Lernens}

\section{Ausgangslage und Relevanz}

Organisationsberatung ist in den vergangenen Jahren verstärkt und aus verschiedensten Perspektiven als Gegenstand pädagogischer Forschung und Praxis wahrgenommen worden. Als pädagogisches Handlungsfeld wird sie beispielsweise in dem Handbuch von Gieseke und Nittel (2016) thematisiert, ebenso in verschiedenen Tagungsbänden der Kommission Organisationspädagogik der DGfE (insbesondere Göhlich, Weber, Seitter und Feld 2010) und zunehmend auch in Studiengangsbeschreibungen (z. B. in pädagogischen Studiengängen der Universitäten Heidelberg, Hildesheim, Koblenz-Landau und anderen). Die Rolle von Lernberatung in Organisationen wird u. a. in dem genannten Band von Göhlich thematisiert, daneben auch in früheren Untersuchungen zu Lernberatung in beruflichen Kontexten (vgl. z. B. Klein und Reutter 2005), auch wenn hier der Bezug zur Gesamtorganisation(-sentwicklung) mitunter implizit bleibt. Schließlich ist organisationales Lernen ein Thema, das in der Erwachsenenpädagogik nicht nur seit längerem aufgegriffen, sondern zunehmend auch mit spezifisch pädagogischen Mitteln wissenschaftlich bearbeitet wird (vgl. z. B. Geißler 2000; Göhlich 2007, 2016; Behrmann 2010; Göhlich, Weber, Schröer u. a. 2014; Engel 2014; Pätzold 2017, 2018).

Die Begrifflichkeit, die dabei verwendet wird, ist vom jeweiligen Referenzhorizont abhängig. Organisationsberatung im Allgemeinen (und Unternehmensberatung im Besonderen) orientiert sich an der historischen Genese des Handlungsfeldes außerhalb der Pädagogik (vgl. Pätzold 2016: 622f.) und an dem bereits weitergehend pädagogisch erschlossenen Handlungsfeld Beratung (vgl. Krause u. a. 2003). Organisationsentwicklung wird ebenso pädagogisch thematisiert (z. B. Schiersmann und Thiel 2014, Macha 2013, Arnold 2000). Auch hier wird an außerpädagogische Konzepte angeschlossen. Daneben etabliert sich aber auch ein breiterer Strom von Arbeiten zur Entwicklung pädagogischer Organisationen (Dollhausen 2008, Feld 2011). Beim organisationalen Lernen schließlich wird der Horizont pädagogischer Theorie- und Begriffsbildung zwar genutzt (s. o.), es finden sich aber auch zahlreiche Arbeiten, in denen gerade das Moment organisationalen Lernens nicht pädagogisch ausgedeutet, sondern gleichsam als geschlossenes Modell aus organisationswissenschaftlicher Standardliteratur entliehen wird (vgl. hierzu Pätzold 2015: 29f.). 
Beratung wird also als pädagogische Aufgabenstellung auf Organisationen bezogen und vielfältig ausgearbeitet. Lernen ist dabei ein wichtiger, aber keineswegs dominanter Bezugspunkt. Viel häufiger findet sich ein Verständnis von Organisationsberatung, bei dem diese auf Organisationsentwicklung abzielt (vgl. z. B. Schiersmann und Thiel 2014: 68). Organisationales Lernen wird dabei zwar mitgedacht, ist aber nicht in gleicher Weise profiliert, stellt es doch - im Gegensatz zu Beratung - keine Handlungsform dar, für deren Ausübung ein professioneller Anspruch erhoben werden kann. Die im Folgenden $\mathrm{zu}$ entfaltende These setzt an diesem Punkt an, indem Organisationsberatung als Lernberatung von Organisationen konzipiert werden soll. Auf diese Weise soll das pädagogische Proprium eines disziplinären und professionellen Beitrags zum Handlungsfeld Organisationsentwicklung herausgearbeitet werden.

Dabei ist es das Verdienst Ortfried Schäffters, diesen möglichen Zusammenhang in einem Beitrag aus dem Jahr 2000 bereits skizziert zu haben. Konzeptionell-systematisch stellt er zunächst Beratung als eine pädagogische Handlungsform dar und nennt dann neben Individuen auch „soziale Gruppen oder [...] Organisationen" (Schäffter 2000: 52) als mögliche Adressaten, womit er die Brücke schlägt zwischen dem Lernen und der Organisationsberatung, welche er ,als pädagogisch intendierte, zielgerichtete Einflussnahme auf organisationale (kollektive) Sinn-, Bedeutungs- und Handlungskontexte" (ebd.: 54) definiert - die Ähnlichkeit zu möglichen Definitionen von Lehre ist dabei unübersehbar.

Schäffters Argumentation macht plausibel, dass Beratung aus pädagogischer Perspektive auf Organisationen bezogen werden kann und dann in einem engen Verhältnis zu Lernprozessen steht. Die Konzepte von Lernberatung wie von organisationalem Lernen sind seither allerdings weiter geschärft worden und so stellt sich die Frage, inwieweit sich hieraus auch neue Konsequenzen für das beschriebene Verhältnis ergeben.

\section{Modelle}

\section{Lernberatung}

Lernberatung begleitet Lernprozesse mit den Mitteln der Beratung und macht diese auch zu deren Gegenstand (vgl. Pätzold 2004). Sie ist eine Einflussnahme ,auf fremde kognitive Strukturen“ (Schäffter 2000: 51), die darauf abzielt, Lernen zu ermöglichen und zu fördern. Beratung verweist dabei auf Merkmale wie zeitliche Befristung, Interaktion zwischen Rat gebenden und 
beratenden Akteurinnen und Akteuren und die Orientierung auf Lösungen in einem Sachbereich, wobei die Verantwortung für den Umgang mit Lösungen aufseiten der Beratenen verbleibt (vgl. Pätzold 2004: 52, 108f). Beratung setzt damit voraus, dass das beratene System über Möglichkeiten verfügt, Lösungen und andere Handlungsoptionen zu bewerten, auszuwählen und umzusetzen und sich dabei selbst zu beobachten. Sie ist eine „Reflexionshilfe für reflexive Systeme“ (Buchinger 2010: 143). Diese Merkmale verweisen - ebenso wie der Begriff des organisationalen Lernens - auf die Frage, ob Organisationen als Lernende (reflexive, entscheidungs- und handlungsfähige) Systeme aufgefasst werden können. Die Auseinandersetzung hierüber wäre ein eigenes Thema. Dass es zumindest analytisch weiterführend sein kann, Organisationen als reflexive, lernfähige Systeme zu konzipieren, sollen die folgenden Darlegungen deutlich machen. Eine ethische Problematik kann sich daraus ergeben, dass das Verständnis von Organisationen als Adressatinnen von Beratung verschleiern kann, dass in den Organisationen einzelne Personen Rat geben und empfangen und darauf Entscheidungen gründen, die dann möglicherweise zu Unrecht der Organisation attribuiert werden. Dieses Problem wird allerdings zumindest entschärft, wenn man Organisationsberatung als Lernberatung von Organisationen fasst, weil damit deutlich wird, dass die Beratung nicht unmittelbar zu Entscheidungen führt, sondern idealerweise Teil eines Lernprozesses ist. Von einem solchen wird kaum die Rede sein können, wenn Beratung und Entscheidung im Wesentlichen „,im stillen Kämmerlein" unter wenigen ausgewählten Personen erfolgen - und dann wäre es auch im klassischen Verständnis nicht angemessen, von Organisationsberatung zu sprechen (vgl. Pätzold 2016).

Dennoch ergibt sich die Frage, wie eigentlich eine Interaktion stattfinden soll, wenn die eine Interaktionspartnerin eine Organisation ist. Wegweisende Antworten hierzu kommen aus der in der Erwachsenenpädagogik intensiv rezipierten systemischen Beratung. Ursprünglich aus der Familientherapie stammend (vgl. Schlippe und Schweitzer 2016: 32ff.), fragt sie gerade danach, wie ein Beratungssetting so entwickelt werden kann, dass nicht Information zwischen der oder dem Beratenden und einer Klientin oder einem Klienten hin und her fließt, sondern in ein System eingeht, das diese Information eigenlogisch verarbeiten und darauf antworten kann. Hierzu entwickelte Interventionsformen, insbesondere das „zirkuläre Fragen“ (ebd.: 251), werden auch für organisationale Kontexte fruchtbar gemacht (vgl. ebd.: 423ff.; König und Vollmer 2014). Wenn gleichzeitig festgestellt wird, dass das Ergebnis nicht mehr unbedingt einen eigenständigen Ansatz innerhalb der Organisationsberatung darstellt (vgl. Elbe und Saam 2010: 87), spricht das eher dafür, dass die systemischen Ansätze innerhalb einer ohnehin häufig eklektizistischen Beratungspraxis ihren Ort gefunden haben. Im Weiteren soll Lernberatung von Organisationen Formen der Beratung beschreiben, die die Organisation im Ganzen als reflexives System in den Blick nehmen und entsprechend Model- 
lierungen und Interventionen vornehmen, die der Organisation vielfältige Reaktionsmöglichkeiten erlauben und sich gerade nicht an einzelne „Entscheider" richten. ${ }^{1}$ Gegenstand dieser Beratung ist, der Definition entsprechend, das Lernen der Organisation.

\section{Lernen: Organisationales Lernen}

Hinsichtlich des Begriffs „lernende Organisation“ ist die oben angesprochene Diskussion deutlich intensiver geführt worden. So kritisiert Faulstich, dass mit dem Begriff unterstellt werde, „,dass Kriterien der Subjekthaftigkeit sich auch auf Gruppen- und Organisationszusammenhänge anwenden lassen“ (Faulstich 2013: 195). Noch deutlich bissiger spricht Miller davon, hier werde lediglich ,auf mystische Weise von einem überindividuellen Subjekt, einem gesellschaftlichen Gesamtsubjekt" (Miller 1986: 210) ausgegangen. Nun ist „mystisch“ hier eine Art Gegenbegriff zu „real“" und damit wiederum ein Versuch, das Problem ontologisch zu lösen. Schwerer wiegt die Frage, ob mit der „Subjektivierung“ der Organisation wiederum ethische Probleme auftreten. Nicht umsonst formuliert Faulstich seine Kritik in einem Band mit dem Titel „Menschliches Lernen“ und auch Illeris (dessen Lernmodell im Folgenden eine zentrale Rolle spielen wird) ist bezüglich der lernenden Organisation skeptisch und nimmt hier mehr die Beschäftigung mit „Management und Floskeln als mit Lernen“" (Illeris 2010: 222) wahr. Es erscheint wichtig, diesem Einwand gegenüber aufmerksam zu sein, indem bei einer Konzeption organisationalen Lernens (ebenso wie bei der Beratung) keine subjektiv-menschlichen Vorgänge der individuellen Verfügung entzogen und bei der Organisation verortet werden. Unter Einhaltung dieser Maßgabe erscheint es allerdings durchaus plausibel, organisationales Lernen auch in seiner historischen Entwicklung als „Veränderung eines organisationalen Ordnungs- und Sinnzusammenhangs" (Behrmann 2010: 96) zu verstehen, die über das Lernen der Individuen hinausgeht.

Folgt man also der Idee, dass es sinnvoll sein kann, von organisationalem Lernen als einem Prozess zu sprechen, bei dem eine Organisation lernt und sich dieses Lernen nicht linear auf Lernprozesse der Organisationsmitglieder zurückführen lässt, so stellt sich auch hier die Frage, wie dieser Prozess im

1 Nur am Rande sei darauf hingewiesen, dass letzteres Vorgehen mit den üblichen Ansprüchen von Organisationsberatung nur begrenzt vereinbar ist. Eine Beratung, die sich nur an Einzelne richtet und nicht auf Einflussmöglichkeiten durch viele andere Organisationsmitglieder bedacht ist, eignet sich eher zur Erfüllung dessen, was als „latente Funktionen“ (von Ameln 2010) bezeichnet wird. 
Einzelnen modelliert und verstanden werden kann. Im vorliegenden Kontext wurde hierzu das Modell des Lerndreiecks von Knud Illeris verwendet (vgl. Illeris 2010: 40; 2006: 32). Das Problem, dass dabei subjektgebundene Begriffe wie „Emotion“ Verwendung finden, wurde dahingehend bearbeitet, dass für die je individuellen Prozesse und Eigenschaften bei der Modellierung organisationalen Lernens funktionale Äquivalente gesucht wurden. Dabei ist es hilfreich, dass Illeris mit seinem Modell eine Heuristik liefert, die es erlaubt, wesentliche Dimensionen des Lernens Erwachsener in den Blick zu nehmen und ins Verhältnis zu setzen. So ist für ihn das Wechselspiel von Emotion und Kognition ein unhintergehbares Moment des Lernens, und es lässt sich nicht nach einer Seite (etwa in Richtung „rein kognitiven“ Lernens) auflösen. In ähnlicher Weise lässt sich auf organisationaler Ebene das Wechselspiel zwischen Wissensmanagement und Organisationskultur als ein dynamischer Ausgleichsprozess beschreiben, bei dem organisationales Lernen immer beide Dimensionen berührt (ausführlicher hierzu sowie zur Darstellung und empirischen Erprobung des Modells vgl. Pätzold 2017, 2018). Abbildung 1 zeigt das Modell des organisationalen Lerndreiecks im Anschluss an Illeris.

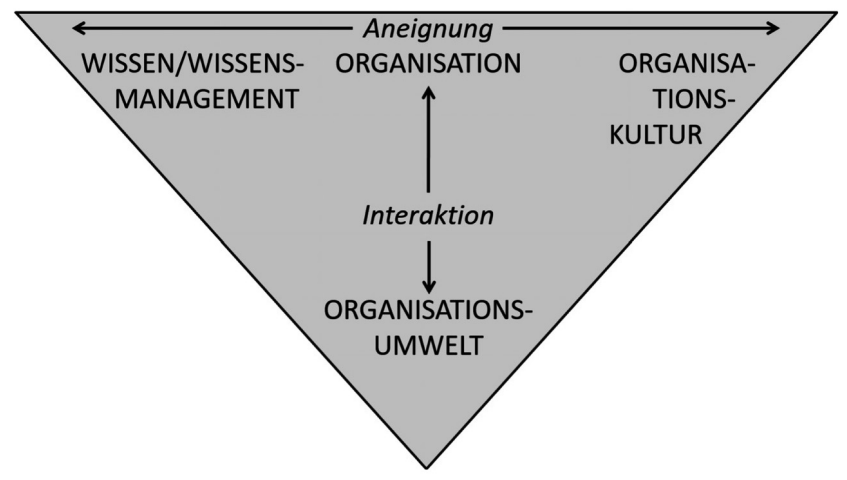

Abb. 1: Organisationales Lerndreieck (Pätzold 2017: 46)

Die Plausibilität des Modells wurde im Rahmen einer ethnografischen Studie getestet. Insbesondere ging es um die Frage, ob es geeignet ist, eine kategoriale Ordnung für die gewonnenen Befunde zu liefern (die Ergebnisse sind in Pätzold 2017 dargestellt). Im Weiteren stellte sich die Frage, inwiefern die daraus gewonnenen Befunde sich auch zu einer erwachsenenpädagogisch fundierten Organisationsberatung eignen. 


\section{Fallbeispiel}

Die oben angesprochene Untersuchung fand in einer kleineren Non-Profit-Organisation statt. Im Rahmen eines zweiwöchigen Praktikums wurde mit einem ethnografischen Zugang eine Vielfalt von Einzelmaterialien und -beobachtungen gewonnen und entlang des Modells ausgewertet. In diesem Zugriff zeigte sich ein überzeugendes strukturierendes Potenzial, es gelang überwiegend leicht und mit intersubjektiv hoher Übereinstimmung, Beobachtungen den Dimensionen und ihren Beziehungen innerhalb des Lerndreiecks zuzuordnen. Im Nachgang der Untersuchung wurde der Organisation angeboten, die Befunde dort vorzustellen, was zunächst zwar begrüßt, aber nicht in Anspruch genommen wurde. Ein gutes halbes Jahr später, in einer für die Organisation insgesamt herausfordernden Gesamtsituation, kamen Mitglieder der Organisationsleitung jedoch auf das Angebot zurück und baten um Beratung in Bezug auf die organisationale Situation unter Berücksichtigung der in der Untersuchung gewonnenen Befunde. Diese fand im Rahmen von zwei Präsentationen statt; bei der ersten nahmen unterschiedliche Mitglieder der NPO teil (einschließlich Leitungsebene), bei der zweiten nur der Gründer, welcher als Stifter die Hauptverantwortung für die Ausrichtung der Organisation hat. Die Beratungen hatten primär das Ziel, die Organisation in ihrem Entwicklungs- und Lernprozess zu unterstützen, weshalb sie nicht umfassend forschungsmethodisch begleitet werden konnten. Während und nach den jeweiligen Beratungen wurden allerdings Notizen angefertigt, die wesentliche Interaktionen festhielten. Interessanterweise zeigte sich hier, dass die Logik des Modells - und damit des Organisationsentwicklungsprozesses als Lernprozess - in wesentlichen Teilen angenommen bzw. im Gespräch genutzt wurde. So wurde geäußert, der „bisherigen Dynamik [fehle] die Reflexion“ (Zitat nach Gesprächsnotiz) und die Organisation wolle „mit der Welt gemeinsam lernen, besser zu werden“ (ebd.). Auch in Bezug auf das Verhältnis der Organisation zu ihrer Umwelt wurde das Reflexionsproblem deutlich: Es wurde festgestellt, dass zu viele Umweltkontakte die Möglichkeiten der Organisation einschränken, sich auch selbst zu beobachten und reflektiert zu positionieren.

Das hier geschilderte Fallbeispiel kann nicht in Anspruch nehmen, ein Konzept der Organisationsberatung als Lernberatung von Organisationen empirisch zu prüfen oder auch nur tiefergehend zu plausibilisieren. Es zeigt jedoch, dass ein solcher Zugang sich grundsätzlich als anschlussfähig erweisen kann und, dass es den Organisationsmitgliedern dabei sehr leichtfällt, die Organisation auch als eigenständige Entität zu erfassen, deren „Verhalten“ sich nicht einfach als aufsummiertes Verhalten der Mitglieder erklärt. So erlaubte die Perspektive auf die lernende Organisation unter anderem eine - bei sehr kleinen Organisationen eher unübliche - Abstraktion von den Handlun- 
gen einzelner Organisationsmitglieder. Leitung und auch Mitarbeitende wurden zwar in den Gesprächen erwähnt und es wurden ihnen selbstverständlich auch Handlungen und Entscheidungen zugeschrieben, gleichwohl wurden diese aber immer in ihrem Einfluss auf das organisationale Geschehen bewertet und so kontextualisiert. Abschließend sollen diese Beobachtungen durch einen Blick auf Möglichkeiten und Grenzen des Verständnisses von Organisationsberatung als Lernberatung von Organisationen ergänzt werden.

\section{Folgerungen - Möglichkeiten und Grenzen}

Versteht man Organisationsentwicklung als Ausdruck organisationalen Lernens und Beratung von Organisationen entsprechend als Lernberatung, so ergeben sich spezifische Perspektiven, aber natürlich auch blinde Flecke. Vor dem Hintergrund der oben dargestellten theoretischen Überlegungen und des ersten empirischen Einblicks lassen sich diese wie folgt zusammenfassen:

Zunächst bestehen die Grenzen, die sich grundsätzlich aus dem Format der Beratung ergeben. Beratung ist ihrem Verständnis nach eine unsichere Intervention, d. h. sie setzt voraus, dass aufseiten des Klientensystems (sei es ein Individuum oder eine Organisation) eigenverantwortliche Entscheidungen getroffen und auch selbst verantwortet werden können. Diese Entscheidungen mögen etwa im Falle von Entlassungen drastischer erscheinen als bei Themen der Lernberatung, aber auch hier werden beispielsweise Ressourcen gebunden, die möglicherweise an anderer Stelle fehlen könnten. Diese Einschränkung gilt aber nicht nur für den vorliegenden Ansatz, sondern für Organisationsberatung generell. Das führt mitunter zu einem Bruch mit den Erwartungen des/der Auftraggebenden, die sich in ähnlicher Weise auch bei individuellen Beratungen immer dann finden, wenn mit der Inanspruchnahme von Beratung die Erwartung verbunden wird, Verantwortung abzugeben. Die Fokussierung auf Lernen verstärkt diesen Aspekt allerdings, insofern Lernen auch hier oft als Prozess verstanden wird, der eher unter Bedingungen geringen Handlungsdrucks möglich ist. Vermutlich wurde auch deshalb in den beschriebenen Beratungsinteraktionen das Konzept des Lernens zwar gelegentlich aufgenommen, aber nicht durchgängig genutzt und mitunter eher als Ideal denn als pragmatisch begründeter Entwicklungspfad verstanden. Schließlich muss konstatiert werden, dass zu dem Konzept - auch 18 Jahre nach Schäffters Artikel - kaum empirische Forschung vorliegt. Dabei ist durchaus an die grundsätzlichen Grenzen einer empirischen Wirkungsforschung zur Organisationsberatung zu erinnern (vgl. Pätzold 2016: 628ff.). Gleichwohl wäre es wünschenswert, wenn zumindest mehr Befunde über die Verläufe, Folgen und Nebenfolgen von Organisationsberatung vorlägen, wie 
es in anderen Bereichen pädagogischer Beratung der Fall ist (vgl. Gieseke und Stimm 2016; Kossack 2006).

Bei allen vorliegenden Einschränkungen zeigen die bisherigen Überlegungen und Erfahrungen aber auch ermutigende Hinweise, das Konzept der Lernberatung von Organisationen weiter zu verfolgen. So ermöglicht es eine Überwindung des „Rationalitätsbias“, der der Organisationsberatung strukturell immer noch oft zu eigen ist. Zwar werden Organisationen längst nicht mehr als Kollektive rational handelnder Akteure verstanden, gleichzeitig folgt aber das Konzept der Organisationsberatung selbst einem sehr rationalen Modell der Arbeitsteilung (vgl. Pätzold 2016: 621f.). Herausforderungen des organisationalen Lernens werden dadurch häufig einseitig bearbeitet (etwa durch die Empfehlung eines organisationalen Wissensmanagements), während Herausforderungen der Organisationskultur als davon separiert betrachtet werden - ähnlich wie eine rationalistische Fokussierung des Lernens auf die Kognition zu recht kritisiert wird, weil sie den emotionalen Aspekt des Lernens marginalisiert (vgl. Arnold 2008).

Die Unterscheidung der Dimensionen Wissensmanagement, Organisationskultur und Organisationsumwelt erlaubt nach den bisherigen Erfahrungen eine umfassende und systematische Analyse und Konzeption von Organisationsberatung und organisationalem Lernen, die sich gleichzeitig nicht in Dichotomien verliert. Insbesondere erlaubt sie auch, die Beziehungen zwischen allen drei Polen - die Seiten des Dreiecks - analytisch zu nutzen. So wurde in den Beratungen vereinzelt angedeutet, dass bestimmte Partner der Organisationsumwelt eher durch die Wissensseite, andere durch die Kulturseite einschließlich der durch die Organisation vertretenden Werte angesprochen würden. Die übergreifende Perspektive des Lernens verweist dabei auf einen im Organisationskontext in der Regel positiv konnotierten Vorgang, der den Blick auf Ressourcen öffnet.

Hieran lässt sich auch anschließen, insofern der beschriebene Ansatz in vielen weiteren Bereichen an bestehende Einsichten zum Lernen (Erwachsener) anknüpfen und diese in einen organisationalen Kontext übersetzen kann. Angefangen von Knowles Konzept des selbstgesteuerten Lernens (Knowles 1975), bei dem u. a. die Rolle der helfenden Ressourcen in durchaus passender Weise angesprochen wird, über Modelle des Oberflächen- oder Tiefenlernens (zum Überblick vgl. Pätzold 2011: 26 und 32f.) bis hin zu lernbezogenen Prozessen wie Training, Übung oder Vergessen eröffnet sich hier eine Vielzahl potenziell ertragreicher theoretischer und analytischer Kategorien, die für die Organisationsberatung fruchtbar gemacht werden können. 


\section{Literatur}

Arnold, Rolf (2000): Das Santiago-Prinzip: Führung und Personalentwicklung im lernenden Unternehmen. Köln: Deutscher Wirtschaftsdienst.

Arnold, Rolf (2008): Die Bildung Erwachsener als emotionale Suchbewegung. In: Arnold, Rolf/Holzapfel, Günther (Hg.): Emotionen und Lernen. Baltmannsweiler: SchneiderVerlag Hohengehren, S. 215-241.

Behrmann, Detlef (2010): Lernen in der Organisation - Rekonstruktionen zum Verhältnis von individuellem und organisationalem Lernen. In: Heidsiek, Charlotte/ Petersen, Jendrik (Hg.): Organisationslernen im 21. Jahrhundert. Festschrift für Harald Geißler. Frankfurt a. M.: Lang, S. 93-103.

Buchinger, Kurt (2010): Beratung von Personen und Organisationen. Unterschied und Verbindung. In: Faßnacht, Michael/Kuhn, Hubert/Schrapper, Chrstian (Hg.): Organisation organisieren. Gruppendynamische Zugänge und Perspektiven für die Praxis. Weinheim, München: Juventa, S. 137-156.

Dollhausen, Karin (2008): Planungskulturen in der Weiterbildung: Angebotsplanungen zwischen wirtschaftlichen Erfordernissen und pädagogischem Anspruch. Bielefeld: Bertelsmann.

Elbe, Martin/Saam, Nicole J. (2010): „Mönche aus Wien, bitte lüftets eure Geheimnisse." Über die Abweichungen der Beratungspraxis von den Idealtypen der Organisationsberatung. In: Kühl, Stefan/Moldaschl, Mandfred (Hg.): Organisation und Intervention: Ansätze für eine sozialwissenschaftliche Fundierung von Organisationsberatung. München: Rainer Hampp Verlag, S. 85-113.

Engel, Nikolas (2014): Der Zwang des Neuen. Organisationales Lernen als Kreation und Simulation von Neuheit. In: Weber, Susanne M./Göhlich, Michael/Schröer, Andreas/Schwarz, Jörg (Hg.): Organisation und das Neue. Wiesbaden: Springer VS, S. 237-246.

Faulstich, Peter (2013): Menschliches Lernen: eine kritisch-pragmatistische Lerntheorie. Bielefeld: Transcript.

Feld, Tim C. (2011): Netzwerke und Organisationsentwicklung in der Weiterbildung. Bielefeld: Bertelsmann.

Geißler, Harald (2000): Organisationspädagogik: Umrisse einer neuen Herausforderung. München: Vahlen.

Gieseke, Wiltrud/Nittel, Dieter (Hg.) (2016): Handbuch Pädagogische Beratung über die Lebensspanne. Weinheim, Basel: Beltz Juventa.

Gieseke, Wiltrud/Stimm, Maria (2016): Praktiken der professionellen Bildungsberatung. Innensichten auf die Entscheidungsfindung im Beratungsprozess. Wiesbaden: Springer VS.

Göhlich, Michael (2007): Organisationales Lernen. In: Göhlich, M.ichael/Wulf, Christian/Zirfas, Jörg (Hg.): Pädagogische Theorien des Lernens. Weinheim, Basel: Beltz, S. 222-232.

Göhlich, Michael (2016): Theories of Organizational Learning as resources of Organizational Education. In: Schröer, Andreas/Göhlich, Michael/Weber, Susanne M./Pätzold, Henning (Hg.): Organisation und Theorie. Wiesbaden: Springer VS, S. 11-21. 
Göhlich, Michael/Weber, Susanne M./Schröer, Andreas et al. (2014): Forschungsmemorandum der Kommission Organisationspädagogik. Erziehungswissenschaft, (49), S. 94-105.

Göhlich, Michael/Weber, Susanne M./Seitter, Wolfgang/Feld, Tim C. (Hg.) (2010): Organisation und Beratung: Beiträge der AG Organisationspädagogik. Wiesbaden: VS Verlag für Sozialwissenschaften.

Illeris, Knud (2006): Das „Lerndreieck“. Rahmenkonzept für ein übergreifendes Verständnis vom menschlichen Lernen. In: Nuissl, Ekkehard (Hg.): Vom Lernen zum Lehren. Lern- und Lehrforschung für die Weiterbildung. Bielefeld: W. Bertelsmann, S. 29-41.

Illeris, Knud (2010): Lernen verstehen. Bedingungen erfolgreichen Lernens. Bad Heilbrunn: Klinkhardt.

Klein, Rosemarie/Reutter, Gerhard (Hg.) (2005): Die Lernberatungskonzeption. Baltmannsweiler: Schneider Verlag Hohengehren.

Knowles, Malcolm S. (1975): Self-directed Learning. A Guide for Learners and Teachers. Englewood Cliffs NJ: Prentice Hall Regents.

König, Eckhard/Volmer, Gerda (2014): Handbuch systemische Organisationsberatung: Grundlagen und Methoden (2. Auflage.). Weinheim: Beltz.

Kossack, Peter (2006): Lernen Beraten. Eine dekonstruktive Analyse des Diskurses zur Weiterbildung. Bielefeld: Transcript.

Krause, Christina/Fittkau, Bernd/Fuhr, Reinhard/Thiel, Hans-Ulrich (Hg.) (2003): Pädagogische Beratung. Grundlagen und Praxisanwendung. Paderborn: F. Schöningh.

Macha, Hildegard (2013): Organisationales Lernen mit Gleichstellung in Organisationen. Vierteljahresschrift für wissenschaftliche Pädagogik, 89(1), S. 42-55.

Miller, Max (1986): Kollektive Lernprozesse. Studien zur Grundlegung einer soziologischen Lerntheorie. Frankfurt a. M.: Suhrkamp.

Pätzold, Henning (2004): Lernberatung und Erwachsenenbildung. Baltmannsweiler: Schneider Verlag Hohengehren. http://www.die-bonn.de/weiterbildung/literaturrecherche/details.aspx?id=11022 [Zugriff: 09.01.2018]

Pätzold, Henning (2011): Learning and teaching in adult education. Contemporary theories. Opladen, Farmington Hills: Barbara Budrich.

Pätzold, Henning (2015): Organisationstheorien in der Erwachsenenbildung: Rezeption und Nutzung. In: Schemmann, Michael (Hg.): Organisationsforschung in der Erwachsenenbildung - Theorie, Methoden, Befunde (Bd. 38). Wien, Köln, Weimar: Böhlau, S. 19-36.

Pätzold, Henning (2016): Organisationsberatung. In: Gieseke, Wiltrud/Nittel, Dieter (Hg.): Handbuch Pädagogische Beratung über die Lebensspanne. Weinheim, Basel: Beltz Juventa, S. 621-632.

Pätzold, Henning (2017): Das organisationale Lerndreieck - eine lerntheoretische Perspektive auf organisationales Lernen. Zeitschrift für Weiterbildungsforschung, 40(1), S. 41-52. doi:10.1007/s40955-017-0087-z [Zugriff: 01.09.2018]

Pätzold, Henning (2018): Bausteine einer Theorie der lernenden Non-Profit-Organisation - eine lerntheoretische Perspektive. In: Schröer, Andreas/Engel, Nikolas/ Fahrenwald, Claudia/Weber, Susanne M. (Hg.): Organisation und Zivilgesellschaft (in Vorber.). Wiesbaden: Springer VS. 
Schäffter, Ortfired (2000): Organisationsberatung als Lernberatung von Organisationen. Literatur- und Forschungsreport Weiterbildung, (46), S. 50-60.

Schiersmann, Chirstiane/Thiel, Hans-Ulrich (2014): Organisationsentwicklung. Prinzipien und Strategien von Veränderungsprozessen (4.). Wiesbaden: Springer VS.

von Ameln, Falko (2010): Latente Funktionen und hidden agendas in der Organisationsberatung. In: Göhlich, Michael/Weber, Susanne M./Seitter, Wolfgang/Feld, Tim C. (Hg.): Organisation und Beratung: Beiträge der AG Organisationspädagogik (1. Aufl.). Wiesbaden: VS Verlag für Sozialwissenschaften, S. 191-199.

Von Schlippe, Arist/Schweitzer, Jochen (2016): Lehrbuch der systemischen Therapie und Beratung. 1: Das Grundlagenwissen: mit 31 Abbildungen und 6 Tabellen (3. Aufl.). Göttingen, Bristol, CT: Vandenhoeck \& Ruprecht. 

Hildegard Macha

\section{Das Konzept der transformativen Beratung in Unternehmen und seine wissenschaftliche Evaluation}

\section{Einleitung}

Pädagogische Beratung ist eine zielgerichtete, vertrauensvolle und Rat suchende Interaktion zwischen Klient(_innen) und Berater(_innen). Probleme eines $/ \mathrm{r}$ Probanden/in bzw. eines Ratsuchenden werden einer Klärung zugeführt (Mutzek 2014: 12; Macha et al. 2012: 18). Somit steht sie im Dienste der Weiterentwicklung von Klient_innen. Kollegiale Beratung hilft zum Beispiel einer Gruppe, thematische Klärungen zu beruflichen Problemen - zum Beispiel des Schulunterrichts $-\mathrm{zu}$ erörtern und ist lösungsorientiert. Beratung in Unternehmen gestaltet sich aufgrund des Kontextes anders als die Face-to-Face-Beratung bzw. Beratung in Gruppen im sozialen Feld. Organisationsberatung und Beratung einzelner Führungskräfte hat zum Ziel, Entwicklungsprozesse oder Bildungsprozesse in den Beteiligten zu initiieren (Göhlich et al. 2010).

In Wirtschaftsunternehmen muss stets das Beratungsziel mit der Wirtschaftlichkeit austariert werden - das Beratungsziel muss entweder die Produktion verbessern oder der Belegschaft und der gesamten Organisation nutzen, am besten beides zugleich (Tomaschek 2014; König/Vollmer 2018). Dennoch gilt es, am Grundsatz der humanen Dienstleistung für die betroffenen Menschen festzuhalten. Um diesen Spagat zwischen Effizienz, Wirtschaftlichkeit und Humanität zu ermöglichen, wurde das Konzept des „Transformativen Organisationalen Lernens - TOL“" anhand von bestehenden Ansätzen entwickelt (Mezirow 2012, Bateson 2014, Schilling/Kluge 2009; Macha/Brendler/Römer 2017; Macha/Brendler/Spiegler 2016). Es verbindet die Beratung einzelner Führungskräfte und Mitarbeitender mit einer Beratung in Gruppen und wirkt so auf die gesamte Organisationsstruktur der Unternehmen im Sinne der festgelegten Ziele ein.

Das Besondere des TOL liegt in der durchgängigen Partizipation der Beteiligten, die während der gesamten Beratung mit in die Verantwortung für die Gestaltung der Entwicklungsprozesse genommen werden. Verantwortung wird so als Anreiz zur motivierten Mitwirkung eingesetzt. Die Elemente sind Selbstermächtigung zur Teilhabe im Prozess, Zutrauen zu den Fähigkeiten der Beteiligten, Feedbackschleifen zur Überprüfung der Projektleiter_innen und Monitoring der Arbeit bei der Organisationsentwicklung. Beratung und thematische Weiterbildung im Sinne der Ziele werden dabei verzahnt. In den 
beiden Projekten, von denen hier berichtet wird, wurden kurzfristige und langfristige Erfolge und Effekte sichtbar, die durch projektinterne kostenlose Angebote der Beratung und Weiterbildung der Unternehmen zu dem Change-Management-Prozess erreicht wurden.

Die Transformation der Unternehmen in Richtung der selbstgesteckten Ziele und im Sinne der humanitären Anliegen wird unter der Leitung von Change Agents - in diesem Fall die Projektleiterinnen - durchgeführt. Insofern stützen wir uns auch auf das „Nudge-Prinzip“ von Thaler und Sunstein (2017, 9. Auflage) sowie Bohnet (2017). Es beruht darauf, dass Menschen durch Verhaltensdesign Ziele leichter erreichen, indem sie darin unterstützt bzw. "geschubst" werden, das ethisch Sinnvolle zu tun (Thaler/Sunstein 2017: 15; Bohnet 2017: 21). Das „Nudge-Prinzip“ besteht darin, lernende Umgebungen zu schaffen, in denen Menschen ermutigt werden, etwas Neues auszuprobieren und zu lernen, auch wenn sie einmal scheitern (Bohnet 2017: 22). Dazu werden Rahmenbedingungen hergestellt, welche die Beteiligten motivieren, ihre Ziele zu erreichen. Allerdings wenden wir im Projekt dieses Nudge-Prinzip nur im Rahmen der verantwortlichen Beratung an.

\section{Beratung in Unternehmen}

Generell muss sich Beratung in Unternehmen an deren komplexer Struktur orientieren und die relevanten Statusvertreter einbeziehen. Bei Interventionsprojekten, die vorgegebene Ziele umsetzen, empfehlen sich ein begleitendes Monitoring und eine Evaluation. Steuerungsinstanzen beim Beratungsprozess sind im hier beschriebenen Fall zum einen das Projektteam, bestehend aus den drei Projekteiterinnen, sowie zum anderen vonseiten der Unternehmen das Change Team, bestehend aus relevanten Gruppen wie zum Beispiel der Unternehmensleitung, Personalverantwortlichen, Personalrat und Vertretern der beteiligten Abteilungen sowie der Frauenbeauftragten.

Thema der Interventionen waren die Verbesserung von Gleichstellung und die Akzeptanz von Diversity. ${ }^{1}$ Das Verhaltensdesign umfasste also Beratung zum Thema und Maßnahmen zur konkreten Erfahrung mit Ungleichheit und Vielfalt der Führungskräfte und beteiligten Mitarbeiterinnen im Unternehmen. Vorurteile und Verhaltensmuster, die Ungleichheit produzieren und perpetuieren, wurden theoretisch reflektiert und praktisch in Übungen mit den Teilnehmenden erfahren, es wurden aber auch alternative Verhaltenswei-

1 Zur Begründung der Strategie Gender und Diversity verweise ich auf Macha/Brendler/ Römer 2017 sowie auf Riegraf 2008. 
sen erprobt. Ziel waren die Änderung des Verhaltens auf der Interaktionsebene und die Definition neuer Regeln für diversitygerechtes Verhalten im Unternehmen auf der Systemebene (Macha 2016; Bohnet 2017). Damit einher ging die Vermittlung eines adäquaten Führungskonzepts, dem Modell der „transformativen Führung“ (Macha et al. 2017: 161-164), das in Zukunft die erarbeiteten Regeln zu verstetigen gestattet.

\subsection{Die Struktur der empirischen Interventionsprojekte}

Zur Umsetzung der Ziele wurde eine feste Struktur in den beiden Projekten entwickelt: Es wurden von 2011 bis 2014 zwei empirische Interventionsprojekte durchgeführt (finanziert durch den Europäischen Sozialfonds, die Universität Augsburg und das Berufsbildungszentrum - BBZ, Augsburg) mit begleitender empirischer Evaluation der Weiterbildung und Beratung: die empirischen Forschungsprojekte „Future is Female - FIF“ und „Die Zukunft im Unternehmen gestalten - ZUG“. Dabei arbeiteten insgesamt 40 kleine und mittlere Unternehmen aus Bayern mit. Die Intervention erfolgte entlang der feministischen Ziele der Gender- und Diversity-Strategie (Riegraf 2008; Macha et al. 2014, a,b; 2015; 2016 a,b; 2017).

Es wurde eine Organisationsberatung zum Thema Gender und Diversity durchgeführt. Das organisationale Lernen bei der Organisationsentwicklung bzw. beim Change Management wurde durch professionelle Trainer begleitet (Macha/Brendler/Römer 2017). Ziel der Projekte war, die Umsetzung von Diversity und Gleichstellung in den 40 Unternehmen zu verbessern und das Modell der transformativen Führung zu vermitteln. Letzteres folgt unmittelbar aus der Anwendung des Transformativen Organisationalen Lernens, der Methode der Beratung. Sie wurde aufgrund des Forschungsstandes entwickelt, im Feld angepasst, erprobt und empirisch evaluiert (Macha et al. 2017; Mezirow 2012; Taylor/Cranton 2012; Lawrence/Maws/Dyck/Kleysen 2005; Schilling/Kluge 2009).

Bei der Beratungsarbeit in den Unternehmen wurden keine Einzelziele zur Implementierung der Gender- und Diversity-Strategie vorgegeben, diese wurden vielmehr von den Unternehmen selbst nach Maßgabe ihres Bedarfs bestimmt. Von den Unternehmensleitungen (UL) identifizierte Probleme ihres Unternehmens wurde dann in Ziele operationalisiert. Zum Beispiel formulierte ein großes Verlagshaus folgende Ziele:

- „Mehr Frauen in Führung durch Potenzialentwicklung“,

- „High Potential Teams zur Förderung des Führungskräftenachwuchses unter Frauen“" sowie

- „mediale Aufklärung über Ungleichheitsstrukturen im Unternehmen“. 
Als Vision für die Beratung wurde in diesem Unternehmen als Ziel projektiert: „30\% Frauen in Führungspositionen im Verlag bis 2015“ (vgl. dazu Macha et al. 2017: 133-174; Macha/Brendler/Hitzler/Spiegler 2016).

Folgende einzelne Methoden von Beratung wurden im Verlauf der beiden Projekte zu Gender und Diversity in den 40 Unternehmen angewendet:

- Coaching für Führungskräfte der beteiligten Unternehmen

- Kollegiale Beratung in Gruppen von Unternehmensvertretern

- Jour fixe als moderierte Leitungsteam-Sitzungen in jedem Unternehmen

- Unterstützung der Lernprozesse durch thematische Weiterbildungsworkshops mit Beratungsanteilen

- Selbstlernmethoden, Rollenspiel, Theater der Unterdrückten u. a. (Macha et al. 2017: 79-90)

\subsection{Prozesssteuerung und Controlling während der Beratung}

Die Steuerung der Beratungsprozesse in den Unternehmen geschah durch zwei Instanzen (vgl. Macha et al. 2017: 69-75):

1. Das Projektteam strukturierte den Prozess und stellte die erste Steuerungsinstanz dar. Es bot Beratung und thematische Weiterbildung an und verzahnte beides. Ferner führte das Projektteam die Evaluation durch.

2. Das Change Team fungierte als zweite Steuerungsinstanz vonseiten der Unternehmen. Es bestand aus Mitgliedern relevanter Unternehmensgruppen wie zum Beispiel der Unternehmensleitung, den beteiligten Abteilungsleiter innen, der Frauenbeauftragten, den Personalentwicklern, Mitarbeitenden sowie Mitgliedern des Personalrats. Seine Aufgabe bestand in der Erstellung einer leitenden Vision, von Einzelzielen und in der Durchführung des Monitorings.

Einzelne thematische Arbeitsgruppen zur Erreichung der Unternehmensziele wurden gegründet (zwischen 6 und 14 Gruppen pro Unternehmen). RegelmäBige Jour fixes dienten der Kontrolle der Arbeitsgruppen und ihrer Fortschritte sowie der Kommunikation der Ergebnisse ins Unternehmen hinein sowie an die Kunden.

Das Beratungsziel bestand darin, die Unternehmen diversitygerecht mit partizipativer Führungskultur zu entwickeln. Ein dynamischer und ergebnisoffener Lernprozess der Beratung und der Weiterbildung in 6 Schritten wurde durchgeführt, der bei den Führungskräften und Mitarbeitenden ansetzte und auch die Ebene der Gruppe und der Organisation mit einbezog. Dabei spielten kognitive, affektive, volitive und spirituelle Dimensionen wie Ziele und Visionen, Wissen, individuelle Motivationen, Kompetenzen, 
Macht-Strukturen und Perspektivenwechsel sowie auftretende Barrieren eine Rolle (Macha/Brendler 2014: 333).

\subsection{Das Beratungskonzept der Interventionsprojekte}

Dem Beratungskonzept der Projekte lag die Methode des „Transformativen Organisationalen Lernens" zugrunde. Sie wurde für die Projekte aus Ergebnissen der Forschung weiterentwickelt (Macha et al. 2011, 2014, 2015; Mezirow 2012; Bateson 2013; Schilling/Kluge 2009). Die Definition des Transformativen Organisationalen Lernens - TOL lautet: Transformatives Organisationales Lernen" - TOL - im Unternehmen ist gekennzeichnet durch ein partizipatives, genderkritisches, pädagogisches und an gleichstellungspolitischen und ethischen Zielen orientiertes Beratungs- und Weiterbildungs-Handeln, das Räume für Lernvorgänge der Stakeholder schafft, anregt und unterstützt und dadurch neue Handlungsoptionen und -Freiräume eröffnet (...). Beratung und Weiterbildung werden verzahnt (vgl. Macha et al. 2017, S. 55).

Die Beratung fand jeweils auf drei Ebenen statt: 1. Auf der Subjektebene wurden die jeweils Einzelnen zum partizipative Lernen mit dem Ziel der Ermächtigung für die Beteiligung an dem Entwicklungsprozess beraten. 2 . Auf der Gruppenebene ermöglichte die Unterstützung des Lernens in den Gruppen - zum Beispiel in der „Kollegialen Beratung“ - einen Perspektivenwechsel und die Spiegelung der eigenen Erfahrungen mit dem Thema Gleichstellung. 3. Auf der organisationalen Ebene der Unternehmen bestand die Beratung in der Begleitung des organisationalen Lernens der Unternehmen - des systemischen Lernens.

Transformatives Organisationales Lernen setzt den Anspruch um, die Beratungsthemen nicht nur theoretisch zu vermitteln und zu untermauern, sondern auch im praktischen Handeln erfahrbar zu machen, wie zu Beispiel Ungleichheit in Interaktionen hervorgebracht wird, wie Vorurteile wirken, aber auch, wie alternative Handlungsmöglichkeiten entwickelt und sogar als neue Regeln im Unternehmen verbindlich gemacht werden können. Die Teilnehmerinnen und Teilnehmer übernahmen Verantwortung in den Beratungs- und Weiterbildungsgruppen, indem sie unter Anleitung selbst alternative Regeln für diversitygerechtes Handeln entwickelten und Programme zu Diversity und Gleichstellung für ihre Unternehmen erarbeiteten. Um diesen hohen Anspruch an Beratung und Lernen umzusetzen, bestand der Beratungsprozess jeweils aus identischen Elementen bzw. Schritten, die aufeinander aufbauten (vgl. Macha et al. 2017: 81-87):

1. Im ersten Schritt wird „Awareness" angestrebt, das ist die Sensibilisierung für Ungleichheitsprozesse in der alltäglichen Interaktion von Mit- 
arbeitenden und Führungskräften zu der Frage: „Wie stellen wir aktiv Ungleichheit her?" Die Prozesse des doing gender und doing difference werden dabei zunächst anhand theoretischer Modelle reflektiert und im konkreten Tun, zum Beispiel in Rollenspielen, erfahrbar.

2. Im zweiten Schritt wird „Deconstruction“ zum Thema, das ist der Perspektivenwechsel vom doing gender und doing difference zum diversitygerechten Handeln unter der Fragestellung: „Wie können wir unser Handeln ändern und gemeinsam diversitygerechte Alternativen finden?"

3. Der dritte Schritt besteht im „Reframing“: Neue Regeln für diversitygerechtes Handeln werden in den Gruppen formuliert und im Handeln erprobt. Hier werden unterschiedliche Methoden angewandt, die Empathie fördern und ethische Werte unterstützen, das Nudge-Prinzip wird wirksam, indem das ethisch als richtig Erkannte auch konkret umsetzbar wird.

4. „Negotiation“ als dritter Schritt bedeutet, dass das Aushandeln von diversitygerechten Regeln für das ganze Unternehmen beginnt. Die Regeln sollen in offizielle Programme und Leitfäden der Unternehmen verbindlich aufgenommen werden. Sie werden deshalb an die Unternehmensleitung zur Genehmigung kommuniziert.

5. Im fünften Schritt, der „Implementation“, werden diese verbindlichen Regeln mit der Unternehmensleitung verhandelt und als Artefakte zu neuen Regelungen mit der Unternehmensleitung verbindlich vereinbart und veröffentlicht, zum Beispiel diversitygerechte Bewerbungsleitfäden, diversitygerechte Unternehmensleitbilder und Home Pages, diversitygerechte Zielvereinbarungsleitfäden usw.

6. Der sechste Schritt, das ,,double loop learning“ nach Argyris und Schön oder die dritte Lernebene nach Bateson auf der individuellen, gruppenbezogenen und organisationalen Ebene, besteht in der Übertragung der neuen Regeln und Bestimmungen auf alle Ebenen - die Organisationsstruktur verändert sich damit. Zum Beispiel wird nun in Konferenzen interveniert, wenn wieder diskriminierende Praktiken angewendet werden.

Diese Prozesse wurden bei allen 40 teilnehmenden Unternehmen absolviert und in unterschiedlichem Tempo auch implementiert. Die Prozesse und Ergebnisse wurden evaluiert.

\section{Die chronologische Struktur des Beratungsprozesses}

Beratungshandeln in den Unternehmen geschah als Prozess der Organisationsentwicklung, er war zielerichtet und erfolgsorientiert. Einzelne typische 
Stufen oder Phasen können dabei identifiziert werden (Macha et al. 2017a, b; unter Bezug auf Kotter 1996; Richman 2015):

1. Ein Problem wurde sichtbar: in diesem Fall strukturelle Ungleichheit in den Unternehmen. Als Grundlage der Beratung wurde eine empirische Erhebung zum Status quo der Diversity-Maßnahmen in den Unternehmen vor Projektbeginn durchgeführt. Der Change-Prozess begann mit der Datenanalyse.

2. Externe Trainerinnen und Trainer aus dem Projektteam wurden beauftragt, die Organisationsentwicklung zur Behebung des Problems zu steuern. Die Durchführung der Intervention mit Beratung und Weiterbildung wurde in Auftaktkonferenzen gestartet und aufgrund der Bedarfe und Ziele der einzelnen Unternehmen gestaltet.

3. Eine Vision für das Entwicklungsziel wurde von den Unternehmensleitungen und Führungskräften formuliert: In diesem Fall bestand die Vision darin, mehr Gleichstellung zu erreichen, um mehr Frauen zu gewinnen, sowie Vielfalt zu akzeptieren, um so mehr Fachkräfte zu rekrutieren und an das Unternehmen zu binden.

4. Das Ziel wurde im Unternehmen kommuniziert und diskutiert (in Kick-off-Konferenzen).

5. Das Change Team als Steuerungsgruppe wurde gegründet (aus relevanten Gruppen der Unternehmen).

6. Thematische Arbeitsgruppen aus verschiedenen Abteilungen wurden gegründet und Monitoring als Steuerungselement eingerichtet: Regelmäßige Jour-fixe-Termine mit dem Projektteam und dem Change Team zu Berichten der Arbeitsgruppen und ggf. Korrekturen wurden vereinbart.

7. Die Evaluation der Maßnahmen der Intervention wurde begleitend durchgeführt; Zwischenergebnisse wurden den Beteiligten präsentiert - zum Beispiel in der Mid Time Konferenz - und kritische Stellungnahmen in Feedbackschleifen angefordert.

8. Ergebnisberichte aller Arbeitsgruppen wurden eingereicht und veröffentlicht und in den Abschlusskonferenzen diskutiert.

In der folgenden Graphik wird der Beratungsprozess zusammengefasst dargestellt: 


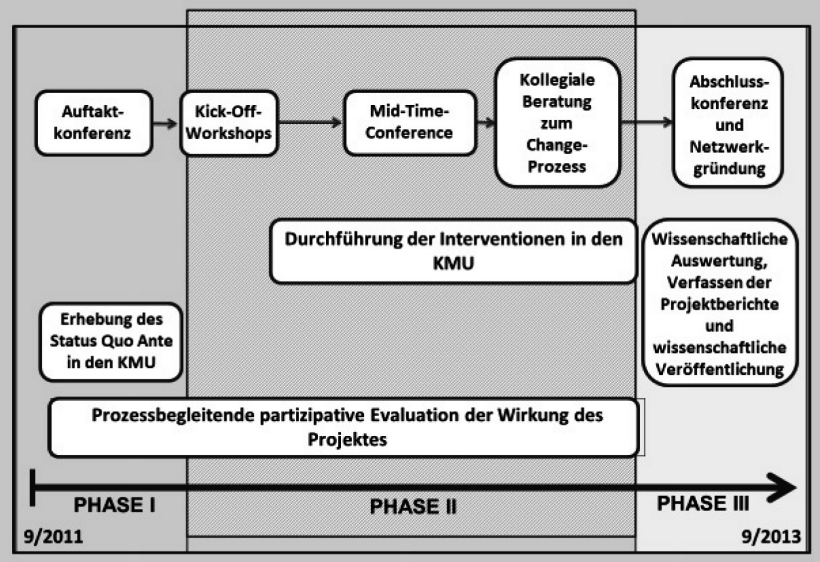

Abb. 1: Ablauf des Beratungsprozesses, Quelle: eigene Darstellung

\section{Partizipative Evaluation der Beratungsprozesse}

Entsprechend dem Verlauf der Beratungsprozesse wurden alle Schritte evaluiert. Dafür wurde nach dem Modell der „Partizipativen Evaluation“ ein trianguliertes Design entworfen (Suarez-Herreira et al. 2009). Das hatte den Vorteil, dass die Beteiligten während der Beratung laufend über Zwischenergebnisse informiert waren und gebeten wurden, zu Methoden der Beratung und Weiterbildung kritisch Stellung zu beziehen.

\subsection{Das Design der Partizipativen Evaluation}

Die Ziele der Unternehmen wurden während der Beratung erfasst, durch Angebote von Beratung und Weiterbildungs-Workshops unterstützt und der Prozess dokumentiert und evaluiert.

Folgende Erhebungsinstrumente wurden dabei genutzt:

1. Online-Fragebogen zur Erhebung des Status quo ante von Gleichstellung und Diversity in den Unternehmen vor Projektbeginn sowie zu den Effekten nach Projektende. 
2. Zielraster zur Dokumentation der Ziele, die in den Unternehmen formuliert und zusammengefasst dokumentiert wurden. Die neu entwickelte Methode der Zielraster besteht in zusammengefassten und inhaltlich strukturierten Zielen aller Firmen in einer Übersichtsgraphik. Sie diente dazu, die Beratungs- und Weiterbildungsangebote zu strukturieren und zu dokumentieren.

3. Feedback-Fragebögen zur Erhebung der Lerneffekte bei Teilnehmenden.

4. Prozessraster zu Verlauf und Effekt der Transformation in den Unternehmen: Die Teilnahme an Beratungs- und Weiterbildungsangeboten wurde empirisch erfasst.

5. Interviews mit relevanten Unternehmensvertretern und -vertreterinnen zum Effekt der Beratung wurden durchgeführt.

6. In Artefaktanalysen wurden die messbaren dokumentierten Effekte in den Unternehmen ausgewertet.

\subsection{Ergebnisse der Evaluation}

Wie oben dargestellt, wurden sowohl Einzelpersonen in der Beratung aktiviert wie auch Gruppen und die Organisation. Daher wurden auch diese drei Ebenen evaluiert.

\section{Ergebnisse auf der Subjekt- und Gruppenebene}

Die Lerneffekte wurden von den Teilnehmenden als hoch bis sehr hoch eingestuft; Lernmethoden wie zum Beispiel die Kollegiale Beratung, das Rollenspiel oder die Selbstlernprogramme mit praktischen Übungen gemäß ihren Aussagen den Prozess der gleichstellungsorientierten Lernkultur. Die Methode des TOL bewirkte bei 79\% der Teilnehmenden und Gruppen Lerneffekte in Bezug auf Wissen und gleichstellungssensible Handlungsoptionen in Interaktionen (vgl. Macha et al. 2017).

Die Akzeptanz der Lernmethoden und Inhalte betrug 80\%, die wissenschaftlichen Informationen waren manchmal jedoch zu abstrakt. Die oberen Führungskräfte und insbesondere die Unternehmensleitungen trugen den Wandel in Bezug auf die Ziele der Gleichstellung: Sie waren die Schlüsselpersonen, die den Erfolg des Beratungsprozesses maßgeblich positiv beeinflussten, indem sie mit Autorität und Überzeugungskraft vorangingen. Sie ließen sich von der Argumentation überzeugen und lernten am meisten und am schnellsten über die Inhalte bis hin zu alternativen Regeln und Verhaltensweisen. 


\section{Ergebnisse auf der organisationalen Ebene der Unternehmen}

Die Lernkultur der Unternehmen wurde hinsichtlich Partizipation und Gleichstellung nachhaltig verändert. Artefakte belegen die nachhaltige Verankerung, zum Beispiel in Bezug auf diversitysensible Unternehmensleitbilder und Diversity-Programme der Unternehmen. 80\% der Unternehmen gaben an, ihre Ziele im Rahmen des Projekts erreicht zu haben. Ein neues partizipatives Führungskonzept folgte der neuen Lernkultur (vgl. Macha et al. 2017).

\section{Ergebnisse zur Gesamtwirkung der Projekte in den Unternehmen}

Während zum Abschluss des Projekts „Future is Female - FIF“ Interviews mit relevanten Unternehmensvertreteinnen und -vertretern geführt wurden, um die Effekte auf die Unternehmensstruktur zu erfassen, wurde im Projekt „Die Zukunft im Unternehmen gestalten mit Gender und Diversity - ZUG“ nach Projektende ein Online-Fragebogen an alle beteiligten Unternehmen verschickt, der die Gesamtwirkung in den einzelnen Unternehmen erhob. Die Unternehmen gaben selbst an, sich nachhaltig gewandelt zu haben. Zum Teil wurde der Prozess der verbesserten Gleichstellung nach Projektende noch weitergeführt. Artefakte belegten die nachhaltige Veränderung der Unternehmenskultur hinsichtlich Gender und Diversity. Das Projekt vergrößerte insgesamt die Akzeptanz von Vielfalt: Das zeigen Beispiele wie das „Fest der Vielfalt" bei einem Unternehmen, dessen Mitarbeitende und Führungskräfte aus insgesamt 38 Nationen stammen. Während vorher dieser Tatsache keine Beachtung geschenkt wurde, kam damit erstmalig die Wertschätzung der Vielfalt im Unternehmen zum Ausdruck.

Andere Effekte waren, dass mehr Frauen in Führungspositionen aufgestiegen sind, dass mehr Führung in Teilzeit für Frauen und Männer ermöglicht wurde, dass $80 \%$ der Ziele in den Unternehmen erreicht wurden und dass die Zufriedenheit mit dem Projektverlauf und den Trainerinnen 91\% betrug (vgl. Macha,Brendler, Hitzler, Spiegler 2017).

\section{Fazit}

Die Ergebnisse des Online-Fragebogens zum Abschluss des Projekts ZUG zeigen eine nachhaltige Transformation hinsichtlich der Verbesserung von Gleichstellung. So gaben die Unternehmen selbst an, sich nachhaltig gewandelt zu haben. Die Artefaktanalyse belegte ebenfalls durch interne und externe Dokumente die nachhaltige Veränderung der Unternehmenskultur. Insgesamt 
sind mehr Frauen in Führungspositionen aufgestiegen, wenngleich bei den kleinen Fallzahlen keine statistisch relevanten Größen erreicht wurden. Es wurde mehr Führung in Teilzeit ermöglicht, obwohl dies vor Projektbeginn bei allen teilnehmenden Führungskräften kategorisch ausgeschlossen wurde.

Es gab jedoch auch erhebliche Widerstände und Barrieren. Insbesondere die mittlere Führungsebene sah in den Anstrengungen zur Gleichstellung eine Bedrohung ihrer Aufstiegschancen. Diese Widerstände wurden als Anregung betrachtet, durch bessere Kommunikation auch diese Führungskräfte stärker in den Change-Prozess einzubinden. Auch wenn nicht jeder Einzelne überzeugt werden konnte, wurde doch die Atmosphäre entspannt und die gegensätzlichen Argumente als eine Bandbreite von Positionen akzeptiert.

\section{Literatur}

Bateson, Gregory (2014): Ökologie des Geistes. Anthropologische, psychologische, biologische und epistemologische Perspektiven. Frankfurt a. M.: Suhrkamp.

Bohnet, Iris (2017): What Works. Wie Verhaltensdesign die Gleichstellung revolutionieren kann. München: C. H. Beck.

Göhlich, Michael/Weber, Susanne Maria/Seitter, Wolfgang/Feld, Timm C. (Hg.) (2010): Organisation und Beratung. Beiträge der AG Organisationspädagogik. Wiesbaden: VS-Verlag.

König, Eckart/Vollmer, Gerda (Hg.) (2014): Handbuch systemische Organisationsberatung (2. Aufl.). Weinheim: Beltz.

Kotter, John P. (1995): Leading Change. Why Transformation Efforts Fail. In: Harvard Business Review, March/April, pp. 1-8.

Lawrence, T. B./ Mauws, M. K./ Dyck, B./Kleysen, R. F. (2005): The Politics of Organizational Learning: Integrating Power into the 4 I Framework. The Academy of Management Review. 30, 1, pp. 180-191.

Macha, Hildegard/Hitzler, Stefanie (2018): Transformative Organisationsforschung mit Gender und Diversity in Unternehmen. In: Ringeisen, Thomas: Handbuch Diversity-Kompetenz. New York: Springer (i. V.).

Macha, Hildegard/Brendler, Hildrun/Römer, Catarina (2017): Gender und Diversity im Unternehmen. Transformatives Organisationales Lernen als Strategie. Opladen: Budrich UniPress.

Macha, Hildegard/Brendler, Hildrun/Hitzler, Stefanie/Spiegler, Elena (2017): Verändern und Forschen - Interventionsprozesse mit Gender und Diversity in Unternehmen steuern und messen. In: Schemme, D./Novak, H.: Gestaltungsorientierte Forschung - Basis für soziale Innovationen. Bonn: Bundesinstitut für Berufsbildung BIBB, S. 569-585.

Macha, Hildegard (2014a): Karrieren von Frauen in Unternehmen - mit Gender und Diversity Gleichstellungschancen erhöhen. In: Zeitschrift Gender, Opladen: Budrich, S. 43-60. 
Macha, Hildegard (2014b): Transformatives Organisationales Lernen in Unternehmen. In: Organisation und das Neue. Beiträge der Kommission Organisationspädagogik, Bd. 15. Weber, S. M./Göhlich, M./Schröer, A./Schwarz, J. (Hg.) Wiesbaden: Springer VS, S. 331-340.

Macha, Hildegard/Brendler, Hildrun (2014): Gleichstellung und Diversity im quartären Bildungssektor - Transformatives Organisationales Lernen in Unternehmen. In: Freiburger Zeitschrift für Geschlechterstudien 20/1: Freiburg, S. 1-16.

Macha, Hildegard/ Lödermann, Anne-Marie/Bauhofer, Wolfgang (2012): Kollegiale Beratung in der Schule: Theoretische, empirische und didaktische Impulse für die Lehrerfortbildung. Weinheim/München: Juventa Verlag.

Mezirow, John (2012): Learning to Think like an Adult. In: Taylor, E./Cranton, P. (Hg.): The Handbook of Transformative Learning: Theory, Research and Practice. San Francisco: Jossey-Bass, pp. 73-96.

Mutzek, Wolfgang (2014): Kooperative Beratung: Grundlagen, Methoden, Training, Effektivität. Weinheim: Beltz.

Richman, Robert (2015): Everything you know about change management is wrong.

http://www.robertrichman.com/everything-you-know-about-change-management-iswrong/ (letzter Aufruf 3.7.2018)

Riegraf, Birgit (2008): Geschlecht und Differenz in Organisationen: Von Gleichstellungspolitik und erfolgreichem Organisationslernen. In: WSI-Mitteilungen, 7, S. $400-405$.

Schilling, J./Kluge, A. (2009): Barriers to Organizational Learning. An Integration of Theory and Research. In: International Journal of Management Reviews, 11, 3, pp. 337-360.

Suarez-Hereira, José Carlos/Springett, Jane/Kagan, Carolyn (2009): Critical Connections between Participatory Evaluation, Organizational Learning and Intentional Change in Pluralistic Organizations. In: Evaluation, 15, Los Angeles: Sage Publications, S. 321-342.

Struthmann, Sandra (2013): Gender \& Diversity im Unternehmen. Wiesbaden: VS Verlag für Sozialwissenschaften.

Taylor, E./Cranton, P. (Hg.): The Handbook of Transformative Learning: Theory, Research and Practice. San Francisco: Jossey-Bass.

Thaler, Richard H./Sunstein, Cass R. (2017): Nudge. Wie man kluge Entscheidungen anstößt. (9. Aufl.) Berlin: Ullstein TB.

Tomaschek, Nino (2006): Systemische Organisationsentwicklung und Beratung bei Veränderungsprozessen. Heidelberg: Karl Auer. 


\section{Beratung im Kontext Hochschule Eigen- und Steuerungslogiken zwischen Emanzipation und Entmündigung?}

1965 konstatiert Klaus Mollenhauer, Vertreter einer kritischen Erziehungswissenschaft, dass Beratung die Möglichkeiten zur Förderung der Mündigkeit im Erziehungsprozess der Ratsuchenden biete:

„Die entscheidende Funktion der Beratung endlich liegt darin, dass sie kritische Aufklärung sein kann. Das Gespräch schafft Distanz, es ermöglicht, das Besprochene objektivierend zu betrachten, es ermöglicht ein rationales Verhalten zu sich selbst und $\mathrm{zu}$ den Bedingungen der eigenen Existenz. In der Beratung werden nicht nur Antworten gegeben, sondern zugleich neue Fragen formuliert; die rationale Erhellung eines Problems wird so weit wie möglich versucht, um eine Entscheidung vorzubereiten, die von Vorurteilen und Verfestigungen frei nach dem Abwägen der vernünftig entscheidbaren Fragen getroffen werden kann." (Mollenhauer 1965, S. 32)

Im Kontrast hierzu behauptet indes der österreichische Politologe und Pädagoge Erich Ribolits 42 Jahre später, Beratung sei ein Instrument der (scheinbaren) Selbstbestimmung; den Beraterinnen und Beratern käme darin „als postmoderne Agentinnen und Agenten die Rolle der Normalisierung zu, die ihren Kund/innen auf sanfte - non-direktive - Art das Gift der Entmündigung einträufeln“" (vgl. Ribolits 2007, S. 8).

Damit eröffnet sich ein Spannungsfeld, das Dimensionen von Mündigkeit und Entmündigung, von Emanzipation und Anpassung, von Fremd- und Selbstbestimmung aufruft. Um sich dieser Vielschichtigkeit anzunähern, werden Grundgedanken der Gouvernementalität von Michel Foucault skizziert (1.). Anhand eines konkreten Praxisbeispiels aus dem Kontext der Hochschulberatung werden sowohl potentielle Folgen von Fremd- und Selbststeuerungsprozessen thematisiert (2.) als auch handlungsorientierte Alternativen diskutiert (3.). Dies geschieht im Rückgriff auf ein pädagogisch-rekonstruktives Beratungsverständnis (4.) und einen kritisch-emanzipativ verorteten Beratungsansatz (5.). 


\section{Grundgedanken der Gouvernementalität}

Ribolits' Ausgangspunkte sind die Gesellschaftsanalysen von Foucault und Deleuze (1993), der Wandel von der Disziplinar- zur Kontrollgesellschaft. Damit sind Mechanismen gemeint, die auf Internalisierung und Selbstdisziplinierung setzen, befeuert durch technische, mediale und auch digitale Kontrollmechanismen. ${ }^{1}$ Diese Normalität, die sich als naturgegeben darstellt, ist von kapitalistischen Bedürfnissen auf neoliberale Weise geformt und führt damit beim Subjekt zur Verinnerlichung der ökonomischen Marktlogik (vgl. Ribolits 2010, S. 209ff; 2007, S. 23f). An Foucault anschließend betont die amerikanische Politikwissenschaftlerin Wendy Brown, dass „das Selbst als Unternehmen mit eigenen Steuerungs- und Managementtechniken agiert" (vgl. Brown 2015, S. 32ff.), oder als - so Bröckling - Unternehmer seiner selbst (vgl. Bröckling 2007). Somit fungiert das Individuum gleichermaßen als Produzent und Konsument neoliberaler Marktlogiken.

\section{Folgen von Fremd- und Selbststeuerungsprozessen für die Bildungsberatung}

Für Stefanie Duttweiler ist die Selbstbestimmung das zentrale Moment in der Beratung, welche mit der Frage der Selbstkonstituierung des Subjekts in Verbindung steht. Diese Selbstkonstituierung ist Teil des Prozesses der Subjektivierung, die sich aus der Verschränkung von Macht- und Wissensformationen mit den Tätigkeiten des Selbstbezugs bestimmt (vgl. dies. 2007, S. 262). Beratung ist dabei ein Knotenpunkt im Netz aus Selbst- und Fremdführungsformen, erstens auf der Ebene der Beratung als institutioneller Einrichtung, zweitens als Interaktions- und Kommunikationsform, aber drittens auch in der Rolle der Berater/innen. In den drei Ebenen spiegeln sich die Interdependenzen aus Fremd- und Selbstführungen; Führende werden zu Geführten und vice versa. Dies gilt gerade für die professionelle Beziehung Berater/ in-Ratsuchende/r. Damit wird die Frage der Selbstbestimmung erneut zum Ausgangspunkt, denn „Selbstbestimmung ist Instrument und Effekt von Be-

1 Dies zeigt sich beispielsweise im sog. Lifelogging und Self-tracking. Die eigene Selbstvermessung, die i. d. R. der Leistungssteigerung und/oder der Selbstoptimierung dient, produziert individuelle Datenmengen, die - digital vermittelt und über soziale Netzwerke geteilt - nicht nur der Idee des gläsernen Menschen näher kommen, sondern auch eine zunehmende Objektivierung des Menschen befördern. Vgl. zum Phänomen des lifelogging und self-tracking exemplarisch Selke 2016, 2014, Mämecke 2016, Duttweiler et al. 2016. 
ratung, ihr Fluchtpunkt und konstitutives, strukturierendes Formprinzip - es wird das vorausgesetzt, was (erst) in der Beratung erzeugt werden soll“" (Duttweiler 2007, S. 272, H. i. O.). Und so muss jene (scheinbare) Selbstbestimmung darauf hinterfragt werden, welche Fremdführung mit ihr einhergeht, wenn Beratende zwar methodisch durch die Gestaltung der Beratungskommunikation lenkend agieren, jedoch nicht mehr diejenigen sind, die - so Ribolits - durch ihr Fach- und Erfahrungswissen Richtungen aufzeigen oder Wertungen vornehmen sollen (vgl. Weber 2018, S. 77). Beratung, gerade auch im Kontext von Bildungsberatung, dient dann dazu, die Ökonomie und die ständige Selbstvermarktung als naturgegeben zu akzeptieren, zu verinnerlichen und sich den verinnerlichten Vorgaben gemäß zu verhalten. Dazu gehört insbesondere auch, Selbstbestimmung als zentrales Moment von Beratung zu postulieren:

„Die Förderung der Selbstbestimmung ist gebunden an die Forderung, einen bestimmten Gebrauch von ihr zu machen - die Verantwortung für gesellschaftliche Risiken in ein Problem der Selbstsorge zu transformieren und in Eigenregie zu managen." (Duttweiler 2004, S. 28).

Ungewissheiten und Kontingenzen sollen nach Bröckling unter neoliberalen Vorgaben als Freiheitsspielraum interpretiert werden, den es ressourcenorientiert zu entdecken und zu erschließen gilt (Bröckling 2000, S. 133, in Pongratz 2005, S. 32).

An das Theorem der Individualisierung anschließend, betont Rauschenbach (1994) dass privat-lebensweltliche Formen der Unterstützung eher abnehmen und soziale und pädagogische Dienste diese ergänzen, wenn nicht sogar ersetzen (Steiner 2007, S. 35). Er stellt die Verschiebung der informellen Solidarität hin zur organisierten, formellen Solidarität fest, was mit einer zunehmenden gesellschaftlichen Entsolidarisierung einhergeht - besonders von Menschen, die hilfs- und unterstützungsbedürftig sind.

Insbesondere in Beratungsfeldern, in denen es um „,regulative Beratung“ geht (vgl. exemplarisch Käpplinger \& Klein 2013), wirkt sich das „doppelte Mandat", der Spagat zwischen Hilfe und Kontrolle, aus. Bereits 1973 konstatierten Böhnisch und Lösch ein ,zentrales Strukturmerkmal“ der Dienstleistungsfunktion des Sozialarbeiters: In dieser ist der Sozialarbeiter angehalten, „ein stets gefährdetes Gleichgewicht zwischen den Rechtsansprüchen, Bedürfnissen und Interessen des Klienten einerseits und den jeweils verfolgten sozialen Kontrollinteressen seitens öffentlicher Steuerungsagenturen andererseits aufrechtzuerhalten“ (Böhnisch \& Lösch 1973, S. 27). Dies wirkt sich unter Umständen auch problematisch auf das konkrete Interaktionsgeschehen in der Beratung aus (vgl. Maier-Gutheit 2015, S. 99). Ausgehend von einem pädagogischen, humanistischen und ressourcenorientierten Beratungsverständnis liegt die Rolle der oder des Beratenden darin, flexibel und individuell auf die Bedürfnisse und die Person der oder des Ratsuchenden einzugehen 
und ihn oder sie im eigenen Lösungsweg zu begleiten. Dies soll - den beraterischen Grundwerten der Emanzipation und Mündigkeit folgend - nicht aktiv eingreifend, vorgebend oder regulativ geschehen. Ribolits hinterfragt diese Prämissen dann, wenn eine als Hilfe zur Selbsthilfe verstandene Beratung eine reine Prozessbegleitung bleibt:

„Indem sich Berater/innen in dieser Form weigern, als Autorität - als jemand, der hinsichtlich der Problemstellung mehr weiß - zu agieren, können sie weder eine Gegenautorität zu den gängigen gesellschaftlichen Erwartungen abgeben oder zu deren Hinterfragen anregen, noch können sie eine Instanz darstellen, an der Klient/innen sich ,messen` und damit ein Hinterfragen der gesellschaftlichen Normalität üben können." (Ribolits 2010, S. 216)

So wird ihnen die Möglichkeit genommen, gewissermaßen in dieser Rahmung als „Autorität“ zu agieren - nämlich als Person, die bezüglich der Problemstellung aufgrund ihres Fach- und Erfahrungswissens in der Regel mehr weiß. In Folge können Beratende - erstens - nicht als Gegenautorität zu gängigen, populären gesellschaftlichen Annahmen und Erwartungen fungieren. Sie können aber zweitens auch nicht den oder die Ratsuchende - etwa durch ihre eigene Position - zu Widerspruch anregen oder in ihren Annahmen irritieren. Drittens stellen sie keine „Instanz“ mehr dar, an der sich die Klientinnen und Klienten „reiben“ oder orientieren können. Damit verbleiben die Ratsuchenden häufig bei den ihnen bekannten Maßstäben, die jedoch den Status quo abbilden und selten Spielraum für innovative Ideen oder widersprüchliche Gedanken lassen.

Hinzu kommt - auf interaktionaler und kommunikativer Ebene in der Beratung - dass die Gestaltung der Beratungsbeziehung im Vordergrund steht. Sie baut auf emotionalen Grundpfeilern wie Empathie, Vertrauen, Wertschätzung auf - menschliche Grundbedürfnisse, die in der Postmoderne mit ihrer Beziehungslosigkeit und ihren brüchigen Bindungen (vgl. Nierobisch 2017) häufig unbefriedigt sind. Umso mehr greift da Beratung auch auf einer (unter Umständen unbewussten) emotionalen Ebene und stillt Bedürfnisse, die originär in den Kontext von Freundschaften, Beziehungen oder Familie gehören. Damit wird sie nicht nur kognitiv, sondern vor allem auch emotional zur selbstverständlichen Lebensbegleiterin, die - wenn nicht herrschaftskritisch reflektiert - ihre neoliberalen Zielsetzungen und Normvorstellungen in den Einzelnen und die Einzelne implantiert (Ribolits 2010, S. 216f).

\section{Hochschulberatung: handlungsorientierte Alternativen}

Das Feld der Hochschulberatung kennzeichnet eine große Heterogenität der Angebote. Diese reichen von Formaten individueller Beratung wie Karriere- 
coaching, Lern- und Schreibberatung, Studien(-fach-)beratung, psychosozialer Beratung, Gruppenangeboten wie Mentoring und Supervision bis hin zu organisationalen Beratungsformen (vgl. Nierobisch \& Schüßler 2018). Das Projekt „Lehren, Organisieren, Beraten (LOB): Gelingensbedingungen von Bologna" wird an der Johannes Gutenberg-Universität Mainz vom Bundesministerium für Bildung und Forschung im Rahmen des Qualitätspakts Lehre seit 2012 gefördert. Es besteht aus drei Teilprojekten, die sich der Institutionalisierung und Professionalisierung der Studienfachberatung (Teilprojekt 1), dem Ausbau und der Professionalisierung der Studienbüros (Teilprojekt 2) sowie der Förderung der Lehrkompetenz (Teilprojekt 3) widmen. Dazu gehört auch die Schulung der Studien(fach)berater/innen und aller in der Beratung Tätigen in Grundlagen professioneller Beratung, was durch das Zentrum für wissenschaftliche Weiterbildung (ZWW) der Mainzer Universität organisiert, wissenschaftlich begleitet und ausgerichtet wird. Die Qualifizierung selbst geschieht in einer zweimoduligen Basisqualifizierung (jeweils drei volle Tage), zwei Reflexionstreffen (Supervision) sowie weiterführenden Fachmodulen. ${ }^{2}$ Bis Ende 2016 wurden 126 Teilnehmende weiterqualifiziert (vgl. Weymann 2018, S. 261).

Neben den Basismodulen werden unterschiedliche Fortbildungsmodule angeboten, die den Bedürfnissen der Teilnehmenden gemäß kontinuierlich ergänzt werden:
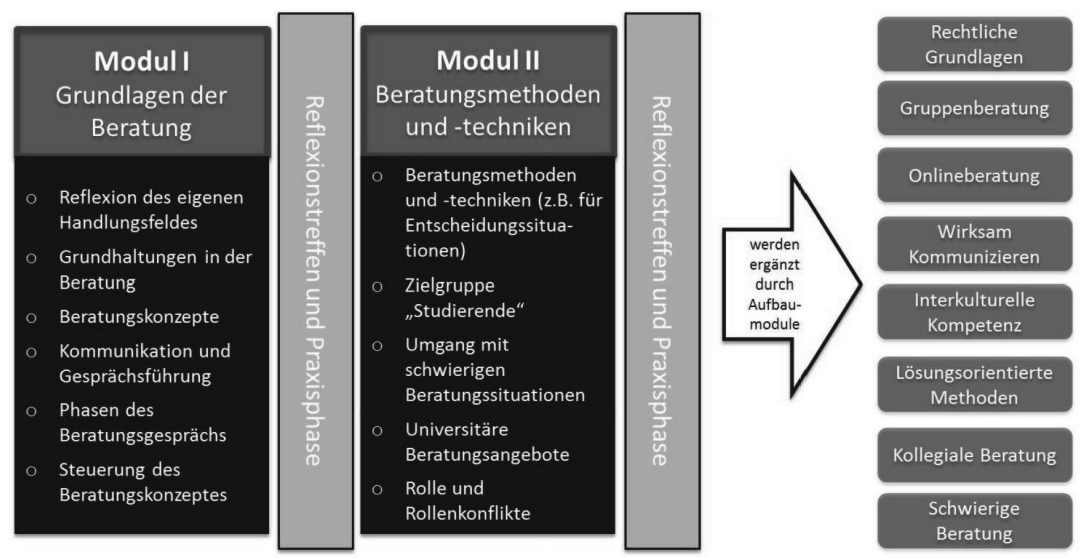

Abb. 1: Aufbau der Qualifizierung (Quelle: Weymann 2018, S. 263) 
Anhand von Tätigkeitsanalysen der Teilnehmenden zeigt sich nicht nur das hohe Maß an Beratung, das formell und informell den Arbeitsalltag der Teilnehmer/innen prägt, sondern es werden auch individuelle und strukturelle Widersprüchlichkeiten zwischen Beratungsverständnis, Beratungsauftrag und methodischem Vorgehen thematisiert. Umso mehr betonen die Teilnehmenden nicht nur die Relevanz des Austauschs mit den Kolleginnen und Kollegen, sondern auch die Notwendigkeit, ihr beraterisches Handeln individuell und gemeinsam zu reflektieren. Dazu gehört ebenso die gemeinsame Auseinandersetzung mit den sozialen und hochschulpolitischen Bedingungen, die Einbettung des Themenfeldes Beratung in aktuelle gesellschaftliche Entwicklungen und das immer wieder Thematisieren einer beraterischen Grundhaltung, die sich im Rückbezug auf ein humanistisch geprägtes Beratungsverständnis als Gegenpart zu aktuellen ökonomisch geprägten Erwartungen offenbaren kann.

\section{Rekonstruktive pädagogische Beratung}

\section{Rekonstruktive Beratungslogiken nehmen}

„,ihren Ausgangspunkt in den inhaltlich-gegenständlichen Problemlagen und Handlungsproblematiken der Ratsuchenden, d. h. in deren Perspektiven, Deutungen und Interpretationen zu der problematisch gewordenen Situation" (Ludwig 2014, S. 560ff)"

Basis ist die Annahme eines an sich widersprüchlichen Verhältnisses von Individuum und Gesellschaft. Das Individuum erfährt in gesellschaftlich vermittelten Bedeutungskonstellationen die Welt in Handlungsmöglichkeiten, die zum Schlüssel der jeweiligen subjektiven Handlungsbegründungen werden. Jene Bedeutungskonstellationen sind jedoch nicht determinierend für das handelnde Subjekt, sondern können selbst Ziel der Reflexion in Welt- und Selbstbeziehung sein:

„Die Handlungsproblematiken bzw. Krisen werden nicht nur als individuelles Problem verstanden, sondern in ihrem Verhältnis zu denjenigen gesellschaftlichen Strukturen rekonstruiert, die das individuelle Handeln rahmen, es ermöglichen und begrenzen" (Ludwig 2015, S. 300).

Diese Lebenssituation muss in „Zusammenhang mit historisch-konkreten Formen gesellschaftlicher Produktion und Reproduktion“ (ebd., S. 306) gesehen werden. Die widersprüchliche Verfasstheit der kapitalistischen Gesell-

3 Zum subjektwissenschaftlich begründeten, rekonstruktiven Beratungsverständnis vgl. exemplarisch auch Ludwig 2014, Ludwig 2012, Dewe \& Schwarz 2011. 
schaft, die sich für das Subjekt in unterschiedlichen Bedeutungsstrukturen ausdrückt, gilt es in der Rekonstruktion der Handlungsproblematik mit aufzudecken (vgl. Weber 2018, S. 86).

\section{Ansätze einer kritisch-emanzipativ verorteten Beratung}

Basis einer kritisch-emanzipativ verorteten Beratung, wie sie nachfolgend verstanden werden soll, bildet ein kritisches Bildungsverständnis, das nicht nur die Widersprüchlichkeit von Individualisierung und Vergesellschaftung in den Blick nimmt, sondern Bildung an sich in ihrer Interdependenz begreift:

„Gegenstand einer kritischen Theorie der Bildung ist die emanzipative Subjektwerdung des Menschen im widerspruchsvollen Beziehungsgeflecht von Individualgenese und gesellschaftlichem Prozess. Sie geht von einer grundlegend unmündigen Gesellschaft aus, die ihre Entwicklungsperspektiven nahezu ausschließlich an ökonomischem Fortschritt und wirtschaftlichem Gewinn anlegt" (Bernhard 2001, S. 20).

Und gleichzeitig ist Bildung die dichotome Kategorie, in welcher die Befreiung aus Abhängigkeitsverhältnissen und zugleich das Instrument zur Anpassung an gesellschaftliche Produktionsverhältnisse stecken (vgl. Drees 2017). Unter Berücksichtigung eines kritischen Bildungsbegriffs beinhaltet dies für die Beratung auch Aufklärung und Auseinandersetzung über Macht- und Abhängigkeitsverhältnisse, die in gesellschaftlichen Sozialisations- und Lebensbedingungen beinhaltet sind.

Erste Aspekte einer sich selbst als kritisch-emanzipativ verortenden Beratung ${ }^{4}$ nehmen nicht nur das Interaktions- und Kommunikationsgeschehen der Beratung in den Blick, sondern verfügen auch über eine (selbst-)kritische beraterische Grundhaltung. Diese umfasst u. a. Fragen der Solidarität, der gesellschaftlichen Einbettung sowie der professionellen Beziehungs- und Bindungsfähigkeit.

So kommt der Beraterin oder dem Berater in einem solchen Beratungsverständnis eine besondere Rolle zu, da er oder sie nicht die von Ribolits kritisierte non-direktive Haltung einnimmt, sondern Wissen und Haltung vermittelt. Sie oder er stellt Normen und Werte zur Diskussion und regt dazu an, in der Auseinandersetzung mit sich selbst eigene Normen und Werte zu entwickeln. Hierzu bedarf es - erstens - einer professionellen Beziehung, die tatsächlich mit dem oder der Ratsuchenden in Kontakt geht, eine präsente, tragfähige Bindung aufbaut sowie Differenzen in den Blick nimmt. Das beinhaltet - zweitens - auch das eigene Eingebunden-sein in gesellschaftliche 
Mechanismen zu thematisieren und zwar im unauflösbaren Widerspruch von Freiheit und Herrschaft (vgl. Euler 2003, S. 416). Neben der Förderung der Selbstverantwortung des oder der Ratsuchenden gilt es auch, die Erfahrungen der Klient/innen anzuerkennen, mitunter Verantwortung für sie zu übernehmen und ihrem Leid eine (öffentliche) Stimme zu geben, um auf strukturelle Defizite aufmerksam zu machen. Das bedeutet ebenso eine progressive Responsibilisierung zu thematisieren, die die Folgen wachsende Ökonomisierung oder gar Streichung öffentlicher Fürsorge in die Eigenverantwortung der Einzelnen legt.

Kritisch-emanzipatorische Beratung wird möglich, wenn ihre eigene Widersprüchlichkeit in Form ihrer Funktionalisierung und Reproduktion von gesellschaftlichen Verhältnissen bei gleichzeitigem Potenzial zu deren Überschreitung sichtbar wird (vgl. Bünger 2009, S. 182).

Eine kritisch-emanzipativ verstandene Beratung knüpft in ihrem Selbstverständnis aber nicht nur an ein kritisch verstandenes Bildungsverständnis an und reflektiert sich selbstkritisch, sondern verweist in sich auch auf ein politisches Selbstverständnis:

„Eine Theorie der politischen Bildung ist nicht neutral. Sie nimmt Partei, und zwar notwendigerweise. Der Gesichtspunkt, unter dem sie ihre Gegenstände zu beschreiben und $\mathrm{zu}$ analysieren sucht, ist politisch-gesellschaftlich nicht exterritorial, sondern ist selbst, auf die eine oder andere Weise, eine Position im Zusammenhang vieler politischer Interessen." (Mollenhauer 1973, S. 151)

Mit Blick auf die aktuellen Professionalisierungsbestrebungen im Kontext der Bildungsberatung zeigen sich Anknüpfungspunkte einer kritisch-emanzipativ verstandenen Beratung sowohl auf disziplinärer und organisationaler Ebene als auch im interaktionalen Beratungshandeln. So gilt es immer wieder zu fragen, wie beispielsweise Erfordernisse von sozialer Teilhabe als Mandat für die Ratsuchenden platziert werden können, wo und in welchem Maße Ökonomisierungszwänge das organisationale und beraterische Handeln beeinflussen oder wie Forderungen nach Emanzipation, Solidarität oder Partizipation pädagogisch gefüllt werden müssen. Umso wichtiger ist - wie das präsentierte LOB Projekt der Universität Mainz exemplarisch zeigt - der Austausch in der Aus-/Weiterbildung der Berater/innen, gerade auch zu Fragen im beraterischen Handeln. Diese thematisieren Widersprüche zwischen institutionellem Vorgaben und eigenem Beratungsprofil oder das Entwickeln einer biographischen Beratungshaltung, die auch Fragen fachlicher Autorität im Sinne von Erich Ribolits beinhalten oder das eigene Beratungshandeln im Kontext politischer Bildung verorten.

Dazu gehört sodann - in Anlehnung an Foucault - den Blick immer wieder auf das Eigene, den eigenen Raum zu lenken. Er nämlich erinnert daran, dass Macht immer die Gegen-Macht, die Möglichkeit zum Widerstand zur 
Seite steht. Allerdings liegt dieser Widerstand nicht in einem machtfreien Raum, einem „Jenseits“ der Macht (vgl. Foucault 1977, S. 113ff.), sondern ist - auch - Teil desselben.

\section{Literatur}

Arnold, Salome (2017): Pädagogische Beratung zwischen Ökonomisierung und Emanzipation. Ludwigsburg (unveröffentlichte Bachelorarbeit).

Bernhard, Armin (2001): Bildung und Erziehung: Grundlagen emanzipativer Subjektwerdung. Beiträge zur kritischen Bildungstheorie und Pädagogik. Kiel.

Böhnisch, Lothar \& Lösch, Hans (1973): Das Handlungsverständnis des Sozialarbeiters und seine institutionelle Determination. In: Otto, Hans-Uwe/Schneider, S. (Hg.): Gesellschaftliche Perspektiven der Sozialarbeit, Bd. 2: Neuwied/Berlin, S. 21-40.

Bröckling Ulrich (2007): Das unternehmerische Selbst. Soziologie einer Subjektivierungsform. Frankfurt a. M.

Brown, Wendy (2015): Die schleichende Revolution: Wie der Neoliberalismus die Demokratie zerstört. Berlin.

Bünger, Carsten/Euler, Peter/Gruschka, Andreas \& Pongratz, Ludwig A. (Hg.) (2009): Heydorn lesen! Herausforderungen kritischer Bildungstheorie. Paderborn.

Deleuze, Gilles (1993): Postskriptum über die Kontrollgesellschaften. In: Ders.: Unterhandlungen 1972-1990, Frankfurt a. M., S. 254-262.

Dewe, Bernd/Schwarz, Martin P. (2011): Beraten als professionelle Handlung und pädagogisches Phänomen. Hamburg.

Drees, Gerhard (2017): Die Sache mit der „List der Vernunft“ - Heinz-Joachim Heydorns Transformationsperspektive und (Erwachsenen)Bildung in der neoliberalen Gesellschaft. In: Ders./Nierobisch, Kira (Hg.): Bildung und gesellschaftliche Transformation. Analysen, Perspektiven, Aktion. Baltmannsweiler, S. 105-158.

Duttweiler, Stefanie/ Gugutzer, Robert / Passoth Jan-Hendrik \& Strübing, Jörg (2016): Leben nach Zahlen: Self-Tracking als Optimierungsprojekt? Bielefeld.

Duttweiler, Stefanie (2007): Beratung als Ort neoliberaler Subjektivierung. In: Anhorn, Roland/Bettinger, Frank \& Stehr, Johannes (Hg.): Foucaults Machtanalytik und Soziale Arbeit. Eine kritische Einführung und Bestandsaufnahme. Wiesbaden, S. 261-275.

Duttweiler, Stefanie (2004): Beratung. In: Bröckling, Ulrich/Krasmann, Susanne/ Lemke, Thomas (Hg.): Glossar der Gegenwart, Frankfurt a. M., S. 23-29.

Euler, Peter (2003): Bildung als „kritische Kategorie“. In: Zeitschrift für Pädagogik. 49 (2003) 3, S. 413-421.

Foucault, Michel (1977): Sexualität und Wahrheit. Bd. 1: Der Wille zum Wissen. Frankfurt a. M.

Käpplinger, B. \& Klein, R. (2013). Beratung bei Weiterbildungsgutscheinen. Zwischen Prüfung, Information und Entscheidungshilfe. In: Käpplinger, B./ Klein, R. \& Haberzeth, E. (Hg.): Weiterbildungsgutscheine. Wirkungen eines Finanzierungsmodells in vier europäischen Ländern. Bielefeld, S. 327-346. 
Ludwig, Joachim (2014): Zur rekonstruktiven Handlungslogik professioneller pädagogischer Beratung. In: Schwarz, Martin P./Ferchhoff, W. \& Vollbrecht, R. (Hg.): Professionalität: Wissen - Kontext. Sozialwissenschaftliche Analysen und pädagogische Reflexionen zur Struktur bildenden und beratenden Handelns. Bad Heilbrunn, S. 550-589.

Ludwig, Joachim (2015): Beratung vom Subjektstandpunkt. In: Allespach, Martin \& Held, Josef (Hg.): Handbuch Subjektwissenschaft. Ein emanzipatorischer Ansatz in Forschung und Praxis, Frankfurt a. M., S. 293-313.

Mämecke Thorben (2016): Die Statistik des Selbst - Zur Gouvernementalität der (Selbst)Verdatung. In: Selke, Stefan (Hg.): Lifelogging zwischen disruptiver Technologie und kulturellem Wandel: Wiesbaden, S. 97-126.

Maier-Gutheil, Cornelia (2015): Beraten. Stuttgart.

Mollenhauer, Klaus (1973): Erziehung und Emanzipation. Polemische Skizzen, München.

Mollenhauer, Klaus (1965): Das pädagogische Phänomen „Beratung“. In: Ders. \& Müller, C. Wolfgang: „Führung“ und „Beratung“ in pädagogischer Sicht. Heidelberg, S. 25-41.

Negt, Oskar (2011): Der politische Mensch. Demokratie als Lebensform. Göttingen.

Nierobisch, Kira (2017): Explosive Bindungen und Nicht-Identitäten - Konstruktionen des Selbst in der späten Moderne. In: Drees, Gerhard/Dies. (Hg.): Bildung und gesellschaftliche Transformation. Analysen, Perspektiven, Aktion. Baltmannsweiler, S. 187-201.

Nierobisch, Kira \& Schüßler, Ingeborg (Hg.) (2018): Hochschulberatung im Spannungsfeld gesellschaftlicher und bildungspolitischer Entwicklungen. Baltmannweiler.

Pongratz, Ludwig (2005): Subjektivität und Gouvernementalität. In: Hafeneger, Benno (Hg.): Subjektdiagnosen. Subjekt, Modernisierung und Bildung. Schwalbach/ Ts., S. 25-38.

Rauschenbach, Thomas (1994): Inszenierte Solidarität: Soziale Arbeit in der Risikogesellschaft. In: Beck, Ulrich \& Beck-Gernsheim, Elisabeth (Hg.): Riskante Freiheiten: Individualisierung in modernen Gesellschaften. Frankfurt a. M., S. 89-111. URL: https://www.ssoar.info/ssoar/bitstream/handle/document/3758/ ssoar-1994-rauschenbach-inszenierte_solidaritat_soziale_arbeit_in.pdf?sequen$\mathrm{ce}=1[02.02 .2018]$

Ribolits, Erich (2010): Führe mich sanft. Beratung, Coaching \& Co - die postmodernen Instrumente der Gouvernementalität. In: Ders.: Bildung ohne Wert. Wider die Humankapitalisierung des Menschen. Wien, S. 205-218.

Ribolits, Erich (2007): Lifelong guidance. Die sanfte Art, Menschen zum Funktionieren zu bringen. In: Christof, Eveline/Ribolits, Erich \& Zuber, Johannes (Hg.): Führe mich sanft. Beratung, Coaching \& Co. - Die postmodernen Instrumente der Gouvernementalität. Schulheft 127/2007. Wien, S. 18-29.

Selke, Stefan (Hg.) (2016): Lifelogging zwischen disruptiver Technologie und kulturellem Wandel: Wiesbaden.

Selke, Stefan (2014): Lifelogging: Wie die digitale Selbstvermessung unsere Gesellschaft verändert. Berlin.

Steiner, Petra (2007): Schöne neue Beratungswelt? Der Beratungstrend als Ausdruck von Individualisierung und Ökonomisierung. In: Christof, Eveline/Ribolits, 
Erich \& Zuber, Johannes (Hg.): Führe mich sanft. Beratung, Coaching \& Co. - Die postmodernen Instrumente der Gouvernementalität. Schulheft 127/2007, Wien, S. 30-38.

Weber, Chris (2018): Selbst- und Fremdbestimmung als Reflexion von Hochschulberatung. In: Nierobisch, Kira \& Schüßler, Ingeborg (Hg.): Hochschulberatung im Spannungsfeld gesellschaftlicher und bildungspolitischer Entwicklungen. Baltmannweiler, S. 73-92.

Weymann, Ulrike (2018): LOB: „Studierende professionell beraten“ - das Qualifizierungsprogramm für Studienfachberatende an der Johannes Gutenberg-Universität Mainz vermittelt Qualitätsstandards der Beratung an der Hochschule. In: Nierobisch, Kira \& Schüßler, Ingeborg (Hg.): Hochschulberatung im Spannungsfeld gesellschaftlicher und bildungspolitischer Entwicklungen. Baltmannweiler, S. 255-268. 



\section{Impact? Whose Impact? \\ A Youth Career Guidance Program's effects on Parents, Teachers and Staff}

\section{Introduction}

This article presents an alternative take on impact evaluation using the results of a transformative evaluation research project (Khalil and Weber, Forthcoming) for an educational career guidance (CG) program which has been implemented in Egypt. Analysing the program's impact from a discourse perspective supports a knowledge-based approach. In this power-knowledge based perspective, impact is regarded as traveling into the self ${ }^{1}$ not only of the program clients (participating students) but as changing the views of parents, teachers and program staff. In this way, impact evaluation becomes a polyphonic, multi-stakeholder impact evaluation and at the same time creates a systemic view, not only of the program outcome, but of its impact and potential. Here, we can see that the analysed CG program not only makes globalized ideas travel to Egypt, but enter the bodies and minds of clients and stakeholders. Program impact seen from a discourse analysis perspective reveals the power of knowledge-changing educational relationships.

In the coming chapter I will discuss impact evaluation and offer an overview on the current debate and, very briefly, the evaluation approach based on the methodological framework of transformative evaluation (Weber 2012) will be presented. What is the resonance and program impact for parents, teachers and program staff? From a Foucauldian perspective the 'gaze' is a crucial, micrological power-knowledge practice: So how is the stakeholders' gaze on students changing and changed by and within the program (Chapter 3)? The empirical section presents the voices of parents, teachers and project staff and focuses on their gaze upon the student and how it transforms within the project. The last chapter discusses the relevance of transforming educational relationships and gives a final outlook relating to program rationale and impact.

1 Impact as traveling into the self is based on Foucault's interest in subjectivity, seeking to understand the power relations which form us as subjects and the strategies by which we might seek to transform the power relations to which we are subject and thus transform ourselves as subjects. The program here enters the level of subjectification (Foucault 1971) and brings about transformation for all stakeholders addressed in this research. 


\section{A Discourse Perspective on Program Impact - Which Impact?}

Within the global trend towards 'evidence', an objectivist paradigm of empirical assessment based on large data sets is promoted, required and requested, especially by supra-state organizations to determine what works in order to create change (Hearn and Buffardi 2016). According to this view, impact evaluation should focus on the efficiency of programs and policies and not on outputs. When defining 'impact evaluation' we see that it varies amongst supra-state organizations. For the Organization for Economic Cooperation and Development (OECD), impact is defined as "Positive and negative, primary and secondary long-term effects produced by a development intervention, directly or indirectly, intended or unintended" (OECD/DAC 2002:24). For the World Bank "an impact evaluation assesses the changes in the well-being of individuals that can be attributed to a particular project, program, or policy. This focus on attribution is the hallmark of impact evaluations" (Gertler et al. 2011:4).

We also find debates among different groups: While some advocate quantitative large scale evidence based approaches assuming that impact is measurable up to cost-benefit analysis (White 2010), Norgbey (2016) questions the idea that quantitative approaches are suitable to reveal deeper dimensions of 'impact'. Constructivist paradigms advocate and assume that 'impact' as measurable in numbers and as a measurement of what was intended and what was achieved, is not enough.

They are contrasted by participatory and dialogical explorations of rationalities, changing patterns of perceptions and interpretations, changing practice and especially changing social relations. By asking in which way 'the gaze' of others changes, program outcomes and impact are analysed. Impact is analysed with regard to the rationalities being brought into existence. In this way, not only the impact and potential for social change at the program level is analysed, but policy level and the potential for social innovation, too, are addressed (Peters, Weber and Britez 2010).

Asking for program impact in the field of a CG program implemented in Egypt, we start with theorizing program knowledge as power-knowledge in a Foucauldian understanding of the concept (Foucault 1971), where the globally relevant knowledge of CG travels into the self (Czarniawska and Sevón 2005; Khalil, Forthcoming (a)). In the context of CG programs in the European Union, Schröder and Karl (2017) see the shift towards economization of the self (Jacquin and Juhel 2017; Sampson et al. 2014; Schröder and Karl 2017). Sultana (2011) problematizes these effects in the case of Egypt. So how does CG 'travel' into Egypt (Czarniawska and Sevón 2005)? And what is the impact of this travelling program knowledge? Will a program, which as such is already 
oriented towards the 'organizational' and towards learning, bring about transformational change not only at the level of students, but at the level of stakeholders, and powerholders like parents, teachers and staff as well? What will be the impact of the program regarding a changing 'gaze' on self and others?

\section{Program, Research Methodology and Program Evaluation Design}

EPP GIZ ${ }^{2}$ introduced an educational program to the Technical Education Sector at the Ministry of Education in Egypt that is grounded in a LifeWork planning approach (Bolles 1998). This resource-oriented, vision- and strength-oriented, individualizing approach was founded in the United States of America and relates not only to CG in a work setting, but as well as and primarily to life. This means that the dimensions of 'life', 'work' and 'planning' are crucial here. As Khalil (2015) explained, the approach goes much beyond a simple pairing/matching process. It rather focuses on facilitating the transition from school to work through a comprehensive process that includes the provision of services. The overall goal of this approach and the program is to enable young people to make work-related decisions based on information and by achieving self-realisation. The approach focuses on the discovery of abilities and strengths, on the analysis of interests, on the recognition of self-awareness, information strategies, employment opportunities and the transition from school to work. Again as discussed in Khalil (2015) the program trained teachers to become facilitators for three years, and teachers go through a learning phase where they refrain from using conventional (traditional) approaches to adopt an interactive style to support clients (15-18 years old students) for the promotion of learning. Not only that, it makes use of the existing experiences of facilitators and builds their capacity to be multipliers to support the diffusion of educational innovations. As a client and resource-centered program, it brings about interactive methods, real-life experience, outreach in regional neighbourhoods and firms, involving family members and potential training locations (Khalil 2015).

2 The Employment Promotion Program, Phase I (EPP I) has been implemented by GIZ on behalf of the Federal Ministry for Economic Cooperation and Development (BMZ) since 2011 in cooperation with the MoE. The program was initiated as a pilot in two governorates - one urban and one rural - in Egypt. 


\section{Transformative Evaluation}

The study is based on the "transformative evaluation" approach (Khalil and Weber Forthcoming; Mertens 2007; Mertens 2012; Weber 2012) which is understood as an inclusive, bottom-up process that creates change by engaging people actively and respectfully with special attention to local contexts and local meaning. The multi-method approach adopted intends to overcome social background differences by focusing on inclusion and takes into account possible limitations of expression. The image-based methodology used (Weber 2015) intends to elicit comments and to support elicitation, articulation and storytelling (Bignante 2010; Hurworth and Sweeney 1995). Applying the core structure of the 'Appreciative Inquiry' (AI) - that is, experience and storytelling / dream / design / delivery - (Cooperrider et al. 2000; Cooperrider and Whitney 1999; Martinetz 2002; Weber 2014a; Weber 2014 b), people were asked to relate important experiences gained throughout the program. Image-based peer interviews supported the combined analysis of images and narration focusing on core metaphors.

\section{Research Methods and Sample}

The multi-stakeholder impact evaluation (Gertler et al. 2016) addressed students, parents, teachers and staff by using participatory Design Research Workshops (teachers), group discussions (GD) (parents) (Bohnsack and Schäffer 2001; Gugglberger et al. 2014) and interviews (staff) (Peräkylä 2005). In general, all approaches intended to reveal narrations of experiences within the program and especially transformational experiences occurring within the program.

\section{Data Analysis}

Within an international and intercultural context, a translational approach has to be taken, in order to connect empirical worlds and research worlds. As 'voicing' and participatory bottom-up articulation required the use of the Arabic language, English translations were needed in order to reconnect and analyse the data within the research group and to allow intersubjectivity and intersubjective validation.

The data analysis (Bourdieu 2007), further discussed in Khalil and Weber (Forthcoming), related to the 'program trajectories' becoming visible in the temporal structure of three core questions commensurate to the 'now', the 'beginning' and the 'desired future'. The analysis took into account the temporal narrations brought about. The data analysis referred to transformational patterns in relation to the self, to institutions and to society. The project data was analysed as a collective trajectory of cohorts of students, having been presented elsewhere (Khalil, Forthcoming (b); Khalil and Weber, Forthcoming). 


\section{Program impact on Parents, Teachers and Staff}

Analysing the EPP GIZ program being implemented in Egypt, elsewhere (Khalil, Forthcoming (b)) we have explained the impact on students participating in the program. ${ }^{3}$ This analysis showed that the program was perceived by students as a transformational educational experience as it helped them become active participants rather than being passive recipients. It also helped students speak about their wishes for the future which contributes to bringing about change. Here we see the impact of the program on students goes deeper as it not only focuses on the program output but on bringing about change, as evidenced by the students themselves. So what is the 'gaze' of the parents, teachers and staff regarding the transformative impact of the program?

The following sections will present the 'gaze' of parents, teachers and staff with regard to how the program transforms educational relationships. The impact evaluation is presented based on three main transformational categories: the personal level, the social level and on the level of family/work relations.

\section{Parents' perspectives on program impact}

Within the group discussions parents relate whether they have noticed changes in their children as they grow up in the program, and what their child said about his/her experiences.

On the personal level, transformative learning and self-development is a strong dimension found in the parents' comments which connects to self-confidence. One parent says "I rediscovered my daughter through the program, she used to be introvert, now she is able to stand and present her work to others, talking about herself... I was surprised". Others say "my son obtained self-confidence", "my son is now able to enter any company to ask about available opportunities", "the experience in the program changed him totally, it changed the way he thinks, and even now he likes to study and to show his strength".

On the level of transformation of social and family relations, parents observed that their children changed: "I felt that this brought change $100 \%$ in my daughter, how she treats us at home, how she treats our neighbours".

And on the level of skills and capabilities the parents noticed that their children learned "how to work with others", "how to do an interview", "She knows how to look for a job".

Finally, on the level of changing institutional relations they say "Someone who found what was lost... The way he thinks changed and now he likes to study", "I am surprised my son got very good grades now and will enter university".

3 Results related to students are discussed in S. Khalil. (Forthcoming): "Imagining Social Innovation: From Individual Career Visions to Multi-Stakeholder Development Strategies”.Weber, Schröder et al. (Ed.). Organisation und Netzwerke. Wiesbaden: Springer VS. 
Parents agree that they themselves like the program and wanted to have a role and learn more about it, and also said how their children became happy, involved, benefited from and liked the program. Knowing about the program from their children and seeing the transformation taking place, most of the parents encouraged them to take part: "the program is good, if there is more take part in it as you will benefit" one parent said. On the other hand, other parents would like to have a bigger role and know more; they say that "we need to know more... I suggest that we receive training to be informed and be able to help our kids at home".

It is obvious that the parents noticed the program's impact on their children and they have observed their development at home.

\section{Teachers' perspectives on program impact}

The assessment of program outcomes shows that facilitators' learning levels are very much oriented towards the transformation of the self. Adopting the image-based approach in this section of the semi-structured interviews, facilitators told each other how they related to the images and what they wanted to express by them.

One of the strongest dimensions found in the comments connects to the discovery of oneself and one's abilities. They commented on how they discovered themselves after they took part in the program, and how this was of help to their personal and professional life. Some said: "I discovered my abilities", "things changed in my life", "the program introduced me to things I had and I didn't know about (I had a boom in my life)". In comparison to how they perceived themselves before the program they said: "I was a person without an objective", "my life was a mess and not planned", "I was lost", "I was a failure", "I didn't know my strength", "I am depressed and frustrated".

A second strongly represented dimension, is that of the facilitators' relation to the students. The facilitators saw themselves getting better on the relationship level with clients after having taken part in the program; they say that they want to "Help clients discover their strength" and that they "feel I can give a helping hand to the youth", "the program changed my relationship with clients to become personal (we tried to understand them)". Compared to this, the reference points before the program started were that "As a teacher I am used to passive teaching, just give, and throw the information", and teaching as well as relations with the children were "was routine".

At the level of skills, "imagination of their future" and "information literacy" seems to become relevant to them as well. Facilitators said the program helped them to "make use of the information I have". In contrast, before the program it was stated that they were "confused and unable to use the information I have", "I didn't understand what the meaning of life is", "I didn't know how to find a job", "I didn't know how to use my skills". 
A fourth dimension refers to societal and labour market capacities. Regarding social and work-life related capabilities, the program helped them learn participation: "I am a person who has social participation", "I am a person who helps others".

It is evident that the facilitators did have transformational learning experiences, which for many of them brought about self-awareness, awareness about their relations with their clients and information literacy.

We also find transformational learning related to others, to groups and to organizations. Here, learning outcomes are related to cooperation and team work: they "Learnt how to work as one team" whereas before the program they stated: "the way I dealt with others was not good enough", "I was a person whose role stopped at helping himself". As we can see, facilitators did have transformational learning experiences, which for many of them brought about teamwork related skills.

The transformation that came about among the facilitators, especially in their relation with students, was validated by clients seeing them as an older brother who listens to them, helps them and cooperates with them. The EPP staff members also commented on the relation between the facilitators and clients, and how this was discussed by the facilitators who informed the staff how different they were when they were wearing the hat of the facilitator.

\section{GIZ Project Staff perspectives on the program - and impact}

Staff were interviewed using semi-structured qualitative interviews. The purpose of the interview was to receive multiple perceptions from them, in order to understand their analysis and narratives and how they perceived that the program developed and emerged, to change and to bring about outcomes over time.

Staff were asked about the ways in which the program could become better and possibly even might become a policy in education. Specifically, they were asked which parts of the program should be maintained, which aspects could be strengthened and which should be changed. The answers show that content and methods have to adapt to conditions. One staff member said "I cannot make sure that things are going to be maintained" this is because by time new things are learned, the material and the approach will need to be developed further. However, the way the program is delivered in this perspective needs to be maintained.

On the level of adapting the material to the needs of the community, it needed to be modified to accommodate the different issues that were raised, such as gender: "the material was targeting male participants more than females, and in order to meet the needs of our clients, the material was adjusted, to consider what they lack". Another staff member added that as the "youth are opting to work abroad, the material should incorporate some information about this topic, in order to help them to be informed". 
Cooperating with different partners is seen as important - even if this might take time - but at least partners should be able to meet on common grounds, which the program managed to do. One of the staff members said, "We need to work in harmony".

Finally, the program staff saw the potential of making a bigger change when better conditions, more political support, and more involvement by more important stakeholders is achieved. As they see it, "policymakers could make a huge change happen by taking brave decisions and take this concept further into structural solutions and diffusion all over the country". The policymakers could develop strategy plans, implement activities to involve other partners and establish institutional structures.

\section{Impact as Transformation of Educational Relationships}

The CG objective is to empower technical education graduates by helping them discover who they are and what they can do to be able to manage their careers by making informed decisions. To be able to do this, the program needed to change the context in which education is taking place, create a new knowledge situation and change the relationship between students, teachers and peers, thus creating a network of relations in the classroom. It also involves parents, actors of civil society and relevant institutions like schools, firms and policymakers. All participants have a core role in creating knowledge by working in 'harmony' to transform society and shift vertical, frontal, teacher-centred approaches in order to build educational systems (Bastenhorst 2005).

From a discourse perspective, the 'gaze' of the different stakeholders involved here shows evidence that CG knowledge is 'travelling into the self'. The concept of travelling here does not refer to going outside the experiences of the students themselves, nor beyond the teachers, family members or places where they live. It is obvious that it is an enabling program where educational patterns change, self-concept changes and the relationship with the educational institution changes as well. We can also see that the program enters the level of subjectification (Foucault 1971) and brings about transformation not only at the level of the students but at the level of teachers, parents and staff as well.

CG here is an educational innovation in the sense that it is transforming educational relationships and shifting the vertical 'pyramid' of teachers' classroom power to the students and their social context. By supporting the self-realization of individuals and helping them voice their needs the program contributes to societal change and democratization (Foucault 1971; Khalil, 
Forthcoming (b)). It is therefore not only for purposes of economization, as discussed by Sultana (2011) and Schröder and Karl (2017).

\section{References}

Bastenhorst, Kai-Olaf. 2005. Die Sustainable University aus der Ressourcenperspektive: Der Sustainability Modus der Wissensproduktion und die nachhaltige Entwicklung der Ressource Wissen. Bd. 2. Hamburg: Lit.

Bignante, Elisa. 2010. "The Use of Photo-Elicitation in Field Research: Exploring Maasai representations and use of natural resources." EchoGéo (11).

Bohnsack, Ralf, and Burkhard Schäffer. 2001. "Gruppendiskussionsverfahren [Group discussion technique]." Pp. 324-41, in Wie kommt Wissenschaft zu Wissen? Band 2: Einführung in die Forschungsmethodik und Forschungspraxis [How does science get its knowledge? Part 2: Introduction into the method and practice of research], edited by T. Hug. Baltmannsweiler: Schneider-Verl. Hohengehren.

Bolles, Richard N. 1998. What Color Is Your Parachute Workbook: How to Create a Picture of Your Ideal Job or Next Career. Berkeley, Calif.: Ten Speed.

Bourdieu, Pierre. 2007. Distinction: A social critique of the judgement of taste. Cambridge, Mass.: Harvard University Press.

Cooperrider, David L., and Diana Whitney. 1999. "Appreciative Inquiry: A Positive Revolution in Change." Pp. 245-61, in The Change Handbook, edited by P. Holman and T. Devane. Oakland, Calif.: Berrett-Koehler Publishers

Cooperrider, David L., Peter F. Sorensen, Diana Whitney, and Therese F. Yaeger, editors. 2000. Appreciative Inquiry: Rethinking Human Organization Toward a Positive Theory of Change. Illinois, USA: Stipes Publishing.

Czarniawska, Barbara, and Guje Sevón, editors. 2005. Advances in Organization Studies, vol. 51, Global Ideas: How Ideas, Objects, and Practices Travel in the Global Economy. Administrative Science Quarterly: Liber \& Copenhagen Business School Press.

Foucault, Michel. 1971. “Orders of Discourse.” Social Science Information 10(2):7-30.

Gertler, Paul J., Sebastian Martinez, Patrick Premand, Laura B. Rawlings, and Christel M. J. Vermeersch. 2011. Impact Evaluation in Practice: The World Bank.

Gertler, Paul J., Sebastian Martinez, Patrick Premand, Laura B. Rawlings, and Christel M. J. Vermeersch. 2016. Impact Evaluation in Practice, Second Edition: The World Bank.

Gugglberger, Lisa, Michaela Adamowitsch, Friedrich Teutsch, Rosemarie Felder-Puig, and Wolfgang Dür. 2014. "The Use of Group Discussions: a case study of learning about organisational characteristics of schools." International Journal of Social Research Methodology 18(2):127-43.

Hearn, Simon, and Anne L. Buffardi. 2016. What is Impact? A Methods Lab Publication. London: Overseas Development Institute

Hurworth, Rosalind, and Martin Sweeney. 1995. "The Use of the Visual Image in a Variety of Australian Evaluations.” Evaluation Practices 16(2):153-64. 
Jacquin, Philippe, and Jacques Juhel. 2017. “An Individual Mixed-Evaluation Method for Career Intervention.” The Career Development Quarterly 65(1):16-28.

Khalil, Shahinaz, and Susanne M. Weber. Forthcoming. "Bridging the Gap Between Program and Policy? Participatory Evaluation for Educational Innovations.", in Organization and Civil Society, edited by A. Schröer, M. Göhlichn, and S. M. Weber. Wiesbaden: Verlag für Sozialwissenschaften.

Khalil, Shahinaz. Forthcoming (a). "Career Guidance as Travelling Idea: Potentials for educational innovations in Egypt.", in Organization and Civil Society, edited by A. Schröer, M. Göhlichn, and S. M. Weber. Wiesbaden: Verlag für Sozialwissenschaften.

Khalil, Shahinaz. Forthcoming (b). "Imagining Social Innovation: From individual Career Visions to Multi-Stakeholder-Development Strategies.”, in Organisation und Netzwerke, edited by S. M. Weber, C. Schröder, I. Truschkat, A. Herz, and L. Peters. Wiesbaden: Springer VS.

Khalil, Shahinaz. 2015. "Career Guidance as Educational Innovation: The Case Study of Egypt." Indian Journal of Career and Livelihood Planning 4(1):5-10

Martinetz, Charles F. 2002. "Appreciative Inquiry as an Organizational Development Tool." Performance Improvement 41(8):34-39.

Mertens, D. M. 2007. "Transformative Paradigm: Mixed Methods and Social Justice." Journal of Mixed Methods Research 1(3):212-25.

Mertens, D. M. 2012. "Transformative Mixed Methods: Addressing Inequities." American Behavioral Scientist 56(6):802-13.

Norgbey, Enyonam B. 2016. "Debate on the Appropriate Methods for Conducting Impact Evaluation of Programs within the Development Context." Journal of MultiDisciplinary Evaluation 12(27):58-66.

OECD/DAC. 2002. Glossary of Key Terms in Evaluation and Results Based Management. Paris: OECD/DAC.

Peräkylä, Anssi. 2005. "Analyzing Talk and Text." Pp. 869-86, in The SAGE handbook of qualitative research, edited by N. K. Denzin and Y. S. Lincoln. 3rd ed. Thousand Oaks: SAGE Publications.

Peters, M. A., Susanne M. Weber, and R. Britez. 2010. "Evaluating Education in Three Policy Eras." Elsevier Evaluation and Program Planning :645-52

Sampson, James P., et al.. 2014. "A Content Analysis of Career Development Theory, Research, and Practice.” The Career Development Quarterly 62(4):290-326.

Schröder, Christian, and Ute Karl. 2017. "On Being Guided - a typology of career guidance in EU discourses." British Journal of Guidance \& Counselling 45(3):356-65.

Weber, Susanne M. 2012. "Transformative Evaluation.” Pp. 120-41, in Erziehungswissenschaftliche Evaluationspraxis: Beispiele - Konzepte-Methoden, edited by U. Kuckartz and S. Rädiker. Wiesbaden.

Weber, Susanne M. 2014a. "Design (Research) Methodologies and Modes of Becoming. Large Group interventions as Practice if Relations, Narrations and Aesthetics." Creative Approaches to Research 7(1):92-116.

Weber, Susanne M. 2014b. "Who speaks? Power Knowledge and the Professional Field: A Discourse Analytical Perspective on Educational Policy Consultancy and Advice." Pp. 111-26, in Special Issue on Michael Peters' work, edited by G. Lazariou. New York: Addleton Academic Publishers. 
Weber, Susanne M. 2015. "Aesthetic Imagination, Organizational Transformation and Organization Research." Knowledge Cultures 3(3):7-18.

White, Howard. 2010. "A Contribution to Current Debates in Impact Evaluation." Evaluation 16(2):153-64. 



\section{Autorinnen und Autoren}

Chyle, Heike, Dipl.-Päd., Europa-Universität Flensburg, Internationales Institut für Management und ökonomische Bildung, Abteilung Wirtschaftswissenschaften und ihre Didaktik, Arbeitsschwerpunkte: Übergänge in Arbeit, Beratungsforschung, Pädagogische Professionsforschung, Qualitative Sozialforschung.

Damm, Christoph, M.A., Universität der Bundeswehr München, Arbeitsschwerpunkte: Organisationen der Erwachsenenbildung, Wissenschaftliche Weiterbildung, Kooperationen und Netzwerke, Qualitative Bildungsforschung, Anrechnungsforschung.

Dittrich, Christiane, M.A., Goethe-Universität Frankfurt am Main, Fachbereich Erziehungswissenschaften, Institut für Sozialpädagogik und Erwachsenenbildung, Arbeitsschwerpunkte: Übergänge in Arbeit, Jugendmobilität, (Übergangs)Beratung, Qualitative Forschungsmethoden.

Franz, Julia, Prof. Dr., Otto-Friedrich-Universität Bamberg, Professur für Erwachsenenbildung und Weiterbildung, Arbeitsschwerpunkte: Didaktik der Erwachsenenbildung; Intergenerationelles Lernen; Qualitative Lehr-Lernforschung; Erwachsenenpädagogische Organisationsforschung.

Hocke, Simone, Dr. (Dipl. Päd.), Zentrum für Arbeit und Politk der Universität Bremen, Betriebliche Interessenvertretung, Beratungsforschung, LehrLern-Forschung und Konfliktforschung.

Käpplinger, Bernd, Prof. Dr., Justus-Liebig-Universität Gießen, Arbeitsschwerpunkte: Bildungsberatung, Programmplanung, Betriebliche Weiterbildung, Zertifizierung und Internationale Weiterbildungsforschung.

Khalil, Shahinaz, Philipps-Universität Marburg; Arbeitsgruppe Future Education and Network Innovation, Arbeitsschwerpunkte: Berufsberatung für Jugendliche.

Koller, Katharina, M.A., Ostbayerische Technische Hochschule (OTH) Amberg-Weiden, Arbeitsschwerpunkte: wissenschaftliche Weiterbildung, Lebenslanges Lernen und Weiterbildung an Hochschulen, Beratungs- und Betreuungsstrukturen an Hochschulen (insb. für nicht-traditionell Studierende), Gender Mainstream und Diversity Management in der wissenschaftlichen Weiterbildung.

Kruse, Carolin, Dipl.-Päd., Westfälische Wilhelms-Universität Münster, Institut für Erziehungswissenschaft, AG Erwachsenenbildung/Weiterbildung, Arbeitsschwerpunkte: Außerschulische Jugendbildung, Bildungspotentiale 
von Freiwilligendiensten, Pädagogische Beratungsforschung, Systemische Beratung.

Lehner, Roland, Mag., LISA - Linzer Institut für soziale Analysen, Arbeitsschwerpunkte: Bildungs- und Berufsforschung; Evaluierungen in Bereichen der Gesundheitsförderung und Präventionsprogrammen; wissenschaftliche Begleitung von Beteiligungs- und Entwicklungsprojekten.

Macha, Hildegard, Prof. em. Dr., Universität Augsburg, Philosophisch-Sozialwissenschaftliche Fakultät, Arbeitsschwerpunkte: Genderforschung in Familie und Gesellschaft; Gleichstellungsforschung: Intervention mit Gleichstellung in Unternehmen; Weiterbildungsforschung; Familienforschung.

Muche, Claudia, Dr. phil., Universität Hildesheim, Institut für Sozial- und Organisationspädagogik, Soziale Dienste am Arbeitsmarkt und Übergänge in Arbeit, Teilhabe von Menschen mit Behinderungen, Organisation(en) Sozialer Arbeit, Organisationaler und institutioneller Wandel.

Maier-Gutheil, Cornelia, Prof. Dr. phil., Evangelische Hochschule Darmstadt, Arbeitsschwerpunkte: Beratungs- und Interaktionsforschung, Professionalisierung, Pädagogische Handlungsformen, Qualitative Forschungsmethodologie.

Nierobisch, Kira, Prof. Dr., Katholische Hochschule Mainz, Arbeitsschwerpunkte: Methoden der Sozialen Arbeit; (Bildungs)Beratung; Jugendarbeit; Identitätsbildung.

Pätzold, Henning, Prof. Dr., Universität Koblenz-Landau, Campus Koblenz, Fachbereich Bildungswissenschaften, Arbeitsschwerpunkte: Organisationspädagogik; Theorie und Empirie des Lernens Erwachsener; Pädagogik und Verantwortung; Systemdenken in organisationspädagogischen Kontexten.

Pelz, Robert, TU Dresden, Arbeitsschwerpunkte: Hochschul- und (Weiter-) Bildungsforschung, Forschung zum Studienerfolg, Absolventenforschung, Forschung zur sozialen Ungleichheit.

Rundnagel, Heike, Philipps-Universität Marburg, Arbeitsschwerpunkte: Wissenschaftliche Weiterbildung, Studiengangkoordination, Professionalisierung, Kooperation, Bildungsmanagement.

Schäfer-Hock, Christian, Dr. phil., TU Dresden, Arbeitsschwerpunkte: Medien- und Kommunikationswissenschaft, Hochschul- und Bildungsforschung, Forschung zum Studienerfolg.

Scheunpflug, Annette, Prof. Dr., Otto-Friedrich-Universität Bamberg, Lehrstuhl für Allgemeine Pädagogik, Arbeitsschwerpunkte: Bildungsqualität, 
Pädagogische Anthropologie, Weltbürgerliche Bildung und Bildung und Religion und Bildung und Religion.

Schiersmann, Christiane, Prof., Universität Heidelberg, Arbeitsschwerpunkte: Analyse und Gestaltung der Beratung von Personen, Teams und Organisationen, Strategien und Instrumente der Kompetenzerfassung, Qualitätsmanagement, Berufliche Weiterbildung.

Schmidtke, Birgit, Dr., Österreichisches Institut für Berufsbildungsforschung - öibf, Arbeitsschwerpunkte: Erwachsenenbildung, Beratungsforschung, Migration.

Schröder, Christian, Dr. phil., Hochschule für Technik und Wirtschaft des Saarlandes, Fakultät für Sozialwissenschaften, Arbeitsschwerpunkte: soziale Dienste, Übergänge und soziale Bewegungen in erziehungswissenschaftlicher Perspektive.

Schulze-Stocker, Franziska, Dr. phil., TU Dresden, Forschung zum Studienerfolg, Lehrerberuf und Lehrerbildung, Hochschulforschung, Schulentwicklungsforschung.

Stanik, Tim, Dr. phil., Universität Tübingen, Arbeitsschwerpunkte: Bildungsberatung, Lehr-Lernforschung, Professionalisierung, Grundbildung, Qualitative Forschungsmethodik.

Sturm, Nico, Dr., Philipps-Universität Marburg, Wissenschaftliche Weiterbildung, Anrechnung außerhochschulisch erworbener Kompetenzen, Durchlässigkeit im Bildungssystem, Beratungsstrukturen.

Wagner, Farina, Martin-Luther-Universität Halle-Wittenberg, Arbeitsschwerpunkte: Subjektivierungsprozesse, Weiterbildungsberatung, Empirie der Erwachsenenbildung/Weiterbildung, Politische Erwachsenenbildung.

Wahl, Johannes, Dr. phil., Goethe-Universität Frankfurt, Institut für Sozialpädagogik und Erwachsenenbildung, Arbeitsschwerpunkte: Komparative pädagogische Berufsgruppenforschung, Methoden der empirischen Sozialforschung, Medienpädagogik, lebenslanges Lernen.

Weber, Peter C., Prof., Hochschule der Bundesagentur für Arbeit, Mannheim, Arbeitsschwerpunkte: Bildungs- und Berufsberatung, Kompetenzentwicklung und Professionalisierung, Qualitätsentwicklung und Organisationsentwicklung in Bildungs- und Beratungskontexten.

Weinhardt, Marc, Prof. Dr., EH Darmstadt, School of Professional Studies, Arbeitsschwerpunkte: Professionalisierungsforschung, Psychosoziale Beratung, Digitalisierung Sozialer Arbeit, Lehren, Lernen und Forschen in Simulationsumgebungen. 
Welser, Stephanie, Dr., Otto-Friedrich-Universität Bamberg, Professur für Erwachsenenbildung und Weiterbildung, Arbeitsschwerpunkte: Professionstheoretische Fragestellungen; Methodologie und Methodik rekonstruktiver Sozialforschung; Ästhetische Bildung und Visualität in der Erwachsenenbildung.

Wienberg, Jana, Dr. phil., Universität Hamburg, Arbeitsbereich Berufliche Bildung und Lebenslanges Lernen, Arbeitsschwerpunkte: Resonanz im Weiterbildungsgeschehen, Beschleunigung und zeitdiagnostische Themen, Lernen in digitalen Umwelten, Bildung im Alter, Berufliche Erwachsenenbildung/Weiterbildung.

Wlassow, Nina, M.A., Universität Tübingen, Institut für Erziehungswissenschaft, Abteilung Sozialpädagogik, Arbeitsschwerpunkte: Übergänge in Arbeit, Hilfen zur Erziehung, Beteiligung und Beschwerde, Careleaver.

Zick, Sebastian, Dipl. Päd., Europa-Universität Flensburg, Arbeitsschwerpunkte: Bildungstheorie, Europäisierungsprozesse von Erwachsenenbildung, Mobilitätspädagogiken und -politiken. 